\title{
An introduction to the theory of nuclear neutron scattering in condensed matter
}

\author{
Helmut Schober \\ Institut Laue Langevin, 71 avenue des Martyrs, 38042 Grenoble Cedex 96, France \\ and \\ Université Joseph Fourier, UFR de Physique, 38041 Grenoble Cedex 9, France \\ E-mail: schober@ill.fr
}

\begin{abstract}
We give an introduction into the theory of neutron scattering in condensed matter with a strong focus on inelastic scattering. Magnetic scattering and polarisation will be excluded from the discussion. The scattering of non-relativistic particles in a potential leads to the concepts of scattering amplitude, partial waves, scattering length, and the Born series. In order to illustrate the formalism with simple examples we allow ourselves a short detour to diffraction. The Born approximation is then augmented to include inelastic scattering from compounds with internal degrees of freedom. This leads to the master equation of neutron scattering that establishes a link between the experimental cross sections and quantum mechanical transition amplitudes. The master equation is reformulated in terms of coherent and incoherent scattering functions, which in turn are expressed in terms of density correlation functions. The abstract formalism is illustrated by explicitly calculating the cross sections for some simple model systems. Considerable attention is given to the scattering involving vibrations in harmonic systems. Explicit expressions are derived that couple inelastic scattering cross sections to the phonon density of states and dispersion relations. We will finish the inelastic part by discussing the effect of multi-phonons and conclude with some remarks concerning the identification of anharmonic effects. The last section is devoted to putting the formalism of scattering theory into the context of a statistical description of the particle beam.
\end{abstract}

Keywords: Non-relativistic scattering theory, elastic and inelastic nuclear neutron scattering, correlation functions, lattice dynamics

\section{Contents}

1. Introduction .......... 111

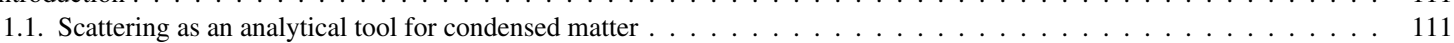

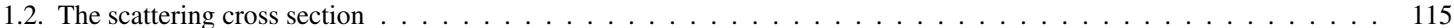

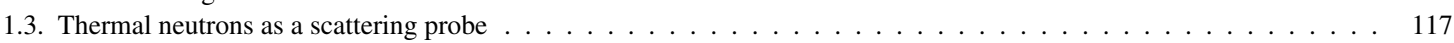

1.4. Particles described by wave packets . . . . . . . . . . . . . . . . . . . . . . . . . 120

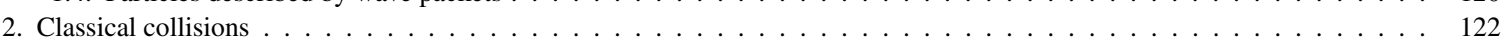

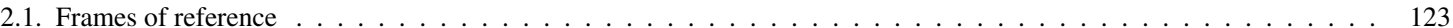

2.2. Elastic and inelastic collisions . . . . . . . . . . . . . . . . . . . . . . . . . . . . . . . . . . 124

2.3. Central potential and impact parameter . . . . . . . . . . . . . . . . . . . . . . . 124

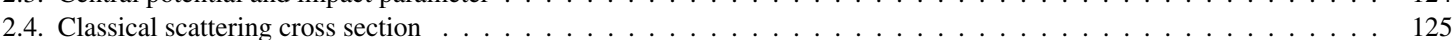

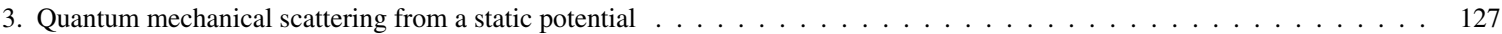

3.1. The wave function in the asymptotic regime . . . . . . . . . . . . . . . . . . . . . . . . . . 128

3.2. Reflection and transmission at a potential barrier as an illustrative example . . . . . . . . . . . . . . . 131

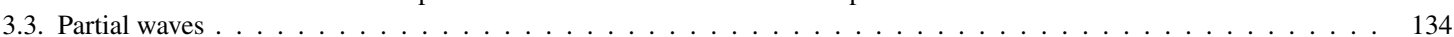

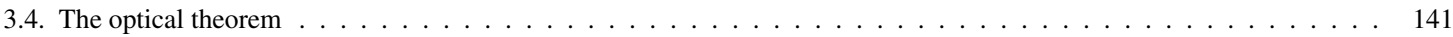

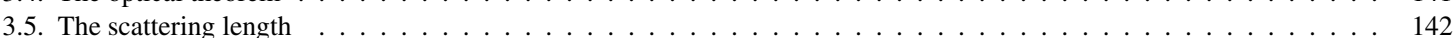

3.6. Model potentials . . . . . . . . . . . . . . . . . . . . . . . . . . . . . . . 144

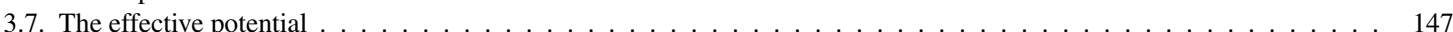

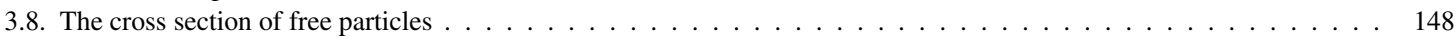

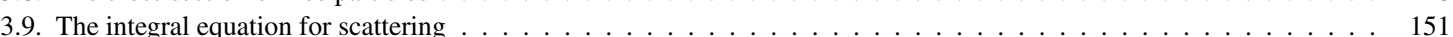

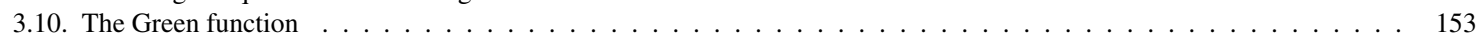

$1023-8166 / 14 / \$ 27.50$ C 2014 - IOS Press and the authors.

This article is published online with Open Access and distributed under the terms of the Creative Commons Attribution Non-Commercial License. 
3.11. The Born series and the Born approximation $\ldots \ldots \ldots \ldots \ldots \ldots \ldots \ldots \ldots \ldots \ldots \ldots \ldots$

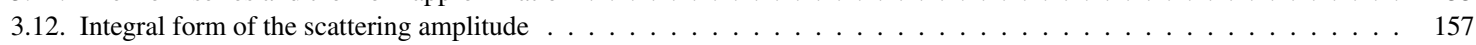

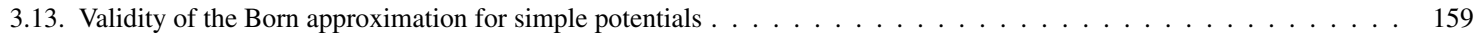

3.14. A word concerning scattering of electro-magnetic radiation $\ldots \ldots \ldots \ldots \ldots \ldots \ldots \ldots$

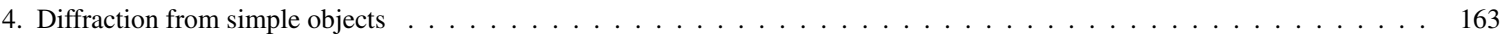

4.1. The Huygens-Fresnel principle . . . . . . . . . . . . . . . . . . . . . . . . . . . . . . . . 164

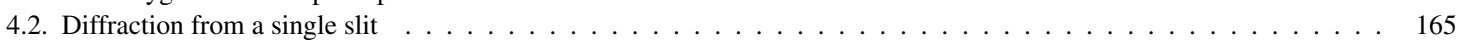

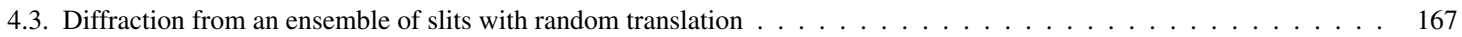

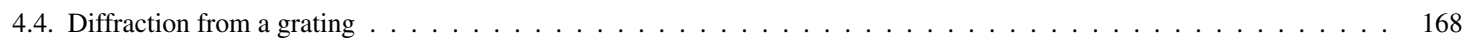

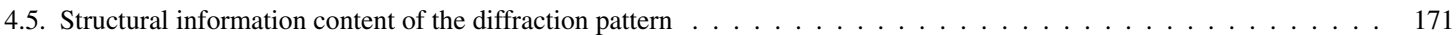

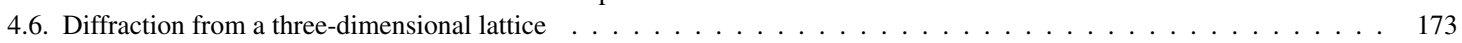

4.7. Longitudinal coherence length . . . . . . . . . . . . . . . . . . . . . . . . . . . . . . . . . 174

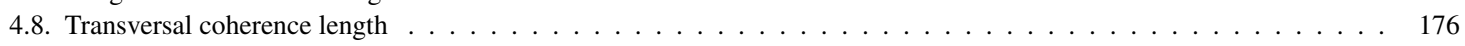

4.9. Coherence volume . . . . . . . . . . . . . . . . . . . . . . . . . . . . . . . . . . . . . . . 177

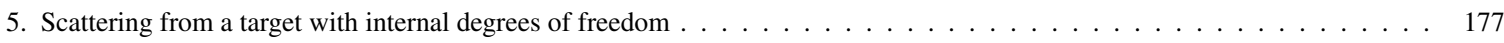

5.1. Wave functions in common Hilbert space . . . . . . . . . . . . . . . . . . . . . . . . . . 178

5.2. Green function of the combined system . . . . . . . . . . . . . . . . . . . . . . . 179

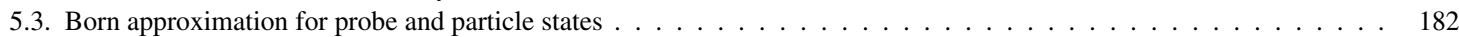

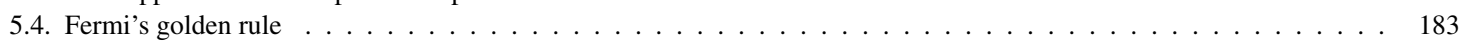

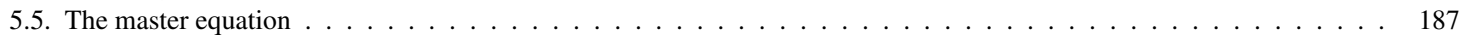

5.6. Statistical density operator and quantum state of the probe particle $\ldots \ldots \ldots \ldots \ldots \ldots \ldots$

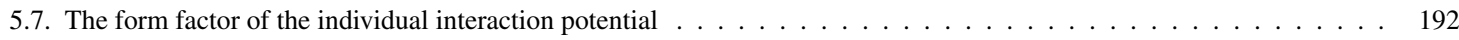

5.8. The Fermi pseudo-potential . . . . . . . . . . . . . . . . . . . . . . . . . . . . . . . . . . . 194

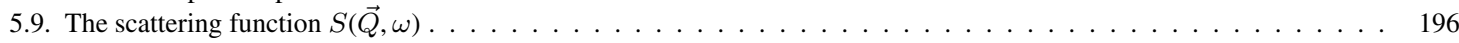

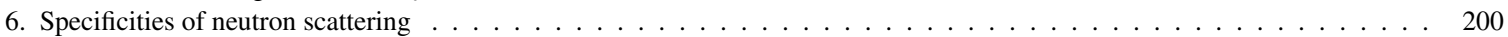

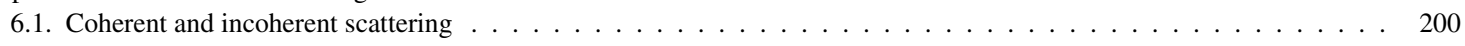

6.2. The dependence of the scattering lengths on the nuclear spin _ . . . . . . . . . . . . . . . . . . . . . . 204

6.3. Neutron scattering from a proton or a deuteron $\ldots \ldots \ldots \ldots \ldots \ldots \ldots \ldots \ldots \ldots \ldots$

6.4. The scattering triangle and the dynamic range . . . . . . . . . . . . . . . . . . . . . . . . . 207

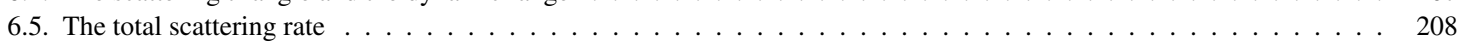

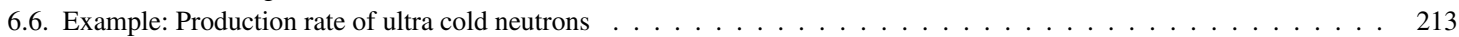

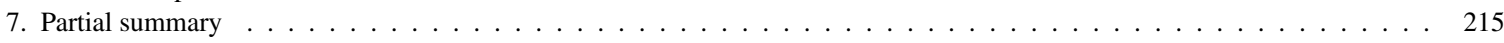

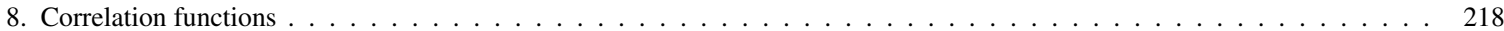

8.1. The autocorrelation function of the atomic density $G(\vec{r}, t) \ldots \ldots \ldots \ldots \ldots \ldots \ldots \ldots \ldots \ldots$

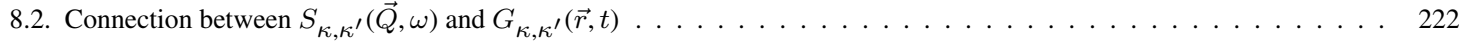

8.3. The relation between the correlation function and the differential cross section . . . . . . . . . . . . . . 223

8.4. Contrast variation as a means of determining partial correlation functions . . . . . . . . . . . . . . . . . 224

8.5. Higher order correlation functions . . . . . . . . . . . . . . . . . . . . . . . . . . . . . . . 224

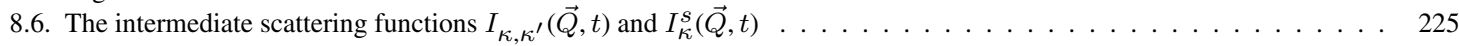

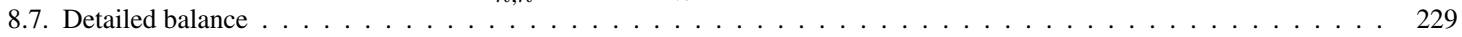

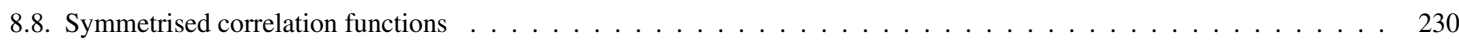

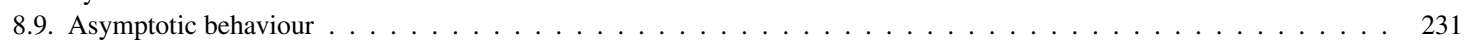

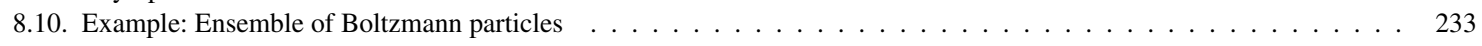

8.11. Response functions and susceptibility . . . . . . . . . . . . . . . . . . . . . . . . . . . . . . 242

8.12. Moments and sum rules for the scattering function $\ldots \ldots \ldots \ldots \ldots \ldots \ldots \ldots \ldots \ldots \ldots \ldots$

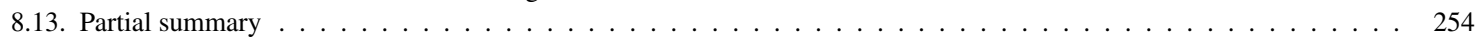

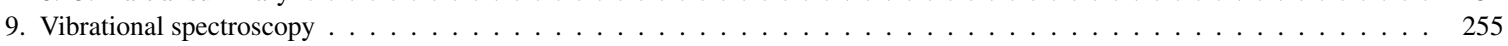

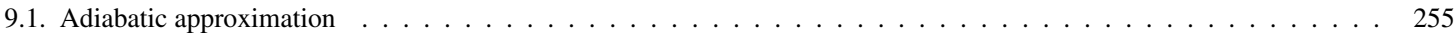

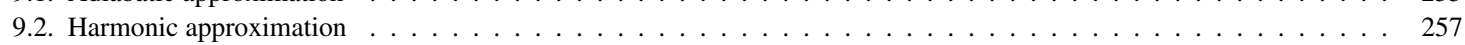

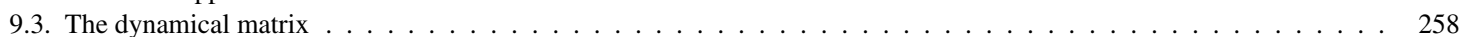

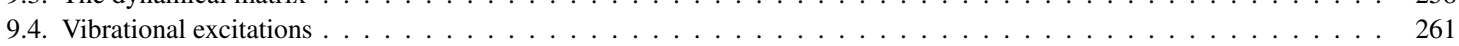

9.5. Lattice modes . . . . . . . . . . . . . . . . . . . . . . . . . . . . . . . . . 263

9.6. Dynamic structure factor $\ldots \ldots \ldots \ldots \ldots \ldots \ldots \ldots \ldots \ldots \ldots \ldots \ldots$

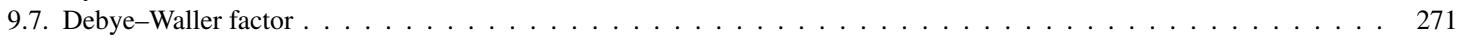

9.8. Coherent elastic scattering . . . . . . . . . . . . . . . . . . . . . . . . . . . . . . . 274

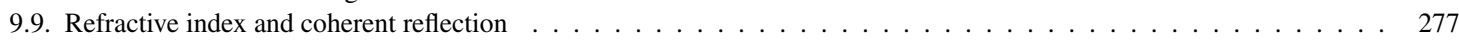

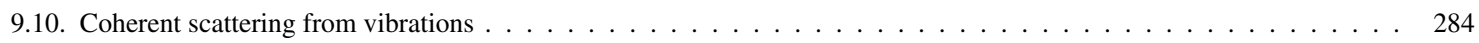

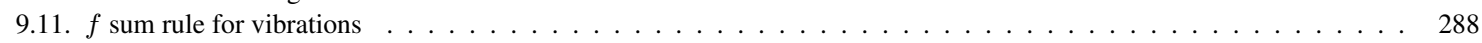


9.12. Coherent scattering of a phonon in a crystal . . . . . . . . . . . . . . . . . . . . . . . 289

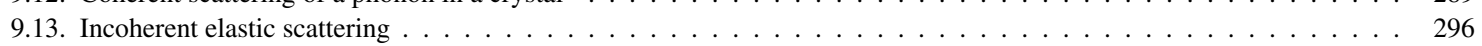

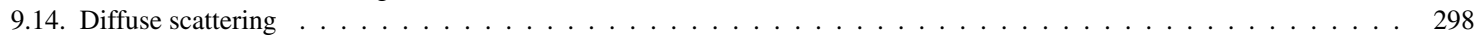

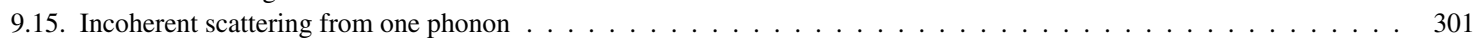

9.16. The incoherent approximation . . . . . . . . . . . . . . . . . . . . . . . . . . . . . . . . . . . . . .

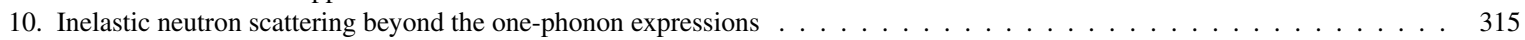

10.1. The integral version of the scattering function $\ldots \ldots \ldots$

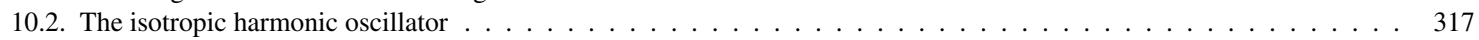

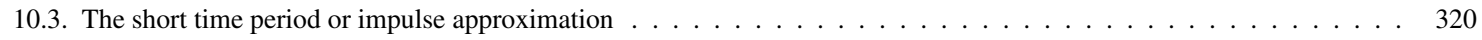

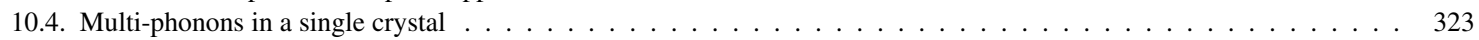

10.5. Iterative calculation of multi-phonon contribution for incoherent monatomic systems . . . . . . . . . . . 327

10.6. Integrating the $p$-phonon terms over angle and $Q \ldots \ldots \ldots \ldots$

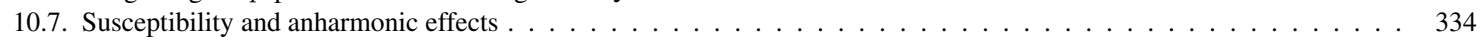

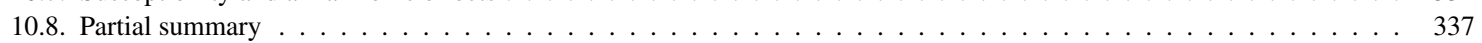

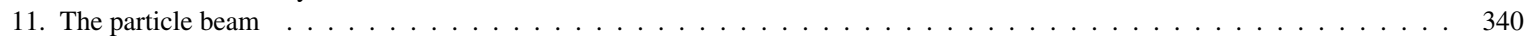

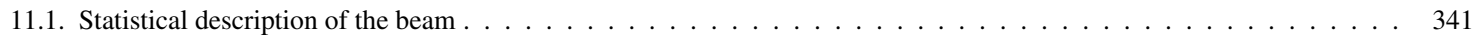

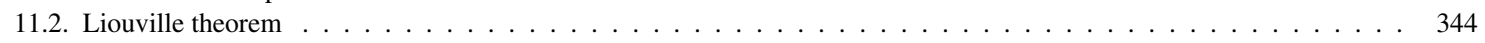

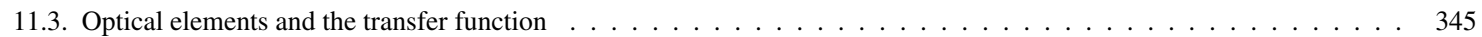

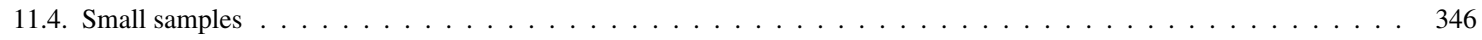

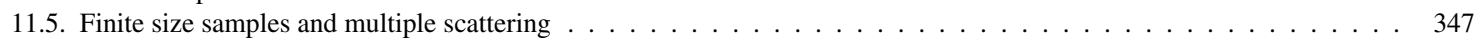

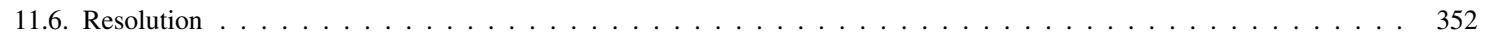

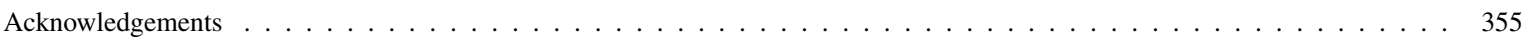

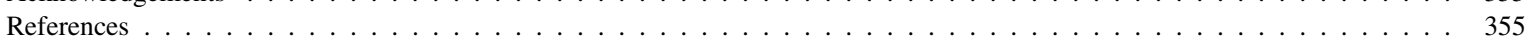

\section{Introduction}

Before developing the formal framework of non-relativistic scattering ${ }^{1}$ we would like to make some general remarks about scattering and in particular scattering with neutrons. We will in particular discuss why neutron scattering is an extremely relevant experimental method when studying materials. Furthermore, we wish to place neutron scattering in a more general context. In practice, the discussion should help the reader to compare different scattering probes, such as neutrons, electrons, X-rays and helium atoms.

\subsection{Scattering as an analytical tool for condensed matter}

To say that our conception of the microscopic world is largely based on scattering experiments is not an exaggeration. This point of view is certainly justified in the research on elementary particles. The fact that we today are convinced that the proton and the neutron have an internal structure is to a large extent the consequence of scattering experiments conducted at very high energies by particle accelerators. However, the statement also holds for the determination of structure and dynamics of condensed matter. Our detailed knowledge of atomic positions and motions is often the fruit of scattering experiments with either electromagnetic waves (light, X-rays or $\gamma$-rays) or with massive particles (electrons, positrons, neutrons or helium atoms).

Clearly, the most intuitive way of understanding a structure is in the first place to look at it, which is the domain of microscopy. Since transmission electron microscopy is unencumbered by the diffraction limits imposed on optical microscopes, it was for a long time the favourite technique when it came to studying structures at the atomic level. With breathtaking recent advances in instrumentation the area is now open for X-ray microscopy and near-field optical microscopy. These microscopes are equally part of a class of devices which work by scanning the object of investigation.

\footnotetext{
${ }^{1}$ The material presented in this article has been used to teach a one semester graduate course in non-relativistic scattering theory. It is based on reference [53]. Familiarity with elementary quantum mechanics in both the Schrödinger and Heisenberg picture is a prerequisite. The reader is equally supposed to possess knowledge in classical electrodynamics and optics, which is used for the purpose of illustration. A sound basis of solid state physics and, in particular, of the quantum mechanical treatment of phonons is indispensable for the understanding of the corresponding sections.
} 
The invention of scanning tunnelling microscopy and atomic force microscopy have radically improved our capacity to analyse but also to manipulate materials at the atomic level. Compared to these experimental techniques, scattering is a more indirect technique since it converts information in real space into information in angular space. In the most simple experiments, like the interference of two spherical waves, the information about the distance between the two sources is encoded in the angular dependence of the scattering profile. It is necessary to consider the positions of two successive maxima in order to retrieve this information. In spite of its indirect character, scattering is still an extremely well used technique in structural studies, in particular when it is important to study the bulk properties of a material and not only the surface.

The principle of a scattering experiment is simple (see Fig. 1). A probe particle, the initial state of which can be controlled, is emitted onto a sample. ${ }^{2}$ In the case of scattering of slow neutrons, the sample can change its internal configuration, but stays otherwise intact. In particular, no secondary particles will be emitted. ${ }^{3}$ Therefore, we can restrict ourselves to the study of the scattering of probe particles. The transition probabilities of going from the initial state to the final state of the probe particle contain all the relevant information concerning the sample. When we talk about scattering in this article we will always limit ourselves to this specific case, which is the dominant one in condensed matter studies.

Since scattering is an interference phenomena it averages all information over the part of the sample that is illuminated by the beam. ${ }^{4}$ This beam spot is large compared to the wave length, unlike the structural information in question, which is of the order of the wave length or even smaller in size. The situation is reversed in case of microscopy that works well above the diffraction limit imposed by the wave length. Scattering can be associated

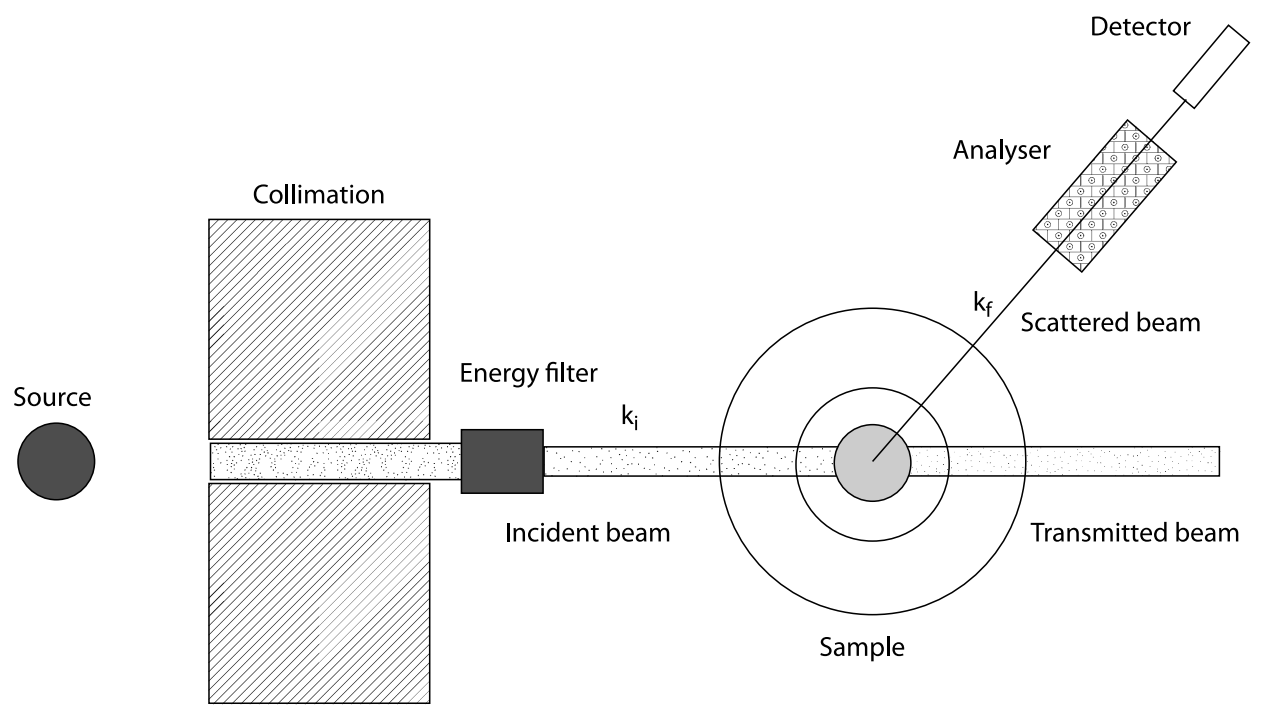

Fig. 1. A schematic illustration of a scattering experiment. A source emits particles that pass through a collimator, which defines their direction. A filter selects an energy band of a certain width. The beam, now monochromatic and collimated, hits the sample. The particles are scattered in space and energy. They are analysed according to their final state (for example their energy) and finally detected at sufficient distance from the sample.

\footnotetext{
${ }^{2}$ In the case of experiments at very high energies it is costume to use the interaction of two beams of particles. This does not change the principle.

${ }^{3}$ It is well known that the collision of two elementary particles can lead to the creation of a bunch of scattered particles at high energies. Similarly the scattering of a particle may be accompanied by the emission of secondary particles. In the case of the scattering of thermal neutrons, the energies involved are such that these effects are virtually inexistant. They occur only in the case of absorption, which can be treated as an extreme case of a scattering experiment. In the case of X-ray scattering, the probe can induce the emission of electrons and photons, because of its energy lying in the range of electronic excitations. This allows for a variety of methods of X-ray spectroscopy.

${ }^{4}$ We will be more precise in Section 11 where we introduce the notion of coherence volume.
} 
with scanning microscopy. It allows us to create contrast in samples that are homogeneous across the illuminated volume (gauge volume). Using holographic principles we can even obtain three-dimensional images using the scattering signal as input and this with a resolution that is set by the gauge volume.

The probe particle and its initial state are chosen carefully according to the purpose of the experiment. The choice should satisfy the following non-exhaustive criteria:

(1) The interaction between the probe and the material should be sufficiently strong in order for the changes to be observable. Neutrinos that can easily traverse kilometres of condensed matter are the counter example of a good scattering probe. When studying condensed matter, the probe should interact with the component of the system that one would like to study. Neutrons interact with the atomic nuclei or with magnetic fields created by internal magnetic moments. All phenomena having their origin in the electronic behaviour of the system can, therefore, only be accessed indirectly with neutrons.

(2) The interaction should on the other hand not be too strong for two reasons. First of all, the strength of the interaction defines the penetration of the probe. As a consequence the choice of probe should be adapted to whether we are interested in surfaces or bulk investigations. Helium atoms are e.g. scattered purely from the surface. Electrons have a limited penetration depth, which is strongly dependent on their energy, and, therefore, probe near-surface phenomena. Thermal neutrons are, as we will see, a perfect probe of the bulk but may equally give surface information when used for reflectometry. Secondly, a very strong interaction will make the interpretation of the data more difficult as it unavoidably leads to multiple scattering. Multiple scattering blurs the relation between the transitions of the sample and those of the probe.

(3) Preferably the particles should be scattered and not absorbed by the sample. Not only does absorption decrease the number of detectable scattering events and thus affect the statistics of the measurement. The very energetic events following absorption can seriously affect the sample, or even destroy it. Radiation damage is a well known problem in X-ray scattering. It is less of an issue for thermal neutrons.

(4) The wave length of the scattered particle defines the length scale on which the properties of materials can be studied with ease. As already mentioned above scattering converts in general terms distance into angle. The wave length of the probe particle is the unique parameter that controls this conversion. The most intuitive way of presenting this argument is via Young's double slit experiment, which can be considered as the parent of all scattering experiments. In its classical version two beams of light emerging from a reasonably coherent source interfere after their passage through two slits in an otherwise opaque plate. Seen from the perspective of a structure investigation, the purpose of the experiment is to determine the distance $d$ between the two slits. The interference pattern observed on a screen at a distance $D$, which is much larger than the slit separation $d$, is given by the expression

$$
I(y) \propto \cos ^{2}\left(\pi \frac{d}{D} \frac{y}{\lambda}\right),
$$

where $y$ is the distance from the centre of the screen to the observation point. The intensity maxima at the screen are found at distances

$$
\Delta y=D \frac{\lambda}{d}
$$

If we convert these distances into angles we obtain

$$
\Delta \phi=\frac{\Delta y}{D}=\frac{\lambda}{d}
$$

Given sufficient intensity the distance between the sample and the observation screen can always be made large. This allows to measure the angle $\Delta \phi$ with the desired precision. If the wave length $\lambda$ is much smaller than the distance $d$ that we would like to determine then the angular deviation will be minimal and risks to 

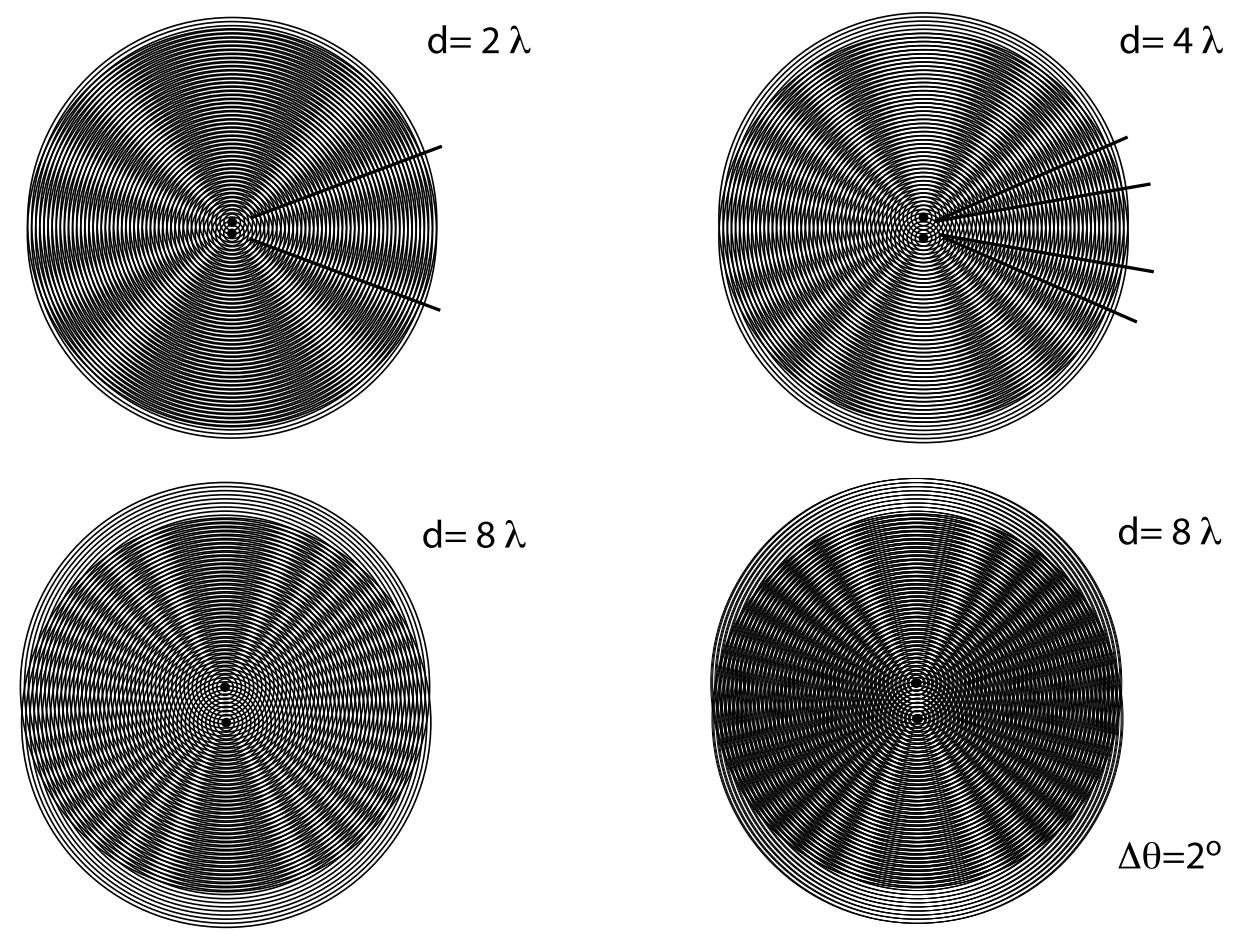

Fig. 2. Illustration of the interference patterns created by two particles (or two slits) that are separated by a distance $d$. If the illumination is coherent then the two particles will emit spherical waves with a precise phase relation. The wave fronts correspond to circles drawn around the two centres. The wave length $\lambda$ corresponds to the distance between the concentric circles. It is clearly seen that the angle of the first maximum increases with the wave length. The detection of these maxima is very easy if $\lambda \approx d$. If $\lambda$ is much smaller than $d$ one can in principle observe a large number of maxima, hence the measurement can be made very precise. However, the beam defining the phase relation should be very well defined in these cases. In the lower right corner we show the interference pattern produced by the superposition of three wave fronts incident at 0,2 and -2 degrees, respectively. The detection of the maxima is practically impossible when $d=8 \lambda$.

be hidden by the direct beam or by experimental imperfections in the setup (see Fig. 2). If $\lambda$ increases, the maximum moves towards higher angles and becomes observable before finally disappearing when the value of $\lambda$ becomes too large. According to (1.3) the measurement will be easiest when $\lambda$ is of the order of $d$. X-rays and thermal neutrons with wave lengths in the order of a few $\AA$ are well suited for the study of condensed matter at an atomic level. For the study of elementary particles it is necessary to work with much smaller wave length and hence much higher energies.

(5) The total energy of the system consisting of the sample and the probe particle is conserved. The change in the energy of the probe, therefore, directly reflects a change of energy of the sample. It is relatively easy to determine the change of a physical quantity if the change is relatively large, that is to say, if it is of the same order of magnitude as the physical quantity itself. As a consequence, in order to determine excitations it is preferable to work with probe particles, that have more or less the same energy. ${ }^{5}$ The energy scale of occupied excitations in condensed matter is given by the temperature $T$. In Section 1.3 we will discover that moderated neutrons can be described as a gas kept at the moderator temperature. As a basic rule, we can

\footnotetext{
${ }^{5}$ There can be good reasons not to follow this simple rule. In the last couple of years inelastic X-ray scattering has brought a lot of new knowledge to the field of excitations in condensed matter despite the fact that the photon energy employed in these experiments is of the order of a few tens of $\mathrm{keV}$, hence 6 or 7 orders of magnitudes larger than the energy of the excitations under scrutiny. One of the reasons for this technique being successful is the fact that the momentum transfer accessible by X-rays for a given energy transfer is much smaller than for neutrons. The experiments require nevertheless that the incident beam is extremely monochromatic, which induces a significant loss in intensity. This type of experiment is, therefore, only feasible at third generation synchrotrons like the ESRF.
} 
assume that the excitations in a material at temperature $T$ should be analysed with neutrons moderated at the same temperature $T$.

(6) It is in general an advantage to control the type of interaction between the probe and the sample. Where a selective control exists it is possible to highlight the signal coming from part of the sample with respect to the rest, creating so-called scattering contrast. In the case of neutrons the control is usually exerted via isotope substitution. In the case of X-rays one can enhance the signal of one type of atoms by choosing a wave length close to its absorption threshold.

\subsection{The scattering cross section}

Within our definition, the only observable in a scattering experiment is the intensity of the scattered particles registered in the detector (see Fig. 1).

Each detector is oriented in such a way that its detection area $\Delta A$ is placed perpendicular to the vector $\vec{r}=$ $(r, \theta, \phi)$ that defines its position with respect to the origin of the coordinate system coinciding with the sample. Ideally this surface should be a part of a sphere with radius $r$. The number of particles that the detector intercepts 6 per second is given by the expression

$$
I(t)=\Delta A \cdot J_{\text {scattered }}(r, \theta, \phi, t)
$$

where we have introduced the flux (or current) $J_{\text {scattered }}(r, \theta, \phi, t)$ of scattered particles. At sufficiently large distance this flux is necessarily radial. It indicates the number of particles that travel through a unitary surface per unit of time. Hence the coordinates that will be used are the polar spherical coordinates $r, \theta$ and $\phi$.

Far from the sample the scattered particles can be considered as free. They are, therefore, fully characterised by their momentum $\vec{p}=\hbar \vec{k}$. For a given detection element the wave vector $\vec{k}_{f}$ of the registered neutrons is parallel to $\vec{r}$ (see Fig. 3). In the same way the incident beam is described by the wave vector $\vec{k}_{i}$. In real experiments the neutron beam is never ideal. It is necessary to deal with a distribution of particles $p\left(\vec{k}_{i}\right)$ and $p\left(\vec{k}_{f}\right)$ centred on their mean values. The secret behind a well constructed instrument is to adapt these distributions to the need of the experiment. Poorly defined beams will have a tendency to smear out the signal. Too well defined beams will lower the flux and thus have a negative effect on statistics. We will treat this point in Section 11.6.

We would like to remark already at this point that scattering correspond to the transition of a particle initially (i.e. before the impact) in the free state $\vec{k}_{i}$ to the final state (after the impact) free state $\vec{k}_{f}$. The mathematical problem consist of finding the probability of this transition. This probability is closely related to the matrix elements

$$
S_{\vec{k}_{f}, \vec{k}_{i}}=\left\langle\vec{k}_{f}\left|\mathbf{U}_{I}(+\infty,-\infty)\right| \vec{k}_{i}\right\rangle
$$

of the time evolution operator $\mathbf{U}_{I}\left(t^{\prime}, t\right)$. This operator describes how a quantum state evolves between the time $t$ and $t^{\prime}$. It depends on the interaction potential acting on the beam. The limits $t \rightarrow+\infty$ and $t \rightarrow-\infty$ correspond to the notions "initial" and "final" in the ideal case. At this point the expression (1.5) is of purely academic value. It casts the experimental situation into an abstract mathematical language. We are going to expand this formalism as we move forward.

Since the flux decreases with increasing distance the signal registered in a detector of a given area falls off as $1 / r^{2}$. We would like to get rid of this trivial $r$ dependence that does not convey any useful information. As we explained in the introduction scattering experiments encode all information in the scattering angle. As a consequence we are interested in the scattered intensity as a function of direction, i.e. the particle flux detected within an angular element $\mathrm{d} \Omega=\sin \theta \mathrm{d} \theta \mathrm{d} \phi$. The relation between the area of the detector and the solid angle $\Omega$ covered is given as

$$
\Delta A=r^{2} \Delta \Omega
$$

\footnotetext{
${ }^{6}$ Here we assume that the detector is ideal, which means that its detection capacity is $100 \%$. In realty this is rarely the case and effects arising from the detector inefficiency have to be corrected for.
} 


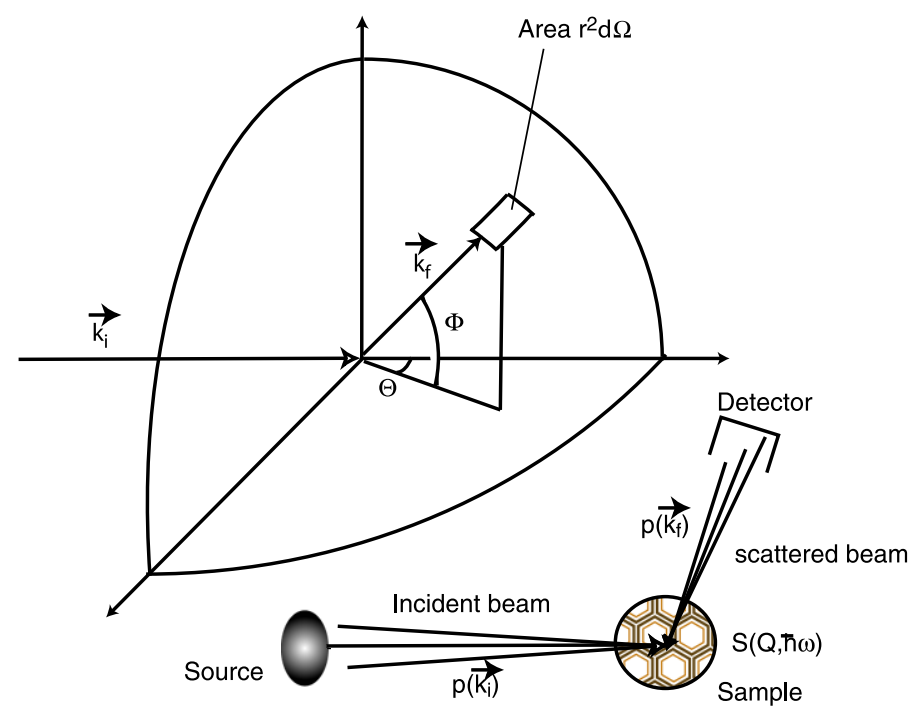

Fig. 3. Scattering of a beam by the sample. In the classical approach the scattering is described by the double differential cross section $\mathrm{d}^{2} \sigma / \mathrm{d} \Omega \mathrm{d} E_{f}$ giving the probability that a normalised flux of neutrons of incident wave-vector $\vec{k}_{i}$ is scattered into a solid angle element $\mathrm{d} \Omega$ perpendicular to the direction $\hat{k}_{f}$ with energies comprised between $E_{f}=\hbar^{2} k_{f}^{2} / 2 m$ and $E_{f}+\mathrm{d} E_{f}$, that is, between $\left(\hbar^{2} / 2 m\right) k_{f}^{2}$ and $\left(\hbar^{2} / 2 m\right)\left(k_{f}^{2}+2 k_{f} \mathrm{~d} k_{f}\right)$. If we choose the coordinate system such that $\hat{k}_{f}=\hat{k}_{z}^{f}$ then $\mathrm{d} \Omega=\mathrm{d} k_{x}^{f} \mathrm{~d} k_{y}^{f} / k_{f}^{2}$. In practice the incoming beam of neutrons has a distribution $p\left(\vec{k}_{i} ; t\right)$ in energy and direction, depending on how the neutrons were moderated and then shaped by the beam transport. In the same way the neutrons detected will have a distribution $p\left(\vec{k}_{f} ; t\right)$ arising from the beam shaping during the transport from the sample to the detector and from the detector efficiency. The beam characteristics described by $p\left(\vec{k}_{i} ; t\right)$ and $p\left(\vec{k}_{f} ; t\right)$ are responsible for both the statistics and the resolution of the measurements. (Colors are visible in the online version of the article; http://dx.doi.org/10.3233/JNR-140016.)

Concerning the distance $r$ we simply assume that the detectors can be placed at a sufficiently large distance from the sample to allow resolving the direction of scattering with the desired precision. The quantity that we wish to determine is finally the ratio between the flux scattered into an angular element $\mathrm{d} \Omega$ and the initial flux that hits the sample

$$
\mathrm{d} \sigma:=\frac{\text { flux scattered into } \mathrm{d} \Omega}{\text { initial flux onto the sample }}=r^{2} \mathrm{~d} \Omega \frac{J_{\text {scattered }}(r, \theta, \phi, t)}{J_{\text {ini }}(t)} .
$$

The quantity $\frac{\mathrm{d} \sigma}{\mathrm{d} \Omega}$ is called the differential scattering cross section. The name is justified by the fact that $\frac{\mathrm{d} \sigma}{\mathrm{d} \Omega}$ has the dimensions of area. Integrating over all angles gives the total scattered flux with respect to the initial flux. This physical quantity is called the total cross section or just the cross section

$$
\sigma_{t}=\int \frac{\mathrm{d} \sigma}{\mathrm{d} \Omega} \mathrm{d} \Omega=\int \mathrm{d} \sigma .
$$

The cross section represent in a simple way the area that the scattering particle opposes to the radiation. For a classical non-transparent sphere of radius $r$ that scatters a homogeneous flux of particles the total cross section corresponds to $\pi r^{2}$ (see Section 2.4). We will later (Section 3.3) see that quantum mechanics modifies this results. Cross sections are in general expressed in barns, where

$$
1 \text { barn }=10^{-24} \mathrm{~cm}^{2} \text {. }
$$

In the introduction we have mentioned the possibility of filtering the particles before their detection. In the case of neutrons, the most common filtering is done with respect to energy. The filter or energy analyser allows identifying 
neutrons that have an energy between $E_{f}$ and $E_{f}+\mathrm{d} E_{f}$. This leads us to the concept of the double differential cross section

$$
\mathrm{d}^{2} \sigma:=\frac{\text { flux scattered into } \mathrm{d} \Omega \text { with an energy between } E_{f} \text { and } E_{f}+\mathrm{d} E_{f}}{\text { initial flux onto the sample with the energy } E_{i}} \mathrm{~d} \Omega \mathrm{d} E_{f} .
$$

The quantity

$$
\frac{\mathrm{d}^{2} \sigma}{\mathrm{d} \Omega \mathrm{d} E_{f}}
$$

has the unity of surface per energy. The total cross section can be obtained by integration

$$
\sigma_{t}=\iint \frac{\mathrm{d}^{2} \sigma}{\mathrm{d} \Omega \mathrm{d} E_{f}} \mathrm{~d} \Omega \mathrm{d} E_{f}
$$

\subsection{Thermal neutrons as a scattering probe}

Before going further into the formalism of scattering we would like to briefly turn our attention to the neutron. The neutron is an elementary particle discovered by James Chadwick [8,9] in 1932. Its quantum mechanical state is determined by its momentum $|\vec{p}\rangle(\vec{p}=\hbar \vec{k}, k=2 \pi / \lambda)$ and its spin $|s\rangle$. The neutrons used as probes of condensed matter are low-energy neutrons and thus can be treated as non-relativistic particles. Their energy as a function of wave vector is given by

$$
E=\frac{\hbar^{2} k^{2}}{2 m}
$$

The mass of the neutron ${ }^{7}$ is 1.008 atomic mass units. This gives the relation between the energy and the neutron wave length

$$
E[\mathrm{meV}] \equiv 2.0725 k^{2}\left[\AA^{-1}\right] \equiv 81.8204 \lambda^{-2}[\AA ̊] \equiv 5.227 v^{2}[\mathrm{~km} / \mathrm{s}],
$$

or inversely

$$
\lambda[\AA] \equiv \frac{2 \pi}{k}\left[\AA^{-1}\right] \equiv 9.045 \frac{1}{\sqrt{E}}[\mathrm{meV}] \equiv 3.956 \frac{1}{v}[\mathrm{~km} / \mathrm{s}] .
$$

The parenthesis give the units used for $E, \lambda, k$ and $v$ respectively.

The spin of the neutron is $1 / 2$ with a magnetic moment of -1.9132 nuclear magnetons.

The neutrons of the universe are either confined within the nucleus or in neutron stars. In order to extract the neutrons from the nuclei one needs to use technical processes like fission in a reactor or spallation in a pulsed source. A description of these methods can be found in [52]. Once produced the free neutrons have a life time of approximately $886 \mathrm{~s}$.

Both the production and the moderation of neutrons proceed via intermediary random processes (see Fig. 4). Since the moderated neutrons are at equilibrium with a thermal bath of temperature $T$ (for example $\mathrm{D}_{2} \mathrm{O}, \mathrm{H}_{2}$ or $\mathrm{D}_{2}$ molecules in the liquid state) their average energy is the same as the average kinetic energy of any material at this

\footnotetext{
${ }^{7}$ Here we use the National Institute of Standards and Technology (http://physics.nist.gov/cuu/Constants/) as a reference for physical constants $h=6.62606896 \times 10^{-34} \mathrm{Js}$ and $m=1.674927211 \times 10^{-27} \mathrm{~kg}$. As $e=1.602176487 \times 10^{-19} \mathrm{C}, 1 \mathrm{Joule}=6.24150965 \times 10^{18} \mathrm{eV}$. Numerous useful relations relating to neutrons can be found in the Neutron Data Booklet [16].
} 


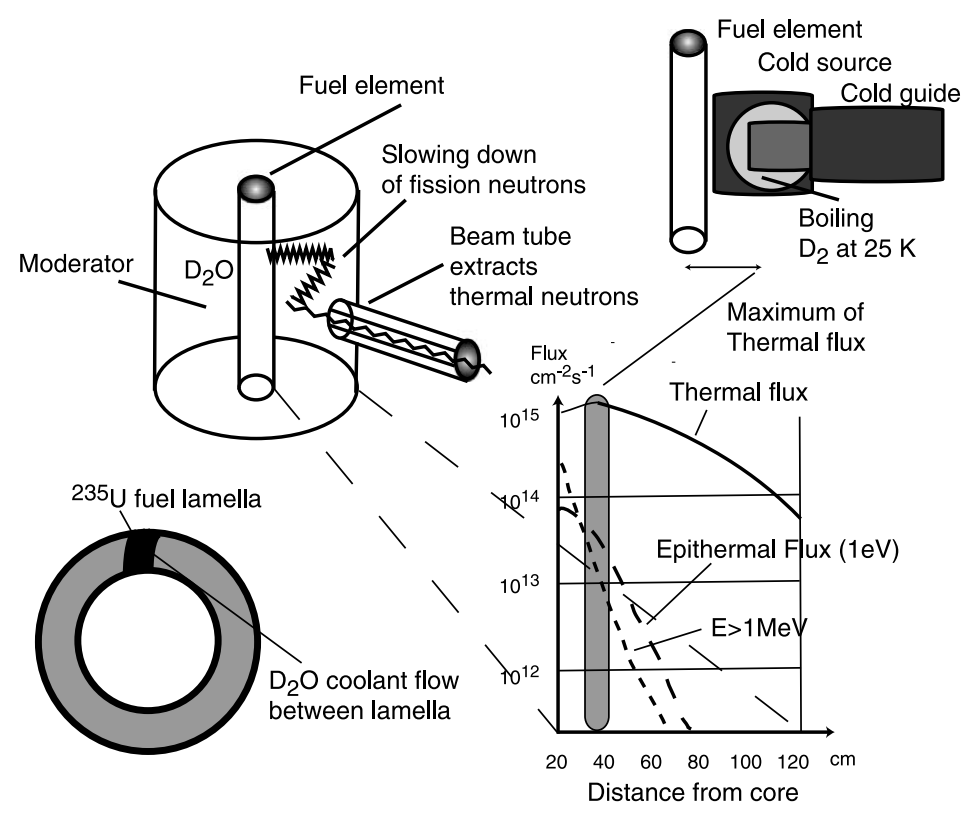

Fig. 4. Schematic view of the neutron source at the Institut Laue-Langevin. A compact fuel element is surrounded by a moderator of heavy water $\mathrm{D}_{2} \mathrm{O}$. High energy neutrons $(E \approx 1 \mathrm{MeV})$ are produced via fission of uranium ${ }^{235} \mathrm{U}$. Their energy drops quickly within the moderator as a consequence of the inelastic collisions with the heavy water molecules. When equilibrated the integrated flux has a maximum of $1.5 \times 10^{15} \mathrm{n}$ per $\mathrm{cm}^{2}$ and per second at approximately $40 \mathrm{~cm}$ from the centre. Neutron beam tubes with their noses placed as close as possible to the region of the flux maximum allow for the extraction of neutrons to the instruments. Some of these beam tubes deliver neutrons to a fan of neutron guides. In order to get an even lower spectrum in terms of energy two cavities filled with boiling $D_{2}$ are introduced into the region of the thermal flux maximum [1]. The cold neutron guides extract the neutrons from these cold sources. A block of graphite heated by the intrinsic $\gamma$ radiation of the core to $2400 \mathrm{~K}$ transposes the spectrum for certain neutron beam tubes to higher energies. These are called hot neutrons.

temperature. In the language of quantum mechanics the energy of the neutrons are comparable to the energy of the populated excitations at this temperature. This fact reveals one of the major advantages of neutron spectroscopy. The argument is nevertheless the same for all moderated particles. The particularity of neutrons resides in the fact that their wave lengths as given by (1.14) are at the same time of the order of interatomic distances. This is a result of the dispersion relation and thus of the specific mass of the neutron.

Identifying the moderated neutrons with a classical gas in thermal equilibrium we may employ classical thermodynamics to calculate the spectrum. It follows statistically a Maxwell-Boltzmann distribution governed by the temperature $T$ of the bath. ${ }^{8}$ The probability of finding a neutron in the state $|\vec{k}\rangle$ is thus given by

$$
p(\vec{k})=\frac{1}{k_{\mathrm{T}}^{3} \sqrt{\pi^{3}}} \mathrm{e}^{-k^{2} / k_{\mathrm{T}}^{2}},
$$

with the mean neutron momentum and energy defined as

$$
\hbar k_{\mathrm{T}}=\sqrt{2 m k_{\mathrm{B}} T}, \quad E_{\mathrm{T}}=\frac{1}{2 m} \hbar^{2} k_{\mathrm{T}}^{2}=k_{\mathrm{B}} T
$$

and the Boltzmann constant

$$
k_{\mathrm{B}}=0.08617 \mathrm{meV} / \mathrm{K} \quad \text { or } \quad k_{\mathrm{B}}^{-1}=11.60 \mathrm{~K} / \mathrm{meV} \text {. }
$$

\footnotetext{
${ }^{8}$ In practice the spectrum will differ from the ideal Maxwell-Boltzmann distribution. This is due to leakage of fast neutrons or incomplete moderation processes. These corrections become more important in the case of spallation sources.
} 
The normalisation is such that the integral over the phase space density (see Section 11.1 for definition)

$$
\mathrm{d} N=p(\vec{k}) \mathrm{d}^{3} r \mathrm{~d}^{3} k=\frac{N}{k_{\mathrm{T}}^{3} \sqrt{\pi^{3}}} \mathrm{e}^{-k^{2} / k_{\mathrm{T}}^{2}} \mathrm{~d}^{3} r \mathrm{~d}^{3} k
$$

gives the total neutron density, which is assumed to vary only slowly with $\vec{r}$. From the phase space density we can calculate the energy distribution as

$$
\Phi(E) \mathrm{d} E=\Phi_{\text {thermal }} \frac{2}{\sqrt{\pi}} \frac{\sqrt{E}}{\left(k_{\mathrm{B}} T\right)^{3 / 2}} \exp \left(-\frac{E}{k_{\mathrm{B}} T}\right) \mathrm{d} E,
$$

with

$$
\Phi_{\text {thermal }}=\frac{2 N}{\sqrt{\pi}} v_{\mathrm{T}}=\frac{2 N}{m \sqrt{\pi}} \hbar k_{\mathrm{T}}
$$

denoting the thermal flux of the source. Typical spectra are shown in Fig. 5. Both at reactors and spallation sources, different moderators at different temperatures are used to give optimised flux distributions for the various scientific applications. At the ILL the thermal spectrum is moderated to lower energies by using boiling $\mathrm{D}_{2}$ sources at $25 \mathrm{~K}$ (see Fig. 4). The up-moderation is achieved via a graphite block heated to $2400 \mathrm{~K}$ via the $\gamma$-radiation produced by the nearby reactor core.

Due to the random production process neutron radiation is by nature completely incoherent. The neutron sources should be compared to incandescent light bulbs and not with lasers. The lack of coherence implies that there is no interference from the waves coming from different regions of the source. The incoherent nature of the source allows us to describe the beam as a distribution $p(\vec{k})$ of the wave vector (see Section 11.1).

In Table 1 the general classification used for moderated neutrons is listed. Besides the moderated neutrons the beam also contains fast and epi-thermal neutrons. Adequate measures are taken in order to avoid that these neutrons become part of the experimental background.

We are going to get to know the properties of the neutron as we proceed with the formalism.

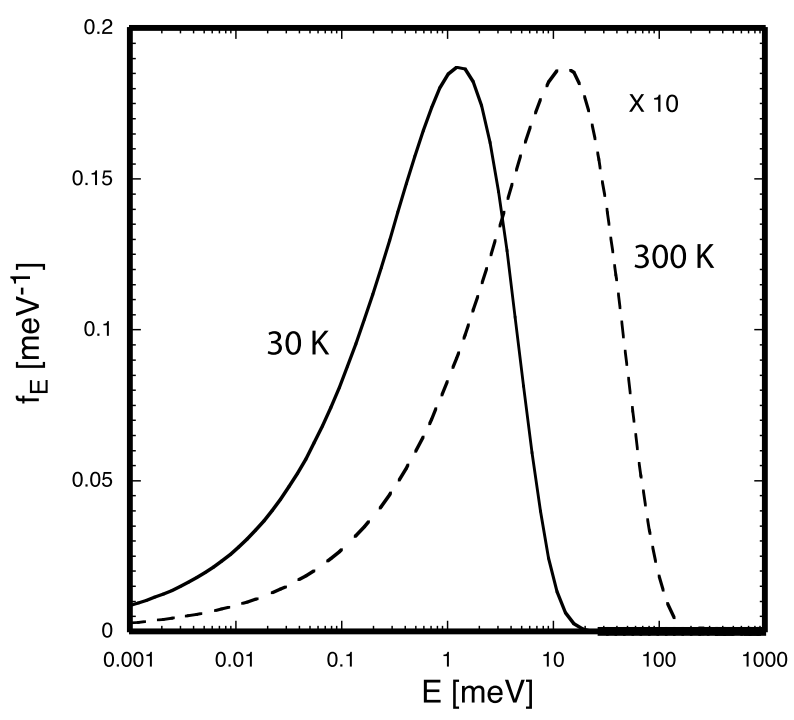

Fig. 5. Maxwell-Boltzmann distribution for two temperatures, 30 and $300 \mathrm{~K}$, according to (1.19). The $300 \mathrm{~K}$ curve has been upscaled by a factor 10 in order to allow for better comparison. The distributions correspond to typical thermal and cold neutron beam spectra. 
Table 1

Standard classification of neutrons after energy

\begin{tabular}{lccc}
\hline Name & Energy $(\mathrm{meV})$ & Wave length $(\AA)$ & Temperature $(\mathrm{K})$ \\
\hline Cold & $0.1-10$ & $30-3$ & $1-120$ \\
Thermal & $10-100$ & $3-1$ & $120-1200$ \\
Hot & $100-500$ & $1-0.4$ & $1200-6000$ \\
\hline Note: The indicated values correspond to the cold, thermal and hot spectrum of the ILL.
\end{tabular}

\subsection{Particles described by wave packets}

The scattering theory that we are going to develop will be based mainly on stationary wave functions. These wave functions describe a time-invariant flow of particles. It will, however, occasionally be necessary to include the particle trajectories into the discussion. Quantum mechanically these trajectories are described by wave packages that we, therefore, want to introduce briefly.

A very general form of a wave package is given by

$$
\psi(x, t)=\frac{1}{\sqrt{2 \pi}} \int g(k) \mathrm{e}^{\mathrm{i}(k x-\omega(k) t)} \mathrm{d} k,
$$

where for reasons of simplicity we restrict ourselves to a single dimension. For a massive free particle the dispersion relation is expressed as

$$
\omega(k)=\frac{\hbar}{2 m} k^{2} .
$$

The function $g(k)$ describes the distribution of the stationary waves

$$
\mathrm{e}^{\mathrm{i}(k x-\omega(k) t)}
$$

in the basis of which the wave package is developed. It is, therefore, responsible for the shape of the wave package at $t=0$. An appropriate form for this distribution is a Gaussian

$$
g(k)=\left(\frac{1}{2 \pi(\Delta k)_{0}^{2}}\right)^{1 / 4} \cdot \exp \left[-\mathrm{i}\left(k-k_{0}\right) x_{0}\right] \cdot \exp \left[-\frac{\left(k-k_{o}\right)^{2}}{4(\Delta k)_{0}^{2}}\right]
$$

where the factor

$$
\exp \left[-\mathrm{i}\left(k-k_{0}\right) x_{0}\right]
$$

assures the correct phase at $t=0$ and

$$
\left(\frac{1}{2 \pi(\Delta k)_{0}^{2}}\right)^{1 / 4}
$$

takes care of the normalisation. $x_{0}$ and $k_{0}$ give the central position and wave vector of the package at $t=0$. If we choose a reference frame in which the particle rests at $t=0$, i.e.

$$
x_{0}=0, \quad k_{0}=0
$$


the notation simplifies considerably. At $t=0$ the wave package is a simple Gaussian of width

$$
(\Delta x)_{0}^{2}=\frac{1}{4} \frac{1}{(\Delta k)_{0}^{2}} .
$$

A Gaussian distribution thus provides a minimum solution of the Heisenberg uncertainty principle

$$
\Delta x \cdot \Delta k \geqslant \frac{1}{2} .
$$

The envelop $\psi(x, t) \psi(x, t)^{*}$ remains Gaussian as the wave package evolves. The time evolution of its width is given as

$$
(\Delta x)^{2}=(\Delta x)_{0}^{2}+\frac{\hbar^{2}}{4 m^{2}(\Delta x)_{0}^{2}} t^{2}=(\Delta x)_{0}^{2}+\frac{(\Delta p)_{0}^{2}}{m^{2}} t^{2}=(\Delta x)_{0}^{2}+(\Delta v)_{0}^{2} t^{2} .
$$

The wave function thus spreads in space (see Fig. 6) in the same way that a Gaussian ensemble of classical particles with an uncertainty in the velocity of $(\Delta v)_{0}$ would do.

In an arbitrary reference system the centre-of-gravity of the wave package moves according to

$$
x_{m}=\left(\frac{\partial \omega}{\partial k}\right)_{k_{0}}=\frac{\hbar k_{0}}{m} t .
$$

This result is well known from wave mechanics. A wave package evolves with the group velocity

$$
\vec{v}_{g}=\frac{1}{\hbar}\left(\frac{\partial E(\vec{k})}{\partial \vec{k}}\right)_{\vec{k}_{0}}=\frac{1}{\hbar}\left(\frac{\partial E(\vec{k})}{\partial k_{x}}, \frac{\partial E(\vec{k})}{\partial k_{y}}, \frac{\partial E(\vec{k})}{\partial k_{z}}\right)_{\vec{k}_{0}}
$$
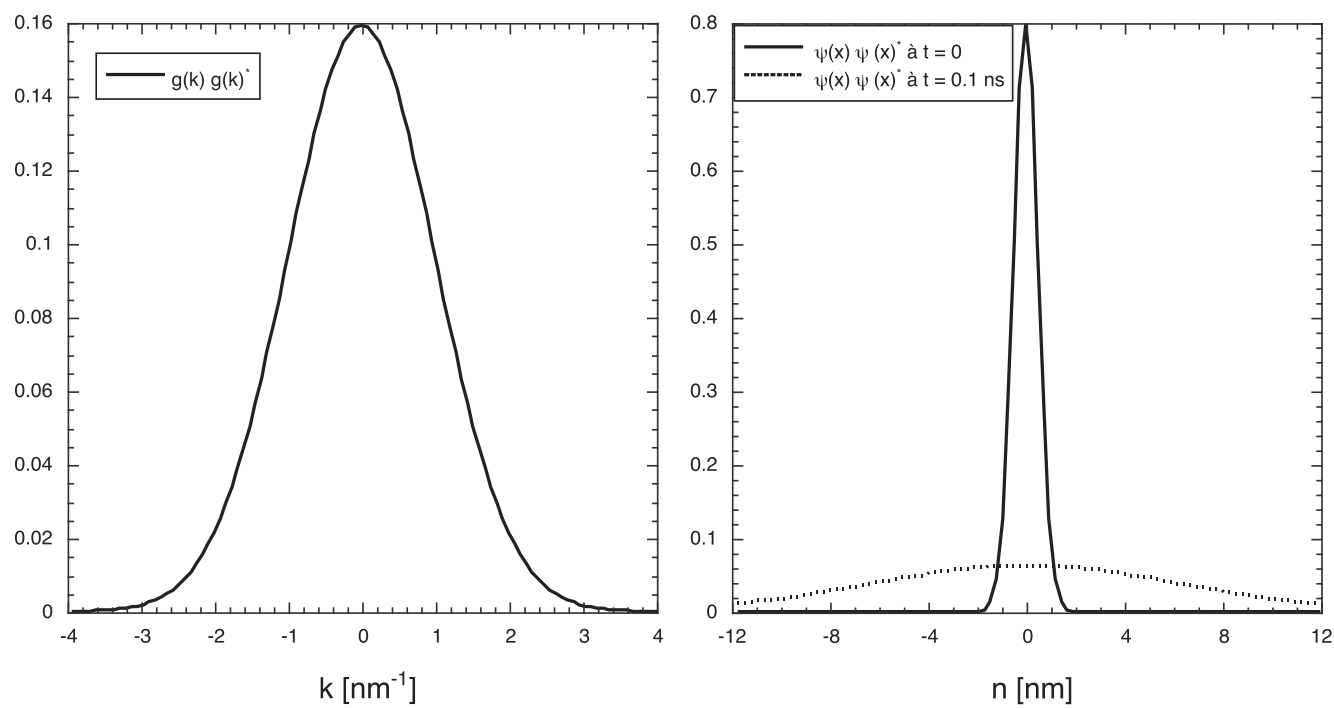

Fig. 6. Wave package. The figure on the left shows a Gaussian distribution $g(k)$. This distribution is invariant as a function of time. The envelop of the corresponding wave function $\psi(x) \psi(x)^{*}$ defined in (1.24) is equally Gaussian. The figure on the right shows the spreading of this wave function for a particle with the mass of the neutron. 
$E(\vec{k})$ describes the energy as a function of $\vec{k}$. In the case of a free particle $v_{g}=\hbar k_{0} / m$.

The concept of wave packages is very precious in particular when we are dealing with transport phenomena between collisions. It allows us to assimilate the particle motion to classical motions. This holds also in external fields, like electromagnetic or gravitational fields, provided these fields vary slowly at the scale of the extension of the wave package. In this way non-relativistic particles in an accelerator follow the equations of motion

$$
\frac{\partial \vec{r}}{\partial t}=\vec{v}_{g}(\vec{k})=\frac{1}{\hbar}\left(\frac{\partial E(\vec{k})}{\partial \vec{k}}\right)
$$

and

$$
\frac{\hbar \partial \vec{k}}{\partial t}=-e\left[\vec{E}(\vec{r}, t)+\frac{1}{c} \vec{v}_{g} \times \vec{H}(\vec{r}, t)\right] .
$$

For a neutron of mass $m=1.6749 \times 10^{-27} \mathrm{~kg}$ we get

$$
\frac{\hbar^{2}}{4 m^{2}}=9.9106 \cdot 10^{-16} \mathrm{~m}^{4} \mathrm{~s}^{-2} \text {. }
$$

To describe a neutron that at $t=0$ occupies a region of about one nanometer in the form of a Gaussian wave package we need according to (1.29) a distribution of wave vectors of width

$$
(\Delta k)_{0}=0.025 \AA^{-1} .
$$

If we choose a typical thermal neutron with $k_{0}=1 \AA^{-1}$ this corresponds to a wave vector spread of $2.5 \%$, which is a typical experimental value. This wave package will spread according to (1.30) by about $30 \mathrm{~nm}$ within one nanosecond. After $1 \mu \mathrm{s}$ it will have attained a width of about $30 \mu \mathrm{m}$. During this time it will have traveled about $2 \mathrm{~cm}$. This means that even for sharply defined momenta of the particles, i.e. for $(\Delta k)_{0} \ll k_{0}$ the uncertainty in the position of the particle remains inferior to the dimensions of optical devices and detectors even after having travelled large distances, e.g. between the target and the detector. This is important in order to be able to perform scattering experiments in the way we have outlined in Section 1.2.

\section{Classical collisions}

Before setting out to develop the quantum mechanical framework of scattering theory we will make a very short excursion into classical mechanics studying the collision of particles. This will help us to put the quantum mechanical concepts into perspective and to clarify the definition of terms. ${ }^{9}$

We speak here of the collision of two classical particles when the following requirements are fulfilled:

- The colliding particles do not experience external forces. Under this condition the centre-of-mass motion is unperturbed and can be decoupled from the relative motion.

- The interaction of the particles is limited to a confined region of space and the relative velocities are such that the motion is unbound. As a consequence the trajectories of the particles outside this region can be described by straight motion. ${ }^{10}$ To describe the system before and after the collision it is, therefore, sufficient to indicate the respective particle momenta $\vec{p}_{i}$ and $\vec{p}_{f} .{ }^{11}$

\footnotetext{
${ }^{9} \mathrm{~A}$ more detailed treatment of classical mechanics can e.g. be found in [64].

${ }^{10}$ If the interaction potential is proportional to $r^{-1}$ then this condition is only met asymptotically by adjusting straight lines to the hyperbola of the motion. Most interaction potentials that we encounter in reality are screened and, therefore, only act in a limited region of space. A typical example is the electric potential of a nucleus screened by the electrons surrounding the nucleus in an atom.

${ }^{11}$ We will come back to the internal degrees of freedom of the particles when dealing with inelastic collisions.
} 
Laboratory frame

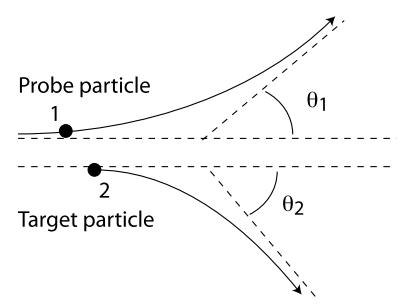

Centre-of-mass frame

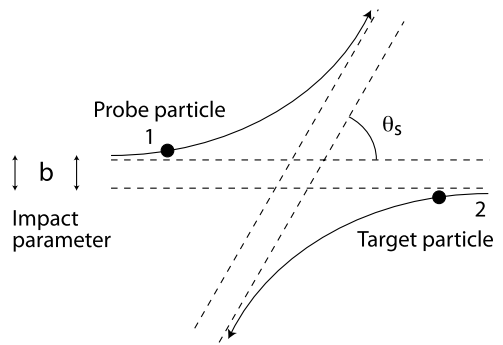

Fig. 7. Schematic motion of two colliding particles. The interaction is assumed to be repulsive. In the laboratory frame the target is at rest. After the collision both particles are scattered at angles $\Theta_{1}$ and $\Theta_{2}$ defined with respect to the asymptotic direction of the probe particle. If the scattering is elastic and both particles have the same mass then the scattering angles add up to $90^{\circ}$ degrees unless one of them remains at rest after the collision (central collision or avoided collision, respectively). In the centre-of-mass frame the situation becomes symmetric. The two particles have oppositely equal momenta before and after the scattering process. The collision can, therefore, be described by a single scattering angle $\Theta_{\mathrm{s}}$. Apart from the interaction potential the decisive parameter determining $\Theta_{\mathrm{s}}$ is the impact parameter $b$.

\subsection{Frames of reference}

To simplify the discussion we will limit the system to two particles (see Fig. 7). In the standard situation encountered in the laboratory one of them will be at rest and considered the target. The probe particle will hit this target with a certain initial velocity $\vec{v}_{i 1}$. In the laboratory frame we, therefore, encounter the following situation before the impact:

$$
\vec{v}_{i 1}^{\mathrm{L}} \neq 0, \quad \vec{v}_{i 2}^{\mathrm{L}}=0, \quad \vec{V}^{\mathrm{L}}=\frac{m_{1}}{m_{1}+m_{2}} \vec{v}_{i 1}^{\mathrm{L}}
$$

where $\vec{V}^{\mathrm{L}}$ denotes the centre-of-mass motion in the laboratory system. As we are dealing with non-relativistic velocities the motions in the two frames are related via a Galilean transformation. If we describe the motion in the centre-of-mass frame we, therefore, obtain

$$
\begin{aligned}
& \vec{v}_{i 1}^{\text {c.m. }}=\vec{v}_{i 1}^{\mathrm{L}}-\vec{V}^{\mathrm{L}}=\frac{m_{2}}{m_{1}+m_{2}} \vec{v}_{i 1}^{\mathrm{L}}, \\
& \vec{v}_{i 2}^{\text {c.m. }}=\vec{v}_{i 2}^{\mathrm{L}}-\vec{V}^{\mathrm{L}}=-\frac{m_{1}}{m_{1}+m_{2}} \vec{v}_{i 1}^{\mathrm{L}},
\end{aligned}
$$

which using the reduced mass $\mu=\left(m_{1} m_{2}\right) /\left(m_{1}+m_{2}\right)$ establishes the following relation among the initial velocities

$$
m_{1} \vec{v}_{i 1}^{\mathrm{c} . \mathrm{m} .}=-m_{2} \vec{v}_{i 2}^{\mathrm{c} . \mathrm{m} .}=\mu \vec{v}_{i 1}^{\mathrm{L}}
$$

As required the particles move in the centre-of-mass frame with opposite momenta. As there are no external forces acting on the system the overall momentum is conserved in the collision. The relation (2.4) between the momenta is, therefore, preserved after the collision

$$
m_{1} \vec{v}_{f 1}^{\text {c.m. }}=-m_{2} \vec{v}_{f 2}^{\text {c.m. }} .
$$

This leads to the symmetric situation depicted in Fig. 7. 


\subsection{Elastic and inelastic collisions}

We can claim that we have acquired full knowledge of the dynamics of interacting particles once their trajectories as a function of time $\left(\vec{r}_{j}(t), j=1, N\right)$ are determined. This is certainly the ultimate goal when e.g. dealing with celestial mechanics. In many laboratory collision problems it is, however, sufficient to deal with the kinematical aspects, i.e. to limit the investigation to the form of the trajectories. In the extreme case where the form of the trajectories within the interaction region is not accessible experimentally all we have to know is the final momenta $\vec{p}_{f j}$ of the scattered particles.

In the case of a two-body collision the momentum conservation imposes 3 constraints on the six unknown quantities that determine $\vec{p}_{f 1}$ and $\vec{p}_{f 2}$. If we work in the centre-of-mass frame then the momenta of target and probe are oppositely equal both before and after the scattering event. The final trajectories of the particles are, therefore, determined if we know the change of direction of the probe given by the scattering angle $\Theta_{\mathrm{s}}$ as well as its final speed $v_{f 1}$ (see Fig. 7).

We call a collision elastic if the sum of the kinetic energies of the colliding partners is a conserved quantity. In an elastic collision energy conservation adds, therefore, another constraint to the momenta of the outgoing particles. A collision is termed inelastic if part of the kinetic energy of the probe particle is converted into other forms of energy like deformation or excitation energy of the colliding particles. If the probe is an elementary particle with no excitable internal degrees of freedom, like the neutron, and if the target is very massive in comparison then elastic scattering implies the conservation of the energy of the target. ${ }^{12}$

\subsection{Central potential and impact parameter}

It is a well known statement of classical mechanics that the relative motion of two particles A and B is the same as that of particle $\mathrm{A}$ in the field of particle $\mathrm{B}$, which is assumed as fixed, provided that the mass $m_{1}$ is replaced by the reduced mass $\mu .{ }^{13}$ Using this formalism the motion of the target drops out of the equation and we are solely concerned with the trajectory of the probe.

If we are dealing with a central force $\vec{F}_{1,2}(\vec{r})=\vec{F}_{1,2}(r)$ then total angular momentum $\vec{J}$ is conserved and the motion is confined to the plane normal to $\vec{J}$. This plane is called the scattering plan. ${ }^{14}$ The angular momentum is easily calculated in the laboratory frame placing the target particle at the origin

$$
\vec{J}=\vec{p}_{i 1}^{\mathrm{L}} \times \vec{r}_{i 1}^{\mathrm{L}}
$$

\footnotetext{
${ }^{12}$ Two billiard balls will collide elastically by exchanging speed. A tennis ball hitting a concrete wall will collide elastically only if it preserves its speed.

${ }^{13}$ The coordinates $\vec{r}_{1}$ and $\vec{r}_{2}$ of the two particles are replaced by the coordinate $\vec{R}$ of the centre-of-mass and the relative coordinate $\vec{r}=$ $\vec{r}_{1}-\vec{r}_{2}$. These coordinates are related via

$$
\begin{aligned}
& \vec{r}_{1}=\vec{R}+\frac{\mu}{m_{1}} \vec{r}, \\
& \vec{r}_{2}=\vec{R}+\frac{\mu}{m_{2}} \vec{r} .
\end{aligned}
$$

Given the homogeneity of space the force between two particles only depends on the relative coordinate $\vec{r}$, i.e. $\vec{F}\left(\vec{r}_{1}, \vec{r}_{2}\right)=\vec{F}(\vec{r})$. In this case the centre-of-mass and relative motions of a closed system (no external forces) are decoupled and follow the equations

$$
\begin{aligned}
& M \ddot{\vec{R}}=0, \\
& \mu \ddot{\vec{r}}=\vec{F}_{1,2}(\vec{r}) .
\end{aligned}
$$

${ }^{14}$ The problem of motion in three dimensions is thus reduced to that of motion in a plane. A typical example is the motion of the planets in the gravitational field of the sun.
} 
Centre-of-mass frame

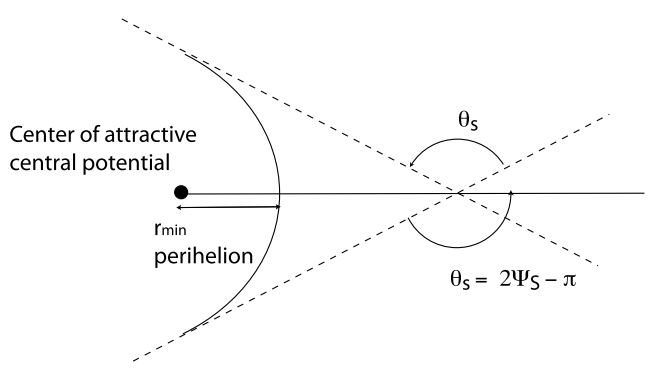

Fig. 8. Schematic motion of a particle in an attractive central potential $v(r)$. The angle $\Psi_{\mathrm{S}}$ of the particle trajectory with respect to the line joining the centre of the potential to the perihelion is directly related to the scattering angle $\Theta_{\mathrm{S}}$.

In the centre-of-mass frame we place the origin at the centre (see Fig. 7) and exploit that the momenta of both particles are oppositely equal

$$
\vec{J}=\vec{p}_{i 1}^{\text {c.m. }} \times \vec{r}_{i 1}^{\text {c.m. }}+\vec{p}_{i 2}^{\text {c.m. }} \times \vec{r}_{i 2}^{\text {c.m. }}=2 \vec{p}_{i 1}^{\text {c.m. }} \times \vec{r}_{i 1}^{\text {c.m. }} .
$$

As the centre-of-mass motion does not contribute to the angular momentum both values are as expected identical.

Defining the distance of one of the scattering partners from the asymptote of the trajectory of the other scattering partner as the impact parameter $b$ (see [64]) we obtain for the modulus of the angular momentum

$$
J=b_{i}\left|p_{i 1}^{\text {c.m. }}\right|=b_{i}\left|p_{i 2}^{\text {c.m. }}\right|=b_{f}\left|p_{f 1}^{\text {c.m. }}\right|=b_{f}\left|p_{f 2}^{\text {c.m. }}\right| .
$$

The situation is depicted in Fig. 8.

If we separate off the centre-of-mass motion and denote the potential of interaction by $V(r)$ then the trajectory of the target as characterised by the angle $\Psi_{\mathrm{S}}$ can be determined unambiguously from $V(r)$ using momentum and energy conservation ${ }^{15}$

$$
\Psi_{\mathrm{S}}=\int_{r_{\min }}^{\infty} \mathrm{d} r \frac{J}{r^{2} \sqrt{2 \mu[E-V(r)]-J^{2} / r^{2}}} .
$$

Using the one-to-one relation of the angular momentum $J$ with the impact parameter $b$ as expressed in Eq. (2.12) this expression can be reformulated as

$$
\Psi_{\mathrm{S}}=\int_{r_{\min }}^{\infty} \mathrm{d} r \frac{b}{r^{2} \sqrt{1-2 m V(r) / p^{2}-b^{2} / r^{2}}} .
$$

The scattering angle $\Psi_{\mathrm{S}}$ is, therefore, a functional of the potential $V(r)$ with $b$ acting as a parameter that contains all the relevant information about the incoming trajectory. By inversion of the problem it should, therefore, be possible to determine the particular form of $V(r)$ from the scattering angle.

\subsection{Classical scattering cross section}

The discussion so far concerned the deviation of a particle from its trajectory when penetrating the force field of another particle and the deterministic calculation of such a deviation. This approach is fully justified when dealing

\footnotetext{
${ }^{15}$ The reader interested in the proof can consult Chapter 5 of Ref. [64].
} 
with isolated macroscopic objects as found in celestial mechanics. In the laboratory it is generally impossible to follow the trajectories of individual particles, i.e. we are obliged to work with the statistical description outlined in Section 1.2. Therefore, provided all the criteria defining a scattering experiment are fulfilled (see Fig. 1) the result has to be expressed in the form of a differential cross section as introduced in Section 1.2. If we assume that each incoming particle is subjected to exactly one collision, i.e. if we exclude multiple scattering events, then the scattering can be described as a superposition of pair-wise collisions. ${ }^{16}$ If we assume in addition that the incoming beam is perfectly well collimated (all incoming particles have identical directions), monochromatic (all incoming particles have the same energies) and homogeneous (the distribution of incoming particles is uniform) and the interaction potential is isotropic then the statistical superposition can be described by the impact parameter $b$. In other words, for every collision what determines the scattering angle is the minimum distance that the incoming trajectory of the probe particle makes with the scattering particle. The situation is depicted in Fig. 9. Probe particles possessing identical $b$ values will experience the same scattering angles $\Theta$. The statistical weight of these particles corresponds to the areas of the ring

$$
\mathrm{d} A_{\text {incident }}=b \mathrm{~d} b \mathrm{~d} \Phi
$$

All these particles will end up in a ring with angular opening $\sin \Theta d \Theta$ with $\Theta=\Theta(b)$ for a monotonously changing potential. ${ }^{17}$ The cross section can, therefore, be written as

$$
\frac{\mathrm{d} \sigma}{\mathrm{d} \Omega}=\left|\frac{b \mathrm{~d} b \mathrm{~d} \Phi}{\sin \Theta \mathrm{d} \Theta \mathrm{d} \Phi}\right|=\left|\frac{b \mathrm{~d} b}{\sin \Theta \mathrm{d} \Theta}\right|=\frac{b}{\sin \Theta}\left|\frac{\mathrm{d} b}{\mathrm{~d} \Theta}\right| .
$$

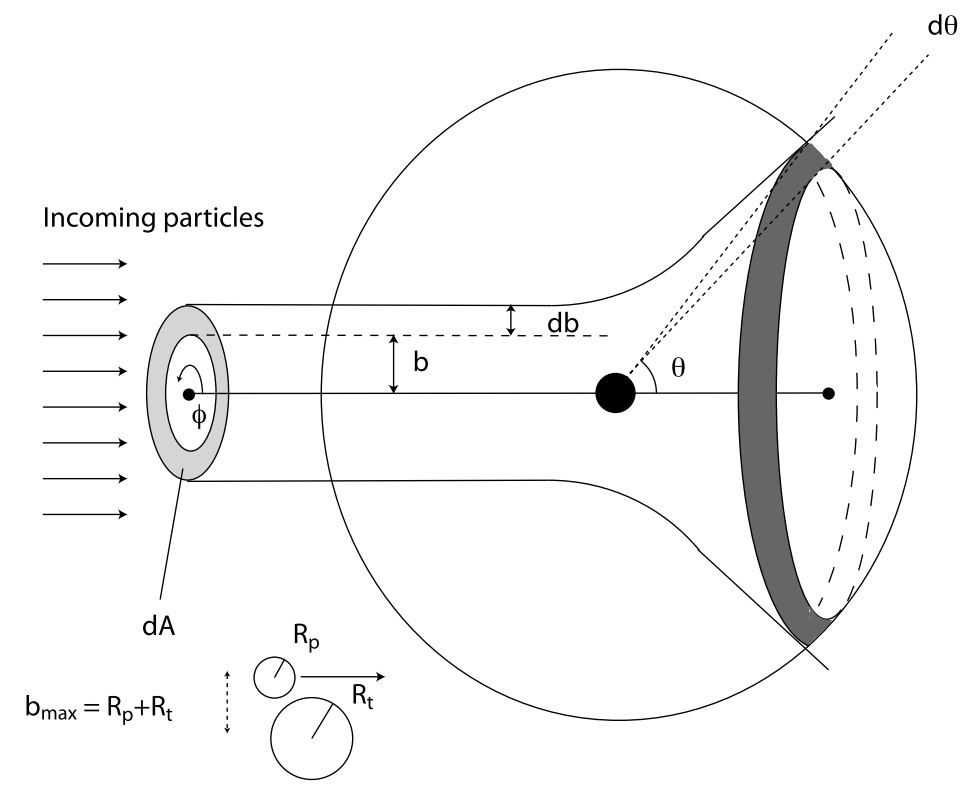

Fig. 9. Schematic presentation of classical scattering. All incoming particles with identical impact parameter are scattered under the same angle $\Theta$. This allows to express the differential cross section via the differential area $\mathrm{d} A_{\text {incident }}=b \mathrm{~d} b \mathrm{~d} \Phi$. It is assumed that the interaction potential is isotropic and that the collimated, monochromatic incoming beam is uniformly distributed over the area perpendicular to its trajectory. For a collision of two hard spheres the maximum impact parameter $b_{\max }$ is given by the sum of radii.

\footnotetext{
${ }^{16}$ Multiple scattering will come up again in the quantum mechanical description. The approximation neglecting higher order events will be termed Born approximation (see Section 3.11).

${ }^{17}$ We work here in the laboratory frame, which is the natural frame for expressing cross sections as it is in the laboratory that the cross sections are measured. We postpone the discussion of the cross section in the centre-of-mass frame to Section 3.8 .
} 
From this expression we can calculate directly the total cross section

$$
\sigma_{t}=\int \frac{\mathrm{d} \sigma}{\mathrm{d} \Omega} \mathrm{d} \Omega=2 \pi \int_{0}^{\pi} \sin \Theta \mathrm{d} \Theta \frac{b}{\sin \Theta}\left|\frac{\mathrm{d} b}{\mathrm{~d} \Theta}\right|=2 \pi \int_{0}^{b_{\max }} b \mathrm{~d} b=\pi b_{\max }^{2},
$$

provided that the interaction has a limited range that leads to negligible scattering beyond a certain maximum impact parameter $b_{\max }$. For a collision of hard spheres the maximum impact parameter is given as $b_{\max }=R_{p}+R_{t}$, where $R-p$ and $R_{t}$ are the radii of the probe and target particles, respectively. If the probe particle is point-like then the total scattering cross section is in this case identical to the classical cross section of the target sphere

$$
\sigma_{t}=\pi R_{t}^{2}
$$

We will see in Section 3.5 that the quantum mechanical treatment gives a different result (see Eq. (3.116)).

\section{Quantum mechanical scattering from a static potential}

In order to take advantage of neutron experiments we need a quantum mechanical formalism that relates the measured intensities to the properties of the sample and this in the most direct way possible. Like for the classical case we start the discussion with the scattering of one particle ${ }^{18}$ of mass $m$ by a static potential ${ }^{19} V(\vec{r})$. Thus scattering will then necessarily be elastic.

From a quantum mechanical point of view the problem is described by the Schrödinger equation ${ }^{20}$

$$
\mathrm{i} \hbar \frac{\partial}{\partial t} \psi(\vec{r}, t)=\mathbf{H} \psi(\vec{r}, t)=\left[-\frac{\hbar^{2}}{2 m} \Delta+V(\vec{r})\right] \psi(\vec{r}, t) .
$$

Since there is no explicit time dependence of the potential the energy of the system is conserved and the solutions are stationary. They have the form

$$
\psi(\vec{r}, t)=u(\vec{r}) \mathrm{e}^{-\mathrm{i}(E / \hbar) t},
$$

where $u(\vec{r})$ is an eigenfunction of the Hamiltonian $\mathbf{H}$

$$
\left[-\frac{\hbar^{2}}{2 m} \Delta+V(\vec{r})\right] u(\vec{r})=E u(\vec{r}) .
$$

Our task can then be described as finding the eigenfunctions $u(\vec{r})$. The first observations that we can make concerns the general nature of these states. As the probe particles have to be free before and after the scattering event we are not concerned with the bound states of the potential $V(\vec{r})$. Hence, scattering can be considered as the spectroscopy of the non-bound states.

\footnotetext{
${ }^{18}$ As we have seen before this includes both the scattering of a particle beam by a fixed target particle as well as the scattering by a free particle that takes into account the possibility of recoil. Mathematical this situation demands that we work in the reference system of the centreof-mass and replace for the relative motion the neutron mass $m$ by its reduced mass $\mu$. We do not find it necessary to add this complication to our general discussion, since for the majority of problems in neutron scattering the mass of the target system can anyway be assumed large compared to that of the probe. We will briefly discuss the difference in scattering by a free and a bound particle in Section 3.8.

${ }^{19}$ Whether in a particular case the interaction between two particles can be described by a static potential has to be checked. For example, the interaction with light requires the use of a vector potential $\vec{A}(\vec{r}, t)$ dependent on time. However, the formalism is still valid with some necessary adaptations.

${ }^{20}$ The Schrödinger equation is a non-relativistic equation. All results derived from it are thus only valid for particles with a small velocity compared to the velocity of light. Despite the similarities existing among all scattering experiments that we have mentioned in the introduction the non-relativistic approach is evidently not applicable to high energy collision experiments.
} 


\subsection{The wave function in the asymptotic regime}

Like in the classical case it is impossible to determine the wave function of the scattered particles in the region of $V(\vec{r})$ without knowledge of the potential. We, therefore, turn our interest towards asymptotic solutions to Eq. (3.3), which means that we are interested in the form of the wave functions in regions far from the location of impact. In accordance with the requirements for scattering experiments we demand that the potential $V(\vec{r})$ decreases rapidly with the distance $\vec{r}$. The most general form of the eigenfunctions is given by

$$
u_{\vec{k}}(\vec{r}) \longrightarrow \frac{1}{\sqrt{V}}\left(\mathrm{e}^{\mathrm{i} \vec{k} \cdot \vec{r}}+f_{\vec{k}}(\theta, \phi) \frac{\mathrm{e}^{\mathrm{i} k r}}{r}\right), \quad r \rightarrow \infty,
$$

with the modulus of the probe particle's wave vector $k$ before and after the scattering given as a function of the conserved energy $E$

$$
k=\frac{1}{\hbar} \sqrt{2 m E}
$$

In order to demonstrate this we insert the expression (3.4) into the Schrödinger equation. Remembering that the Laplace operator in spherical coordinates is written as

$$
\Delta=\frac{\partial^{2}}{\partial r^{2}}+\frac{2}{r} \cdot \frac{\partial}{\partial r}+\frac{1}{r^{2}}\left(\frac{1}{\sin \theta} \cdot \frac{\partial}{\partial \theta} \sin \theta \frac{\partial}{\partial \theta}+\frac{1}{\sin ^{2} \theta} \frac{\partial^{2}}{\partial \phi^{2}}\right),
$$

which simplifies to

$$
\frac{\partial^{2}}{\partial r^{2}} \text { for } r \rightarrow \infty
$$

we find

$$
\frac{\partial^{2}}{\partial r^{2}}\left(\frac{\mathrm{e}^{\mathrm{i} k r}}{r}\right)+\frac{2 m E}{\hbar^{2}}\left(\frac{\mathrm{e}^{\mathrm{i} k r}}{r}\right)=0+\mathrm{O}\left(\frac{1}{r^{2}}\right) .
$$

The functions (3.4), whose existence are in principle to be proved, are thus asymptotic and stationary solutions to the Schrödinger equation. The role of the normalisation volume $V$ for free particle states is discussed in all text books of quantum mechanics [10,41].

At this point we briefly have to discuss the fact that the scattering process is time dependent. An incident particle is created at $t \rightarrow-\infty$, propagates freely, experiences the potential of the target at $t \approx 0$, is scattered with a certain probability, propagates again freely, and is finally registered in a detector at $t \rightarrow+\infty$. One formalism capable of explicitly taking this time dependence into account are wave packages that can be constructed from stationary states via superposition

$$
\psi(\vec{r}, t)=\frac{1}{\sqrt{V}} \int \mathrm{d}^{3} k w(\vec{k}) u_{\vec{k}}(\vec{r}) \exp \left(-\mathrm{i} \omega_{k} t\right) .
$$

The wave packages, as the particles, travel in time and thus allow us to determine the dynamics of the scattering process. A typical piece of relevant dynamical information is the time delay that a particle experiences when reflected from a potential barrier due to the finite penetration of the wave function into the barrier (see Ref. [10]). The price for the ease of interpretation and dynamical information is a rather heavy formalism. The main information of the wave package is fortunately contained in the function $w(\vec{k})$ that describes the composition of the package 
in terms of stationary waves. This distribution is in itself time independent. In particular, it is not modified by the scattering process. In other words, each function $u_{\vec{k}}(\vec{r})$ constitutes a scattering channel or scattering path and the different channels can be superimposed. ${ }^{21}$ This is the reason why we can circumvent the wave packages and get a good kinematic description of the scattering process if we limit ourselves to stationary processes.

The stationary wave functions are interpreted in terms of a probability current. These currents describe the flux of particles. They allows us to make the link to the cross sections defined previously. The probability density of a particle is given by the square of the norm of the wave function

$$
\rho(\vec{r}, t)=\psi(\vec{r}, t) \psi(\vec{r}, t)^{*}=|\psi(\vec{r}, t)|^{2}
$$

The probability of finding the particle at time $t$ in the volume $\mathrm{d}^{3} r$ around $\vec{r}$ is then given by $\rho(\vec{r}, t) \mathrm{d}^{3} r$. The integral of $\rho(\vec{r}, t)$ over the entire space is by definition constant, but its local value can vary. These variations give rise to a probability current $\vec{J}(\vec{r}, t)$, which can be calculated as

$$
\vec{J}(\vec{r}, t)=\frac{\hbar}{2 m \mathrm{i}}\left(\psi^{*} \vec{\nabla} \psi-\psi \vec{\nabla} \psi^{*}\right)
$$

and obeys the continuity equation

$$
\frac{\partial}{\partial t} \rho(\vec{r}, t)+\vec{\nabla} \cdot \vec{J}(\vec{r}, t)=0
$$

Relations of this type govern the flow of any conserved quantity. They simply states that the probability of finding a particle in a given region only evolves if it is accompanied by a probability current that crosses the surface of the region in question. We can always write wave functions in the form

$$
\psi(\vec{r}, t)=A(\vec{r}, t) \exp (\mathrm{i} \Phi(\vec{r}, t))
$$

With this notation the probability density is related to the square of the amplitude

$$
\rho(\vec{r}, t)=A^{2}(\vec{r}, t)
$$

and the probability current to the probability density multiplied by the gradient of the phase

$$
\vec{J}(\vec{r}, t)=\rho \vec{\nabla}\left(\frac{\hbar \Phi}{m}\right)
$$

of the wave function. Hence the expression $\vec{\nabla}(\hbar \Phi / m)$ can be interpreted as the velocity of the probability flow. For a plane wave

$$
\psi(\vec{r}, t)=A \exp (\mathrm{i}(\vec{k} \cdot \vec{r}-\omega t))
$$

we find

$$
\rho(\vec{r}, t)=|A|^{2}
$$

\footnotetext{
${ }^{21}$ Attention, this is not the case if we construct the wave packages from plane waves $\psi(\vec{r}, t) \propto \exp (\mathrm{i}(\vec{k} \cdot \vec{r}-\omega t))$. In this case the form of the wave package is only conserved if the scattering amplitude $f_{\vec{k}}(\theta, \phi)$ varies sufficiently slowly with $\vec{k}$. This is not always the case. A typical example is Bragg scattering from a perfect crystal (see Sections 4.6 and 11.5), that can make the scattered wave package much better defined than the initial wave package.
} 


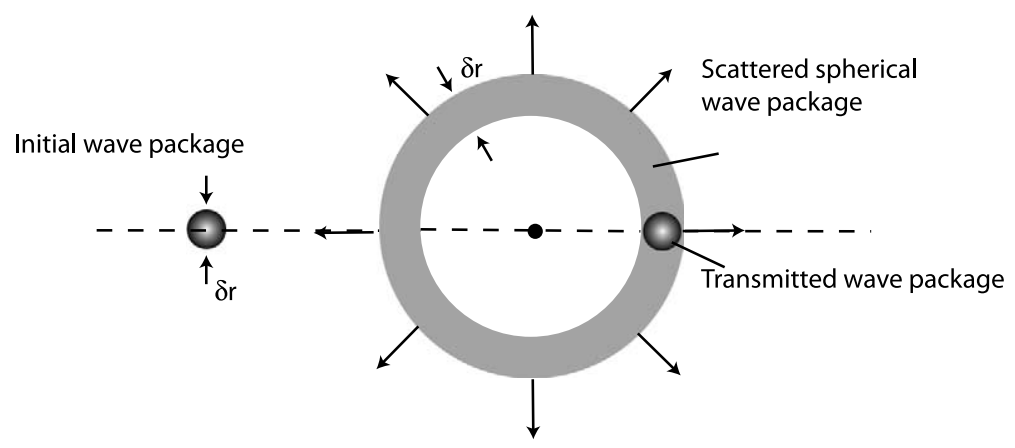

Fig. 10. Schematic illustration of the wave function corresponding to a scattering experiment. A source emits particles that pass through a collimator. This defines the direction and the lateral extension of the wave packet. An appropriate filter selects an energy band, which among other things affects the extension of the wave packet. The beam now collimated and monochromatic hits the sample, which scatters the particles in space. The scattered particles propagate and are then analysed (filtered) with respect to their final state (for example their energy) before being detected. All these processes are described by a spherical wave.

and

$$
\vec{J}(\vec{r}, t)=|A|^{2} \vec{\nabla}(\hbar \Phi / m)=\rho(\vec{r}, t) \frac{\vec{p}}{m}=\rho(\vec{r}, t) \vec{v}_{g}
$$

$v_{g}$ is the group velocity associated with the momentum $\hbar \vec{k}$. In the case of a plane wave the flow is therefore stationary. It can be identified with a uniform, continuous beam of non-interacting, identical particles.

The solutions (3.4) to the Shrödinger equation can be interpreted in the following way (see also Fig. 10).

(1) The function

$$
\frac{1}{\sqrt{V}} \mathrm{e}^{\mathrm{i} \vec{k} \cdot \vec{r}}
$$

describes a flux of free particles with momentum $\vec{p}=\hbar \vec{k}$. If we chose to normalise the wave functions describing free particle with respect to a unit box of dimensions $L_{x}=L_{y}=L_{z}=1$ then the normalisation volume $V$ becomes

$$
V=(2 \pi)^{3}
$$

and the wave vectors take on the simple values

$$
\vec{k}=\left(k_{x}, k_{y}, k_{z}\right)=\left(n_{x}, n_{y}, n_{z}\right), \quad n_{x}, n_{y}, n_{z}=\cdots-2,-1,0,1,2, \ldots
$$

In this normalisation the associated probability flux is expressed as

$$
\vec{J}(\vec{r}, t)=\frac{1}{(2 \pi)^{3}} \frac{\hbar}{m} \vec{k} .
$$

In the formalism of wave packages, it is this part that survives at negative times, i.e. long before the impact. Formally the extension in the lateral dimension, i.e. the dimension perpendicular to the propagation direction, is infinite for a plane wave. In practice we require that the beam is sufficiently large, in order to entirely illuminate the sample. At the same time the beam should be sufficiently narrow in order not to interfere with the scattered beam at the place of detection. 
(2) The wave function

$$
\frac{1}{\sqrt{V}} f_{\vec{k}}(\theta, \phi) \frac{\mathrm{e}^{\mathrm{i} k r}}{r}
$$

is the mathematical expression for a propagating spherical wave with wave vector $k=2 \pi / \lambda$. It is essential to remark that it is the absolute values $r$ and $k$ that are present in this expression. The propagation direction is always from the sample $(r=0)$ towards the detector $(r \rightarrow \infty)$. The spherical wave describes the scattered particles. Given that the potential is time independent the scattering is elastic, this means that the moduli of the wave vectors before and after the scattering are identical. The amplitude of the scattered wave function decreases as $r^{-1}$ as is required in order for the norm

$$
\int \rho(\vec{r}, t) r^{2} \mathrm{~d} r
$$

of the probability density

$$
\rho(\vec{r})=\frac{1}{V} \frac{1}{r^{2}}\left|f_{\vec{k}}(\theta, \phi)\right|^{2}
$$

to be preserved as a function of $r$. Expressed differently, the probability of finding a scattered particle under a given angle cannot depend on the distance between the detector and the sample.

The wave package associated with the spherical wave possesses the same group velocity as the initial particle. Its amplitude evolves in the same way as the transmitted wave package (see Fig. 10). Hence it is not surprising the flux associated with the scattered wave is given by

$$
J_{\text {scatt }}=J_{\text {ini }} \frac{1}{r^{2}}|f(\theta, \phi)|^{2}, \quad r \rightarrow \infty
$$

All the information concerning the interaction of particle with the potential that survives in the asymptotic regime is contained in the function

$$
f_{\vec{k}}(\theta, \phi)
$$

$f_{\vec{k}}(\theta, \phi)$ has the dimension of length and is called the scattering amplitude. $f_{\vec{k}}(\theta, \phi)$ modulates the spherical wave as a function of angles $\theta$ and $\phi$. It expresses in a mathematical way the fact that scattering encodes the interaction potential in the angular dependence of the scattered beam. It is clear that the scattering amplitude $f_{\vec{k}}(\theta, \phi)$ cannot take on any arbitrary form. In particular it has to be ensured that the probability of scattering and of transmission in the absence of absorption add up to one.

Using the expressions (3.25) and (1.7) we get the relation between the cross section and the scattering amplitude

$$
\frac{\mathrm{d} \sigma}{\mathrm{d} \Omega}=r^{2} \frac{J_{\text {scatt }}(r, \theta, \phi, t)}{J_{\text {ini }}(t)}=|f(\theta, \phi)|^{2} .
$$

We see that the normalisation volume $V$ is no longer present in this expression.

\subsection{Reflection and transmission at a potential barrier as an illustrative example}

We will continue with an example that allows us to explicitly calculate the wave functions and thus illustrates the concepts developed above. The most simple scattering problem we can think of is a potential barrier in one 
dimension. Incoming particles can either pass the barrier or be reflected. The problem is solved when we have determined the coefficients of transmission and reflection. The scattering potential has the simple form

$$
\begin{aligned}
& V(x)=V_{0}>0, \quad \frac{a}{2} \geqslant x \geqslant-\frac{a}{2}, \\
& V(x)=0, \quad \text { otherwise. }
\end{aligned}
$$

The stationary wave functions in the different regions are determined as

$$
\begin{aligned}
& u_{1}(x)=A_{1} \mathrm{e}^{\mathrm{i} k x}+A_{1}^{\prime} \mathrm{e}^{-\mathrm{i} k x}, \quad x<-\frac{a}{2}, \\
& u_{2}(x)=A_{2} \mathrm{e}^{\mathrm{i} k^{\prime} x}+A_{2}^{\prime} \mathrm{e}^{-\mathrm{i} k^{\prime} x}, \quad \frac{a}{2} \geqslant x \geqslant-\frac{a}{2}, \\
& u_{3}(x)=A_{3} \mathrm{e}^{\mathrm{i} k x}, \quad x>\frac{a}{2},
\end{aligned}
$$

with

$$
\begin{aligned}
k & =\sqrt{\frac{2 m E}{\hbar^{2}}}, \\
k^{\prime} & =\sqrt{\frac{2 m\left(E-V_{0}\right)}{\hbar^{2}}} .
\end{aligned}
$$

$E$ and $k$ are the energy and wave vector of the incoming particles, respectively. The wave number $k^{\prime}$ is real for particle energies $E$ greater than the barrier height and complex otherwise. A real wave vector corresponds to a propagating wave while a complex wave vector leads to an exponentially decaying wave function.

In the region $x<-a / 2$ the wave function $u_{1}(x)$ gives rise to two probability currents that are travelling in opposite direction.

- The current propagating towards the potential barrier is characterised by the density $\rho_{\text {in }}=\left|A_{1}\right|^{2}$. It is to be identified with the incoming particle flux.

- The current propagating backwards, away from the potential barrier, is characterised by the density $\rho_{\text {ref }}=$ $\left|A_{1}^{\prime}\right|^{2}$. It is to be identified with the reflected, i.e. scattered particle flux.

In the region $x>a / 2$ the wave function $u_{3}(x)$ gives rise to a single probability current propagating towards infinity. It is characterised by the density $\rho_{\text {trans }}=\left|A_{3}\right|^{2}$. It is to be identified with the transmitted particle flux.

By requiring the overall wave function to be square integrable ${ }^{22}$ we impose 4 conditions on the five amplitudes, which allows us to express all of them in terms of the incoming particle density $\rho_{\text {in }}$ (see [10]). We may in particular calculate the coefficients of reflection $R$ and transmission $T$ for $E>V_{0}$

$$
\begin{aligned}
& R=\frac{\rho_{\text {ref }}}{\rho_{\text {in }}}=\left|\frac{A_{1}^{\prime}}{A_{1}}\right|^{2}=\frac{\left(k^{2}-k^{\prime 2}\right)^{2} \sin ^{2} k^{\prime} a}{4 k^{2} k^{\prime 2}+\left(k^{2}-k^{\prime 2}\right)^{2} \sin ^{2} k^{\prime} a}, \\
& T=\frac{\rho_{\text {trans }}}{\rho_{\text {in }}}=\left|\frac{A_{3}}{A_{1}}\right|^{2}=\frac{4 k^{2} k^{\prime 2}}{4 k^{2} k^{\prime 2}+\left(k^{2}-k^{\prime 2}\right)^{2} \sin ^{2} k^{\prime} a} .
\end{aligned}
$$

As required by particle number conservation the sum of reflected and transmitted current is equal to the incident current

$$
R+T=1 .
$$

\footnotetext{
${ }^{22}$ This simply implies that both $u(x)$ and $\mathrm{d} u / \mathrm{d} x$ have to be continuous.
} 
If we express the transmission coefficient as a function of energy we obtain

$$
\begin{aligned}
& T=\frac{4 E\left(E-V_{0}\right)}{4 E\left(E-V_{0}\right)+V_{0}^{2} \sin ^{2}\left[\sqrt{2 m\left(E-V_{0}\right)} a / \hbar\right]}, \quad E \geqslant V_{0}, \\
& T=\frac{4 E\left(V_{0}-E\right)}{4 E\left(V_{0}-E\right)+V_{0}^{2} \sinh ^{2}\left[\sqrt{2 m\left(V_{0}-E\right)} a / \hbar\right]}, \quad E<V_{0} .
\end{aligned}
$$

This result is shown in Fig. 11. Contrary to the classical situation there is finite transmission even for energies smaller than the barrier height due to quantum mechanical tunnelling. Similarly, for energies larger than the barrier height we find oscillations in the transmission coefficient. Transmission equals one at energies associated with quantum mechanical resonances characterised by

$$
k^{\prime}=n \frac{\pi}{a}
$$

Resonances are, therefore observed when the wave length $\lambda^{\prime}$ fits into the barrier of width $a$

$$
n \lambda^{\prime}=2 a
$$

leading to

$$
T=\frac{4 E\left(E-V_{0}\right)}{4 E\left(E-V_{0}\right)+V_{0}^{2}}
$$

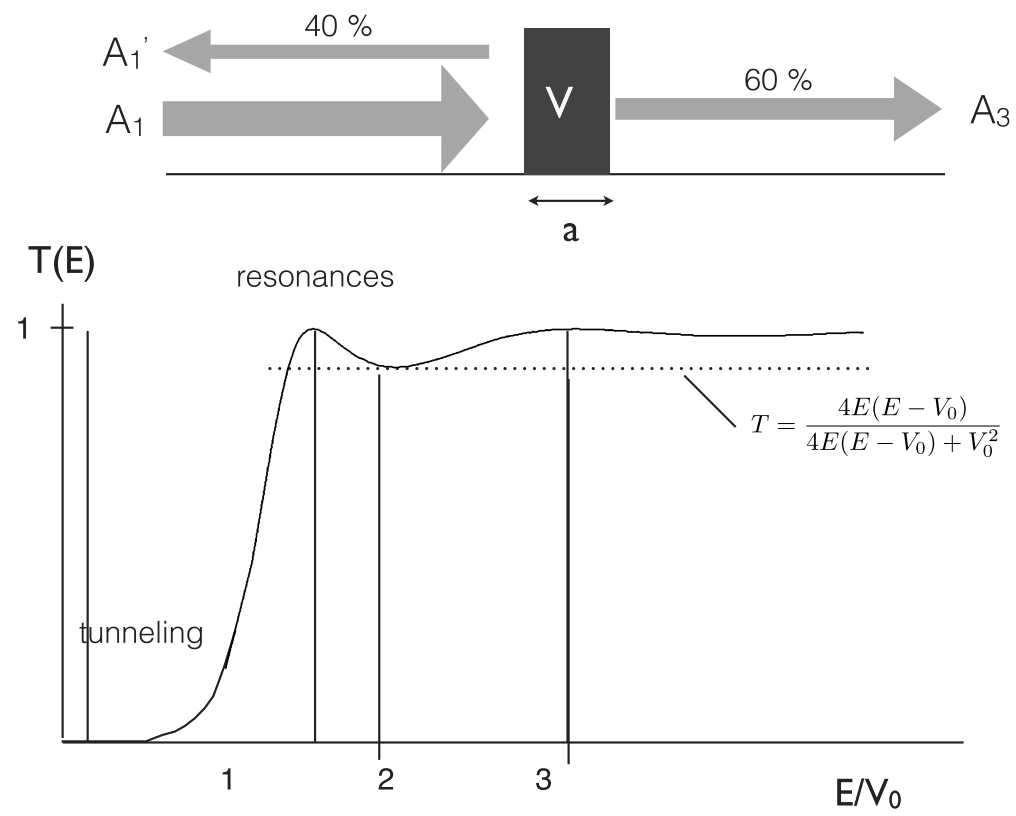

Fig. 11. Schematic presentation of a transmission function for a one-dimensional potential barrier. Contrary to the classical expectation there is transmission for incoming energies smaller than the barrier height due to quantum tunnelling. Similarly, for energies larger than the barrier height we find oscillations in the transmission coefficient (for more details see the text). 


\subsection{Partial waves}

In order to become more familiar with the concepts developed in the previous sections and in particular with the scattering amplitude we turn our attention towards isotropic potentials, i.e. spherical symmetric potentials where $V(\vec{r})=V(r) .^{23}$

We will see that in analogy to the classical treatment of Section 2.3 the scattering can be classified according to the angular momentum. Given the quantum nature of the angular momentum this leads in the quantum mechanical case to discrete scattering channels. Each channel is characterised by a wave function equally called partial wave. In our approach to determine the partial waves we are going to be guided by the quantum mechanical treatment of the hydrogen atom. It is well known that the wave functions of the electron bound in the Coulomb potential originating from the proton can be labelled with respect to the angular momentum operator $\vec{L}$. This is just a particular manifestation of the fact that for an isotropic potential the eigenfunctions $u(\vec{r})$ of the Hamiltonian can always be written as

$$
u(\vec{r})=u_{\operatorname{lm}}(r, \theta, \phi)=R_{l}(r) Y_{\operatorname{lm}}(\theta, \phi)
$$

with $r, \theta$ and $\phi$ denoting spherical coordinates

$$
\begin{aligned}
& x=r \sin \theta \cos \phi, \\
& y=r \sin \theta \sin \phi, \\
& z=r \cos \phi .
\end{aligned}
$$

The spherical harmonics $Y_{\operatorname{lm}}(\theta, \phi)$ are the eigenfunctions of the operators $\mathbf{L}^{2}$ and $\mathbf{L}_{z}$.

$$
\mathbf{L}^{2} Y_{\operatorname{lm}}(\theta, \phi)=l(l+1) \hbar^{2} Y_{\operatorname{lm}}(\theta, \phi)
$$

with

$$
\mathbf{L}^{2}=\hbar^{2}\left(\frac{1}{\sin \theta} \cdot \frac{\partial}{\partial \theta} \sin \theta \frac{\partial}{\partial \theta}+\frac{1}{\sin ^{2} \theta} \cdot \frac{\partial^{2}}{\partial \phi^{2}}\right)
$$

and

$$
\mathbf{L}_{z} Y_{\operatorname{lm}}(\theta, \phi)=m \hbar Y_{\operatorname{lm}}(\theta, \phi)
$$

with

$$
\mathbf{L}_{z}=-\mathrm{i} \hbar \frac{\partial}{\partial \phi}
$$

Thus, we can separate the angular and radial dependence of the wave functions. The angular dependence is described by the well known spherical harmonics that equally describe the electronic orbitals in hydrogen and as such is independent of the potential. The angular momentum quantum number $l$ acts as a parameter for the radial wave function and thus indeed will allow us classifying the scattering according to scattering channels. Let us now turn our attention to the problem that consists in determining the radial wave function for each $l$ value.

\footnotetext{
${ }^{23}$ The formalism that we present can from time to time seem tedious. However this mathematical complexity is unavoidable if we wish to clearly establish important concepts such as the scattering length. The reader only interested in the use of the formalism can proceed directly to Section 3.9 .
} 
The radial function $R_{l}(r)$ has to satisfy the equation

$$
\left(-\frac{\hbar^{2}}{2 m}\left[\frac{\mathrm{d}^{2}}{\mathrm{~d} r^{2}}+\frac{2}{r} \frac{\mathrm{d}}{\mathrm{d} r}-\frac{l(l+1)}{r^{2}}\right]+V(r)\right) R_{l}(r)=E R_{l}(r)
$$

with the boundary conditions

$$
R_{l}(r) \propto r^{l+1}, \quad \text { for } r \rightarrow 0
$$

We can simplify this differential equation significantly by factoring out $R_{l}(r)$ into $w_{l}(r) / r$

$$
\left(-\frac{\hbar^{2}}{2 m}\left[\frac{\mathrm{d}^{2}}{\mathrm{~d} r^{2}}-\frac{l(l+1)}{r^{2}}\right]+V(r)\right) w_{l}(r)=E w_{l}(r)
$$

The term

$$
\frac{\hbar^{2}}{2 m} \frac{l(l+1)}{r^{2}}
$$

is called the centrifugal barrier in analogy to classical mechanics.

In the absence of a potential the radial equation (3.46) reduces to

$$
\left[\frac{\mathrm{d}^{2}}{\mathrm{~d} \rho^{2}}+\frac{2}{\rho} \frac{\mathrm{d}}{\mathrm{d} \rho}+1-\frac{l(l+1)}{\rho^{2}}\right] R_{l}(\rho)=0,
$$

which is the defining equation of the spherical Bessel functions ${ }^{24} j_{l}(\rho)$ and $n_{l}(\rho)$, provided that we express the distance $r$ in units of $\lambda / 2 \pi$, which is equivalent to setting $\rho=r \cdot k$. For the lowest values of $l$ these functions are given as

$$
\begin{aligned}
& j_{0}(\rho)=\frac{\sin \rho}{\rho}, \\
& n_{0}(\rho)=\frac{\cos \rho}{\rho}, \\
& j_{1}(\rho)=\frac{\sin \rho}{\rho^{2}}-\frac{\cos \rho}{\rho}, \\
& n_{1}(\rho)=\frac{\cos \rho}{\rho^{2}}+\frac{\sin \rho}{\rho} .
\end{aligned}
$$

\footnotetext{
${ }^{24}$ The complete basis of solutions to this equation comprises both the spherical Bessel functions in the proper sense and the spherical von Neumann functions. These functions are connected to the ordinary Bessel functions $J(\rho)$ through

$$
\begin{aligned}
& j_{l}(\rho)=\sqrt{\frac{\pi}{2 \rho}} J_{l+1 / 2}(\rho), \\
& n_{l}(\rho)=(-1)^{l} \sqrt{\frac{\pi}{2 \rho}} J_{-(l+1 / 2)}(\rho) .
\end{aligned}
$$


The functions for higher values of $l$ can be obtained by differentiation

$$
\begin{aligned}
& j_{l}(\rho)=(-\rho)^{l}\left(\frac{1}{\rho} \cdot \frac{\mathrm{d}}{\mathrm{d} \rho}\right)^{l} \frac{\sin \rho}{\rho}, \\
& n_{l}(\rho)=(-\rho)^{l}\left(\frac{1}{\rho} \cdot \frac{\mathrm{d}}{\mathrm{d} \rho}\right)^{l} \frac{\cos \rho}{\rho} .
\end{aligned}
$$

A general solution of the radial part of the Schrödinger equation (3.48) can be expressed by a superposition of these functions

$$
R_{l}(\rho)=\sum_{l}\left[a_{l} j_{l}(\rho)+b_{l} n_{l}(\rho)\right]
$$

or alternatively, by posing $a_{l}=B_{l} \cos \delta_{l}$ and $b_{l}=B_{l} \sin \delta_{l}$ in the form

$$
R_{l}(\rho)=\sum_{l}\left[B_{l}\left(\cos \delta_{l} j_{l}(\rho)+\sin \delta_{l} n_{l}(\rho)\right] .\right.
$$

The usefulness of representing the coefficients of expansion in terms of an amplitude and phase is not clear at this stage of the discussion but will be justified later.

The functions $j_{l}(\rho)$ are shown for $l \leqslant 5$ in Fig. 12. The maxima for $l>0$ are close to the value

$$
r k \approx \sqrt{l(l+1)}
$$

that corresponds to the minimum distance between the trajectory of a classical particle with angular momentum

$$
|\vec{J}|=|\vec{r} \times \vec{p}|=\hbar \sqrt{l(l+1)}
$$

and the origin, i.e. to its impact parameter as defined in Section 2.3. The quantum mechanical $l$-channels are, therefore, the analog of the classical impact parameter channels for scattering. It is obvious that higher $l$ values will contribute only if the potential is sufficiently long range.

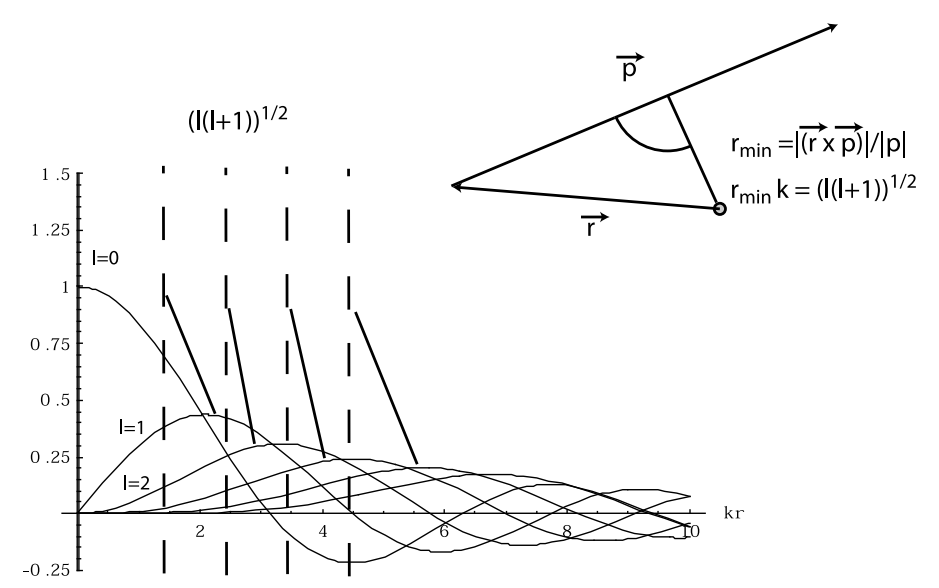

Fig. 12. Spherical Bessel functions. The maxima for $l>0$ are close to the value $r k \approx \sqrt{l(l+1)}$ that corresponds to the minimum distance between the trajectory of a classical particle with angular momentum $|\vec{J}|=|\vec{r} \times \vec{p}|=\hbar \sqrt{l(l+1)}$ and the origin. The case $l=0$ has to be assimilated with a scattering channel centred on the target particle. For targets that interact with the probe at distances very much shorter than the wave length $\lambda$ only this so-called $s$-type scattering channel survives. 


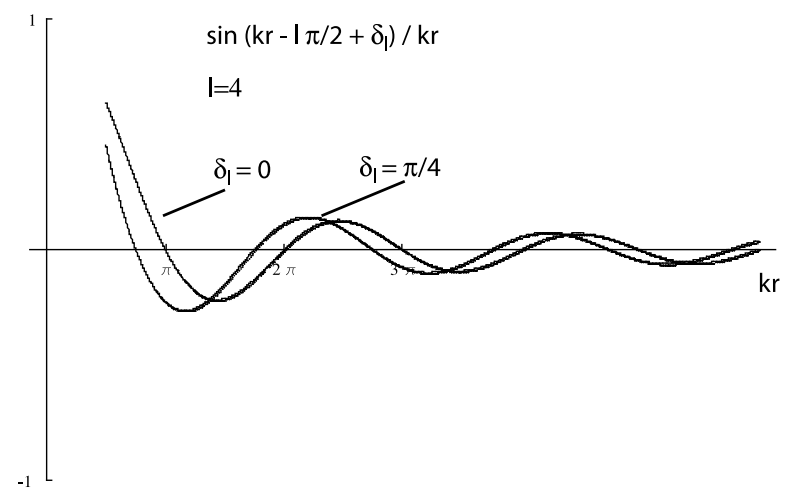

Fig. 13. Stationary spherical waves for $l=4$ with and without phase shift.

It can be clearly seen that for distances $r \ll \lambda$ the function with $l=0$ (called $s$-type) dominates all the others. Mathematically we find in the limit $\rho \rightarrow 0$

$$
\begin{aligned}
& j_{l}(\rho)=\frac{1}{1 \cdot 2 \cdot 3 \cdots(2 l+1)} \rho^{l}, \\
& n_{l}(\rho)=\frac{1 \cdot 2 \cdot 3 \cdots(2 l+1)}{(2 l+1)} \frac{1}{\rho^{l+1}} .
\end{aligned}
$$

Having developed expressions for the general form of the wave function (3.60) we now turn our attention to the asymptotic regime. For $\rho \gg l(l+1)$ the Bessel functions simplify to

$$
\begin{aligned}
& j_{l}(\rho)=\frac{\sin (\rho-l \pi / 2)}{\rho}, \\
& n_{l}(\rho)=\frac{\cos (\rho-l \pi / 2)}{\rho} .
\end{aligned}
$$

We are dealing with simple stationary spherical waves ${ }^{25}$ (see Fig. 13). The stationary character of the solution is not a surprise. It reflects the fact that a quantum mechanical state with a fixed angular momentum cannot propagate in space.

We can use the approximation (3.63) and (3.64) in order to reformulate the expansion (3.60) of the radial function in the case of large distances as

$$
R_{l}(\rho) \approx \sum_{l} B_{l} \frac{\sin \left(\rho-l \pi / 2+\delta_{l}\right)}{\rho}
$$

If we restrict ourselves to the scattering of non-polarised particles, it is clear that the result of the experiment cannot depend on the angle $\phi$. In other words, the intrinsic symmetry of the problem imposes that the scattering probability possesses cylindrical symmetry around the direction of the incident beam. ${ }^{26}$ In this case it suffices to

\footnotetext{
${ }^{25}$ The adjective "stationary" is here employed in the sense of stationary waves and not in the sense of quantum mechanics.

${ }^{26}$ If the incoming particles possess a fixed polarisation with a component perpendicular to the trajectory the cylindrical symmetry about the incoming direction is broken. This will be reflected in the scattering if it is spin dependent.
} 
keep the functions $u_{l 0}$ in the expansion (3.40) as they are the only ones independent of $\phi$. Far from the sample we can then, using expressions (3.65) and (3.40), express the wave function as

$$
u(\vec{r})=\sum_{l=0}^{\infty} B_{l} \frac{\sin \left(\rho-l \pi / 2+\delta_{l}\right)}{\rho} Y_{l 0}(\theta) .
$$

Therefore, far away from the interaction region the wave function of a particle interacting with an isotropic potential can be described by a superposition of spherical waves. The amplitude and phase of each spherical wave is characterised by the angular momentum quantum number $l$. The angular dependence is fully contained in the respective spherical harmonics.

Everything we did so far was to find the most general form of the wave function of a particle in an isotropic potential. The scattering geometry, however, imposes additional constraints on the wave function. These constraints will allow us determining the functional dependence of the amplitudes $B_{l}$ with respect to the phase shift $\delta_{l}$. Having done so we will be in a position to express the scattering amplitude $f(\theta)$ purely as a function of the set of phase shifts $\left\{\delta_{l}\right\}$.

The scattering amplitude had been defined in general terms by expression (3.4). This expression describes the incoming free particle flux as a plane wave. To connect it with the partial wave formalism that we have developed here we must briefly revisit the free Schrödinger equation, however, imposing the requirement that the solutions posses well-defined angular momenta $l$ (with respect to a given reference point, which we identify with the origin of the coordinate system). This exercise will allow us to relate the wave function of the incident particle to that of the scattered particle in the same basis.

The functions $n_{l}(\rho)$ diverge at the origin. Hence they cannot contribute to the expansion of $R_{l}(\rho)$ at the origin, and in particular not to the expansion of the wave function of a free particle in this basis. The basis for all possible states of a free particle is thus given by the functions

$$
u_{\operatorname{lm}}(r, \theta, \phi)=k \sqrt{\frac{2}{\pi}} j_{l}(k r) Y_{\operatorname{lm}}(\theta, \phi) .
$$

In particular, the wave function of a particle with its momentum parallel to the direction $\hat{z}$ is expressed as

$$
\begin{aligned}
\frac{1}{\sqrt{V}} \mathrm{e}^{\mathrm{i} k z} & =\frac{1}{\sqrt{V}} \mathrm{e}^{\mathrm{i} k r \cos \theta} \\
& =\sqrt{\frac{4 \pi}{V}} \sum_{l=0}^{\infty} \sqrt{2 l+1} \mathrm{i}^{l} j_{l}(k r) Y_{l 0}(\theta) .
\end{aligned}
$$

Using the connection

$$
Y_{l 0}(\theta)=\sqrt{\frac{2 l+1}{4 \pi}} P_{l}(\cos \theta)
$$

between the spherical harmonics and the Legendre polynomials this expression can be put to the equivalent form

$$
\frac{1}{\sqrt{V}} \mathrm{e}^{\mathrm{i} k z}=\frac{1}{\sqrt{V}} \sum_{l=0}^{\infty}(2 l+1) \mathrm{i}^{l} j_{l}(k r) P_{l}(\cos \theta) .
$$

The form of $u(\vec{r})$ found here for an isotropic potential in terms of spherical waves has to be consistent with the expression (3.4) that was derived for the general case in terms of the scattering amplitude (repeated here for 
convenience)

$$
u_{\vec{k}}(\vec{r}) \longrightarrow \frac{1}{\sqrt{V}}\left(\mathrm{e}^{\mathrm{i} \vec{k} \cdot \vec{r}}+f_{\vec{k}}(\theta, \phi) \frac{\mathrm{e}^{\mathrm{i} k r}}{r}\right), \quad r \rightarrow \infty .
$$

Using the identity (3.68) this expression translates into

$$
u_{\vec{k}}(\vec{r}) \longrightarrow \sqrt{\frac{1}{V}}\left\{\left[\sum_{l=0}^{\infty} \sqrt{4 \pi(2 l+1)} i^{l} j_{l}(k r) Y_{l 0}(\theta)\right]+\left[f_{\vec{k}}(\theta, \phi) \frac{\mathrm{e}^{\mathrm{i} k r}}{r}\right]\right\} .
$$

As we are working in the asymptotic regime we may apply the approximation (3.63), which leads us to

$$
u_{\vec{k}}(\vec{r}) \longrightarrow \sqrt{\frac{1}{V}}\left\{\left[\sum_{l=0}^{\infty} \sqrt{4 \pi(2 l+1)} \mathrm{i} l \frac{\sin (k r-l \pi / 2)}{k r} Y_{l 0}(\theta)\right]+\left[f_{\vec{k}}(\theta) \frac{\mathrm{e}^{\mathrm{i} k r}}{r}\right]\right\} .
$$

Expressing the sinus function with the help of exponentials this expression can be reformulated as

$$
\begin{aligned}
u_{\vec{k}}(\vec{r}) \longrightarrow & \frac{1}{2 \mathrm{i}} \sqrt{\frac{1}{V}}\left\{\left[\sum_{l=0}^{\infty} \sqrt{4 \pi(2 l+1)} \mathrm{i}^{l} \mathrm{e}^{-\mathrm{i} l \pi / 2} Y_{l 0}(\theta)\right]+2 \mathrm{i} k f_{\vec{k}}(\theta)\right\} \frac{\mathrm{e}^{\mathrm{i} k r}}{k r} \\
& -\frac{1}{2 \mathrm{i}} \sqrt{\frac{1}{V}}\left[\sum_{l=0}^{\infty} \sqrt{4 \pi(2 l+1)} \mathrm{i}^{l} \mathrm{e}^{\mathrm{i} l \pi / 2} Y_{l 0}(\theta)\right] \frac{\mathrm{e}^{-\mathrm{i} k r}}{k r}
\end{aligned}
$$

Expression (3.66) can be brought into a similar form

$$
u(\vec{r})=\frac{1}{2 \mathrm{i}}\left[\sum_{l=0}^{\infty} B_{l} \mathrm{e}^{-\mathrm{i} l \pi / 2} \mathrm{e}^{\mathrm{i} \delta_{l}} Y_{l 0}(\theta)\right] \frac{\mathrm{e}^{\mathrm{i} k r}}{k r}-\frac{1}{2 \mathrm{i}}\left[\sum_{l=0}^{\infty} B_{l} \mathrm{e}^{\mathrm{i} l \pi / 2} \mathrm{e}^{-\mathrm{i} \delta_{l}} Y_{l 0}(\theta)\right] \frac{\mathrm{e}^{-\mathrm{i} k r}}{k r} .
$$

Comparing the two expressions for $u(\vec{r})$ allows us to determine the coefficients $B_{l}$ from the term proportional to $\frac{\mathrm{e}^{-\mathrm{i} k r}}{k r}$ as

$$
B_{l}=\frac{1}{\sqrt{V}} \mathrm{i}^{l} \mathrm{e}^{\mathrm{i} \delta_{l}} \sqrt{4 \pi(2 l+1)}=\frac{1}{\sqrt{V}} \mathrm{e}^{\mathrm{i} l \pi / 2+\mathrm{i} \delta_{l}} \sqrt{4 \pi(2 l+1)} .
$$

$B_{l}$ being determined the scattering amplitude can be obtained from comparing the terms proportional to $\frac{\mathrm{e}^{-\mathrm{i} k r}}{k r}$

$$
\begin{aligned}
f(\theta) & =\frac{1}{2 \mathrm{i} k} \sum_{l=0}^{\infty} \sqrt{4 \pi(2 l+1)}\left(\mathrm{e}^{2 \mathrm{i} \delta_{l}}-1\right) Y_{l 0}(\theta) \\
& =\frac{1}{2 \mathrm{i} k} \sum_{l=0}^{\infty}(2 l+1)\left(\mathrm{e}^{2 \mathrm{i} \delta_{l}}-1\right) P_{l}(\cos \theta) \\
& =\frac{1}{k} \sum_{l=0}^{\infty}(2 l+1)\left(\mathrm{e}^{\mathrm{i} \delta_{l}} \sin \delta_{l}\right) P_{l}(\cos \theta) \\
& =\sum_{l=0}^{\infty}(2 l+1) f_{l} P_{l}(\cos \theta),
\end{aligned}
$$


where all information concerning physics is contained in the intrinsically complex numbers

$$
f_{l} \equiv \frac{\mathrm{e}^{2 \mathrm{i} \delta_{l}}-1}{2 \mathrm{i} k}
$$

We have thus succeeded in expanding the scattering amplitude in the basis of the functions $Y_{l 0}(\theta)$, which are also called partial waves. The expansion coefficients are expressed as a function of the phases $\delta_{l}$. This is an important result, that shows us, that the scattering process can be described by a discrete parameter set that can be determined experimentally without any precise knowledge of the interaction potential. The phase shifts $\delta_{l}(k)$ depend explicitly on the modulus of the wave vector $k$. In this context, the abbreviation

$$
S_{l}(k)=\mathrm{e}^{2 \mathrm{i} \delta_{l}(k)}
$$

is often used. $S_{l}(k)$ is called matrix element of the partial wave $l$. We state without proving it that we are actually dealing with the matrix elements already encountered in Section 1.2 (see expression (1.5)). ${ }^{27}$

In order to clarify the role of the phase shift in a scattering process we will now compare (in the partial wave basis) the wave function $u(\vec{r})$ (3.66) with a plane wave. Inserting the expression (3.77) for $B_{l}$ into the expression (3.76) the wave function in the asymptotic regime can be found to be of the alternate form

$$
u(\vec{r})=\frac{1}{\sqrt{V}} \frac{\mathrm{i}}{2 k} \sum_{l=0}^{\infty}(2 l+1) P_{l}(\cos \theta)\left[(-1)^{l} \frac{\mathrm{e}^{-\mathrm{i} k r}}{r}-S_{l}(k) \frac{\mathrm{e}^{\mathrm{i} k r}}{r}\right] .
$$

The radial part of the terms in this sum is the superposition of an outgoing spherical wave

$$
u^{\text {out }}(r) \propto \frac{\mathrm{e}^{\mathrm{i} k r}}{r}
$$

and an incoming spherical wave

$$
u^{\text {in }}(r) \propto \frac{\mathrm{e}^{-\mathrm{i} k r}}{r} .
$$

This superposition is controlled by the factor $S_{l}$. For $V(r)=0$ no scattering takes place. Hence, the wave function should correspond to a plane wave. A plane wave can in full analogy to expression (3.81) be written in a basis of spherical waves as

$$
u(\vec{r})=\frac{1}{\sqrt{V}} \frac{\mathrm{i}}{2 k} \sum_{l=0}^{\infty}(2 l+1) P_{l}(\cos \theta)\left[(-1)^{l} \frac{\mathrm{e}^{-\mathrm{i} k r}}{r}-\frac{\mathrm{e}^{\mathrm{i} k r}}{r}\right] .
$$

It is again a superposition of an incoming and an outgoing spherical wave. Comparing (3.81) with (3.84) we can see that the difference introduced by the potential is the multiplication of the outgoing wave with the unitary complex factor $S_{l}(k)$. As a consequence, the potential produces a phase shift in the outgoing wave with respect

\footnotetext{
${ }^{27}$ The scattering matrix (or S-matrix) is a concept of fundamental importance in physics. Expressed in its most general form the S-matrix relates the initial state and the final state of a physical system undergoing a scattering process. It was historically introduced by John Archibald Wheeler in the article "On the mathematical description of light nuclei by the method of resonating group structure" that was published in 1937. From its definition it follows that the elements of the S-matrix, which are called scattering amplitudes, are closely related to the quantum mechanical transition probability amplitudes. Poles of the S-matrix in the complex-energy plane are identified with bound states, virtual states or resonances. From a historical point of view S-matrix theory, which was further developed by Werner Heisenberg, can be considered the cradle of modern string theory.
} 
to the incoming wave. The interference between the phase shifted waves creates a spherical stationary wave in the same way that two phonons can produce a plane stationary wave. It is this stationary wave that describes the scattering. Without any potential and hence without any phase shift the superpositions of partial waves results in a cancellation of the outgoing spherical wave.

Integrating the differential cross section (expression (3.27)) over the solid angle we can calculate the total cross section

$$
\sigma_{t}=2 \pi \int_{0}^{\pi}|f(\theta)|^{2} \sin \theta \mathrm{d} \theta
$$

The Legendre polynomials are orthogonal. This facilitates the calculation considerably. In the square of expression (3.78) only the diagonal terms in $l$ survive. These can easily be integrated. With the help of the relation

$$
\int_{0}^{\pi} P_{l}^{2}(\cos \theta) \sin \theta \mathrm{d} \theta=\frac{2}{2 l+1}
$$

we find

$$
\sigma_{t}=\frac{4 \pi}{k^{2}} \sum_{l=0}^{\infty}(2 l+1) \sin ^{2} \delta_{l}=4 \pi \sum_{l=0}^{\infty}(2 l+1)\left|f_{l}\right|^{2}=\sum_{l=0}^{\infty} \sigma_{t}(l) .
$$

The total cross section is thus the sum of the partial cross sections

$$
\sigma_{t}(l)=\frac{4 \pi}{k^{2}}(2 l+1) \sin ^{2} \delta_{l}=4 \pi(2 l+1)\left|f_{l}\right|^{2}
$$

In other words, each value of $l$ contributes a distinct scattering channel. This is not surprising since the angular momentum is a conserved quantity for an isotropic potential.

The phases $\delta_{l}$ are real. This implies that $\left|S_{l}\right|=1$. In expression (3.81) the amplitude associated with the incoming wave is then identical to the amplitude associated with the outgoing wave. This is the necessary condition for getting a stationary wave. The unitarity of $S_{l}(k)$ reflects the physical situation that we describe: The number of particles before and after the scattering is identical. If we had a sample capable of absorbing probe particles we would have $\left|S_{l}\right|<1$ for at least some values of $l$. This would imply that $\delta_{l}$ was complex.

The fact that the $\delta_{l}$ are real imposes an upper limit on the associated total cross sections

$$
\sigma_{t}(l)=4 \pi(2 l+1)\left|f_{l}\right|^{2}=\frac{4 \pi}{k^{2}}(2 l+1) \sin ^{2} \delta_{l} \leqslant \frac{4 \pi}{k^{2}}(2 l+1)
$$

These conditions are called the unitarity conditions of the partial cross sections. Within this unitarity limit the maximum values of the cross sections are obtained for

$$
\delta_{l}= \pm \frac{\pi}{2}
$$

\subsection{The optical theorem}

The scattering amplitude

$$
f(\theta)=\frac{1}{k} \sum_{l=0}^{\infty}(2 l+1)\left(\mathrm{e}^{\mathrm{i} \delta_{l}} \sin \delta_{l}\right) P_{l}(\cos \theta)
$$


is an intrinsically complex function. It can thus be decomposed into its real and imaginary part

$$
f(\theta)=\Re(f(\theta))+\mathrm{i} \Im(f(\theta))
$$

with

$$
\Re(f(\theta))=\frac{1}{k} \sum_{l=0}^{\infty}(2 l+1)\left(\cos \delta_{l} \sin \delta_{l}\right) P_{l}(\cos \theta)
$$

and

$$
\Im(f(\theta))=\frac{1}{k} \sum_{l=0}^{\infty}(2 l+1)\left(\sin ^{2} \delta_{l}\right) P_{l}(\cos \theta) .
$$

With the known property of the associated Legendre polynomials

$$
P_{l}(0)=1
$$

we obtain

$$
\Im(f(0))=\frac{1}{k} \sum_{l=0}^{\infty}(2 l+1)\left(\sin ^{2} \delta_{l}\right) .
$$

Comparing this with expression (3.88) we arrive at

$$
\sigma_{t}(l)=\frac{4 \pi}{k} \Im(f(0)) .
$$

This result is know as the optical theorem. It states that the amount of particles scattered under all angles is equivalent to the imaginary part of the scattering amplitude in the forward direction. The scattering, therefore, creates a shadow of the incident beam. This is a very general result given that it can be derived from the pure preservation of quantum mechanical probabilities.

\subsection{The scattering length}

The cut-off $l_{\max }$ - up to which partial waves have to be included in the expansion of the scattering amplitude $f(\theta)$ - scales with the range of the interaction potential. In this context the relevant length scale is given by the wave length of the probe particle. In fact, as we have seen in detail, the functions $j_{l}(r k)$ do not contribute significantly in the region of the potential $\left(r<r_{0}\right)$ unless $\sqrt{l(l+1)}<k r_{0}=2 \pi \frac{r_{0}}{\lambda}$. The partial waves that possess negligible presence in the interaction region of the potential can clearly not be strongly modified by the scattering process and as a consequence are of little importance in the expansion of $f(\theta)$.

The above statement can be cast into a rigorous mathematical form. It can be shown [41] that in all generality

$$
\delta_{l}(k) \propto k^{(2 l+1)}, \quad \text { for } k \rightarrow 0,
$$

and this independent of the shape of the potential. Hence we can without any ambiguity define

$$
b=-\lim _{k \rightarrow 0} \frac{\delta_{0}(k)}{k} .
$$


The quantity $b$ is called the scattering length. It is real when there is no absorption in the scattering process. When there is absorption the scattering length becomes complex and $k$ dependent. Hence the scattering length has to be specified for each $k$.

We would like to stress that the definition of the scattering length given here relies on working in the centre-ofmass frame. We will treat the cross section of free particles in Section 3.8.

As a consequence of the scaling expressed in (3.97) only the phase shift $\delta_{0}$ survives in the expression for the scattering amplitude if $k$ goes to 0

$$
\begin{aligned}
f(\theta)_{\vec{k} \rightarrow 0}^{l=0} & =\frac{1}{2 \mathrm{i} k} \sqrt{4 \pi}\left(\mathrm{e}^{2 \mathrm{i} \delta_{0}}-1\right) Y_{00}(\theta) \\
& =\frac{1}{2 \mathrm{i} k} \sqrt{4 \pi} Y_{00}(\theta)\left(2 \mathrm{i} \delta_{0}+\mathrm{O}\left(k^{2}\right)\right) \\
& =\frac{-1}{2 \mathrm{i} k} \sqrt{4 \pi} 2 \mathrm{i} b k \frac{1}{\sqrt{4 \pi}}+\mathrm{O}(k) \\
& =-b+\mathrm{O}(k)
\end{aligned}
$$

where we have used

$$
Y_{00}=\frac{1}{\sqrt{4 \pi}} .
$$

The scattering length can, therefore, equally be defined as

$$
b=-\lim _{k \rightarrow 0} f(\theta)
$$

with

$$
f(\theta)=\frac{\mathrm{e}^{2 \mathrm{i} \delta_{0}}-1}{2 \mathrm{i} k}=\frac{1}{k} \mathrm{e}^{\mathrm{i} \delta_{0}} \sin \delta_{0} .
$$

$f(\theta)$ is in particular independent of the scattering angle and thus isotropic for $k \ll r$. This result confirms that the structure of a sample can only be studied at length scales longer than the wave length of the probe particle.

In arguments involving the imaginary part of the scattering amplitude we cannot limit ourselves to terms of leading order. E.g. expression (3.93) becomes in the limit $k \rightarrow 0$

$$
\Im(f(0))_{\vec{k} \rightarrow 0}^{l=0}=\frac{1}{k}\left(b^{2} k^{2}\right)=k b^{2} .
$$

This is what we expect according to the optical theorem. As the total cross section for $k \rightarrow 0$ is given by

$$
\sigma_{t}^{l=0}=4 \pi b^{2}
$$

the optical theorem implies

$$
\Im(f(0))^{l=0}=\frac{k}{4 \pi} \sigma_{t}^{l=0}=k b^{2} \quad \text { for } k \rightarrow 0 .
$$

In Fig. 14 we give the scattering length for neutrons. They are generally of the order of a few femtometre $\left(10^{-15} \mathrm{~m}\right)$ leading to cross sections of a few barns. From the scattering length we can calculate the associated phase shift 


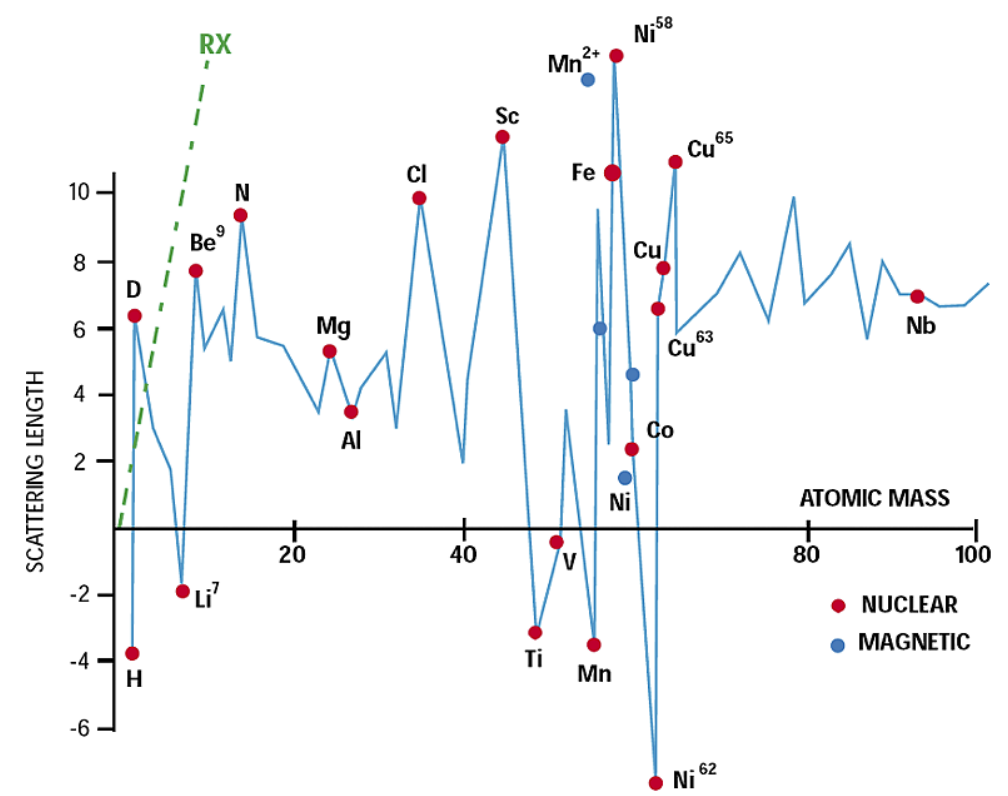

Fig. 14. Nuclear neutron scattering length as a function of isotope (red points). The units are femtometre leading to cross sections of a few barn ( 1 barn is equivalent to $10^{-24} \mathrm{~cm}^{2}$ ). The line denoted RX gives the scattering length for X-rays when scattered by the electronic cloud of the corresponding atoms. (Picture courtesy of Laboratoire Léon Brillouin.) (Colors are visible in the online version of the article; http://dx.doi.org/10.3233/JNR-140016.)

according to expression (3.104). For thermal neutrons the length of the $k$-vector is close to one $\AA^{-1}$. This leads to phase shifts of the order of

$$
\left|\delta_{0}\right| \approx 10^{-6}-10^{-4}
$$

The phase shifts associated with neutron scattering are, therefore, really tiny.

\subsection{Model potentials}

We now will explicitly calculate the phase shift for simple isotropic model potentials. The most extreme case is hard sphere scattering. The potential is described via

$$
V(r)= \begin{cases}\infty, & r \leqslant r_{0} \\ 0, & r>r_{0}\end{cases}
$$

With the potential barrier being infinitely high the wave function cannot penetrate into the interior of the sphere. If we assume that the wave length has been chosen such that $\lambda \gg r_{0}$ we may restrict ourselves to the $s$-type wave. We are, therefore looking for the radial solution

$$
R(r)=w_{0}(r) / r
$$

of the Schrödinger equation (expression (3.48))

$$
\left(-\frac{\hbar^{2}}{2 m} \frac{\mathrm{d}^{2}}{\mathrm{~d} r^{2}}+V(r)\right) w_{0}(r)=E w_{0}(r),
$$




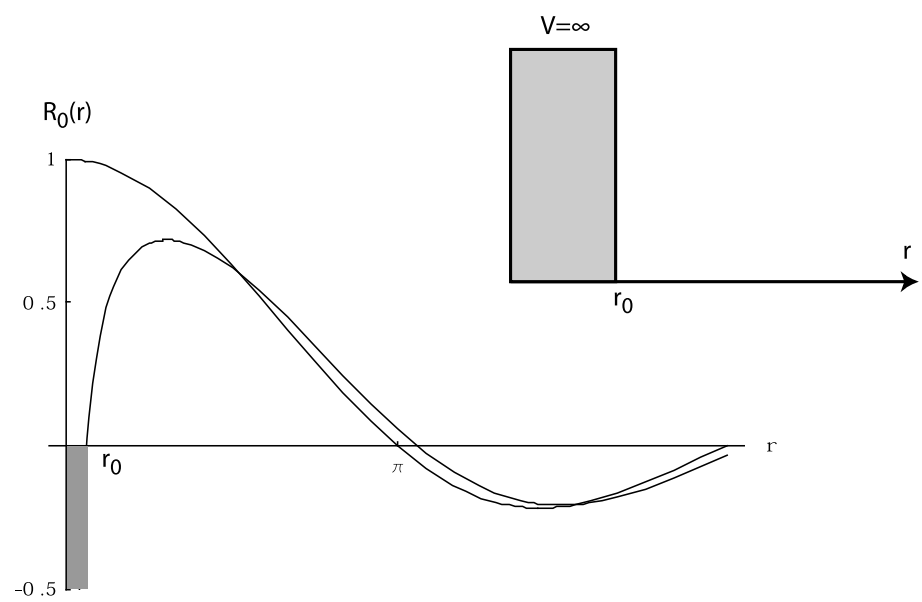

Fig. 15. A hard sphere expels the wave function of the probe particle from its interior. Here we show the radial part $R(r)=\sin (k r+\delta) /(k r)$ for a finite phase shift compared to a zero phase shift.

which becomes

$$
\begin{array}{ll}
r>r_{0}: & \left(\frac{\mathrm{d}^{2}}{\mathrm{~d} r^{2}}+k^{2}\right) w_{0}(r)=0, \\
r \leqslant r_{0}: & w_{0}(r)=0 .
\end{array}
$$

The solutions to this equation is clearly of the form (see Fig. 15)

$$
\begin{array}{ll}
r>r_{0}: & w_{0}(r)=C \sin \left(k r+\delta_{0}\right), \\
r<r_{0}: & w_{0}(r)=0 .
\end{array}
$$

From the continuity condition at the boundary of the potential we derive that

$$
k r_{0}+\delta_{0}=n \pi .
$$

For sufficiently small $k$ this implies a linear phase shift in $k$

$$
\delta_{0}=-k r_{0},
$$

in agreement with the general expression (3.97). For the case of the hard sphere we find for the scattering length

$$
b=r_{0} .
$$

The scattering length is thus defined by convention in such a way that it is positive for a hard sphere.

For the total cross section we obtain

$$
\sigma_{t}=4 \pi b^{2}=4 \pi r_{0}^{2} .
$$

This cross section is four times larger than the geometric section of the sphere that we had found to represent the classical scattering cross section in Section 2.4. A quantum mechanical treatment is, therefore, essential even in this very simple case. 
In the case of the hard sphere, the scattering length is completely defined by the characteristic length of the potential $r_{0}$. This is no longer the case when we add a bit more complexity by going to the soft sphere

$$
V(r)= \begin{cases}V_{0}, & r \leqslant r_{0} \\ 0, & r>r_{0}\end{cases}
$$

We can easily determine the radial functions that satisfy the Schrödinger equation of this problem if we limit ourselves to either being at the interior or at the exterior of the sphere. Demanding that the total function should be continuous and differentiable at the surface of the sphere we can fix the phase $\delta_{0}$ for the $s$-type waves. From this phase we can determine the scattering length in the limit where $k \rightarrow 0$. The result can be expressed as

$$
b=r_{0}\left(1+\frac{\tanh \left(k_{0} r_{0}\right)}{k_{0} r_{0}}\right)
$$

with

$$
k_{0}=\sqrt{\frac{2 m V_{0}}{\hbar^{2}}}
$$

The hyperbolic tangent

$$
\tanh x=\frac{\mathrm{e}^{x}+\mathrm{e}^{-x}}{\mathrm{e}^{x}-\mathrm{e}^{-x}}
$$

is always smaller than one. Hence, the scattering length is positive and always smaller than the characteristic length of the potential, as seen in Fig. 16.

The situation changes for spherical potential wells

$$
V(r)= \begin{cases}-V_{0}, & r \leqslant r_{0} \\ 0, & r>r_{0}\end{cases}
$$

The scattering length can again be easily calculated from the continuity conditions

$$
b=r_{0}\left(1+\frac{\tan k_{0} r_{0}}{k_{0} r_{0}}\right) .
$$

We state without prove that the scattering length is always positive in the case of a repulsive potential. In the case of an attractive potential, which is normally the case for neutron scattering by a nucleus, the sign of the scattering length depends on the details (length, depth) of the potential. This variation of the scattering length plays a very important role for neutron scattering (see Fig. 14). We would like to remark as well that the scattering length diverges for

$$
\sqrt{2 m V_{0} / \hbar^{2}} r_{0} \approx \pi / 2+n \pi .
$$

This is an effect called resonant scattering. It happens each time when - by raising either $V_{0}$ or $r_{0}$ - the system accepts another bound state. 

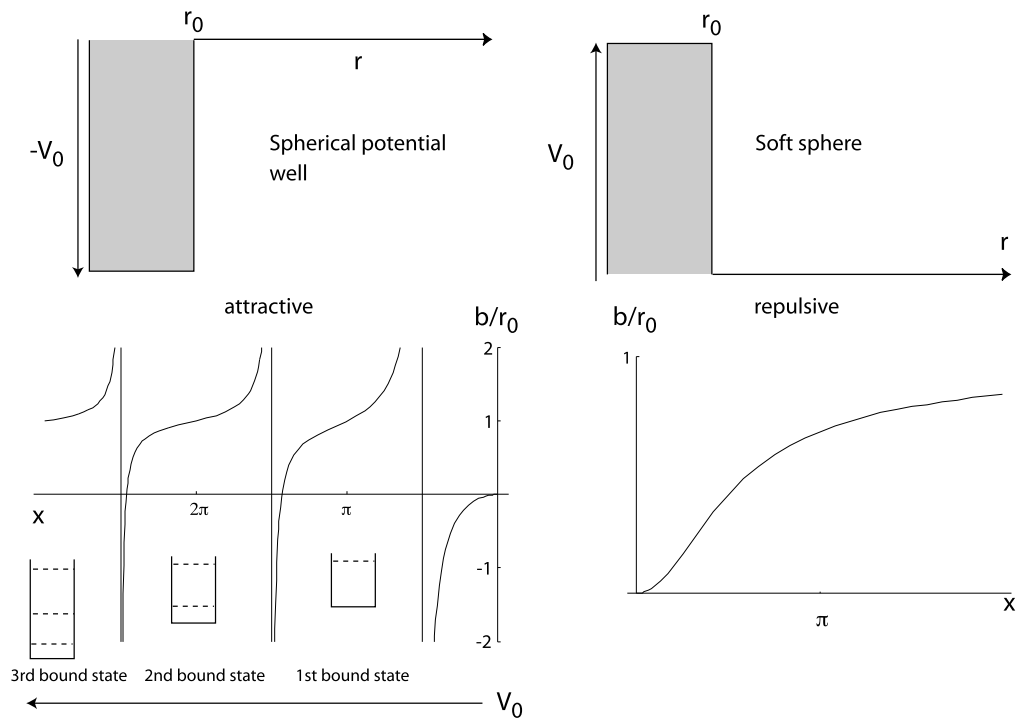

Fig. 16. The scattering length $b$ in the case of a potential wall of depth $V_{0}$ and radius $r_{0} . b / r_{0}$ is shown as a function of $x=k_{0} r_{0}=\sqrt{2 m V_{0} / \hbar^{2}} r_{0}$. It is seen that in contrast to a repulsive potential, the attractive potential is compatible with all scattering lengths. The sign of $b$ depends in a crucial manner on the parameters $V_{0}$ and $r_{0}$. Negative and very large values of $b$ indicate the imminent appearance of a bound state.

\subsection{The effective potential}

We have seen that in the case where the energy of the probe particle is so low that the scattering is purely of the $s$-type it is entirely described by the scattering length $b$. In many practical cases the exact form of the potential is in this limit without importance. Hence, it is useful to create a potential that gives the right phase shift for the $s$-wave and that is otherwise as simple as possible. In other words, we seek a potential without any internal structure. The obvious choice is a point.

$$
V_{\mathrm{eff}}(r) \psi(r)=\left.\frac{2 \pi \hbar^{2}}{m} b \delta(\vec{r}) \frac{\mathrm{d}}{\mathrm{d} r}\right|_{r=0}(r \psi(r))
$$

is an effective potential that fulfills the required criteria. In fact, the $s$-wave function

$$
\psi(r)=\frac{\sin \left(k r+\delta_{0}\right)}{k r}
$$

is an eigenfunction of the Schrödinger equation

$$
\frac{\hbar^{2}}{2 m}\left[\Delta+V_{\mathrm{eff}}(r)\right] \psi(r)=\frac{\hbar^{2} k^{2}}{2 m} \psi(r)
$$

provided that

$$
k \tan ^{-1} \delta_{0}(k)=-\frac{1}{b}
$$


The proof of this statement is not trivial. The identity

$$
\Delta f(r)=\frac{1}{r} \frac{\mathrm{d}^{2}}{\mathrm{~d} r^{2}}(r f(r))
$$

only holds if $f(r)$ is not singular at $r=0$. We can construct such a regular function by posing

$$
f(r):=\psi(r)-\psi(0)=\frac{\sin \left(k r+\delta_{0}\right)}{k r}-\frac{\sin \delta_{0}}{k r} .
$$

In order to calculate the term

$$
\frac{\sin \delta_{0}}{k} \Delta \frac{1}{r}
$$

we use the equation

$$
\Delta \frac{1}{r}=-4 \pi \delta(\vec{r})
$$

which is well known from electrostatics.

In the limit $k \rightarrow 0$ condition (3.125) reduces to

$$
\lim _{k \rightarrow 0} \frac{\delta_{0}}{k}=-b
$$

which corresponds to the correct definition of the scattering length $b$.

The effective potential only depends on one parameter

$$
g=\frac{2 \pi \hbar^{2}}{m} b
$$

which determines the scattering power without need for knowing the range or depth of the potential. It is called the coupling constant between probe and target.

\subsection{The cross section of free particles}

If the scatterer is found in a free state we have to take into consideration the two-particle character of the motion. The concepts of partial waves and in particular the scattering length have been developed for scattering by a static potential. To apply these concepts to the two-particle problem we have to work in the centre-of-mass frame (see also footnote 18 at the beginning of Section 3$).^{28}$

The cross section is generally measured in the laboratory frame (see Sections 1.2 and 2.4). Since $s$-wave scattering is isotropic in the centre-of-mass frame it is generally no longer isotropic in the laboratory frame. In order to go beyond this qualitative statement and to retrieve the specific form of the cross section in the laboratory frame we have to go into the details of the relation between the two reference systems. The scattering angles $\theta$ and $\phi$ are defined by the direction of the outgoing wave vector $\vec{k}_{f}$ with respect to the incoming wave vector $\vec{k}_{i}$. As both wave vectors depend on the reference frame so do a priori the scattering angles. We start by determining how the angles $\theta^{\mathrm{L}}$ and $\phi^{\mathrm{L}}$ in the laboratory frame change under the Galilean transformation into the centre-of-mass frame.

\footnotetext{
${ }^{28}$ In addition, we have to be aware that whenever the mass of the particle, which is considered the probe, appears in the formalism describing the motion in the potential it has to be replaced by the reduced mass

$$
\mu=\left(\frac{1}{m_{p}}+\frac{1}{m_{t}}\right)^{-1}
$$




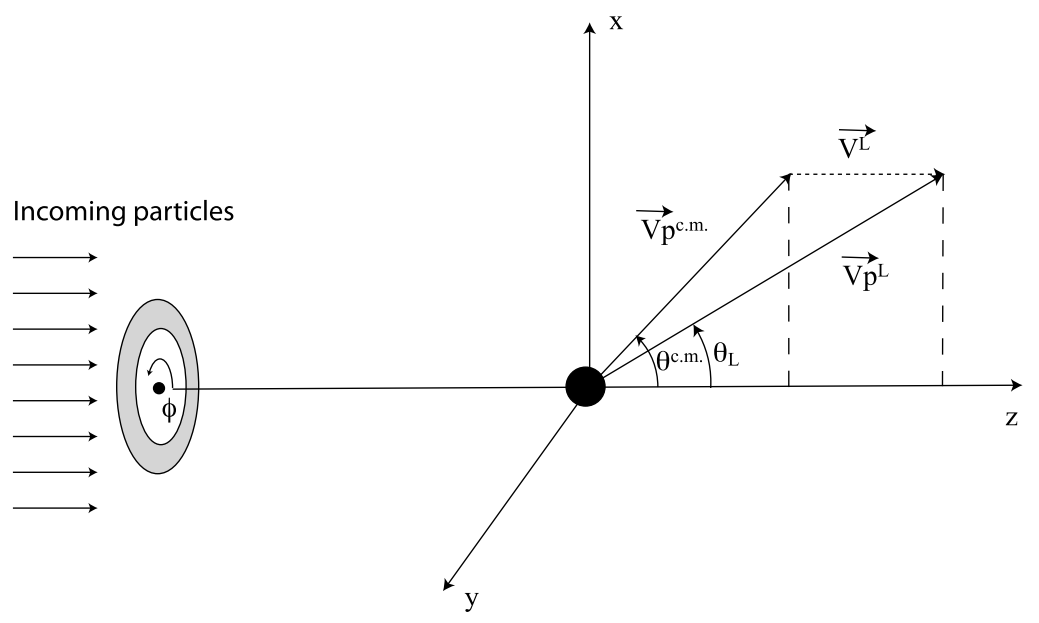

Fig. 17. Transformation of scattering from the laboratory frame to the centre-of-mass frame. Due to the symmetry around $\phi$ the scattering plane may be chosen as the $x-z$ plane without loss of generality. $\vec{V}^{\mathrm{L}}$ is the velocity of the centre-of-mass system in the laboratory frame. The target particle is assumed initially at rest in the laboratory frame. $\vec{v}_{p}^{\mathrm{c} . \mathrm{m}}$. and $\vec{v}_{p}^{\mathrm{L}}$ denote the velocities of the probe particle after the scattering in the centre-of-mass and laboratory frame, respectively. The scattering angles $\theta^{\text {c.m. and }} \theta^{\mathrm{L}}$ are determined unambiguously by these velocities.

The angle $\phi^{\mathrm{L}}$ defines the orientation of the scattering plane. As the transformation to the centre-of-mass frame leaves the scattering plane invariant we deduce

$$
\phi:=\phi^{\mathrm{L}}=\phi^{\mathrm{c} \cdot \mathrm{m} .} .
$$

To determine the transformation properties of $\theta$ we may, without loss of generality, place the scattering plane into the $x-z$ plane of the reference system (see Fig. 17). We recall that the origin of the laboratory frame coincides with the position of the target before the scattering, this means that the target is at rest in this reference system. From the addition of velocities (see Section 2.1) and basic geometric considerations we obtain for elastic scattering ${ }^{29}$

$$
\begin{aligned}
& \cos \theta^{\mathrm{L}}=\frac{v_{p}^{\text {c.m. }}}{v_{p}^{\mathrm{L}}}\left(\cos \theta^{\text {c.m. }}+\frac{V^{\mathrm{L}}}{v_{p}^{\text {c.m. }}}\right), \\
& \sin \theta^{\mathrm{L}}=\frac{v_{p}^{\text {c.m. }}}{v_{p}^{\mathrm{L}}} \sin \theta^{\text {c.m. }},
\end{aligned}
$$

with $\vec{v}_{p}^{\mathrm{c} . \mathrm{m} .}$ and $\vec{v}_{p}^{\mathrm{L}}$ the velocities of the probe particle after the scattering in the centre-of-mass and laboratory frame, respectively. $\vec{V}^{\mathrm{L}}$ denotes as before the velocity of the centre-of-mass motion.

From these identities we obtain

$$
\tan \theta^{\mathrm{L}}=\frac{\sin \theta^{\text {c.m. }}}{\cos \theta^{\text {c.m. }}+V^{\mathrm{L}} / v_{p}^{\text {c.m. }}}=\frac{\sin \theta^{\text {c.m. }}}{\cos \theta^{\text {c.m. }}+\gamma}
$$

or equivalently

$$
\cos \theta^{\mathrm{L}}=\frac{\gamma+\cos \theta^{\mathrm{c} . \mathrm{m} .}}{\sqrt{1+2 \gamma \cos \theta^{\mathrm{c} \cdot \mathrm{m} .}+\gamma^{2}}}
$$

\footnotetext{
${ }^{29} \mathrm{We}$ assume that neither probe nor target have internal degrees of freedom. This does not mean that the probe preserves its energy in the laboratory frame. To the contrary, in the laboratory frame energy and momentum conservation lead necessarily to a change of energy of the probe apart from the special case of infinitely massive targets, for which laboratory and centre-of-mass frames coincide.
} 
with

$$
\gamma:=\frac{V^{\mathrm{L}}}{v_{p}^{\mathrm{c} . \mathrm{m} .}}=\frac{m_{p}}{m_{t}}
$$

the ratio between the mass of the probe $m_{p}$ and the mass of the target particle $m_{t}$.

Therefore, the elastic cross section for the collision in the laboratory frame is related to the cross section in the centre-of-mass frame via $[33,41]$

$$
\begin{aligned}
\left(\frac{\mathrm{d} \sigma}{\mathrm{d} \Omega}\right)_{\mathrm{lab}}\left(\theta^{\mathrm{L}}\left(\theta^{\text {c.m. }}\right)\right) & =\left(\frac{\mathrm{d} \sigma}{\sin \theta^{\mathrm{L}} \mathrm{d} \theta^{\mathrm{L}} \mathrm{d} \phi^{\mathrm{L}}}\right)_{\mathrm{lab}}\left(\theta^{\mathrm{L}}\left(\theta^{\text {c.m. }}\right)\right) \\
& =\left(\frac{\mathrm{d} \sigma}{\mathrm{d} \cos \theta^{\mathrm{L}} \mathrm{d} \phi^{\mathrm{L}}}\right)_{\mathrm{lab}}\left(\theta^{\mathrm{L}}\left(\theta^{\text {c.m. }}\right)\right) \\
& =\left|\frac{\mathrm{d} \cos \theta^{\text {c.m. }}}{\mathrm{d} \cos \theta^{\mathrm{L}}}\right|\left(\frac{\mathrm{d} \sigma}{\mathrm{d} \Omega}\right)_{\text {c.m. }}\left(\theta^{\text {c.m. }}\right) \\
& =\frac{\left(1+\gamma^{2}+2 \gamma \cos \theta^{\text {c.m. }}\right)^{3 / 2}}{\left|1+\gamma \cos \theta^{\text {c.m. }}\right|}\left(\frac{\mathrm{d} \sigma}{\mathrm{d} \Omega}\right)_{\text {c.m. }} .
\end{aligned}
$$

Therefore, the transformation is uniquely determined by the parameter $\gamma$. If we take the case of a neutron being scattered by a free proton then $\gamma \approx 1$. Thus

$$
\cos \theta^{\mathrm{L}}=\frac{1}{\sqrt{2}} \sqrt{1+\cos \theta^{\mathrm{c} . \mathrm{m}}}
$$

and

$$
\left(\frac{\mathrm{d} \sigma}{\mathrm{d} \Omega}\right)_{\mathrm{lab}}\left(\theta^{\mathrm{L}}\right)=2 \sqrt{2} \sqrt{\left(1+\cos \theta^{\text {c.m. }}\right)}\left(\frac{\mathrm{d} \sigma}{\mathrm{d} \Omega}\right)_{\text {c.m. }}\left(\theta^{\text {c.m. }}\right) .
$$

If we restrict ourselves to $s$-wave scattering then we get in the centre-of-mass frame isotropic scattering of the form

$$
\left(\frac{\mathrm{d} \sigma}{\mathrm{d} \Omega}\right)_{\text {c.m. }}\left(\theta^{\mathrm{c} \cdot \mathrm{m} \cdot}\right)=\overline{|b|^{2}}
$$

where we have taken into consideration that for the proton there are two scattering channels due to the spin states. $\overline{|b|^{2}}$ is the mean squared scattering length derived from these two states (see Section 6.3 for further discussion). This leads to the following form of scattering in the laboratory frame

$$
\begin{aligned}
\left(\frac{\mathrm{d} \sigma}{\mathrm{d} \Omega}\right)_{\text {lab }}^{\text {free }}\left(\theta^{\mathrm{L}}\right) & =\overline{|b|^{2}} \cos \theta^{\mathrm{L}} ; & & \theta^{\mathrm{L}} \leqslant \frac{\pi}{2}, \\
& =0 ; & & \theta^{\mathrm{L}}>\frac{\pi}{2} .
\end{aligned}
$$

In the laboratory frame the scattering is, therefore, limited to the forward half-sphere. It evolves from its maximum value in the forward direction proportional to the cosine of the scattering angle. For the extreme case of $\theta^{\mathrm{L}}=\pi / 2$ we obtain in the centre-of-mass frame $\theta^{\text {c.m. }}=\pi$. The neutron experiences back-scattering in the centre-of-mass frame. Its velocity in the centre-of-mass frame is not impacted by the scattering. It, therefore corresponds to half 
the velocity of the incoming neutrons in the laboratory frame. In consequence the neutron necessarily stops in the laboratory frame by transferring all its energy to the proton. ${ }^{30}$

By integrating equation (3.140) over $\mathrm{d} \Omega$ we obtain the total cross section

$$
\sigma_{\text {free }}=\pi \overline{|b|^{2}} \text {. }
$$

We now can fix the proton in position by hypothetically letting its mass tend to infinity. In that case $\gamma$ will tend to zero and as expected the centre-of-mass cross section is identical to the cross section in the laboratory frame

$$
\left(\frac{\mathrm{d} \sigma}{\mathrm{d} \Omega}\right)_{\text {lab }}^{\text {bound }}\left(\theta^{\mathrm{L}}\right)=\overline{|b|^{2}} .
$$

We obtain by integration for the total cross section

$$
\sigma_{\text {bound }}=4 \pi \overline{|b|^{2}}=4 \sigma_{\text {free }}
$$

The total cross section measured in the laboratory for a neutron hitting a free proton is thus only a quarter of the total cross section of a neutron hitting a bound proton.

In the remainder of the article we will work predominantly with bound particles. The possibility of recoil is automatically taken into account in the formalism that deals with inelastic effects (see Section 8.10).

\subsection{The integral equation for scattering}

All information about the sample is contained in the scattering amplitude $f_{\vec{k}}(\theta, \phi)$. In order to take advantage of the experimental results we need to find a method that allows us to relate the measured scattered amplitude to the microscopic properties of the sample. Analytic solutions only exist for simple potentials of the kind that we have encountered in the previous section. For potentials of real practical value we need to perform numerical calculations or use perturbation theory. In the two cases it is desirable to establish a direct link between the scattering amplitude and the interaction potential of the form

$$
f_{\vec{k}}(\theta, \phi)=\mathbf{L}_{\vec{k}} V
$$

with an operator $\mathbf{L}$ that preferentially does not contain a differential operator but sums and integrals. Relations of this type exist almost everywhere in physics. A well known example is the calculation of an electric field from a given charge distribution.

We would now like to establish such a relation for the scattering of neutrons. To keep the formalism reasonably simple we retain for the moment the static character of the potential, which means that we are as in the previous sections looking for stationary solutions to the Schrödinger equation

$$
\left[-\frac{\hbar^{2}}{2 m} \Delta+V(\vec{r})\right] u(\vec{r})=E u(\vec{r}) .
$$

In order to find approximate solutions to this equation we turn to perturbation theory. This requires that the nonperturbed system can be solved. In the case of scattering the unperturbed system corresponds to the free particles. In other words, it is the specificity of scattering that all potentials constitute a perturbation. Due to this, scattering is

\footnotetext{
${ }^{30}$ There is no classical analog for $s$-wave scattering from a point-like potential. Only a head-on collision with an impact parameter equal to zero would lead to scattering for a point-like classical target. In this case energy and momentum conservation imply that the probe stops and that the target continues along the same direction with equal speed. The quantum scenario leads to scattering over the full forward half-sphere.
} 
an extreme case in terms of perturbation calculations. As a first step towards an approximative solution we isolate all the terms including the potential in the Schrödinger equation

$$
-\frac{\hbar^{2}}{2 m}\left(\Delta+k^{2}\right) u_{\vec{k}}(\vec{r})=-V(\vec{r}) u_{\vec{k}}(\vec{r}) .
$$

Guided by the language of electrostatics and consistent with the mathematical language of differential equations we introduce the concept of a source $e^{31}$

$$
j_{\vec{k}}(\vec{r}):=V(\vec{r}) u_{\vec{k}}(\vec{r})
$$

The Schrödinger then becomes

$$
\mathbf{D} u_{\vec{k}}(\vec{r})=j_{\vec{k}}(\vec{r})
$$

with the operator

$$
\mathbf{D}:=-\left(\mathbf{H}_{0}-E\right) .
$$

The source $j_{\vec{k}}(\vec{r})$ can be considered as the origin of the scattering field that we need to determine. ${ }^{32}$

Since the Schrödinger equation is linear the solutions are additive. We can exploit this fact in order to formally integrate (3.150). Still guided by the analogy with electrostatics we start from the principle that all smooth potentials $V(\vec{r})$ and thus the source $j_{\vec{k}}(\vec{r})$ can be expressed as a superposition of point sources ${ }^{33}$ ( $\delta$-functions or Dirac distributions)

$$
j_{\vec{k}}(\vec{r})=\int \mathrm{d}^{3} r^{\prime} j_{\vec{k}}\left(\vec{r}^{\prime}\right) \delta\left(\vec{r}-\vec{r}^{\prime}\right)
$$

We introduce the Green function $G_{0}\left(\vec{r}, \vec{r}^{\prime}\right)$ as the solution to the equation

$$
\mathbf{D} G_{0}\left(\vec{r}, \vec{r}^{\prime}\right)=\frac{\hbar^{2}}{2 m}\left(\Delta+k^{2}\right) G_{0}\left(\vec{r}, \vec{r}^{\prime}\right)=\delta\left(\vec{r}-\vec{r}^{\prime}\right) .
$$

\footnotetext{
${ }^{31}$ A typical example of a differential equation that has a source term is the first Maxwell equation. For static systems$$
\vec{\nabla} \cdot \vec{E}(\vec{r})=\frac{\rho(\vec{r})}{\varepsilon_{0}} .
$$

The electric charge density $\rho(\vec{r})$ acts as the source of the electric field $\vec{E}(\vec{r})$. It leads to Poisson's equation

$$
\Delta \phi(\vec{r})=-\frac{\rho(\vec{r})}{\varepsilon_{0}},
$$

which allows to determine an electrostatic potential from a given charge distribution.

${ }^{32}$ As we have included the term $\left(\hbar^{2} / 2 m\right) k^{2} u_{\vec{k}}(\vec{r})$ on the left-hand side of the equation the scattering field $u_{\vec{k}}(\vec{r})$ is nevertheless not trivial even in the absence of a source. For $V(\vec{r})=0$ the source of the scattering field disappears but the Schrödinger equation still contains non-trivial solutions

$$
\mathbf{H}_{0} u_{\vec{k}}^{0}(\vec{r})=-\frac{\hbar^{2}}{2 m} \Delta u_{\vec{k}}(\vec{r})=E u_{\vec{k}}^{0}(\vec{r}) .
$$

${ }^{33}$ In the case of the Maxwell equation cited above the charge distribution is expressed as an integral over point charges $q . \rho(\vec{r})=q \delta(\vec{r})$ is the mathematical expression for a charge that is found at the origin. 
Hence the Green functions are solutions to the Schrödinger equation for single point sources. If this function is known the solution $u_{\vec{k}}(\vec{r})$ for any source can be calculated using superposition

$$
u_{\vec{k}}(\vec{r})=C(\vec{r})+\int \mathrm{d}^{3} r^{\prime} G_{0}\left(\vec{r}, \vec{r}^{\prime}\right) j_{\vec{k}}\left(\vec{r}^{\prime}\right) .
$$

In fact, as the operator $\mathbf{D}$ acts on the variable $\vec{r}$ we obtain by permuting derivation and integration, and by using the defining property of $G_{0}\left(\vec{r}, \vec{r}^{\prime}\right)$

$$
\begin{aligned}
\mathbf{D}_{\vec{r}} u_{\vec{k}}(\vec{r}) & =\mathbf{D}_{\vec{r}} \int \mathrm{d}^{3} r^{\prime} G_{0}\left(\vec{r}, \vec{r}^{\prime}\right) j_{\vec{k}}\left(\vec{r}^{\prime}\right) \\
& =\int \mathrm{d}^{3} r^{\prime} \mathbf{D}_{\vec{r}} G_{0}\left(\vec{r}, \vec{r}^{\prime}\right) j_{\vec{k}}\left(\vec{r}^{\prime}\right) \\
& =\int \mathrm{d}^{3} r^{\prime} \delta\left(\vec{r}-\vec{r}^{\prime}\right) j_{\vec{k}}\left(\vec{r}^{\prime}\right) \\
& =j_{\vec{k}}(\vec{r})
\end{aligned}
$$

under the condition that

$$
\mathbf{D}_{\vec{r}} C(\vec{r})=\frac{\hbar^{2}}{2 m}\left(\Delta+k^{2}\right) C(\vec{r})=0 .
$$

$C(\vec{r})$ therefore has to correspond to the wave function of the free incident particle

$$
C(\vec{r})=u_{\vec{k}}^{0}(\vec{r})
$$

Using the definition (expression (3.149)) for the source $j_{\vec{k}}(\vec{r})$ the final result can be written as

$$
u_{\vec{k}}(\vec{r})=u_{\vec{k}}^{0}(\vec{r})+\int \mathrm{d}^{3} r^{\prime} G_{0}\left(\vec{r}, \vec{r}^{\prime}\right) V\left(\vec{r}^{\prime}\right) u_{\vec{k}}\left(\vec{r}^{\prime}\right) .
$$

The wave function (the field) that we have to determine is defined by this so-called integral form of the Schrödinger equation. As the parameter $\vec{k}$ samples the full Hilbert space we are dealing with an infinite set of equations. Each equation pertains to a different free particle state $\left|u_{\vec{k}}^{0}\right\rangle$. It determines how this state evolves when switching on the interaction $V(\vec{r})$. There is a one-to-one correspondence of the unperturbed state $\left|u_{\vec{k}}^{0}\right\rangle$ with the eigenstate $\left|u_{\vec{k}}\right\rangle$ that develops in the presence of the potential. The set of integral equations in its totality is completely equivalent to the original set of stationary Schrödinger equations in their differential form.

The advantages of working with the integral form of the equation are not a priori obvious. The wave functions that we are trying to determine are referred to themselves as they enter on both sides of Eq. (3.159). The solutions, therefore, have to be developed self-consistently. In consequence we have not achieved our original goal that consisted in developing a formalism that allows calculating the wave-function in a straightforward manner with the potential as the only input. The real advantage of the integral equation resides in the fact that it is ideally suited for iteration and thus constitutes, as we will see, a perfect entry point for a perturbation treatment.

\subsection{The Green function}

Before embarking on perturbation theory we are going to give the explicit form of the Green function

$$
G_{0}\left(\vec{r}, \vec{r}^{\prime}\right)=-\frac{1}{4 \pi} \cdot \frac{2 m}{\hbar^{2}} \cdot \frac{\mathrm{e}^{\left(\mathrm{i} k \cdot\left|\vec{r}-\vec{r}^{\prime}\right|\right)}}{\left|\vec{r}-\vec{r}^{\prime}\right|}
$$


Not surprisingly the Green function corresponds to a spherical wave with the point source as its origin. We could have intuitively derived this result using the arguments exposed in Section 3.1 for deciding on the asymptotic form of the wave function. ${ }^{34}$

A proper mathematical derivation is unfortunately a bit more involved. We start by reformulating Eq. (3.154)

$$
\left(E-\mathbf{H}_{0}\right) G_{0}\left(\vec{r}, \vec{r}^{\prime}\right)=\delta\left(\vec{r}-\vec{r}^{\prime}\right) .
$$

In the following it is useful to be very precise with respect to the notation and to clearly indicate the fact that we work in the real space basis of the Hilbert space. Thus

$$
\left\langle\vec{r}\left|\left(E-\mathbf{H}_{0}\right) G_{0}\left(\vec{r}, \vec{r}^{\prime}\right)\right| \vec{r}^{\prime}\right\rangle=\left\langle\vec{r} \mid \vec{r}^{\prime}\right\rangle .
$$

This result shows that formally 35

$$
\mathbf{G}_{0}=\frac{1}{\left(E-\mathbf{H}_{0}\right)},
$$

which means that Green's operator is the inverse of the operator $\left(E-\mathbf{H}_{0}\right)$. For the Green function formulated in real space this implies that

$$
G_{0}\left(\vec{r}, \vec{r}^{\prime}\right)=\left\langle\vec{r}\left|\frac{1}{\left(E-\mathbf{H}_{0}\right)}\right| \vec{r}^{\prime}\right\rangle .
$$

The solutions to the Schrödinger equation in absence of a potential are plane waves

$$
\langle\vec{r} \mid \vec{k}\rangle=u_{\vec{k}}^{0}(\vec{r})=\frac{1}{\sqrt{V}} \mathrm{e}^{\mathrm{i} \vec{k} \cdot \vec{r}}
$$

with

$$
\mathbf{H}_{\mathbf{0}}|\vec{k}\rangle=\frac{\hbar^{2} k^{2}}{2 m}|\vec{k}\rangle .
$$

Using $E=\hbar^{2} k^{2} / 2 m$ and the fact that

$$
\left(E-\mathbf{H}_{0}\right) \frac{2 m}{\hbar^{2}\left(k^{2}-k^{\prime 2}\right)}\left|\vec{k}^{\prime}\right\rangle=\left|\vec{k}^{\prime}\right\rangle
$$

identifies $2 m / \hbar^{2}\left(k^{2}-k^{\prime 2}\right)$ with the inverse of $\left(E-\mathbf{H}_{0}\right)$ in the basis of the Hilbert space formed by the momentum eigenstates $\left\{\left|\vec{k}^{\prime}\right\rangle\right\}$. We may, therefore, express Green's operator in the alternate form

$$
\frac{1}{\left(E-\mathbf{H}_{0}\right)}=\int \mathrm{d}^{3} k^{\prime} \frac{1}{\left(E-\mathbf{H}_{0}\right)}\left|\vec{k}^{\prime}\right\rangle\left\langle\vec{k}^{\prime}\left|=\frac{2 m}{\hbar^{2}} \int \mathrm{d}^{3} k^{\prime} \frac{1}{\left(k^{2}-k^{\prime 2}\right)}\right| \vec{k}^{\prime}\right\rangle\left\langle\vec{k}^{\prime}\right|,
$$

\footnotetext{
${ }^{34}$ For a point-like potential that has no extension any distance is asymptotically far away.

${ }^{35}$ Often the Green function for scattering is defined as

$$
\mathbf{G}_{0}=\lim _{\varepsilon \rightarrow+0} \frac{1}{\left(E-\mathbf{H}_{0}\right)+\mathrm{i} \varepsilon} .
$$

The term $\mathrm{i} \varepsilon$ in the denominator moves the poles of the Green function away from the real axis and allows for a simplified integration path in the complex plane when calculating the residues. We will not dwell on these mathematical details as in our opinion they do not add to the comprehension of the underlying physics.
} 
which in real space gives us

$$
\left\langle\vec{r}\left|\frac{1}{\left(E-\mathbf{H}_{0}\right)}\right| \vec{r}^{\prime}\right\rangle=\frac{1}{V} \frac{2 m}{\hbar^{2}} \int \mathrm{d}^{3} k^{\prime} \frac{\mathrm{e}^{\mathrm{i} \vec{k}^{\prime} \cdot\left(\vec{r}-\vec{r}^{\prime}\right)}}{\left(k^{2}-k^{\prime 2}\right)} .
$$

The remaining mathematical difficulty resides in the evaluation of this integral in the presence of poles at the points $k^{2}=k^{\prime 2}$. This is the field of residue calculation. The interested readers is asked to consult specialised books. The applicable solution to the scattering problem is given by expression (3.160) with a normalisation volume chosen to be $V=(2 \pi)^{3}$.

Inserting (3.160) into (3.159) we get the following expression for the wave function

$$
\begin{aligned}
u_{\vec{k}}(\vec{r}) & =u_{\vec{k}}^{0}(\vec{r})+\int \mathrm{d}^{3} r^{\prime} G_{0}\left(\vec{r}, \vec{r}^{\prime}\right) V\left(\vec{r}^{\prime}\right) u_{\vec{k}}\left(\vec{r}^{\prime}\right) \\
& =u_{\vec{k}}^{0}(\vec{r})-\frac{1}{4 \pi} \frac{2 m}{\hbar^{2}} \int \mathrm{d}^{3} r^{\prime} \frac{\exp \left(\mathrm{i} k \cdot\left|\vec{r}-\vec{r}^{\prime}\right|\right)}{\left|\vec{r}-\vec{r}^{\prime}\right|} V\left(\vec{r}^{\prime}\right) u_{\vec{k}}\left(\vec{r}^{\prime}\right) .
\end{aligned}
$$

\subsection{The Born series and the Born approximation}

The expression (3.171) has a precise physical interpretation (see Fig. 18). In absence of a potential the incident particle behaves as a free particle described by the wave function $u_{\vec{k}}^{0}(\vec{r})$. The potential is a source of perturbation.

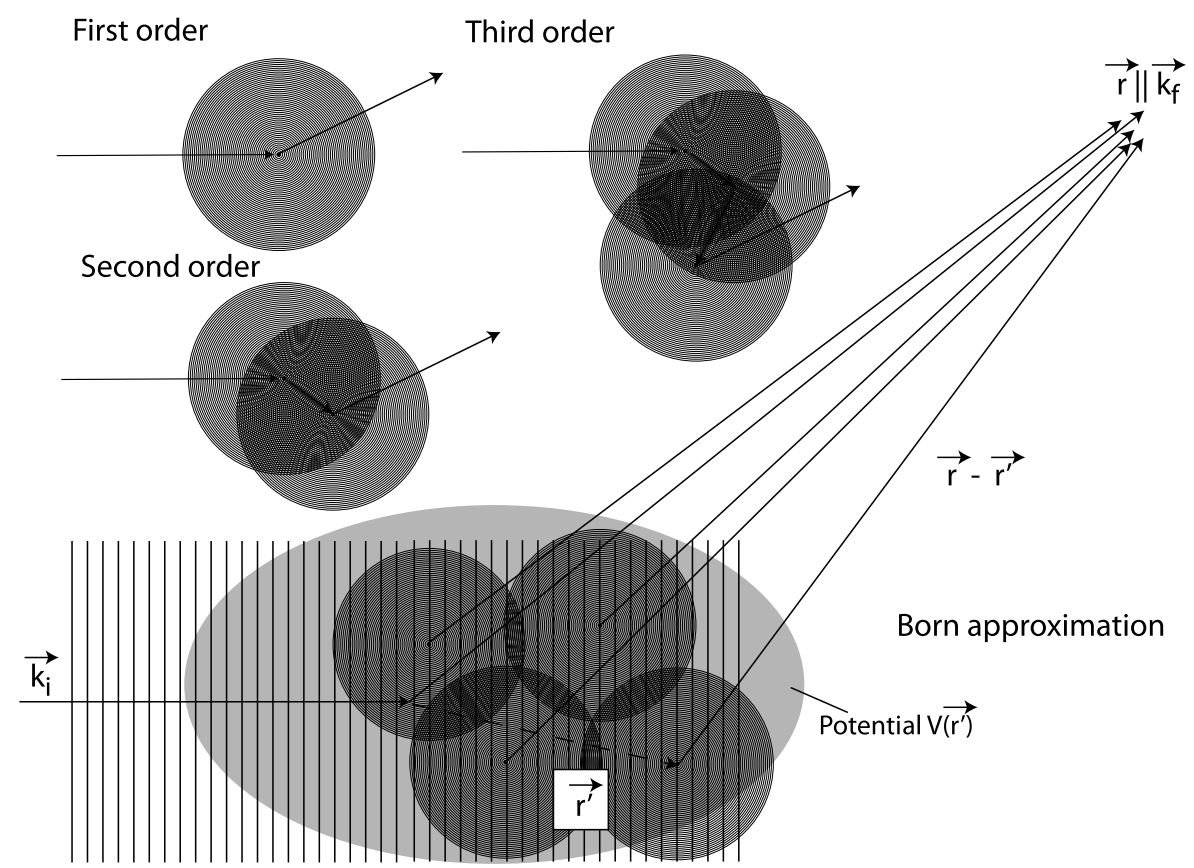

Fig. 18. Schematic representation of the Born series. Without a potential $V\left(\vec{r}^{\prime}\right)$ the incident beam is described by a plane wave with a wave vector $\vec{k}$. Each point in the interaction region is a potential source of scattering and can thus generate spherical waves. The probability of such an event depends both on the potential and the wave function itself. In other words, the wave function is coupled to itself by the intermediary of the potential. The created spherical waves become sources of scattering in their own right. The complete process can be resumed in an infinite series of scattering processes (see expression (3.175)). This series can be truncated if the interaction is sufficiently weak. Retaining only the first term means working in the Born approximation (see expression (3.177)). In this case the scattered wave is the sum of all the spherical waves created by the unperturbed incident wave. It is observed in a direction $\vec{r}$ that is defined by the final wave vector $\vec{k}_{f}$. In this approximation the summation of spherical waves corresponds to a Fourier transformation of the potential (see expression (3.188)). 
Each point $\vec{r}$ in space where the potential $V(\vec{r})$ is non-zero is a potential source of a spherical wave. The amplitude of this wave is proportional to both the interaction force of perturbation (the potential) and the wave function at this point. This is plausible if the wave function is considered as the probability of the presence of a particle at this point. If this probability is high the probability of generating a perturbation is also high. If on the other hand the probability of presence is zero at a given place then there is no creation of a spherical wave even if the potential is non-zero. Now we understand why the wave function appears in the expression (3.149) for the source. All the mathematical problems come from this intricacy: The wave function we are looking for is both the source and the result of the perturbation.

The integral expression has the advantage over the differential expression that it is amenable to iteration. By simple substitution we find

$$
\begin{aligned}
u_{\vec{k}}(\vec{r})= & u_{\vec{k}}^{0}(\vec{r})+\int \mathrm{d}^{3} r^{\prime} G_{0}\left(\vec{r}, \vec{r}^{\prime}\right) V\left(\vec{r}^{\prime}\right) u_{\vec{k}}\left(\vec{r}^{\prime}\right) \\
= & u_{\vec{k}}^{0}(\vec{r})+\int \mathrm{d}^{3} r^{\prime} G_{0}\left(\vec{r}, \vec{r}^{\prime}\right) V\left(\vec{r}^{\prime}\right) u_{\vec{k}}^{0}\left(\vec{r}^{\prime}\right) \\
& +\int \mathrm{d}^{3} r^{\prime \prime} \int \mathrm{d}^{3} r^{\prime} G_{0}\left(\vec{r}, \vec{r}^{\prime}\right) V\left(\vec{r}^{\prime}\right) G_{0}\left(\vec{r}^{\prime}, \vec{r}^{\prime \prime}\right) V\left(\vec{r}^{\prime \prime}\right) u_{\vec{k}}\left(\vec{r}^{\prime \prime}\right) .
\end{aligned}
$$

This way of explicitly writing the wave function becomes very fast impractical. By working with Green's operator that acts as follows on the wave functions

$$
\mathbf{G}_{0}\left|u_{\vec{k}}\right\rangle=\int \mathrm{d}^{3} r^{\prime} G_{0}\left(\vec{r}, \vec{r}^{\prime}\right) u_{\vec{k}}\left(\vec{r}^{\prime}\right)
$$

we can reformulate expression (3.172) in a more compact way

$$
\begin{aligned}
\left|u_{\vec{k}}\right\rangle & =\left|u_{\vec{k}}^{0}\right\rangle+\mathbf{G}_{0} V\left|u_{\vec{k}}\right\rangle \\
& =\left|u_{\vec{k}}^{0}\right\rangle+\mathbf{G}_{0} V\left|u_{\vec{k}}^{0}\right\rangle+\mathbf{G}_{0} V \mathbf{G}_{0} V\left|u_{\vec{k}}\right\rangle
\end{aligned}
$$

This equation is known as the Lippmann-Schwinger equation. It is of fundamental importance in all perturbation calculations.

Pushing the iteration to the extreme leads to the Born series.

$$
\left|u_{\vec{k}}\right\rangle=\sum_{n=0}^{\infty}\left(\mathbf{G}_{0} V\right)^{n}\left|u_{\vec{k}}^{0}\right\rangle .
$$

The operator

$$
\Omega:=\sum_{n=0}^{\infty}\left(\mathbf{G}_{0} V\right)^{n},
$$

that produces the scattered function $\left|u_{\vec{k}}\right\rangle$ from the unperturbed wave function $\left|u_{\vec{k}}^{0}\right\rangle$ is called the wave operator. It depends on the interaction between probe and target whether and where to truncate this series. If we assume that the perturbation experienced by the initial wave function in the course of scattering is small then we may use the unperturbed function to describe the source term in the integral expression. In this case we can limit ourselves to the lowest order of perturbation

$$
\left|u_{\vec{k}}\right\rangle=\left|u_{\vec{k}}^{0}\right\rangle+\mathbf{G}_{0} V\left|u_{\vec{k}}^{0}\right\rangle .
$$


This is called working in the Born approximation. If the Born approximation is applicable then we have made a great leap forward. We are capable of calculating the wave function of the scattered particles starting from the interaction potential.

We will discuss in some detail what small means in Section 3.13 that deals with the conditions of validity of the Born approximation. We will see that the Born approximation is applicable to most experimental situations where slow neutrons are scattered from condensed matter. Working in the Born approximation we assume that all the atoms in the sample see the arriving neutrons as if all the other atoms where not present. In particular the number of scattered neutrons is so low that in practice there is no attenuation effect on the incident flux over the irradiated volume. $^{36}$

\subsection{Integral form of the scattering amplitude}

In order to make the link with the scattering amplitude we need to determine the asymptotic form of the wave function. We will start from the general form given by expression (3.171) that we reproduce here for convenience

$$
\begin{aligned}
u_{\vec{k}}(\vec{r}) & =u_{\vec{k}}^{0}(\vec{r})+\int \mathrm{d}^{3} r^{\prime} G_{0}\left(\vec{r}, \vec{r}^{\prime}\right) V\left(\vec{r}^{\prime}\right) u_{\vec{k}}\left(\vec{r}^{\prime}\right) \\
& =u_{\vec{k}}^{0}(\vec{r})-\frac{1}{4 \pi} \frac{2 m}{\hbar^{2}} \int \mathrm{d}^{3} r^{\prime} \frac{\exp \left(\mathrm{i} k \cdot\left|\vec{r}-\vec{r}^{\prime}\right|\right)}{\left|\vec{r}-\vec{r}^{\prime}\right|} V\left(\vec{r}^{\prime}\right) u_{\vec{k}}\left(\vec{r}^{\prime}\right)
\end{aligned}
$$

and then will introduce the specificities related to the Born approximation along the way. For the positions $\vec{r}$ very far from the sample, this means for the $|\vec{r}|$ that are large compared to the length $\left|\vec{r}^{\prime}\right|$ that characterise the potential, ${ }^{37}$ we can expand the distance $\left|\vec{r}-\vec{r}^{\prime}\right|$ as

$$
\left|\vec{r}-\vec{r}^{\prime}\right|=\sqrt{r^{2}+r^{\prime 2}-2 \vec{r} \cdot \vec{r}^{\prime}} \approx r\left(1-\frac{\vec{r} \cdot \vec{r}^{\prime}}{r^{2}}\right) \text {. }
$$

The denominator $\left|\vec{r}-\vec{r}^{\prime}\right|$ of the function

$$
\frac{\exp \left(\mathrm{i} k \cdot\left|\vec{r}-\vec{r}^{\prime}\right|\right)}{\left|\vec{r}-\vec{r}^{\prime}\right|}
$$

varies very slowly with $r^{\prime}$. Hence it can be replaced by $r$ to a very good approximation. This is not the case for the exponential $\exp \left(\mathrm{i} k \cdot\left|\vec{r}-\vec{r}^{\prime}\right|\right)$. It varies strongly for distances $r^{\prime}$ that are comparable to the wave length $\lambda=2 \pi / k .^{38}$ As a consequence of these fast variations we have to keep the linear term in $r^{\prime}$ even for very long distances $r$. The wave function then becomes

$$
r \rightarrow \infty: \quad u_{\vec{k}}(\vec{r})=\frac{1}{(2 \pi)^{3 / 2}} \mathrm{e}^{\mathrm{i} \vec{k} \cdot \vec{r}}-\frac{1}{4 \pi} \frac{2 m}{\hbar^{2}} \frac{\mathrm{e}^{\mathrm{i} k \cdot r}}{r} \int \mathrm{d}^{3} r^{\prime} \exp \left(-\mathrm{i} k \cdot\left(\hat{r} \cdot \vec{r}^{\prime}\right)\right) V\left(\vec{r}^{\prime}\right) u_{\vec{k}}\left(\vec{r}^{\prime}\right) .
$$

\footnotetext{
${ }^{36}$ There are experimental situations where this is not necessarily the case and this despite a small scattering length. A typical example is the Bragg scattering of a high-quality crystal. The scattering power is so high for the selective set of $\vec{Q}$ values that satisfy Bragg's law, that the incident beam is strongly attenuated even after only a few atomic layers. This is called an extinction effect. In this case one should use a more complex theory which is the dynamic scattering theory (see Section 11.5).

${ }^{37}$ In optics this condition corresponds to Fraunhofer diffraction (see Section 4.1).

${ }^{38}$ As we will see later it is these variations that produce the interference phenomena in the scattered beam and thus are at the origin of the transmission of structural information into the scattering amplitude. These interference phenomena will depend crucially on the wave length, which, therefore, has to be chosen with great care.
} 
This is the asymptotic form that we have expected. The comparison to expression (3.4) allows to identify the scattering amplitude as

$$
f_{\vec{k}}(\Omega)=-\frac{\sqrt{2 \pi} m}{\hbar^{2}} \int \mathrm{d}^{3} r^{\prime} \exp \left(-\mathrm{i} k \hat{r} \cdot \vec{r}^{\prime}\right) V\left(\vec{r}^{\prime}\right) u_{\vec{k}}\left(\vec{r}^{\prime}\right)
$$

It only depends on the direction $\hat{r}$ and on the wave vector $\vec{k}$ of the sample. At this point it is advantageous to introduce the vectors

$$
\begin{aligned}
\vec{k}_{i} & :=\vec{k}, \\
\vec{k}_{f} & :=k \hat{r}, \\
\vec{Q} & :=\vec{k}_{i}-\vec{k}_{f} .
\end{aligned}
$$

With this notation and

$$
\langle r \mid \vec{k}\rangle=\frac{1}{(2 \pi)^{3 / 2}} \mathrm{e}^{\mathrm{i} \vec{k} \cdot \vec{r}}
$$

we find

$$
f\left(\vec{k}_{f}, \vec{k}_{i}\right)=-\frac{4 \pi^{2} m}{\hbar^{2}}\left\langle\vec{k}_{f}|V| u_{\vec{k}_{i}}\right\rangle=-\frac{4 \pi^{2} m}{\hbar^{2}}\left\langle\vec{k}_{f}|V \Omega| \vec{k}_{i}\right\rangle .
$$

This expression allows us to define the transition operator $\mathbf{T}$ via

$$
f\left(\vec{k}_{f}, \vec{k}_{i}\right)=-\frac{4 \pi^{2} m}{\hbar^{2}}\left\langle\vec{k}_{f}|\mathbf{T}| \vec{k}_{i}\right\rangle
$$

In the Born approximation $(\Omega \rightarrow 1, \mathbf{T} \rightarrow V)$ and, therefore,

$$
f\left(\vec{k}_{f}, \vec{k}_{i}\right)=-\frac{4 \pi^{2} m}{\hbar^{2}}\left\langle\vec{k}_{f}|V| \vec{k}_{i}\right\rangle
$$

which we can write as

$$
f_{\vec{Q}}(\Omega)=-\frac{1}{2 \pi} \frac{m}{\hbar^{2}} \int \mathrm{d}^{3} r^{\prime} \mathrm{e}^{\mathrm{i} \vec{Q} \cdot \vec{r}^{\prime}} V\left(\vec{r}^{\prime}\right) .
$$

This is a remarkable result. The scattering amplitude is simply proportional to the Fourier transform of the interaction potential. Therefore, in cases where the Born approximation is applicable we fully reached our goal, which was to connect the scattering amplitude to an intrinsic property of the sample (see expression (3.144)).

The total wave function is obtained in the asymptotic region $r \rightarrow \infty$ as

$$
\begin{aligned}
u_{\vec{k}}(\vec{r}) & =\frac{1}{\sqrt{(2 \pi)^{3}}}\left(\mathrm{e}^{\mathrm{i} \vec{k} \cdot \vec{r}}+f_{\vec{Q}}(\Omega) \frac{\mathrm{e}^{\mathrm{i} k r}}{r}\right) \\
& =\frac{1}{\sqrt{(2 \pi)^{3}}}\left(\mathrm{e}^{\mathrm{i} \vec{k} \cdot \vec{r}}-\frac{1}{2 \pi} \frac{m}{\hbar^{2}} \frac{\mathrm{e}^{\mathrm{i} k r}}{r} \int \mathrm{d}^{3} r^{\prime} \mathrm{e}^{\mathrm{i} \vec{Q} \cdot \vec{r}^{\prime}} V\left(\vec{r}^{\prime}\right)\right) .
\end{aligned}
$$




\subsection{Validity of the Born approximation for simple potentials}

A necessary condition for the applicability of the Born approximation is the rapid convergence of the Born series

$$
\left\langle\vec{r} \mid u_{\vec{k}}\right\rangle=\left\langle\vec{r}\left|\sum_{n=0}^{\infty}\left(\mathbf{G}_{0} V\right)^{n}\right| u_{\vec{k}}^{0}\right\rangle .
$$

This implies that the first term should be small at all points with respect to the incoming plane wave. Formally,

$$
\left|\left\langle\vec{r}\left|\mathbf{G}_{0} V\right| u_{\vec{k}}^{0}\right\rangle\right| \ll\left|\left\langle\vec{r} \mid u_{\vec{k}}^{0}\right\rangle\right| \text {. }
$$

Writing this expression explicitly as an integral over the Green function this condition reads

$$
\left|-\frac{1}{4 \pi} \frac{2 m}{\hbar^{2}} \int \mathrm{d}^{3} r^{\prime} \frac{\exp \left(\mathrm{i} k \cdot\left|\vec{r}-\vec{r}^{\prime}\right|\right)}{\left|\vec{r}-\vec{r}^{\prime}\right|} V\left(\vec{r}^{\prime}\right) u_{\vec{k}}^{0}\left(\vec{r}^{\prime}\right)\right| \ll\left|u_{\vec{k}}^{0}(\vec{r})\right| .
$$

The condition has to be fulfilled for all $\vec{r}$ lying inside the range of the potential $V(\vec{r})$ and, therefore, in particular for $\vec{r}=0$

$$
\left|\frac{1}{4 \pi} \frac{2 m}{\hbar^{2}} \int \mathrm{d}^{3} r^{\prime} \frac{\exp \left(-\mathrm{i} k \cdot r^{\prime}\right)}{r^{\prime}} V\left(\vec{r}^{\prime}\right) \exp \left(\mathrm{i} \vec{k} \cdot \vec{r}^{\prime}\right)\right| \ll 1 .
$$

If the potential is spherically symmetric $V(\vec{r})=V(r)$ then we can integrate the angular part of this expression

$$
\begin{aligned}
\Delta & :=\left|\frac{1}{4 \pi} \frac{2 m}{\hbar^{2}} \int \mathrm{d}^{3} r^{\prime} \frac{\exp \left(-\mathrm{i} k \cdot r^{\prime}\right)}{r^{\prime}} V\left(r^{\prime}\right) \exp \left(\mathrm{i} \vec{k} \cdot \vec{r}^{\prime}\right)\right| \\
& =\left|\frac{1}{4 \pi} \frac{2 m}{k \hbar^{2}} \int_{0}^{r_{0}} r^{\prime 2} \mathrm{~d} r^{\prime} \frac{\exp \left(-\mathrm{i} k \cdot r^{\prime}\right)}{r^{\prime}} V\left(r^{\prime}\right) \int_{0}^{2 \pi} \mathrm{d} \phi \int_{0}^{\pi} \sin \theta \mathrm{d} \theta \exp \left(\mathrm{i} k \cdot r^{\prime} \cos \theta\right)\right| \\
& =\left|\frac{m}{k \hbar^{2}} \int_{0}^{r_{0}} r^{\prime 2} \mathrm{~d} r^{\prime} \frac{\exp \left(-\mathrm{i} k \cdot r^{\prime}\right)}{r^{\prime}} V\left(r^{\prime}\right) \int_{0}^{\pi} \mathrm{d}(\cos \theta) \exp \left(\mathrm{i} k \cdot r^{\prime} \cos \theta\right)\right| \\
& =\left|\frac{m}{k \hbar^{2}} \int_{0}^{r_{0}} r^{\prime 2} \mathrm{~d} r^{\prime} \frac{\exp \left(-\mathrm{i} k \cdot r^{\prime}\right)}{r^{\prime}} V\left(r^{\prime}\right)\left[\frac{1}{\mathrm{i} k \cdot r^{\prime}}\left[\exp \left(-\mathrm{i} k \cdot r^{\prime}\right)-\exp \left(\mathrm{i} k \cdot r^{\prime}\right)\right]\right]\right| \\
& =\left|\frac{2 m}{k \hbar^{2}} \int_{0}^{r_{0}} \mathrm{~d} r^{\prime} \exp \left(-\mathrm{i} k \cdot r^{\prime}\right) \sin \left(k \cdot r^{\prime}\right) V\left(r^{\prime}\right)\right| \\
& =\left|\frac{m}{2 \mathrm{i} k \hbar^{2}} \int_{0}^{r_{0}} \mathrm{~d} r^{\prime}\left[\exp \left(2 \mathrm{i} k \cdot r^{\prime}\right)-1\right] V\left(r^{\prime}\right)\right|,
\end{aligned}
$$

with $r_{0}$ denoting the range of the potential. If the energy of the probe particle is low, i.e. if $k r_{0} \ll 1$, then

$$
\left[\exp \left(2 \mathrm{i} k \cdot r^{\prime}\right)-1\right] \approx 2 \mathrm{i} k r^{\prime}
$$

Therefore, $\Delta \ll 1$ under the condition that

$$
\frac{2 m}{\hbar^{2}} \int_{0}^{r_{0}} r V(r) \mathrm{d} r \ll 1
$$


This is called the low-energy validity condition for the Born approximation. For a square well potential of depth $V_{0}$ (see definition (3.120)) this condition reduces to

$$
\frac{m V_{0} r_{0}^{2}}{\hbar^{2}} \ll 1
$$

We recall that the more we confine a particle the stronger become the zero point fluctuations and, therefore, the higher is the ground state energy.

To get a better feeling for the condition we introduce the energy

$$
E\left(\lambda=r_{0} / 2 \pi\right)=\frac{\hbar^{2}}{2 m r_{0}^{2}}
$$

of a probe particle that features a wave vector

$$
k=\frac{1}{r_{0}} .
$$

Then the low-energy condition becomes

$$
\frac{V_{0}}{2 E} \ll 1
$$

The condition is fulfilled if the potential is very much deeper than the energy of a probe particle that possess a wave length of the order of the potential range $r_{0}$.

For a potential well to be able to host a particle in a bound state the condition

$$
\frac{m V_{0} r_{0}^{2}}{2 \hbar^{2}} \approx \frac{\pi^{2}}{8} \approx 1
$$

has to hold. E.g. in the case of a narrow deep potential $\left(V_{0} \rightarrow \infty, r_{0} \rightarrow 0\right.$ with $V_{0} \cdot r_{0}$ fixed) we get exactly one bound state at

$$
E_{0} \approx-\frac{m V_{0} r_{0}^{2}}{2 \hbar^{2}} V_{0}
$$

A potential accepting a bound state is, therefore, a very bad candidate for applying the Born approximation in the low energy limit. This implies in particular that the Born approximation is not suitable for treating the scattering of low-energy neutrons in the potential of nuclei. The range $r_{0}$ of the nuclear interaction lies at about $10^{-15} \mathrm{~m}$. For neutrons with wave lengths of a few $\AA$

$$
r_{0} \cdot k<10^{-4}
$$

We are, therefore, clearly in the low-energy regime (see Section 5.8). However, typical values for the potential strength $V_{0}$ are of the order of the nuclear binding energy, i.e. tens of MeVs and, therefore,

$$
\frac{m V_{0} r_{0}^{2}}{\hbar^{2}}=\frac{V_{0}}{2 E\left(k=1 \AA^{-1}\right)} r_{0}^{2}[\AA] \approx \frac{\text { tens of } \mathrm{MeV}}{4 \mathrm{meV}} 10^{-10} \approx 1 .
$$

As a consequence multiple scattering is strong within the range of the potential. The problem of a neutron scattered by a nucleus is, therefore, way better treated within the partial wave method that we have developed in Section 3.3 
and that let to the description of this scattering by a simple scattering length $b$ (see Section 3.5). The result is not surprising given the fact that the neutron interacts with the nuclei via the strong interaction, however, over a very short distance on the length scale of the neutron wave length.

This result may surprise given that nearly the totality of all neutron scattering experiments are evaluated under the Born approximation. This is possible because the scattering from a single nucleus can be described with the help of the effective potential using the experimentally determined scattering length (see Sections 3.7 and 5.8). The scattering from an ensemble of nuclei in condensed matter can then be dealt with in the Born approximation because the distance between the scatterers leads to such a dilution of scattering strength that the perturbation of the incoming wave front is weak for most experimental situations (see Sections 11.5 and 6.5).

The situation changes when we go to very energetic particles. In this case the function

$$
\exp \left(2 \mathrm{i} k \cdot r^{\prime}\right)
$$

oscillates strongly over regions where $V(r)$ can be assumed to be constant. It thus does not contribute to the integral. Therefore, for $k r \gg 1$

$$
\Delta=\left|\frac{m}{k \hbar^{2}} \int \mathrm{d} r^{\prime} V\left(r^{\prime}\right)\right|
$$

For a square well potential the condition $\Delta \ll 1$ is fulfilled for

$$
\frac{m r_{0} V_{0}}{k \hbar^{2}}=\frac{r_{0} V_{0}}{\hbar v} \ll 1,
$$

with $v=\hbar k / m$ the velocity of the scattered particle, where we have to make sure that we stay in the non-relativistic regime. This is called the high-energy condition for the validity of the Born approximation. In the high-energy regime the Born approximation is, therefore valid even for potentials that support bound states. ${ }^{39}$ If we were trying to work in this regime with the partial wave approach we would be obliged to include a very large number of terms. We, therefore, see the interest of developing both techniques even in the case of simple central potentials.

\subsection{A word concerning scattering of electro-magnetic radiation}

We have repeatedly stressed that the concepts developed here are transposable to the scattering of photons and in particular X-rays. This may surprise given that everything we have done so far is based on the non-relativistic Schrödinger equation while electromagnetic radiation is governed by the relativistic Maxwell equations. While it cannot be the purpose of this article to develop a full scattering theory for electromagnetic radiation we would like to outline very briefly the principle reason for the similarity of non-relativistic particle and photon scattering from condensed matter.

We start by writing down the Maxwell equations using the elegant form of the electromagnetic potential given as the four-vector

$$
\bar{A}(\vec{r}, t)=(\Phi(\vec{r}, t), \vec{A}(\vec{r}, t))
$$

from which we can derive the electric

$$
\vec{E}(\vec{r}, t)=-\vec{\nabla} \Phi(\vec{r}, t)-\frac{1}{c} \frac{\partial \vec{A}(\vec{r}, t)}{\partial t}
$$

\footnotetext{
${ }^{39}$ It should be remembered that when treating the bonding in two particle systems the mass $m$ has to be replaced by the reduced mass $\mu$.
} 
and magnetic fields

$$
\vec{B}(\vec{r}, t)=\vec{\nabla} \times \vec{A}(\vec{r}, t) .
$$

Introducing the four-current density

$$
\bar{J}=\left(\rho(\vec{r}, t), \frac{1}{c} \vec{j}(\vec{r}, t)\right)=\left(\rho(\vec{r}, t), \frac{1}{c}, \vec{j}_{x}(\vec{r}, t), \vec{j}_{y}(\vec{r}, t), \vec{j}_{z}(\vec{r}, t)\right),
$$

with $\rho(\vec{r}, t)$ the electric charge density and $\vec{J}(\vec{r}, t)$ the electric current density, the Maxwell equations can be written in the compact form

$$
\left(\Delta-\frac{1}{c^{2}} \frac{\partial^{2}}{\partial t^{2}}\right) A_{\mu}(\vec{r}, t)=-4 \pi J_{\mu}(\vec{r}, t) .
$$

We are dealing with four inhomogeneous wave equations. The current densities $J_{\mu}$ constitute the source terms. The main difference of these equations with respect to the Schrödinger equation is the fact that they are not only quadratic in the derivatives with respect to space but equally with respect to time, which is a natural consequence of Lorentz invariance. Without current, i.e. in source-free space the associated wave functions are separable

$$
A_{\mu}(\vec{r}, t)=\mathrm{e}^{\mathrm{i} \hbar \omega t} A_{\mu}^{\omega}(\vec{r})
$$

with

$$
E=\hbar \cdot \omega=h \cdot \nu=h \frac{c}{\lambda}=\hbar \cdot c \cdot k .
$$

The stationary wave functions $\psi_{\mu}^{\omega}(\vec{r})$ have to satisfy the equations

$$
\Delta A_{\mu}^{\omega}(\vec{r})+k^{2} A_{\mu}^{\omega}(\vec{r})=0 .
$$

These equations are known as the Helmholtz equations. They are identical to the Schrödinger equation for free particles (3.3). The two unperturbed stationary systems are, therefore, described by the same kind of wave function.

The electric and magnetic fields in free space are, therefore, given as

$$
\vec{E}=\vec{E}_{0} \exp \mathrm{i}(\vec{k} \cdot \vec{r}-\omega t), \quad \vec{B}=\vec{B}_{0} \exp \mathrm{i}(\vec{k} \cdot \vec{r}-\omega t) .
$$

The 6 components of $\vec{E}$ and $\vec{B}$ are not independent. The Maxwell equations imply that

$$
\vec{\nabla} \cdot \vec{E}_{0}=\mathrm{i} \vec{k} \cdot \vec{E}_{0}=0
$$

and

$$
\vec{\nabla} \cdot \vec{B}_{0}=\mathrm{i} \vec{k} \cdot \vec{B}_{0}=0 .
$$

The field amplitudes $\vec{E}_{0}$ and $\vec{B}_{0}$ are, therefore, perpendicular to the propagation vector $\vec{k}$. Light has transverse character. In addition

$$
\vec{\nabla} \times \vec{E}=-\partial_{t} \vec{B}
$$


leads to

$$
\vec{k} \times \vec{E}=\omega \vec{B} .
$$

Therefore, $\vec{E} \perp \vec{B}$. The magnetic field is perpendicular to the electric field. All this is specific to light scattering in the same way as spin polarisation is specific to non-relativistic fermion scattering.

Let us now come back to the commonalities and check what happens in the case of a perturbation. For this we need the Green function as defined via

$$
\left[\Delta_{r}+k^{2}\right] G^{\text {e.m. }}\left(\vec{r}, \vec{r}^{\prime}\right)=\delta\left(\vec{r}-\vec{r}^{\prime}\right) .
$$

It is found as

$$
G_{0}^{\text {e.m. }}\left(\vec{r}, \vec{r}^{\prime}\right)=-\frac{1}{4 \pi} \cdot \frac{\mathrm{e}^{\left(\mathrm{i} k \cdot\left|\vec{r}-\vec{r}^{\prime}\right|\right)}}{\left|\vec{r}-\vec{r}^{\prime}\right|},
$$

which is identical to the Green function for non-relativistic particles of expression (3.214)

$$
G_{0}\left(\vec{r}, \vec{r}^{\prime}\right)=-\frac{1}{4 \pi} \cdot \frac{2 m}{\hbar^{2}} \cdot \frac{\mathrm{e}^{\left(\mathrm{i} k \cdot\left|\vec{r}-\vec{r}^{\prime}\right|\right)}}{\left|\vec{r}-\vec{r}^{\prime}\right|}
$$

apart from the pre-factor. Knowing the Green function we can solve the inhomogeneous or "source-full" wave equations by simple integration over space

$$
\begin{aligned}
& \Phi(\vec{r}, t)=\int_{V} \frac{\rho\left(\vec{r}^{\prime}, t-\left|\vec{r}-\vec{r}^{\prime}\right| / c\right)}{\left|\vec{r}-\vec{r}^{\prime}\right|} \mathrm{d}^{3} \vec{r}^{\prime}, \\
& \vec{A}(\vec{r}, t)=\int_{V} \frac{\vec{j}\left(\vec{r}^{\prime}, t-\left|\vec{r}-\vec{r}^{\prime}\right| / c\right)}{\left|\vec{r}-\vec{r}^{\prime}\right|} \mathrm{d}^{3} \vec{r}^{\prime},
\end{aligned}
$$

where for time-dependent four-current densities we have taken into account the retardation of the signal when travelling from $\vec{r}$ to $\vec{r}^{\prime}$. If instead of external charges and currents we want to calculate the perturbation that experience stationary electromagnetic fields due to scattering then the source terms $J_{\mu}$ have to be replaced by $V(\vec{r}) A_{\mu}^{\omega}(\vec{r}, t)$. In this way we recover the expressions for the Born series in complete analogy to the non-relativistic particle case.

\section{Diffraction from simple objects}

To demonstrate how expression (3.188) is applied in practice we will embark on a short excursion into the world of diffraction. By using simple diffracting objects like slits and gratings we will familiarise ourselves with the principle concepts that underlie structural determination from diffraction patterns. Using expression (3.188) we could calculate these patterns directly for massive probe particles like neutrons. We will choose a slightly more indirect route. Exploiting the fact that expression (3.188) is in nearly all aspects equivalent to the Huygens-Fresnel principle for optical diffraction we will calculate the diffraction patterns for light. We consider this pedagogical advantageous as most of the readers will be familiar with the diffraction of light. The results obtained are without modification transferable to X-ray, neutron or electron diffraction. As no new concepts will be developed in Sections 4.1-4.6 readers already familiar with the application of expression (3.188) to diffraction may directly proceed to Section 4.7 . 


\subsection{The Huygens-Fresnel principle}

In optics the interpretation of diffraction and refraction is based on the Huygens-Fresnel principle [32,44]. According to this principle, each surface element that the luminous wave front meets acts itself as a light source emitting spherical wavelets. The amplitude of theses wavelets is proportional to both the intensity of the incoming light and the surface area of the emitting element. The complex light amplitude produced at any given point by the ensemble of secondary sources is simply the sum of the complex amplitudes of the wavelets. Without perturbation the interference among the wavelets is such that it leads to straight propagation. This is the regime of geometric optics. When the light meets an obstacle the propagation is perturbed. It is the absence of wavelets at the places of perturbation that produces diffraction effects. According to this principle the wave function ${ }^{40}$ at a point $\vec{r}$ generated by a monochromatic wave $(k=2 \pi / \lambda)$ is found from the wave function over a generating surface $S$ according to $[32,44]$

$$
\psi_{\vec{k}}(\vec{r})=\int_{S} \Gamma(\vec{r}) \cdot \psi_{\vec{k}}^{0}(\vec{r}) \cdot \frac{\exp \left(\mathrm{i} k \cdot\left|\vec{r}-\vec{r}^{\prime}\right|\right)}{\left|\vec{r}-\vec{r}^{\prime}\right|} \mathrm{d} S .
$$

The vectors $\vec{r}$ and $\vec{r}^{\prime}$ are defined as in Fig. 18 with the only difference that they do not run over a scattering volume $V$ but over a generating surface $S$ that contains the wave front. The factor $\Gamma$ takes into account that the surface $S$ may be inclined at the point $\vec{r}^{\prime}{ }^{41}$ We immediately realise that apart from this particularity the Huygens-Fresnel principle resembles the integral form of the Schrödinger equation (see (3.171)). The principal difference consist in the fact that the interference created by scattering is due to the creation of wavelets and not to their omission, as this is the case in optical diffraction. One of the major consequences of this difference is the absence of higher order effects in optical diffraction. Since optical diffraction is not the result of the reemission of light by matter there are by definition no multiple reemission phenomena. Hence we are automatically in the Born approximation. ${ }^{42}$

In the Fraunhofer approximation the observation plane is placed at infinity (see Fig. 19). In other words, the distance between the diffracting object and the point of observation is considered to be very large compared to all the other dimensions of the problem. The light rays that have to be retained when considering images at infinity must leave the diffracting plane along parallel directions. Hence they are completely characterised by the vector $\vec{k}_{f} \| \hat{r}$. This corresponds exactly to the scattering conditions in the Frauenhofer picture. In practice, the light rays can be returned to the observation plane by a lens. In the Fraunhofer approximation the diffracted wave function at a point $\vec{r}$ of the observation plane is given for $\vec{k}_{i} \| \hat{z}$ by

$$
\psi_{\vec{k}}(\vec{r}) \propto \Gamma \frac{\exp (\mathrm{i} k \cdot r)}{r} \int_{S} \psi_{\vec{k}}^{0}(\vec{r}) \exp \left(\overrightarrow{\mathrm{i}}_{f} \cdot \vec{r}^{\prime}\right) \mathrm{d} S,
$$

which we may rewrite in the more general form

$$
\psi_{\vec{k}}(\vec{r}) \propto \Gamma \frac{\exp (\mathrm{i} k \cdot r)}{r} \int_{S} t\left(\vec{r}^{\prime}\right) \psi_{\vec{k}}^{0}(\vec{r}) \exp \left(\mathrm{i}_{f} \cdot \vec{r}^{\prime}\right) \mathrm{d} S,
$$

where we have formally introduced the transmittance $t(\vec{r})$ of the aperture. It is given by the ratio of the complex amplitudes just after and before the optical obstacle

$$
t(x, y)=\frac{\psi_{0}^{\text {after }}(x, y)}{\psi_{0}^{\text {before }}(x, y)}
$$

\footnotetext{
${ }^{40}$ As for neutrons we ignore phenomena associated with polarisation. This allows us describing light with the help of a scalar complex amplitude, which may correspond to any of the components of the electromagnetic wave field.

${ }^{41}$ The Huygens-Fresnel principle can be derived rigorously from Kirchhoff's diffraction formula. The diffraction formula in particular account for the factor $\Gamma$ and equally explain why the Huygens-Fresnel principle works only in the forward direction.

${ }^{42}$ This is strictly speaking only true as long as we stay with two-dimensional generating surfaces over which the light front can be considered unperturbed.
} 


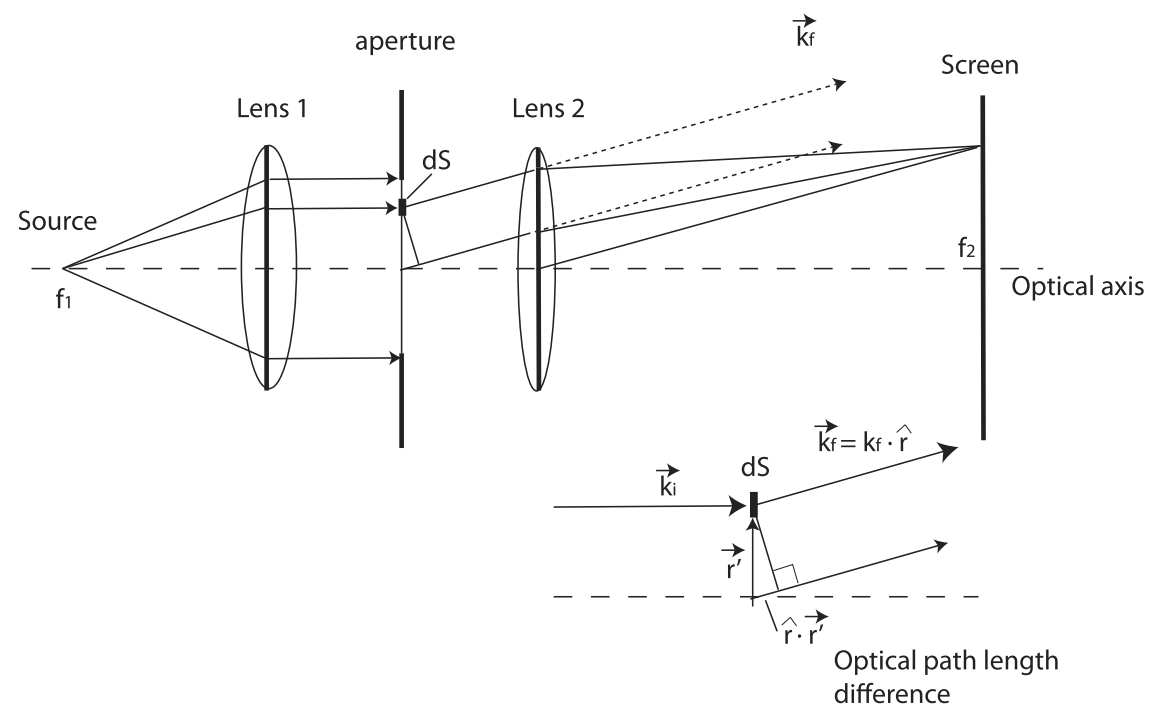

Fig. 19. Schematics of an optical set-up creating conditions for Frauenhofer diffraction. A coherent plane wave front is produced over the aperture via a point source placed in the focal point of a lens. Parallel rays emerging from the aperture are focused onto the observation screen via a second lens. Interference is created via the optical path length differences that experience parallel rays that have their origin in different surface elements d $S$.

Introducing the transmittance allows treating equally cases where the aperture is not ideal, i.e. where it is attenuating the light amplitude. In the Fraunhofer approximation the image created by the diffracted light, therefore, gives the square of the modulus of the Fourier transform of the diffracting motive.

This expression is equivalent to the scattered part of the wave function (3.189)

$$
u_{\vec{k}}(\vec{r}) \longrightarrow-\frac{1}{2 \pi} \frac{m}{\hbar^{2}} \frac{\exp (\mathrm{i} k \cdot r)}{r} \int \mathrm{d}^{3} r^{\prime} \mathrm{e}^{\mathrm{i} \vec{Q} \cdot \vec{r}^{\prime}} V\left(\vec{r}^{\prime}\right)
$$

(obtained for scattering in the Born approximation) provided we identify the generating light amplitude

$$
t\left(\vec{r}^{\prime}\right) \psi_{0}\left(\vec{r}^{\prime}\right)
$$

with the source term (see expression (3.149)) of scattering in the Born approximation ${ }^{43}$

$$
V\left(\vec{r}^{\prime}\right) u_{\vec{k}}\left(\vec{r}^{\prime}\right)
$$

We will illustrate the Huygens-Fresnel formalism with two examples: A simple slit and a grating of slits.

\subsection{Diffraction from a single slit}

We consider a slit $F=2 a \times 2 b$ illuminated by a monochromatic, plane light front in normal incidence $\left(\vec{k}_{i}=\right.$ $(2 \pi / \lambda) \hat{z}$; see Fig. 20). In this case the wave front $\psi_{0}$, which acts as the source of the diffracted wavelets, can be identified with the transmittance of the slit in the diffracting plane.

\footnotetext{
${ }^{43}$ This makes perfect sense as $t\left(\vec{r}^{\prime}\right) \psi_{0}\left(\vec{r}^{\prime}\right)$ is the source of a spherical light wave in the same way as $V\left(\vec{r}^{\prime}\right) u_{\vec{k}^{(}}\left(\vec{r}^{\prime}\right)$ is the source of a spherical scattered particle wave.
} 


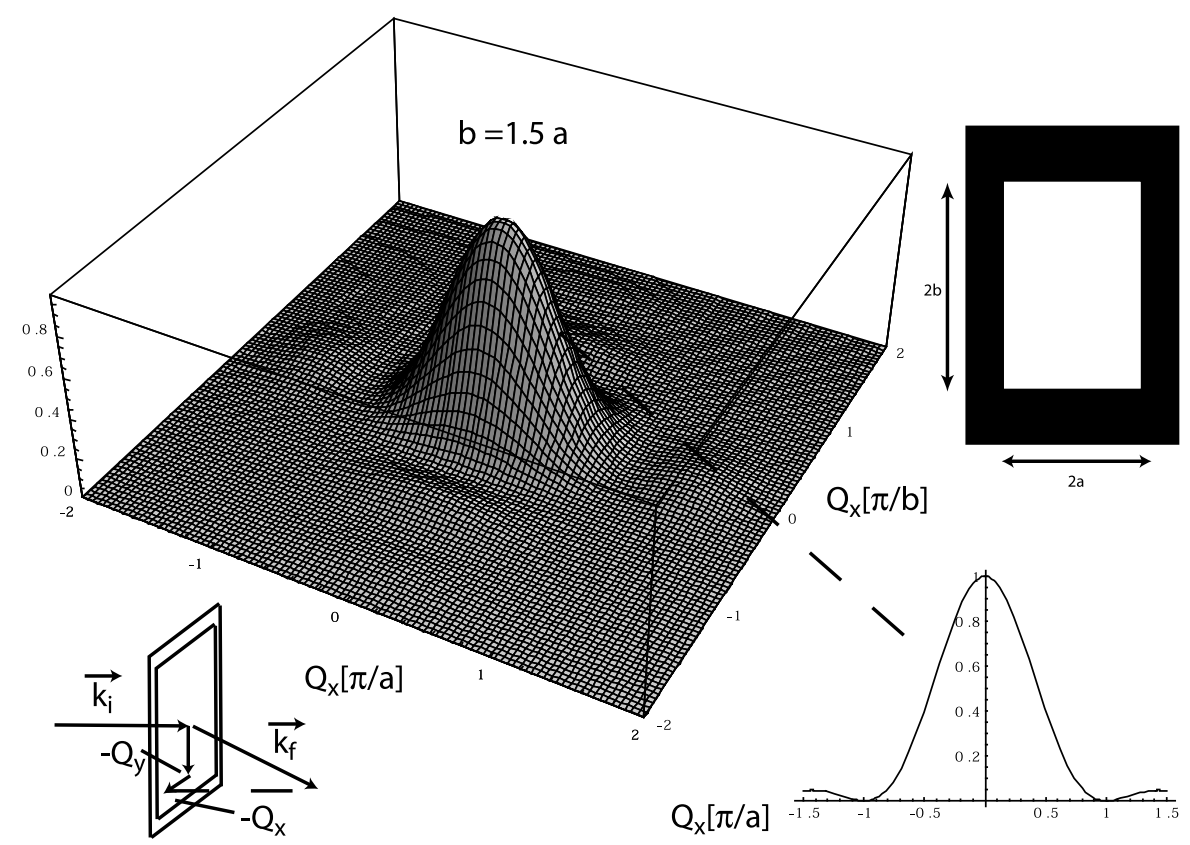

Fig. 20. Diffraction from a single ideal slit. The widths of the diffraction peak are inversely proportional to the widths of the slit. In the case presented here $b=1.5 a$. The peak is, therefore, narrower along $Q_{y}$ than along $Q_{x}$.

In the case of an ideal slit $F$, i.e. of a slit characterised by a perfect transparency of the aperture, the transmittance is necessarily binary. It is one for all points within the aperture $F$ and zero otherwise

$$
t(x, y)=\operatorname{rect}\left(\frac{x}{2 a}\right) \operatorname{rect}\left(\frac{y}{2 b}\right)= \begin{cases}1, & -a<x<a \text { and }-b<y<b \\ 0, & \text { otherwise }\end{cases}
$$

By defining $\vec{Q}=\vec{k}_{i}-\vec{k}_{f}$ we obtain at normal incidence for the projections of $\vec{k}_{f}$ onto the diffracting plane

$$
k_{x}^{f}=-Q_{x}=\frac{2 \pi}{\lambda} \sin \theta_{x} \quad \text { and } \quad k_{y}^{f}=-Q_{y}=\frac{2 \pi}{\lambda} \sin \theta_{y} .
$$

This notation has the merit of being very close to the notation used for scattering. In addition, formulated in terms of $\vec{Q}$ the expressions possess the additional benefit of being also valid for oblique incidence of the generating light front.

The Fourier transform of the transmittance of the slit is given by

$$
\begin{aligned}
\tilde{t}\left(Q_{x}, Q_{y}\right): & =F(t(x, y)) \\
& =\int_{-a}^{a} \int_{-b}^{b} \exp \left(\mathrm{i}\left(Q_{x} x+Q_{y} y\right)\right) \mathrm{d} x \mathrm{~d} y \\
& =\int_{-a}^{a} \exp \left(\mathrm{i} Q_{x} x\right) \mathrm{d} x \int_{-b}^{b} \exp \left(\mathrm{i} Q_{y} y\right) \mathrm{d} y \\
& =4 a b\left[\frac{\sin \left(Q_{x} a\right)}{Q_{x} a}\right]\left[\frac{\sin \left(Q_{y} b\right)}{Q_{y} b}\right] .
\end{aligned}
$$


The diffraction pattern, i.e. the intensity on the observation screen is obtained as the square of the amplitude

$$
I\left(Q_{x}, Q_{y}\right)=16 a^{2} b^{2}\left[\frac{\sin \left(Q_{x} a\right)}{Q_{x} a}\right]^{2}\left[\frac{\sin \left(Q_{y} b\right)}{Q_{y} b}\right]^{2} .
$$

This is shown in Fig. 20. The widths of the peaks are inversely proportional to the widths of the slit. In practice, all of the intensity is concentrated in the central peak, which has a full width at half maximum of $0.89 \pi \cdot a^{-1}$ and $0.89 \pi \cdot b^{-1}$, respectively. The angular deviations corresponding to these widths can be calculated as

$$
\theta_{x}=\arcsin \left(\frac{0.89 \cdot \lambda}{2 a}\right) \text { and } \theta_{y}=\arcsin \left(\frac{0.89 \cdot \lambda}{2 b}\right)
$$

Hence, important diffraction phenomena are only to be expected when the dimensions of the slit do not considerably exceed the wave length of the radiation.

In the following we will assume that $b \gg a$ and $b \gg \lambda$ as well. Diffraction is then limited to the $Q_{x}$ direction.

\subsection{Diffraction from an ensemble of slits with random translation}

If we shift the slit a distance $\Delta x$ along the $\hat{x}$ direction we introduce, which is easily shown, a phase factor in the diffraction amplitude

$$
\tilde{t}\left(Q_{x}\right)=2 a \exp \left(\mathrm{i} Q_{x} \Delta x\right)\left[\frac{\sin \left(Q_{x} a\right)}{Q_{x} a}\right] .
$$

Thus the translation leaves the diffraction pattern unchanged. As we will show now, this is also true for a large number of slits with random translation along $\hat{x}$.

The amplitude of the diffracted wave is the sum of the amplitudes conveyed by each individual aperture. Let $x_{m}$ be the coordinates of the centres of the apertures. The Fourier transform of the transmittance of the ensemble is given as

$$
\tilde{t}\left(Q_{x}\right)=2 a\left[\frac{\sin \left(Q_{x} a\right)}{Q_{x} a}\right] \sum_{m=1}^{N} \exp \left(\mathrm{i} Q_{x} x_{m}\right) .
$$

Taking the square of this expression in order to get the diffraction pattern leads to a double sum

$$
I \propto \sum_{m=1}^{N} \exp \left(\mathrm{i} Q_{x} x_{m}\right) \sum_{n=1}^{N} \exp \left(-\mathrm{i} Q_{x} x_{n}\right) .
$$

For $m=n$ we obtain $N$ terms each contributing to the sum with a value of one. In the terminology, that we will introduce in order to describe the scattering by complex targets, this is the eigen or self terms. The part that is left writes as

$$
I \propto 2 \operatorname{Re}\left[\sum_{m \neq n} \exp \left(\mathrm{i} Q_{x}\left(x_{m}-x_{n}\right)\right)\right] .
$$

For a large number of slits with random positions this sum will average out to zero. Hence the random multiplication of slits increases the intensity proportional to the number of slits. The diffraction figure itself remains unchanged since the arbitrary superposition of phases does not produce any constructive interference between the contributions stemming from different slits. We will see later that this phenomenon is found in diffuse neutron scattering (Section 9.14) and is equally reminiscent of incoherent neutron scattering (Section 6.1). 


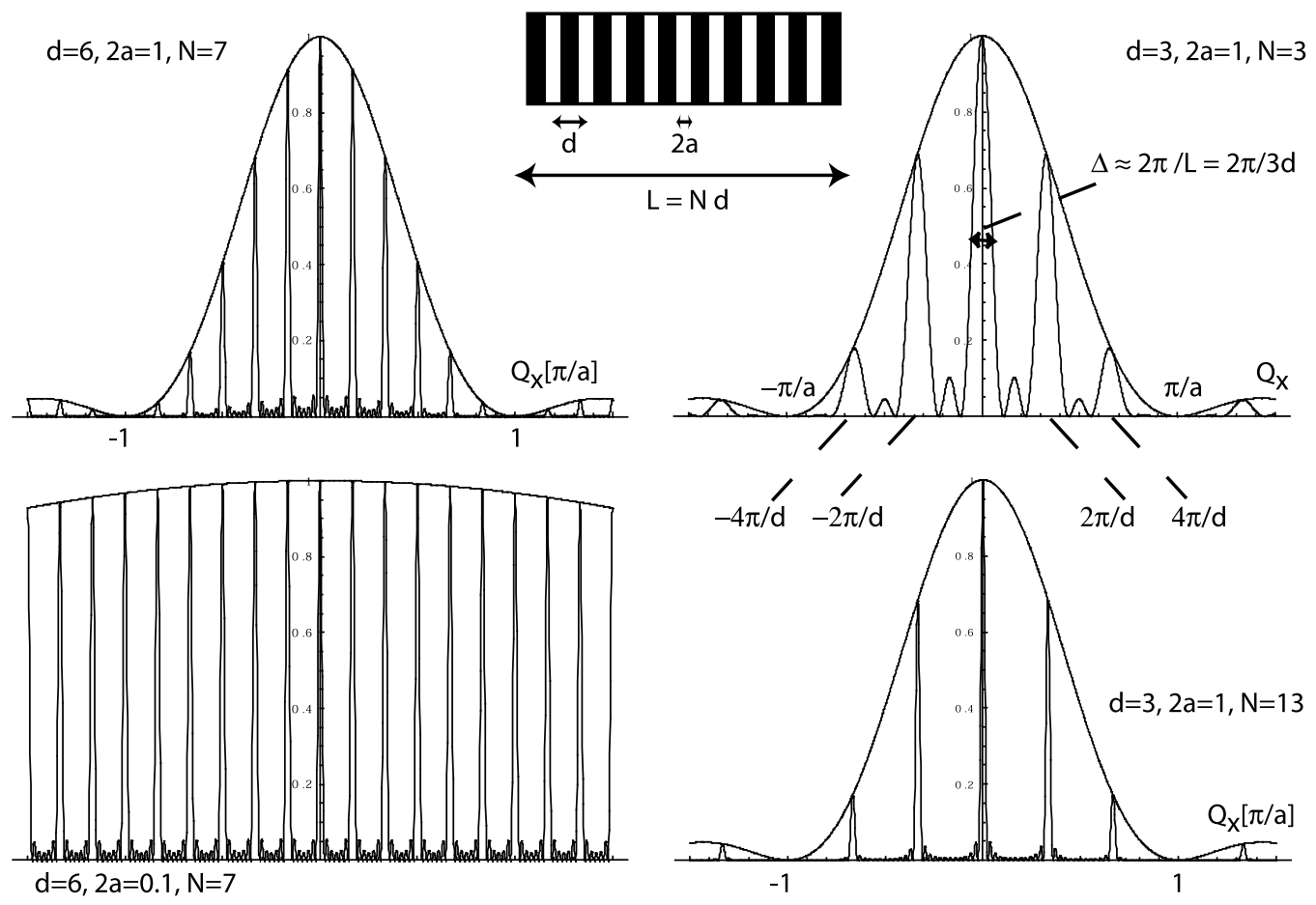

Fig. 21. Diffraction patterns as a function of $Q_{x}$ for various gratings.

\subsection{Diffraction from a grating}

The situation changes completely when we go to an ordered grating. Let us consider a regular arrangement of $N$ slits of width $2 a$ and distance $d$ (see Fig. 21). The transmittance of the ensemble is the sum of the transmittance $t_{a}$ of the individual slits

$$
t(x)=\sum_{m=-n}^{m=n} t_{a}\left(x-x_{m}\right),
$$

where $N=2 n+1 . x_{m}$ designates the $x$-coordinate at the centre of the $m$ th slit. The Fourier transform can be written as

$$
\begin{aligned}
\tilde{t}\left(Q_{x}\right) & =\int t(x) \exp \left(\mathrm{i} Q_{x} x\right) \mathrm{d} x \\
& =\sum_{m=-n}^{m=n} \int t_{a}\left(x-x_{m}\right) \exp \left(\mathrm{i} Q_{x} x\right) \mathrm{d} x \\
& =\sum_{m=-n}^{m=n} \exp \left(\mathrm{i} Q_{x} x_{m}\right) \int t_{a}\left(x-x_{m}\right) \exp \left(\mathrm{i} Q_{x}\left(x-x_{m}\right)\right) \mathrm{d}\left(x-x_{m}\right) \\
& =\tilde{t}_{a}\left(Q_{x}\right) \sum_{m=-n}^{m=n} \exp \left(\mathrm{i} Q_{x} x_{m}\right)
\end{aligned}
$$




$$
\begin{aligned}
& =\tilde{t}_{a}\left(Q_{x}\right) \sum_{m=-n}^{m=n}\left(\exp \left(\mathrm{i} Q_{x} d\right)\right)^{m} \\
& =2 a\left[\frac{\sin \left(Q_{x} a\right)}{Q_{x} a}\right] \sum_{m=-n}^{m=n}\left(\exp \left(\mathrm{i} Q_{x} d\right)\right)^{m} .
\end{aligned}
$$

The expression

$$
\sum_{m=-n}^{m=n}\left(\exp \left(\mathrm{i} Q_{x} d\right)\right)^{m}
$$

is a geometrical series

$$
\sum_{k=-n}^{n} r^{k}=\frac{r^{n+1}-r^{-n}}{r-1}=r^{-n} \frac{1-r^{N}}{1-r} .
$$

Hence it has the solution

$$
\sum_{m=-n}^{m=n}\left(\exp \left(\mathrm{i} Q_{x} d\right)\right)^{m}=\exp \left(\mathrm{i} n Q_{x} d\right) \frac{1-\exp \left(\mathrm{i} N Q_{x} d\right)}{1-\exp \left(\mathrm{i} Q_{x} d\right)}=\frac{\sin \left(N Q_{x} d / 2\right)}{\sin \left(Q_{x} d / 2\right)}
$$

According to expression (4.16) the result for the diffraction amplitude of the grating can be found as

$$
\tilde{t}\left(Q_{x}\right)=N 2 a\left[\frac{\sin \left(Q_{x} a\right)}{Q_{x} a}\right]\left[\frac{\sin \left(N Q_{x} d / 2\right)}{N \sin \left(Q_{x} d / 2\right)}\right] .
$$

The intensity $I\left(Q_{x}\right)$ is the square of the amplitude

$$
I\left(Q_{x}\right)=4 N^{2} a^{2}\left[\frac{\sin \left(Q_{x} a\right)}{Q_{x} a}\right]^{2}\left[\frac{\sin \left(N Q_{x} d / 2\right)}{N \sin \left(Q_{x} d / 2\right)}\right]^{2}
$$

We show several diffraction patterns in Fig. 21 as a function of $Q_{x}$ and in Fig. 22 as function of $\theta_{x}$.

Before interpreting these results we would like to establish an alternative procedure. We introduce the Dirac comb as a periodic suite of Dirac functions

$$
\sum_{m=-\infty}^{\infty} \delta(x-m \cdot d)
$$

Then we recall that the convolution of two functions $g(x)$ and $h(x)$ is defined as

$$
g(x) \star h(x):=\int g\left(x^{\prime}\right) h\left(x-x^{\prime}\right) \mathrm{d} x^{\prime},
$$

and that the Fourier transform of two convoluted functions is the product of their individual Fourier transforms

$$
\mathrm{FT}[g(x) \star h(x)]=\mathrm{FT}[g(x)] \cdot \mathrm{FT}[h(x)]
$$



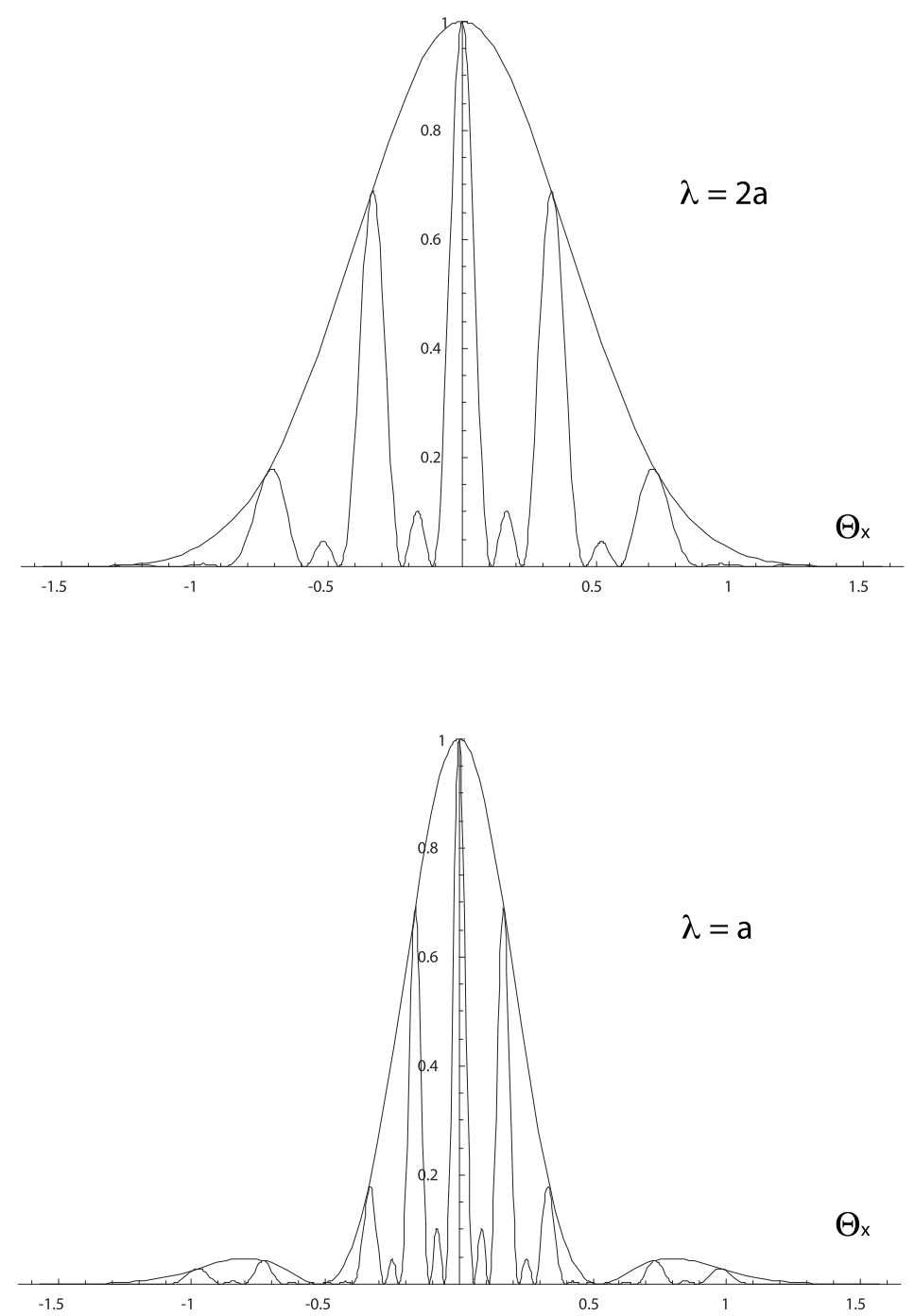

Fig. 22. Diffraction figures as a function of $\theta_{x}$ for two selected wave lengths. The grating is composed of three slits of width $2 a$ spaced at $d=6 a$ from each other. For $\lambda=2 a$ only the central peak of the envelope is observable. The dynamic range of the diffraction experiment is very limited. When the wave length decreases the dynamic range increases.

The transmittance of the grating can, therefore, be written in the form

$$
\begin{aligned}
t(x) & =\operatorname{rect}\left(\frac{x}{L}\right) \cdot\left[\int_{-\infty}^{\infty} \mathrm{d} x^{\prime} \operatorname{rect}\left(\frac{x^{\prime}}{2 a}\right) \sum_{m=-\infty}^{\infty} \delta\left(x^{\prime}-(x-m \cdot d)\right)\right] \\
& =\operatorname{rect}\left(\frac{x}{L}\right) \cdot\left[\operatorname{rect}\left(\frac{x}{2 a}\right) \star \sum_{m=-\infty}^{\infty} \delta(x-m \cdot d)\right]
\end{aligned}
$$

The Dirac comb corresponding to the centres of the slits is convoluted with the transmittance of the individual slit and finally multiplied by the transmittance of a rectangle, which takes into account the finite extension of the grating. The process is a bit like placing tiles onto the floor of a room. The tiles correspond to the slits, the 
Dirac comb indicates the grid onto which the tiles should be placed and the ensemble is delimited by the walls of the room. The Fourier transform of function (4.25) is the convolution of the diffraction function produced by the envelope (the room)

$$
\mathrm{FT}\left[\operatorname{rect}\left(\frac{x}{L}\right)\right]=L\left[\frac{\sin \left(Q_{x} L / 2\right)}{\left(Q_{x} L / 2\right)}\right]
$$

with the product of the diffraction functions of the individual slits (the tiles)

$$
\mathrm{FT}\left[\operatorname{rect}\left(\frac{x}{2 a}\right)\right]=2 a\left[\frac{\sin \left(Q_{x} a\right)}{Q_{x} a}\right]
$$

and the Dirac comb (the grid)

$$
\mathrm{FT}[t]=\mathrm{FT}\left[\operatorname{rect}\left(\frac{x}{L}\right)\right] \star\left\{\mathrm{FT}\left[\operatorname{rect}\left(\frac{x}{2 a}\right)\right] \cdot \mathrm{FT}\left[\sum_{m=-\infty}^{\infty} \delta(x-m \cdot d)\right]\right\} .
$$

The Fourier transform of a Dirac comb is simply a Dirac comb in reciprocal space with a period of $2 \pi / d$. Formally

$$
\mathrm{FT}\left[\sum_{m=-\infty}^{\infty} \delta(x-m \cdot d)\right]=\frac{1}{d} \sum_{m=-\infty}^{\infty} \delta\left(\frac{Q_{x}}{2 \pi}-\frac{m}{d}\right)
$$

The diffraction patterns is obtained from the square of expression (4.28), which is nothing else but expression (4.21).

\subsection{Structural information content of the diffraction pattern}

Already from this very simple one-dimensional examples we can judge that diffraction patterns are rich in structural information. This is particularly true for ordered structures. This wealth is at the origin of the immense contribution of modern diffraction-based crystallography in fields ranging from material science to structural biology. It is all based on the simple expressions (3.188) that relates the scattering amplitude to the Fourier transform of the potential. We will later see how by incorporating thermal motion the information content of diffraction patterns can be pushed even further. We may summarise our findings as follows:

- The diffraction peaks are found at distances $2 \pi / d$. Their positions thus give us information about the periodicity $d$ of the lattice.

- The width of an individual diffraction peak is given by $2 \pi / L$, i.e. it is determined by the envelope of the lattice. The peaks become sharper with either the number of peaks or their spacing increasing. But beware, finally it is the extension of the lattice and not the number of slits that defines the width of the individual diffraction peaks. In the limit of a lattice that can be considered infinitely large on the scale of the wave length of the diffracted radiation we obtain Dirac $\delta$-peaks (simply because the Fourier transform of the envelope becomes a $\delta$-function in this case). As a consequence the width of the peaks holds information on the extension of the periodic regions (e.g. the size of microcrystals).

- The intensity of the peaks is modulated by the diffraction figure or form factor (see Section 5.7) of the slit. If the slit is very narrow the periodicity $\pi / a$ of this modulation will be very large. For slits described by a Dirac function, the form factor is a simple constant. In scattering, this is the case for the effective potential of Section 3.7. For X-rays, the scattering is produced by the electronic cloud. This introduces a form factor that decreases the intensity for large wave vectors $\vec{Q}$ in the same way as finite sized optical apertures. 


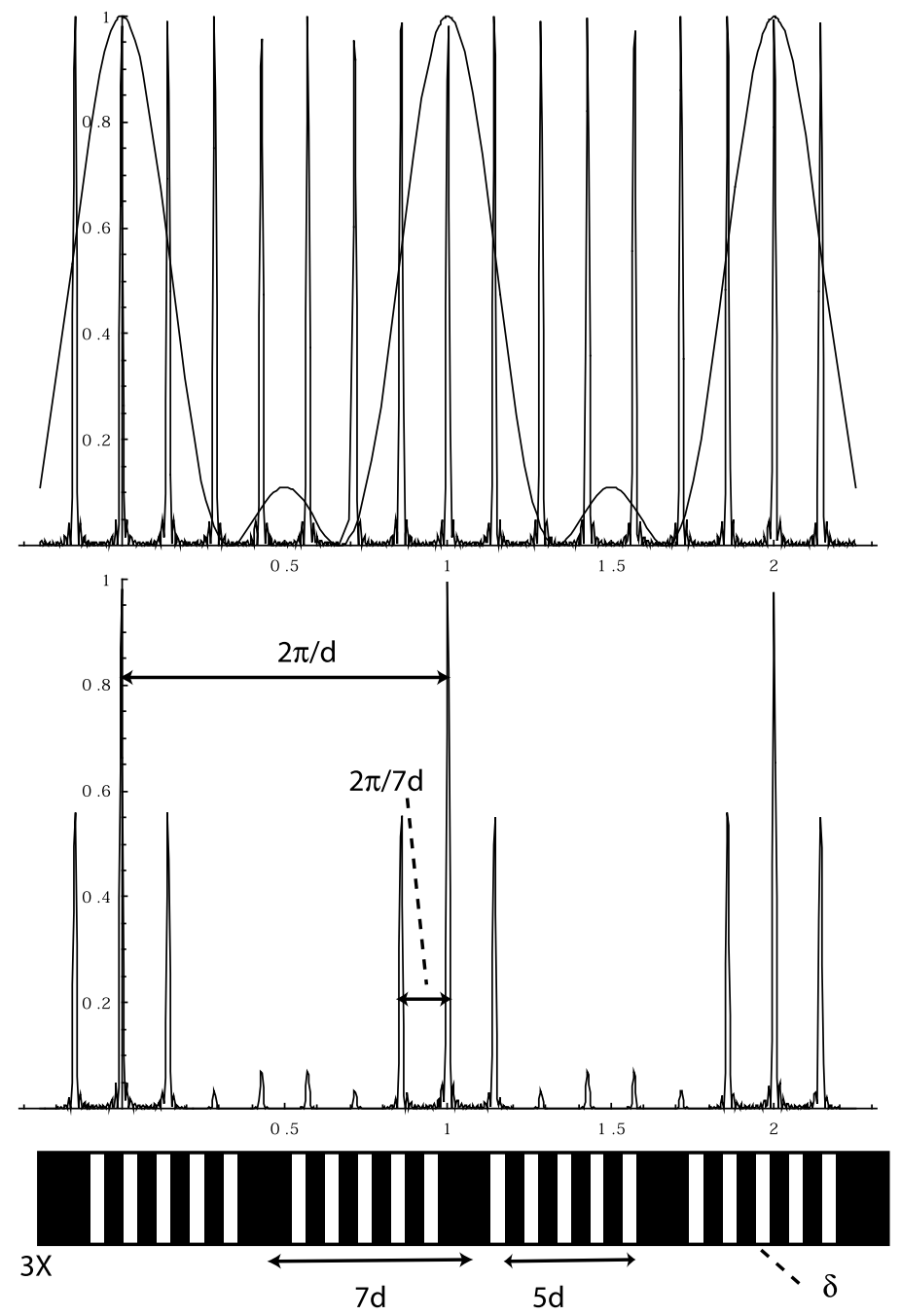

Fig. 23. Diffraction pattern of a lattice with a motive. If we neglect the finite extension of the lattice the diffraction pattern is obtained by multiplying the diffraction pattern of the motive, here a system of 5 Dirac slits a distance $d$ apart, with the diffraction pattern of the periodic lattice, here a Dirac comb with a spacing of $7 d$. The diffraction pattern of the infinite periodic lattice is a Dirac comb with a spacing of $2 \pi / 7 d$. The diffraction pattern of the individual motive is a periodic function with periodicity $2 \pi / d$. Both function are shown in the upper picture. Multiplying both patterns results in a modulation of the peak heights. For slits with a finite aperture the thus obtained function should be multiplied with the diffraction figure of the aperture itself. This diffraction figure is equivalent to the form factor for an atom in X-ray scattering. It introduces, in particular, a loss in intensity at high $Q$ values. The information on the periodicity of the lattice (the unit cell) is thus contained in the peak positions. The information on the form of the motive, or in the case of a crystal on the position of the atoms in the unit cell, is obtained from the intensity of the peaks.

- If we want to go even further we have to check what will happen if we replace a simple slit by a complex motive. One simple and instructive way of forming such a motive consists in periodically removing slits (see Figs 23 and 24) from the grating. In this way we create a lattice with a larger periodicity. The individual peaks now appear at shorter intervals. Again the grating can be considered a convolution of a Dirac comb with the transmittance of, this time not the slits, but the motive. The total diffraction function is, therefore, obtained as the product of the diffraction function of the individual motive with the diffraction function of the lattice. The intensity of the peaks is thus modulated according to the diffraction function of the motive. Hence it is 


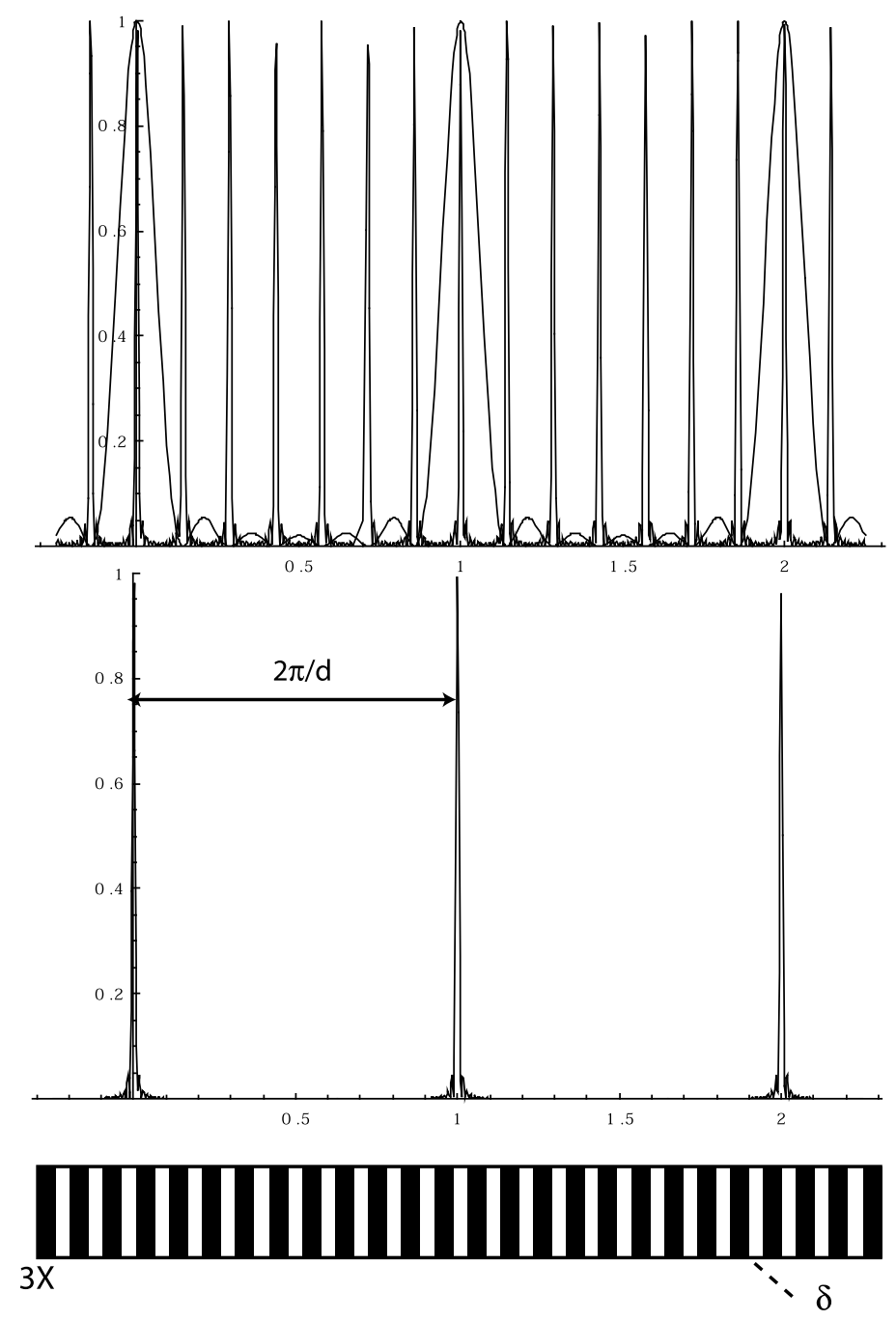

Fig. 24. Same as Fig. 23 with the only difference that we have chosen a motive that restores periodicity at a distance $d$. As required all the intermediate peaks are made extinct by the multiplication with the form factor of the motive.

possible to determine the form of the motive by studying these intensities.

\subsection{Diffraction from a three-dimensional lattice}

If we go from a lattice defined on a plane to a three dimensional lattice we get Bragg scattering. The fundamental relation between the scattering angle $2 \Theta$ (see Fig. 25) and the wave length of the diffracted light is given by Bragg's law

$$
n \lambda=2 \cdot d \cdot \sin \Theta
$$

where $d$ is the distance between the diffracting planes and $n$ is the order of the Bragg reflection. Bragg's law expresses the fact, that in order to have positive interference from the individual lattice planes, the optical paths must differ by an integer multiple $n$ of the wave length (see Fig. 25). Such three-dimensional lattices are created 

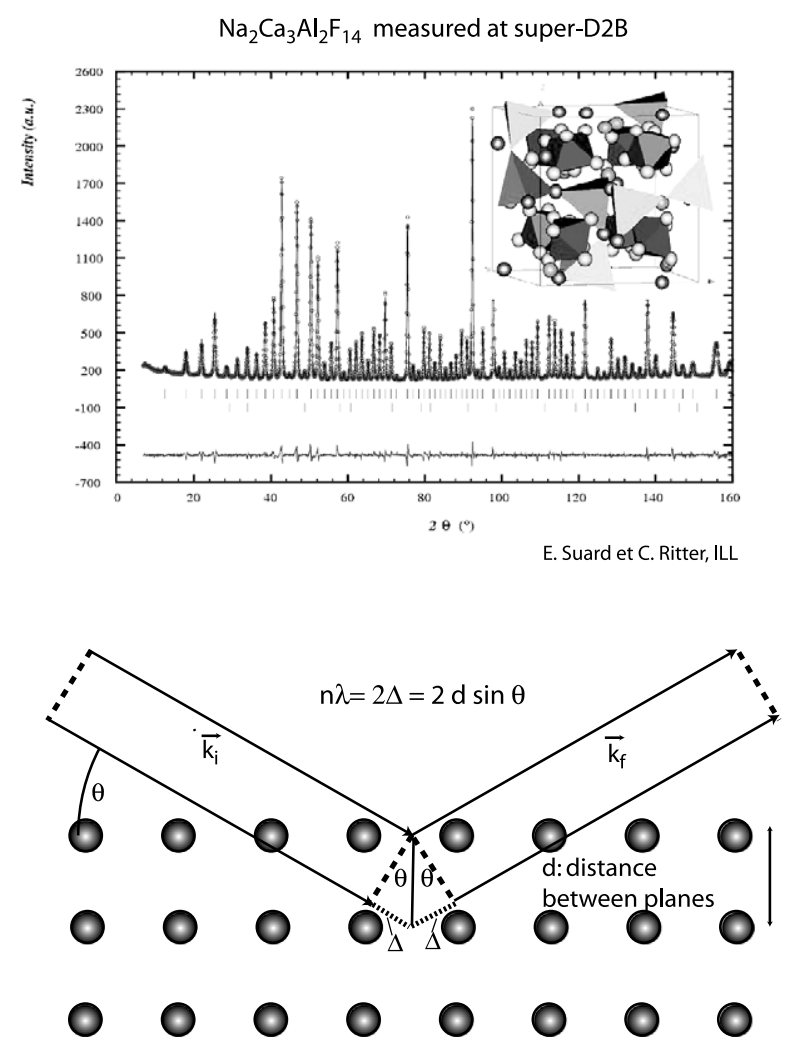

Fig. 25. Bragg scattering. The top figure shows a typical example of a diffraction pattern obtained for a crystalline powder on a high resolution neutron diffractometer. The bottom figure justifies Bragg's law from the difference in optical paths $\Delta$ between adjacent lattice planes.

by single crystals. These lattices are suitable for X-ray or neutron diffraction since the distance between the atoms is of the order of a few $\AA$.

\subsection{Longitudinal coherence length}

Until now we have considered that the incident radiation can be described perfectly by monochromatic waves. In practice this is never the case. Imperfections originate either from the spacial extension or the spectral distribution of the primary radiation source. Interference phenomena rely on exact phase relations between the wavelets originating in the various regions of the diffracting object. The interference patters will be sharp if the generation of the wavelets at different points is synchronised. This is e.g. the case for a monochromatic wave front produced by a point source. Any random fluctuation in the wave front will weaken the interference effects. Such fluctuations cannot be avoided in extended radiation sources where the emittance is random, i.e. unsynchronised. This is e.g. the case for incandescent light sources such as light bulbs. Lasers on the other hand are coherent sources of radiation. Independent of phase fluctuations the diffraction pattern is also dependent on the wave length. If the primary radiation source contains a spectrum of frequencies the superposition of diffraction patterns for the various wave lengths weakens the interference effects.

We first will discuss the effect of the polychromatic nature of the primary radiation source. For a given frequency $\nu$ the phase of the complex amplitude changes by a factor $2 \pi$ over the distance $L=\lambda=c / \nu$ where $c$ is the velocity of light ( $c$ has to be replaced by the phase velocity $v_{p}$ for other forms of radiation). Two waves that are 
simultaneously generated at the same point in space and that differ in frequency by $\Delta \nu$ will be completely out of phase after they have covered an optical path of length

$$
L_{c}=\frac{c}{\Delta \nu}=\frac{\lambda^{2}}{\Delta \lambda}
$$

$L_{c}$ is called the temporal coherence length or the longitudinal coherence length (see Fig. 26). It is a measure of the spectral purity of the primary radiation source. If the variation in the length of the optical path traveled when reaching the point $P$ exceeds the coherence length of the primary radiation source then these wavelets no longer contribute to the construction of interference patterns. To illustrate this phenomenon we use the example of a grating. The diffraction maxima are found at

$$
Q_{m}=m \frac{2 \pi \sin \theta}{\lambda}=m \frac{2 \pi}{d}
$$

If the uncertainty in wave length is chosen to be $\Delta \lambda=\frac{\lambda^{2}}{d}$ expression (4.31) gives a longitudinal coherence length

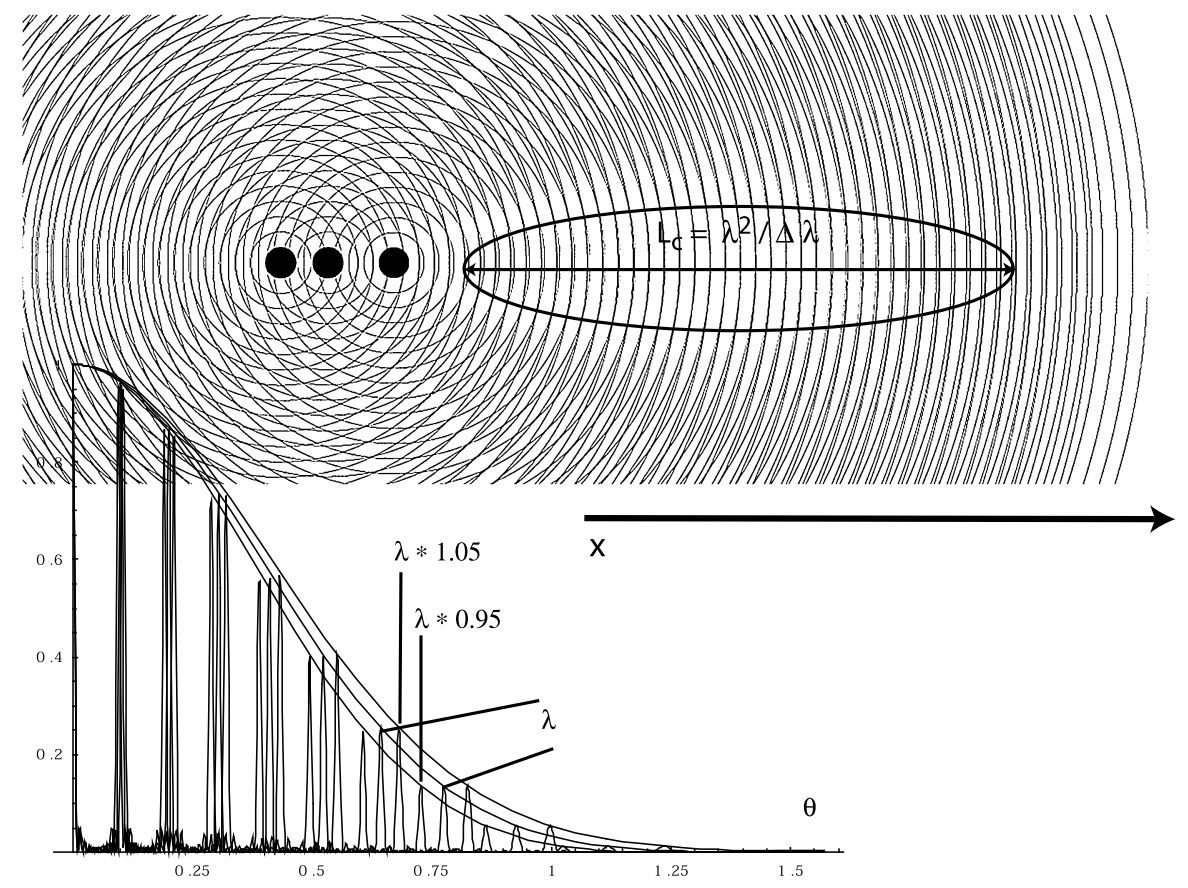

Fig. 26. Longitudinal coherence length. The illuminated object is found at a certain distance along $x$. The polychromatic wave trains will get out of phase with respect to each other over the distance $L_{c}$. It is important to realise that the absolute phase of the wavelets does not matter since each wave train produces a distinct diffraction pattern of its own. All these individual diffraction patterns can simply be summed up as there is no interference between wave trains that possess different frequencies. This is due to the fact that such interferences oscillate in time and, therefore, cancel out when integrating over time. Profiting from the freedom in choosing the absolute phase the point sources have here been translated along $\hat{x}$ for the purpose of illustration. This translation allows us to illustrate the de-phasing at a certain distance. The choice of this distance is arbitrary. It is the superposition of the diffraction patterns that blurs the interferences. The bottom figure illustrates this blurring effect by superposing three diffraction patterns calculated as a function of diffraction angle $\theta$ for a system of 11 slits of width $2 a=\lambda$ and spaced at $d=10 \cdot \lambda$. The $\Delta \lambda$ of $10 \%$ gives a longitudinal coherence length $L_{\mathrm{c}}=10 \cdot \lambda$, i.e. just compatible with the spacing of the slits. It can be seen that the regions between the peaks are successively filled with intensity for large diffraction angles as we add patterns from additional wave lengths. For small diffraction angles the difference in optical path length of the wavelets constituting the diffraction pattern are sufficiently small compared to the coherence length for the fringes to remain separated. 
that is equal to the distance between the slits $d$. For a given diffraction order $m$ the maxima of the diffraction pattern will, as a function of wave length, sweep the range from $Q_{m}(\lambda)$ to $Q_{m}\left(\lambda^{\prime}\right)$ with

$$
\lambda^{\prime}=\lambda \pm \frac{\Delta \lambda}{2}=\lambda \pm \frac{\lambda^{2}}{2 d}
$$

For $\Delta \lambda \ll \lambda$ we get

$$
Q_{m=1}\left(\lambda^{\prime}\right)=\frac{2 \pi \sin \theta}{\lambda \pm \lambda^{2} /(2 d)} \approx Q \pm \frac{1}{2} \frac{2 \pi \sin \theta}{d} .
$$

For a typical diffraction angle of $\theta=\pi / 4$ we find $\sin \theta=1 / \sqrt{2}$ and hence $Q_{m=1}\left(\lambda^{\prime}\right) \approx Q \pm \pi / d$. The signal, therefore, sweeps the complete region between the peaks. The interferences are hardly observable (see Fig. 26). Simply speaking, the maxima generated with one frequency are superimposed onto the minima generated with another frequency erasing the interference patterns. The coherence length hence defines the resolution of the diffraction setup in real space.

\subsection{Transversal coherence length}

Even if the source is monochromatic the resolution of the optical setup will be limited by the lateral extension of the source. To illustrate this phenomena we will study how the displacement of a point source effects the interference figure observed on the screen (see Fig. 27). Assuming that the displacements are large compared to the wave length allows us to use wave fronts in our argumentation. To first order, only the lateral displacements contribute to the modification of the optical path lengths. Purely longitudinal displacements, i.e. displacements along the axis that joins the source to the diffracting object, do not modify the phase relations of the secondary wavelets. We chose as diffracting object a pair of Young slits, which we have already encountered in the introduction. We
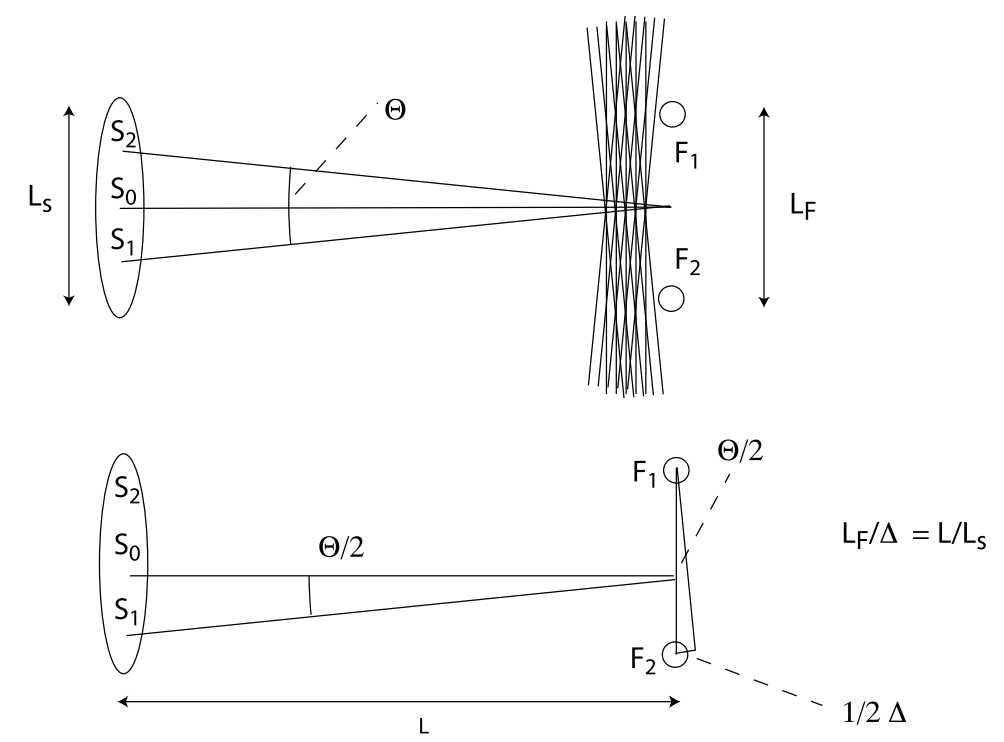

Fig. 27. Lateral coherence length due to the finite extension of the primary source. Both $L_{S}$ and $L$ are assumed large on the scale of $L_{F}$, and in addition $L \gg L_{S}$. The wave fronts originating from different points of the primary source will be inclined with respect to each other at the location of the slits. This inclination is characterised by the distance $\Delta$, which translates into a "delay" of emission of the wavelets and, therefore, into a phase shift. If the distance $\Delta$ approaches the wave length $\lambda$ then the interference fringes become blurred. This defined the lateral coherence length. 
demand that the distance $L$ between the primary source and the Young slits is large compared to the extension $L_{S}$ of the source and compared to the distance between the slits $L_{F}$. The secondary part of the setup remaining unchanged when replacing the source laterally, any change of optical path lengths has to take place in the primary part, that is to say between the primary source and the slits. Wave fronts having their origin in different points of the source will hit the two slits with a spatial displacement $\Delta$ of

$$
\Delta \approx \frac{L_{F}}{L} L_{S}
$$

As soon as this difference in path length corresponds to the wave length the interference effects will disappear. We define the lateral coherence length as

$$
L_{c}=\lambda \frac{L}{L_{S}}=\frac{\lambda}{\Theta}
$$

where $\Theta$ is the angle sustained by the source as seen from the position of the slits (see Fig. 27). The lateral coherence length increases with the distance between the primary source and the diffracting object. This phenomenon is called creation of coherence via distance. For example the lateral coherence length of the sun due to its distance is a remarkable $60 \mu \mathrm{m}$. If we use diffraction as an analytic instrument for structural determination then it is impossible to determine the distance between slits separated by a distance that exceeds $L_{c}$.

\subsection{Coherence volume}

We want now to discuss how the longitudinal and transverse coherence lengths define the resolution of the experimental set up in real space. ${ }^{44}$ To start we pick out one of the diffracting objects and denote it by $D_{m}$. In the next step we determine the set of objects $D_{n}$ that produce scattering amplitudes capable of interfering constructively with the scattering amplitude produced by $D_{m}$. We find these diffraction objects within a perimeter surrounding $D_{m}$ given by the coherence lengths of the primary radiation. This corresponding volume is called the coherence volume. Objects found outside the coherence volume surrounding $D_{m}$, i.e. at distances from $D_{m}$ exceeding the coherence length, will not contribute to the diffraction pattern. Similar to the random arrangement of slits in a grating (see Section 4.3) they will, with their own coherence volumes, just add extra intensity to the scattering.

\section{Scattering from a target with internal degrees of freedom}

A condensed matter sample is composed of a large number of scatterers (atomic nuclei and magnetic moments for the neutrons, electrons for the X-rays, etc.). In order to calculate how a given sample scatters an incident beam we have to solve the Schrödinger equation for the combined system of the target and probe. This will lead us to a quantum mechanical description of inelastic scattering. The formalism that we will employ is analogous to the one that we have developed for scattering from a static potential, i.e. we will try to express the problem in the form of an integral equation using the concept of a Green function, which will allow us to determine the scattering cross section in the Born approximation. The difficulty resides in the mathematical complexity introduced by the necessity to carry along the wave functions describing the target states.

\footnotetext{
${ }^{44}$ We will return to this point in the discussion on resolution in the case of neutron scattering (see Section 11.6).
} 


\subsection{Wave functions in common Hilbert space}

Let us consider the scattering of a probe particle of mass $m$ by a complex target system $\mathrm{T}$, which is assumed to possess a certain number of internal degrees of freedom. Transitions between the quantum states of the target open up the possibility of inelastic scattering. We, therefore, have to design a mathematical formalism capable of taking on board inelastic scattering.

To avoid any complication related to the choice of frames of reference we assume that the mass of the target system is largely superior to that of the probe particle $m$. In order not to be bothered by questions of quantum mechanical exchange we, in addition, require that the probe particle is different from the particles that make up the target. This is e.g. the case if neutrons interact with compound nuclei. Let us denote the coordinates describing the target T collectively as $\{\xi\}$. The coordinate describing the probe particle is represented as before by $\vec{r}$. Then, if $\mathbf{H}_{t}$ denotes the Hamiltonian of the target system, the combined system is described by the wave function $\psi(\vec{r}, \xi)$ satisfying the following stationary Schrödinger equation

$$
\left(E_{\mathrm{tot}}-\mathbf{H}_{t}(\xi)+\mathrm{i} \frac{\hbar^{2}}{2 m} \Delta\right) \psi(\vec{r}, \xi)=V(\vec{r}, \xi) \psi(\vec{r}, \xi)
$$

with $E_{\text {tot }}$ the total energy of the system and $V(\vec{r}, \xi)$ the interaction potential of the probe with the target.

We will aim for a perturbative treatment with the uncoupled systems serving as the unperturbed reference. The isolated target system be described by the wave functions $\phi_{\nu}(\xi)$, which are eigenfunctions to the Hamilton operator $\mathbf{H}_{t}$ with eigenvalues $E_{\nu}$

$$
\mathbf{H}_{t}(\xi) \phi_{\nu}(\xi)=E_{\nu} \phi_{\nu}(\xi)
$$

The index $\nu$ collectively denotes all the quantum numbers of the target states.

For the combined system of probe and target the direct product states

$$
\left|\Theta_{\nu}(\vec{k})\right\rangle=\left|\phi_{\nu}\right\rangle \otimes|\vec{k}\rangle
$$

form a basis. They are eigenfunctions of the system in the case of vanishing interaction

$$
\left(\mathbf{H}_{t}(\xi)+\mathrm{i} \frac{\hbar^{2}}{2 m} \Delta_{\vec{r}}\right) \Theta_{\nu, \vec{k}}(\vec{r}, \xi)=\left(E_{\nu}+\frac{\hbar^{2} k^{2}}{2 m}\right) \Theta_{\nu, \vec{k}}(\vec{r}, \xi),
$$

which we may write in the Dirac notation

$$
\mathbf{H}_{0}\left|\Theta_{\nu}(\vec{k})\right\rangle=E_{0}\left|\Theta_{\nu}(\vec{k})\right\rangle .
$$

In a scattering configuration with the action of the potential limited in spacial extent the total energy $E_{\text {tot }}$ will be identical to the energy $E_{0}$ that the combined system possesses before and after the scattering.

As the wave functions for the free probe particle are explicitly known we get in the real space representation

$$
\left\langle\vec{r}, \xi \mid \Theta_{\nu}(\vec{k})\right\rangle=\Theta_{\nu, \vec{k}}(\vec{r}, \xi)=\frac{1}{\sqrt{2 \pi}^{3}} \mathrm{e}^{\mathrm{i} \vec{k} \cdot \vec{r}} \phi_{\nu}(\xi) .
$$

We now state a few useful relations.

Orthogonality of the eigenfunctions is expressed via

$$
\left\langle\Theta_{\nu^{\prime}}\left(\vec{k}^{\prime}\right) \mid \Theta_{\nu}(\vec{k})\right\rangle=\delta_{\nu, \nu^{\prime}} \cdot \delta\left(\vec{k}-\vec{k}^{\prime}\right) .
$$


Completeness leads to the closure relation

$$
\sum_{\nu} \int \mathrm{d}^{3} k\left|\Theta_{\nu}(\vec{k})\right\rangle\left\langle\Theta_{\nu}(\vec{k})\right|=1
$$

Normalisation is such that

$$
\sum_{\nu} \int \mathrm{d}^{3} k\left\langle\vec{r}, \xi \mid \Theta_{\nu}(\vec{k})\right\rangle\left\langle\Theta_{\nu}(\vec{k}) \mid \vec{r}^{\prime}, \xi^{\prime}\right\rangle=\left\langle\vec{r}, \xi \mid \vec{r}^{\prime}, \xi^{\prime}\right\rangle=\delta\left(\xi-\xi^{\prime}\right) \cdot \delta\left(\vec{r}-\vec{r}^{\prime}\right),
$$

which may be expressed in the alternative form

$$
\frac{1}{(2 \pi)^{3}} \sum_{\nu} \int \phi_{\nu}\left(\xi^{\prime}\right) \phi_{\nu}^{*}(\xi) \mathrm{e}^{\mathrm{i} \vec{k} \cdot\left(\vec{r}-\vec{r}^{\prime}\right)} \mathrm{d}^{3} k=\delta\left(\xi-\xi^{\prime}\right) \cdot \delta\left(\vec{r}-\vec{r}^{\prime}\right) .
$$

Please note that the quantum states of the target are discrete and bound due to the fact that the particles constituting the target are confined in space. The orthogonality condition, therefore, employs the sum over these states, while in the case of the target particle we have to integrate over all free states.

\subsection{Green function of the combined system}

Following the successful route employed to determine the scattering amplitude for static potentials we will recast the problem in the integral form using the concept of Green functions. From Eq. (5.1) we derive the source term in complete analogy with expression (3.149) as

$$
j_{\vec{k}}(\vec{r}, \xi):=V(\vec{r}, \xi) \psi(\vec{r}, \xi)
$$

This allows expressing the Schrödinger equation as

$$
\mathbf{D} \psi(\vec{r}, \xi)=j_{\vec{k}}(\vec{r}, \xi)
$$

with the operator

$$
\mathbf{D}:=-\left(\mathbf{H}_{0}-E_{0}\right) \text {. }
$$

The defining equation of the Green function now reads

$$
\mathbf{D} G_{0}\left(\vec{r}, \vec{r}^{\prime} \mid \xi, \xi^{\prime}\right)=\delta\left(\vec{r}-\vec{r}^{\prime}\right) \cdot \delta\left(\xi-\xi^{\prime}\right) .
$$

The main difference with respect to the previous expression (3.154) resides in the presence of additional "point sources" represented by the functions $\delta\left(\xi-\xi^{\prime}\right)$. These point sources translate the physical fact that the interaction potential is no longer a pure function of the probe particle position $\vec{r}$ but equally of the simultaneous ${ }^{45}$ position of all the reciprocally interacting target particles.

Formally the Green function we are looking for satisfies

$$
\left(E_{0}-\mathbf{H}_{0}\right) G_{0}\left(\vec{r}, \vec{r}^{\prime} \mid \xi, \xi^{\prime}\right)=\delta\left(\vec{r}-\vec{r}^{\prime}\right) \cdot \delta\left(\xi-\xi^{\prime}\right)
$$

\footnotetext{
${ }^{45} \mathrm{We}$ assume instantaneous interaction and thus do not include retardation effects.
} 
Therefore,

$$
\left\langle\vec{r}, \xi\left|\left(E_{0}-\mathbf{H}_{0}\right) G_{0}\left(\vec{r}, \vec{r}^{\prime}\right)\right| \vec{r}^{\prime}, \xi^{\prime}\right\rangle=\left\langle\vec{r}, \xi \mid \vec{r}^{\prime}, \xi^{\prime}\right\rangle,
$$

which leads us again to formally identify Green's operator with the inverse of $\left(E_{0}-\mathbf{H}_{0}\right)$

$$
\mathbf{G}_{0}=\frac{1}{\left(E_{0}-\mathbf{H}_{0}\right)} \text {. }
$$

Using the orthogonally (5.7), closure (5.8) and normalisation (5.10) conditions we may calculate the Green function as follows

$$
\begin{aligned}
G_{0}\left(\vec{r}, \xi \mid \vec{r}^{\prime}, \xi^{\prime}\right) & =\left\langle\vec{r}, \xi\left|\frac{1}{\left(E_{0}-\mathbf{H}_{0}\right)}\right| \vec{r}^{\prime}, \xi^{\prime}\right\rangle \\
& =\sum_{\nu \nu^{\prime}} \iint \mathrm{d}^{3} k \mathrm{~d}^{3} k^{\prime}\left\langle\vec{r}, \xi \mid \Theta_{\nu}(\vec{k})\right\rangle\left\langle\Theta_{\nu}(\vec{k})\left|\frac{1}{\left(E_{0}-\mathbf{H}_{0}\right)}\right| \Theta_{\nu^{\prime}}\left(\vec{k}^{\prime}\right)\right\rangle\left\langle\Theta_{\nu^{\prime}}\left(\vec{k}^{\prime}\right) \mid \vec{r}^{\prime}, \xi^{\prime}\right\rangle \\
& =\sum_{\nu \nu^{\prime}} \iint \mathrm{d}^{3} k \mathrm{~d}^{3} k^{\prime}\left\langle\vec{r}, \xi \mid \Theta_{\nu}(\vec{k})\right\rangle \frac{\left\langle\Theta_{\nu}(\vec{k}) \mid \Theta_{\nu^{\prime}}\left(\vec{k}^{\prime}\right)\right\rangle}{\left(E_{0}-E_{\nu}(\vec{k})\right)}\left\langle\Theta_{\nu^{\prime}}\left(\vec{k}^{\prime}\right) \mid \vec{r}^{\prime}, \xi^{\prime}\right\rangle \\
& =\sum_{\nu \nu^{\prime}} \iint \mathrm{d}^{3} k \mathrm{~d}^{3} k^{\prime}\left\langle\vec{r}, \xi \mid \Theta_{\nu}(\vec{k})\right\rangle \frac{\delta_{\nu, \nu^{\prime}} \cdot \delta\left(\vec{k}-\vec{k}^{\prime}\right)}{\left(E_{0}-E_{\nu}(\vec{k})\right)}\left\langle\Theta_{\nu^{\prime}}\left(\vec{k}^{\prime}\right) \mid \vec{r}^{\prime}, \xi^{\prime}\right\rangle \\
& =\sum_{\nu} \int \mathrm{d}^{3} k\left\langle\vec{r}, \xi \mid \Theta_{\nu}(\vec{k})\right\rangle \frac{1}{\left(E_{0}-E_{\nu}(k)\right)}\left\langle\Theta_{\nu}(\vec{k}) \mid \vec{r}^{\prime}, \xi^{\prime}\right\rangle \\
& =\frac{1}{(2 \pi)^{3}} \sum_{\nu} \int \mathrm{d}^{3} k \frac{\phi_{\nu}(\xi) \phi_{\nu}^{*}\left(\xi^{\prime}\right)}{\left(E_{0}-E_{\nu}(k)\right)} \mathrm{e}^{\mathrm{i} \vec{k} \cdot\left(\vec{r}-\vec{r}^{\prime}\right)} .
\end{aligned}
$$

The energy $E_{\nu}(k)$ is given as

$$
E_{\nu}(k)=E_{\nu}+\frac{\hbar^{2} k^{2}}{2 m}
$$

The total energy $E_{0}$ can be expressed by adding the kinetic energy that the probe particle possesses before the scattering to the energy of the initial state of the sample

$$
E_{0}=E_{\nu_{\text {ini }}}+\frac{\hbar^{2} k_{i}^{2}}{2 m}
$$

With this we may reformulate the denominator of the above expression for the Green function as

$$
\frac{\phi_{\nu}(\xi) \phi_{\nu}^{*}\left(\xi^{\prime}\right)}{E_{0}-E_{\nu}(k)}=\frac{2 m}{\hbar^{2}} \cdot \frac{\phi_{\nu}(\xi) \phi_{\nu}^{*}\left(\xi^{\prime}\right)}{k_{f}^{2}-k^{2}}
$$

with $k_{f}$ defined as ${ }^{46}$

$$
k_{f}^{2}=k_{f}^{2}(\nu)=\frac{2 m}{\hbar^{2}}\left(E_{0}-E_{\nu}\right)=\frac{2 m}{\hbar^{2}}\left(E_{\nu_{\mathrm{ini}}}-E_{\nu}+\frac{\hbar^{2} k_{i}^{2}}{2 m}\right),
$$

\footnotetext{
${ }^{46}$ We will see later under which conditions $k_{f}$ relates to the wave vector of the probe particle state after the scattering.
} 
where the notation $k_{f}^{2}(\nu)$ renders the dependence of this quantity on the energy of the target state $\left|\phi_{\nu}\right\rangle$ explicit.

Using the methods of analytical algebra we can integrate the pole ${ }^{47}$

$$
\frac{1}{2 \pi^{3}} \int \mathrm{d}^{3} k \frac{\mathrm{e}^{\mathrm{i} \vec{k} \cdot \vec{r}}}{k_{f}^{2}-k^{2}}=-\frac{\mathrm{e}^{\mathrm{i} k_{f} \cdot r}}{4 \pi r},
$$

which leads to the final form of the Green function

$$
\begin{aligned}
G_{0}\left(\vec{r}, \xi \mid \vec{r}^{\prime}, \xi^{\prime}\right) & =\frac{1}{(2 \pi)^{3}} \sum_{\nu} \int \mathrm{d}^{3} k \frac{\phi_{\nu}(\xi) \phi_{\nu}^{*}\left(\xi^{\prime}\right)}{\left(E_{0}-E_{\nu}(k)\right)} \mathrm{e}^{\mathrm{i} \vec{k} \cdot\left(\vec{r}-\vec{r}^{\prime}\right)} \\
& =\frac{2 m}{(2 \pi)^{3} \hbar^{2}} \sum_{\nu} \int \mathrm{d}^{3} k \frac{\phi_{\nu}(\xi) \phi_{\nu}^{*}\left(\xi^{\prime}\right)}{k_{f}^{2}-k^{2}} \mathrm{e}^{\mathrm{i} \vec{k} \cdot\left(\vec{r}-\vec{r}^{\prime}\right)} \\
& =-\frac{m}{2 \pi \hbar^{2}} \sum_{\nu} \phi_{\nu}(\xi) \phi_{\nu}^{*}\left(\xi^{\prime}\right) \frac{\mathrm{e}^{\mathrm{i} k f \cdot\left|\vec{r}-\vec{r}^{\prime}\right|}}{\left|\vec{r}-\vec{r}^{\prime}\right|} .
\end{aligned}
$$

The Green function is a superposition of spherical waves. Each spherical wave is characterised by a wave number $k_{f}(\nu)$ and has to be seen in conjunction with the accompanying target state $\left|\phi_{\nu}\right\rangle$. The relative weight of a spherical wave in this superposition is given by the norm of the wave function $\phi_{\nu}(\xi) \phi_{\nu}^{*}\left(\xi^{\prime}\right)$.

It is obvious that the developed Green function can only describe scattering if $k_{f}$ is real and greater zero, which implies that $k_{f}^{2}>0$. This is the case if

$$
E_{\nu}<E_{\nu_{\text {ini }}}+\frac{\hbar^{2} k_{i}^{2}}{2 m}
$$

In other words, the sample states entering into the Green function have to possess an energy that is smaller than the total initial energy of the combined system. When this is the case the scattering channel associated with $\left|\phi_{\nu}\right\rangle$ is called an open channel. Also not relevant for our purpose closed channels are important in many collision problems. They correspond to situations where the probe particle is incorporated (absorbed) into the target depositing not only all of its kinetic energy but contributing in addition to the internal energy of the target.

Having determined the Green function we can write down the integral form of the Schrödinger equation according to expression (3.159). If we choose to express this equation in the basis of the unperturbed states $\left|\Theta_{\mu_{i} \vec{k}_{i}}\right\rangle$ we obtain the following set of equations

$$
\begin{aligned}
& \psi_{\mu_{i} \vec{k}_{i}}(\vec{r}, \xi) \\
& =\Theta_{\mu_{i} \vec{k}_{i}}(\vec{r}, \xi)+\iint \mathrm{d}^{3} r^{\prime} \mathrm{d} \xi^{\prime} G_{0}\left(\vec{r}, \xi \mid \vec{r}^{\prime}, \xi^{\prime}\right) V\left(\vec{r}^{\prime}, \xi^{\prime}\right) \psi_{\vec{k}_{i}}\left(\vec{r}^{\prime}, \xi^{\prime}\right) \\
& =\Theta_{\mu_{i} \vec{k}_{i}}(\vec{r}, \xi)+\iint \mathrm{d}^{3} r^{\prime} \mathrm{d} \xi^{\prime} G_{0}\left(\vec{r}, \xi \mid \vec{r}^{\prime}, \xi^{\prime}\right) V\left(\vec{r}^{\prime}, \xi^{\prime}\right) \Theta_{\mu_{i} \vec{k}_{i}}\left(\vec{r}^{\prime}, \xi^{\prime}\right) \\
& \quad+\iint \mathrm{d}^{3} r^{\prime \prime} \mathrm{d} \xi^{\prime \prime} \iint \mathrm{d}^{3} r^{\prime} \mathrm{d} \xi^{\prime} G_{0}\left(\vec{r}, \xi \mid \vec{r}^{\prime}, \xi^{\prime}\right) V\left(\vec{r}^{\prime}, \xi^{\prime}\right) G_{0}\left(\vec{r}^{\prime}, \xi^{\prime} \mid \vec{r}^{\prime \prime} \xi^{\prime \prime}\right) V\left(\vec{r}^{\prime \prime}, \xi^{\prime \prime}\right) \psi_{\mu_{i} \vec{k}_{i}}\left(\vec{r}^{\prime \prime}, \xi^{\prime \prime}\right) .
\end{aligned}
$$

\footnotetext{
${ }^{47}$ In a rigorous mathematical treatment we have to make sure that we turn around the pole with the right sense of rotation.
} 
$\Theta_{\mu_{i} \vec{k}_{i}}(\vec{r}, \xi)$ denotes any of the wave functions of the unperturbed system. $\psi_{\mu_{i} \vec{k}_{i}}(\vec{r}, \xi)$ are the wave functions that develop from the unperturbed states when we switch on the interaction. The above expression thus constitutes an operation that maps unperturbed states $\left|\Theta_{\mu_{i} \vec{k}_{i}}\right\rangle$ onto exact stationary solutions $\left|\psi_{\mu_{i} \vec{k}_{i}}\right\rangle$. The corresponding operator is an extension of the wave operator introduced in Section 3.11.

\subsection{Born approximation for probe and particle states}

The perturbation created by the interaction concerns both the probe and target states. They are, therefore, both evolving away from their unperturbed eigenstates under the influence of the mutual interaction. Like in the case of elastic scattering the right hand side of the integral equation (5.26) refers back to the wave function $\left\langle\vec{r}, \xi \mid \psi_{\mu_{i}} \vec{k}_{i}\right\rangle$, which it is actually supposed to express. As we had seen when treating the case of a static potential this problem can be circumvented by going to the Born approximation. To do so we make the following substitution

$$
\psi_{\mu_{i} \vec{k}_{i}}\left(\vec{r}^{\prime}, \xi^{\prime}\right) \rightarrow \Theta_{\mu_{i} \vec{k}_{i}}\left(\vec{r}^{\prime}, \xi^{\prime}\right)
$$

under the integral, which leads us to

$$
\begin{aligned}
\psi_{\mu_{i} \vec{k}_{i}}(\vec{r}, \xi)= & \Theta_{\mu_{i} \vec{k}_{i}}(\vec{r}, \xi) \\
& -\frac{m}{2 \pi \hbar^{2}} \sum_{\nu} \phi_{\nu}(\xi) \iint \mathrm{d}^{3} r^{\prime} \mathrm{d} \xi^{\prime} \phi_{\nu}^{*}\left(\xi^{\prime}\right) \frac{\mathrm{e}^{\mathrm{i} k_{f} \cdot\left|\vec{r}-\vec{r}^{\prime}\right|}}{\left|\vec{r}-\vec{r}^{\prime}\right|} V\left(\vec{r}^{\prime}, \xi^{\prime}\right) \Theta_{\mu_{i} \vec{k}_{i}}\left(\vec{r}^{\prime}, \xi^{\prime}\right) .
\end{aligned}
$$

In Sections 3.11 and 3.13 we had elaborated at length on the conditions that warrant the use of the Born approximation from the point of view of the probe particle. When dealing with inelastic scattering the Born approximation is equally employed to the quantum states of the target. These states as we have seen enter directly into the amplitudes of the spherical waves that describe the scattered probe particles. When calculating these amplitudes in the Born approximation we use the target states of the unperturbed system. In the same way that we assume that the incoming probe wave can be assumed unperturbed by the scattering upstream we assume that the target states are left unchanged. We assume that the interaction is so weak that the probe does not influence the states $\left|\phi_{\nu}\right\rangle$ of the sample. Its role is reduced to inducing transitions between these states. This approach is certainly justified for slow neutrons far from resonance.

In the Born approximation we now exploit the fact that the initial wave function factorises and that the form of the probe particle's wave function is known explicitly. This leads us to

$$
\begin{aligned}
& \psi_{\mu_{i} \vec{k}_{i}}(\vec{r}, \xi) \\
& =\Theta_{\mu \vec{k}}(\vec{r}, \xi) \\
& \quad-\frac{m}{4 \pi^{2} \sqrt{2 \pi} \hbar^{2}} \sum_{\nu} \phi_{\nu}(\xi) \iint \mathrm{d}^{3} r^{\prime} \mathrm{d} \xi^{\prime} \phi_{\nu}^{*}\left(\xi^{\prime}\right) \frac{\mathrm{e}^{\mathrm{i} k_{f} \cdot\left|\vec{r}-\vec{r}^{\prime}\right|}}{\left|\vec{r}-\vec{r}^{\prime}\right|} V\left(\vec{r}^{\prime}, \xi^{\prime}\right) \mathrm{e}^{\mathrm{i} \vec{k}_{i} \cdot \vec{r}^{\prime}} \phi_{\mu_{i}}\left(\xi^{\prime}\right) .
\end{aligned}
$$

Using the approximation (3.178) this expression simplifies in the asymptotic limit of $r \rightarrow \infty$ to

$$
\begin{aligned}
& \psi_{\mu_{i} \vec{k}_{i}}(\vec{r}, \xi) \\
& =\Theta_{\mu_{i} \vec{k}_{i}}(\vec{r}, \xi) \\
& \quad-\frac{m}{4 \pi^{2} \sqrt{2 \pi} \hbar^{2}} \sum_{\nu} \phi_{\nu}(\xi) \frac{\mathrm{e}^{\mathrm{i} k_{f} \cdot r}}{r} \iint \mathrm{d}^{3} r^{\prime} \mathrm{d} \xi^{\prime} \phi_{\nu}^{*}\left(\xi^{\prime}\right) \mathrm{e}^{\mathrm{i} k_{f} \cdot\left|\hat{r}^{\cdot} \cdot \vec{r}^{\prime}\right|} V\left(\vec{r}^{\prime}, \xi^{\prime}\right) \mathrm{e}^{\mathrm{i} \vec{k}_{i} \cdot \vec{r}^{\prime}} \phi_{\mu_{i}}\left(\xi^{\prime}\right) .
\end{aligned}
$$


Employing the notation introduced in (3.184) this expression can be written as

$$
\begin{aligned}
\psi_{\mu_{i} \vec{k}_{i}}(\vec{r}, \xi) & =\Theta_{\mu_{i} \vec{k}_{i}}(\vec{r}, \xi)-\frac{m}{4 \pi^{2} \sqrt{2 \pi} \hbar^{2}} \sum_{\nu} \phi_{\nu}(\xi) \frac{\mathrm{e}^{\mathrm{i} k_{f} \cdot r}}{r} \iint \mathrm{d}^{3} r^{\prime} \mathrm{d} \xi^{\prime} \phi_{\nu}^{*}\left(\xi^{\prime}\right) \mathrm{e}^{\mathrm{i} \vec{Q} \cdot \vec{r}^{\prime}} V\left(\vec{r}^{\prime}, \xi^{\prime}\right) \phi_{\mu_{i}}\left(\xi^{\prime}\right) \\
& =\Theta_{\mu_{i} \vec{k}_{i}}(\vec{r}, \xi)-\frac{m}{4 \pi^{2} \sqrt{2 \pi} \hbar^{2}} \sum_{\nu} \phi_{\nu}(\xi) \frac{\mathrm{e}^{\mathrm{i} k_{f} \cdot r}}{r} \int \mathrm{d}^{3} r^{\prime} \mathrm{e}^{\mathrm{i} \vec{Q} \cdot \vec{r}^{\prime}} \int \mathrm{d} \xi^{\prime} \phi_{\nu}^{*}\left(\xi^{\prime}\right) V\left(\vec{r}^{\prime}, \xi^{\prime}\right) \phi_{\mu_{i}}\left(\xi^{\prime}\right) \\
& =\left|\Theta_{\mu_{i} \vec{k}_{i}}\right\rangle-\frac{m}{4 \pi^{2} \sqrt{2 \pi} \hbar^{2}} \sum_{\nu}\left|\phi_{\nu}\right\rangle \frac{\mathrm{e}^{\mathrm{i} k_{f} \cdot r}}{r} \int \mathrm{d}^{3} r^{\prime} \mathrm{e}^{\mathrm{i} \vec{Q}^{\prime} \cdot \vec{r}^{\prime}}\left\langle\phi_{\nu}|V| \phi_{\mu_{i}}\right\rangle_{\vec{r}^{\prime}}
\end{aligned}
$$

and can be conveniently expressed in Dirac notation as

$$
\left|\psi_{\mu_{i} \vec{k}_{i}}\right\rangle=\left|\Theta_{\mu_{i} \vec{k}_{i}}\right\rangle+\sum_{\nu} A_{\nu \mu_{i}}\left|\phi_{\nu}\right\rangle
$$

with the effective transition amplitude $A_{\nu \mu_{i}}$ defined as

$$
\begin{aligned}
A_{\nu \mu_{i}} & =-\frac{m}{4 \pi^{2} \sqrt{2 \pi} \hbar^{2}} \frac{\mathrm{e}^{\mathrm{i} k_{f} \cdot r}}{r} \int \mathrm{d}^{3} r^{\prime} \mathrm{e}^{\mathrm{i} \vec{Q}^{\cdot} \cdot \vec{r}^{\prime}}\left\langle\phi_{\nu}|V| \phi_{\mu_{i}}\right\rangle_{\vec{r}^{\prime}} \\
& =-\frac{m \sqrt{2 \pi}}{\hbar^{2}} \frac{\mathrm{e}^{\mathrm{i} k_{f} \cdot r}}{r}\left\langle\phi_{\nu}, \vec{k}_{f}|V| \phi_{\mu_{i}}, \vec{k}_{i}\right\rangle .
\end{aligned}
$$

\subsection{Fermi's golden rule}

The scattering amplitude, the way that we had defined it, is purely defined in terms of the probe particle's wave function. ${ }^{48}$ For elastic scattering from a potential we had obtained

$$
u_{\vec{k}}(\vec{r}) \longrightarrow \frac{1}{\sqrt{V}}\left(\mathrm{e}^{\mathrm{i} \vec{k} \cdot \vec{r}}+f_{\vec{k}}(\theta, \phi) \frac{\mathrm{e}^{\mathrm{i} k r}}{r}\right), \quad r \rightarrow \infty .
$$

The concept has now to be carried over to the case of a target with internal degrees of freedom. Inspecting the wave functions $\left|\psi_{\mu_{i} \vec{k}_{i}}\right\rangle$ we realise immediately that they all contain the probe particle in the state $\left|\vec{k}_{i}\right\rangle$ while the target is present in the state $\left|\phi_{\mu_{i}}\right\rangle$. If we forget for a moment the stationary character of the wave functions then we may identify these states with the initial states of the probe and target. In the correction term

$$
-\frac{m}{4 \pi^{2} \sqrt{2 \pi} \hbar^{2}} \sum_{\nu}\left|\phi_{\nu}\right\rangle \frac{\mathrm{e}^{\mathrm{i} k_{f} \cdot r}}{r} \int \mathrm{d}^{3} r^{\prime} \mathrm{e}^{\mathrm{i} \vec{Q} \cdot \vec{r}^{\prime}}\left\langle\phi_{\nu}|V| \phi_{\mu_{i}}\right\rangle_{\vec{r}^{\prime}}
$$

that is added to the wave function by the perturbation we find the scattered probe particles in the form of outgoing spherical waves of wave number $k_{f}$. These spherical waves are accompanied by individual target states $\left|\phi_{\mu_{f}}\right\rangle$ that respect the energy conservation law

$$
E_{\mu_{i}}-E_{\mu_{f}}=-\left(\frac{\hbar^{2} k_{i}^{2}}{2 m}-\frac{\hbar^{2} k_{f}^{2}}{2 m}\right)
$$

\footnotetext{
${ }^{48}$ See Section 5.6 for an in-depth discussion on this point.
} 
This expression simply states that the energy lost or taken up by the probe particle is transmitted to, or retrieved from, the target. The wave function $\left\langle\xi \mid \phi_{\mu_{f}}\right\rangle$ describes the target at the final stage after the probe has left the region of interaction.

We may calculate the flux corresponding to the probe particle according to

$$
\vec{J}(\vec{r})=\frac{\hbar}{2 m \mathrm{i}} \int \mathrm{d} \xi\left(\psi_{\mu_{i} \vec{k}_{i}}^{*}(\vec{r}, \xi) \vec{\nabla}_{\vec{r}} \psi_{\mu_{i} \vec{k}_{i}}(\vec{r}, \xi)-\psi_{\mu_{i} \vec{k}_{i}}(\vec{r}, \xi) \vec{\nabla}_{\vec{r}} \psi_{\mu_{i} \vec{k}_{i}}^{*}(\vec{r}, \xi)\right) .
$$

In writing down this expression we have exploited the fact that what we determine experimentally is the flux of the probe particles at $\vec{r}$ independent of where the target particles might be found. This justifies the integration over all possible target coordinates $\xi$ (see Section 5.6).

We now inspect the probe particle flux arising from the sum of outgoing spherical waves. As the operator $\vec{\nabla}_{\vec{r}}$ does not act on the states $\left|\phi_{\nu}\right\rangle$ the expression for the outgoing flux is of the form

$$
\sum_{\nu^{\prime}} \sum_{\nu}\left\langle\phi_{\nu^{\prime}} \mid \phi_{\nu}\right\rangle\left(A_{\nu^{\prime} \mu_{i}}^{*} \nabla_{\vec{r}} A_{\nu \mu_{i}}\right)=\sum_{\nu^{\prime}} \sum_{\nu} \delta_{\nu^{\prime} \nu} \cdot\left(A_{\nu^{\prime} \mu_{i}}^{*} \nabla_{\vec{r}} A_{\nu \mu_{i}}\right)=\sum_{\nu} A_{\nu \mu_{i}}^{*} \nabla_{\vec{r}} A_{\nu \mu_{i}}
$$

plus its complex conjugate. There is, therefore, no interference between terms pertaining to different final target states $\left|\phi_{\nu}\right\rangle$. Each such state thus defines a specific scattering channel and the total scattering is just the sum of the individual channels.

In order to isolate a specific scattering channel in a rigorous mathematically way we project the total wave function $\left|\psi_{\mu_{i}} \vec{k}_{i}\right\rangle$ onto a given final target state $\left|\phi_{\mu_{f}}\right\rangle$. We formally express this projection onto the subspace of target wave functions using the projector

$$
P_{\phi_{\mu_{f}}}=\left|\phi_{\mu_{f}}\right\rangle\left\langle\phi_{\mu_{f}}\right| \otimes 1_{\vec{k}}
$$

with

$$
1_{\vec{k}}=\sum_{\vec{k}}|\vec{k}\rangle\langle\vec{k}|
$$

the unit operator in the subspace of the probe particle states. We have to distinguish two cases. If the final target state coincides with the initial target state, i.e. if $\mu_{f}=\mu_{i}$ then

$$
\begin{aligned}
\left\langle\vec{r}, \xi\left|P_{\phi_{\mu_{f}}}\right| \psi_{\mu_{i} \vec{k}_{i}}\right\rangle & =\left\langle\vec{r} \mid \vec{k}_{i}\right\rangle\left|\phi_{\mu_{i}}\right\rangle-\frac{4 \pi^{2} m}{\hbar^{2}} \frac{\mathrm{e}^{\mathrm{i} k_{i} \cdot r}}{r}\left\langle\xi \mid \phi_{\mu_{i}}\right\rangle\left\langle\phi_{\mu_{i}}, \vec{k}_{f}|V| \phi_{\mu_{i}}, \vec{k}_{i}\right\rangle \\
& =\frac{1}{\sqrt{2 \pi^{3}}} \mathrm{e}^{\mathrm{i} \vec{k}_{i} \cdot \vec{r}} \phi_{\mu_{i}}(\xi)-\frac{m}{2 \pi \hbar^{2}} \frac{\mathrm{e}^{\mathrm{i} k_{i} \cdot r}}{r} \int \mathrm{d}^{3} r^{\prime} \mathrm{e}^{\mathrm{i} \vec{Q} \cdot \vec{r}^{\prime}} \phi_{\mu_{i}}(\xi)\left\langle\phi_{\nu}|V| \phi_{\mu_{i}}\right\rangle_{\vec{r}^{\prime}} .
\end{aligned}
$$

If the target state changes, i.e. if $\mu_{f} \neq \mu_{i}$ then

$$
\begin{aligned}
\left\langle\vec{r}, \xi\left|P_{\phi_{\mu_{f}}}\right| \psi_{\mu_{i} \vec{k}_{i}}\right\rangle & =-\frac{4 \pi^{2} m}{\hbar^{2}} \frac{\mathrm{e}^{\mathrm{i} k_{f} \cdot r}}{r}\left\langle\xi \mid \phi_{\mu_{f}}\right\rangle\left\langle\phi_{\mu_{f}}, \vec{k}_{f}|V| \phi_{\mu_{i}}, \vec{k}_{i}\right\rangle \\
& =-\frac{m}{2 \pi \hbar^{2}} \frac{\mathrm{e}^{\mathrm{i} k_{f} \cdot r}}{r} \int \mathrm{d}^{3} r^{\prime} \mathrm{e}^{\mathrm{i} \vec{Q} \cdot \vec{r}^{\prime}} \phi_{\mu_{f}}(\xi)\left\langle\phi_{\nu}|V| \phi_{\mu_{i}}\right\rangle_{\vec{r}^{\prime}} .
\end{aligned}
$$

The wave function $\left\langle\vec{r}, \xi \mid \psi_{\mu_{i} \vec{k}_{i}}\right\rangle$ allows us to determine the probability of finding the probe particle at $\vec{r}$ and the target particles at $\xi$. Following the prescription of expression (5.38) we have to integrate over $\xi$. This leaves us with the following wave functions $u(\vec{r})$ for the probe particle. 
If $\mu_{f}=\mu_{i}$ then

$$
\begin{aligned}
\left\langle\vec{r} \mid u_{\vec{k}_{i} \rightarrow \vec{k}_{f}, \mu_{i} \rightarrow \mu_{i}}\right\rangle & =\left\langle\vec{r} \mid \vec{k}_{i}\right\rangle-\frac{4 \pi^{2} m}{\hbar^{2}} \frac{\mathrm{e}^{\mathrm{i} k_{i} \cdot r}}{r}\left\langle\phi_{\mu_{i}}, \vec{k}_{f}|V| \phi_{\mu_{i}}, \vec{k}_{i}\right\rangle \\
& =\frac{1}{\sqrt{2 \pi^{3}}} \mathrm{e}^{\mathrm{i} \vec{k}_{i} \cdot \vec{r}}-\frac{m}{2 \pi \hbar^{2}} \frac{\mathrm{e}^{\mathrm{i} k_{i} \cdot r}}{r} \int \mathrm{d}^{3} r^{\prime} \mathrm{e}^{\mathrm{i} \vec{Q} \cdot \vec{r}^{\prime}}\left\langle\phi_{\nu}|V| \phi_{\mu_{i}}\right\rangle_{\vec{r}^{\prime}} .
\end{aligned}
$$

If $\mu_{f} \neq \mu_{i}$ then

$$
\begin{aligned}
\left\langle\vec{r} \mid u_{\vec{k}_{i} \rightarrow \vec{k}_{f}, \mu_{i} \rightarrow \mu_{f}}\right\rangle & =-\frac{4 \pi^{2} m}{\hbar^{2}} \frac{\mathrm{e}^{\mathrm{i} k_{f} \cdot r}}{r}\left\langle\phi_{\mu_{f}}, \vec{k}_{f}|V| \phi_{\mu_{i}}, \vec{k}_{i}\right\rangle \\
& =-\frac{m}{2 \pi \hbar^{2}} \frac{\mathrm{e}^{\mathrm{i} k_{f} \cdot r}}{r} \int \mathrm{d}^{3} r^{\prime} \mathrm{e}^{\mathrm{i} \vec{Q} \cdot \vec{r}^{\prime}}\left\langle\phi_{\nu}|V| \phi_{\mu_{i}}\right\rangle_{\vec{r}^{\prime}} .
\end{aligned}
$$

From these wave functions we now calculate the current associated with the probe particle according to

$$
\vec{J}(\vec{r})=\frac{\hbar}{2 m \mathrm{i}}\left(u^{*} \vec{\nabla}_{\vec{r}} u-u \vec{\nabla}_{\vec{r}} u^{*}\right) .
$$

Using for $r \rightarrow \infty$ the fact that

$$
\vec{\nabla}\left(\frac{\mathrm{e}^{\mathrm{i} k \cdot r}}{r} f(\theta, \phi)\right)=\mathrm{i} \vec{k} \cdot \hat{r} \frac{\mathrm{e}^{\mathrm{i} k \cdot r}}{r} f(\theta, \phi)+\mathrm{O}\left(\frac{1}{r^{2}}\right)
$$

we obtain if $\mu_{f}=\mu_{i}$

$$
\vec{J}_{\vec{k}_{i} \rightarrow \vec{k}_{f}, \mu_{i} \rightarrow \mu_{i}}(\vec{r})=\frac{\hbar \vec{k}_{i}}{2 m}+\frac{\hbar \vec{k}_{f}}{2 m} \frac{m^{2}}{r^{2}}\left(\frac{2 \pi}{\hbar}\right)^{4}\left|\left\langle\phi_{\mu_{i}}, \vec{k}_{f}|V| \phi_{\mu_{i}}, \vec{k}_{i}\right\rangle\right|^{2}
$$

and if $\mu_{f} \neq \mu_{i}$

$$
\vec{J}_{\vec{k}_{i} \rightarrow \vec{k}_{f}, \mu_{i} \rightarrow \mu_{f}}(\vec{r})=\frac{\hbar \vec{k}_{f}}{2 m} \frac{m^{2}}{r^{2}}\left(\frac{2 \pi}{\hbar}\right)^{4}\left|\left\langle\phi_{\mu_{f}}, \vec{k}_{f}|V| \phi_{\mu_{i}}, \vec{k}_{i}\right\rangle\right|^{2}
$$

As the incoming neutrons are necessarily associated with the target state $\left|\phi_{\mu_{i}}\right\rangle$ it is not surprising that in this stationary description there is no incoming flux for $\mu_{f} \neq \mu_{i}$.

The cross section is defined in the stationary regime as

$$
\frac{\mathrm{d} \sigma}{\mathrm{d} \Omega}=r^{2} \frac{J_{\text {scatt }}(r, \theta, \phi)}{J_{\text {ini }}}
$$

which leads us for $\mu_{f}=\mu_{i}$ to

$$
\left(\frac{\mathrm{d} \sigma}{\mathrm{d} \Omega}\right)_{\vec{k}_{i} \rightarrow \vec{k}_{f}, \mu_{i} \rightarrow \mu_{i}}=m^{2}\left(\frac{2 \pi}{\hbar}\right)^{4}\left|\left\langle\phi_{\mu_{f}}, \vec{k}_{f}|V| \phi_{\mu_{i}}, \vec{k}_{i}\right\rangle\right|^{2}
$$

and if $\mu_{f} \neq \mu_{i}$

$$
\left(\frac{\mathrm{d} \sigma}{\mathrm{d} \Omega}\right)_{\vec{k}_{i} \rightarrow \vec{k}_{f}, \mu_{i} \rightarrow \mu_{f}}=m^{2}\left(\frac{2 \pi}{\hbar}\right)^{4} \frac{k_{f}}{k_{i}}\left|\left\langle\phi_{\mu_{f}}, \vec{k}_{f}|V| \phi_{\mu_{i}}, \vec{k}_{i}\right\rangle\right|^{2} .
$$


As clearly indicated by the notation we are dealing with the conditional scattering cross section of the probe changing its state from $\left|\vec{k}_{i}\right\rangle$ to $\left|\vec{k}_{f}\right\rangle$ with the target simultaneously passing from the initial state $\left|\phi_{\mu_{i}}\right\rangle$ to the final state $\left|\phi_{\mu_{f}}\right\rangle$. We equally see that expression (5.50) is only a special case of the general form (5.51).

From this the scattering amplitude is obtained as

$$
f_{\vec{k}_{i} \rightarrow \vec{k}_{f}, \mu_{i} \rightarrow \mu_{f}}(\Omega)=-\frac{4 \pi^{2} m}{\hbar^{2}}\left\langle\phi_{\mu_{f}}, \vec{k}_{f}|V| \phi_{\mu_{i}}, \vec{k}_{i}\right\rangle,
$$

where the minus sign can be obtained by direct inspection of the wave function $u(\vec{r})$ as given in expressions (5.43) and (5.44). The differential cross section can be interpreted as

$$
\mathrm{d} \sigma_{\vec{k}_{i} \rightarrow \vec{k}_{f}, \mu_{i} \rightarrow \mu_{f}}=\frac{w_{\vec{k}_{i} \rightarrow \vec{k}_{f}, \mu_{i} \rightarrow \mu_{f}}}{J_{\vec{k}_{i}}} \mathrm{~d} \Omega,
$$

where $w_{\vec{k}_{i} \rightarrow \vec{k}_{f}, \mu_{i} \rightarrow \mu_{f}}$ designates the probability density of a transition from the states $\left|\vec{k}_{i}\right\rangle$ and $\left|\phi_{\mu_{i}}\right\rangle$ to the states $\left|\vec{k}_{f}\right\rangle$ and $\left|\phi_{\mu_{f}}\right\rangle$. Comparing expression (5.51) and (5.53) we identify

$$
w_{\vec{k}_{i} \rightarrow \vec{k}_{f}, \mu_{i} \rightarrow \mu_{f}}=\frac{2 \pi}{\hbar}\left|\left\langle\phi_{\mu_{f}}, \vec{k}_{f}|V| \phi_{\mu_{i}}, \vec{k}_{i}\right\rangle\right|^{2} \rho\left(E_{f}\right)
$$

where we have introduced the density of final states

$$
\rho_{\vec{k}}\left(E_{f}\right)=\frac{m k_{f}}{\hbar^{2}} .
$$

Expression (5.54) is known as Fermi's golden rule. It is such an important and general result of perturbation theory that many textbooks dealing with scattering theory actually start with it. This is perfectly letigimate as long as one is aware of the approximations that enter into its derivation.

One of the strengths of Fermi's golden rule is the fact that all its terms lend themselves to a very intuitive interpretation. The square of the matrix element

$$
\left|\left\langle\phi_{\mu_{f}}, \vec{k}_{f}|V| \phi_{\mu_{i}}, \vec{k}_{i}\right\rangle\right|^{2}=\left|\int \mathrm{d}^{3} r \mathrm{e}^{\mathrm{i} \vec{Q} \cdot \vec{r}} \int \mathrm{d} \xi \phi_{\mu_{f}}^{*}(\xi) V(\vec{r}, \xi) \phi_{\mu_{i}}(\xi)\right|^{2}
$$

gives the probability of the transition from the state $\left|\phi_{\mu_{i}}, \vec{k}_{i}\right\rangle$ to the state $\left|\phi_{\mu_{f}}, \vec{k}_{f}\right\rangle$ in the proper sense of the term. This transition is induced by the interaction potential $V$. As the states $|\vec{k}\rangle$ form a continuum we need to make sure that all expressions are properly normalised. The continuity of the states $|\vec{k}\rangle$ implies that the experimentalist cannot determine them individually. All measurements correspond imperatively to an integration over a set of final states defined by the experimental conditions. This is the reason why we have chosen to call $w_{\vec{k}_{i} \rightarrow \vec{k}_{f}}$ a transition probability density and not a transition probability in short. In the case of scattering the integration space is given by the energy and the solid angle. Formally we demand that the double differential cross section integrated over all final states gives the total cross section. All functions included in the double differential cross section should then be defined on the steradian element $\mathrm{d} \Omega$ and the final energy $\mathrm{d} E_{f}$. In order to go from $\mathrm{d}^{3} k$ to $\mathrm{d} \Omega \mathrm{d} E_{f}$ the density of states $|\vec{k}\rangle$ are necessary or in other words we need a function that indicates how the states $|\vec{k}\rangle$ are distributed in an element $\mathrm{d} \Omega \mathrm{d} E_{f}$. This function is $\rho_{\vec{k}}\left(E_{f}\right)$. This is illustrated in the Fig. 28.

At this point it may be instructive to reflect a bit on the notion of a transition in a stationary theory, in which time does not appear explicitly. In particular Fermi's golden rule (5.54) is a result from a stationary treatment of the Schrödinger equation. The state $\left|\vec{k}_{i}\right\rangle$ corresponds, therefore, to a continuous flux of particles with momentum $\vec{p}=\hbar \vec{k}_{i}$. This is the particle flux that exists in the absence of any perturbation. Hence it is natural to consider it as the starting point for the scattering. To it is associated the initial target state $\left|\phi_{\mu_{i}}\right\rangle$. It is from this initial particle flux 


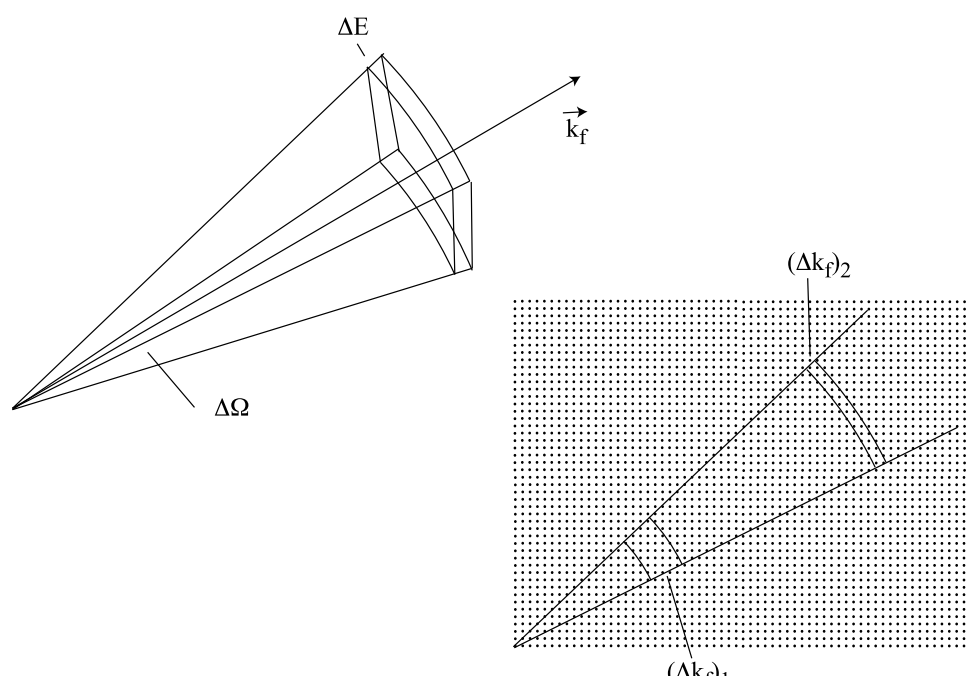

$\left(\Delta \mathrm{k}_{\mathrm{f}}\right)_{1}$

Fig. 28. Illustration of the function $\rho_{\vec{k}}\left(E_{f}\right)$. This function denotes the density of states $\left|\vec{k}_{f}\right\rangle$ in the space spanned by $\left(\Omega, E_{f}\right)$. To evaluate this density, we simply have to count the number of states $\left|\vec{k}_{f}\right\rangle$ in an element $\mathrm{d} \Omega \mathrm{d} E_{f}$. This element is shown in the figure on the left in the space spanned by the eigenstates $\left|\vec{k}_{f}\right\rangle$. The figure at the right corresponds to a two dimensional cut of this three-dimensional element. The states $\left|\vec{k}_{f}\right\rangle$ are shown schematically by the points that are uniformly distributed in space. With a constant radial thickness, the volume of $\mathrm{d} \Omega \mathrm{d} k_{f}$ would increase as the surface of a sphere, which is as $k_{f}^{2}$. However, given that the thickness is required to correspond to a constant $\mathrm{d} E_{f}, \mathrm{~d} k_{f}$ must effectively decrease with $k_{f}$. With $E_{f}=\hbar^{2} k_{f}^{2} /(2 m)$ we can calculate $\mathrm{d} k_{f}\left(E_{f}\right)=\hbar^{2} /\left(m k_{f}\right)$. This implies that $\mathrm{d} k_{f}\left(E_{f}\right)$ is proportional to $k_{f}^{-1}$. The density of states (number of points in $\mathrm{d} \Omega \mathrm{d} E_{f}$ ) is, therefore, only linear in $k_{f}$. This is the result of expression (5.55). The presence of $\rho_{\vec{k}}\left(E_{f}\right)$ in the expression for the cross section has some important practical consequences. If $k_{f}$ becomes very small, as it is the case for neutrons that are almost stopped by the scattering process, then there are very few final states available. As a consequence the scattering rate for a given band of final energy will be very low. The opposite statement can be made for $k_{i}$. The cross section actually increases if $k_{i}$ decreases, i.e. if the incoming probe particles slow down. Since the particle flux is proportional to the density multiplied by the velocity (3.15) the density increases when the velocity decreases at constant flux. There are simply more and more neutrons simultaneous present in the sample when the velocity is lowered.

that we create the scattered wave $|u\rangle$ by introducing the target. We assume that the potential can be switched on sufficiently slow with respect to the relevant time scales to consider the system at any time quasi-stationary. This process is equivalent to placing a stick slowly into a stream of running water. Far away from the target the scattered wave cannot be distinguished from a plane wave $\left|\vec{k}_{f}\right\rangle$. This is the final state of the scattering. It is associated with the target state $\left|\phi_{\mu_{f}}\right\rangle$. In order for the probability fluid to reach a steady state time will have to pass. Hence the transition that we are talking about formally corresponds to a time evolution of the system from $t \rightarrow-\infty$ to $t \rightarrow+\infty$.

\subsection{The master equation}

In the preceding section we tried to be as rigorous and precise as possible with the notation. This choice was motivated by the complexity of the arguments that had to be developed. For the following considerations we judge a lighter formalism more adequate. In particular we would like to denominate the states of the target as $|\lambda\rangle$ with $\lambda$ denoting an element of the complete set of quantum numbers characterising the target. In this notation the initial and final states of the combined system are expressed by the direct products

$$
\begin{aligned}
& |i\rangle=\left|\vec{k}_{i}\right\rangle \otimes\left|\lambda_{i}\right\rangle=\left|\vec{k}_{i} \lambda_{i}\right\rangle, \\
& |f\rangle=\left|\vec{k}_{f}\right\rangle \otimes\left|\lambda_{f}\right\rangle=\left|\vec{k}_{f} \lambda_{f}\right\rangle .
\end{aligned}
$$


The conservation of energy (5.36) is expressed via

$$
\delta\left(\left(E_{f}-E_{i}\right)-\left(E_{\lambda_{i}}-E_{\lambda_{f}}\right)\right)
$$

with $E_{i}$ and $E_{f}$ denoting the initial and final kinetic energy of the probe particle, respectively.

This allows us to express the cross section

$$
\frac{\mathrm{d} \sigma_{\vec{k}_{i} \rightarrow \vec{k}_{f}, \lambda_{i} \rightarrow \lambda_{f}}}{\mathrm{~d} \Omega}=m^{2}\left(\frac{2 \pi}{\hbar}\right)^{4} \frac{k_{f}}{k_{i}}\left|\left\langle\lambda_{f}, \vec{k}_{f}|V| \lambda_{i}, \vec{k}_{i}\right\rangle\right|^{2}
$$

in a double differential form

$$
\frac{\mathrm{d}^{2} \sigma_{\vec{k}_{i} \rightarrow \vec{k}_{f}, \lambda_{i} \rightarrow \lambda_{f}}}{\mathrm{~d} \Omega \mathrm{d} E_{f}}=\frac{k_{f}}{k_{i}} \frac{(2 \pi)^{4} m^{2}}{\hbar^{4}}\left|\left\langle\lambda_{f}, \vec{k}_{f}|V| \lambda_{i}, \vec{k}_{i}\right\rangle\right|^{2} \delta\left(\left(E_{f}-E_{i}\right)-\left(E_{\lambda_{i}}-E_{\lambda_{f}}\right)\right) .
$$

In experiments we do not observe the final states of the sample. The conditional cross sections - the scattering only takes place if the sample goes from the state $\left|\lambda_{i}\right\rangle$ to $\left|\lambda_{f}\right\rangle$ - are as a consequence inaccessible in a simple scattering experiment. ${ }^{49}$ Ignoring the final states we can thus sum ${ }^{50}$ the corresponding cross sections.

A similar argument holds for the initial states. In a thermodynamic system we only know their statistical distribution $p\left(\lambda_{i}\right)$. A concrete example is the occupation of phonons as a function of temperature. In order to obtain the observable cross section we have to sum over the initial states with their statistical weights $p\left(\lambda_{i}\right)$ properly taken into consideration.

With these considerations we end up with the equation

$$
\frac{\mathrm{d}^{2} \sigma_{\vec{k}_{i} \rightarrow \vec{k}_{f}}}{\mathrm{~d} \Omega \mathrm{d} E_{f}}=\frac{k_{f}}{k_{i}} \frac{(2 \pi)^{4} m^{2}}{\hbar^{4}} \sum_{\lambda_{i}, \lambda_{f}} p\left(\lambda_{i}\right)\left|\left\langle\lambda_{f}, \vec{k}_{f}|V| \lambda_{i}, \vec{k}_{i}\right\rangle\right|^{2} \delta\left(\left(E_{f}-E_{i}\right)-\left(E_{\lambda_{i}}-E_{\lambda_{f}}\right)\right) .
$$

This expression is called the master equation of scattering. We wish to recall that it is based on the Born approximation and that the pre-factors hold for the functions $\langle\vec{r} \mid \vec{k}\rangle$ normalised according to (3.19). As we will see, almost all neutron experiments can be interpreted in the framework of this equation.

\subsection{Statistical density operator and quantum state of the probe particle}

At this point of the discussion we want to briefly touch upon the nature of the quantum state of the probe particle after scattering. Starting from the expressions (5.41) and (5.42) we argued that

$$
\begin{aligned}
\left\langle\vec{r} \mid u_{\vec{k}_{i} \rightarrow \vec{k}_{f}, \mu_{i} \rightarrow \mu_{i}}\right\rangle & =\left\langle\vec{r} \mid \vec{k}_{i}\right\rangle-\frac{4 \pi^{2} m}{\hbar^{2}} \frac{\mathrm{e}^{\mathrm{i} k_{i} \cdot r}}{r}\left\langle\phi_{\mu_{i}}, \vec{k}_{f}|V| \phi_{\mu_{i}}, \vec{k}_{i}\right\rangle \\
& =\frac{1}{\sqrt{2 \pi^{3}}} \mathrm{e}^{\mathrm{i} \vec{k}_{i} \cdot \vec{r}}-\frac{m}{2 \pi \hbar^{2}} \frac{\mathrm{e}^{\mathrm{i} k_{i} \cdot r}}{r} \int \mathrm{d}^{3} r^{\prime} \mathrm{e}^{\mathrm{i} \vec{Q} \cdot \vec{r}^{\prime}}\left\langle\phi_{\nu}|V| \phi_{\mu_{i}}\right\rangle_{\vec{r}^{\prime}}
\end{aligned}
$$

$\left(\mu_{f}=\mu_{i}\right)$ and

$$
\begin{aligned}
\left\langle\vec{r} \mid u_{\vec{k}_{i} \rightarrow \vec{k}_{f}, \mu_{i} \rightarrow \mu_{f}}\right\rangle & =-\frac{4 \pi^{2} m}{\hbar^{2}} \frac{\mathrm{e}^{\mathrm{i} k_{f} \cdot r}}{r}\left\langle\phi_{\mu_{f}}, \vec{k}_{f}|V| \phi_{\mu_{i}}, \vec{k}_{i}\right\rangle \\
& =-\frac{m}{2 \pi \hbar^{2}} \frac{\mathrm{e}^{\mathrm{i} k_{f} \cdot r}}{r} \int \mathrm{d}^{3} r^{\prime} \mathrm{e}^{\mathrm{i} \vec{Q} \cdot \vec{r}^{\prime}}\left\langle\phi_{\nu}|V| \phi_{\mu_{i}}\right\rangle_{\vec{r}^{\prime}}
\end{aligned}
$$

\footnotetext{
${ }^{49}$ One could imagine a complex scattering experiment where the final state is identified by a supplementary measurement, for example if it is accompanied by the emission of another particle such as a photon or an electron.

${ }^{50}$ We can always assume discrete states. Where this is not the case we can replace the sum by an integral.
} 
$\left(\mu_{f} \neq \mu_{i}\right.$ ) should be considered the wave functions of the scattered probe particles. Our arguments were rewarded by the fact that these functions lead to the correct currents for the scattered particles. ${ }^{51}$ While this use of language is practical we have to be conscious of the fact that it is not rigorously correct ${ }^{52}$ and can lead to wrong conclusion if applied without care.

Let us consider in all generality a combined quantum system $H_{A B} . H_{A}$ may in our case represent the probe and $H_{B}$ the target. Often $H_{A}$ is called the system of observation and $H_{B}$ the environment. The most general quantum state of $H_{A B}$ can be written as a superposition of direct product stares

$$
\left|\phi_{A B}\right\rangle=\sum_{k, \lambda} c_{k, \lambda}(|k\rangle \otimes|\lambda\rangle)=\sum_{k, \lambda} c_{k, \lambda}|k \otimes \lambda\rangle
$$

Let us now assume that we measure a physical quantity $A$ of the system $H_{A}$. This may e.g. be the position, momentum or spin of the scattered particles. In the combined quantum space this quantity will be represented by the operator $A \otimes I_{B}$ with

$$
\left(A \otimes I_{B}\right)\left|\phi_{A B}\right\rangle=\sum_{k, \lambda} c_{k, \lambda}(|A k \otimes \lambda\rangle) .
$$

If we calculate the expectation value we obtain

$$
\begin{aligned}
\left\langle\phi_{A B}\left|\left(A \otimes I_{B}\right)\right| \phi_{A B}\right\rangle & =\sum_{k, \lambda} \sum_{k^{\prime}, \lambda^{\prime}} c_{k^{\prime}, \lambda^{\prime}}^{*} c_{k, \lambda}\left\langle k^{\prime} \otimes \lambda^{\prime} \mid A k \otimes \lambda\right\rangle \\
& =\sum_{k, \lambda} \sum_{k^{\prime}, \lambda^{\prime}} c_{k^{\prime}, \lambda^{\prime}}^{*} c_{k, \lambda}\left\langle k^{\prime}|A| k\right\rangle \delta_{\lambda, \lambda^{\prime}} \\
& =\sum_{k, k^{\prime}} \sum_{\lambda} c_{k^{\prime}, \lambda}^{*} c_{k, \lambda}\left\langle k^{\prime}|A| k\right\rangle \\
& =\sum_{k, k^{\prime}} \rho_{k, k^{\prime}}\left\langle k^{\prime}|A| k\right\rangle \\
& =\operatorname{Tr}(\hat{\rho} A)
\end{aligned}
$$

with the operator $\hat{\rho}$

$$
\rho_{k, k^{\prime}}=\sum_{\lambda} c_{k^{\prime}, \lambda}^{*} c_{k, \lambda}
$$

acting in the space $H_{A}$. This operator possesses all the characteristics of a statistical density operator. It is Hermitian, i.e. $\hat{\rho}=\hat{\rho}^{+}$. It has unit trace, i.e. the sum of its eigenvalues is equal to one, and it is positive, i.e. $\langle\psi|\hat{\rho}| \psi\rangle \geqslant 0 \forall|\psi\rangle$.

In the special case of a pure state of the simple form

$$
\left|\phi_{A B}\right\rangle=|k \otimes \lambda\rangle
$$

\footnotetext{
${ }^{51}$ Like the wave function the probability current is in itself not a physical observable. It is converted into a measured intensity via the physical processes taking place at the time of detection.

${ }^{52}$ We follow strictly the excellent discussion given by Michel Le Bellac [37].
} 
we get $\rho^{2}=1$ and

$$
\left\langle\phi_{A B}\left|\left(A \otimes I_{B}\right)\right| \phi_{A B}\right\rangle=\left\langle k^{\prime}|A| k\right\rangle .
$$

This is the usual form of an expectation value obtained from a well-defined quantum state. In all other cases the expectation value of the physical quantity cannot be attributed to a quantum state of the probe particle. Therefore, by ignoring in a measurement the target states we arrive at a statistical description of the probe particle state that no longer can be represented by a pure quantum state. We get, therefore a description of the quantum system that strongly resembles the one introduced by statistical disorder, e.g. via the coupling of the quantum system to a thermal bath. Despite this similarity one should be aware of the fact that the statistical operator obtained by ignoring quantum coupling and the one obtained from quantum statistical mechanics differ. We speak of improper and proper mixtures.

We can easily introduce statistical disorder into the above outlined formalism. Let $\hat{\rho}_{A B}$ be the statistical density operator of the combined system. Then we can obtain the so-called reduced statistical density operator of subsystem $H_{A}$ via contraction of the $H_{B}$ indices

$$
\rho_{k, k^{\prime}}^{A}=\sum_{\lambda} \rho_{k^{\prime}, \lambda ; k, \lambda}^{A B}
$$

or in matrix notation

$$
\hat{\rho}_{A}=\operatorname{Tr}_{B} \hat{\rho}_{A B}
$$

$\operatorname{Tr}_{B}$ is called partial trace over the subsystem $H_{B}$.

Let us for illustration calculate the statistical density operator for a pure state, which as stated above writes in the most general form as

$$
\left|\Phi_{A B}\right\rangle=\sum_{k, \lambda} c_{k, \lambda}|k \otimes \lambda\rangle .
$$

The statistical density operator of $H_{A B}$ is then given as

$$
\left|\Phi_{A B}\right\rangle\left\langle\Phi_{A B}\left|=\sum_{k, k^{\prime} ; \lambda, \lambda^{\prime}} c_{k, \lambda} c_{k^{\prime}, \lambda^{\prime}}^{*}\right| k \otimes \lambda\right\rangle\left\langle k^{\prime} \otimes \lambda^{\prime}\right| .
$$

We calculate the reduced statistical density operator as

$$
\begin{aligned}
\rho_{A} & =\operatorname{Tr}_{B}\left|\Phi_{A B}\right\rangle\left\langle\Phi_{A B}\right| \\
& =\sum_{\lambda^{\prime \prime}} \sum_{k, k^{\prime} ; \lambda, \lambda^{\prime}} c_{k, \lambda} c_{k^{\prime}, \lambda^{\prime}}^{*}|k\rangle\left\langle\lambda^{\prime \prime} \mid \lambda\right\rangle\left\langle k^{\prime}\right|\left\langle\lambda^{\prime} \mid \lambda^{\prime \prime}\right\rangle \\
& =\sum_{\lambda^{\prime \prime}} \sum_{k, k^{\prime} ; \lambda, \lambda^{\prime}} c_{k, \lambda} c_{k^{\prime}, \lambda^{\prime}}^{*}|k\rangle\left\langle k^{\prime}\right|\left\langle\lambda^{\prime} \mid \lambda^{\prime \prime}\right\rangle\left\langle\lambda^{\prime \prime} \mid \lambda\right\rangle \\
& =\sum_{k, k^{\prime} ; \lambda, \lambda^{\prime}} c_{k, \lambda} c_{k^{\prime}, \lambda^{\prime}}^{*}|k\rangle\left\langle k^{\prime}\right|\left\langle\lambda^{\prime} \mid \lambda\right\rangle .
\end{aligned}
$$

If we reorder the terms by attributing to an index $m$ the pairs of indices of $k(m)$ and $\lambda(m)$ then

$$
\left|\Phi_{A B}\right\rangle=\sum_{k, \lambda} c_{k(m), \lambda(m)}\left|k_{m} \otimes \lambda_{m}\right\rangle=\sum_{m} c_{m}\left|k_{m} \otimes \lambda_{m}\right\rangle
$$


and

$$
\rho_{A}=\operatorname{Tr}_{B}\left|\Phi_{A B}\right\rangle\left\langle\Phi_{A B}\left|=\sum_{m, m^{\prime}} c_{m} c_{m^{\prime}}^{*}\right| k_{m}\right\rangle\left\langle k_{m^{\prime}}\right|\left\langle\lambda_{m^{\prime}} \mid \lambda_{m}\right\rangle .
$$

If the target states $|\lambda\rangle$ are chosen orthonormal then all the off-diagonal elements vanish and

$$
\rho_{A}=\operatorname{Tr}_{B}\left|\Phi_{A B}\right\rangle\left\langle\left.\Phi_{A B}\left|=\sum_{m}\right| c_{m}\right|^{2} \mid k_{m}\right\rangle\left\langle k_{m}\right| .
$$

Expressions (5.75) and (5.76) give the mathematical justification for deducing the particle wave functions (5.43) and (5.44) from the combined wave functions (5.41) and (5.42) via integration over the variables $\xi$ of the target, which is equivalent to calculating $\left\langle\lambda_{m^{\prime}} \mid \lambda_{m}\right\rangle$ in (5.75). This integration is, therefore, the signature of calculating the partial trace over the target states.

As the information on the phases of the complex numbers $c_{m}$ is lost the statistical density operator in expression (5.76) corresponds to a superposition of states without phase coherence. Therefore, if the states of the probe particle are perfectly correlated with eigenstates of the target then they loose their phase coherence among themselves. This phenomenon is called decoherence of particle states by interaction with an environment. It has to be stressed that the phases are preserved and can be retrieved if we keep control of the complete probe plus target system. In other words decoherence means that information leaks into the target.

Information content may be quantified by the Von Neumann entropy $S$. Let us remember that the statistical density operator is positive semi-definite and thus can be spectrally decomposed into eigenvalues

$$
\hat{\rho}=\sum_{i} p_{i}\left|\psi_{i}\right\rangle\left\langle\psi_{i}\right|
$$

with the $\left|\psi_{i}\right\rangle$ orthonormal, all the eigenvalues $p_{i}$ positive and $\sum_{i} p_{i}=1$. Then the entropy of the mixture is given as

$$
S=-\sum_{i} p_{i} \ln p_{i}=-\operatorname{Tr} \hat{\rho} \ln \hat{\rho}
$$

While the entropy of the combined system of probe and particle is preserved entropy will decrease if we consider the probe on its own.

In summary, if a pure system $H_{A}$ is coupled to an environment via an interaction described by a Hamiltonian then it will suffer so-called decoherence. Its time evolution will be non-unitary and thus irreversible. The nonunitarity will persist for system $H_{A}$ even if the total system $H_{A B}$ evolves in a unitary fashion. This implies that when a neutron is scattered inelastically from a complex target possessing internal degrees of freedom then we have to pass from a wave function to a statistical description of the neutron state in terms of a density matrix if we want to capture the full physics of the scattering process. We will come back to this discussion in Section 11.2 that deals with the Liouville theorem. We will see how the loss of coherence introduced into the probe particle state allows bypassing the Liouville theorem. This fact has important consequences for designing neutron instrumentation.

The difference of a statistical with respect to a pure quantum state description is so important that we want to illustrate it by an example. Let us consider the polarisation of a neutron beam. We may decompose the statistical density matrix of the spin states in terms of the Pauli spin matrices

$$
\hat{\rho}=\frac{1}{2}[I+\vec{b} \cdot \vec{\sigma}]
$$

with

$$
\sigma_{x}=\left[\begin{array}{cc}
0 & 1 \\
1 & 0
\end{array}\right], \quad \sigma_{y}=\left[\begin{array}{cc}
0 & -\mathrm{i} \\
\mathrm{i} & 0
\end{array}\right], \quad \sigma_{z}=\left[\begin{array}{cc}
1 & 0 \\
0 & -1
\end{array}\right]
$$


and the spin operator defined as

$$
\overrightarrow{\mathbf{S}}=\frac{\hbar}{2} \vec{\sigma}
$$

The vector $\vec{b}$ is called the Bloch vector. For $\rho$ to fulfil the above outlined requirements of a statistical density operator (e.g. the sum of the probabilities, i.e. of the eigenvalues of $\hat{\rho}$, has to be equal to one, etc.) the Bloch vector has to satisfy

$$
|\vec{b}| \leqslant 1
$$

If $|\vec{b}|=1$ we speak of a fully polarised beam. If $|\vec{b}|=0$ we speak of a fully unpolarised beam.

If for an unpolarised beam we measure the expectation value of $\overrightarrow{\mathbf{S}}$, e.g. by an adequate spin separator, which may be a simple Stern-Gerlach apparatus, then we will always count $50 \%$ of the overall intensity in the spin up and $50 \%$ in the spin down channel, and this independent of the direction, i.e. independent of the orientation of our spin separator.

The situation is completely different for a pure state. Let us describe the beam in the form of a wave function that superimposes coherently the two spin states of the neutron

$$
|\psi\rangle_{ \pm}=\frac{1}{\sqrt{2}}\left(|\uparrow\rangle_{z} \pm|\downarrow\rangle_{z}\right)
$$

where we have chosen $\hat{z}$ as the quantisation axis. As long as the $\vec{B}$ field of our Stern-Gerlach apparatus is aligned along $\hat{z}$ we will get $50 \%$ of the neutrons in each channel. However, as we immediately can show by using the Pauli matrices, $|\psi\rangle_{+}$is now an eigenstate of $\mathbf{S}_{x}$

$$
\mathbf{S}_{x}|\psi\rangle_{+}=\frac{\hbar}{2}|\psi\rangle_{+}
$$

Therefore, all the neutrons will be deviated along the positive $x$-direction if $\vec{B}$ is parallel to $\hat{x}$. In the same way $|\psi\rangle_{-}$is an eigenstate of $\mathbf{S}_{x}$ with eigenvalue $-\hbar / 2 .{ }^{53}$ We are, therefore, dealing with fully polarised states. The reason is the perfect coherence of the superposition of states that leads to a well-defined phase relation between the amplitudes of the up and down states. When a fully polarised neutron state couples to an environment we will witness depolarisation. The Bloch vector will be mapped from the unitary surface towards the interior and finally towards the origin of the Bloch sphere.

\subsection{The form factor of the individual interaction potential}

In the discussion so far the potential $V(\vec{r}, \xi)$ was not further specified. We will now exploit the fact that the interactions that we are generally dealing with are of the two-particle form. The scattering potential that the probe particle experiences from one specific target particle is, therefore, not influenced by the presence of other target particles. As a consequence we can express the interaction potential as a sum of individual contributions

$$
V(\vec{r})=\sum_{j=1}^{N} V_{j}\left(\vec{r}-\vec{R}_{j}\right)=\int_{V} \mathrm{~d} r^{\prime 3} \sum_{j=1}^{N} V_{j}\left(\vec{r}^{\prime}\right) \delta\left(\vec{r}-\vec{R}_{j}\right),
$$

\footnotetext{
${ }^{53}$ The eigenvectors of $S_{y}$ are given as

$$
|\psi\rangle_{ \pm}=\frac{1}{\sqrt{2}}\left(|\uparrow\rangle_{z} \pm \mathrm{i}|\downarrow\rangle z\right)
$$
}


where $V_{j}\left(\vec{r}-\vec{R}_{j}\right)$ is the interaction potential of an individual scatterer characterised by its position operator $\vec{R}_{j} .{ }^{54}$ The second equality underlines the fact that we are dealing with a convolution of the positions with the interaction potentials.

The first step in the evaluation of the master equation consists in the integration over the variable $\vec{r}$ of the matrix element $\left\langle\lambda_{f}, \vec{k}_{f}|V| \lambda_{i}, \vec{k}_{i}\right\rangle$, which is at the heart of the master equation. Using the above expression for the potential the matrix element takes on the form

$$
\begin{aligned}
& \left\langle\lambda_{f}, \vec{k}_{f}|V| \lambda_{i}, \vec{k}_{i}\right\rangle \\
& \quad=\sum_{j=1}^{N} \frac{1}{(2 \pi)^{3}} \int \mathrm{d}^{3} R_{1} \cdots \int \mathrm{d}^{3} R_{N} \int \mathrm{d}^{3} r \phi_{\lambda_{f}}^{*}\left(\mathrm{e}^{-\mathrm{i} \vec{k}_{f} \cdot \vec{r}} V_{j}\left(\vec{r}-\vec{R}_{j}\right) \mathrm{e}^{\mathrm{i} \vec{k}_{i} \cdot \vec{r}}\right) \phi_{\lambda_{i}} .
\end{aligned}
$$

The eigenfunctions of the target

$$
\phi_{\lambda}=\langle r \mid \lambda\rangle=\phi_{\lambda}\left(\left\{\vec{R}_{j}\right\}\right)
$$

only depend on the coordinates $\vec{R}_{j}$.

Given that the integral extends over all space we can for each individual $j$-term featuring in the sum of expression (5.85) freely choose the origin of the integration variable pertaining to the probe particle. In particular by defining (see Fig. 29)

$$
\vec{r}_{j}=\vec{r}-\vec{R}_{j}
$$

we find

$$
\begin{aligned}
& \int \mathrm{d}^{3} R_{1} \cdots \int \mathrm{d}^{3} R_{N} \int \mathrm{d}^{3} r \phi_{\lambda_{f}}^{*}\left(\mathrm{e}^{-\mathrm{i} \vec{k}_{f} \cdot \vec{r}} V_{j}\left(\vec{r}-\vec{R}_{j}\right) \mathrm{e}^{\mathrm{i} \vec{k}_{i} \cdot \vec{r}}\right) \phi_{\lambda_{i}} \\
& =\int \mathrm{d}^{3} R_{1} \cdots \int \mathrm{d}^{3} R_{N} \int \mathrm{d}^{3} r_{j} \phi_{\lambda_{f}}^{*}\left(\mathrm{e}^{-\mathrm{i} \vec{k}_{f} \cdot\left(\vec{r}_{j}+\vec{R}_{j}\right)} V_{j}\left(\vec{r}_{j}\right) \mathrm{e}^{\mathrm{i} \vec{k}_{i} \cdot\left(\vec{r}_{j}+\vec{R}_{j}\right)}\right) \phi_{\lambda_{i}} \\
& =\int \mathrm{d}^{3} R_{1} \cdots \int \mathrm{d}^{3} R_{N} \phi_{\lambda_{f}}^{*} \mathrm{e}^{\mathrm{i} \vec{Q} \cdot \vec{R}_{j}} \phi_{\lambda_{i}} \int \mathrm{d}^{3} r_{j} \mathrm{e}^{\mathrm{i} \vec{Q} \cdot \vec{r}_{j}} V_{j}\left(\vec{r}_{j}\right) .
\end{aligned}
$$

With (5.88) the matrix element appearing in the master equation can be written in a more compact manner

$$
\left\langle\lambda_{f}, \vec{k}_{f}|V| \lambda_{i}, \vec{k}_{i}\right\rangle=\frac{1}{(2 \pi)^{3}} \sum_{j=1}^{N} V_{j}(\vec{Q})\left\langle\lambda_{f}\left|\mathrm{e}^{\mathrm{i} \vec{Q} \cdot \vec{R}_{j}}\right| \lambda_{i}\right\rangle .
$$

The function

$$
V_{j}(\vec{Q})=\int \mathrm{d}^{3} r \mathrm{e}^{\mathrm{i} \vec{Q} \cdot \vec{r}} V_{j}(\vec{r})
$$

is the Fourier transform of the interaction potential of the $j$ th scatterer. It reminds us of the expression (3.188) that we had obtained for the scattering of a particle by a static potential. The function $V_{j}(\vec{Q})$ can be thought of as a form factor of the potential $V_{j}\left(\vec{r}_{j}\right)$ of the $j$ th scatterer.

\footnotetext{
${ }^{54}$ The set of $\left\{\vec{R}_{j}\right\}$ is equivalent to the set of coordinates $\{\xi\}$ that we have used so far and that was formulated as unspecific as possible in order not to render the notation too cumbersome.
} 


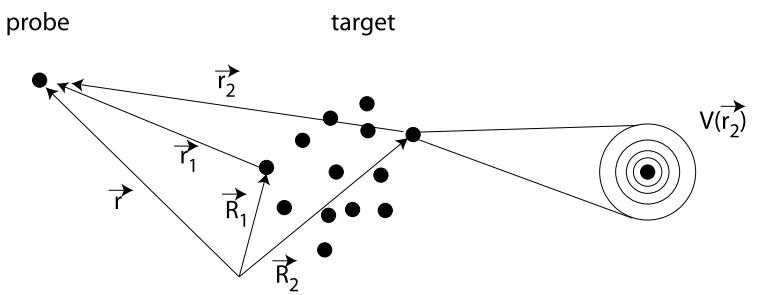

Fig. 29. Coordinates used for the calculation of $V_{j}(\vec{Q})$ (expression (5.90)) starting from the interaction potential $V_{j}\left(\vec{R}_{j}\right)$.

This is again a remarkable result in full analogy with what we had found for optical diffraction. ${ }^{55}$ We have succeeded in factorising the matrix element into two terms. The first, the form factor of the potential

$$
V_{j}(\vec{Q})
$$

is a function that characterises the individual scatterers. ${ }^{56}$ The interaction of a scatterer with the probe particles is entirely described by its form factor. Therefore the potential is no longer present in the second factor

$$
\left\langle\lambda_{f}\left|\mathrm{e}^{\mathrm{i} \vec{Q} \cdot \vec{R}_{j}}\right| \lambda_{i}\right\rangle
$$

which is a function that purely depends on the target.

Using the form factors $V_{j}(\vec{Q})$ of the interaction potential the master equation (5.62) becomes

$$
\frac{\mathrm{d} \sigma_{\vec{k}_{i} \rightarrow \vec{k}_{f}}}{\mathrm{~d} \Omega \mathrm{d} E_{f}}=\frac{k_{f}}{k_{i}} \frac{m^{2}}{(2 \pi)^{2} \hbar^{4}} \sum_{\lambda_{i}, \lambda_{f}} p\left(\lambda_{i}\right)\left|\sum_{j=1}^{N} V_{j}(\vec{Q})\left\langle\lambda_{f}\left|\mathrm{e}^{\mathrm{i} \vec{Q} \cdot \vec{R}_{j}}\right| \lambda_{i}\right\rangle\right|^{2} \delta\left(\left(E_{f}-E_{i}\right)-\left(E_{\lambda_{i}}-E_{\lambda_{f}}\right)\right) .
$$

\subsection{The Fermi pseudo-potential}

We would now like to evaluate the form factor for extremely short-ranged potentials. This is notably the case for neutron scattering from a nucleus, which is happening on the nuclear length scale of the femptometre. If we use the effective potential developed in Section 3.7 we find ${ }^{57}$

$$
V_{j}(\vec{Q})=\int \mathrm{d}^{3} r V(\vec{r}) \mathrm{e}^{\mathrm{i} \vec{Q} \cdot \vec{r}}=\left.\frac{2 \pi \hbar^{2}}{m} b_{j} \int \mathrm{d}^{3} r \delta(\vec{r}) \frac{\mathrm{d}}{\mathrm{d} r}\right|_{r=0} r \mathrm{e}^{\mathrm{i} \vec{Q} \cdot \vec{r}}
$$

\footnotetext{
${ }^{55}$ We could simply have anticipated this result from the fact that the scattering amplitude in the Born approximation is the Fourier transform of the scattering potential (see (3.188). For a potential which is, as seen in (5.84), the convolution of two functions, the Fourier transform is the product of the individual Fourier transforms as discussed in Section 4).

${ }^{56}$ There is no ambiguity in the definition of the potential if the scattering particle is an elementary particle without any internal degrees of freedom (we recall that we neglect spin in this discussion). In general, what we call a scatterer is nevertheless itself a composite particle. This is true both for the nucleus in nuclear scattering and the electron cloud for magnetic scattering. We can only neglect these internal structures under the condition that they are stable at the level of the scattering. In other words the interaction with the composite object can be described by an effective potential as long as the neutron does not trigger a transition between different excited states. For the nucleus this is the case when we are off resonance. For the electronic levels, it is in general the case for the typical thermal neutron scattering experiments, which are below some $100 \mathrm{meV}$. At higher energies the transitions between multiplets should be taken into consideration in the formal treatment of scattering (see [3]).

${ }^{57}$ We place the scatterer at the origin for convenience.
} 
We can evaluate this expression by expanding the function $\mathrm{e}^{-\mathrm{i} \vec{Q} \cdot \vec{r}}$ in partial waves (see (3.68)) and by remembering the behaviour of the radial part of the wave function for $\rho \rightarrow 0$ (see (3.61)). We obtain

$$
V_{j}(\vec{Q})=\frac{2 \pi \hbar^{2}}{m} b_{j} .
$$

This result is not surprising. The Fourier transform of a point should give a constant. We could have found the same result by using the simpler potential

$$
V_{\text {eff }}(r)=\frac{2 \pi \hbar^{2}}{m} b_{j} \delta(\vec{r})
$$

which is known as the Fermi pseudo-potential. The conditional cross section (5.91) then takes the form

$$
\frac{\mathrm{d}^{2}{\sigma_{\vec{k}} \rightarrow \vec{k}_{f}, \lambda_{i} \rightarrow \lambda_{f}}_{k_{i}}}{\mathrm{~d} \Omega \mathrm{d} E_{f}}=\frac{k_{f}}{k_{i}}\left|\sum_{j=1}^{N} b_{j}\left\langle\lambda_{f}\left|\mathrm{e}^{\mathrm{i} \vec{Q} \cdot \vec{R}_{j}}\right| \lambda_{i}\right\rangle\right|^{2} \delta\left(\left(E_{\lambda_{i}}-E_{\lambda_{f}}\right)+\hbar \omega\right),
$$

where we have introduced

$$
\hbar \omega:=E_{i}-E_{f}
$$

in order to express the energy transferred from the probe particle to the target.

A word of further explanation is in order to better understand the term pseudo-potential. When calculating the form factor we work strictly in the Born approximation. However, as we have seen in Section 3.13 the Born approximation is actually not valid when it comes to calculating the scattering of a neutron from a nuclear potential. For neutrons with wave lengths of a few $\AA$

$$
r_{0} \cdot k<10^{-4}
$$

which means that we are in the low-energy regime. The low-energy condition for validity of the Born approximation

$$
m V_{0} r_{0}^{2} / \hbar^{2} \ll 1
$$

is, however, not fulfilled. If we insist on the Born approximation, we have to apply a mathematical trick. Let us first see what form factor we obtain with the Born approximation from a short-range spherically symmetric potential.

$$
V(\vec{Q})=\int \mathrm{d}^{3} r V(\vec{r}) \mathrm{e}^{\mathrm{i} \vec{Q} \cdot \vec{r}}=\int_{0}^{r_{0}} r^{2} \mathrm{~d} r V(r)
$$

where in order to obtain the last equality we have exploited the fact that $|\vec{Q}|$ is small on the scale of $1 / r_{0}$. The result can be expressed as a product

$$
V(\vec{Q})=V_{\text {const }}=\frac{4 \pi}{3} r_{0}^{3} \cdot \bar{V},
$$

with the average potential defined via

$$
\bar{V}=\int_{0}^{1} x^{2} \mathrm{~d} x V(x)
$$


As only the product of the volume of the interaction region with the average potential comes into play Fermi proposed [25] to choose an effective potential range $r_{\text {eff }}$ such that it preserves the form factor, while still remaining in the low-energy regime but equally fulfilling the validity condition of the Born approximation. All these conditions are e.g. respected if we choose

$$
r_{\text {eff }}:=10^{2} \cdot r_{0}
$$

and

$$
\bar{V}_{\text {eff }}:=10^{-6} \cdot \bar{V} \text {. }
$$

In this case

$$
r_{\mathrm{eff}} \cdot k \approx 10^{-2} \ll 1
$$

and

$$
\frac{m \bar{V}_{\mathrm{eff}} r_{\mathrm{eff}}^{2}}{\hbar^{2}} \approx 10^{-2} \ll 1,
$$

while the form factor $V_{\text {const }}$ remains unchanged. To free ourselves from the arbitrariness inherent in the choice of $r_{\text {eff }}$ we may define a pseudo-potential that gives the correct form factor in the Born approximation while its range is described by a Dirac function. The result is given in expression (5.94).

\subsection{The scattering function $S(\vec{Q}, \omega)$}

The master equation (5.91) that we have obtained for the partial differential cross section contains a sum, which depends explicitly on the set of all the final quantum states $\left\{\left|\phi_{\lambda}\right\rangle\right\}$ of the target. We certainly would have preferred an expression that was based on expectation values of the thermodynamic equilibrium state of the target. These expectation values are of the form

$$
\langle\mathbf{A}\rangle=\operatorname{Tr}\left[\hat{\rho}_{\lambda} \mathbf{A}\right]=\sum_{\lambda} p_{\lambda}\langle\lambda|\mathbf{A}| \lambda\rangle,
$$

with the density operator in thermodynamic equilibrium defined as

$$
\hat{\rho}=\frac{\exp \left(-\mathbf{H} /\left(k_{\mathrm{B}} T\right)\right)}{\operatorname{Tr}\left[\exp \left(-\mathbf{H} /\left(k_{\mathrm{B}} T\right)\right)\right]},
$$

which in the basis of eigenvectors of $\mathbf{H}$ is diagonal with elements

$$
p_{\lambda}=\frac{1}{Z} \exp \left(-\frac{E_{\lambda}}{k_{\mathrm{B}} T}\right)
$$

and the canonical partition function defined as

$$
Z=\sum_{\lambda} \exp \left(-\frac{E_{\lambda}}{k_{\mathrm{B}} T}\right)=\sum_{\lambda}\left\langle\lambda\left|\exp \left(-\frac{\mathbf{H}}{k_{\mathrm{B}} T}\right)\right| \lambda\right\rangle=\operatorname{Tr}\left[\exp \left(-\frac{\mathbf{H}}{k_{\mathrm{B}} T}\right)\right] .
$$


It would be tempting to invoke the closure relation

$$
\sum_{\lambda_{f}}\left|\lambda_{f}\right\rangle\left\langle\lambda_{f}\right|=\mathbf{I}_{\lambda}
$$

with $\mathbf{I}_{\lambda}$ being the identity operator in the target subspace, in order to achieve this. The presence of the $\delta\left(\left(E_{\lambda_{i}}-\right.\right.$ $\left.\left.E_{\lambda_{f}}\right)+\hbar \omega\right)$ )-function in the expression (5.62) unfortunately prevents us from doing so. ${ }^{58}$ To overcome this obstacle we have to use a trick. We recall that a Dirac function of energy can be expressed as a Fourier transform of a constant in time

$$
\begin{aligned}
& \delta\left(E_{\lambda_{i}}-E_{\lambda_{f}}+\hbar \omega\right) \\
& \quad=\frac{1}{2 \pi \hbar} \int_{-\infty}^{\infty} \mathrm{d} t \exp \left(-\frac{\mathrm{i}}{\hbar}\left(E_{\lambda_{i}}-E_{\lambda_{f}}+\hbar \omega\right) t\right) \\
& \quad=\frac{1}{2 \pi \hbar} \int_{-\infty}^{\infty} \mathrm{d} t \exp \left(-\frac{\mathrm{i}}{\hbar}\left(E_{\lambda_{i}}-E_{\lambda_{f}}\right) t\right) \cdot \exp (-\mathrm{i} \omega t) .
\end{aligned}
$$

The energies $E_{\lambda}$, listed in this expression, are the eigenvalues of the target Hamiltonian

$$
\mathbf{H}|\lambda\rangle=E_{\lambda}|\lambda\rangle .
$$

For any function $f(\mathbf{H})$ of this Hamiltonian acting on one of its eigenfunctions we get

$$
f(\mathbf{H})|\lambda\rangle=f\left(E_{\lambda}\right)|\lambda\rangle .
$$

In particular,

$$
\exp \left(\frac{\mathrm{i}}{\hbar} \mathbf{H} t\right)|\lambda\rangle=\exp \left(\frac{\mathrm{i}}{\hbar} E_{\lambda} t\right)|\lambda\rangle
$$

We now return to the master equation (5.95). The central part of this equation is the square of the sum of the matrix elements. We express this square as a double sum

$$
\left|\sum_{j=1}^{N} b_{j}\left\langle\lambda_{f}\left|\mathrm{e}^{\mathrm{i} \vec{Q} \cdot \vec{R}_{j}}\right| \lambda_{i}\right\rangle\right|^{2}=\sum_{j, j^{\prime}=1}^{N} b_{j} b_{j^{\prime}}^{*}\left\langle\lambda_{f}\left|\mathrm{e}^{\mathrm{i} \vec{Q} \cdot \vec{R}_{j}}\right| \lambda_{i}\right\rangle\left\langle\lambda_{i}\left|\mathrm{e}^{-\mathrm{i} \vec{Q} \cdot \vec{R}_{j^{\prime}}}\right| \lambda_{f}\right\rangle .
$$

For each term associated with a $\delta(E)$-function, we apply expression (5.105) in the following way

$$
\begin{aligned}
& \left\langle\lambda_{f}\left|\mathrm{e}^{\mathrm{i} \vec{Q} \cdot \vec{R}_{j}}\right| \lambda_{i}\right\rangle\left\langle\lambda_{i}\left|\mathrm{e}^{-\mathrm{i} \vec{Q} \cdot \vec{R}_{j^{\prime}}}\right| \lambda_{f}\right\rangle \delta\left(E_{\lambda_{i}}-E_{\lambda_{f}}-\hbar \omega\right) \\
& \quad=\frac{1}{2 \pi \hbar} \int_{-\infty}^{\infty} \mathrm{d} t\left\langle\lambda_{f}\left|\mathrm{e}^{\mathrm{i}\left(E_{\lambda_{f}} / \hbar\right) t} \mathrm{e} \mathrm{e}^{\mathrm{i} \cdot \overrightarrow{R_{j}} \vec{R}_{j}} \mathrm{e}^{-\mathrm{i}\left(E_{\lambda_{i}} / \hbar\right) t}\right| \lambda_{i}\right\rangle\left\langle\lambda_{i}\left|\mathrm{e}^{-\mathrm{i} \vec{Q} \cdot \vec{R}_{j^{\prime}}}\right| \lambda_{f}\right\rangle \mathrm{e}^{-\mathrm{i} \omega t} .
\end{aligned}
$$

\footnotetext{
${ }^{58}$ The closure relation expresses the fact that any target state can be expressed as a linear combination of the states $|\lambda\rangle$. The $\delta$-function in the expression (5.62) introduces a drastic selection of the states entering the sum. It is no longer possible to constitute a basis from this reduced set of target sates.
} 
The energies written here can following (5.108) be replaced by the Hamilton operator. We find

$$
\begin{aligned}
& \left\langle\lambda_{f}\left|\mathrm{e}^{\mathrm{i} \vec{Q} \cdot \vec{R}_{j}}\right| \lambda_{i}\right\rangle\left\langle\lambda_{i}\left|\mathrm{e}^{-\mathrm{i} \vec{Q} \cdot \vec{R}_{j^{\prime}}}\right| \lambda_{f}\right\rangle \delta\left(E_{\lambda_{i}}-E_{\lambda_{f}}-\hbar \omega\right) \\
& =\frac{1}{2 \pi \hbar} \int_{-\infty}^{\infty} \mathrm{d} t\left\langle\lambda_{f}\left|\mathrm{e}^{\mathrm{i}(\mathbf{H} / \hbar) t} \mathrm{e}^{\mathrm{i} \vec{Q} \cdot \vec{R}_{j}} \mathrm{e}^{-\mathrm{i}(\mathbf{H} / \hbar) t}\right| \lambda_{i}\right\rangle\left\langle\lambda_{i}\left|\mathrm{e}^{-\mathrm{i} \vec{Q} \cdot \vec{R}_{j^{\prime}}}\right| \lambda_{f}\right\rangle \mathrm{e}^{-\mathrm{i} \omega t} \\
& =\frac{1}{2 \pi \hbar} \int_{-\infty}^{\infty} \mathrm{d} t\left\langle\lambda_{i}\left|\mathrm{e}^{-\mathrm{i} \vec{Q} \cdot \vec{R}_{j^{\prime}}}\right| \lambda_{f}\right\rangle\left\langle\lambda_{f}\left|\mathrm{e}^{\mathrm{i}(\mathbf{H} / \hbar) t} \mathrm{e}^{\mathrm{i} \vec{Q} \cdot \vec{R}_{j}} \mathrm{e}^{-\mathrm{i}(\mathbf{H} / \hbar) t}\right| \lambda_{i}\right\rangle \mathrm{e}^{-\mathrm{i} \omega t},
\end{aligned}
$$

where we have inverted the order of the matrix elements in the last line. We have thus reached the point where we can apply the closure relation (5.104)

$$
\begin{aligned}
& \sum_{\lambda_{f}}\left\langle\lambda_{f}\left|\mathrm{e} \mathrm{e}^{\mathrm{i} \vec{Q} \cdot \vec{R}_{j}}\right| \lambda_{i}\right\rangle\left\langle\lambda_{i}\left|\mathrm{e}^{-\mathrm{i} \vec{Q} \cdot \vec{R}_{j^{\prime}}}\right| \lambda_{f}\right\rangle \delta\left(E_{\lambda_{i}}-E_{\lambda_{f}}-\hbar \omega\right) \\
& \quad=\frac{1}{2 \pi \hbar} \int_{-\infty}^{\infty} \mathrm{d} t\left\langle\lambda_{i}\left|\mathrm{e}^{-\mathrm{i} \vec{Q} \cdot \vec{R}_{j^{\prime}}} \mathrm{e}^{\mathrm{i}(\mathbf{H} / \hbar) t} \mathrm{e}^{\mathrm{i} \vec{Q} \cdot \vec{R}_{j}} \mathrm{e}^{-\mathrm{i}(\mathbf{H} / \hbar) t}\right| \lambda_{i}\right\rangle \mathrm{e}^{-\mathrm{i} \omega t}
\end{aligned}
$$

At this point it is important to mention that the order in which the $\vec{R}_{j}$ appear is fixed since the $\vec{R}_{j}$ as operators do not commute with the Hamiltonian $\mathbf{H}{ }^{59}$ We have liberated ourselves from the sum over the final states but at the price of introducing exponential functions of the Hamilton operator in the master equation. The operators $\exp (-(\mathrm{i} / \hbar) \mathbf{H} t)$ are nevertheless well known in quantum mechanics. They are the time evolution operators that we have already mentioned in Section 1.2

$$
\mathbf{U}\left(t, t_{0}\right)=\mathrm{e}^{-(\mathrm{i} / \hbar) \mathbf{H}\left(t-t_{0}\right)} .
$$

Applied to an operator $\mathbf{A}$

$$
\mathbf{A}(t)=\mathbf{U}^{+}\left(t, t_{0}\right) \mathbf{A}\left(t_{0}\right) \mathbf{U}\left(t, t_{0}\right)=\mathrm{e}^{(\mathrm{i} / \hbar) \mathbf{H}\left(t-t_{0}\right)} \mathbf{A}\left(t_{0}\right) \mathrm{e}^{-(\mathrm{i} / \hbar) \mathbf{H}\left(t-t_{0}\right)}
$$

the operator $\mathbf{U}$ describes the time evolution of $\mathbf{A} .{ }^{60}$

Identifying A with $\overrightarrow{\mathbf{R}}_{j}^{0}:=\overrightarrow{\mathbf{R}}_{j}(t=0)$ we see that ${ }^{61}$

$$
\mathrm{e}^{\mathrm{i}(\mathbf{H} / \hbar) t} \mathrm{e}^{\mathrm{i} \mathbf{Q} \cdot \vec{R}_{j}} \mathrm{e}^{-\mathrm{i}(\mathbf{H} / \hbar) t}=\mathrm{e}^{\mathrm{i} \vec{Q} \cdot \vec{R}_{j}(t)}
$$

As a consequence the terms in the master equation take the form

$$
\begin{gathered}
\sum_{\lambda_{f}}\left\langle\lambda_{f}\left|\mathrm{e}^{\mathrm{i} \vec{Q} \cdot \vec{R}_{j}}\right| \lambda_{i}\right\rangle\left\langle\lambda_{i}\left|\mathrm{e}^{-\mathrm{i} \vec{Q} \cdot \vec{R}_{j^{\prime}}}\right| \lambda_{f}\right\rangle \delta\left(E_{\lambda_{i}}-E_{\lambda_{f}}-\hbar \omega\right) \\
=\frac{1}{2 \pi \hbar} \int_{-\infty}^{\infty} \mathrm{d} t\left\langle\lambda_{i}\left|\mathrm{e}^{-\mathrm{i} \vec{Q} \cdot \vec{R}_{j^{\prime}}^{0}} \mathrm{e}^{\mathrm{i} \vec{Q} \cdot \vec{R}_{j}(t)}\right| \lambda_{i}\right\rangle \mathrm{e}^{-\mathrm{i} \omega t} .
\end{gathered}
$$

\footnotetext{
${ }^{59} \mathbf{H}$ contains momentum operators $\vec{P}_{j}$ that do not commute with $\vec{R}_{j}$.

${ }^{60}$ The reader motivated to know the formal justification for this relation can find the explanation in every book on quantum mechanics.

${ }^{61}$ In order to lighten the formalism we will in most formulas refrain from explicitly indicating the operator character of $\vec{R}_{j}$.
} 
The partial differential cross section becomes

$$
\frac{\mathrm{d}^{2} \sigma_{\vec{k}_{i} \rightarrow \vec{k}_{f}}}{\mathrm{~d} \Omega \mathrm{d} E_{f}}=\frac{k_{f}}{k_{i}} \frac{1}{2 \pi \hbar} \sum_{\lambda_{i}} p\left(\lambda_{i}\right) \sum_{j, j^{\prime}=1}^{N} b_{j} b_{j^{\prime}}^{*} \int_{-\infty}^{\infty} \mathrm{d} t\left\langle\lambda_{i}\left|\mathrm{e}^{-\mathrm{i} \vec{Q} \cdot \vec{R}_{j^{\prime}}^{0}} \mathrm{e}^{\mathrm{i} \vec{Q} \cdot \vec{R}_{j}(t)}\right| \lambda_{i}\right\rangle \mathrm{e}^{-\mathrm{i} \omega t} .
$$

We can write this result in the more compact form

$$
\frac{\mathrm{d}^{2} \sigma_{\vec{k}_{i} \rightarrow \vec{k}_{f}}}{\mathrm{~d} \Omega \mathrm{d} E_{f}}=\frac{k_{f}}{k_{i}} S(\vec{Q}, \omega)
$$

where

$$
S(\vec{Q}, \omega)=\frac{1}{2 \pi \hbar} \sum_{\lambda_{i}} p\left(\lambda_{i}\right) \sum_{j, j^{\prime}=1}^{N} b_{j} b_{j^{\prime}}^{*} \int_{-\infty}^{\infty} \mathrm{d} t\left\langle\lambda_{i}\left|\mathrm{e}^{-\mathrm{i} \vec{Q} \cdot \vec{R}_{j^{\prime}}^{0}} \mathrm{e}^{\mathrm{i} \vec{Q} \cdot \vec{R}_{j}(t)}\right| \lambda_{i}\right\rangle \mathrm{e}^{-\mathrm{i} \omega t} .
$$

$S(\vec{Q}, \omega)$ is called the scattering function or the dynamic structure factor. ${ }^{62}$ As closer inspection shows the scattering function is expressed in terms of expectation values (see expression (5.100)). We, therefore, have attained our original goal.

We will briefly comment on this result.

- We recall that the expression (5.119) describes the scattering within the Born approximation.

- The expression (5.118) is valid for very short ranged interaction potentials that are expressed via the scattering length $b_{j}$. The generalisation is done by substituting the scattering lengths in (5.91) with the form factors of the interaction potential (5.90) according to

$$
b_{j} \rightarrow \frac{m}{2 \pi \hbar^{2}} V_{j}(\vec{Q}) .
$$

- The scattering function is an extensive quantity of the sample. In other words, the scattering function is proportional to the number of scatterers. The explicit dependence on the number of scatterers is mostly an inconvenience. The scattering function is, therefore, often given in a normalised form. The normalisation is performed with respect to the number of scatterers either in the chemical unit or in the unit cell (for samples of crystalline character).

- The proportionality between the scattering function and the number of scatterers clearly shows the limits of the Born approximation. It is not possible that the number of scattered probe particles increases without bounds for a given incident flux. There will be a point at which the majority of incident particles have been scattered. Beyond this point it does not make sense to increase the sample size further. This is called the extinction effect. In practice extinction is observed mainly for Bragg scattering. When a crystal is of good quality then the incident neutron flux giving rise to the Bragg scattering will be exhausted after only a few thousand atomic layers. It is evident that beyond this point the incident beam can no longer be considered to be homogeneous over the sample volume. This is nevertheless exactly what the Born approximation amounts to. When dealing with such cases we have to turn to dynamical scattering theory [49,58]. We will come back to this discussion in Section 11.5. Another example where the Born approximation fails by definition is total scattering or grazing incident scattering (see Section 9.9). As the probability of scattering approaches $100 \%$ it is no longer possible to consider the scattering a weak perturbation to the incident beam. This case is treated within the distorted-wave Born approximation [11,60].

\footnotetext{
${ }^{62}$ The way we have defined the scattering function it possesses units of [area/energy]. If we stuck to a rigorous notation we would, therefore, use the energy as a variable and write $S(\vec{Q}, \hbar \omega)$. Following the main stream of the literature we opt for the shorter $S(\vec{Q}, \omega)$.
} 
- The scattering function is a physical quantity that relates to the equilibrium state of the sample. We will exploit this fact when relating the scattering function to correlation functions and susceptibilities making use of linear response theory.

- The scattering function does not depend explicitly on the final sates of the sample. At thermodynamic equilibrium these states are nevertheless present in the factors $p_{\lambda_{i}}$. As we have seen these probabilities can be expressed as

$$
p_{\lambda}=\frac{1}{Z} \exp \left(-\frac{E_{\lambda}}{k_{\mathrm{B}} T}\right)
$$

with the canonical partition function $Z$ defined as

$$
Z=\sum_{\lambda} \exp \left(-\frac{E_{\lambda}}{k_{\mathrm{B}} T}\right)
$$

In order to calculate $Z$ we need to know the complete spectrum of excitations.

- If the probe particles have themselves internal degrees of freedom this necessarily adds an extra layer of complexity to the problem. It equally provides additional means to retrieve valuable information from the scattering. For the neutron these channels are linked to its two spin states $|\sigma\rangle=| \pm\rangle$. If $p\left(\sigma_{i}\right)$ denotes the probability of finding the neutron in the spin state $\left|\sigma_{i}\right\rangle$ before the collision and if we do not reveal the final spin state $\left|\sigma_{f}\right\rangle$ before the detection (no spin analysis) then the scattering function becomes

$$
S(\vec{Q}, \omega)=\frac{1}{2 \pi \hbar} \sum_{\lambda_{i}, \sigma_{i}, \sigma_{f}} p\left(\lambda_{i}\right) p\left(\sigma_{i}\right) \sum_{j, j^{\prime}=1}^{N} b_{j} b_{j^{\prime}}^{*} \int_{-\infty}^{\infty} \mathrm{d} t\left\langle\sigma_{f} ; \lambda_{i}\left|\mathrm{e}^{-\mathrm{i} \vec{Q} \cdot \vec{R}_{j^{\prime}}^{0}} \mathrm{e}^{\mathrm{i} \vec{Q} \cdot \vec{R}_{j}(t)}\right| \lambda_{i} ; \sigma_{i}\right\rangle \mathrm{e}^{-\mathrm{i} \omega t}
$$

\section{Specificities of neutron scattering}

With the intention of developing a general formalism we have so far avoided, whenever possible, specificities related to neutron scattering. For this reason the results obtained can be applied with little modification to other scattering techniques provided that these satisfy the criteria required by the approximations used. In this section we will deal with aspects that are specific to neutron scattering. Among these features prominently the distinction of coherent and incoherent neutron scattering.

\subsection{Coherent and incoherent scattering}

The scattering function (5.119) contains a double sum over the scatterers that formally can be expressed as

$$
S(\vec{Q}, \omega)=\sum_{j, j^{\prime}=1}^{N}\left(b_{j} b_{j^{\prime}}^{*}\right) S_{j, j^{\prime}}(\vec{Q}, \omega)
$$

with

$$
S_{j, j^{\prime}}(\vec{Q}, \omega)=\frac{1}{2 \pi \hbar} \sum_{\lambda_{i}} p\left(\lambda_{i}\right) \int_{-\infty}^{\infty} \mathrm{d} t\left\langle\lambda_{i}\left|\mathrm{e}^{-\mathrm{i} \vec{Q} \cdot \vec{R}_{j^{\prime}}^{0}} \mathrm{e}^{\mathrm{i} \vec{Q} \cdot \vec{R}_{j}(t)}\right| \lambda_{i}\right\rangle \mathrm{e}^{-\mathrm{i} \omega t}
$$

While the term $b_{j} b_{j^{\prime}}^{*}$ depends on the nuclei the function $S_{j, j^{\prime}}(\vec{Q}, \omega)$ depends on the atomic coordinates and electronic states of the sample and thus generally reflects the chemical state of the sample. It is a particularity of 


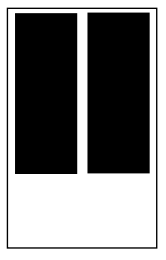

12

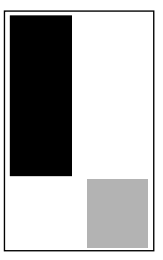

12

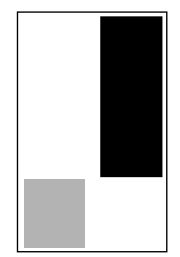

12

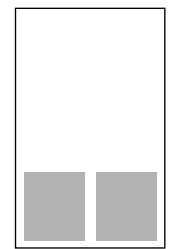

12

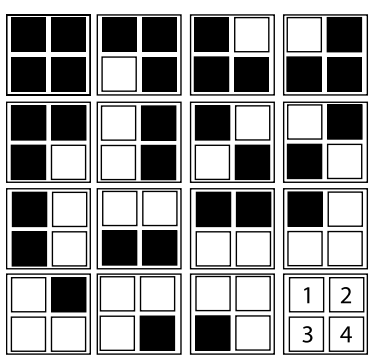

Fig. 30. Illustration of the statistical distribution of the scattering lengths. In the top example we imagine a system composed of two identical atoms (for example a bi-atomic molecule). We assume that the nucleus of the atom has two equally probable isotopes. The two isotopes have different scattering lengths. Here, one is assumed positive and the other negative. There is no correlation between the position and the isotope state of the atom. When we do the calculation of the cross section we need to average over all four possible configurations. If we remain with the picture of a molecule then we do so by adding the cross sections of the ensemble of molecules, which is representative for the isotope distribution. In the lower figure we treat the case of four atoms with two scattering lengths equally distributed. The sixteen configurations shown are representative for this distribution. We can imagine that the four atoms correspond to the correlation volume of the sample. By sweeping this volume over the sample we will perfectly average over the isotope distribution.

neutron scattering that the nuclear scattering potential can vary from one atom to the next for the same chemical element. These variations are due to the fact that neutrons are sensitive to nuclear isotopes and to the nuclear spin. The nuclear isotopes as well as the nuclear spins are in general distributed in a statistical manner. The interactions between the nuclei capable of aligning the spins are very weak due to their indirect nature. In condensed matter they are mediated by the electrons (hyperfine interactions) ${ }^{63}$ It is clear that the same argument does not hold for scattering of X-rays or neutron scattering from the magnetic moments created by the electronic cloud, which both relate to the chemical elements.

A random distribution of the scattering length on the atomic site $\vec{R}_{j}$ implies that any information on the value of the scattering length at the site $\vec{R}_{j}$ is of statistical nature. Randomness in particular implies that there is no statistical correlation between the values of the scattering lengths $b_{j}$ and the sites $\vec{R}_{j}$. The randomness present in the nuclear system concerns exclusively the terms $\left(b_{j} b_{j^{\prime}}^{*}\right)$ in expression (6.1) but not $S_{j, j^{\prime}}(\vec{Q}, \omega)$.

We would like to illustrate these general statements by a simple example. We consider a sample composed of two atoms of the same chemical element. The nuclei of these two atoms are present in two isotopic forms, thus we denote the respective scattering lengths by $c_{1}$ and $c_{2}$. The scattering lengths are assumed to be real. We replicate the system in the four possible isotopic combinations. Each replica corresponds to a sample for which we calculate the scattering function. From these particular scattering functions we determine the average scattering function. We will first examine the distinct parts of the sum (6.1). For a given pair $j, j^{\prime}$ we are dealing with four individual cases (see Fig. 30)

$$
j \neq j^{\prime}: \quad b_{j} b_{j^{\prime}}=c_{1} \cdot c_{1}, \quad b_{j} b_{j^{\prime}}=c_{1} \cdot c_{2}, \quad b_{j} b_{j^{\prime}}=c_{2} \cdot c_{1}, \quad b_{j} b_{j^{\prime}}=c_{2} \cdot c_{2} .
$$

\footnotetext{
${ }^{63}$ However we would like to emphasise that there exist systems with correlations between the nuclear spins at very low temperatures. Neutrons are an ideal probe of this nuclear magnetic ordering. For a review see the article by Steiner [63] who pioneered this field.
} 
Hence we can write

$$
j \neq j^{\prime}: \quad\left(b_{j} b_{j^{\prime}}\right) S_{j, j^{\prime}}(\vec{Q}, \omega) \rightarrow \frac{1}{4}\left(c_{1} \cdot c_{1}+2 c_{1} \cdot c_{2}+c_{2} \cdot c_{2}\right) S_{j, j^{\prime}}(\vec{Q}, \omega)=(\bar{c})^{2} S_{j, j^{\prime}}(\vec{Q}, \omega) .
$$

The distinct terms of the scattering function are thus proportional to the square of the average scattering length $\bar{c}=(1 / 2)\left(c_{1}+c_{2}\right)$. Similarly for the self terms $j=j^{\prime}$ we find

$$
j=j^{\prime}: \quad b_{j} b_{j}=c_{1} \cdot c_{1}(2 \text { times }), \quad b_{j} b_{j}=c_{2} \cdot c_{2}(2 \text { times }),
$$

which brings us to

$$
j=j^{\prime}: \quad\left(b_{j} b_{j}\right) S_{j, j}(\vec{Q}, \omega)=\rightarrow \frac{1}{2}\left(c_{1} \cdot c_{1}+c_{2} \cdot c_{2}\right) S_{j, j}(\vec{Q}, \omega)=\overline{c^{2}} S_{j, j}(\vec{Q}, \omega)
$$

The self terms of the scattering function are thus proportional to the average of the square of the scattering length $\overline{c^{2}}=(1 / 2)\left(c_{1}^{2}+c_{2}^{2}\right)$.

We will now generalise this result. We start from a macroscopic system with a large number of scatterers. This system yields a priori a precisely defined and unique scattering function. ${ }^{64}$ As we are going to see later (Section 11.5) we rarely get interference from macroscopic volumes of our sample. The volume fraction of the sample that gives rise to interference effects, the so-called correlation volume. Hence we can consider that the macroscopic system is composed of an ensemble of replica of the correlation volume. Given the large number of replicas we can safely assume that every possible nuclear isotope or spin configuration appears with its correct statistical weight in this ensemble. As in our previous example we find the scattering function by averaging over all possible replicas

$$
S(\vec{Q}, \omega)=\sum_{j, j^{\prime}=1}^{N} \overline{\left(b_{j} b_{j^{\prime}}^{*}\right)} S_{j, j^{\prime}}(\vec{Q}, \omega) .
$$

Due to the absence of correlation between the scattering lengths and the positions of the atoms

$$
\begin{array}{ll}
j \neq j^{\prime}: & \overline{\left(b_{j} b_{j^{\prime}}^{*}\right)}=\overline{b_{j} b_{j^{\prime}}^{*}}, \\
j=j^{\prime}: & \overline{\left(b_{j} b_{j}^{*}\right)}=\overline{b_{j}^{2}},
\end{array}
$$

with

$$
\begin{aligned}
& \overline{b_{j}}=\sum_{i} p_{j}^{i} \cdot b_{j}^{i}, \\
& \overline{b_{j}^{2}}=\sum_{i} p_{j}^{i} \cdot\left(b_{j}^{i}\right)^{2} .
\end{aligned}
$$

The coefficients $p_{j}^{i}$ give the probability that the scattering length of the element at position $\vec{R}_{j}$ has the value $b_{j}^{i}$. Naturally

$$
\sum_{i} p_{j}^{i}=1
$$

\footnotetext{
${ }^{64}$ The interference of all the wave fronts produced in the illuminated sample volume is e.g. exploited in the case of Photon Correlation Spectroscopy (PCS). It requires that the light front is coherent over this volume (see Section 4.7).
} 
where the index $i$ runs over all possible isotope or spin states of the corresponding nucleus.

With this result we can calculate the scattering function from the statistical distributions of the scattering lengths $p\left(b_{j}\right)$, which in general are well known,

$$
\begin{aligned}
S(\vec{Q}, \omega) & =\sum_{j \neq j^{\prime}}^{N} \overline{b_{j} b_{j^{\prime}}^{*}} S_{j, j^{\prime}}(\vec{Q}, \omega)+\sum_{j=1}^{N} \overline{b_{j}^{2}} S_{j, j}(\vec{Q}, \omega) \\
& =\sum_{j, j^{\prime}=1}^{N} \overline{b_{j} b_{j^{\prime}}^{*}} S_{j, j^{\prime}}(\vec{Q}, \omega)+\sum_{j=1}^{N}\left(\overline{b_{j}^{2}}-\left(\bar{b}_{j}\right)^{2}\right) S_{j, j}(\vec{Q}, \omega) .
\end{aligned}
$$

To obtain the last line we have added the term $\sum_{j=1}^{N}\left(\bar{b}_{j}\right)^{2} S_{j, j}(\vec{Q}, \omega)$ to the first sum and subtracted it from the second.

We define

$$
\frac{\mathrm{d}^{2} \sigma}{\mathrm{d} \Omega \mathrm{d} E_{f}}=\left(\frac{\mathrm{d}^{2} \sigma}{\mathrm{d} \Omega \mathrm{d} E_{f}}\right)_{\mathrm{coh}}+\left(\frac{\mathrm{d}^{2} \sigma}{\mathrm{d} \Omega \mathrm{d} E_{f}}\right)_{\mathrm{inc}}
$$

where the coherent contribution to the cross section is given by

$$
\left(\frac{\mathrm{d}^{2} \sigma}{\mathrm{d} \Omega \mathrm{d} E_{f}}\right)_{\text {coh }}=\frac{k_{f}}{k_{i}} \frac{1}{2 \pi \hbar} \sum_{\lambda_{i}} p\left(\lambda_{i}\right) \sum_{j, j^{\prime}=1}^{N} \overline{b_{j} b_{j^{\prime}}^{*}} \int_{-\infty}^{\infty} \mathrm{d} t\left\langle\lambda_{i}\left|\mathrm{e}^{-\mathrm{i} \vec{Q} \cdot \vec{R}_{j^{\prime}}^{0}} \mathrm{e}^{\mathrm{i} \vec{Q} \cdot \vec{R}_{j}(t)}\right| \lambda_{i}\right\rangle \mathrm{e}^{-\mathrm{i} \omega t},
$$

while the incoherent contribution is

$$
\left(\frac{\mathrm{d}^{2} \sigma}{\mathrm{d} \Omega \mathrm{d} E_{f}}\right)_{\text {inc }}=\frac{k_{f}}{k_{i}} \frac{1}{2 \pi \hbar} \sum_{\lambda_{i}} p\left(\lambda_{i}\right) \sum_{j}^{N}\left(\overline{b_{j}^{2}}-\left(\bar{b}_{j}\right)^{2}\right) \int_{-\infty}^{\infty} \mathrm{d} t\left\langle\lambda_{i}\left|\mathrm{e}^{-\mathrm{i} \vec{Q} \cdot \vec{R}_{j}^{0}} \mathrm{e}^{\mathrm{i} \vec{Q} \cdot \vec{R}_{j}(t)}\right| \lambda_{i}\right\rangle \mathrm{e}^{-\mathrm{i} \omega t} .
$$

If the system is monatomic we can simplify these expressions by introducing the total coherent and incoherent cross sections

$$
\begin{aligned}
\sigma_{\mathrm{coh}} & =4 \pi(\bar{b})^{2}, \\
\sigma_{\mathrm{inc}} & =4 \pi\left[\overline{b^{2}}-(\bar{b})^{2}\right] .
\end{aligned}
$$

The expressions (6.16) and (6.17) then become

$$
\left(\frac{\mathrm{d}^{2} \sigma}{\mathrm{d} \Omega \mathrm{d} E_{f}}\right)_{\mathrm{coh}}=\frac{\sigma_{\mathrm{coh}}}{4 \pi} \frac{k_{f}}{k_{i}} \frac{1}{2 \pi \hbar} \sum_{\lambda_{i}} p\left(\lambda_{i}\right) \sum_{j, j^{\prime}=1}^{N} \int_{-\infty}^{\infty} \mathrm{d} t\left\langle\lambda_{i}\left|\mathrm{e}^{-\mathrm{i} \vec{Q} \cdot \vec{R}_{j^{\prime}}^{0}} \mathrm{e}^{\mathrm{i} \vec{Q} \cdot \vec{R}_{j}(t)}\right| \lambda_{i}\right\rangle \mathrm{e}^{-\mathrm{i} \omega t}
$$

and

$$
\left(\frac{\mathrm{d}^{2} \sigma}{\mathrm{d} \Omega \mathrm{d} E_{f}}\right)_{\mathrm{inc}}=\frac{\sigma_{\text {inc }}}{4 \pi} \frac{k_{f}}{k_{i}} \frac{1}{2 \pi \hbar} \sum_{\lambda_{i}} p\left(\lambda_{i}\right) \sum_{j}^{N} \int_{-\infty}^{\infty} \mathrm{d} t\left\langle\lambda_{i}\left|\mathrm{e}^{-\mathrm{i} \vec{Q} \cdot \vec{R}_{j}^{0}} \mathrm{e}^{\mathrm{i} \vec{Q} \cdot \vec{R}_{j}(t)}\right| \lambda_{i}\right\rangle \mathrm{e}^{-\mathrm{i} \omega t} .
$$

The coherent scattering is qualitatively very different from the incoherent scattering. We are going to discuss these differences after having equipped ourselves with the important tool of correlation functions.

We equally want to stress the fundamental difference between incoherent scattering and diffuse scattering that we are going to discuss in a bit more detail in Section 9.14. 


\subsection{The dependence of the scattering lengths on the nuclear spin}

The statistical distribution of nuclear scattering lengths can, as mentioned before, originate in the atomic isotopes but also in the nuclear spin. The distribution of isotopes is a complicated subject in nuclear physics, which we have no intention to develop further. The distribution of scattering channels due to nuclear spin is on the other hand a consequence of the angular momentum decomposition that falls within the basics of ordinary quantum mechanics $[10,37]$. We, therefore, are capable of deriving explicit expressions for $\bar{b}$ and $\overline{b^{2}}$ for a given isotope with the nuclear $\operatorname{spin} I$.

As already mentioned in the context of partial waves the angular momentum is a conserved quantity in systems with isotropic symmetry. When both the probe particle and the target possess spin their total angular momentum $\vec{J}$ is conserved. For $s$-type scattering there is no orbital contribution to the scattering. The total angular momentum operator is then simply the sum of the spin $\overrightarrow{\mathbf{S}}$ of the probe particle and of the spin $\overrightarrow{\mathbf{I}}$ of the target

$$
\overrightarrow{\mathbf{J}}=\overrightarrow{\mathbf{I}}+\overrightarrow{\mathbf{S}}
$$

The neutron has a spin of $S=1 / 2$ and the combined system can take the values of

$$
\begin{aligned}
& J^{+}=I+1 / 2 \quad \text { and } \\
& J^{-}=I-1 / 2 .
\end{aligned}
$$

For each value of $J$ we, in principle, expect a different scattering length. The degeneracy of the $\overrightarrow{\mathbf{J}}$ levels are given by

$$
n_{J}=2 J+1
$$

We find

$$
\begin{aligned}
& n^{+}=2(I+1 / 2)+1=2 I+2, \\
& n^{-}=2(I-1 / 2)+1=2 I
\end{aligned}
$$

If the incident beam is not polarised and if the spin states of the sample are distributed in a statistical manner each of the $\overrightarrow{\mathbf{J}}$ states is equally probable. The conditional probability distribution that indicates the probability for the scattering to proceed either via the channel $(+)$ or $(-)$ is then expressed as

$$
\begin{aligned}
& p^{+}=\frac{2 I+2}{4 I+2}=\frac{I+1}{2 I+1}, \\
& p^{-}=\frac{2 I}{4 I+2}=\frac{I}{2 I+1} .
\end{aligned}
$$

This enables us to calculate the statistical mean values

$$
\begin{aligned}
& \bar{b}=\frac{1}{2 I+1}\left((I+1) b^{+}+I b^{-}\right), \\
& \overline{b^{2}}=\frac{1}{2 I+1}\left((I+1)\left(b^{+}\right)^{2}+I\left(b^{-}\right)^{2}\right) .
\end{aligned}
$$




\subsection{Neutron scattering from a proton or a deuteron}

As a concrete example of this formalism we will look at the neutron scattering from a proton. Both the neutron and the proton have a spin of $S=1 / 2$. The Hilbert space of the common system is the tensor product of the two dimensional space of the two spins. Hence it has four dimensions and a complete basis is given by

$$
\begin{aligned}
& |\uparrow \uparrow\rangle=|\uparrow\rangle_{n} \otimes|\uparrow\rangle_{p}, \\
& |\downarrow \uparrow\rangle=|\downarrow\rangle_{n} \otimes|\uparrow\rangle_{p}, \\
& |\uparrow \downarrow\rangle=|\uparrow\rangle_{n} \otimes|\downarrow\rangle_{p}, \\
& |\downarrow \downarrow\rangle=|\downarrow\rangle_{n} \otimes|\downarrow\rangle_{p} .
\end{aligned}
$$

The indices $n$ and $p$ refer to the neutron and the proton, respectively. The operator $\overrightarrow{\mathbf{S}}^{2}$ where $\overrightarrow{\mathbf{S}}=\overrightarrow{\mathbf{S}}_{n}+\overrightarrow{\mathbf{S}}_{p}$ is not diagonal in this basis. Hence this basis is not very useful since it is the total spin that is the conserved quantity and not the isolated spins of the neutron and the proton. By applying the rules of combining spins we find two eigenvalues for $\overrightarrow{\mathbf{S}}^{2}$

$$
S=S_{n}+S_{p}=1 \quad \text { and } \quad S=\left|S_{n}-S_{p}\right|=0
$$

Each value of $S$ corresponds to a sub-space of eigenvectors of dimension $2 S+1$. In the case of two spin $1 / 2$ particles these eigenvectors are give by

$$
\begin{aligned}
S=1: & |1,1\rangle=|\uparrow \uparrow\rangle \\
: & |1,0\rangle=\frac{1}{\sqrt{2}}(|\uparrow \downarrow\rangle+|\downarrow \uparrow\rangle) \\
: & |1,-1\rangle=|\downarrow \downarrow\rangle, \\
S=0: & |0,0\rangle=\frac{1}{\sqrt{2}}(|\uparrow \downarrow\rangle-|\downarrow \uparrow\rangle) .
\end{aligned}
$$

The functions inside a multiplet can be distinguished by their projection onto $S_{z}$

$$
\mathbf{S}_{z}\left|S, M_{s}\right\rangle=M_{s}\left|S, M_{s}\right\rangle
$$

For the neutron-proton system, we find a triplet and a singlet.

In a system without preferred direction ${ }^{65}$ the value of $M_{s}$ cannot influence the scattering. In other words, the scattering length has to be a function of $S$ only. In the present case the scattering length can, therefore take on two different values

$$
b^{+}=b(S=1)
$$

and

$$
b^{-}=b(S=0)
$$

\footnotetext{
${ }^{65}$ The only direction that breaks isotropic symmetry is the direction of the incident beam. However, this direction has no influence on the spin system. The situation changes if an external magnetic field is applied.
} 
These scattering lengths are determined experimentally for bound protons as

$$
\begin{aligned}
& b^{+}\left({ }^{1} H\right)=1.04 \times 10^{-12} \mathrm{~cm}=10.4 \mathrm{fm}, \quad p^{+}=\frac{3}{4}, \\
& b^{-}\left({ }^{1} H\right)=-4.74 \times 10^{-12} \mathrm{~cm}=47.4 \mathrm{fm}, \quad p^{-}=\frac{1}{4} .
\end{aligned}
$$

The scattering length of the singlet is appreciable and negative. Following the arguments developed in Section 3.3 we can deduce that the system is close to the formation of a bound state. In the triplet state there exists a true bound state $(n-p)$ the deuteron, which explains why the corresponding scattering length is very large and positive. From these scattering lengths we obtain

$$
\begin{aligned}
& \bar{b}=\frac{3}{4} b^{+}+\frac{1}{4} b^{-}=-0.38 \times 10^{-12} \mathrm{~cm}, \\
& \overline{|b|^{2}}=\frac{3}{4}\left|b^{+}\right|^{2}+\frac{1}{4}\left|b^{-}\right|^{2}=6.49 \text { barn. }
\end{aligned}
$$

The fact that

$$
\frac{b^{+}}{b^{-}} \approx-\frac{p^{+}}{p^{-}}=-\frac{1}{3}
$$

has the consequence that the average scattering length is close to zero. Hence we expect a very weak coherent scattering. The incoherent scattering is on the other hand appreciable due to the large cross section of the singlet state.

With (6.18)-(6.19) we numerical can calculate the total coherent and incoherent cross sections

$$
\begin{aligned}
& \sigma_{\text {coh }}\left({ }^{1} \mathrm{H}\right)=1.8 \text { barn, } \\
& \sigma_{\text {inc }}\left({ }^{1} \mathrm{H}\right)=79.8 \text { barn, } \\
& \sigma_{\text {tot }}\left({ }^{1} \mathrm{H}\right)=81.7 \text { barn. }
\end{aligned}
$$

If we replace the proton by a deuteron the situation changes considerably. The spin of deuterium is $I=1$. The combined states of the neutron and the deuteron possess the spin states $S=3 / 2$ and $S=1 / 2$. Hence, we have a quadruplet and a doublet. The experimental scattering lengths are

$$
\begin{aligned}
& b^{+}\left({ }^{2} \mathrm{H}\right)=0.95 \times 10^{-12} \mathrm{~cm}, \quad p^{+}=\frac{4}{6}, \\
& b^{-}\left({ }^{2} \mathrm{H}\right)=0.10 \times 10^{-12} \mathrm{~cm}, \quad p^{-}=\frac{2}{6} .
\end{aligned}
$$

We can calculate

$$
\begin{aligned}
& \bar{b}=\frac{2}{3} b^{+}+\frac{1}{3} b^{-}=0.67 \times 10^{-12} \mathrm{~cm}, \\
& \overline{|b|^{2}}=\frac{2}{3}\left|b^{+}\right|^{2}+\frac{1}{3}\left|b^{-}\right|^{2}=0.61 \mathrm{barn}
\end{aligned}
$$


and find the cross sections

$$
\begin{aligned}
\sigma_{\text {coh }}\left({ }^{2} \mathrm{H}\right) & =5.6 \text { barn, } \\
\sigma_{\text {inc }}\left({ }^{2} \mathrm{H}\right) & =2.0 \text { barn, } \\
\sigma_{\text {tot }}\left({ }^{2} \mathrm{H}\right) & =7.6 \text { barn. }
\end{aligned}
$$

By substituting the proton by a deuteron we can, therefore, change the scattering characteristics of a sample considerably without influencing its chemical and physical properties significantly. This possibility is frequently used in practice, for example in order to change the contrast between different parts of the sample or simply to play with the ratio between coherent and incoherent scattering (see Section 8.4).

The different isotopes of hydrogen also have different absorption levels. The absorption is very weak for the deuteron but it is no longer negligible for the proton $\left(\sigma_{\mathrm{abs}} \approx 0.33\right.$ barn for neutrons of $\left.2200 \mathrm{~m} / \mathrm{s}\right)$. This is one of the reasons why research reactors are moderated with heavy water instead of light water.

Another very important element in neutron scattering is Vanadium. Its cross section of

$$
\sigma_{\text {tot }}(\text { Vanadium }) \equiv \sigma_{\text {inc }}(\text { Vanadium })=4.95 \text { barn }
$$

is nearly purely incoherent. As a consequence the scattering of a Vanadium sample at low temperatures is purely isotropic and, therefore, constitutes a perfect standard for calibrating spectrometers.

\subsection{The scattering triangle and the dynamic range}

The scattering function (5.119) does a priori no longer depend on the intrinsic properties of the neutron (or the probe particle in general). Forgetting the spin the properties of the neutron are summed up in the wave vector $\vec{k}$. The scattering function does nevertheless still include the interaction potential between the target and the probe, which means the nuclear scattering lengths $b_{j}$. We can only get rid of this dependence if the interaction is identical for all the scatterers involved (see expression (6.20) and (6.21) for the example of a monatomic sample). Apart from this formal complication the scattering function is a function of the sample only, i.e. it depends solely on the positions and motions of the scatterers.

We will now discuss some practical aspects of this rather abstract discussion. We begin with some definitions. We have already introduced the scattering vector

$$
\begin{aligned}
& \vec{Q}=\vec{k}_{i}-\vec{k}_{f}, \\
& Q^{2}=k_{i}^{2}+k_{f}^{2}-2 k_{i} k_{f} \cos 2 \Theta,
\end{aligned}
$$

as well as the energy transfer

$$
\hbar \omega=E_{i}-E_{f}=\frac{\hbar^{2}}{2 m}\left(k_{i}^{2}-k_{f}^{2}\right) .
$$

The scattering function is defined in the space of $(\vec{Q}, \omega)$. This four dimensional space is also called reciprocal space. One often just uses the expression $(\vec{Q}, \omega)$ space to denote it. $(\vec{Q}, \omega)$ space is infinite. From a purely mathematical point of view the function $S(\vec{Q}, \omega)$ is defined everywhere in $(\vec{Q}, \omega)$. However, we have to remember that the scattering function has been derived under the condition that the Born approximation is applicable. When basing the calculation of the differential cross section on the scattering function we have to make sure that these conditions are satisfied. This translates into a "region of validity" for $S(\vec{Q}, \omega)$ in $(\vec{Q}, \omega)$ space specific to the experimental set up (sample size, wave length used etc.).

The triplet of vectors $\left(\vec{Q}, \vec{k}_{i}, \vec{k}_{f}\right)$ form a triangle lying within the scattering plane (see Fig. 31). In an experiment the true variables are the initial wave vector $\vec{k}_{i}$ and the final wave vector $\vec{k}_{f}$. In general, there exists an infinite 

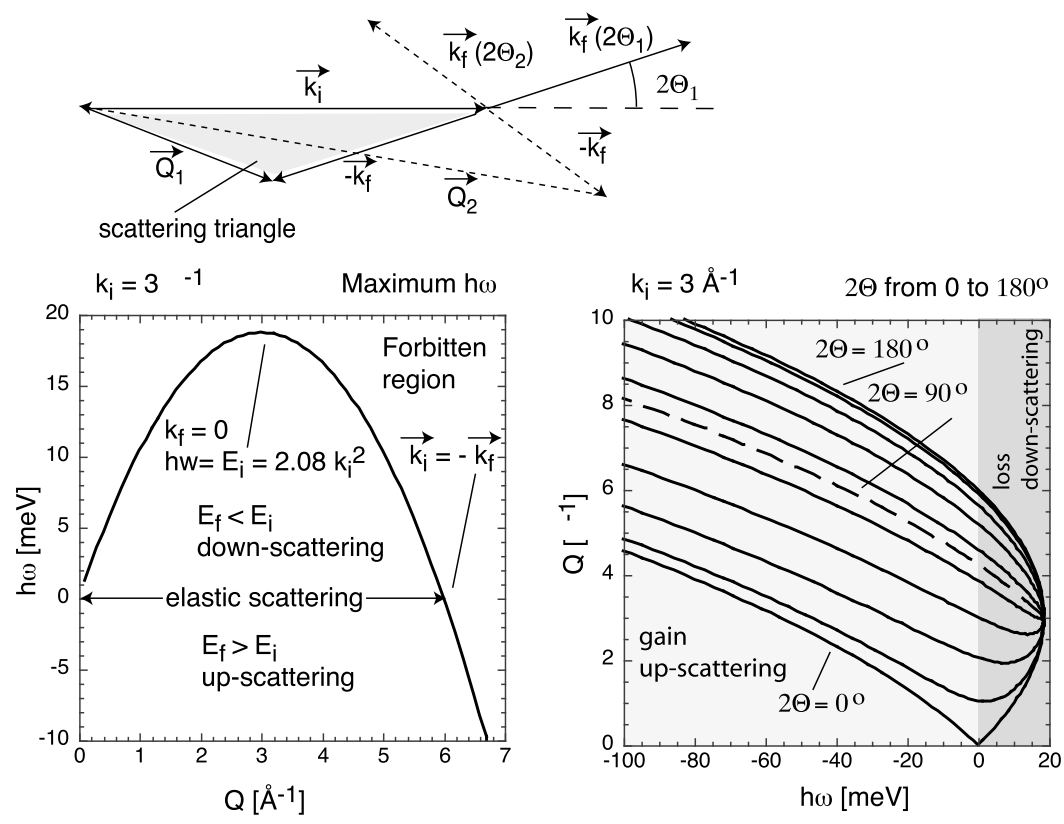

Fig. 31. The dynamic range of a neutron scattering experiment. For a given $k_{i}$ there will always be points in $(\vec{Q}, \hbar \omega)$ space for which the scattering triangle (shown at the top of the figure) will not close, i.e. where the kinematic conditions cannot be satisfied. Left figure: The maximum energy transfer as a function of $Q=|\vec{Q}|$ for a $k_{i}$ of $3 \AA^{-1}$. Right figure: Energy transfer corresponding to a specific $Q$ value for various scattering angles $2 \Theta\left(2 \Theta\right.$ increases from 0 to $180^{\circ}$ in steps of $\left.20^{\circ}\right)$. The solid lines correspond to $(Q, \hbar \omega)$ trajectories where $k_{i}$ and the angle $2 \Theta$ are fixed. All regions outside the lines for $\Theta=0$ and $\Theta=180^{\circ}$ are not accessible. Hence these lines define the field of view for the measurement.

number of combinations of $\left(\vec{k}_{i}, \vec{k}_{f}\right)$ that give access to the same $(\vec{Q}, \omega)$ point in reciprocal space. For example one can fix the direction and energy of the incident beam and reach a $(\vec{Q}, \hbar \omega)$ point by detecting the final energy

$$
E_{f}=\frac{\hbar^{2}}{2 m} k_{f}^{2}=E_{i}-\hbar \omega
$$

at a scattering angle

$$
\cos 2 \Theta=\frac{k_{i}^{2}+k_{f}^{2}-Q^{2}}{2 k_{i} k_{f}} .
$$

This is called an experimental configuration with $\vec{k}_{i}$ fixed. In perfect analogy one can work with $\vec{k}_{f}$ fixed.

For a given $k_{i}$ or $k_{f}$ only a restricted region in reciprocal space is accessible experimentally. This restriction is due to the fact that the kinematic constraints (6.60) and (6.61) cannot always be satisfied. One simple example is elastic scattering. For $\hbar \omega=0$ we see from (6.60) that $k_{i}=k_{f}$. The largest $Q=|\vec{Q}|$ value that can be reached according to (6.61) is equal to $2 k_{i}$ in backscattering $\left(\Theta=90^{\circ}\right)$. The physical explanation is simple. The neutron can at maximum transfer twice its initial momentum. In the same way the neutron cannot loose more than its total energy in an inelastic experiment.

\subsection{The total scattering rate}

With the formalism that we have developed it is possible to calculate the total scattering rate, i.e. the percentage of neutrons that are effectively scattered. This is a very important number for the preparation of an experiment. 
It e.g. constitutes a coarse indicator for the importance of multiple scattering and thus the validity of the Born approximation.

Let $n_{i}(t)$ denote the number of neutrons hitting the sample during the period $\Delta t$.

$$
n_{i}(t)=A \cdot \int_{t}^{t+\Delta t} \mathrm{~d} t^{\prime} \phi_{i}\left(t^{\prime}\right)=A \cdot \Delta t \cdot \phi_{i}
$$

where the last equality holds for stationary conditions and $A$ is the geometrical cross section of the sample. The scattering rate can then be obtained from the cross section

$$
\frac{\mathrm{d}^{2} \sigma}{\mathrm{d} \Omega \mathrm{d} E_{f}}=\frac{k_{f}}{k_{i}} S(\vec{Q}, \omega)
$$

which gives the probability per unity of time and per unity of incident flux of scattering a neutron $\vec{k}_{i}$ into $\mathrm{d} \Omega \mathrm{d} E_{f}$. Therefore the number of scattered neutrons $n_{s}(t)$ over the period $\Delta t$ is equal to

$$
n_{s}(t)=\Delta t \cdot \Phi_{i} \cdot \int \mathrm{d}^{2} \sigma=\frac{k_{f}}{k_{i}} \cdot \Delta t \cdot \Phi_{i} \cdot \int \mathrm{d} \Omega \mathrm{d} E_{f} S(\vec{Q}, \omega)
$$

The rate is obtained as

$$
\begin{aligned}
\xi_{\text {scatt }}\left(\vec{k}_{i}\right) & =\frac{n_{s}}{n_{i}} \\
& =\frac{1}{A} \frac{k_{f}}{k_{i}} \int \mathrm{d} \Omega \mathrm{d} E_{f} S(\vec{Q}, \omega) \\
& =\frac{1}{A} \frac{\hbar}{m k_{i}} \int \mathrm{d}^{3} k_{f} S(\vec{Q}, \omega),
\end{aligned}
$$

where we have used

$$
\mathrm{d}^{3} k_{f}=k_{f}^{2} \mathrm{~d} k_{f} \mathrm{~d} \Omega=\frac{m k_{f}}{\hbar} \mathrm{d} \Omega \mathrm{d} E_{f}
$$

The last integration runs over all $k_{f}$ and the expression is valid in the Born approximation. The scattering rate is thus expressed as the ratio of two areas: the total scattering cross section and the total area of the sample illuminated by the beam.

Since the scattering function is the result expected from the experiment it is generally not know up front. An approximate knowledge is, however, sufficient for estimating the scattering rates, which in turn allow deducing the optimum experimental conditions concerning data statistics. This is why we want to introduce a simple approximative method for the estimation of the scattering rate.

We will see later that elastic scattering is the dominant contribution for the vast majority of experimental conditions (liquids are a noticeable exception). Elastic scattering is related to the correlations between particle positions that persist for $t \rightarrow \infty$ (see Section 8.9). An approximate way of describing these correlations consists in assuming that the positions of the scatterers are fixed in time. In this case, the expressions for the cross sections simplify significantly. The operator $\overrightarrow{\mathbf{R}}_{j}$ at $t=0$ acts on the wave functions like a simple multiplying number. In particular we get

$$
\left\langle\lambda_{i}\left|\mathrm{e}^{\mathrm{i} \vec{Q} \cdot\left(\vec{R}_{j}(t=0)-\vec{R}_{j^{\prime}}^{0}\right)}\right| \lambda_{i}\right\rangle \rightarrow \mathrm{e}^{\mathrm{i} \vec{Q} \cdot\left(\vec{R}_{j}-\vec{R}_{j^{\prime}}\right)}\left\langle\lambda_{i} \mid \lambda_{i}\right\rangle=\mathrm{e}^{\mathrm{i} \vec{Q} \cdot\left(\vec{R}_{j}-\vec{R}_{j^{\prime}}\right)} .
$$


We recall that

$$
\sum_{\lambda_{i}} p\left(\lambda_{i}\right)=1
$$

and that

$$
\frac{1}{2 \pi \hbar} \int_{-\infty}^{\infty} \mathrm{d} t \mathrm{e}^{-\mathrm{i} \omega t}=\delta(\hbar \omega)
$$

By limiting ourselves to a monatomic system these three relations allow us to reformulate the partial differential cross sections (6.20) and (6.21). We get

$$
\left(\frac{\mathrm{d} \sigma}{\mathrm{d} \Omega \mathrm{d} E_{f}}\right)_{\text {coh }}=\frac{\sigma_{\text {coh }}}{4 \pi} \frac{k_{f}}{k_{i}} \sum_{j, j^{\prime}=1}^{N} \mathrm{e}^{\mathrm{i} \vec{Q} \cdot\left(\vec{R}_{j}-\vec{R}_{j^{\prime}}\right)} \delta(\hbar \omega)
$$

and

$$
\left(\frac{\mathrm{d} \sigma}{\mathrm{d} \Omega \mathrm{d} E_{f}}\right)_{\text {inc }}=\frac{\sigma_{\text {inc }}}{4 \pi} \frac{k_{f}}{k_{i}} \sum_{j}^{N} \mathrm{e}^{\mathrm{i} \vec{Q} \cdot\left(\vec{R}_{j}-\vec{R}_{j^{\prime}}\right)} \delta(\hbar \omega)=N \frac{\sigma_{\text {inc }}}{4 \pi} \frac{k_{f}}{k_{i}} \delta(\hbar \omega) .
$$

Effectuating the trivial integral over the energy leads to the differential cross sections

$$
\begin{aligned}
\left(\frac{\mathrm{d} \sigma}{\mathrm{d} \Omega}\right)_{\text {coh }} & =\frac{\sigma_{\text {coh }}}{4 \pi} \sum_{j, j^{\prime}=1}^{N} \mathrm{e}^{\mathrm{i} \vec{Q} \cdot\left(\vec{R}_{j}-\vec{R}_{j^{\prime}}\right)} \\
& =\frac{\sigma_{\mathrm{coh}}}{4 \pi}\left|\sum_{j=1}^{N} \mathrm{e}^{\mathrm{i} \vec{Q} \cdot \vec{R}_{j}}\right|^{2}
\end{aligned}
$$

and

$$
\left(\frac{\mathrm{d} \sigma}{\mathrm{d} \Omega}\right)_{\mathrm{inc}}=N \frac{\sigma_{\mathrm{inc}}}{4 \pi} .
$$

Hence the coherent scattering is given by the static structure factor

$$
F_{\text {stat }}(\vec{Q}):=\left|\sum_{j=1}^{N} \mathrm{e}^{\mathrm{i} \vec{Q} \cdot \vec{R}_{j}}\right|^{2}
$$

For a monatomic crystal (see Section 9.8)

$$
F(\vec{Q}):=N \frac{(2 \pi)^{3}}{V_{\text {unit cell }}} \sum_{\vec{G}} \delta(\vec{Q}-\vec{G})
$$

$V_{\text {unit cell }}$ is the volume of the unit cell and $\vec{G}$ corresponds to a reciprocal lattice vector. 
Introducing the concept of a scattering length density

$$
\rho_{\mathrm{b}}(\vec{r})=\frac{1}{V} \sum_{j=1}^{N} \bar{b}_{j} \cdot \delta\left(\vec{r}-\vec{R}_{j}\right)
$$

we may express the coherent cross section in terms of the Fourier transform of $\rho_{\mathrm{b}}(\vec{r})$

$$
\left(\frac{\mathrm{d} \sigma}{\mathrm{d} \Omega}\right)_{\text {coh }}=\left|\sum_{j=1}^{N} \bar{b}_{j} \cdot \mathrm{e}^{\mathrm{i} \vec{Q} \cdot \vec{R}_{j}}\right|^{2}=\left|\int_{V} \mathrm{~d}^{3} r \rho_{\mathrm{b}}(\vec{r}) \cdot \mathrm{e}^{\mathrm{i} \vec{Q} \cdot \vec{r}}\right|^{2}
$$

This expression is often used in small angle scattering where atomic details do not matter and where the scattering length density for that reason becomes the more appropriate concept for describing the system.

In order to make the cross sections more intuitive we propose to estimate the scattering rate (see also Fig. 32) simply from the one-particle properties. If we ignore the structure factor then we have to limit our consideration from a formal point of view rigorously to incoherent scattering. In practice it is often possible to obtain good estimates even for coherent systems with the formula that we are going to develop. For this we simply have to replace the incoherent cross section $\sigma_{\text {inc }}$ by the total cross section $\sigma_{\text {tot }}{ }^{66}$ If we remain in the one-particle picture then the correlation volume can be condensed to the space occupied by a single scatterer and the neutron can be considered to collide with individual atoms.

Let our samples be characterised by

(1) the molar mass $m_{\mathrm{mol}}$,

(2) the mass density $\rho_{\mathrm{m}}$,

(3) the incoherent cross section $\sigma_{\text {inc. }}$.

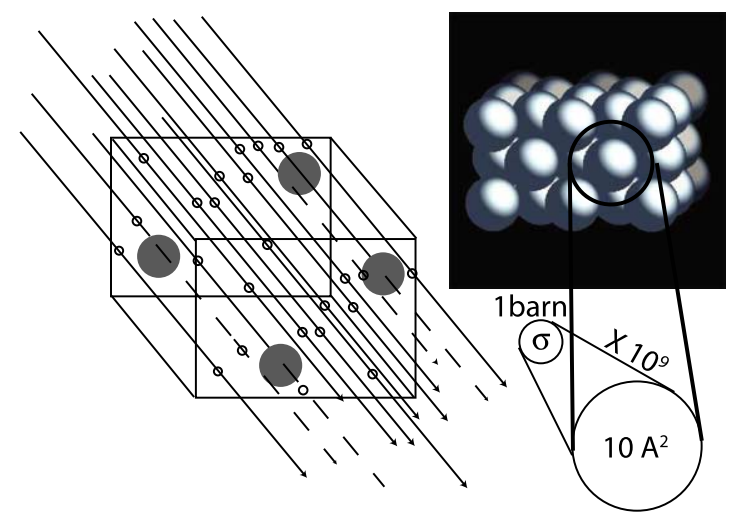

Fig. 32. Schematic illustration of individual cross sections. Each scatterer is indicated by a sphere. The section of that sphere is meant to represent the cross section. A neutron intercepted by this section is assumed to be scattered while the others will pass. The ratio between the scattering cross section and the section of a sphere that corresponds to the volume occupied by an individual atom is typically of the order of $10^{7}-10^{9}$. Hence it takes about 1 billion atomic layers in order to make a sample "opaque" to neutrons. This corresponds to a macroscopic dimension ranging from a fraction of a $\mathrm{mm}$ to a few $\mathrm{cm}$. (Colors are visible in the online version of the article; http://dx.doi.org/10.3233/JNR-140016.)

\footnotetext{
${ }^{66}$ Attention, this implies that the wave length is sufficiently small. When the wave length becomes very long elastic scattering in the form of Bragg peaks cannot be produced even in back scattering. This is called working below the Bragg threshold. A purely coherent sample will appear almost transparent below this threshold.
} 
The microscopic volume corresponding to an individual scatterer is given by

$$
V_{\text {ind }}=\frac{m_{\mathrm{mol}}}{N_{A} \cdot \rho_{\mathrm{m}}},
$$

where Avogadro's constant is $N_{A}=6.022 \cdot 10^{23}$. We can assume that $V_{\text {ind }}$ is cubic without hampering the result. The individual surface areas of this volume are given by

$$
a_{\text {ind }}=V_{\text {ind }}^{2 / 3} .
$$

The scattering rate $\xi_{\text {ind }}$ for a single target particle is determined according to expression (6.65) by the ratio between the cross section $\sigma_{\text {inc }}$ and the area $a_{\text {ind }}$ that the particle opposes to the neutron flux

$$
\xi_{\text {ind }}=\frac{\sigma_{\text {inc }}}{a_{\text {ind }}} .
$$

We would like to study the situation where a large number of scatterers are aligned along the direction of the incident neutron beam that we choose parallel to $\hat{z}$. If we assume that scattering is weak then we can neglect the fact that the incident flux decreases as a function of penetration (Born approximation). In order to obtain at certain scattering rate $\xi$ it is necessary to fill the surface $a_{\text {ind }}$ with

$$
n=\xi \frac{a_{\text {ind }}}{\sigma_{\text {inc }}}
$$

individual "tiles" each corresponding in area to the cross section $\sigma_{\text {inc }}$. This condition yields a penetration length

$$
l=n \cdot \sqrt{a_{\text {ind }}}=\xi \frac{\sqrt{a_{\text {ind }}} a_{\text {ind }}}{\sigma_{\text {inc }}}=\frac{V_{\text {ind }}}{\sigma_{\text {inc }}} .
$$

This is the mean free path of the neutron that we will meet again in a more general form in expression (11.34). In the context of multiple scattering the expression (6.83) is a very simple formula for the calculation of the appropriate thickness of the sample.

We will now treat two concrete examples that are particularly important for neutron scattering: water and vanadium.

(1) Water $\left(\mathrm{H}_{2} \mathrm{O}\right)$ is a universal solvent in chemistry and an indispensable component in biological matter. It is characterised by the parameters:

1. $m_{\mathrm{mol}}=16 \mathrm{~g}$,

2. $\rho_{m}=1 \mathrm{~g} / \mathrm{cm}^{3}$,

3. $\sigma_{\mathrm{inc}} \approx 165 \mathrm{barn}=165 \times 10^{-10} \mathrm{~nm}^{2}$,

4. $V_{\mathrm{H}_{2} \mathrm{O}}=3 \times 10^{-2} \mathrm{~nm}^{3}$,

5. $33 \mathrm{H}_{2} \mathrm{O}$ molecules in a $\mathrm{nm}^{3}$.

We obtain

$$
\frac{V_{\mathrm{H}_{2} \mathrm{O}}}{\sigma_{\text {inc }}}=1.875 \mathrm{~mm} \text {. }
$$

In order to get $10 \%$ of scattering and thus $90 \%$ of transmission the thickness should not exceed around $0.2 \mathrm{~mm}$ along the trajectory of the incident neutron. Water is one of the strongest scatterers. We see that even 
in this extreme case neutrons possess great penetration power. This penetration power constitutes one of the major advantages of neutron scattering. ${ }^{67}$

(2) Vanadium is as already mentioned a completely incoherent scatterer. It is very often used to calibrate spectrometers.

1. $m_{\mathrm{mol}}=51 \mathrm{~g}$,

2. $\rho_{m}=6.1 \mathrm{~g} / \mathrm{cm}^{3}$,

3. $\sigma_{\text {inc }}=5.08$ barn $=5.08 \times 10^{-10} \mathrm{~nm}^{2}$,

4. $V_{\mathrm{V}}=1.4 \times 10^{-2} \mathrm{~nm}^{3}$,

5. 71 Vanadium atoms in a $\mathrm{nm}^{3}$.

Hence we obtain

$$
\frac{V_{\mathrm{V}}}{\sigma_{\mathrm{inc}}}=28 \mathrm{~mm} .
$$

In order to get $10 \%$ of scattering we need a slice of vanadium with a thickness of about $2.8 \mathrm{~mm}$ along the trajectory. We would like to emphasise that the shape of the sample besides its dimensions matters a lot for multiple scattering (see [57]).

\subsection{Example: Production rate of ultra cold neutrons}

We would now like to illustrate the usefulness of the scattering function $S(\vec{Q}, \omega)$ with a concrete example. Doing so we wish to emphasise the fundamentally different character of the scattering function and the partial differential cross section. This difference may surprise given the simple relation that exists between the two quantities.

- The cross section gives the scattering rate in barns for a concrete experimental set-up. This rate can be calculated with the help of the scattering function.

- The scattering function can be determined under an infinite variety of experimental conditions. The only requirement for its transferability from one condition to another resides in the validity of the Born approximation.

The example chosen is the production of ultra cold neutrons (UCNs) starting from a cold or thermal beam of neutrons. UCNs have a great importance in fundamental physics and the improvement in the production rate is a problem of great relevance [27]. The quantity of interest is the total number of neutrons converted to ultra cold neutrons, i.e. to neutrons with an energy below $E_{\mathrm{u}}^{\max } \approx 300 \mathrm{neV}$, starting from a cold or thermal neutron beam. This threshold is chosen since neutrons below around $300 \mathrm{neV}$ no longer penetrate most materials and thus can be trapped easily, e.g. in order to measure their life time.

We set the initial energy of the neutrons to $E_{i}$. The cross section of the production of ultra cold neutrons - this means the number of neutrons produced per second and per unity of incident flux - is given by

$$
\begin{aligned}
\sigma_{\text {prod }}\left(E_{i}\right) & =\int_{0}^{E_{\mathrm{u}}^{\max }} \frac{k_{f}}{k_{i}} S(Q, \omega) \mathrm{d} E_{f} \mathrm{~d} \Omega \\
& =4 \pi \int_{0}^{E_{\mathrm{u}}^{\max }} \frac{k_{f}}{k_{i}} S(Q, \omega) \mathrm{d} E_{f},
\end{aligned}
$$

\footnotetext{
${ }^{67}$ Attention, the total cross section that we have used is the one for bound atoms. This is logical given that our starting point consisted in assuming that the atoms are fixed. If we work with a very short wave length or very light atoms like hydrogen then the static approximation is poor. It becomes necessary to take the fact into account that the cross sections decrease (see Section 3.8) in order to calculate the right sample thickness. Thanks to Bruno Dorner for raising my attention to this point.
} 
where we have taken into account that the production process covers the full solid angle of $4 \pi$ and that the scattering is isotropic in a liquid or in a powder.

With respect to the energy $E_{i}$ of the incident neutrons (a few meV) the energy $E_{\mathrm{u}}$ of the ultra cold neutrons (a few $\mathrm{neV}$ ) is extremely low. The scattering function - in addition convoluted with the resolution (see Section 11.6) only varies little over this energy range. Hence we can assume

$$
\begin{aligned}
& Q \approx k_{i}, \\
& \hbar \omega \approx \frac{\hbar^{2} k_{i}^{2}}{2 m} .
\end{aligned}
$$

The $Q$ and $\omega$ values that interest us correspond to the parabola $\hbar \omega=\hbar^{2} Q^{2} / 2 m$ of free neutrons. Thus

$$
\begin{aligned}
\sigma_{\text {prod }}\left(E_{0}\right) & =4 \pi \int_{0}^{E_{\mathrm{u}}^{\max }} \frac{k_{f}}{k_{i}} S\left(k_{i}, \frac{\hbar^{2} k_{i}^{2}}{2 m}\right) \mathrm{d} E \\
& =4 \pi \frac{1}{k_{i}} S\left(k_{i}, \frac{\hbar^{2} k_{i}^{2}}{2 m}\right) \int_{0}^{E_{\mathrm{u}}^{\max }} \frac{k_{f}^{2} \hbar^{2}}{m} \mathrm{~d} k_{f} \\
& =4 \pi \frac{1}{k_{i}} S\left(k_{i}, \frac{\hbar^{2} k_{i}^{2}}{2 m}\right) \frac{2}{3} k_{\mathrm{u}}^{\max } E_{\mathrm{u}}^{\max }
\end{aligned}
$$

This expression is the product of three factors. The first factor

$$
\frac{8 \pi}{3} k_{\mathrm{u}}^{\max } E_{\mathrm{u}}^{\max }
$$

measures the phase space volume of the final states. This volume is strongly dependent on the wave vector of the converted neutrons. Since the final energy is so low this volume is correspondingly small for ultra cold neutrons. The second factor

$$
\frac{1}{k_{i}}
$$

measures the incident flux. When calculating the absolute production rate the cross section will be multiplied with the spectrum of the incident flux and this factor will disappear. The last factor

$$
S\left(k_{i}, \frac{\hbar^{2} k_{i}^{2}}{2 m}\right)
$$

is the scattering function evaluated along the parabola of the free neutron. It gives the probability of producing an ultra cold neutron from a cold neutron of energy $E_{i}$. The scattering function can be extracted from any scattering experiment provided that the energy used exceeds $E_{i}$ and that the experiment satisfies the Born approximation criteria.

A very promising UCN converter is solid deuterium [27]. In Fig. 33 we show the scattering function $S(Q, \omega)$ determined from the partial differential cross section. The sample is a powder of solid $\mathrm{D}_{2}$ with a $33 \%$ content of para-deuterium. The incident energy is $17.4 \mathrm{meV}$. The total cross section is determined as $\sigma_{\mathrm{tot}}\left(E_{i}=17.4 \mathrm{meV}\right) \approx$ 23 barn. Naturally this value depends on the incident energy. The scattering function allows us to calculate the partial differential cross sections for any energy, including the ones necessary for the production of ultra cold neutrons. For example in order to calculate how many neutrons we can get from cold neutrons with an incident energy of $5 \mathrm{meV}$ it is necessary to know $S\left(Q=1.55 \AA^{-1}, \hbar \omega=5 \mathrm{meV}\right)$. This value is extracted from the 

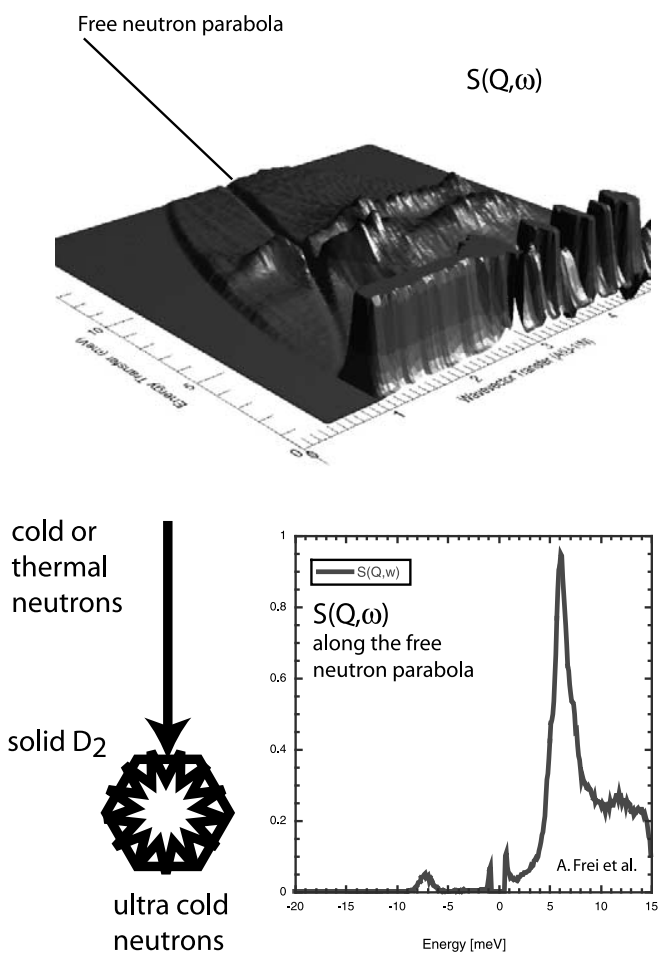

Fig. 33. $S(Q, \omega)$ for a sample of $D_{2}$ powder measured with a incident energy of $E_{i}=17.288 \mathrm{meV} \equiv k_{i}=2.888 \AA^{-1}$ at the IN4 instrument at ILL [27]. The deuterium content of para-deuterium is $33 \%$. We only present the side of the spectrum that corresponds to an energy loss of the neutron. The probability of a neutron loosing all its energy $\hbar \omega$ is proportional to the scattering function $S(Q, \omega)$ along the parabola of the free neutron. Starting from the measurement we can thus predict the scattering rate for all energies below $\sim 15 \mathrm{meV}$.

measured partial differential cross section by considering the transition of thermal neutrons from 17.4 to $12.4 \mathrm{meV}$ and with $\left|\vec{k}_{i}-\vec{k}_{f}\right|=1.55 \AA^{-1}$.

The situation changes when we look at the inverse process, i.e. the loss of ultra cold neutrons in an inelastic process. In this case the phase space of potential final states is very large. In order to limit the loss the scattering function should tend to zero on the energy gain side. This is the case at low temperature due to the principle of detailed balance (see Section 8.7).

\section{Partial summary}

We will now briefly summarise the formalism that we have developed so far.

The quantity characterising a scattering process is the differential cross section. It is defined by (1.7)

$$
\mathrm{d} \sigma:=\frac{\text { scattered flux into } \mathrm{d} \Omega}{\text { initial flux onto the sample }}=r^{2} \mathrm{~d} \Omega \frac{J_{\text {scattered }}(r, \theta, \phi, t)}{J_{\text {ini }}(t)} .
$$

For a static potential the scattering is elastic. The wave function that describes the scattered neutrons has to satisfy the stationary Schrödinger equation (3.3)

$$
\left[-\frac{\hbar^{2}}{2 m} \Delta+V(\vec{r})\right] u_{\vec{k}}(\vec{r})=E(k) u_{\vec{k}}(\vec{r}) .
$$


In the asymptotic regime it takes the form (3.4)

$$
u_{\vec{k}}(\vec{r}) \longrightarrow \frac{1}{\sqrt{V}}\left(\mathrm{e}^{\mathrm{i} \vec{k} \cdot \vec{r}}+f_{\vec{k}}(\theta, \phi) \frac{\mathrm{e}^{\mathrm{i} k r}}{r}\right), \quad r \rightarrow \infty,
$$

with $f_{\vec{k}}(\theta, \phi)$ the scattering amplitude. This amplitude is directly linked to the cross section via (3.27)

$$
\frac{\mathrm{d} \sigma}{\mathrm{d} \Omega}=r^{2} \frac{J_{\text {scattered }}(r, \theta, \phi, t)}{J_{\text {ini }}(t)}=|f(\theta, \phi)|^{2} .
$$

For static and isotropic potentials the scattering amplitude is expressed in terms of spherical harmonics (or partial waves) as (3.78)

$$
f(\theta)=\frac{1}{2 \mathrm{i} k} \sum_{l=0}^{\infty} \sqrt{4 \pi(2 l+1)}\left(\mathrm{e}^{2 \mathrm{i} \delta_{l}}-1\right) Y_{l 0}(\theta) .
$$

For wave lengths that are much smaller than the extension of the potential it is sufficient to keep the s-type partial wave. The scattering amplitude can then be expressed as a function of the phase shift of this wave (3.105)

$$
f(\theta)=\frac{\mathrm{e}^{2 \mathrm{i} \delta_{0}}-1}{2 \mathrm{i} k}=\frac{1}{k} \mathrm{e}^{\mathrm{i} \delta_{0}} \sin \delta_{0} .
$$

The scattering length is defined as the limit of the ratio between the phase shift and the wave vector (3.98)

$$
b=-\lim _{k \rightarrow 0} f(\theta)=-\lim _{k \rightarrow 0} \frac{\delta_{0}(k)}{k} .
$$

For a hard sphere this is identical to the radius (3.115)

$$
b=r_{0} .
$$

In general the scattering length can take both positive and negative values. Absorption away from resonance is taken into account by a complex scattering length.

In order to allow for a perturbation treatment the Schrödinger equation that defines the wave function of the scattering process is transformed into its integral form (3.159)

$$
u_{\vec{k}}(\vec{r})=u_{\vec{k}}^{0}(\vec{r})+\int \mathrm{d}^{3} r^{\prime} G_{0}\left(\vec{r}, \vec{r}^{\prime}\right) V\left(\vec{r}^{\prime}\right) u_{\vec{k}}\left(\vec{r}^{\prime}\right),
$$

with the Green function (3.160)

$$
G_{0}\left(\vec{r}, \vec{r}^{\prime}\right)=-\frac{1}{4 \pi} \cdot \frac{2 m}{\hbar^{2}} \cdot \frac{\mathrm{e}^{\left(\mathrm{i} k \cdot\left|\vec{r}-\vec{r}^{\prime}\right|\right)}}{\left|\vec{r}-\vec{r}^{\prime}\right|} .
$$

Replacing $u_{\vec{k}}(\vec{r})$ by the non-perturbed wave $u_{\vec{k}}^{0}(\vec{r})$ in the integral

$$
u_{\vec{k}}(\vec{r})=u_{\vec{k}}^{0}(\vec{r})-\frac{1}{4 \pi} \frac{2 m}{\hbar^{2}} \int \mathrm{d}^{3} r^{\prime} \frac{\exp \left(\mathrm{i} k \cdot\left|\vec{r}-\vec{r}^{\prime}\right|\right)}{\left|\vec{r}-\vec{r}^{\prime}\right|} V\left(\vec{r}^{\prime}\right) u_{\vec{k}}^{0}\left(\vec{r}^{\prime}\right)
$$


is equivalent to working in the Born approximation. In this approximation the scattering amplitude can be calculated as the Fourier transform of the potential (3.188)

$$
f_{\vec{Q}^{(}}(\Omega)=-\frac{1}{2 \pi} \frac{m}{\hbar^{2}} \int \mathrm{d}^{3} r^{\prime} \mathrm{e}^{\mathrm{i} \vec{Q} \cdot \vec{r}^{\prime}} V\left(\vec{r}^{\prime}\right) .
$$

Composite samples with internal dynamics allow for inelastic scattering. This scattering is accompanied by transitions within the sample states. Fermi's golden rule (5.54)

$$
w_{\vec{k}_{i} \rightarrow \vec{k}_{f}, \lambda_{i} \rightarrow \lambda_{f}}=\frac{2 \pi}{\hbar}\left|\left\langle\lambda, \vec{k}_{f}|V| \lambda_{i}, \vec{k}_{i}\right\rangle\right|^{2} \rho\left(E_{f}\right),
$$

establishes a link between these transitions and the partial differential cross section. In the Born approximation this link is given by (5.62)

$$
\frac{\mathrm{d}^{2} \sigma_{\vec{k}_{i} \rightarrow \vec{k}_{f}}}{\mathrm{~d} \Omega \mathrm{d} E_{f}}=\frac{k_{f}}{k_{i}} \frac{(2 \pi)^{4} m^{2}}{\hbar^{4}} \sum_{\lambda_{i}, \lambda_{f}} p\left(\lambda_{i}\right)\left|\left\langle\lambda_{f}, \vec{k}_{f}|V| \lambda_{i}, \vec{k}_{i}\right\rangle\right|^{2} \delta\left(\left(E_{f}-E_{i}\right)-\left(E_{\lambda_{i}}-E_{\lambda_{f}}\right)\right) .
$$

This expression is called the master equation of scattering. The interaction potential between the probe and the target is in general a sum of individual contributions. In that case the master equation becomes (5.91)

$$
\frac{\mathrm{d} \sigma_{\vec{k}_{i} \rightarrow \vec{k}_{f}}}{\mathrm{~d} \Omega \mathrm{d} E_{f}}=\frac{k_{f}}{k_{i}} \frac{m^{2}}{(2 \pi)^{2} \hbar^{4}} \sum_{\lambda_{i}, \lambda_{f}} p\left(\lambda_{i}\right)\left|\sum_{j=1}^{N} V_{j}(\vec{Q})\left\langle\lambda_{f}\left|\mathrm{e}^{\mathrm{i} \vec{Q} \cdot \vec{R}_{j}}\right| \lambda_{i}\right\rangle\right|^{2} \delta\left(\left(E_{f}-E_{i}\right)-\left(E_{\lambda_{i}}-E_{\lambda_{f}}\right)\right),
$$

with the scattering form factors defined in terms of their Fourier transforms (5.90)

$$
V_{j}(\vec{Q})=\int \mathrm{d}^{3} r_{j} \mathrm{e}^{\mathrm{i} \vec{Q} \cdot \vec{r}_{j}} V_{j}\left(\vec{r}_{j}\right)
$$

For nuclear scattering the adapted potential is called the Fermi pseudo-potential (5.94)

$$
V_{\text {eff }}(r) \psi(r)=\frac{2 \pi \hbar^{2}}{m} b_{j} \delta(\vec{r})
$$

The form factor then reduces to a simple constant

$$
V_{j}(\vec{Q})=\frac{2 \pi \hbar^{2}}{m} b_{j} .
$$

The sum over the final states in the master equation can be eliminated introducing a Fourier transform in time. For the partial differential cross section we find (5.118)

$$
\frac{\mathrm{d}^{2} \sigma_{\vec{k}_{i} \rightarrow \vec{k}_{f}}}{\mathrm{~d} \Omega \mathrm{d} E_{f}}=\frac{k_{f}}{k_{i}} S(\vec{Q}, \omega)
$$

with (5.119)

$$
S(\vec{Q}, \omega)=\frac{1}{2 \pi \hbar} \sum_{\lambda_{i}} p\left(\lambda_{i}\right) \sum_{j, j^{\prime}=1}^{N} b_{j} b_{j^{\prime}}^{*} \int_{-\infty}^{\infty} \mathrm{d} t\left\langle\lambda_{i}\left|\mathrm{e}^{-\mathrm{i} \vec{Q} \cdot \vec{R}_{j^{\prime}}^{0}} \mathrm{e}^{\mathrm{i} \vec{Q} \cdot \vec{R}_{j}(t)}\right| \lambda_{i}\right\rangle \mathrm{e}^{-\mathrm{i} \omega t} .
$$


$S(\vec{Q}, \omega)$ is called the scattering function.

The nuclear scattering of neutrons depends on the composition of isotopes and spin states of the nucleus. It is necessary to distinguish between the coherent scattering and the incoherent scattering. The coherent scattering depends on the average scattering length of a scatterer (6.16)

$$
\left(\frac{\mathrm{d}^{2} \sigma}{\mathrm{d} \Omega \mathrm{d} E_{f}}\right)_{\mathrm{coh}}=\frac{k_{f}}{k_{i}} \frac{1}{2 \pi \hbar} \sum_{\lambda_{i}} p\left(\lambda_{i}\right) \sum_{j, j^{\prime}=1}^{N} \overline{b_{j} b_{j^{\prime}}^{*}} \int_{-\infty}^{\infty} \mathrm{d} t\left\langle\lambda_{i}\left|\mathrm{e}^{-\mathrm{i} \vec{Q} \cdot \vec{R}_{j^{\prime}}^{0}} \mathrm{e}^{\mathrm{i} \vec{Q} \cdot \vec{R}_{j}(t)}\right| \lambda_{i}\right\rangle \mathrm{e}^{-\mathrm{i} \omega t} .
$$

The incoherent scattering is determined by the deviation from the average of the scattering length (6.17)

$$
\left(\frac{\mathrm{d}^{2} \sigma}{\mathrm{d} \Omega \mathrm{d} E_{f}}\right)_{\text {inc }}=\frac{k_{f}}{k_{i}} \frac{1}{2 \pi \hbar} \sum_{\lambda_{i}} p\left(\lambda_{i}\right) \sum_{j}^{N}\left(\overline{b_{j}^{2}}-\left(\bar{b}_{j}\right)^{2}\right) \int_{-\infty}^{\infty} \mathrm{d} t\left\langle\lambda_{i}\left|\mathrm{e}^{-\mathrm{i} \vec{Q} \cdot \vec{R}_{j}^{0}} \mathrm{e}^{\mathrm{i} \vec{Q} \cdot \vec{R}_{j}(t)}\right| \lambda_{i}\right\rangle \mathrm{e}^{-\mathrm{i} \omega t}
$$

\section{Correlation functions}

During the discussion of coherent and incoherent scattering we had already hinted at an important connection between the scattering function and quantum statistical correlation functions. We will in the following establish a precise mathematical formulation of this connection. In our derivation we will closely follow the route proposed by Van Hove [68].

Our starting point is expression (5.119) for the scattering function $S(\vec{Q}, \omega)$

$$
S(\vec{Q}, \omega)=\frac{1}{2 \pi \hbar} \sum_{\lambda_{i}} p\left(\lambda_{i}\right) \sum_{j, j^{\prime}=1}^{N} V_{j}(\vec{Q}) V_{j^{\prime}}^{*}(\vec{Q}) \int_{-\infty}^{\infty} \mathrm{d} t\left\langle\lambda_{i}\left|\mathrm{e}^{-\mathrm{i} \vec{Q} \cdot \vec{R}_{j^{\prime}}^{0}} \mathrm{e}^{\mathrm{i} \vec{Q} \cdot \vec{R}_{j}(t)}\right| \lambda_{i}\right\rangle \mathrm{e}^{-\mathrm{i} \omega t} .
$$

We will lighten the notation by exploiting the fact that the quantum statistical average of a physical quantity $A$ is defined by

$$
\langle A\rangle=\sum p_{n}\left\langle\psi_{n}|\mathbf{A}| \psi_{n}\right\rangle
$$

As the states $\left|\lambda_{i}\right\rangle$ constitute a complete basis set of the target system we may write

$$
\sum_{\lambda_{i}} p\left(\lambda_{i}\right)\left\langle\lambda_{i}\left|\mathrm{e}^{-\mathrm{i} \vec{Q} \cdot \vec{R}_{j^{\prime}}^{0}} \mathrm{e}^{\mathrm{i} \vec{Q} \cdot \vec{R}_{j}(t)}\right| \lambda_{i}\right\rangle=\left\langle\mathrm{e}^{-\mathrm{i} \vec{Q} \cdot \vec{R}_{j^{\prime}}^{0}} \mathrm{e}^{\mathrm{i} \vec{Q} \cdot \vec{R}_{j}(t)}\right\rangle
$$

Hence the scattering function becomes

$$
S(\vec{Q}, \omega)=\sum_{j, j^{\prime}}\left(V_{j}(\vec{Q}) V_{j^{\prime}}^{*}(\vec{Q})\right) S_{j, j^{\prime}}(\vec{Q}, \omega)
$$

with

$$
S_{j, j^{\prime}}(\vec{Q}, \omega)=\frac{1}{2 \pi \hbar} \sum_{j, j^{\prime}=1}^{N} \int_{-\infty}^{\infty} \mathrm{d} t\left\langle\mathrm{e}^{-\mathrm{i} \vec{Q} \cdot \vec{R}_{j^{\prime}}^{0}} \mathrm{e}^{\mathrm{i} \vec{Q} \cdot \vec{R}_{j}(t)}\right\rangle \mathrm{e}^{-\mathrm{i} \omega t}
$$


The total scattering function is, therefore, a superposition of functions that involve pairs $\left(j, j^{\prime}\right)$ of scatterers. In general the number of distinct interaction potentials $V_{j}$ is quite limited. In the case of a monatomic system we are even dealing with only a single type of scatterer. This fact brings us to a natural classification scheme of the terms $\left(j, j^{\prime}\right)$. We distinguish between the different types of scatterers using the index $\kappa$. Each of the $N_{\kappa}$ scatterers belonging to the same type $\kappa$ are labeled $j_{\kappa}$. The sum over the scatterers in this way becomes

$$
\sum_{j}=\sum_{\kappa} \sum_{j_{\kappa}}:=\sum_{\kappa} \sum_{j \in\left\{j_{\kappa}\right\}} .
$$

We now reformulate the scattering function (8.4) as

$$
S(\vec{Q}, \omega)=\sum_{\kappa, \kappa^{\prime}}\left(V_{\kappa}(\vec{Q}) V_{\kappa^{\prime}}^{*}(\vec{Q})\right) S_{\kappa, \kappa^{\prime}}(\vec{Q}, \omega)
$$

with the partial scattering functions defined as

$$
S_{\kappa, \kappa^{\prime}}(\vec{Q}, \omega)=\frac{1}{2 \pi \hbar} \sum_{j \in\left\{j_{\kappa}\right\}, j^{\prime} \in\left\{j_{\kappa^{\prime}}\right\}} \int_{-\infty}^{\infty} \mathrm{d} t\left\langle\mathrm{e}^{-\mathrm{i} \vec{Q} \cdot \vec{R}_{j^{\prime}}^{0}} \mathrm{e}^{\mathrm{i} \vec{Q} \cdot \vec{R}_{j}(t)}\right\rangle \mathrm{e}^{-\mathrm{i} \omega t}
$$

In the same way we define the the partial self scattering functions

$$
S_{\kappa}^{s}(\vec{Q}, \omega)=\frac{1}{2 \pi \hbar} \sum_{j \in\left\{j_{\kappa}\right\}} \int_{-\infty}^{\infty} \mathrm{d} t\left\langle\mathrm{e}^{-\mathrm{i} \vec{Q} \cdot \vec{R}_{j}^{0}} \mathrm{e}^{\mathrm{i} \vec{Q} \cdot \vec{R}_{j}(t)}\right\rangle \mathrm{e}^{-\mathrm{i} \omega t}
$$

The later functions are particularly useful in the context of incoherent scattering.

We could simplify our notation immensely if we restricted ourselves to monatomic systems, which for pedagogical reasons could be an advantage. We nevertheless have to refrain from doing so as real systems are rarely monatomic. In order for the developed formula to be widely applicable we need to take into account the diversity of scatterers.

\subsection{The autocorrelation function of the atomic density $G(\vec{r}, t)$}

We will show that the partial scattering functions $S_{\kappa, \kappa^{\prime}}(\vec{Q}, \omega)$ can be expressed in terms of two-point autocorrelation functions.

Ignoring the spin the scattering particle is fully characterised by its position operator in space $\overrightarrow{\mathbf{R}}_{j}$. It is, therefore, natural to have a closer look at correlation functions based on these operators. The autocorrelation function ${ }^{68}$ of a

\footnotetext{
${ }^{68}$ In statistics the autocorrelation of a process $X_{t}$, which may be discrete or continuous in time with $t$ an integer or real number, respectively, is defined as the normalised correlation function of the fluctuation, i.e. the deviation from average

$$
X_{t}-\langle X\rangle
$$

taken at time $t$ with the same deviation taken at time $t^{\prime}$. Formally

$$
R(t):=\frac{\left\langle\left(X_{t^{\prime}}-\langle X\rangle\right)\left(X_{t^{\prime}+t}-\langle X\rangle\right)\right\rangle}{\left\langle\left(X_{t^{\prime}}-\langle X\rangle\right)^{2}\right\rangle} .
$$

The autocorrelation function, therefore, gives a measure of the similarity of the process as a laps of time and thus is ideally suited to detect patterns of periodicity in the process $X(t)$. Without the normalisation to the variance

$$
\sigma^{2}=\left\langle\left(X_{t^{\prime}}-\langle X\rangle\right)^{2}\right\rangle
$$
}


physical quantity $A$ is defined as the crossed correlation of $A$ with itself. Formally this function is equivalent to a convolution in time and space of $A^{*}(-\vec{r},-t)$ with $A(\vec{r}, t)$

$$
A \star A=A(\vec{r}, t) * A^{*}(-\vec{r},-t)
$$

or written in explicit form

$$
\Gamma_{A, A}(\vec{r}, t)=A \star A=\lim _{T \rightarrow \infty} \frac{1}{2 T} \frac{1}{V} \int_{-T}^{T} \mathrm{~d} t^{\prime} \int \mathrm{d}^{3} r^{\prime}\left\langle A\left(\vec{r}^{\prime}, t^{\prime}\right) A^{*}\left(\vec{r}^{\prime}-\vec{r}, t^{\prime}-t\right)\right\rangle,
$$

where we assume that the system is ergodic in order to assure that the $\operatorname{limit}_{T \rightarrow \infty}$ exists. The autocorrelation function is a powerful tool to detect regularities in signals such as its fundamental oscillations.

For a stationary system the origin of time can be chosen arbitrarily. Hence the expression

$$
\left\langle A\left(\vec{r}^{\prime}, t^{\prime}\right) A^{*}\left(\vec{r}^{\prime}-\vec{r}, t^{\prime}-t\right)\right\rangle
$$

does not depend on $t^{\prime}$. As a consequence the average over $t^{\prime}$ is trivial and the autocorrelation function simplifies to

$$
\Gamma_{A, A}(\vec{r}, t)=\frac{1}{V} \int \mathrm{d}^{3} r^{\prime}\left\langle A\left(\vec{r}^{\prime}, t=0\right) A^{*}\left(\vec{r}^{\prime}-\vec{r}, t\right)\right\rangle=\frac{1}{V} \int \mathrm{d}^{3} r^{\prime}\left\langle A\left(\vec{r}^{\prime}, t\right) A^{*}\left(\vec{r}^{\prime}-\vec{r}, t=0\right)\right\rangle .
$$

The exact physical quantity that determines the correlations in the scattering function remain to be identified. We start with the case of a single particle. Following the postulates of quantum mechanics the probability density of a particle at position $\vec{R}$ at time $t$ in the quantum state $|\lambda\rangle$ is given by

$$
\begin{aligned}
\psi_{\lambda}^{*}(\vec{R}(t)) \psi_{\lambda}(\vec{R}(t)) & =\int \mathrm{d}^{3} r \int \mathrm{d}^{3} r^{\prime} \delta(\vec{r}-\vec{R}(t)) \delta\left(\vec{r}^{\prime}-\vec{R}(t)\right) \psi_{\lambda}^{*}(\vec{r}) \psi_{\lambda}\left(\vec{r}^{\prime}\right) \\
& =\int \mathrm{d}^{3} r \delta(\vec{r}-\vec{R}(t)) \psi_{\lambda}^{*}(\vec{r}) \psi_{\lambda}(\vec{r}) \\
& =\langle\lambda|\hat{\rho}(\vec{r}, t)| \lambda\rangle
\end{aligned}
$$

one usually uses the term auto-covariance. Two statistical variables are considered uncorrelated if

$$
\langle X \cdot Y\rangle=\langle X\rangle\langle Y\rangle .
$$

This implies that correlation functions are strictly zero if and only if they are formulated in terms of deviations from mean values, like this is the case for expression (8.10). One should be aware that the van Hove type correlation functions used in the theory of scattering do in general not satisfy this criterium.

The notion of statistical independence, which is directly connected to the notion of correlation of two random variables, is not always intuitive. The reader is invited to consult the book by Konstantin Protassov [47] on this subject. The reader may particularly enjoy the telling example of the 'king of hearts' that we will briefly outline here. In a deck of 52 cards the event 'king' and the event 'heart' are statistically independent. Formally this independence is expressed by the fact that the probability of drawing a 'king of hearts' is the product of the probability of drawing a 'king' multiplied by the probability of drawing the colour 'heart'.

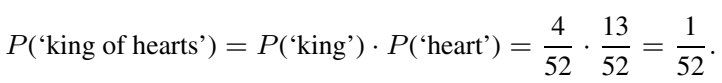

This relation is violated if we add a joker to the game

$$
P(\text { 'king of hearts' })=\frac{1}{53}>P\left(\text { 'king' }^{\prime}\right) \cdot P(\text { 'heart' })=\frac{4}{53} \cdot \frac{13}{53}=\frac{52}{53} \frac{1}{53} .
$$

The joker, therefore, modifies the statistical independence of two events in which he has a priori no part. This correlation may be explained by the fact that when drawing cards we eliminate immediately the possibility that we are dealing with a joker from the moment we know that the card has a colour. This exclusion raises the probability of having drawn a king. 
with

$$
\hat{\rho}(\vec{r}, t)=\delta(\vec{r}-\vec{R}(t)) .
$$

$\hat{\rho}(\vec{r}, t)$ is called the number density operator. It is not to be confused with the statistical density operator (see Section 5.6). The matrix elements of $\hat{\rho}(\vec{r}, t)$ give the probability of the particle to be found in the volume element $\mathrm{d}^{3} r$ around $\vec{r}$.

This concept can be generalised to a system with several particles by introducing the operator

$$
\hat{\rho}_{\kappa}(\vec{r}, t)=\sum_{j \in\left\{j_{\kappa}\right\}} \delta\left(\vec{r}-\vec{R}_{j}(t)\right) .
$$

$\left\{j_{\kappa}\right\}$ denotes the ensemble of indices that characterise scatterers of type $\kappa$. The operator $\hat{\rho}_{\kappa}(\vec{r}, t)$ extracts the probability of finding a scatterer of type $\kappa$ in the volume element $\mathrm{d}^{3} r$ around $\vec{r}$.

The spatial Fourier transform of $\hat{\rho}_{\kappa}(\vec{r}, t)$ is given by

$$
\hat{\rho}_{\kappa}(\vec{Q}, t)=\int_{V} \mathrm{~d}^{3} r \exp (-\mathrm{i} \vec{Q} \cdot \vec{r}) \hat{\rho}_{\kappa}(\vec{r}, t)=\sum_{j \in\left\{j_{\kappa}\right\}} \exp \left(-\mathrm{i} \vec{Q} \cdot \vec{R}_{j}(t)\right) .
$$

This is exactly the type of expression that we find in the expectation values that constitute the scattering function (see (8.8)).

We can now define the correlation functions of the number density operator

$$
\begin{aligned}
G_{\kappa, \kappa^{\prime}}(\vec{r}, t) & =\int \mathrm{d}^{3} \vec{r}^{\prime}\left\langle\hat{\rho}_{\kappa}\left(\vec{r}^{\prime}-\vec{r}\right) \hat{\rho}_{\kappa^{\prime}}\left(\vec{r}^{\prime}, t\right)\right\rangle \\
& =\sum_{j \in\left\{j_{\kappa}\right\}, j^{\prime} \in\left\{j_{\kappa^{\prime}}\right\}} \int \mathrm{d}^{3} \vec{r}^{\prime}\left\langle\delta\left(\vec{r}^{\prime}-\vec{r}-\vec{R}_{j}(t=0)\right) \delta\left(\vec{r}^{\prime}-\vec{R}_{j^{\prime}}(t)\right)\right\rangle .
\end{aligned}
$$

The function $G_{\kappa, \kappa^{\prime}}(\vec{r}, t)$ relates the probability of finding a scatterer of type $\kappa$ at time $t=0$ at $\vec{r}^{\prime}-\vec{r}$ to the presence of a scatterer of type $\kappa^{\prime}$ at time $t$ at $\vec{r}^{\prime}$.

Before establishing the link between the correlation functions and the scattering functions some supplementary concepts have to be introduced

- It will be useful to define a function that only takes the correlation of a scatterer with itself into account

$$
G_{\kappa}^{s}(\vec{r}, t):=\sum_{j \in\left\{j_{\kappa}\right\}} \int \mathrm{d}^{3} \vec{r}^{\prime}\left\langle\delta\left(\vec{r}^{\prime}-\vec{r}-\vec{R}_{j}(t=0)\right) \delta\left(\vec{r}^{\prime}-\vec{R}_{j}(t)\right)\right\rangle .
$$

$G_{\kappa}^{s}(\vec{r}, t)$ is called the partial self pair correlation function. It is a function that relates the probability of finding a scatterer of type $\kappa$ at time $t=0$ at $\vec{r}^{\prime}-\vec{r}$ to that of finding the same scatterer at $t$ at $\vec{r}^{\prime}$.

- When the number density operator $\hat{\rho}$ was introduced we have without explicitly mentioning it used the Heisenberg representation for the position operators $[10,37]$. The Heisenberg operators $\overrightarrow{\mathbf{R}}_{j}(t)$ are particularly welladapted when describing correlation functions in space and time. In the Heisenberg representation the system is described by stationary wave functions and it is the operators that, in the same way as the classical trajectory, trace the evolution of particles in time.

- We are not obliged to place the operator $\overrightarrow{\mathbf{R}}_{j}(t=0)$ at the left of $\overrightarrow{\mathbf{R}}_{j^{\prime}}(t)$ when defining $G(\vec{r}, t)$. This choice can, therefore, be considered arbitrary. The two options are related by

$$
G(\vec{r}, t)=G(-\vec{r},-t),
$$

which can be shown using basic quantum mechanical calculus. 
- One might be astonished of the complicated form that take the correlation functions (8.19) and (8.20) when it is well-known that

$$
\int \mathrm{d}^{3} \vec{r}^{\prime} \delta\left(\vec{r}^{\prime}-\vec{r}\right) \delta\left(\vec{r}^{\prime}\right)=\delta(\vec{r})
$$

It is the operator character of $\mathbf{R}_{j}(t)$ that prevents us from using this relation when carrying out the integration in the expressions (8.19) and (8.20). The two $\delta$ functions will in general not commute with each other at different times. This is easily seen when looking at their Fourier transforms (8.18). If we remove this constraint imposed by quantum mechanics we obtain the classical correlation functions

$$
\begin{aligned}
& { }^{\mathrm{cl}} G_{\kappa, \kappa^{\prime}}(\vec{r}, t)=\sum_{j \in\left\{j_{\kappa}\right\}, j^{\prime} \in\left\{j_{\kappa^{\prime}}\right\}}\left\langle\delta\left(\vec{r}+\vec{R}_{j}(t=0)-\vec{R}_{j^{\prime}}(t)\right)\right\rangle, \\
& { }^{\mathrm{cl}} G_{\kappa}^{S}(\vec{r}, t)=\sum_{j \in\left\{j_{\kappa}\right\}}\left\langle\delta\left(\vec{r}+\vec{R}_{j}(t=0)-\vec{R}_{j}(t)\right)\right\rangle .
\end{aligned}
$$

The interpretation of the classical correlation functions is considerably simpler. Apart from a factor that takes the number of scatterers of type $\kappa$ into account this function simply states the probability of finding at time $t$ a scatterer of type $\kappa$ at $\vec{r}$ given that a scatterer of type $\kappa^{\prime}$ was at the origin at time $t=0$.

8.2. Connection between $S_{\kappa, \kappa^{\prime}}(\vec{Q}, \omega)$ and $G_{\kappa, \kappa^{\prime}}(\vec{r}, t)$

In the same way as we had expressed $\delta\left(E_{i}-E_{f}-\hbar \omega\right)$ as an integral over time $\hat{\rho}(\vec{r}, t)$ can be expressed as an integral over $\vec{Q}$, i.e. in terms of its Fourier transforms

$$
\delta\left(\vec{r}^{\prime}-\vec{r}-\vec{R}_{j}(t)\right)=\frac{1}{(2 \pi)^{3}} \int \mathrm{d}^{3} Q \exp \left(\mathrm{i} \vec{Q} \cdot\left(\vec{r}^{\prime}-\vec{r}-\vec{R}_{j}(t)\right)\right) .
$$

This allows us to reformulate the correlation function $G(\vec{r}, t)$ in the following way

$$
\begin{aligned}
G_{\kappa, \kappa^{\prime}}(\vec{r}, t)= & \int \mathrm{d}^{3} r^{\prime}\left\langle\hat{\rho}_{\kappa}\left(\vec{r}^{\prime}-\vec{r}\right) \hat{\rho}_{\kappa^{\prime}}\left(\vec{r}^{\prime}, t\right)\right\rangle \\
= & \sum_{j \in\left\{j_{\kappa}\right\}, j^{\prime} \in\left\{j_{\kappa^{\prime}}\right\}} \int \mathrm{d}^{3} r^{\prime}\left\langle\delta\left(\vec{r}^{\prime}-\vec{r}-\vec{R}_{j}\right) \delta\left(\vec{r}^{\prime}-\vec{R}_{j^{\prime}}(t)\right)\right\rangle \\
= & \sum_{j \in\left\{j_{\kappa}\right\}, j^{\prime} \in\left\{j_{\kappa^{\prime}}\right\}} \int \mathrm{d}^{3} r^{\prime}\left\langle\frac{1}{(2 \pi)^{3}} \int \mathrm{d}^{3} Q \exp \left(\mathrm{i} \vec{Q} \cdot\left(\vec{r}^{\prime}-\vec{r}-\vec{R}_{j}\right)\right) \delta\left(\vec{r}^{\prime}-\vec{R}_{j^{\prime}}(t)\right)\right\rangle \\
= & \frac{1}{(2 \pi)^{3}} \int \mathrm{d}^{3} Q \exp (-\mathrm{i} \vec{Q} \cdot \vec{r}) \\
& \times \sum_{j \in\left\{j_{\kappa}\right\}, j^{\prime} \in\left\{j_{\kappa^{\prime}}\right\}} \int \mathrm{d}^{3} r^{\prime}\left\langle\exp \left(\mathrm{i} \vec{Q} \cdot \vec{r}^{\prime}-\mathrm{i} \vec{Q} \cdot \vec{R}_{j}\right) \delta\left(\vec{r}^{\prime}-\vec{R}_{j^{\prime}}(t)\right)\right\rangle \\
= & \frac{1}{(2 \pi)^{3}} \int \mathrm{d}^{3} Q \exp (-\mathrm{i} \vec{Q} \cdot \vec{r}) \\
& \times \sum_{j \in\left\{j_{\kappa}\right\}, j^{\prime} \in\left\{j_{\kappa^{\prime}}\right\}}\left\langle\exp \left(-\mathrm{i} \vec{Q} \cdot \vec{R}_{j}\right) \int \mathrm{d}^{3} r^{\prime} \exp \left(\mathrm{i} \vec{Q} \cdot \vec{r}^{\prime}\right) \delta\left(\vec{r}^{\prime}-\vec{R}_{j^{\prime}}(t)\right)\right\rangle \\
= & \frac{1}{(2 \pi)^{3}} \int \mathrm{d}^{3} Q \exp (-\mathrm{i} \vec{Q} \cdot \vec{r}) \sum \sum_{j \in\left\{j_{\kappa}\right\}, j^{\prime} \in\left\{j_{\kappa^{\prime}}\right\}}\left\langle\exp \left(-\mathrm{i} \vec{Q} \cdot \vec{R}_{j}\right) \exp \left(\mathrm{i} \vec{Q} \cdot \vec{R}_{j^{\prime}}(t)\right)\right\rangle .
\end{aligned}
$$


We have been very careful in keeping the correct time ordering of expressions involving $\overrightarrow{\mathbf{R}}_{j}(t)$ for the reason that they do not commute at different times $t$ as stated earlier.

Comparing the expression found for $G(\vec{r}, t)$ with the expression (8.8) for $S_{\kappa, \kappa^{\prime}}(\vec{Q}, \omega)$ and using the properties of Fourier transforms, we arrive at

$$
G_{\kappa, \kappa^{\prime}}(\vec{r}, t)=\frac{\hbar}{(2 \pi)^{3}} \int_{V_{Q}} \mathrm{~d}^{3} Q \int_{-\infty}^{\infty} \mathrm{d} \omega \mathrm{e}^{-\mathrm{i}(\vec{Q} \cdot \vec{r}-\omega t)} S_{\kappa, \kappa^{\prime}}(\vec{Q}, \omega) .
$$

Thus $G_{\kappa, \kappa^{\prime}}(\vec{r}, t)$ turns out to be the double Fourier transform of $S_{\kappa, \kappa^{\prime}}(\vec{Q}, \omega)$.

Using the inverse Fourier transform the roles of the scattering function $S_{\kappa, \kappa^{\prime}}(\vec{Q}, \omega)$ and of the correlation function $G_{\kappa, \kappa^{\prime}}(\vec{r}, t)$ can be reversed

$$
S_{\kappa, \kappa^{\prime}}(\vec{Q}, \omega)=\frac{1}{2 \pi \hbar} \int_{V} \mathrm{~d}^{3} r \int_{-\infty}^{\infty} \mathrm{d} t \mathrm{e}^{\mathrm{i}(\vec{Q} \cdot \vec{r}-\omega t)} G_{\kappa, \kappa^{\prime}}(\vec{r}, t)
$$

We have thus reached our goal. The scattering function is identified as a double Fourier transform in space and time of the autocorrelation function of the atomic number density. Thus scattering experiments give us a Fourier analysis of correlations in space and time. This is an extremely important result for data analysis.

\subsection{The relation between the correlation function and the differential cross section}

The differential cross section can be expressed using the scattering function $S(\vec{Q}, \omega)$

$$
\frac{\mathrm{d}^{2} \sigma_{\vec{k}_{i} \rightarrow \vec{k}_{f}}}{\mathrm{~d} \Omega \mathrm{d} E_{f}}=\frac{k_{f}}{k_{i}} S(\vec{Q}, \omega)
$$

with

$$
S(\vec{Q}, \omega)=\sum_{\kappa, \kappa^{\prime}}\left(V_{\kappa}(\vec{Q}) V_{\kappa^{\prime}}^{*}(\vec{Q})\right) S_{\kappa, \kappa^{\prime}}(\vec{Q}, \omega)
$$

and

$$
S_{\kappa, \kappa^{\prime}}(\vec{Q}, \omega)=\frac{1}{2 \pi \hbar} \sum_{j \in\left\{j_{\kappa}\right\}, j^{\prime} \in\left\{j_{\kappa^{\prime}}\right\}} \int_{-\infty}^{\infty} \mathrm{d} t\left\langle\lambda_{i}\left|\mathrm{e}^{-\mathrm{i} \vec{Q} \cdot \vec{R}_{j^{\prime}}^{0}} \mathrm{e}^{\mathrm{i} \vec{Q} \cdot \vec{R}_{j}(t)}\right| \lambda_{i}\right\rangle \mathrm{e}^{-\mathrm{i} \omega t}
$$

With (8.28) it is possible to establish a link between the cross sections and the correlation functions

$$
\frac{\mathrm{d}^{2} \sigma_{\vec{k}_{i} \rightarrow \vec{k}_{f}}}{\mathrm{~d} \Omega \mathrm{d} E_{f}}=\frac{k_{f}}{k_{i}} \frac{1}{2 \pi \hbar} \sum_{\kappa, \kappa^{\prime}}\left[\left(V_{\kappa}(\vec{Q}) V_{\kappa^{\prime}}^{*}(\vec{Q})\right) \int_{V} \mathrm{~d}^{3} r \int_{-\infty}^{\infty} \mathrm{d} t \mathrm{e}^{\mathrm{i}(\vec{Q} \cdot \vec{r}-\omega t)} G_{\kappa, \kappa^{\prime}}(\vec{r}, t)\right]
$$

If we know the correlation functions, e.g. from a calculation or a theoretical model, we are thus capable of determining the cross section and hence predicting the results of a scattering experiment. Expression (8.32), therefore, plays a very important role in data analysis.

Unfortunately this is in general a one-way street. The expression (8.32) cannot be inverted, which means that the measurement of a cross section does not allow us to determine the correlation functions $G_{\kappa, \kappa^{\prime}}(\vec{r}, t)$ unambiguously. The information needed is lost in the superposition of contributions coming from different types of scatterers (chemical elements) in (8.32). The severity of this problem varys a lot with the kind of scientific question investigated. Due to the constraints imposed by translational symmetry the information contained in the coherent diffraction signal is in most cases sufficient in order to determine with high precision the positions of atoms in a 
crystal. This is no longer the case for liquids and amorphous materials. The loss of translational symmetry leads to a smearing of the measured signal over the entire reciprocal space. The extraction of correlation functions in order to describe the structure of a disordered system is, therefore, a very complex task that does not possess a unique solution.

The situation changes obviously when we deal with a monatomic system. In this case it is the scattering function itself that is related to the correlation function through a Fourier transform. Hence the correlation function can be directly determined from the measurements. The total scattering cross section is then

$$
\frac{\mathrm{d}^{2} \sigma_{\vec{k}_{i} \rightarrow \vec{k}_{f}}}{\mathrm{~d} \Omega \mathrm{d} E_{f}}=\frac{\sigma}{4 \pi} \frac{k_{f}}{k_{i}} \frac{1}{2 \pi \hbar} \int_{V} \mathrm{~d}^{3} r \int_{-\infty}^{\infty} \mathrm{d} t \mathrm{e}^{\mathrm{i}(\vec{Q} \cdot \vec{r}-\omega t)} G(\vec{r}, t) .
$$

The coherent (6.20) and the incoherent (6.21) contributions are given by

$$
\left(\frac{\mathrm{d}^{2} \sigma_{\vec{k}_{i} \rightarrow \vec{k}_{f}}}{\mathrm{~d} \Omega \mathrm{d} E_{f}}\right)_{\mathrm{coh}}=\frac{\sigma_{\text {coh }}}{4 \pi} \frac{k_{f}}{k_{i}} \frac{1}{2 \pi \hbar} \int_{V} \mathrm{~d}^{3} r \int_{-\infty}^{\infty} \mathrm{d} t \mathrm{e}^{\mathrm{i}(\vec{Q} \cdot \vec{r}-\omega t)} G(\vec{r}, t)
$$

and

$$
\left(\frac{\mathrm{d}^{2} \sigma_{\vec{k}_{i} \rightarrow \vec{k}_{f}}}{\mathrm{~d} \Omega \mathrm{d} E_{f}}\right)_{\text {inc }}=\frac{\sigma_{\text {inc }}}{4 \pi} \frac{k_{f}}{k_{i}} \frac{1}{2 \pi \hbar} \int_{V} \mathrm{~d}^{3} r \int_{-\infty}^{\infty} \mathrm{d} t \mathrm{e}^{\mathrm{i}(\vec{Q} \cdot \vec{r}-\omega t)} G^{s}(\vec{r}, t) .
$$

Coherent scattering thus gives us information about the correlations that exist between all the scatterers. Incoherent scattering is much more restricted in its information content. It is related to the self correlation function and therefore only contains information on the correlation of a particle with itself. For an immobile particle this information reduces to a proof of existence without further interest. However, when the position of particles evolves with time then the incoherent scattering is a precise and unencumbered measure of the dynamics of the individual particles.

\subsection{Contrast variation as a means of determining partial correlation functions}

The only way to overcome the difficulties associated with partial correlation functions is contrast variation, i.e. the possibility of changing the visibility of different types of scatterers. In an ideal world it would be possible to smoothly turn on the scattering power of each chemical element one by one. This process would allow us to isolate the different contributions to (8.32) (see Fig. 34). With neutrons there is two ways of doing this. (i) We can play with the isotopic composition or (ii) we can use the dependence of the scattering length on the nuclear spin. Doing so we do not interfere with the chemical composition of the sample. The change in contrast, therefore does not alter the physical quantities that the experiment is supposed to expose. ${ }^{69}$ Variation of contrast turns out to be essential in all systems composed of hydrogen (soft matter, organic chemistry, biology and bio-materials). The subject of contrast variation can in the details be quite sophisticated. We are, therefore, not going further into the discussion.

\subsection{Higher order correlation functions}

We would like to conclude with a remark on higher order correlation functions. Until now we have only looked at two-point functions, i.e. functions that only depend on the distance $\vec{r}$. Nothing prevents us from defining more complex correlation functions, e.g. $G\left(\vec{r}, \vec{r}^{\prime}, t\right)$. These functions have the undebatable advantage of taking the influence of the environment on pair correlations into account. They, therefore, have a elevated information content, admittedly with the unavoidable down-side of increased complexity. They are thus of great interest when trying to understand the structure of liquids and molecules. They can e.g. inform us about the orientation of a molecule

\footnotetext{
${ }^{69}$ As the isotope variation has an influence on the atomic mass it does actually influence the motion of the particles. This can be an issue for very light elements like hydrogen and has to be properly taken into account.
} 


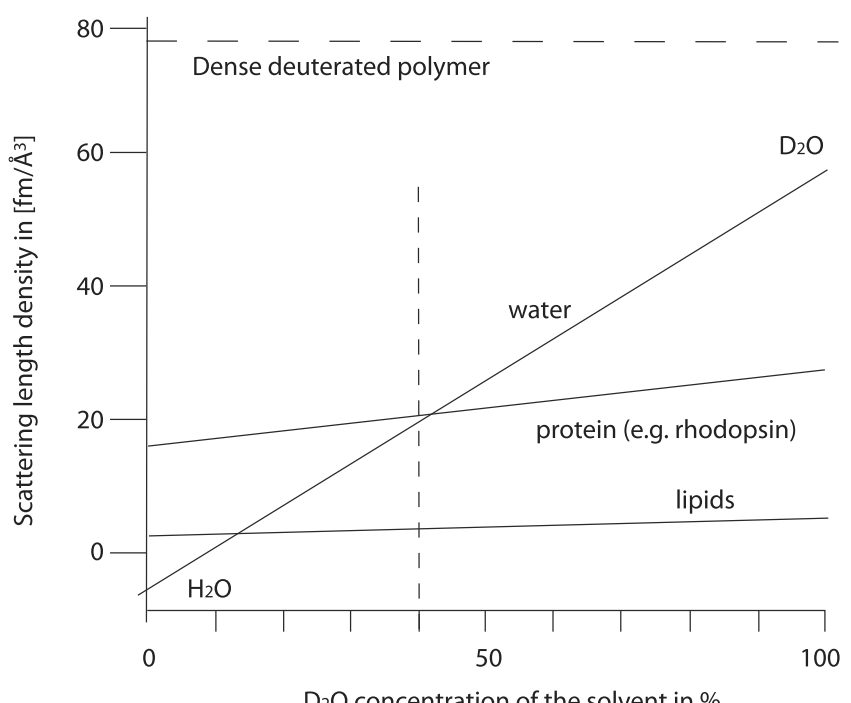

Fig. 34. Contrast matching for biological samples in solution. Exploiting the difference in scattering length of hydrogen and deuterium (see Section 6.3) it is possible to match the scattering length density of the water solvent to that of the dissolved macromolecules. The scattering length density of the dissolved molecules will depend strongly on their hydrogen and deuterium content, which due to exchange with the water solvent will depend itself on the $\mathrm{D}_{2} \mathrm{O}$ concentration. At the points where the lines of the macromolecules cross the water line we get perfect contrast matching. These points are, therefore, called match-points. The match points for proteins lie in the range of $40-50 \%$ of $\mathrm{D}_{2} \mathrm{O}$. At the match point the macromolecules become invisible in a small angle neutron experiment. The figure is based on data from reference [70].

with respect to its neighbours. In the Born approximation the higher order correlation functions are not accessible. It is possible to show that they come into play when higher order terms are added to the Born series (3.175) [26]. To access them it would be necessary to work with very large coherence volumes (see Section 11.6) leading to very challenging experiments. Even if one met the necessary conditions required to observe second order terms in the Born series the advantage would in practice be of limited value. The second order contribution would be superimposed on the first order contributions. Separating the two would amount to a problem similar in complexity to the extraction of two-point correlation functions in a system with several types of atoms. And even if it was possible to overcome this obstacle there exists no simple relation between the higher order effects and the multi-point correlation functions that we seek. It appears that this information will remain experimentally inaccessible. ${ }^{70}$

\subsection{The intermediate scattering functions $I_{\kappa, \kappa^{\prime}}(\vec{Q}, t)$ and $I_{\kappa}^{s}(\vec{Q}, t)$}

If we perform a spacial Fourier transform on $G(\vec{r}, t)$ we obtain what is called the intermediate scattering function $I_{\kappa, \kappa^{\prime}}(\vec{Q}, t)[48]{ }^{71}$ Each of the following expressions can serve as its definition

$$
I_{\kappa, \kappa^{\prime}}(\vec{Q}, t)=\sum_{j \in\left\{j_{\kappa}\right\}, j^{\prime} \in\left\{j_{\kappa^{\prime}}\right\}}\left\langle\exp \left(-\mathrm{i} \vec{Q} \cdot \vec{R}_{j}\right) \exp \left(\mathrm{i} \vec{Q} \cdot \vec{R}_{j^{\prime}}(t)\right)\right\rangle=\left\langle\hat{\rho}_{\kappa}(\vec{Q}, t=0) \hat{\rho}_{\kappa^{\prime}}(-\vec{Q}, t)\right\rangle,
$$

\footnotetext{
${ }^{70}$ One possible way of progress on this issue is the direct manipulation of wave packets as proposed by $\mathrm{H}$. Rauch. This could permit, in analogy to spin-echo for the two point correlation function, to measure the higher order correlation functions directly. A more indirect way consists in exploiting quantum statistical relations between three-point correlation functions and the derivative of two-point correlation functions, e.g. as a function of temperature.

${ }^{71}$ In same way as for $G(\vec{r}, t)$ we try to remain as general as possible and, therefore, define partial intermediate scattering functions. Logically, these functions are not normalised to the number of scatterers. This common characteristic of the partial functions $S_{\kappa, \kappa^{\prime}}(\vec{r}, t), I_{\kappa, \kappa^{\prime}}(\vec{r}, t)$ and $G_{\kappa, \kappa^{\prime}}(\vec{r}, t)$ has to be taken into account if one compares them with the total scattering functions $(S(\vec{r}, t), I(\vec{r}, t)$ and $G(\vec{r}, t))$ found in literature, which in general are normalised to the number of scatterers $N$.
} 


$$
\begin{aligned}
& I_{\kappa, \kappa^{\prime}}(\vec{Q}, t)=\int_{V} \mathrm{~d}^{3} r \mathrm{e}^{-\mathrm{i} \vec{Q} \cdot \vec{r}} G_{\kappa, \kappa^{\prime}}(\vec{r}, t), \\
& I_{\kappa, \kappa^{\prime}}(\vec{Q}, t)=\hbar \int_{-\infty}^{\infty} \mathrm{d} \omega \mathrm{e}^{\mathrm{i} \omega t} S_{\kappa, \kappa^{\prime}}(\vec{Q}, \omega) .
\end{aligned}
$$

The first one is the most appealing since it defines the correlation function directly in terms of Fourier transforms of the density operator. We recall that the correlation function is a convolution (see (8.19)) of $\rho_{\kappa}(\vec{r}, t=0)$ with $\rho_{\kappa^{\prime}}(-\vec{r}, t)$ in space. The Fourier transform of this convolution is then the product of the individual Fourier transforms (see (4.23) and (4.24)).

Inverting the last equation the scattering function is expressed in terms of the intermediate scattering function

$$
\begin{aligned}
S_{\kappa, \kappa^{\prime}}(\vec{Q}, \omega) & =\frac{1}{2 \pi \hbar} \int_{-\infty}^{\infty} \mathrm{d} t I_{\kappa, \kappa^{\prime}}(\vec{Q}, t) \mathrm{e}^{-\mathrm{i} \omega t} \\
& =\frac{1}{2 \pi \hbar} \int_{-\infty}^{\infty} \mathrm{d} t\left\langle\hat{\rho}_{\kappa}(\vec{Q}, t=0) \hat{\rho}_{\kappa^{\prime}}(-\vec{Q}, t)\right\rangle \mathrm{e}^{-\mathrm{i} \omega t} .
\end{aligned}
$$

To total scattering function can thus be written as

$$
\begin{aligned}
S(\vec{Q}, \omega) & =\sum_{\kappa, \kappa^{\prime}}\left(V_{\kappa}(\vec{Q}) V_{\kappa^{\prime}}^{*}(\vec{Q})\right) S_{\kappa, \kappa^{\prime}}(\vec{Q}, \omega) \\
& =\frac{1}{2 \pi \hbar} \sum_{\kappa, \kappa^{\prime}}\left(V_{\kappa}(\vec{Q}) V_{\kappa^{\prime}}^{*}(\vec{Q})\right) \int_{-\infty}^{\infty} \mathrm{d} t I_{\kappa, \kappa^{\prime}}(\vec{Q}, t) \mathrm{e}^{-\mathrm{i} \omega t} \\
& =\frac{1}{2 \pi \hbar} \int_{-\infty}^{\infty} \mathrm{d} t I(\vec{Q}, t) \mathrm{e}^{-\mathrm{i} \omega t},
\end{aligned}
$$

with the total intermediate scattering function defined as

$$
I(\vec{Q}, t):=\sum_{\kappa, \kappa^{\prime}}\left(V_{\kappa}(\vec{Q}) V_{\kappa^{\prime}}^{*}(\vec{Q})\right) I_{\kappa, \kappa^{\prime}}(\vec{Q}, t)
$$

In the same way we can introduce the intermediate self scattering function.

$$
\begin{aligned}
& I_{\kappa}^{s}(\vec{Q}, t)=\sum_{j \in\left\{j_{\kappa}\right\}}\left\langle\exp \left(-\mathrm{i} \vec{Q} \cdot \vec{R}_{j}\right) \exp \left(\mathrm{i} \vec{Q} \cdot \vec{R}_{j}(t)\right)\right\rangle, \\
& I_{\kappa}^{s}(\vec{Q}, t)=\int_{V} \mathrm{~d}^{3} r \mathrm{e}^{-\mathrm{i} \vec{Q} \cdot \vec{r}} G_{\kappa}^{S}(\vec{r}, t) .
\end{aligned}
$$

Starting from its definition in terms of correlation function we can derive some relevant relations for the intermediate scattering functions.

- We begin with

$$
I_{\kappa, \kappa^{\prime}}(\vec{Q}, t)=I_{\kappa^{\prime}, \kappa}^{*}(\vec{Q},-t)
$$

This relation follows from the fact that for every pair of operators

$$
\langle\mathbf{A B}\rangle=\sum_{\lambda} p_{\lambda}\langle\lambda|\mathbf{A B}| \lambda\rangle=\sum_{\lambda} p_{\lambda}\left\langle\lambda\left|\mathbf{B}^{+} \mathbf{A}^{+}\right| \lambda\right\rangle^{*}=\left\langle\mathbf{B}^{+} \mathbf{A}^{+}\right\rangle^{*} .
$$


With

$$
\hat{\rho}_{\kappa}^{+}(\vec{Q}, t)=\frac{1}{(2 \pi)^{3}} \sum_{j_{\kappa}} \exp \left(\mathrm{i} \vec{Q} \cdot \vec{R}_{j}(t)\right)=\hat{\rho}_{\kappa}(-\vec{Q}, t)
$$

we obtain

$$
\begin{aligned}
I_{\kappa, \kappa^{\prime}}(\vec{Q}, t) & =\left\langle\hat{\rho}_{\kappa}(\vec{Q}, t=0) \hat{\rho}_{\kappa^{\prime}}(-\vec{Q}, t)\right\rangle \\
& =\left\langle\hat{\rho}_{\kappa^{\prime}}^{+}(-\vec{Q}, t) \hat{\rho}_{\kappa}^{+}(\vec{Q}, t=0)\right\rangle^{*} \\
& =\left\langle\hat{\rho}_{\kappa^{\prime}}(\vec{Q}, t) \hat{\rho}_{\kappa}(-\vec{Q}, t=0)\right\rangle^{*} \\
& =\left\langle\hat{\rho}_{\kappa^{\prime}}(\vec{Q}, t=0) \hat{\rho}_{\kappa}(-\vec{Q},-t)\right\rangle^{*} \\
& =I_{\kappa^{\prime}, \kappa}^{*}(\vec{Q},-t),
\end{aligned}
$$

where when going from the third to the fourth line we invoke the homogeneity of the system in time. In other words, the origin of time can be chosen arbitrarily. The only relevant time is the delay between the events when calculating the matrix elements

$$
\left\langle\lambda\left|\mathbf{A}(t) \mathbf{B}\left(t^{\prime}\right)\right| \lambda\right\rangle=\left\langle\lambda\left|\mathbf{A}(t=0) \mathbf{B}\left(t^{\prime}-t\right)\right| \lambda\right\rangle .
$$

The relation (8.44) teaches us that it is sufficient to know the intermediate scattering functions along the positive time axis.

- Until now the intermediate scattering function has been defined on the real time axis. This definition can be reformulated using the time evolution operator

$$
\mathbf{U}(t)=\exp (-\mathrm{i} \mathbf{H} t / \hbar)
$$

We arrive at

$$
\begin{aligned}
I_{\kappa, \kappa^{\prime}}(\vec{Q}, t) & =\left\langle\hat{\rho}_{\kappa}(\vec{Q}, t=0) \hat{\rho}_{\kappa^{\prime}}(-\vec{Q}, t)\right\rangle \\
& =\sum_{\lambda} p_{\lambda}\left\langle\lambda\left|\hat{\rho}_{\kappa}(\vec{Q}, 0) \hat{\rho}_{\kappa^{\prime}}(-\vec{Q}, t)\right| \lambda\right\rangle \\
& \left.=\sum_{\lambda} p_{\lambda}\langle\lambda| \hat{\rho}_{\kappa}(\vec{Q}) \mathbf{U}^{+}(t) \hat{\rho}_{\kappa^{\prime}}(-\vec{Q}) \mathbf{U}(t)\right)|\lambda\rangle .
\end{aligned}
$$

This definition allows us extend the function in a natural way to the complex plane. We postulate

$$
\begin{aligned}
I_{\kappa, \kappa^{\prime}}(\vec{Q}, t+\mathrm{i} \tau) & =\sum_{\lambda} p_{\lambda}\left\langle\lambda\left|\hat{\rho}_{\kappa}(\vec{Q}) \mathbf{U}^{+}(t+\mathrm{i} \tau) \hat{\rho}_{\kappa^{\prime}}(-\vec{Q}) \mathbf{U}(t+\mathrm{i} \tau)\right| \lambda\right\rangle \\
& =\sum_{\lambda} p_{\lambda}\left\langle\lambda\left|\hat{\rho}_{\kappa}(\vec{Q}) \exp (\mathrm{i} \mathbf{H}(t+\mathrm{i} \tau) / \hbar) \hat{\rho}_{\kappa^{\prime}}(-\vec{Q}) \exp (-\mathrm{i} \mathbf{H}(t+\mathrm{i} \tau))\right| \lambda\right\rangle
\end{aligned}
$$

At first glance this extension into the complex plane seems to be of purely academic interest. However the concept turns out to be very powerful when we identify the complex time with the temperature as

$$
\tau:=\hbar \beta=\frac{\hbar}{k_{B} T} .
$$


For any expectation value in thermodynamical equilibrium we derive the relation

$$
\begin{aligned}
\langle\mathbf{A}(t=0) \mathbf{B}(t)\rangle & =\sum_{\lambda} p_{\lambda}\langle\lambda|\mathbf{A}(0) \mathbf{B}(t)| \lambda\rangle \\
& =\frac{1}{Z} \sum_{\lambda} \exp \left(-\beta E_{\lambda}\right)\left\langle\lambda\left|\mathbf{A \mathbf { U } ^ { + }}(t) \mathbf{B U}(t)\right| \lambda\right\rangle \\
& =\frac{1}{Z} \sum_{\lambda}\left\langle\lambda\left|\mathbf{A \mathbf { U } ^ { + }}(t) \mathbf{B} \mathbf{U}(t) \exp (-\beta \mathbf{H})\right| \lambda\right\rangle \\
& =\frac{1}{Z} \sum_{\lambda, \lambda^{\prime}}\left\langle\lambda|\mathbf{A}| \lambda^{\prime}\right\rangle\left\langle\lambda^{\prime}\left|\left(\mathbf{U}^{+}(t) \mathbf{B} \mathbf{U}(t)\right) \exp (-\beta \mathbf{H})\right| \lambda\right\rangle \\
& =\frac{1}{Z} \sum_{\lambda, \lambda^{\prime}}\left\langle\lambda^{\prime}\left|\left(\mathbf{U}^{+}(t) \mathbf{B} \mathbf{U}(t)\right) \exp (-\beta \mathbf{H})\right| \lambda\right\rangle\left\langle\lambda|\mathbf{A}| \lambda^{\prime}\right\rangle \\
& =\frac{1}{Z} \sum_{\lambda}\left\langle\lambda\left|\left(\mathbf{U}^{+}(t) \mathbf{B} \mathbf{U}(t)\right) \exp (-\beta \mathbf{H}) \mathbf{A}\right| \lambda\right\rangle \\
& =\frac{1}{Z} \sum_{\lambda} \exp \left(-\beta E_{\lambda}\right)\left\langle\lambda\left|\exp (\beta \mathbf{H})\left(\mathbf{U}^{+}(t) \mathbf{B} \mathbf{U}(t)\right) \exp (-\beta \mathbf{H}) \mathbf{A}\right| \lambda\right\rangle \\
& =\sum_{\lambda} p_{\lambda}\left\langle\lambda\left|\left(\mathbf{U}^{+}(t-\mathrm{i} \hbar \beta) \mathbf{B U}(t-\mathrm{i} \hbar \beta)\right) \mathbf{A}(0)\right| \lambda\right\rangle \\
& =\sum_{\lambda} p_{\lambda}\langle\lambda|\mathbf{B}(t-\mathrm{i} \hbar \beta) \mathbf{A}(0)| \lambda\rangle \\
& =\langle\mathbf{B}(t-\mathrm{i} \hbar \beta) \mathbf{A}(0)\rangle \\
& =\langle\mathbf{B}(t) \mathbf{A}(\mathrm{i} \hbar \beta)\rangle .
\end{aligned}
$$

If the system is not in equilibrium then the probability factors $p_{\lambda}$ are not described by Boltzmann factors and as a consequence the reasoning that we have used in order to derive relation (8.55) is not applicable.

Applying expression (8.55) to the intermediate scattering function leads to

$$
I_{\kappa, \kappa^{\prime}}(\vec{Q}, t)=I_{\kappa^{\prime}, \kappa}(-\vec{Q},-t+\mathrm{i} \hbar \beta)
$$

and for the total intermediate scattering function to

$$
\begin{aligned}
I(\vec{Q}, t) & =\sum_{\kappa \leqslant \kappa^{\prime}}\left[\left(V_{\kappa}(\vec{Q}) V_{\kappa^{\prime}}^{*}(\vec{Q})\right) I_{\kappa, \kappa^{\prime}}(\vec{Q}, t)+\left(V_{\kappa^{\prime}}(\vec{Q}) V_{\kappa}^{*}(\vec{Q})\right) I_{\kappa^{\prime}, \kappa}(\vec{Q}, t)\right] \\
& =\sum_{\kappa \leqslant \kappa^{\prime}}\left[\left(V_{\kappa}(\vec{Q}) V_{\kappa^{\prime}}^{*}(\vec{Q})\right) I_{\kappa^{\prime}, \kappa}(-\vec{Q},-t+\mathrm{i} \hbar \beta)+\left(V_{\kappa^{\prime}}(\vec{Q}) V_{\kappa}^{*}(\vec{Q})\right) I_{\kappa, \kappa^{\prime}}(-\vec{Q},-t+\mathrm{i} \hbar \beta)\right] \\
& =I(-\vec{Q},-t+\mathrm{i} \hbar \beta)
\end{aligned}
$$

provided that $V_{\kappa}(\vec{Q})=V_{\kappa}^{*}(\vec{Q}), \forall \kappa$, which means in the absence of absorption.

Hence there exists an exact relation between the intermediate scattering function on the real time axis and the one defined on the complex time axis. Knowing one we automatically also know the other. 
We will now use these relations in order to derive an important property of the scattering function.

\subsection{Detailed balance}

Using (8.39) with (8.56) the following expression is found for the scattering function

$$
\begin{aligned}
S_{\kappa, \kappa^{\prime}}(\vec{Q}, \omega) & =\frac{1}{2 \pi \hbar} \int_{-\infty}^{\infty} \mathrm{d} t I_{\kappa, \kappa^{\prime}}(\vec{Q}, t) \mathrm{e}^{-\mathrm{i} \omega t} \\
& =\frac{1}{2 \pi \hbar} \int_{-\infty}^{\infty} \mathrm{d} t I_{\kappa^{\prime}, \kappa}(-\vec{Q},-t+\mathrm{i} \hbar \beta) \mathrm{e}^{-\mathrm{i} \omega t} \\
& =\frac{1}{2 \pi \hbar} \int_{-\infty}^{\infty} \mathrm{d} t^{\prime} I_{\kappa^{\prime}, \kappa}\left(-\vec{Q}, t^{\prime}\right) \mathrm{e}^{-\mathrm{i} \omega\left(-t^{\prime}+\mathrm{i} \hbar \beta\right)} \\
& =\mathrm{e}^{\hbar \omega \beta} \frac{1}{2 \pi \hbar} \int_{-\infty}^{\infty} \mathrm{d} t I_{\kappa^{\prime}, \kappa}(-\vec{Q}, t) \mathrm{e}^{\mathrm{i} \omega t} \\
& =\mathrm{e}^{\hbar \omega \beta} S_{\kappa^{\prime}, \kappa}(-\vec{Q},-\omega) .
\end{aligned}
$$

If the system is invariant under inversion of the direction of $\vec{r}$ and hence also $\vec{Q}$ this expression can be simplified to

$$
S_{\kappa, \kappa^{\prime}}(\vec{Q},-\omega)=\mathrm{e}^{-\hbar \omega \beta} S_{\kappa^{\prime}, \kappa}(\vec{Q}, \omega)
$$

This is especially the case for disordered systems like liquids and glasses and holds also for crystals that possess an inversion centre.

When applied to the total scattering function, we find

$$
\begin{aligned}
S(\vec{Q}, \omega) & =\sum_{\kappa \leqslant \kappa^{\prime}}\left[\left(V_{\kappa}(\vec{Q}) V_{\kappa^{\prime}}^{*}(\vec{Q})\right) S_{\kappa, \kappa^{\prime}}(\vec{Q}, \omega)+\left(V_{\kappa^{\prime}}(\vec{Q}) V_{\kappa}^{*}(\vec{Q})\right) S_{\kappa^{\prime}, \kappa}(\vec{Q}, \omega)\right] \\
& =\mathrm{e}^{\hbar \omega \beta} \sum_{\kappa \leqslant \kappa^{\prime}}\left[\left(V_{\kappa}(\vec{Q}) V_{\kappa^{\prime}}^{*}(\vec{Q})\right) S_{\kappa^{\prime}, \kappa}(-\vec{Q},-\omega)+\left(V_{\kappa^{\prime}}(\vec{Q}) V_{\kappa}^{*}(\vec{Q})\right) S_{\kappa, \kappa^{\prime}}(-\vec{Q},-\omega)\right] \\
& =\mathrm{e}^{\hbar \omega \beta} \sum_{\kappa \leqslant \kappa^{\prime}}\left[\left(V_{\kappa}(\vec{Q}) V_{\kappa^{\prime}}^{*}(\vec{Q})\right) S_{\kappa^{\prime}, \kappa}^{*}(-\vec{Q},-\omega)+\left(V_{\kappa^{\prime}}(\vec{Q}) V_{\kappa}^{*}(\vec{Q})\right) S_{\kappa, \kappa^{\prime}}^{*}(-\vec{Q},-\omega)\right] \\
& =\mathrm{e}^{\hbar \omega \beta} S^{*}(-\vec{Q},-\omega)=\mathrm{e}^{\hbar \omega \beta} S(-\vec{Q},-\omega),
\end{aligned}
$$

where we have taken advantage of the fact that the scattering functions are real.

As we can see, the scattering function is asymmetric in terms of the frequency $\omega$. The factor $\mathrm{e}^{-\hbar \omega \beta}$ is always smaller than one for $\omega>0$. Therefore the negative side of $S(\vec{Q}, \omega)$ will always be inferior to the positive side.

In order to get a better understanding of the significance of this result we will return to the partial differential cross sections (see Fig. 35). Comparing

$$
\frac{\mathrm{d}^{2} \sigma_{\vec{k}_{i} \rightarrow \vec{k}_{f}}}{\mathrm{~d} \Omega \mathrm{d} E_{f}}=\frac{k_{f}}{k_{i}} S(\vec{Q}, \omega)
$$

with

$$
\frac{\mathrm{d}^{2} \sigma_{\vec{k}_{i} \rightarrow \vec{k}_{f}}}{\mathrm{~d} \Omega \mathrm{d} E_{f}}=\frac{k_{f}}{k_{i}} \frac{m^{2}}{(2 \pi)^{2} \hbar^{4}} \sum_{\lambda_{i}, \lambda_{f}} p\left(\lambda_{i}\right)\left|\sum_{j=1}^{N} V_{j}(\vec{Q})\left\langle\lambda_{f}\left|\mathrm{e}^{\mathrm{i} \vec{Q} \cdot \vec{R}_{j}}\right| \lambda_{i}\right\rangle\right|^{2} \delta\left(\hbar \omega+\left(E_{\lambda_{i}}-E_{\lambda_{f}}\right)\right)
$$




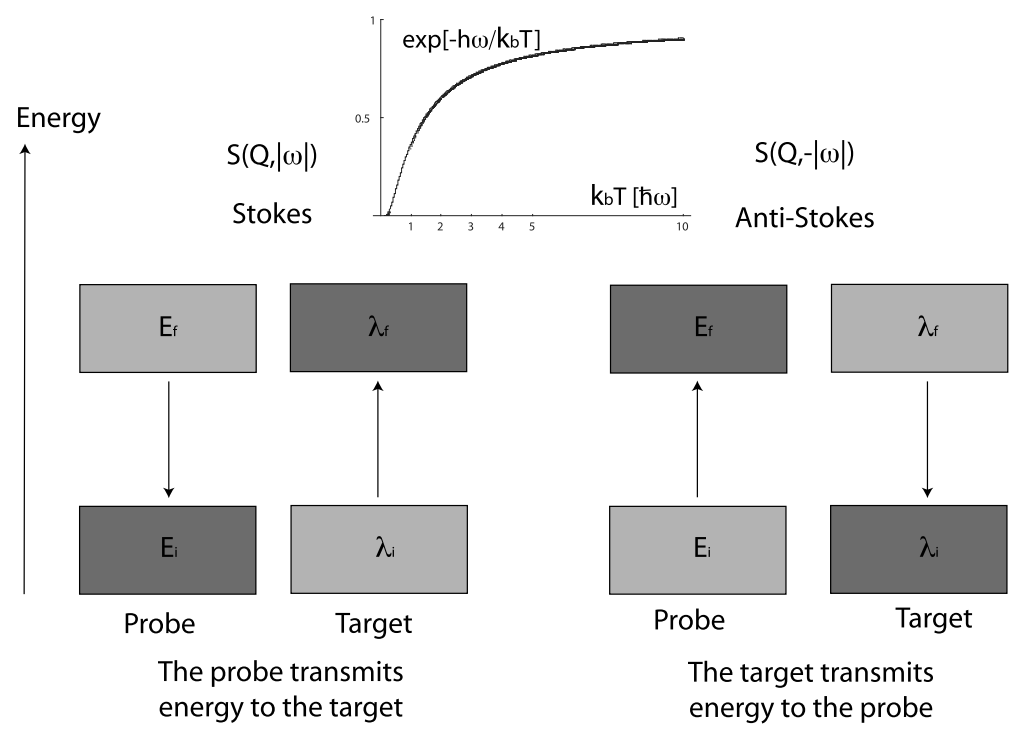

Fig. 35. Schematic presentation of detailed balance. For temperatures $k_{\mathrm{B}} T$ much smaller than the energy transfer the anti-Stokes side of the scattering goes to zero. There are simply no occupied states at the required energy levels. For $k_{\mathrm{B}} T \approx \hbar \omega$ the value of the anti-Stokes side is about $30 \%$ of the Stokes side. At very high temperatures the ratio between the two sides approaches one.

we remind ourselves that the scattering function $S(\vec{Q}, \omega)$ (apart from the factor $k_{f} / k_{i}$ ) gives the probability of a neutron passing from the state $\vec{k}_{i}$ to the state $\vec{k}_{f}$ while exchanging the energy $\hbar \omega=E_{f}-E_{i}$ with the sample. If $\hbar \omega<0$ then $E_{f}>E_{i}$ and, therefore, the neutron gains energy from the sample. The neutron gets "excited" whereas the sample gets de-excited by the scattering. Borrowing the vocabulary of optical spectroscopy we find ourselves on the anti-Stokes side of the spectrum. If on the contrary $\hbar \omega>0$ then $E_{f}<E_{i}$ and the neutron, therefore, transfers energy to the sample. This corresponds to the Stokes side of the spectrum. The intrinsic probability for passing from one state to another is identical for excitation and de-excitation. The difference is due to the occupation of the initial states of the sample. ${ }^{72}$ This rate is expressed by the factor $\mathrm{e}^{-\hbar \omega \beta}$. At very low temperature the sample is in its ground state. No de-excitations are possible and $S(\vec{Q}, \omega)=0$ for $\omega<0$. At very high temperatures $(T \gg \hbar \omega)$ the factor $\mathrm{e}^{-\hbar \omega \beta} \approx 1$ and the spectrum becomes more and more symmetric.

\subsection{Symmetrised correlation functions}

The scattering function $S(\vec{Q}, \omega)$ is as we have shown asymmetric in the variable $\omega$. We can derive a symmetric function [48] via

$$
S^{\mathrm{sym}}(\vec{Q}, \omega):=\exp \left(-\frac{1}{2} \hbar \omega \beta\right) S(\vec{Q}, \omega) .
$$

In fact

$$
S^{\mathrm{sym}}(\vec{Q}, \omega)=S^{\mathrm{sym}}(-\vec{Q},-\omega) .
$$

In the same way we define

$$
I_{\kappa, \kappa^{\prime}}^{\mathrm{sym}}(\vec{Q}, t):=I_{\kappa, \kappa^{\prime}}\left(\vec{Q}, t+\frac{1}{2} \hbar \omega \beta\right),
$$

\footnotetext{
${ }^{72}$ The neutron is always a very weak perturbation even at very high flux. It will not change the thermodynamic properties of the sample.
} 
which gives a function

$$
I^{\mathrm{sym}}(\vec{Q}, t)=I^{\mathrm{sym}}(-\vec{Q},-t)\left(=I^{\mathrm{sym} *}(\vec{Q},-t)\right),
$$

that is symmetric in $t$ and real for systems with inversion symmetry.

The practical value of these functions becomes manifest when we have to compare scattering data to calculations or to theoretical predictions. Knowledge of the wave functions of the sample, which are necessary in order to calculate the expectation values in the expressions for $S(\vec{Q}, \omega)$ is more the exception than the rule. In most cases the measurement has to be compared to classical calculations, e.g. obtained from molecular dynamics simulations or stochastic models (diffusion models). These calculations provide the classical correlation functions ${ }^{\mathrm{cl}} G(\vec{r}, t)$ (8.23) and (8.24). Often these functions are real and even in time, which implies that $S(\vec{Q}, \omega)$ will be symmetric in $\omega$, in contradiction to the principle of detailed balance. One possibility for reestablishing the correct balance is to compare the classical predictions to the symmetrised scattering functions. This procedure was first proposed by Schofield [56].

\subsection{Asymptotic behaviour}

It is often very instructive to study the behaviour of a function for extreme values of its argument. In this way we can hope to identify useful relations that are independent of the exact form of the functions.

Let us consider the intermediate scattering function $I_{\kappa, \kappa^{\prime}}(\vec{Q}, t)$. For very long times the correlations will have to become necessarily independent of time. Formally this implies that the limit $I_{\kappa, \kappa^{\prime}}(\vec{Q}, t \rightarrow \pm \infty)$ exists. $^{73}$

Let us define

$$
I_{\kappa, \kappa^{\prime}}^{\prime}(\vec{Q}, t):=I_{\kappa, \kappa^{\prime}}(\vec{Q}, t)-I_{\kappa, \kappa^{\prime}}(\vec{Q}, \infty)
$$

The scattering function is found by Fourier transformation of the intermediate function

$$
\begin{aligned}
S_{\kappa, \kappa^{\prime}}(\vec{Q}, \omega) & =\frac{1}{2 \pi \hbar} \int_{-\infty}^{\infty} \mathrm{d} t I_{\kappa, \kappa^{\prime}}(\vec{Q}, t) \mathrm{e}^{-\mathrm{i} \omega t} \\
& =\frac{1}{2 \pi \hbar} \int_{-\infty}^{\infty} \mathrm{d} t\left[I_{\kappa, \kappa^{\prime}}^{\prime}(\vec{Q}, t)+I_{\kappa, \kappa^{\prime}}(\vec{Q}, \infty)\right] \mathrm{e}^{-\mathrm{i} \omega t} \\
& =I_{\kappa, \kappa^{\prime}}(\vec{Q}, \infty) \delta(\hbar \omega)+\int_{-\infty}^{\infty} \mathrm{d} t I_{\kappa, \kappa^{\prime}}^{\prime}(\vec{Q}, t) \mathrm{e}^{-\mathrm{i} \omega t}
\end{aligned}
$$

From (8.29) we deduce that the partial differential coherent cross section contains an elastic component, i.e. a component proportional to $\delta(\hbar \omega)$, which is given by

$$
\left(\frac{\mathrm{d}^{2} \sigma}{\mathrm{d} \Omega \mathrm{d} E_{f}}\right)_{\mathrm{el}}^{\mathrm{coh}}=\sum_{\kappa, \kappa^{\prime}} \bar{b}_{\kappa} \bar{b}_{\kappa^{\prime}} I_{\kappa, \kappa^{\prime}}(\vec{Q}, \infty) \delta(\hbar \omega)
$$

\footnotetext{
${ }^{73}$ In theory one can construct situations where this is not the case. E.g. in a system described by a harmonic excitation the intermediate scattering function will oscillate for all $t$ around its mean value. This is by the way the reason why the dynamical response of such a system is described by Dirac $\delta$-functions in energy (see Sections 9.10 and 9.12). In reality physical systems are normally immersed in a thermal bath and the oscillations are damped, which guaranties the existence of a limiting value that the intermediate scattering functions decay to.
} 
The difference between the partial differential cross section and the elastic cross section corresponds to the inelastic cross section. Attention, the inelastic cross section is not necessarily zero when $\hbar \omega=0$. One speaks of quasi-elastic intensity. ${ }^{74}$ Quasi-elastic scattering can be clearly distinguished from elastic scattering since

$$
\int_{-\varepsilon}^{\varepsilon} \mathrm{d}(\hbar \omega)\left(\frac{\mathrm{d}^{2} \sigma}{\mathrm{d} \Omega \mathrm{d} E_{f}}\right)_{\mathrm{el}}=\sum_{\kappa, \kappa^{\prime}} \bar{b}_{\kappa} \bar{b}_{\kappa^{\prime}}^{*} I_{\kappa, \kappa^{\prime}}(\vec{Q}, \infty)
$$

is independent of the choice of $\varepsilon$ while

$$
\int_{-\varepsilon}^{\varepsilon} \mathrm{d}(\hbar \omega)\left(\frac{\mathrm{d}^{2} \sigma}{\mathrm{d} \Omega \mathrm{d} E_{f}}\right)_{\text {quasi-el }} \rightarrow 0
$$

for $\varepsilon \rightarrow 0$. In practice this difference manifests itself in the observation that the intensity of elastic scattering is independent of the experimental resolution (see Section 11.6).

Let us look a bit more into the details of the function $I_{\kappa, \kappa^{\prime}}(\vec{Q}, \infty)$, defined by $(8.38)$ as

$$
\begin{aligned}
I_{\kappa, \kappa^{\prime}}(\vec{Q}, \infty) & =\sum_{j \in\left\{j_{\kappa}\right\}, j^{\prime} \in\left\{j_{\kappa^{\prime}}\right\}}\left\langle\exp \left(-\mathrm{i} \vec{Q} \cdot \vec{R}_{j}\right) \exp \left(\mathrm{i} \vec{Q} \cdot \vec{R}_{j^{\prime}}(\infty)\right)\right\rangle \\
& =\left\langle\hat{\rho}_{\kappa}(\vec{Q}, t=0) \hat{\rho}_{\kappa^{\prime}}(-\vec{Q}, \infty)\right\rangle \\
& =\int_{V} \mathrm{~d}^{3} r \mathrm{e}^{-\mathrm{i} \vec{Q} \cdot \vec{r}} \int \mathrm{d}^{3} \vec{r}^{\prime}\left\langle\hat{\rho}_{\kappa}\left(\vec{r}^{\prime}-\vec{r}\right) \hat{\rho}_{\kappa^{\prime}}\left(\vec{r}^{\prime}, \infty\right)\right\rangle .
\end{aligned}
$$

If the limit $I_{\kappa, \kappa^{\prime}}(\vec{Q}, \infty)$ exists then the correlation between $\vec{R}_{j}$ and $\vec{R}_{j^{\prime}}(t)$ has to become independent of $t$ when $t \rightarrow \infty$. In other words, $\vec{R}_{j}$ and $\vec{R}_{j^{\prime}}(t)$ should de-correlate as $t \rightarrow \infty$. Formally

$$
\int \mathrm{d}^{3} \vec{r}^{\prime}\left\langle\hat{\rho}_{\kappa}\left(\vec{r}^{\prime}-\vec{r}\right) \hat{\rho}_{\kappa^{\prime}}\left(\vec{r}^{\prime}, \infty\right)\right\rangle=\int \mathrm{d}^{3} \vec{r}^{\prime}\left\langle\hat{\rho}_{\kappa}\left(\vec{r}^{\prime}-\vec{r}\right)\right\rangle\left\langle\hat{\rho}_{\kappa^{\prime}}\left(\vec{r}^{\prime}\right)\right\rangle,
$$

where we have exploited the fact that $\left\langle\hat{\rho}_{\kappa}\left(\vec{r}^{\prime}-\vec{r}\right)\right\rangle$ is independent of $t$.

In a liquid the density $\rho=N / V$ is homogeneous, which is the same as saying that it is independent of position. Hence

$$
\int \mathrm{d}^{3} \vec{r}^{\prime}\left\langle\hat{\rho}_{\kappa}\left(\vec{r}^{\prime}-\vec{r}\right)\right\rangle\left\langle\hat{\rho}_{\kappa^{\prime}}\left(\vec{r}^{\prime}\right)\right\rangle=\int \mathrm{d}^{3} \vec{r}^{\prime} \rho^{2}=V \rho^{2}=N \rho .
$$

The Fourier transform of this expression gives the intermediate scattering function

$$
I_{\kappa, \kappa^{\prime}}(\vec{Q}, \infty) \propto \delta(\vec{Q})
$$

A constant density in space does, therefore, not give rise to scattering. The same result is found for incoherent scattering. Hence a liquid or a gas does not produce elastic scattering. When the liquid crystallises or vitrifies the atoms become trapped in their equilibrium positions, which are stable on the time scale of the experimental observation. This stability gives rise to a finite value of $I_{\kappa, \kappa^{\prime}}(\vec{Q}, \infty)$ and hence produces elastic scattering (see Fig. 36).

\footnotetext{
${ }^{74}$ It seems that the expression has been introduced by [48].
} 


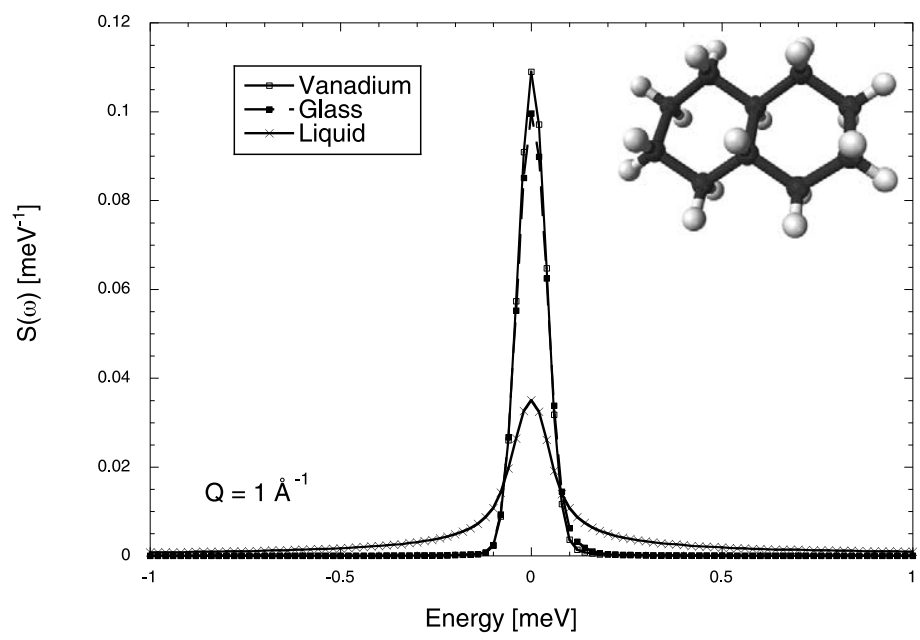

Fig. 36. In this figure the scattering of trans-Decalin in the liquid and glassy state are compared. The molecules $\mathrm{C}_{10} \mathrm{H}_{18}$ consist of two fused cyclohexane rings and is an excellent glass former. The quasi-elastic signal characterising the liquid state disappears in favour of an elastic peak in the glass state, where the width of the signal is given by the instrument resolution as measured with a Vanadium standard. Contrary to a liquid the atoms keep their equilibrium positions in a glass.

\subsection{Example: Ensemble of Boltzmann particles}

We follow Squires [62] and Lovesey [39] and illustrate the correlation functions that we have just defined using the ideal gas as an example. In an ideal gas there is no interaction between the particles. Hence the wave functions correspond to the wave functions of an ensemble of free particles. In the Heisenberg description

$$
|\lambda\rangle=|\vec{P}(1), \ldots, \vec{P}(N)\rangle=|\vec{P}(1)\rangle \cdots|\vec{P}(N)\rangle
$$

with $^{75}$

$$
|\vec{P}(j)\rangle=\frac{1}{\sqrt{V}} \exp \left(\mathrm{i} \vec{q}(j) \cdot \vec{R}_{j}\right), \quad \vec{q}(j)=\frac{\vec{P}(j)}{\hbar}
$$

The ensemble of particles is coupled to a thermal bath. We assume that all the particles can be distinguished. As a consequence the probability that a particle $j$ is found in a state $\vec{P}_{j}$ is given by the Boltzmann distribution

$$
f_{\vec{p}} \mathrm{~d}^{3} p=f\left(p_{x}, p_{y}, p_{z}\right) \mathrm{d} p_{x} \mathrm{~d} p_{y} \mathrm{~d} p_{z}=\sqrt{\left(\frac{1}{2 \pi m k_{\mathrm{B}} T}\right)^{3}} \exp \left(-\frac{p_{x}^{2}+p_{y}^{2}+p_{z}^{2}}{2 m k_{\mathrm{B}} T}\right) \mathrm{d}^{3} p
$$

If we proceed via a direct calculation of the partial differential cross section from the master equation then we need to evaluate

$$
\begin{aligned}
\frac{\mathrm{d}^{2} \sigma_{\vec{k}_{i} \rightarrow \vec{k}_{f}}}{\mathrm{~d} \Omega \mathrm{d} E_{f}}= & \frac{k_{f}}{k_{i}} \sum_{\lambda_{i}, \lambda_{f}} p\left(\lambda_{i}\right)\left|\sum_{j=1}^{N} b_{j}\left\langle(\vec{P}(1), \ldots, \vec{P}(N))_{f}\left|\mathrm{e}^{\mathrm{i} \vec{Q} \cdot \vec{R}_{j}}\right|(\vec{P}(1), \ldots, \vec{P}(N))_{i}\right\rangle\right|^{2} \\
& \times \delta\left(\left(E_{\lambda_{i}}-E_{\lambda_{f}}\right)+\hbar \omega\right) .
\end{aligned}
$$

\footnotetext{
${ }^{75}$ In order to avoid confusion between the wave vectors of the neutron and the wave vectors of the scattering particles we denote the later by $\vec{q}$.
} 
The matrix elements in this expression can be calculated with (8.77) as

$$
\begin{aligned}
\langle & \left.(\vec{P}(1), \ldots, \vec{P}(N))_{f}\left|\mathrm{e}^{\mathrm{i} Q \vec{Q} \cdot \vec{R}_{j}}\right|(\vec{P}(1), \ldots, \vec{P}(N))_{i}\right\rangle \\
= & \left\langle\vec{P}_{f}(1) \mid \vec{P}_{i}(1)\right\rangle \cdots\left\langle\vec{P}_{f}(j)\left|\mathrm{e}^{\mathrm{i} \vec{Q} \cdot \vec{R}_{j}}\right| \vec{P}_{i}(j)\right\rangle \cdots\left\langle\vec{P}_{f}(N) \mid \vec{P}_{i}(N)\right\rangle \\
= & \frac{1}{V^{N}} \int_{V} \mathrm{~d}^{3} R_{1} \exp \left(\mathrm{i}\left(\vec{q}_{i}(1)-\vec{q}_{f}(1)\right) \cdot \vec{R}_{1}\right) \cdots \int_{V} \mathrm{~d}^{3} R_{j} \exp \left(\mathrm{i}\left(\vec{Q}+\vec{q}_{i}(j)-\vec{q}_{f}(j)\right) \cdot \vec{R}_{j}\right) \cdots \\
& \quad \times \int_{V} \mathrm{~d}^{3} R_{N} \exp \left(\mathrm{i}\left(\vec{q}_{i}(N)-\vec{q}_{f}(N)\right) \cdot \vec{R}_{N}\right) \\
= & \delta\left(\vec{q}_{i}(1)-\vec{q}_{f}(1)\right) \cdots \cdots \delta\left(\vec{Q}+\vec{q}_{i}(j)-\vec{q}_{f}(j)\right) \cdots \delta\left(\vec{q}_{i}(N)-\vec{q}_{f}(N)\right) .
\end{aligned}
$$

This is the expression for the conservation of momentum in a collision that only involves single particles. Thus, if the initial wave function and the final wave function differ in more than one quantum number the contribution to the cross section is zero. In all the other cases the matrix element is equal to one provided that

$$
\hbar \omega=-\left(E_{\lambda_{i}}-E_{\lambda_{f}}\right)=-\frac{\hbar^{2}}{2 m_{j}}\left(q_{i}^{2}-q_{f}^{2}\right)=\frac{\hbar^{2}}{2 m}\left(Q^{2}+2 \vec{Q} \cdot \vec{q}_{i}(j)\right) .
$$

Replacing (8.80) and (8.81) in (8.79) we find

$$
\begin{aligned}
\frac{\mathrm{d}^{2} \sigma_{\vec{k}_{i} \rightarrow \vec{k}_{f}}}{\mathrm{~d} \Omega \mathrm{d} E_{f}} & =\frac{k_{f}}{k_{i}} \sum_{j=1}^{N} b_{j} b_{j}^{*} \sum_{\vec{q}_{i}(j)} p\left(\vec{q}_{i}(j)\right) \delta\left(\hbar \omega-\frac{\hbar^{2}}{2 m_{j}}\left(Q^{2}+2 \vec{Q} \cdot \vec{q}_{i}(j)\right)\right) \\
& =\frac{k_{f}}{k_{i}} \sum_{j=1}^{N} b_{j} b_{j}^{*} \int \mathrm{d}^{3} q_{i}(j) p\left(\vec{q}_{i}(j)\right) \delta\left(\hbar \omega-\frac{\hbar^{2}}{2 m_{j}}\left(Q^{2}+2 \vec{Q} \cdot \vec{q}_{i}(j)\right)\right) .
\end{aligned}
$$

The integral here can be expressed in terms of the Boltzmann probability (8.78) as

$$
\begin{aligned}
& \int \mathrm{d}^{3} q p(\vec{q}) \delta\left(\hbar \omega-\frac{\hbar^{2}}{2 m_{j}}\left(Q^{2}+2 \vec{Q} \cdot \vec{q}\right)\right) \\
& =\hbar^{3} \sqrt{\left(\frac{1}{2 \pi m_{j} k_{\mathrm{B}} T}\right)^{3}} \int \mathrm{d}^{3} q \exp \left(-\frac{\hbar^{2} q^{2}}{2 m_{j} k_{\mathrm{B}} T}\right) \delta\left(\hbar \omega-\frac{\hbar^{2}}{2 m_{j}}\left(Q^{2}+2 \vec{Q} \cdot \vec{q}\right)\right) .
\end{aligned}
$$

This integral can be evaluated using a Cartesian reference system with its axis aligned in the $\vec{Q}$ direction. ${ }^{76}$ We find

$$
\frac{\mathrm{d}^{2} \sigma_{\vec{k}_{i} \rightarrow \vec{k}_{f}}}{\mathrm{~d} \Omega \mathrm{d} E_{f}}=\frac{k_{f}}{k_{i}} \sum_{j=1}^{N} b_{j} b_{j}^{*} \frac{1}{\sqrt{2 \pi} \sigma_{j}} \exp \left(-\frac{\left(\hbar \omega-E_{r}(j)\right)^{2}}{2 \sigma_{j}^{2}}\right)
$$

$$
\begin{aligned}
& { }^{76} \text { We choose } \hat{z} \| \vec{Q} \text {. The Dirac function is written as } \\
& \begin{aligned}
\delta\left(\hbar \omega-\frac{\hbar^{2}}{2 m_{j}}\left(Q^{2}+2 \vec{Q} \cdot \vec{q}\right)\right) & =\delta\left(\hbar \omega-\frac{\hbar^{2}}{2 m_{j}}\left(Q^{2}+2 Q \cdot q z\right)\right) \\
& =\delta\left(\hbar \omega-E_{r}(j)-2 \frac{E_{r}(j)}{Q} \cdot q z\right) \\
& =\frac{Q}{2 E_{r}(j)} \delta\left(\left(\frac{\hbar \omega Q}{2 E_{r}(j)}-\frac{Q}{2}\right)-q z\right) .
\end{aligned}
\end{aligned}
$$


with the recoil energy of the particle $j$

$$
E_{r}(j):=\frac{\hbar^{2} Q^{2}}{2 m_{j}}
$$

and the standard deviation

$$
\sigma_{j}:=\sqrt{2 k_{\mathrm{B}} T E_{r}(j)}
$$

The recoil energy is calculated with (1.13) as

$$
E_{r}(j)[\mathrm{meV}]=\left(\frac{m_{n}}{m_{j}}\right) 2.0725 \cdot Q^{2}\left[\AA^{-1}\right]
$$

where $m_{n}$ is the mass of the neutron. Hence the partial differential cross section is the sum of $N$ Gaussians. Each Gaussian is centred on the recoil energy of the particle in question. The width of the Gaussians is proportional to the square root of the temperature and the square root of the recoil energy as well. The dynamic response of a heavy particle will always be more centred than the response of a light particle. With

$$
\delta(x)=\lim _{\sigma \rightarrow 0} \frac{1}{\sqrt{2 \pi \sigma}} \exp \left(-\frac{x^{2}}{2 \sigma}\right)
$$

in the limit $T \rightarrow 0$ we find

$$
\frac{\mathrm{d}^{2} \sigma_{\vec{k}_{i} \rightarrow \vec{k}_{f}}}{\mathrm{~d} \Omega \mathrm{d} E_{f}}=\frac{k_{f}}{k_{i}} \sum_{j=1}^{N} b_{j} b_{j}^{*} \delta\left(\hbar \omega-E_{r}(j)\right) .
$$

This is the zero temperature response of an ensemble of Boltzmann particles.

We see that there is no interference terms between the contributions from the different particles. This is not surprising. We are dealing with plane waves and hence each particle is distributed homogeneously over the gas volume. The lack of interaction including exchange interactions has the consequence that a given particle is not

Integration over $q_{x}$ and $q_{y}$ gives us twice the factor

$$
\hbar \sqrt{\frac{1}{2 \pi m_{j} k_{\mathrm{B}} T}} .
$$

This is a direct consequence of the normalisation of probabilities. The integration over $q_{z}$ is done using the Dirac function. We find

$$
\begin{aligned}
\hbar & \sqrt{\frac{1}{2 \pi m_{j} k_{\mathrm{B}} T}} \int \mathrm{d} q_{z} \exp \left(-\frac{\hbar^{2} q_{z}^{2}}{2 m_{j} k_{\mathrm{B}} T}\right) \frac{Q}{2 E_{r}(j)} \delta\left(\left(\frac{\hbar \omega Q}{2 E_{r}(j)}-\frac{Q}{2}\right)-q_{z}\right) \\
& =\hbar \sqrt{\frac{1}{2 \pi m_{j} k_{\mathrm{B}} T}} \frac{Q}{2 E_{r}(j)} \exp \left(-\frac{\hbar^{2}}{2 m_{j} k_{\mathrm{B}} T}\left(\frac{\hbar \omega Q}{2 E_{r}(j)}-\frac{Q}{2}\right)^{2}\right) \\
& =\sqrt{\frac{1}{4 \pi E_{r}(j) k_{\mathrm{B}} T}} \exp \left(\frac{\left(\hbar \omega-E_{r}(j)\right)^{2}}{4 E_{r}(j) k_{\mathrm{B}} T}\right) .
\end{aligned}
$$


aware of the existence of other particles, which means that there are no correlations between the particles. Thus the coherent scattering from an ensemble of Boltzmann particles without interactions is indistinguishable from its incoherent scattering. One often says that the response of the system is incoherent, even though the correct term would be un- or de-correlated.

We should nevertheless be aware of the fact that at very low temperatures the level of occupation is very low. Which means that the Boltzmann distribution is no longer a good approximation. We can no longer ignore the correlations introduced by the exchange interactions between undistinguishable particles. These forces are especially pronounced for fermions. In this case the Boltzmann gas should be replaced by a Fermi liquid. A particle in a Fermi liquid is aware of the presence of other particles due to the Pauli exclusion principle. This principle introduces correlations of occupation levels.

Despite of its limitations we will in this section stay with the concept of Boltzmann particles. In the limit where $m_{j} \rightarrow \infty$ we can derive that

$$
\frac{\mathrm{d}^{2} \sigma_{\vec{k}_{i} \rightarrow \vec{k}_{f}}}{\mathrm{~d} \Omega \mathrm{d} E_{f}}=\frac{k_{f}}{k_{i}} \sum_{j=1}^{N} b_{j} b_{j}^{*} \delta(\hbar \omega) .
$$

We obtain the known result for elastic scattering from fixed particles.

We would now like to derive the correlation functions for the Boltzmann gas. Instead of proceeding via a direct calculation we pass via the cross section (8.87) from which we can identify the partial self scattering functions as

$$
S_{\kappa}^{S}(\vec{Q}, \omega)=\frac{N_{\kappa}}{\sqrt{2 \pi} \sigma_{\kappa}} \exp \left(-\frac{\left(\hbar \omega-E_{r}(\kappa)\right)^{2}}{2 \sigma_{\kappa}^{2}}\right)
$$

They are shown in Fig. 37. The $S_{\kappa}^{s}(\vec{Q}, \omega)$ satisfy detailed balance. The fact that we have used the Boltzmann distribution in our calculations, which a priori is only valid for classical distinguishable particles, changes nothing about this fact. This can be understood if one considers that we would have obtained the same expression for an

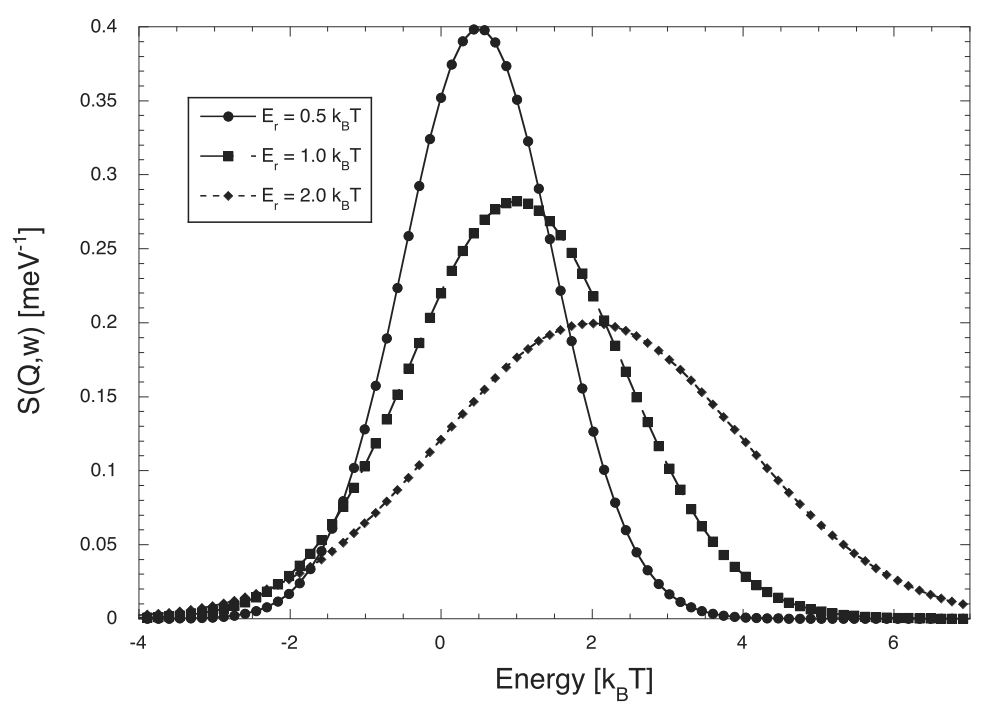

Fig. 37. Scattering function for Boltzmann particle for different recoil energies $E_{r}$. The natural unit of the energy is $\left(k_{\mathrm{B}} T\right)$. 


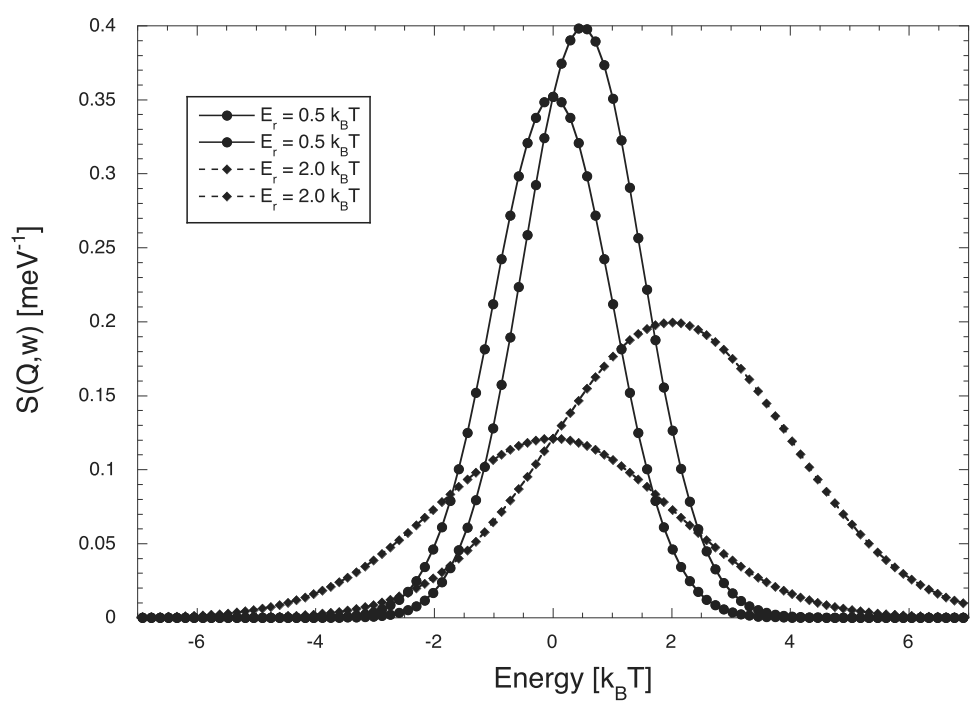

Fig. 38. Comparison between the scattering function and the symmetrised scattering function for two distinct recoil energies $E_{r}$.

isolated particle or for an ensemble of completely different particles. The symmetrised function is calculated as

$$
\begin{aligned}
S_{\kappa}^{\mathrm{sym}}(\vec{Q}, \omega) & =\exp \left(-\frac{1}{2} \hbar \omega \beta\right) S_{\kappa}^{2}(\vec{Q}, \omega) \\
& =\frac{N_{\kappa}}{\sqrt{2 \pi} \sigma_{\kappa}} \exp \left(-\frac{\hbar \omega}{2 k_{\mathrm{B}} T}\right) \exp \left(-\frac{\left(\hbar \omega-E_{r}(\kappa)\right)^{2}}{2 \sigma_{\kappa}^{2}}\right) \\
& =\frac{N_{\kappa}}{\sqrt{2 \pi} \sigma_{\kappa}} \exp \left(\frac{-2 E_{r}(\kappa) \hbar \omega-\left(\hbar \omega-E_{r}(\kappa)\right)^{2}}{2 \sigma_{\kappa}^{2}}\right) \\
& =\frac{N_{\kappa}}{\sqrt{2 \pi} \sigma_{\kappa}} \exp \left(-\frac{\hbar^{2} \omega^{2}+E_{r}(\kappa)^{2}}{2 \sigma_{\kappa}^{2}}\right) .
\end{aligned}
$$

Attention, these functions are no longer normalised (see Fig. 38). The intermediate scattering functions are obtained by Fourier transforming the partial scattering functions. Since

$$
\int_{-\infty}^{\infty} \mathrm{d} x \exp \left(-a x^{2}+b x\right)=\sqrt{\frac{\pi}{a}} \exp \left(\frac{b^{2}}{4 a}\right),
$$

they become

$$
I_{\kappa}^{s}(\vec{Q}, t)=\hbar \int_{-\infty}^{\infty} \mathrm{d} \omega \exp (-\mathrm{i} \omega t) S_{\kappa}^{s}(\vec{Q}, \omega)=\exp \left(-\frac{1}{2} Q^{2} \sigma_{\kappa}(t)^{2}\right)
$$

with

$$
\sigma_{\kappa}(t)^{2}=\frac{k_{\mathrm{B}} T}{m_{\kappa}} \cdot t\left(t-\mathrm{i} \frac{\hbar}{k_{\mathrm{B}} T}\right) .
$$

We could have found the same result by evaluating

$$
I_{\kappa}^{s}(\vec{Q}, t)=\sum_{j \in\left\{j_{\kappa}\right\}}\left\langle\exp \left(-\mathrm{i} \vec{Q} \cdot \vec{R}_{j}\right) \exp \left(\mathrm{i} \vec{Q} \cdot \vec{R}_{j}(t)\right)\right\rangle
$$


with the methods of quantum mechanical calculus [39]. The intermediate scattering function is thus an intrinsically complex function. The symmetrised form can be calculated as

$$
I_{\kappa}^{\mathrm{sym}}(\vec{Q}, t):=I_{\kappa}^{S}\left(\vec{Q}, t+\frac{1}{2} \hbar \omega \beta\right)=\exp \left(-\frac{1}{2} Q^{2} \sigma_{\text {sym }}^{2}(\kappa)\right)
$$

with

$$
\sigma_{\mathrm{sym}}^{2}(\kappa)=\frac{k_{\mathrm{B}} T}{m_{\kappa}}\left(t^{2}+\frac{\hbar^{2}}{4\left(k_{\mathrm{B}} T\right)^{2}}\right)
$$

which is a real and even function.

Finally the pair correlation function $G_{\kappa}^{s}(\vec{r}, t)$ can be calculated from the intermediate scattering function by a Fourier transformation in space

$$
G_{\kappa}^{S}(\vec{r}, t)=\frac{1}{(2 \pi)^{3}} \int \mathrm{d}^{3} Q \mathrm{e}^{-\mathrm{i} \vec{Q} \cdot \vec{r}} I_{\kappa}^{S}(\vec{Q}, t)=\frac{1}{\sqrt{\left(2 \pi \sigma_{\kappa}(t)^{2}\right)^{3}}} \exp \left(-\frac{r^{2}}{2 \sigma_{\kappa}(t)^{2}}\right) .
$$

This is a normalised Gaussian where the width is complex and increases with time. The symmetrised version of $G_{\kappa}^{s}(\vec{r}, t)$ is found as

$$
G_{\kappa}^{\text {sym }}(\vec{r}, t)=\frac{1}{\sqrt{\left(2 \pi \sigma_{\kappa}^{\text {sym }}(t)^{2}\right)^{3}}} \exp \left(-\frac{r^{2}}{2 \sigma_{\kappa}^{\text {sym }}(t)^{2}}\right) .
$$

The interpretation of a complex correlation function is not easy. Therefore, we turn our attention to classical systems. For an ideal gas the pair correlation function has the same form as (8.102). It is determined by

$$
G_{\kappa}^{\mathrm{cl}}(\vec{r}, t)=\frac{1}{\sqrt{\left(2 \pi \sigma_{\kappa}^{\mathrm{cl}}(t)^{2}\right)^{3}}} \exp \left(-\frac{r^{2}}{2 \sigma_{\kappa}^{\mathrm{cl}}(t)^{2}}\right) .
$$

The standard deviation is calculated as

$$
\sigma_{\kappa}^{\mathrm{cl}}(t)=\sqrt{\frac{k_{\mathrm{B}} T}{m_{\kappa}}} t .
$$

The two functions $G_{\kappa}^{s}(\vec{r}, t)$ and $G_{\kappa}^{\mathrm{cl}}(r, t)$ are connected by substitution of variables.

$$
\left.G_{\kappa}^{s}(\vec{r}, t)=G_{\kappa}^{\mathrm{cl}}(r, \sqrt{t(t+\mathrm{i} \beta \hbar})\right)
$$

This substitution is an alternative approach to the method of Schofield of creating a scattering function that respects the detail balance principle starting from a classical calculation. It was proposed by Egelstaff [20]. Its validity is discussed in [21].

The function $G_{\kappa}^{\mathrm{cl}}(\vec{r}, t)$ constitutes the classical limit of $G_{\kappa}^{s}(\vec{r}, t)$. One can easily verify this with (8.98) by letting $\hbar \rightarrow 0$ or $\beta \rightarrow 0(T \rightarrow \infty)$. The significance of the function $G_{\kappa}^{\mathrm{cl}}(r, t)$ is simple (see Fig. 39).

$$
4 \pi r^{2} G_{\kappa}^{\mathrm{cl}}(\vec{r}, t) \mathrm{d} r
$$




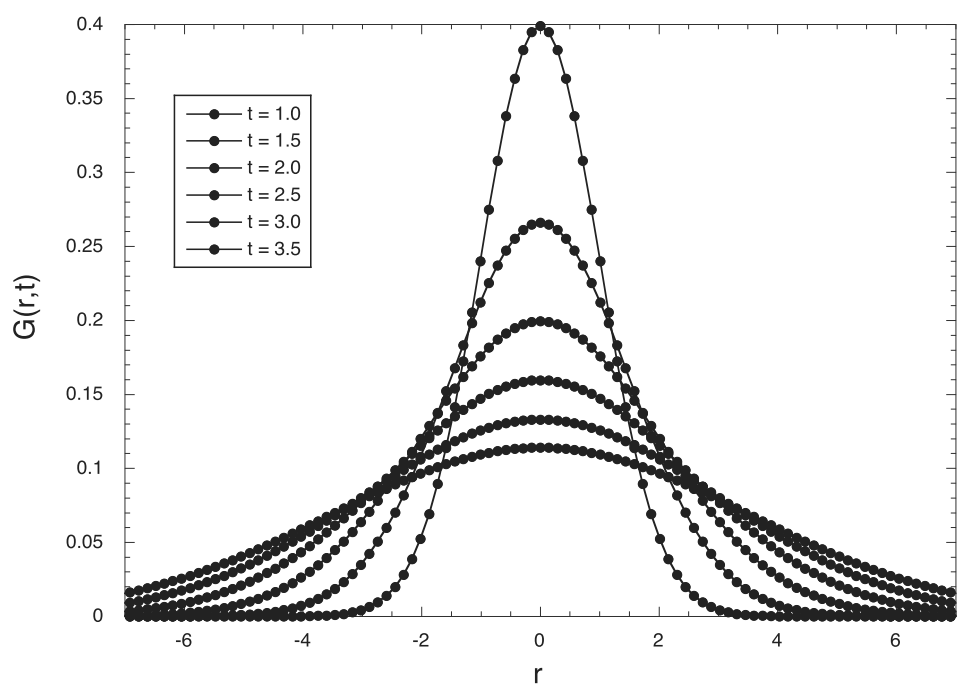

Fig. 39. The function $G_{\kappa}^{\mathrm{cl}}(\vec{r}, t)$ for different times. The units are chosen in such a way so the velocity $\sqrt{k_{\mathrm{B}} T / m_{\kappa}}=1$.

gives the probability of finding a particle of type $\kappa$ at time $t$ at a position between $r$ and $r+\mathrm{d} r$, if the same particle was at time $t=0$ at the origin. In the ballistic regime this probability is directly related to the probability $p(v) \mathrm{d} v$ that the particle possesses a velocity between $v$ and $v+\mathrm{d} v$ with $v=r / t . p(v)$ is given by the Maxwell distribution

$$
p(v) \propto v^{2} \exp \left(-\frac{m_{\kappa} v^{2}}{2 k_{\mathrm{B}} T}\right) .
$$

The width of $G_{\kappa}^{\mathrm{cl}}(\vec{r}, t)$ increases with $v$, which is equivalent to a specific $r$ at a given time $t$.

Systems of Boltzmann particles may be of pedagogical value but their scientific interest is limited. If we do not want to handle the interaction between particles at the atomic level one way of taking their existence into account is to introduce diffusion. We have already mentioned that the dynamics of a fluid is governed by a continuity equation (3.12)

$$
\frac{\partial}{\partial t} n(\vec{r}, t)+\vec{\nabla} \cdot \vec{J}(\vec{r}, t)=0
$$

which relates the changes in the atomic density $n(r, t)$ to a current $\vec{J}(\vec{r}, t)$. The first of Fick's laws states that this current is proportional to the gradient of the concentration

$$
\vec{J}(\vec{r}, t)=\vec{\nabla} n(\vec{r}, t)
$$

It is evident that this law can only by applied to a system in the steady state. At very short times we remain necessary in a ballistic regime. Deviations become important as time goes on. If we combine the continuity equation with Fick's first law we get the diffusion equation or Fick's second law

$$
\frac{\partial n(\vec{r}, t)}{\partial t}=D \vec{\nabla} \cdot \vec{\nabla} n(\vec{r}, t)=D \Delta n(\vec{r}, t) .
$$


$D$ is the diffusion coefficient. This equation resembles in its form very much the Schrödinger equation for a free particle. The solution are nevertheless very different. If we only keep the isotropic solutions ${ }^{77}$ the classical correlation functions corresponding to the diffusion equation are found to be

$$
G_{\kappa}^{\mathrm{cl}}(\vec{r}, t)=\frac{1}{\sqrt{4 \pi D|t|}^{3}} \exp \left(-\frac{r^{2}}{4 D|t|}\right) .
$$

We would like to emphasise that $G_{\kappa}^{\mathrm{cl}}(\vec{r}, t)$ describes the correlation of a particle with itself. The correlation function is, as in the case of the Boltzmann particles, a Gaussian. However, we see that the width of this Gaussian does only increase with $\sqrt{t}$. The classical intermediate self scattering function can be determined by Fourier transformation

$$
\begin{aligned}
I_{s}^{\mathrm{cl}}(\vec{r}, t) & =\int \mathrm{d}^{3} r \exp (\mathrm{i} \vec{Q} \cdot \vec{r}) G_{\kappa}^{\mathrm{cl}}(\vec{r}, t) \\
& =\exp \left(-\frac{1}{2} Q^{2} \sigma^{2}(t)\right) \\
& =\exp \left(-Q^{2} D|t|\right) .
\end{aligned}
$$

${ }^{77}$ The $\nabla^{2}$ operator is well known from the Schrödinger equation. The isotropic solutions of the Fick law are of $s$-type (see Section 3.3). In this case we can set (see (3.46))

$$
\Delta=\left[\frac{\partial^{2}}{\partial r^{2}}+\frac{2}{r} \frac{\partial}{\partial r}\right]
$$

Using the Ansatz

$$
n(\vec{r}, t)=\frac{1}{\sqrt{(2 \pi)^{3}}} \frac{1}{\sigma(t)^{3}} \exp \left(-\frac{r^{2}}{2 \sigma(t)^{2}}\right)
$$

we verify that

$$
\left[\frac{\partial^{2}}{\partial r^{2}}+\frac{2}{r} \frac{\partial}{\partial r}\right] n(r, t)=\left[-\frac{3}{\sigma(t)^{2}}-\frac{r^{2}}{\sigma(t)^{4}}\right] n(\vec{r}, t)
$$

together with

$$
\frac{\partial}{\partial t} n(r, t)=\left[-\frac{3 \mathrm{~d} \sigma(t) / \mathrm{d} t}{\sigma(t)}-\frac{r^{2} \mathrm{~d} \sigma(t) / \mathrm{d} t}{\sigma(t)^{3}}\right] n(\vec{r}, t) .
$$

Hence we find a solution to the diffusion equation (8.110) provided that

$$
\sigma(t) \frac{\mathrm{d} \sigma(t)}{\mathrm{d} t}=D .
$$

This is the case if

$$
\frac{\mathrm{d}}{\mathrm{d} t} \sigma(t)^{2}=2 D
$$

or

$$
\sigma(t)=2 D|t|+C .
$$

For $t$ large, which here is of interest, we can neglect the constant $C$. The pair correlation function is found from $n(\vec{r}, t)$ with the boundary condition $n(0, t)=n_{0} \delta(\vec{r})$. 


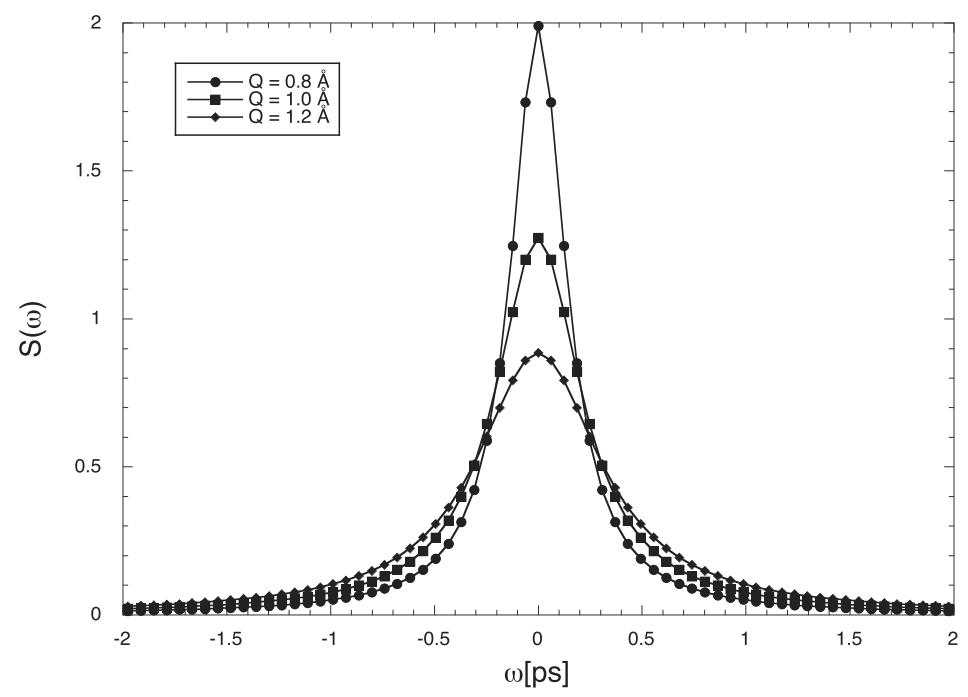

Fig. 40. The function $S_{\kappa}^{\mathrm{cl}}(Q, \omega)$ defined in (8.120) for different $Q$ values. The diffusion constant is chosen to be $2.5 \cdot 10^{-5} \mathrm{~cm}^{2} / \mathrm{s}$ corresponding to $0.25 \AA^{2} / \mathrm{ps}$. This is close to the diffusion constant of water at 25 degrees Celsius. One can compare the shape of these curves to the ones measured in Fig. 36 for liquid Decalin.

The Fourier transform of this function, and as a consequence the scattering function, is a Lorentzian

$$
\begin{aligned}
S_{s}^{\mathrm{cl}}(\vec{r}, t) & =\frac{1}{2 \pi \hbar} \int_{-\infty}^{\infty} I_{s}^{\mathrm{cl}}(\vec{r}, t) \exp (-\mathrm{i} \omega t) \mathrm{d} t \\
& =\frac{1}{2 \pi \hbar}\left\{\int_{0}^{\infty} \exp \left(-\left(Q^{2} D+\mathrm{i} \omega\right) t\right) \mathrm{d} t+\int_{-\infty}^{0} \exp \left(\left(Q^{2} D-\mathrm{i} \omega\right) t\right) \mathrm{d} t\right\} \\
& =\frac{1}{\pi \hbar} \frac{D Q^{2}}{\left(D Q^{2}\right)^{2}+\omega^{2}} .
\end{aligned}
$$

The function (see Fig. 40) is centred on $\omega=0$ and has a width (FWHM) of

$$
\hbar \Delta \omega=2 \hbar D Q^{2} .
$$

Thus we can clearly distinguish between the ballistic regime and the diffusive regime just from the shape of the dynamic response.

If we identify $S_{s}^{\mathrm{cl}}(\vec{r}, t)$ with the self scattering function $S^{s}(\vec{r}, t)$ this function will be symmetric and hence violate the principle of detailed balance. One solution to this problem is to identify $S_{s}^{\mathrm{cl}}(\vec{r}, t)$ with the symmetrised self scattering function $S_{s}^{\text {sym }}(\vec{r}, t)$ and to derive from there the self scattering function.

We will finally turn to the total cross section of an ensemble of Boltzmann particles. We need to calculate

$$
\sigma_{\text {tot }}=4 \pi \frac{k_{f}}{k_{i}} \int_{-\infty}^{\infty} \mathrm{d}(\hbar \omega) \sum_{\kappa} \overline{\left|b_{\kappa}\right|^{2}} S_{\kappa}(Q, \omega) .
$$

The presence of the factor $k_{f} / k_{i}$ makes the calculation quite tedious. For the reader who is keen to go through this in detail we refer to Lovesey [39]. Here we continue by stating the result in the limit of sufficiently large incident 
energy

$$
E_{i} \gg \frac{m_{\kappa}}{m_{n}}\left(k_{\mathrm{B}} T\right)
$$

In this case we find

$$
\sigma_{\text {tot }} \approx 4 \pi \sum_{\kappa} N_{\kappa} \overline{\left|b_{\kappa}\right|^{2}}\left(1+\frac{m_{n}}{m_{\kappa}}\right)^{-2} .
$$

For protons this cross section is a quarter of the bound cross section. This is the result that we expected. For very high energies the motion of the particles can be neglected. We, therefore, recognise the free particle case from Section 3.8. The expression (8.123) confirms the fact that in the developed formalism scattering lengths are "bound" scattering lengths.

\subsection{Response functions and susceptibility}

Linear response theory describes how a physical system reacts if perturbed weakly. Since most scattering probes are rather gentle we can hope that this theory will be useful when describing scattering processes. As the name indicates the theory is based on the assumption that the change in an observable is linear in the perturbation. ${ }^{78}$

For $t \rightarrow-\infty$, hence way before the interaction with the probe particle takes place, the system is described by the unperturbed Hamiltonian $\hat{H}_{0} \cdot{ }^{79}$ The perturbation is supposed to be switched on in an adiabatic way, which means that it builds up sufficiently slowly for the system to remain permanently in its equilibrium state. ${ }^{80}$ The perturbation adds the term $\hat{H}_{p}=-\hat{B} F(t)$ to the Hamiltonian

$$
\hat{H}=\hat{H}_{0}-\hat{B} F(t)
$$

$\hat{B}$ is a time independent operator that characterises the perturbation. The externally imposed time evolution of the perturbation is fully contained within the function $F(t)$. The changes induced in the system by the perturbation will be monitored via an observable $\hat{A}$. The expectation value of $\hat{A}$ can be determined with the help of the statistical density operator. Without perturbation

$$
\begin{aligned}
& \langle\hat{A}\rangle=\operatorname{Tr}\left\{\rho_{0} \hat{A}\right\}, \\
& \rho_{0}=\frac{1}{Z} \exp \left(-\frac{\hat{H}_{0}}{k_{\mathrm{B}} T}\right), \quad Z=\operatorname{Tr} \exp \left(-\frac{\hat{H}_{0}}{k_{\mathrm{B}} T}\right),
\end{aligned}
$$

$\langle\hat{A}\rangle$ is independent of time. When we switch on the perturbation the statistical density operator will change and so will the expectation value of $\hat{A}$

$$
\begin{aligned}
& \langle\hat{A}(t)\rangle=\operatorname{Tr}\{\rho(t) \hat{A}\}, \\
& \rho(t)=\frac{1}{Z} \exp \left(-\frac{\hat{H}}{k_{\mathrm{B}} T}\right), \quad Z=\operatorname{Tr} \exp \left(-\frac{\hat{H}}{k_{\mathrm{B}} T}\right) .
\end{aligned}
$$

\footnotetext{
${ }^{78}$ The subject is very well described in the book by Jensen and Mackintosh [34].

${ }^{79}$ We use in this section the $\hat{A}$ notation to designate quantum mechanical observables.

${ }^{80}$ We have already encountered this problem when we described scattering in terms of stationary states. These states correspond to a stationary, i.e. time invariant flux of particles. The question we had to answer was, therefore, how to initiate this flux in the first place. The solution we had proposed was to employ an adiabatic process.
} 
This evolution can be described in terms of a response function $\Phi_{A, B}\left(t-t^{\prime}\right)$. Formally

$$
\Delta\langle\hat{A}(\vec{r}, t)\rangle=\langle\hat{A}(\vec{r}, t)\rangle-\langle\hat{A}(\vec{r})\rangle=\int_{-\infty}^{t} \mathrm{~d} t^{\prime} \int \mathrm{d}^{3} \vec{r}^{\prime} \Phi_{A, B}\left(\vec{r}-\vec{r}^{\prime} ; t-t^{\prime}\right) F\left(\vec{r}^{\prime}, t^{\prime}\right) .
$$

The response function

$$
\Phi_{A, B}\left(\vec{r}-\vec{r}^{\prime} ; t-t^{\prime}\right)
$$

describes the reaction of the observable $\hat{A}$ at the position $\vec{r}$ and at time $t$ to a perturbation $\hat{B} F$ induced at the position $\vec{r}^{\prime}$ at time $t^{\prime}$. The response is in general neither local in space nor local in time given that a perturbation at $\vec{r}^{\prime}$ may in principle produce an instantaneous or a delayed reaction at any other position $\vec{r}$. The only stringent condition that we have to impose onto the response function is the respect of causality. Reaction cannot precede perturbation. We impose causality via the limits used for the integration in time. To render the notation digestible we will in the following refrain from explicitly mentioning the dependence of the observables on $\vec{r}$.

With the Kubo formula the response function can be calculated as ${ }^{81}$

$$
\Phi_{A, B}\left(t-t^{\prime}\right)=\frac{\mathrm{i}}{\hbar}\left\langle\left[\hat{A}(t), \hat{B}\left(t^{\prime}\right)\right]\right\rangle \Theta\left(t-t^{\prime}\right),
$$

with the special case

$$
\Phi_{A, B}(t)=\frac{\mathrm{i}}{\hbar}\langle[\hat{A}(t), \hat{B}]\rangle \Theta(t)
$$

The presence of $\Theta(t)$ assures as mentioned above causality. These response functions are, therefore, called causal response functions. For the calculations that follow it is helpful to define also the more general response functions

$$
K_{A, B}(t)=\frac{\mathrm{i}}{\hbar}\langle[\hat{A}(t), \hat{B}]\rangle
$$

We verify that

$$
K_{A, B}(t)= \begin{cases}K_{A, B}(t) & \text { for } t>0 \\ -K_{B, A}(-t) & \text { for } t<0\end{cases}
$$

In the case of nuclear neutron scattering the perturbation of the target system is created via the interaction of the neutron wave with the Fermi potential (5.94). This interaction involves the atomic density operator $\hat{\rho}_{\kappa}(\vec{r}, t)(8.17)$. Seen from the point of view of the system the perturbation is caused within the density. Preferring to work in reciprocal space we, therefore, identify

$$
B(t) \equiv \hat{\rho}_{\kappa}(\vec{Q}, t) .
$$

The perturbation induced in the atomic density will propagate throughout the system. We, therefore, decide to monitor the response to the disturbance equally in terms of the atomic number density. ${ }^{82}$ The conjugate of the operator $\hat{\rho}_{\kappa}(\vec{Q}, t)$ is $\hat{\rho}_{\kappa}^{+}(\vec{Q}, t)=\hat{\rho}_{\kappa}(-\vec{Q}, t)$. As consequence

$$
A(t) \equiv \hat{B}^{+}(t)=\hat{\rho}_{\kappa}(-\vec{Q}, t)
$$

\footnotetext{
${ }^{81}$ The Heaviside step function is defined by $\Theta(t)=1, t>0$, and 0 otherwise. The Kubo formula can be derived with quantum statistical methods.

${ }^{82}$ For example via the creation or absorption of a phonon.
} 
where we implicitly allow the index $\kappa$ to vary between $A=B^{+}$and $B$, which means that we include the response of the atoms of the sub-system of type $\kappa$ to a perturbation within the sub-system of type $\kappa^{\prime}$.

In this notation the partial scattering functions can be written as

$$
\begin{aligned}
S_{\kappa, \kappa^{\prime}}(\vec{Q}, \omega) & =\frac{1}{2 \pi \hbar} \int_{-\infty}^{\infty} \mathrm{d} t \exp (-\mathrm{i} \omega t) I_{\kappa, \kappa^{\prime}}(\vec{Q}, t) \\
& =\frac{1}{2 \pi \hbar} \int_{-\infty}^{\infty} \mathrm{d} t \exp (-\mathrm{i} \omega t)\left\langle\hat{B}, \hat{B}^{+}(t)\right\rangle .
\end{aligned}
$$

To establish a relation between the scattering function and the response function $\Phi_{B^{+} B}(t)$ we evaluate the Fourier transform of $K_{B+B}(t)$.

$$
\begin{aligned}
\frac{1}{2 \pi} \int_{-\infty}^{\infty} \mathrm{d} t \exp (-\mathrm{i} \omega t) K_{B^{+} B}(t) & =\frac{\mathrm{i}}{2 \pi \hbar} \int_{-\infty}^{\infty} \mathrm{d} t \exp (-\mathrm{i} \omega t)\left\langle\hat{B}^{+}(t) \hat{B}-\hat{B} \hat{B}^{+}(t)\right\rangle \\
& =-\mathrm{i} S_{\kappa, \kappa^{\prime}}(\vec{Q}, \omega)+\frac{\mathrm{i}}{2 \pi \hbar} \int_{-\infty}^{\infty} \mathrm{d} t \exp (-\mathrm{i} \omega t)\left\langle\hat{B}^{+}(t) \hat{B}\right\rangle .
\end{aligned}
$$

With the help of expression (8.55) we can reverse the order of the operators. We find

$$
\left\langle\hat{B}^{+}(t) \hat{B}\right\rangle=\left\langle\hat{B} \hat{B}^{+}\left(t+\mathrm{i} \hbar \frac{\hbar \omega}{k_{\mathrm{B}} T}\right)\right\rangle
$$

and as a consequence

$$
\begin{aligned}
\frac{1}{2 \pi \hbar} \int_{-\infty}^{\infty} \mathrm{d} t \exp (-\mathrm{i} \omega t)\left\langle\hat{B}^{+}(t) \hat{B}\right\rangle & =\frac{1}{2 \pi \hbar} \int_{-\infty}^{\infty} \mathrm{d} t \exp (-i \omega t)\left\langle\hat{B} \hat{B}^{+}\left(t+\mathrm{i} \frac{\hbar}{k_{\mathrm{B}} T}\right)\right\rangle \\
& =\frac{1}{2 \pi \hbar} \exp \left(-\frac{\hbar \omega}{k_{\mathrm{B}} T}\right) \int_{-\infty}^{\infty} \mathrm{d} t \exp (-\mathrm{i} \omega t)\left\langle\hat{B} \hat{B}^{+}(t)\right\rangle \\
& =\frac{1}{2 \pi \hbar} \exp \left(-\frac{\hbar \omega}{k_{\mathrm{B}} T}\right) S_{\kappa, \kappa^{\prime}}(\vec{Q}, \omega) .
\end{aligned}
$$

Combining the expressions (8.134) and (8.136) we arrive at the expression

$$
S_{\kappa, \kappa^{\prime}}(\vec{Q}, \omega)=(1+n(\omega)) \frac{\mathrm{i}}{2 \pi} \int_{-\infty}^{\infty} \mathrm{d} t \exp (-\mathrm{i} \omega t) K_{B^{+} B^{(}}(t)
$$

We have in an explicit manner derived a version of the fluctuation-dissipation theorem for neutrons. This expression has the advantage of being formulated in terms of a regular Fourier transform. This is possible at the price of working with response functions that are not causal.

To arrive at the usual version of the fluctuation-dissipation theorem we remind ourselves that the general susceptibility is defined in terms of a Laplace transform ${ }^{83}$ of the causality respecting response function

$$
\chi_{B^{+} B}[\omega]=\lim _{\varepsilon \rightarrow 0^{+}} \chi_{B^{+} B}[\mathrm{i} p=\omega+\mathrm{i} \varepsilon]=\lim _{\varepsilon \rightarrow 0^{+}} \int_{0}^{\infty} \mathrm{d} t \Phi_{B^{+} B}(t) \exp (p t) .
$$

\footnotetext{
${ }^{83}$ The Laplace transform of a function $f(t)$ of a real and positive variable $t$ is the function $F(p)$ of the complex variable $p$ defined by

$$
F(p):=\int_{0}^{\infty} \mathrm{d} t f(t) \exp (-p t)
$$
}


The notation $[\omega]$ emphasises the fact that we are dealing with a Laplace transform and not with a Fourier transform.

The susceptibility relates the Fourier transform of the observable $\hat{A}$

$$
A(\omega)=\langle\hat{A}(\omega)\rangle=\lim _{\varepsilon \rightarrow 0^{+}} \int_{-\infty}^{\infty} \mathrm{d} t \mathrm{e}^{\mathrm{i} \omega t} \mathrm{e}^{-\varepsilon t}(\langle\hat{A}(t)\rangle-\langle\hat{A}(t \rightarrow-\infty)\rangle),
$$

to the Fourier transform of the perturbation $F(\omega)$ via

$$
A(\omega)=\chi[\omega] F(\omega)
$$

In the definition of $A(\omega)$ we have made sure that the perturbation is switched on adiabatically. For a periodic perturbation this can only be done by ramping up the amplitude. The ramp is provided by the factor $\exp (-\varepsilon t)$.

The susceptibility is composed of two parts

$$
\chi_{B^{+} B_{B}}[p]=\chi_{B^{+} B_{B}}^{\prime}[p]+\mathrm{i} \chi_{B^{+} B}^{\prime \prime}[p] .
$$

The first part

$$
\chi_{B^{+} B^{\prime}}^{\prime}[p]:=\frac{1}{2}\left(\chi_{B^{+} B}[p]+\chi_{B B^{+}}\left[-p^{*}\right]\right)
$$

is reactive and the second part

$$
\chi_{B^{+} B^{\prime \prime}}^{\prime \prime}[p]:=\frac{1}{2 \mathrm{i}}\left(\chi_{B^{+} B}[p]-\chi_{B B^{+}}\left[-p^{*}\right]\right)
$$

is dissipative. The two parts are related by the Kramers-Kronig relation

$$
\chi^{\prime}[\omega]=P \int_{-\infty}^{\infty} \mathrm{d} \omega^{\prime} \frac{1}{\pi} \frac{\chi^{\prime \prime}\left[\omega^{\prime}\right]}{\omega^{\prime}-\omega}
$$

with $P$ denoting the Cauchy principle value.

To see why it is justified to call the real part reactive and the imaginary part dissipative we consider the dynamic response to a periodic perturbation. If we choose for the time evolution a simple oscillatory form

$$
F(t)=F_{0} \cos \left(\omega_{0} t\right)
$$

then the Fourier transform

$$
F(\omega)=\int_{-\infty}^{\infty} F(t) \mathrm{e}^{\mathrm{i} \omega t} \mathrm{~d} t
$$

is given by

$$
F(\omega)=\frac{F_{0}}{2}\left(\delta\left(\omega-\omega_{0}\right)+\delta\left(\omega+\omega_{0}\right)\right) .
$$

The spectral response is obtained via

$$
A(\omega)=\chi[\omega] \frac{F_{0}}{2}\left(\delta\left(\omega-\omega_{0}\right)+\delta\left(\omega+\omega_{0}\right)\right) .
$$


Inverse Fourier transformation brings us back into the time domain

$$
\begin{aligned}
\delta\langle\hat{A}(t)\rangle & =\frac{1}{2 \pi} \int_{-\infty}^{\infty} \mathrm{d} \omega \mathrm{e}^{-\mathrm{i} \omega t} A(\omega) \\
& =\frac{1}{2 \pi} \int_{-\infty}^{\infty} \mathrm{d} \omega \mathrm{e}^{-\mathrm{i} \omega t} \chi[\omega] F(\omega) \\
& =\frac{F_{0}}{4 \pi}\left[\chi\left[\omega_{0}\right] \mathrm{e}^{-\mathrm{i} \omega_{0} t}+\chi\left[-\omega_{0}\right] \mathrm{e}^{\mathrm{i} \omega_{0} t}\right] \\
& =\frac{F_{0}}{4 \pi}\left[\cos \omega_{0} t\left\{\chi\left[\omega_{0}\right]+\chi\left[-\omega_{0}\right]\right\}+\mathrm{i} \sin \omega_{0} t\left\{\chi\left[\omega_{0}\right]-\chi\left[-\omega_{0}\right]\right\}\right] \\
& =\frac{F_{0}}{2 \pi}\left[\chi^{\prime}\left[\omega_{0}\right] \cos \omega_{0} t+\chi^{\prime \prime}\left[-\omega_{0}\right] \sin \omega_{0} t\right]
\end{aligned}
$$

where we have used (see (8.143) and (8.144))

$$
\chi^{\prime}[p]:=\frac{1}{2}\left(\chi[p]+\chi\left[-p^{*}\right]\right)
$$

and

$$
\chi^{\prime \prime}[p]:=\frac{1}{2 \mathrm{i}}\left(\chi[p]-\chi\left[-p^{*}\right]\right)
$$

These properties can be rigorously demonstrated starting from the definition (8.139) of the susceptibility. We see that the real part of the susceptibility $\chi^{\prime}$ describes a response that is in phase (reactive) with the perturbation while the imaginary part gives a response in anti-phase with the perturbation (dissipative).$^{84}$

The Fourier transform of the function $K_{B^{+} B}(t)$ found in the expression (8.137) is closely related to the susceptibility. It is

$$
\begin{aligned}
& \int_{-\infty}^{\infty} \mathrm{d} t \exp (-\mathrm{i} \omega t) K_{B^{+} B}(t) \\
& \quad=\lim _{\varepsilon \rightarrow 0^{+}} \int_{-\infty}^{\infty} \mathrm{d} t \exp (-\mathrm{i} \omega t-\varepsilon|t|) K_{B^{+} B}(t) \\
& \quad=\lim _{\varepsilon \rightarrow 0^{+}}\left\{\int_{0}^{\infty} \mathrm{d} t \exp (-\mathrm{i} \omega t-\varepsilon t) K_{B^{+} B}(t)+\int_{-\infty}^{0} \mathrm{~d} t \exp (-\mathrm{i} \omega t+\varepsilon t) K_{B^{+} B}(t)\right\} \\
& =\lim _{\varepsilon \rightarrow 0^{+}}\left\{\int_{0}^{\infty} \mathrm{d} t \exp (-\mathrm{i} \omega t-\varepsilon t) K_{B^{+} B}(t)-\int_{0}^{\infty} \mathrm{d} t \exp (+\mathrm{i} \omega t-\varepsilon t) K_{B B^{+}}(t)\right\} \\
& =\chi_{B^{+} B}[\omega]-\chi_{B B^{+}}[-\omega] \\
& =2 \mathrm{i} \chi_{B^{+} B^{\prime \prime}}[\omega],
\end{aligned}
$$

where we have used the identity

$$
K_{B^{+B}}(-t)=-K_{B B^{+}}(t)
$$

\footnotetext{
${ }^{84}$ If the response is in phase with the driving force then the driving force does not do work. For an electrical motor to do work the rotor has to be phase delayed with respect to the driving magnetic field of the stator.
} 
which can be verified easily on the basis of Eq. (8.132).

Inserting (8.153) into (8.137) we find

$$
S_{\kappa, \kappa^{\prime}}(\vec{Q}, \omega)=(1+n(\omega)) \cdot \frac{1}{\pi} \cdot \chi_{\vec{Q}}^{\prime \prime}[\omega]
$$

This is the commonly known form of the fluctuation-dissipation theorem.

We would like to illustrate the general formalism with a simple example. Let us look at a damped harmonic oscillator under the influence of an external force. The equation of motion for the expectation value $\langle x\rangle$ that we formulate as a classical equation of motion ${ }^{85}$ reads

$$
m\langle\ddot{x}(t)\rangle+m \gamma\langle\dot{x}(t)\rangle+m \omega_{0}^{2}\langle x(t)\rangle=f(t)
$$

where we have introduced a frictional damping that is proportional to the velocity. Its strength is given by the damping constant $\gamma$. The quantity

$$
m \omega_{0}^{2}=K
$$

is the force constant. Going into Fourier space the equation of motion becomes

$$
\left(-\omega^{2}+\omega_{0}^{2}+\mathrm{i} \gamma \omega\right) x(\omega)=\frac{f(\omega)}{m} .
$$

This leads us to identify the susceptibility according to expression (8.141) as

$$
\chi[\omega]=\frac{1}{m} \frac{1}{\omega_{0}^{2}-\omega^{2}+\mathrm{i} \gamma \omega} .
$$

The imaginary part that describes dissipation is given by

$$
\chi^{\prime \prime}[\omega]=\frac{1}{m} \frac{\gamma \omega}{\left(\omega_{0}^{2}-\omega^{2}\right)^{2}+(\gamma \omega)^{2}}
$$

and the real part describing reaction as

$$
\chi^{\prime}[\omega]=\frac{1}{m} \frac{\left(\omega_{0}^{2}-\omega^{2}\right)}{\left(\omega_{0}^{2}-\omega^{2}\right)^{2}+(\gamma \omega)^{2}} .
$$

$\chi^{\prime \prime}[\omega]$ is an odd function of $\omega$ that for large $\omega_{0}$ and small damping can be assimilated to the sum of two Lorentzian functions (see expression (10.108)). Their half width at half maximum width is given by the damping constant (see Fig. 41). It is confirmed that the susceptibility of a harmonic oscillator is strictly independent of temperature.

The validity of the Kramers-Kronig relation (8.145) is easily verified for the static part $\chi^{\prime}[0]$

$$
\int_{-\infty}^{\infty} \mathrm{d} \omega \frac{\chi^{\prime \prime}[\omega]}{\omega}=\frac{\pi}{m \omega_{0}^{2}}=\frac{\pi}{K}=\pi \chi^{\prime}[0] .
$$

\footnotetext{
${ }^{85}$ The Ehrenfest theorem [10] stipulates that the expectation values of position and momentum operators $\left\langle\mathbf{Q}_{i}\right\rangle$ and $\left\langle\mathbf{P}_{i}\right\rangle$ satisfy the same equations as the classical variables provided that the operators $\mathbf{Q}_{i}$ and $\mathbf{P}_{i}$ appear only to first and second order in the Hamiltonian, which is the case for a harmonic system. But attention, the quantum theory of the damped harmonic oscillator is a subject of current research. Quantisation encounters an obstacle in the dissipation of energy introduced by the damping term.
} 

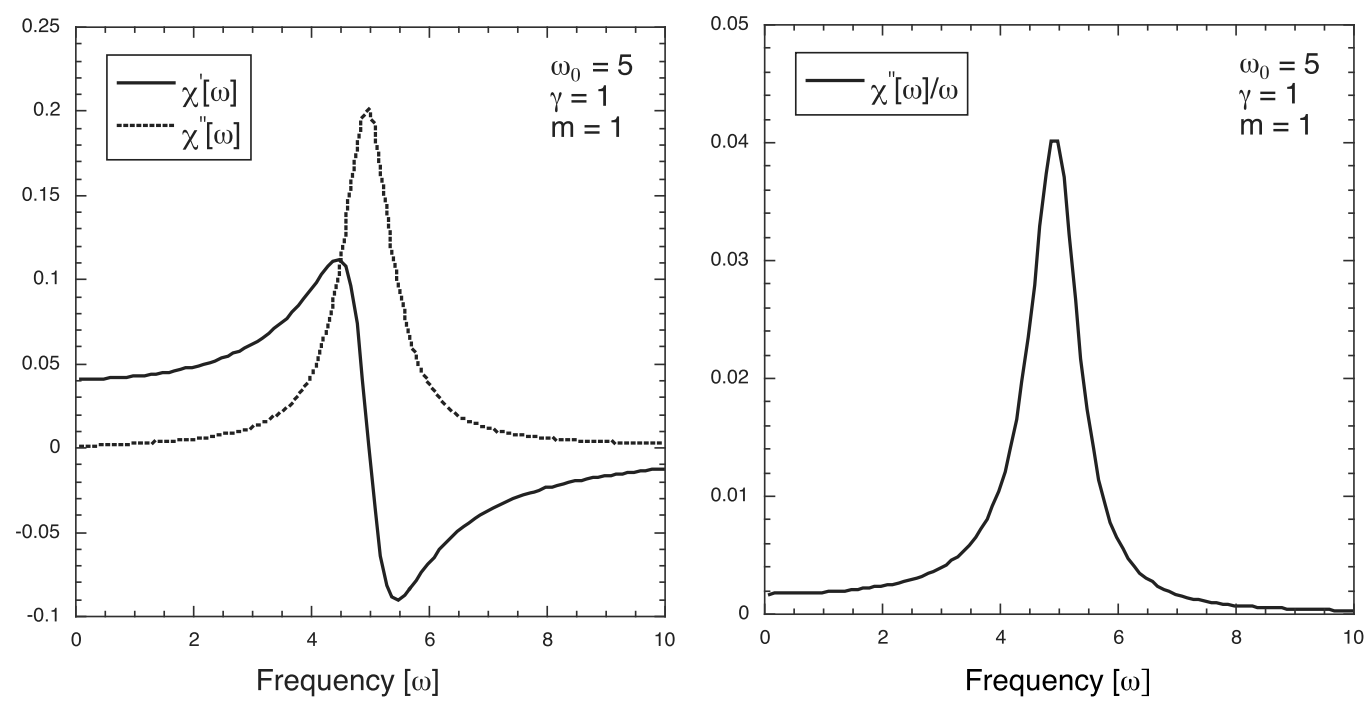

Fig. 41. Susceptibility of a damped harmonic oscillator. To the left the real and imaginary parts of $\chi[\omega]$. To the right the function $\chi^{\prime \prime} / \omega$. The surface below this function is $\chi^{\prime}[0]=K^{-1}$ and thus temperature independent.

The force constant $K$ is expressed in units of $\mathrm{Nm}^{-1} . K^{-1}$ gives the change in position $\langle x\rangle$ per unit of applied force, which is the static response of the system.

Knowing the susceptibility the fluctuation-dissipation theorem (8.155) gives immediately access to the fluctuations of the variable $x$

$$
S_{x x}(\omega)=(1+n(\omega)) \cdot \frac{1}{\pi m} \cdot \frac{\gamma \omega}{\left(\omega^{2}-\omega_{0}^{2}\right)^{2}+\gamma^{2} \omega^{2}} .
$$

When the damping tends to zero then the Lorentzian functions become Dirac functions

$$
S_{x x}(\omega)=\frac{1}{2 m \omega_{0}}\left[(1+n(\omega)) \delta\left(\omega-\omega_{0}\right)+n(\omega) \delta\left(\omega+\omega_{0}\right)\right],
$$

where we have used

$$
1+n(\omega)+n(-\omega)=0 .
$$

This concept will be generalised in Section 10.7 .

\subsection{Moments and sum rules for the scattering function}

For a function $f(x)$, continuous on an interval $I$ (that is not reduced to a point), we define (under the assumption of existence) the $n$th moment of $f$ as

$$
m_{n}(f):=\int_{I} x^{n} f(x) \mathrm{d} x
$$

where $n$ is a natural number. For the partial scattering function the moments with respect to the energy are written as

$$
S_{\kappa, \kappa^{\prime}}^{n}(\vec{Q}, \omega):=\int_{-\infty}^{\infty} S_{\kappa, \kappa^{\prime}}(\vec{Q}, \omega)(\hbar \omega)^{n} \mathrm{~d}(\hbar \omega)
$$


Using the definition (8.38) the zeroth moment is directly related to the intermediate scattering function

$$
\begin{aligned}
S_{\kappa, \kappa^{\prime}}(\vec{Q}) & :=S_{\kappa, \kappa^{\prime}}^{0}(\vec{Q}, \omega) \\
& =\int_{-\infty}^{\infty} S_{\kappa, \kappa^{\prime}}(\vec{Q}, \omega) \mathrm{d}(\hbar \omega) \\
& =\int_{-\infty}^{\infty} \mathrm{d} t I_{\kappa, \kappa^{\prime}}(\vec{Q}, t) \frac{1}{2 \pi \hbar} \int_{\infty}^{\infty} \mathrm{d}(\hbar \omega) \mathrm{e}^{-\mathrm{i} \omega t} \\
& =\int_{-\infty}^{\infty} \mathrm{d} t I_{\kappa, \kappa^{\prime}}(\vec{Q}, t) \delta(t) \\
& =I_{\kappa, \kappa^{\prime}}(\vec{Q}, t=0) .
\end{aligned}
$$

In Fig. 42 we show an example of an intermediate scattering function $I(Q, t)$. This function is the result of a classical molecular dynamics simulation. For $t=0$ we obtain the structure factor $S(q)$.

$S_{\kappa, \kappa^{\prime}}(\vec{Q})$ is the static partial structure factor or in the case of an monatomic system the (static) structure factor in short. It is of great importance when studying structures. In the case of diffraction the final energy is not analysed and hence the measurement corresponds to an integration of the signal over the energy. ${ }^{86}$ Furthermore the cross section contains the factor $k_{f} / k_{i}$. Such a measurement yields the structure factor only in the case where $k_{f} \approx$

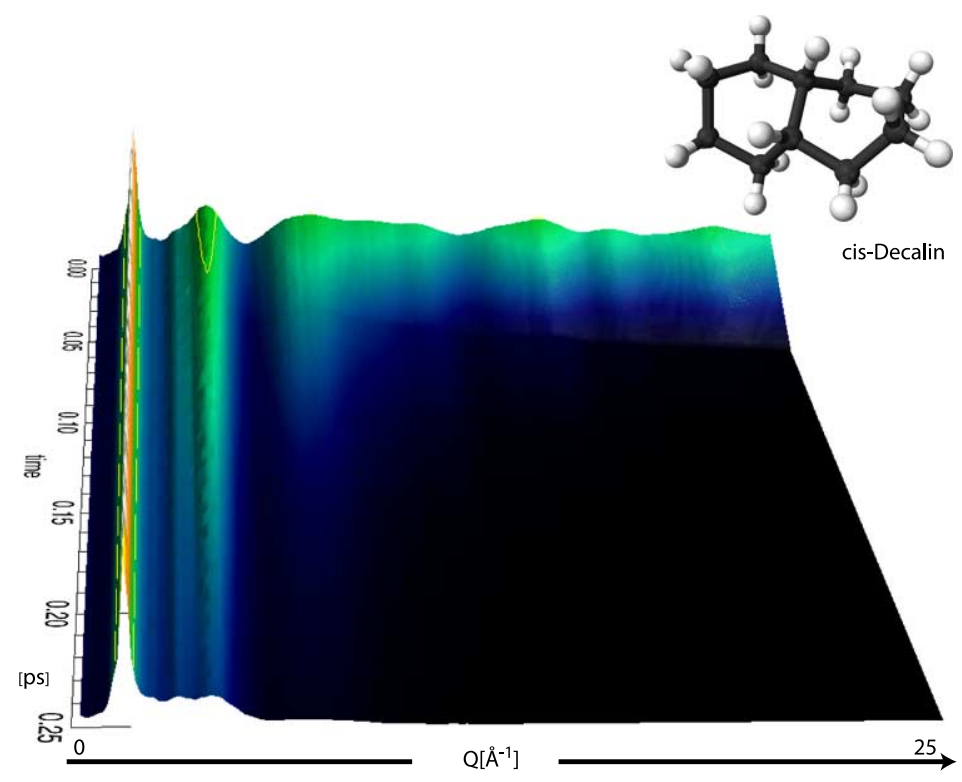

Fig. 42. The intermediate scattering function of deuterated cis-Decalin $\left(\mathrm{C}_{10} \mathrm{D}_{18}\right)$ in its liquid state normalised to the number of scatterers. Time is given in units of picoseconds. The scattering vector $Q$ covers the range from 1 to $25 \AA^{-1}$. For $t=0$ the intermediate scattering function corresponds to the structure factor $S(Q)$. With time the correlations decay. The relaxation time is visibly longest for $Q$ values that correspond to maxima of the static structure factor $S(Q)$. For large $Q$ values the static structure factor oscillates around the value of one and this with continuously diminishing amplitude as we increase $Q$. In addition $S(Q, \omega)$ decreases fast with time. The expected value for the incoherent scattering is equally one (see (8.171)) in this normalisation. From this fact we can conclude that no correlations persist at very short distances. We only observe the average density at high $Q$ (image courtesy of S. Eibl). (Colors are visible in the online version of the article; http://dx.doi.org/10.3233/JNR-140016.)

${ }^{86}$ Attention, it is an integration made with a fixed scattering angle. 
$k_{i}$ over the whole excitation spectrum. ${ }^{87}$ This is certainly the case for X-rays. For neutrons this condition can be assumed valid for large $k_{i}$. But even in this case inelastic corrections should be taken into account. These corrections are known as Placzek corrections [4].

We would like to emphasise that the integration over the final energy in diffraction experiments corresponds to the superposition of the cliches obtained via a representative series of snapshots of the structure. The exposure time for each snapshot is defined by the integration interval in energy. The larger the energy interval covered the shorter the exposure time. The limit of purely elastic intensity corresponds to infinite exposure times.

The expressions for the moments of the scattering function have the advantage of not involving time-ordering of operators. As a consequence we find

$$
\begin{aligned}
I_{\kappa, \kappa^{\prime}}(\vec{Q}, t=0) & =\sum_{j \in\left\{j_{\kappa}\right\}, j^{\prime} \in\left\{j_{\kappa^{\prime}}\right\}}\left\langle\exp \left(-\mathrm{i} \vec{Q} \cdot \vec{R}_{j}\right) \exp \left(\mathrm{i} \vec{Q} \cdot \vec{R}_{j^{\prime}}\right)\right\rangle \\
& =\sum_{j \in\left\{j_{\kappa}\right\}, j^{\prime} \in\left\{j_{\kappa^{\prime}}\right\}}\left\langle\exp \left(-\mathrm{i} \vec{Q} \cdot\left(\vec{R}_{j}-\vec{R}_{j^{\prime}}\right)\right)\right\rangle \\
& =\int_{V} \mathrm{~d}^{3} r \exp (-\mathrm{i} \vec{Q} \cdot \vec{r}) G_{\kappa, \kappa^{\prime}}(\vec{r}, t=0) \\
& =\int_{V} \mathrm{~d}^{3} r \exp (-\mathrm{i} \vec{Q} \cdot \vec{r})^{\mathrm{cl}} G_{\kappa, \kappa^{\prime}}(\vec{r}, t=0),
\end{aligned}
$$

with the classical version of the density correlation function $G_{\kappa, \kappa^{\prime}}(\vec{r}, t)$ defined in (8.23). If the system under investigation is monatomic this result can be expressed in terms of the static pair distribution function $g(\vec{r})$

$$
\frac{I(\vec{Q}, t=0)}{N}=1+\int_{V} \mathrm{~d}^{3} r \exp (-\mathrm{i} \vec{Q} \cdot \vec{r}) g(\vec{r})
$$

with

$$
{ }^{\mathrm{cl}} G(\vec{r}, t=0)=\delta(\vec{r})+g(\vec{r})=\delta(\vec{r})+\sum_{j \neq 0}\left\langle\delta\left(\vec{r}-\left(\vec{R}_{j}-\vec{R}_{j=0}\right)\right)\right\rangle .
$$

$g(\vec{r})$ provides the average atomic number density as a function of position.

The moment of the self scattering function is

$$
\begin{aligned}
S_{\kappa}^{s}(\vec{Q}): & =\int_{\infty}^{\infty} S_{\kappa}^{s}(\vec{Q}, \omega) \mathrm{d}(\hbar \omega) \\
& =I_{\kappa, \kappa^{\prime}}^{s}(\vec{Q}, t=0) \\
& \left.=\int_{V} \mathrm{~d}^{3} r \exp (-\mathrm{i} \vec{Q} \cdot \vec{r})\right) G_{\kappa}^{s}(\vec{r}, t=0) \\
& =N_{\kappa} .
\end{aligned}
$$

The integral over energy of the incoherent scattering function, normalised to the number of scatterers, is therefore equal to unity. The signal that is lost in the elastic channel has to show up imperatively in the inelastic channel at the same $\vec{Q}$ value. Attention, this does not mean that the integrated intensity for a specific scattering angle should

${ }^{87}$ Often this factor is implicitly corrected for by the efficiency of the detectors, which diminishes in proportion to the velocity of the neutrons. 
be constant. Apart from the fact that the integration is not done for a constant $\vec{Q}$ only part of the inelastic signal is covered by the measurement due to kinematic constraints.

With the rules of calculation for Fourier transforms we can relate the higher order moments of the scattering functions to the derivatives of the intermediate scattering functions at $t=0$ [14]

$$
S_{\kappa, \kappa^{\prime}}^{n}(\vec{Q}, \omega)=\left(\frac{\hbar}{\dot{\mathrm{i}}}\right)^{n}\left[\frac{\partial^{n}}{\partial t^{n}} I_{\kappa, \kappa^{\prime}}(\vec{Q}, t)\right]_{t=0} .
$$

These moments are of great practical use. They permit both to test the quality of the experimental data and at the same time of the models used to interpret them.

Of the higher order moments the first moment is of particular interest. It is related to the first derivative of the intermediate scattering function

$$
\begin{aligned}
\frac{\partial}{\partial t} I_{\kappa, \kappa^{\prime}}(\vec{Q}, t) & =\left\langle\hat{\rho}_{\kappa}(\vec{Q}) \frac{\partial}{\partial t} \hat{\rho}_{\kappa^{\prime}}(-\vec{Q}, t)\right\rangle \\
& =-\frac{\mathrm{i}}{\hbar}\left\langle\hat{\rho}_{\kappa}(\vec{Q})\left[\hat{\rho}_{\kappa^{\prime}}(-\vec{Q}), \hat{H}\right]\right\rangle,
\end{aligned}
$$

where, in order to arrive at the last line, we have applied the usual formula that expresses the derivative of a Heisenberg operator in terms of its commutator with the Hamiltonian.

To calculate this moment we prefer nevertheless to use the non-causal response function

$$
K_{\hat{\rho}_{\kappa}(-\vec{Q}) \hat{\rho}_{\kappa^{\prime}}(\vec{Q})}(t)=\frac{\mathrm{i}}{\hbar}\left\langle\left[\hat{\rho}_{\kappa}(-\vec{Q}, t), \hat{\rho}_{\kappa^{\prime}}(\vec{Q})\right]\right\rangle
$$

that we have introduced in Section 8.11, and not the intermediate scattering function. By using an inverse Fourier transformation we obtain from the fluctuation dissipation theorem (8.137) an expression for $K_{\kappa, \kappa^{\prime}}(\vec{Q}, t)$ in terms of the scattering function

$$
-\mathrm{i} \int_{-\infty}^{\infty} \mathrm{d} \omega \exp (\mathrm{i} \omega t)(1+n(\omega))^{-1} S_{\kappa, \kappa^{\prime}}(\vec{Q}, \omega)=K_{\kappa, \kappa^{\prime}}(\vec{Q}, t)
$$

and as a consequence

$$
\int_{-\infty}^{\infty} \mathrm{d} \omega \omega(1+n(\omega))^{-1} S_{\kappa, \kappa^{\prime}}(\vec{Q}, \omega)=\left.\frac{\partial}{\partial t} K_{\kappa, \kappa^{\prime}}(\vec{Q}, t)\right|_{t=0}
$$

With the detailed balance principle (8.59) we are able to show that

$$
\int_{-\infty}^{\infty} \mathrm{d} \omega \omega(1+n(\omega))^{-1} S_{\kappa, \kappa^{\prime}}(\vec{Q}, \omega)=2 \int_{-\infty}^{\infty} \mathrm{d} \omega \omega S_{\kappa, \kappa^{\prime}}(\vec{Q}, \omega)
$$

under the condition that $S_{\kappa, \kappa^{\prime}}(\vec{Q}, \omega)=S_{\kappa, \kappa^{\prime}}(-\vec{Q}, \omega)$. Hence we get a direct relationship between the derivative of the response function and the first moment of the scattering function

$$
\int_{-\infty}^{\infty} \mathrm{d} \omega \omega S_{\kappa, \kappa^{\prime}}(\vec{Q}, \omega)=\left.\frac{1}{2} \frac{\partial}{\partial t} K_{\kappa, \kappa^{\prime}}(\vec{Q}, t)\right|_{t=0}
$$


We calculate the derivative with respect to time from the commutator with the Hamiltonian

$$
\begin{aligned}
\frac{\partial}{\partial t} K_{\kappa, \kappa^{\prime}}(\vec{Q}, t) & =\frac{\mathrm{i}}{\hbar}\left\langle\left[\frac{\partial}{\partial t} \hat{\rho}_{\kappa^{\prime}}(-\vec{Q}, t), \hat{\rho}_{\kappa}(\vec{Q})\right]\right\rangle \\
& =\frac{1}{\hbar^{2}}\left\langle\left[\left[\hat{\rho}_{\kappa^{\prime}}(-\vec{Q}), \hat{H}\right], \hat{\rho}_{\kappa}(\vec{Q})\right]\right\rangle .
\end{aligned}
$$

In many cases we can safely assume that the interaction between atoms is independent of the moments $\vec{P}_{j}$ of the atoms. In this case the Hamiltonian takes the form

$$
\hat{H}=\sum_{\kappa} \sum_{j \in\left\{j_{\kappa}\right\}} \frac{\left(\vec{P}_{j}\right)^{2}}{2 m_{\kappa}}+V\left(\left\{\vec{R}_{j}\right\}\right) .
$$

The commutator of the number density operator and the Hamiltonian is defined as

$$
\left[\hat{\rho}_{\kappa^{\prime}}(-\vec{Q}), \hat{H}\right]=\sum_{\kappa} \sum_{j^{\prime} \in\left\{j_{\kappa}\right\}}\left[\exp \left(\mathrm{i} \vec{Q} \cdot \vec{R}_{j^{\prime}}\right), \hat{H}\right] .
$$

In this expression all the operators are operators in the Schrödinger picture, i.e. not dependent on time. As a consequence

$$
\left[\exp \left(\mathrm{i} \vec{Q} \cdot \vec{R}_{j^{\prime}}\right), V\left(\left\{\vec{R}_{j}\right\}\right)\right]=0 \quad \forall \vec{R}_{j}
$$

and hence

$$
\begin{aligned}
{\left[\hat{\rho}_{\kappa^{\prime}}(-\vec{Q}), \hat{H}\right] } & =\sum_{\kappa} \sum_{j \in\left\{j_{\kappa}\right\}, j^{\prime} \in\left\{j_{\kappa^{\prime}}\right\}} \frac{1}{2 m_{\kappa}}\left[\exp \left(\mathrm{i} \vec{Q} \cdot \vec{R}_{j^{\prime}}\right),\left(\vec{P}_{j}\right)^{2}\right] \\
& =\sum_{j \in\left\{j_{\kappa^{\prime}}\right\}} \frac{1}{2 m_{\kappa^{\prime}}}\left[\exp \left(\mathrm{i} \vec{Q} \cdot \vec{R}_{j}\right),\left(\vec{P}_{j}\right)^{2}\right] \\
& =\sum_{j \in\left\{j_{\kappa^{\prime}}\right\}} \frac{1}{2 m_{\kappa^{\prime}}}\left\{\left[\exp \left(\mathrm{i} \vec{Q} \cdot \vec{R}_{j}\right), \vec{P}_{j}\right] \vec{P}_{j}+\vec{P}_{j}\left[\exp \left(\mathrm{i} \vec{Q} \cdot \vec{R}_{j}\right), \vec{P}_{j}\right]\right\},
\end{aligned}
$$

where we have used the identity

$$
\left[A, B^{2}\right]=[A, B] B-B[B, A]=[A, B] B+B[A, B] .
$$

Knowing that the commutator of a function $F\left(\vec{R}_{j}\right)$ with $\vec{P}_{j}$ gives the gradient of this function according to

$$
\left[f\left(\vec{R}_{j}\right), \vec{P}_{j}\right]=\mathrm{i} \hbar \vec{\nabla}_{\vec{R}_{j}} f\left(\vec{R}_{j}\right)
$$

the commutator can be calculated as

$$
\begin{aligned}
{\left[\hat{\rho}_{\kappa^{\prime}}(-\vec{Q}), \hat{H}\right] } & =\sum_{j \in\left\{j_{\kappa^{\prime}}\right\}} \frac{1}{2 m_{\kappa^{\prime}}}\left\{-\hbar \vec{Q} \cdot \exp \left(\mathrm{i} \vec{Q} \cdot \vec{R}_{j}\right) \vec{P}_{j}-\hbar \vec{Q} \cdot \vec{P}_{j} \exp \left(\mathrm{i} \vec{Q} \cdot \vec{R}_{j}\right)\right\} \\
& =-\sum_{j \in\left\{j_{\kappa^{\prime}}\right\}} \frac{1}{2 m_{\kappa^{\prime}}} \exp \left(\mathrm{i} \vec{Q} \cdot \vec{R}_{j}\right)\left(2 \hbar \vec{Q} \cdot \vec{P}_{j}+\hbar^{2} Q^{2}\right) .
\end{aligned}
$$


Inserting (8.185) into (8.173) gives

$$
\begin{aligned}
{\left[\left[\hat{\rho}_{\kappa^{\prime}}(-\vec{Q}), \hat{H}\right], \hat{\rho}_{\kappa}(\vec{Q})\right] } & =-\left[\hat{\rho}_{\kappa}(\vec{Q}),\left[\hat{\rho}_{\kappa^{\prime}}(-\vec{Q}), \hat{H}\right]\right] \\
& =\sum_{j \in\left\{j_{\kappa}\right\}} \sum_{j^{\prime} \in\left\{j_{\kappa^{\prime}}\right\}} \frac{1}{2 m_{\kappa^{\prime}}} \exp \left(\mathrm{i} \vec{Q} \cdot \vec{R}_{j^{\prime}}\right)\left(2 \hbar \vec{Q} \cdot\left[\exp \left(-\mathrm{i} \vec{Q} \cdot \vec{R}_{j}\right), \vec{P}_{j^{\prime}}\right]\right) \\
& =\sum_{j \in\left\{j_{\kappa}\right\}} \sum_{j^{\prime} \in\left\{j_{\kappa^{\prime}}\right\}} \frac{\hbar^{2} Q^{2}}{m_{\kappa^{\prime}}} \delta_{j, j^{\prime}} \exp \left(\mathrm{i} \vec{Q} \cdot \vec{R}_{j^{\prime}}\right) \exp \left(-\mathrm{i} \vec{Q} \cdot \vec{R}_{j}\right) \\
& =\sum_{j \in\left\{j_{\kappa}\right\}} \delta_{\kappa, \kappa^{\prime}} \frac{1}{m_{\kappa^{\prime}}} \hbar^{2} Q^{2}=\delta_{\kappa, \kappa^{\prime}} N_{\kappa} \frac{\hbar^{2} Q^{2}}{m_{\kappa}},
\end{aligned}
$$

where we have used identity (8.184) a second time. We are finally in a position to express the first moment. According to (8.177)

$$
\int_{-\infty}^{\infty} \mathrm{d} \hbar \omega \hbar \omega S_{\kappa, \kappa^{\prime}}(\vec{Q}, \omega)=\left.\frac{\hbar^{2}}{2} \frac{\partial}{\partial t} K_{\kappa, \kappa^{\prime}}(\vec{Q}, t)\right|_{t=0}=\delta_{\kappa, \kappa^{\prime}} N_{\kappa} \frac{\hbar^{2} Q^{2}}{2 m_{\kappa}}=\delta_{\kappa, \kappa^{\prime}} N_{\kappa} E_{\kappa}^{r}(Q)
$$

with

$$
E_{\kappa}^{r}(Q):=\frac{\hbar^{2} Q^{2}}{2 m_{\kappa}}
$$

the recoil energy of atoms of type $\kappa$. The recoil energy is the average energy exchanged under a collision with momentum transfer $\hbar \vec{Q}$. We would like to emphasise once more that this result has been obtained under the sole condition that the interactions are independent of $\vec{P}_{j}$. It is, therefore, of very general nature. It holds in particular for non-interacting particles, i.e. the ideal gas. As a consequence coherence effects do not enter into the first moment. The expression (8.187) is widely known as the $f$-sum rule. It is of special importance in fluid mechanics. E.g. the $f$-sum rule allows us to conclude that the dispersion of the excitations in ${ }^{4} \mathrm{He}$ has to have a minimum, the roton mode, where the structure factor has its maximum [28].

The first moment is strictly zero for symmetric functions. As a consequence it constitutes a measure of the asymmetry of the scattering function. The expression (8.187) shows us that this asymmetry is independent of temperature. In particular, the first moment can always be evaluated at $T=0$. In this case $S(\vec{Q}, \omega)=0$ for $\omega<0$. $S_{T=0}(\vec{Q}, \omega)$ constitutes the base, upon which the signal at higher temperatures builds on in a symmetric manner. With the fluctuation-dissipation theorem (8.155) a sum-rule for the susceptibility can be derived

$$
\int_{0}^{\infty} \mathrm{d} \hbar \omega \hbar \omega S_{\kappa, \kappa^{\prime}}(\vec{Q}, \omega ; T=0)=\frac{1}{\pi} \int_{0}^{\infty} \mathrm{d} \hbar \omega \hbar \omega \chi_{\vec{Q}}^{\prime \prime}[\omega ; T=0]=\delta_{\kappa, \kappa^{\prime}} N_{\kappa} E_{\kappa}^{r} .
$$

Expressions for the higher order moments can be found in [14,46,48]. One should nevertheless carefully distinguish the moments of the classical scattering functions from those obtained with the quantum mechanical expressions. As we already have mentioned the classical $S^{\mathrm{cl}}(\vec{Q}, \omega)$ is in general an even function of $\omega$. As a consequence all the moments for odd $n$ are necessarily zero. The classical moment that comes closest to the quantum mechanical $f$-sum rule is the second order moment. Under the assumption that the velocity of the different atoms are not correlated, which is a good approximation for liquids, it is given by [14]

$$
\int_{-\infty}^{\infty} \mathrm{d} \omega \omega^{2} S^{\mathrm{cl}}(\vec{Q}, \omega)=N \frac{Q^{2} k_{\mathrm{B}} T}{m}
$$


Pierre Gilles de Gennes has used this sum rule in order to derive the slowing-down of the structural relaxation in liquids for $Q$ values that correspond to the maxima of the structure factor (de Gennes narrowing) [14] (see Fig. 42).

\subsection{Partial summary}

We will again briefly pause and repeat the main concepts introduced in this section.

Nuclear scattering gives access to the auto correlation function of the atomic number density (8.19)

$$
G_{\kappa, \kappa^{\prime}}(\vec{r}, t)=\int \mathrm{d}^{3} \vec{r}^{\prime}\left\langle\hat{\rho}_{\kappa}\left(\vec{r}^{\prime}-\vec{r}\right) \hat{\rho}_{\kappa^{\prime}}\left(\vec{r}^{\prime}, t\right)\right\rangle .
$$

This correlation function is related to the intermediate scattering function (8.38)

$$
I_{\kappa, \kappa^{\prime}}(\vec{Q}, t)=\left\langle\hat{\rho}_{\kappa}(\vec{Q}, t=0) \hat{\rho}_{\kappa^{\prime}}(-\vec{Q}, t)\right\rangle
$$

by a Fourier transformation in space

$$
I_{\kappa, \kappa^{\prime}}(\vec{Q}, t)=\int_{V} \mathrm{~d}^{3} r \mathrm{e}^{-\mathrm{i} \vec{Q} \cdot \vec{r}} G_{\kappa, \kappa^{\prime}}(\vec{r}, t) .
$$

The scattering function is obtained by Fourier transforming the intermediate scattering function (8.39) in time

$$
S_{\kappa, \kappa^{\prime}}(\vec{Q}, \omega)=\frac{1}{2 \pi \hbar} \int_{-\infty}^{\infty} \mathrm{d} t I_{\kappa, \kappa^{\prime}}(\vec{Q}, t) \mathrm{e}^{-\mathrm{i} \omega t} .
$$

Hence the scattering function is a double Fourier transform of the auto correlation function of the atomic number density (8.28)

$$
S_{\kappa, \kappa^{\prime}}(\vec{Q}, \omega)=\frac{1}{2 \pi \hbar} \int_{V} \mathrm{~d}^{3} r \int_{-\infty}^{\infty} \mathrm{d} t \mathrm{e}^{\mathrm{i}(\vec{Q} \cdot \vec{r}-\omega t)} G_{\kappa, \kappa^{\prime}}(\vec{r}, t)
$$

This relation allows us to express the coherent partial differential cross sections in terms of the auto correlation function of the atomic number density (8.32)

$$
\frac{\mathrm{d}^{2} \sigma}{\mathrm{d} \Omega \mathrm{d} E_{f}}=\frac{k_{f}}{k_{i}} \frac{1}{2 \pi \hbar} \sum_{\kappa, \kappa^{\prime}}\left[\left(V_{\kappa}(\vec{Q}) V_{\kappa^{\prime}}^{*}(\vec{Q})\right) \int_{V} \mathrm{~d}^{3} r \int_{-\infty}^{\infty} \mathrm{d} t \mathrm{e}^{\mathrm{i}(\vec{Q} \cdot \vec{r}-\omega t)} G_{\kappa, \kappa^{\prime}}(\vec{r}, t)\right] .
$$

The auto correlation function of the atomic number density is the double Fourier transform in time and space of the scattering function (8.27)

$$
G_{\kappa, \kappa^{\prime}}(\vec{r}, t)=\frac{\hbar}{(2 \pi)^{3}} \int_{V_{Q}} \mathrm{~d}^{3} Q \int_{-\infty}^{\infty} \mathrm{d} \omega \mathrm{e}^{-\mathrm{i}(\vec{Q} \cdot \vec{r}-\omega t)} S_{\kappa, \kappa^{\prime}}(\vec{Q}, \omega) .
$$

When dealing with incoherent scattering the distinct correlation functions are replaced by the self correlation functions.

The analytic properties of the scattering function impose the principle of detailed balance (8.58)

$$
S_{\kappa, \kappa^{\prime}}(\vec{Q}, \omega)=\mathrm{e}^{\hbar \omega \beta} S_{\kappa^{\prime}, \kappa}(-\vec{Q},-\omega) .
$$


The correlation functions are tightly connected to linear response functions. In statistical mechanics the response functions describe the reaction of a system to a perturbation in the linear regime. We define the general response function as

$$
K_{\hat{\rho}_{\kappa^{\prime}}^{+}, \hat{\rho}_{\kappa}}(t)=\frac{\mathrm{i}}{\hbar}\left\langle\left[\hat{\rho}_{\kappa^{\prime}}(-\vec{Q}, t), \hat{\rho}_{\kappa}(\vec{Q}, t=0)\right]\right\rangle
$$

This function is related to the scattering function by (8.137)

$$
S_{\kappa, \kappa^{\prime}}(\vec{Q}, \omega)=(1+n(\omega)) \frac{\mathrm{i}}{2 \pi} \int_{-\infty}^{\infty} \mathrm{d} t \exp (-\mathrm{i} \omega t) K_{\hat{\rho}_{\kappa^{\prime}}^{+}, \hat{\rho}_{\kappa}}(t)
$$

Causality is guarantied by limiting the response to positive times (8.130)

$$
\Phi_{\hat{\rho}_{\kappa^{\prime}}^{+}, \hat{\rho}_{\kappa}}(t)=\frac{\mathrm{i}}{\hbar}\left\langle\left[\hat{\rho}_{\kappa^{\prime}}(-\vec{Q}, t), \hat{\rho}_{\kappa}(\vec{Q}, t=0)\right]\right\rangle \Theta(t) .
$$

The transition to the frequency regime is done by a Laplace transform (8.139)

$$
\chi_{\hat{\rho}_{\kappa^{\prime}}^{+}, \hat{\rho}_{\kappa}}[\omega]=\lim _{\varepsilon \rightarrow 0^{+}} \chi_{\hat{\rho}_{\kappa^{\prime}}^{+}, \hat{\rho}_{\kappa}}[\mathrm{i} p=\omega+\mathrm{i} \varepsilon]=\lim _{\varepsilon \rightarrow 0^{+}} \int_{0}^{\infty} \mathrm{d} t \Phi_{\hat{\rho}_{\kappa^{\prime}}^{+}, \hat{\rho}_{\kappa}}(t) \exp (p t) .
$$

The generalised susceptibility $\chi_{\hat{\rho}_{\kappa^{\prime}}{ }^{\prime}, \hat{\rho}_{\kappa}}[\omega]$ is connected to the scattering function via the fluctuation-dissipation theorem (8.155)

$$
S_{\kappa, \kappa^{\prime}}(\vec{Q}, \omega)=(1+n(\omega)) \frac{1}{\pi} \chi_{\hat{\rho}_{\kappa^{\prime}}^{+}, \hat{\rho}_{\kappa}}^{\prime \prime}[\omega] .
$$

\section{Vibrational spectroscopy}

We will now apply the developed formalism to the scattering of neutrons involving vibrations. In order to do so we have to express the operators $\vec{R}_{j}(t)$ that enter the dynamical structure factor in terms of vibrational creation and annihilation operators. It can certainly not be the purpose of this article to give a full introduction into the subject of structural excitations. We will, therefore, be as concise as possible. The reader interested in the details is referred to the abundant literature that exists in this field. ${ }^{88}$

To make atoms vibrate we have to fix them in equilibrium positions at least on the time scale of the vibrational period. This condition is fulfilled for crystals and amorphous systems in the glassy state. We will consider in the following only systems for which the structural correlations do not decay in time, i.e. for which the structural relaxation is complete. Diffusional motions can always be added as a perturbation provided that they are slow on the time scale of the vibrational period.

\subsection{Adiabatic approximation}

To fix the equilibrium positions of the atoms we have to place them into the minima of a potential. If we include zero-point vibrations then the nuclei fluctuate about these minima at all temperatures. The Coulomb potential not being capable of providing stable atomic configurations on its own the potentials necessarily include electronic

\footnotetext{
${ }^{88} \mathrm{An}$ introduction to vibrations in crystals can be found in the books of Kittel [35] and Ashcroft and Mermin [2]. A complete discussion of the subject is given in reference [40].
} 
contributions. This is not surprising given the fact that the atomic structure is the result of chemical bonding, which in turn is provided by the overlap of electronic orbitals. Any vibrational study is, therefore, above all an investigation of the electronic system. Despite the intimate connection of electrons and ions ${ }^{89}$ it is possible to decouple the two systems via the adiabatic approximation that we are going to comment briefly.

The Hamilton operator $\mathbf{H}$ contains both the ionic $\left\{\vec{R}_{I}\right\}$ and the electronic degrees $\left\{\vec{r}_{i}\right\}$ of freedom. The Schrödinger equation reads

$$
\mathrm{i} \hbar \frac{\partial}{\partial t} \Psi\left(\left\{\vec{R}_{I}\right\},\left\{\vec{r}_{i}\right\} ; t\right)=\mathbf{H} \Psi\left(\left\{\vec{R}_{I}\right\},\left\{\vec{r}_{i}\right\} ; t\right)
$$

with the Hamilton operator 90

$$
\begin{aligned}
\mathbf{H} & =\sum_{i} \frac{\hbar^{2}}{2 m_{e}} \vec{\nabla}_{i}^{2}+\sum_{I} \frac{\hbar^{2}}{2 m_{I}} \vec{\nabla}_{I}^{2}+\sum_{i \leqslant j} \frac{e^{2}}{\left|\vec{r}_{i}-\vec{r}_{j}\right|}+\sum_{I \leqslant J} \frac{Z_{I} Z_{J} e^{2}}{\left|\vec{R}_{I}-\vec{R}_{J}\right|}+\sum_{i, I} \frac{Z_{I} e^{2}}{\left|\vec{r}_{i}-\vec{R}_{I}\right|} \\
& =\mathbf{T}_{i}+\mathbf{T}_{I}+\mathbf{V}_{i, j}+\mathbf{V}_{I, J}+\mathbf{V}_{i, I} .
\end{aligned}
$$

- $\mathbf{T}_{i}$ represents the kinetic energy of the electrons,

- $\mathbf{T}_{I}$ represents the kinetic energy of the ions,

- $\mathbf{V}_{i, j}$ the electron-electron interaction,

- $\mathbf{V}_{I, J}$ the ion-ion interaction, and

- $\mathbf{V}_{i, I}$ the electron-ion interaction.

The stationary wave functions of the coupled system are given by the eigenfunctions of $\mathbf{H}$

$$
\mathbf{H} \Psi_{n}\left(\left\{\vec{R}_{I}\right\},\left\{\vec{r}_{i}\right\} ; t\right)=E_{n} \Psi_{n}\left(\left\{\vec{R}_{I}\right\},\left\{\vec{r}_{i}\right\} ; t\right) .
$$

The $E_{n}$ are the excitation energies of the coupled system. Even if the nuclei were frozen in position resolving only the electronic part of the problem amounts to a formidable challenge. Directly solving equation (9.4) is, therefore, an impossible endeavour. Fortunately an exact solution is not necessary to capture the physics of condensed matter systems. This holds in particular for the description of the ionic motion. The vastly smaller electronic mass implies that the dynamics of the electrons is in general very much faster than that of the ions. The electrons, therefore, adapt nearly instantaneously to any change in the ionic positions. This allows us to separate the ionic from the electronic variables. The wave function becomes a direct product of the form

$$
\Psi\left(\left\{\vec{R}_{I}\right\},\left\{\vec{r}_{i}\right\} ; t\right)=\psi\left(\left\{\vec{r}_{i}\right\}, t ;\left\{\vec{R}_{I}\right\}\right) \cdot \Phi\left(\left\{\vec{R}_{I}\right\} ; t\right)
$$

with the electronic part defined by the wave equation

$$
\left(\mathbf{T}_{i}+\mathbf{V}_{i, j}+\mathbf{V}_{i, I}\right) \psi\left(\left\{\vec{r}_{i}\right\}, t ;\left\{\vec{R}_{I}\right\}\right)=E_{\mathrm{el}}\left(\left\{\vec{R}_{I}\right\}\right) \cdot \psi\left(\left\{\vec{r}_{i}\right\}, t ;\left\{\vec{R}_{I}\right\}\right)
$$

which contains the ionic variables only as parameters but not as dynamic variables. For the ionic part we obtain the equation

$$
\left(\mathbf{T}_{I}+E_{\mathrm{el}}\left(\left\{\vec{R}_{I}\right\}\right)+\mathbf{V}_{I, J}\right) \Phi\left(\left\{\vec{R}_{I}\right\}, t\right)=E \cdot \Phi\left(\left\{\vec{R}_{I}\right\}, t\right)
$$

\footnotetext{
${ }^{89}$ We denote as ions the nuclei surrounded by their core electrons.

${ }^{90}$ Whenever there can be an ambiguity we will use capital letters to designate ions and small characters to designate electrons. $Z_{I}$ and $M_{I}$ denote the ionic charge and mass, respectively.
} 
in which the electronic energy $E_{\mathrm{el}}\left(\left\{\vec{R}_{I}\right\}\right)$ acts as an external potential. In general this adiabatic approximation works well in most materials (see e.g. [2]). It should, however, not be taken for granted. From a quantum mechanical point of view there is a priori no reason why electronic excitations should not couple to ionic excitations, i.e. form resonances, unless the energy scales of both systems are vastly different. This condition is generally fulfilled in insulators with a large electronic gap of a few eV, which is to be compared to vibrational energies of at most several hundred meV. The separation of energy scale argument can, however, not be used for metals. Close to the Fermi surface electronic excitations of arbitrarily small energies can be created. Nothing prevents a priori these excitations to resonate with lattice excitations. In practice, this is normally not happening and most metallic states can be well described by the adiabatic approximation. Exceptions exist and phonon mediated superconductivity is one of them.

\subsection{Harmonic approximation}

Now that we have separated off the electronic degrees of freedom we can turn our attention to calculating the ionic excitation spectrum.

We denote the potential governing the ionic wave equation (9.7) by

$$
U\left(\vec{R}_{1}, \ldots, \vec{R}_{N}\right)=E_{\mathrm{el}}\left(\left\{\vec{R}_{I}\right\}\right)+\mathbf{V}_{I, J}
$$

While the ionic positions $\vec{R}_{n}(n=1, \ldots, N)$ are time dependent the corresponding equilibrium positions $\vec{R}_{n}^{0}$ $(n=1, \ldots, N)$ are stationary. We introduce the displacements

$$
\vec{u}(n \mid t)=\vec{R}_{n}-\vec{R}_{n}^{0}, \quad n=1, \ldots, N
$$

with respect to the equilibrium positions $R_{n}^{0}$. The potential $U\left(\vec{R}_{1}, \ldots, \vec{R}_{N}\right)$ can then be expanded in terms of these displacements

$$
\vec{u}(n \mid t)=\vec{R}_{n}-\vec{R}_{n}^{0}, \quad n=1, \ldots, N
$$

For small displacements we can limit ourselves to terms of second order

$$
U\left(\vec{R}_{1}, \ldots, \vec{R}_{N}\right)=U_{0}+\left.\sum_{\alpha, n=1}^{3, N} \frac{\partial U}{\partial u_{\alpha}(n)}\right|_{0} u_{\alpha}(n)+\left.\frac{1}{2} \sum_{\alpha, n=1}^{3, N} \sum_{\beta, n^{\prime}=1}^{3, N} \frac{\partial^{2} U}{\partial u_{\alpha}(n) \partial u_{\beta}\left(n^{\prime}\right)}\right|_{0} u_{\alpha}(n) u_{\beta}\left(n^{\prime}\right)
$$

with $\alpha=1,2$ or 3 denoting the three space directions. The derivatives have to be taken with respect to the equilibrium positions, which do not always coincide with the potental minima. This holds even for $T \rightarrow 0$ due to quantum fluctuations. The first term in Eq. (9.11) is a constant. The second term involves the first derivatives of the potential, i.e. the forces acting on the ions. These forces have to cancel in equilibrium. The first non-trivial term is, therefore, of second order. If we treat the problem up to second order in the ionic displacements then we work in the so-called harmonic approximation. This approximation holds if the displacements are small. ${ }^{91}$ What "small" means depends a lot on the system studied.

\footnotetext{
${ }^{91}$ We would like to point out that for the harmonic approximation to hold it is the relative displacements of the ions that have to be small. The potential experienced by the ions depends only on the distances between them and not on their absolute positions. The absolute displacements can, therefore, be quite considerable. For an acoustic wave the condition of small relative displacements is generally fulfilled if the wave length is considerably larger than the range of the inter-ionic potentials. The vibrations of a piano cord are an excellent example to illustrate this point. These vibrations are harmonic despite the fact that the atoms along the cord move by several mm, which is immense on the atomic scale. This is possible because the wave length is equally of a few $\mathrm{cm}$, which is again enormous on the atomic scale.
} 


\subsection{The dynamical matrix}

To determine the vibrations within the harmonic approximation we have to calculate the eigenfunctions

$$
\psi_{I}\left(\vec{R}_{1}, \ldots, \vec{R}_{N}\right)
$$

of the Hamiltonian

$$
\mathbf{H}=\sum_{i} \frac{\hbar^{2}}{2 m_{i}} \vec{\nabla}_{i}^{2}+U\left(\vec{R}_{1}, \ldots, \vec{R}_{N}\right)
$$

with the potential given as

$$
U\left(\vec{R}_{1}, \ldots, \vec{R}_{N}\right)=U_{0}+\left.\sum_{\alpha, n=1}^{3, N} \frac{\partial U}{\partial u_{\alpha}(n)}\right|_{0} u_{\alpha}(n)+\left.\frac{1}{2} \sum_{\alpha, n=1}^{3, N} \sum_{\beta, n^{\prime}=1}^{3, N} \frac{\partial^{2} U}{\partial u_{\alpha}(n) \partial u_{\beta}\left(n^{\prime}\right)}\right|_{0} u_{\alpha}(n) u_{\beta}\left(n^{\prime}\right)
$$

We will determine these eigenfunctions by separating the variables in analogy to the quantum mechanical harmonic oscillator. We will do so by carrying out the quantisation in the space of the classical eigenfunctions. The first step in this direction consists in determining these eigenmodes by diagonalising the classical equations of motion.

This diagonalisation is best done working with matrices. The second derivatives

$$
\Phi_{\alpha \beta}\left(n, n^{\prime}\right)=-\left.\frac{\partial^{2} U}{\partial u_{\alpha}(n) \partial u_{\beta}\left(n^{\prime}\right)}\right|_{0}
$$

are the so-called force constants of the material. They determine the restoring force that acts on the ion $n$ along the direction $\vec{a}_{\alpha}$ when the ion $n^{\prime}$ is displaced along $\vec{a}_{\beta}$ (see Fig. 43). The force constants form a matrix $\tilde{F}$ of dimension

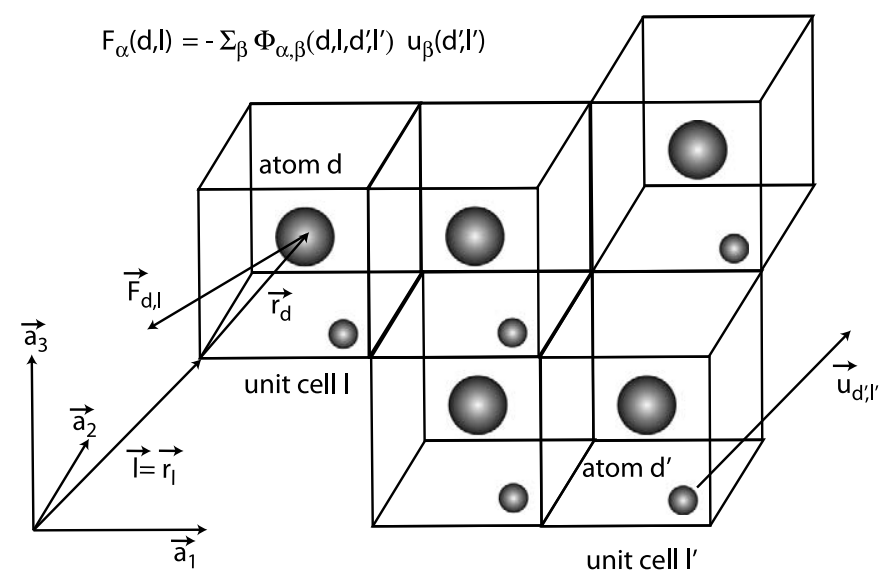

Fig. 43. Schematic presentation of the force constants for a crystal with two atoms $(\kappa=1,2)$ in the primitive cell. When the atom $\kappa^{\prime}$ of cell $l^{\prime}$ is displaced by $\vec{u}\left(\kappa^{\prime}, \vec{l}^{\prime}\right)$ a force acts on atom $\kappa$ of cell $l$. This force can be calculated with the help of the force constants $\Phi_{\alpha, \beta}\left(d, l, d^{\prime}, l^{\prime}\right)$ according to the formula given in the figure. 
$(3 N \times 3 N)^{92}$

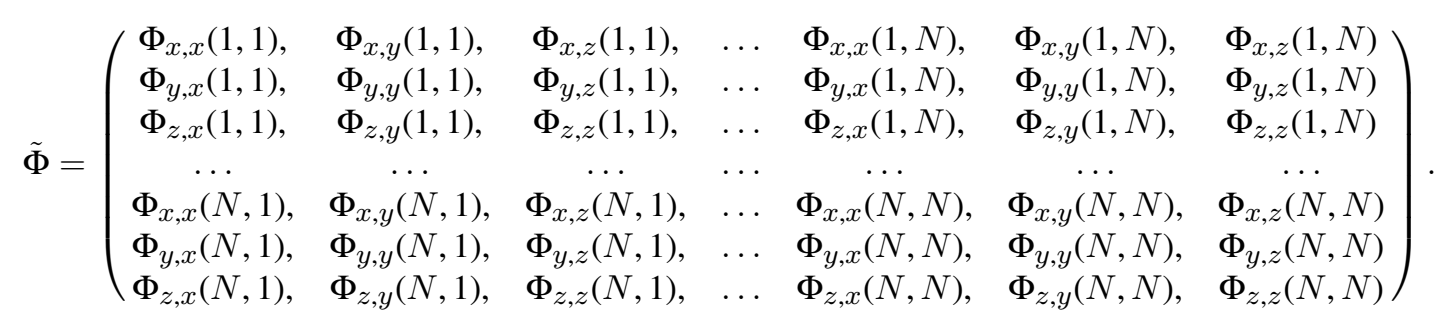

Particular attention has to be given to the so-called self-terms $\Phi_{\alpha \beta}(n, n)$. They often have to be evaluated indirectly invoking Newton's third law of action equalling reaction

$$
\Phi_{\alpha \beta}(n, n)=-\sum_{n^{\prime} \neq n} \Phi_{\alpha \beta}\left(n, n^{\prime}\right) .
$$

Details can be found in [40]. We define the dynamical matrix in terms of the force constant matrix via

$$
D_{\alpha \beta}\left(n, n^{\prime}\right)=\left.\frac{1}{\sqrt{m_{n} \cdot m_{n^{\prime}}}} \frac{\partial^{2} U}{\partial u_{\alpha}(n) \partial u_{\beta}\left(n^{\prime}\right)}\right|_{0}=-\frac{\Phi_{\alpha \beta}\left(n, n^{\prime}\right)}{\sqrt{m_{n} \cdot m_{n^{\prime}}}} .
$$

$m_{n}$ denotes the mass of the ion $n$.

In matrix notation

$$
\tilde{D}=-\tilde{M}^{-1 / 2} \tilde{F} \tilde{M}^{-1 / 2} \quad \text { with } \tilde{M}_{\alpha, \beta}^{-1 / 2}\left(n, n^{\prime}\right)=\frac{1}{\sqrt{m_{n}}} \delta_{\alpha, \beta} \delta_{n, n^{\prime}}
$$

We introduce the displacement vector

$$
\overrightarrow{\vec{u}}_{3 N}=\left(\sqrt{m_{1}} \vec{u}(1), \sqrt{m_{2}} \vec{u}(2), \ldots, \sqrt{m_{N}} \vec{u}(N)\right) .
$$

The $3 N$ components of $\overrightarrow{\vec{u}}_{3 N}$ are weighted by the square root of the mass of the ions.

In the basis of these vectors the classical equations of motion

$$
m_{i} \ddot{u}_{\alpha}(n \mid t)=\sum_{\beta, n^{\prime}=1}^{3, N} \Phi_{\alpha \beta}\left(n, n^{\prime}\right) u_{\beta}\left(n^{\prime} \mid t\right)
$$

become

$$
\ddot{\bar{u}}_{\alpha}(n \mid t)=-\sum_{n^{\prime}=1, \beta=1}^{N, 3} D_{\alpha \beta}\left(n, n^{\prime}\right) \bar{u}_{\beta}\left(n^{\prime} \mid t\right) .
$$

We are looking for oscillatory solutions characterised by a frequency $\omega$ and a phase $\phi$ and, therefore, pose

$$
\bar{u}_{\alpha}(n \mid t) \propto \cos (\omega t+\phi), \quad \forall n=1, \ldots N, \forall \alpha=1,2,3 .
$$

\footnotetext{
${ }^{92} \mathrm{We}$ will here denote matrices by a tilde.
} 
Plucked into the classical equations of motions we get

$$
\omega^{2} \vec{u}_{3 N}=\tilde{D} \vec{u}_{3 N}
$$

$\tilde{D}$ being a real symmetric matrix

$$
\tilde{D}=\tilde{D}^{T}
$$

it can be diagonalised. Its $3 N$ eigenvalues $\omega_{j}^{2}$ are real. If they are in addition positive then we end up with $3 N$ positive frequencies $\omega_{j}$. Negative eigenvalues $\omega_{j}^{2}$ produce imaginary $\omega_{j}$ and, therefore, describe relaxation. They are forbidden by the stability of the equilibrium positions. The $3 N$ eigenvectors of the dynamical matrix $\vec{e}_{j}$ describe the pattern of the displacements corresponding to a particular mode. A very simple example is given in Fig. 44. The $3 N$ eigenvectors $\vec{e}_{j}$ form a complete real basis that can be chosen orthonormal [40]

$$
\begin{aligned}
& \vec{e}_{j} \cdot \vec{e}_{j^{\prime}}=\sum_{\alpha, n=1}^{3, N} e_{j}(\alpha, n) e_{j^{\prime}}(\alpha, n)=\delta_{j, j^{\prime}}, \\
& \sum_{j=1}^{3 N} e_{j}(\alpha, n) e_{j}\left(\beta, n^{\prime}\right)=\delta_{\alpha, \beta} \delta_{n, n^{\prime}} .
\end{aligned}
$$

Therefore, the motion of the atoms can be expressed in this basis

$$
\bar{u}_{\alpha}(n \mid t)=\sum_{j=1}^{3 N} Q_{j} \cdot e_{j}(\alpha, n) \cdot \cos \left(\omega_{j} t+\phi_{j}\right), \quad n=1, \ldots, N, \alpha=1,2,3
$$

with $Q_{j}$ and $\phi_{j}$ the expansion coefficients, which are determined by the initial conditions. We have, therefore, performed a transformation into the coordinate space of eigenmodes each characterised by its eigenfrequency. The

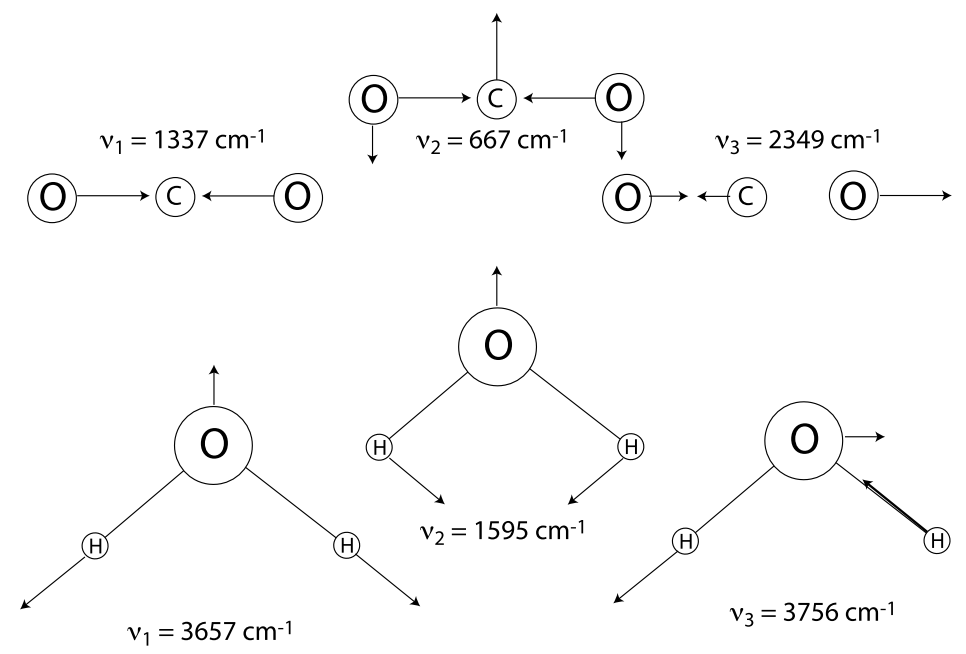

Fig. 44. Normal modes for a $\mathrm{CO}_{2}$ and a water molecule. The vibrations $\nu_{1}$ are symmetric modes that stretch the chemical bonds. The vibrations $\nu_{2}$ are asymmetric modes that bend the bond angles. The vibrations $\nu_{3}$ are asymmetric modes that stretch the chemical bonds. All these modes are internal, i.e. they leave the centre of gravity invariant. 
classical problem can be considered solved once the dynamical matrix is diagonalised. For this we have to find the roots of the characteristic polynomial

$$
P\left(\omega^{2}\right)=\left|\left(\tilde{D}-\omega^{2} \tilde{I}_{3 N}\right)\right|
$$

with $\tilde{I}_{3 N}$ the unit matrix of dimension $(3 N \times 3 N)$. The obtained result does not require translational symmetry and, therefore, applies to crystals as well as to amorphous systems.

\subsection{Vibrational excitations}

To make the transition back to quantum mechanics we rewrite the Hamiltonian (9.13) in matrix form

$$
\mathbf{H}=\frac{1}{2} \overrightarrow{\mathbf{p}}_{3 N} \cdot \overrightarrow{\mathbf{p}}_{3 N}+\overrightarrow{\mathbf{u}}_{3 N} \tilde{D} \overrightarrow{\mathbf{u}}_{3 N},
$$

with the $3 N$-dimensional vectorial position and momentum operators

$$
\begin{aligned}
\overrightarrow{\mathbf{u}}_{3 N} & =\left(\sqrt{m_{1}} \overrightarrow{\mathbf{u}}(1), \sqrt{m_{2}} \overrightarrow{\mathbf{u}}(2), \ldots, \sqrt{m_{N}} \overrightarrow{\mathbf{u}}(N)\right), \\
\overrightarrow{\mathbf{p}}_{3 N} & =\left(\left(1 / \sqrt{m_{1}}\right) \overrightarrow{\mathbf{p}}(1),\left(1 / \sqrt{m_{2}}\right) \overrightarrow{\mathbf{p}}(2), \ldots,\left(1 / \sqrt{m_{N}}\right) \overrightarrow{\mathbf{p}}(N)\right) .
\end{aligned}
$$

Performing a coordinate transformation into the eigenmodes of the dynamical matrix $\tilde{D}$ we obtain

$$
\mathbf{H}=\frac{1}{2}\left(\sum_{j=1}^{3 N} \mathbf{P}_{j} \cdot \mathbf{P}_{j}+\omega_{j}^{2}\left(\mathbf{Q}_{j} \cdot \mathbf{Q}_{j}\right)\right)
$$

with $\mathbf{Q}_{j}$ and $\mathbf{P}_{j}$ the transformed $3 N$-dimensional position and momentum operators in eigenmode space. ${ }^{93}$ Formally

$$
\mathbf{Q}_{j}=\sum_{\alpha, n}^{3, N} e_{j}(\alpha, n) \cdot \overline{\mathbf{u}}_{\alpha}(n)
$$

or in matrix notation

$$
\begin{aligned}
& \overrightarrow{\mathbf{Q}}_{3 N}=\tilde{S} \overrightarrow{\mathbf{u}}_{3 N}, \\
& \overrightarrow{\mathbf{P}}_{3 N}=\tilde{S} \overrightarrow{\mathbf{p}}_{3 N}
\end{aligned}
$$

with

$$
\tilde{S}=\left(\begin{array}{ccccccc}
e_{1}(x, 1) & e_{1}(y, 1) & e_{1}(z, 1) & \ldots & e_{1}(x, N) & e_{1}(y, N) & e_{1}(z, N) \\
& & & \ldots & & & \\
e_{3 N}(x, 1) & e_{3 N}(y, 1) & e_{3 N}(z, 1) & \ldots & e_{3 N}(x, N) & e_{3 N}(y, N) & e_{3 N}(z, N)
\end{array}\right)
$$

the orthogonal matrix governing the coordinate transformation. $\tilde{S}$ is a matrix of dimension $3 N \times 3 N$, the rows of which are given by the eigenvectors.

\footnotetext{
${ }^{93}$ The components of $\mathbf{Q}_{j}$ are linear combinations of the position operators $\overrightarrow{\mathbf{u}}_{3 N}$ and the components of $\mathbf{P}_{j}$ are linear combinations of the momentum operators $\overrightarrow{\mathbf{p}}_{3 N}$, respectively.
} 
The Hamiltonian (9.32) describes a sum of $3 N$ uncoupled harmonic oscillators. We may, therefore, diagonalise it in complete analogy to the simple harmonic oscillator treated in every text book of quantum mechanics. Formally we introduce creation $\mathbf{a}_{j}^{+}$and annihilation $\mathbf{a}_{j}$ operators for each mode $j$ :

$$
\begin{aligned}
& \mathbf{a}_{j}^{+}=\frac{1}{\sqrt{2}}\left(\hat{\xi}_{j}-\mathrm{i} \hat{\pi}_{j}\right), \quad j=1, \ldots, 3 N \\
& \mathbf{a}_{j}=\frac{1}{\sqrt{2}}\left(\hat{\xi}_{j}+\mathrm{i} \hat{\pi}_{j}\right), \quad j=1, \ldots, 3 N
\end{aligned}
$$

with

$$
\begin{aligned}
& \hat{\xi}_{j}=\alpha_{j} \mathbf{Q}_{j}, \\
& \hat{\pi}_{j}=\frac{1}{\alpha_{j} \hbar} \mathbf{P}_{j},
\end{aligned}
$$

and

$$
\alpha_{j}=\sqrt{\frac{\omega_{j}}{\hbar}}
$$

It is at this point that we realise the usefulness of working with displacements that are weighted by the square root of the mass.

In terms of the creation and annihilation operators the Hamiltonian becomes

$$
\mathbf{H}=\sum_{j=1}^{3 N} \hbar \omega_{j}\left(\mathbf{a}_{j}^{+} \mathbf{a}_{j}+\frac{1}{2}\right)
$$

As expected it describes a sum of decoupled quantum mechanical harmonic oscillators. The excited states are obtained from the ground state by applying the $3 N$ creation operators

$$
\left|\psi_{n_{1}, \ldots, n_{3 N}}\right\rangle \equiv\left|n_{1}, \ldots, n_{3 N}\right\rangle=\prod_{j=1}^{3 N} \frac{\left(\mathbf{a}_{j}^{+}\right)^{n_{j}}}{\sqrt{n_{j} !}}|0, \ldots, 0\rangle .
$$

The function

$$
\left|n_{1}, \ldots, n_{3 N}\right\rangle
$$

specifies for each of the $3 N$ normal modes of vibration the occupation level. The energy of a stationary mode is calculated as

$$
E=\left\langle n_{1}, \ldots, n_{3 N}|\mathbf{H}| n_{1}, \ldots, n_{3 N}\right\rangle=\sum_{j=1}^{3 N}\left(n_{j}+\frac{1}{2}\right) \hbar \omega .
$$

Knowing the transformation matrix $\tilde{S}$ we may invert expression (9.37) using the fact that the inverse of an orthogonal matrix $\tilde{S}$ is given by its transpose

$$
\tilde{S}^{-1}=\tilde{S}^{t} .
$$


The displacement operator $\mathbf{u}_{\alpha}(n)$ of the $n$th ion along the direction $\alpha$ is related to the components of the eigenvectors $\vec{e}_{j}$ via $^{94}$

$$
\mathbf{u}_{\alpha}(n)=\sum_{j} \sqrt{\frac{\hbar}{2 m_{n} \omega_{j}}}\left[e_{j}(\alpha, n) \mathbf{a}_{j}+e_{j}^{*}(\alpha, n) \mathbf{a}_{j}^{+}\right] .
$$

As we can show using expressions (9.37) and (9.38) this relation corresponds to

$$
\sqrt{m_{n}} \mathbf{u}_{\alpha}(n)=\sum_{j} e_{j}(\alpha, n) \mathbf{Q}_{j}
$$

and is, therefore, the quantum analog of (9.27).

In similar fashion we obtain for the momentum operators

$$
\mathbf{p}_{\alpha}(n)=-\mathrm{i} \sum_{j} \sqrt{\frac{\hbar \omega_{j} m_{n}}{2}}\left[e_{j}(\alpha, n) \mathbf{a}_{j}^{+}-e_{j}^{*}(\alpha, n) \mathbf{a}_{j}\right]
$$

As vibrational excitations satisfy Bose-Einstein statistics the occupation of the modes in thermal equilibrium at temperature $T$ is given as

$$
\left\langle\mathbf{a}_{j}^{+} \mathbf{a}_{j}\right\rangle=n\left(\omega_{j}\right)=\frac{1}{\mathrm{e}^{\hbar \omega_{j} /\left(k_{B} T\right)}-1} .
$$

\subsection{Lattice modes}

In crystals the translational symmetry puts additional constraints on the wave functions of elementary excitations. This holds both for electrons and ions. To determine the consequences of these constraints we return to the classical equations of motion. The position of an atom in a crystal is given as

$$
\vec{R}(n)=\vec{R}(d, \vec{l})=\vec{l}+\vec{r}(d) .
$$

The vector $\vec{l}$ indicates the origin of the primitive cell and $\vec{r}_{d}$ the position of the atom within this cell (see Fig. 43). The index $d$ ranges from 1 to $r$ with $r$ the number of atoms in the primitive cell. We denote the displacement of atom $\vec{R}(d, \vec{l})$ with respect to its equilibrium position $\vec{R}^{0}(d, \vec{l})$ by $\vec{u}(d, \vec{l})$. Looking again for stationary oscillatory solutions we try the Ansatz

$$
\vec{u}(d, \vec{l} \mid t)=\vec{u}(d \mid \vec{k}) \mathrm{e}^{\mathrm{i}(\vec{q} \cdot \vec{l}-\omega t)}
$$

The vectors $\vec{u}(d \mid \vec{k})$ depend only on the primitive cell and the vector $\vec{q}$ is chosen within the first Brillouin zone.

The second derivatives

$$
\Phi_{\alpha \beta}\left(\begin{array}{ll}
\vec{l} & \overrightarrow{l^{\prime}} \\
d & d^{\prime}
\end{array}\right)=\left.\frac{\partial^{2} U}{\partial u_{\alpha}(d, \vec{l}) \partial u_{\beta}\left(d^{\prime}, \overrightarrow{l^{\prime}}\right)}\right|_{0}
$$

\footnotetext{
${ }^{94}$ To keep the expressions as general as possible we formulate them for complex eigenvectors knowing that the eigenvectors of the dynamical matrix in real space are real.
} 
are the force constants in the notation adapted to the translational symmetry of the crystal. In analogy to the general case we define weighted displacements

$$
\vec{u}(d, \vec{l} \mid t)=\sqrt{m_{d}} \vec{u}(d, \vec{l} \mid t)=\sqrt{m_{d}} \vec{u}(d \mid \vec{k}) \mathrm{e}^{\mathrm{i}(\vec{q} \cdot \vec{l}-\omega t)}=\overrightarrow{\vec{u}}(d \mid \vec{k}) \mathrm{e}^{\mathrm{i}(\vec{q} \cdot \vec{l}-\omega t)}
$$

In this basis the classical equations of motion

$$
m_{d} \ddot{u}_{\alpha}(d, \vec{l} \mid t)=\sum_{\beta, d^{\prime}, \vec{l}^{\prime}} \Phi_{\alpha \beta}\left(\begin{array}{cc}
\vec{l} & \overrightarrow{l^{\prime}} \\
d & d^{\prime}
\end{array}\right) u_{\beta}\left(d^{\prime}, \overrightarrow{l^{\prime}} \mid t\right)
$$

read

$$
\ddot{\bar{u}}_{\alpha}(d, \vec{l} \mid t)=-\sum_{\beta, d^{\prime}, \overrightarrow{l^{\prime}}} D_{\alpha \beta}\left(\begin{array}{cc}
\vec{l} & \overrightarrow{l^{\prime}} \\
d & d^{\prime}
\end{array}\right) \bar{u}_{\beta}\left(d^{\prime}, \overrightarrow{l^{\prime}} \mid t\right)
$$

with, in analogy to Eq. (9.18),

$$
D_{\alpha \beta}\left(\begin{array}{cc}
\vec{l} & \overrightarrow{l^{\prime}} \\
\kappa & \kappa^{\prime}
\end{array}\right)=\frac{\Phi_{\alpha \beta}\left(\begin{array}{ll}
\vec{l} & \overrightarrow{l^{\prime}} \\
\kappa & \kappa^{\prime}
\end{array}\right)}{\sqrt{m_{\kappa} m_{\kappa^{\prime}}}} .
$$

Using the Ansatz (9.49) for $\vec{u}(\kappa, \vec{l} \mid t)$ these equations become

$$
\omega^{2} \bar{u}_{\alpha}(d \mid \vec{q})=\sum_{d^{\prime}, \beta} D_{\alpha \beta}\left(d, d^{\prime} \mid \vec{q}\right) \cdot \bar{u}_{\beta}\left(d^{\prime} \mid \vec{q}\right),
$$

with the dynamical matrix $\tilde{D}(\vec{q})$ in reciprocal space defined for every $\vec{q}$ of the first Brillouin zone as

$$
D_{\alpha \beta}\left(d, d^{\prime} \mid \vec{q}\right)=\sum_{\vec{l}-\overrightarrow{l^{\prime}}} D_{\alpha \beta}\left(\begin{array}{cc}
\vec{l} & \overrightarrow{l^{\prime}} \\
d & d^{\prime}
\end{array}\right) \exp \left(\mathrm{i} \vec{q} \cdot\left(\overrightarrow{l^{\prime}}-\vec{l}\right)\right)
$$

$\tilde{D}(\vec{q})$ can be considered the Fourier transform of the force constant matrix.

The problem has finally taken the form

$$
\omega_{j}^{2}(\vec{q}) \cdot \vec{e}_{j}(\vec{q})=\tilde{D}(\vec{q}) \cdot \vec{e}_{j}(\vec{q})
$$

It is solved once we have determined for every $\vec{q}$ the $3 r$ eigenvalues $\omega_{j}^{2}(\vec{q})$ of the dynamical matrix which then will also give the corresponding eigenvectors $\vec{e}_{j}(\vec{q})$.

The dynamical matrix $\tilde{D}(\vec{q})$ being by definition Hermitian

$$
D_{\alpha \beta}\left(d, d^{\prime} \mid \vec{q}\right)=D_{\beta \alpha}^{*}\left(d, d^{\prime} \mid \vec{q}\right)
$$

the eigenvalues $\omega_{j}^{2}$ exist and are real. As $\tilde{D}(\vec{q})$ is in general not symmetric the eigenvectors are complex. They form a basis that can be chosen orthonormal

$$
\vec{e}_{j}^{*}(\vec{q}) \cdot \vec{e}_{j^{\prime}}(\vec{q})=\sum_{\alpha, d=1}^{3, r} e_{j}^{*}(\alpha, d \mid \vec{q}) e_{j^{\prime}}(\alpha, d \mid \vec{q})=\delta_{j, j^{\prime}}
$$

In a crystal with $N$ atoms respecting the usual Born-von Karman boundary conditions there exist $N / r$ distinct values $\vec{q}$ in the Brillouin zone. For each value of $\vec{q}$ we obtain $3 r$ eigenmodes. These eigenmodes are termed phonons. 


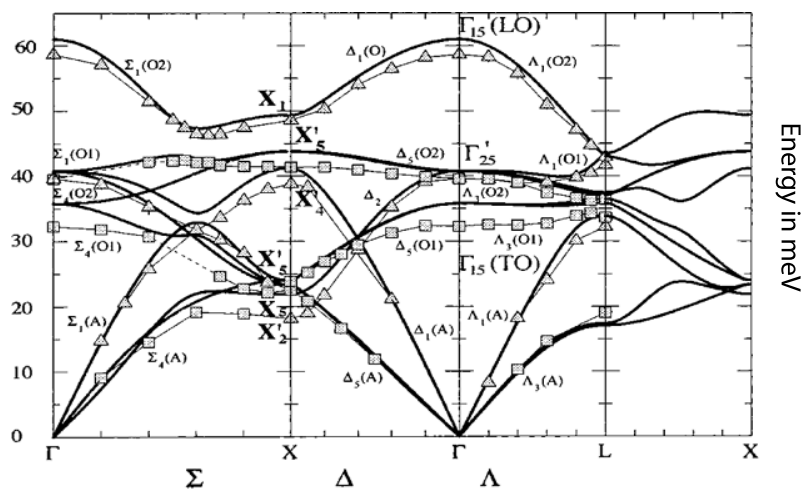

Directions of high symmetry in Brillouin zone

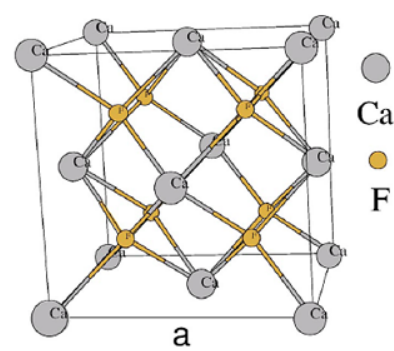

Fig. 45. Dispersion relations for the super-ionic conductor $\mathrm{CaF}_{2}$ [51]. The points connected by thin lines were measured by inelastic neutron scattering (see Fig. 52). The measurements are compared to predictions from ab-initio density-functional calculations (full lines). Calcium fluoride $\left(\mathrm{CaF}_{2}\right)$ crystallises in the fluorite fcc structure with three atoms in the primitive cell (the lower right picture shows a cube containing 4 primitive units). The crystal volume can be viewed as being composed of tetrahedra and octahedra with the Ca ions at the corners and the $\mathrm{F}$ ions at the centres of the tetrahedra. The 3 atoms in the primitive cell lead to 9 dispersion branches. For high-symmetry directions these branches can be classified using the irreducible representations of the primitive cell (denoted by capital Greek letters according to the high-symmetry directions) (images courtesy K. Schmalzl). (Colors are visible in the online version of the article; http://dx.doi.org/10.3233/JNR-140016.)

The functions $\omega_{j}(\vec{q})$ are called the phonon dispersion relations of the system. Every $j$ defines one out of $3 r$ socalled phonon branches. An example of a phonon dispersion is shown in Fig. 45 for the super-ionic conductor $\mathrm{CaF}_{2}$. There are $3 N$ phonon modes in total. This is the number of eigenmodes that we would have obtained by diagonalising directly $\tilde{D}$ ignoring the translational symmetry. Despite this formal equivalence the practical advantage of diagonalising $N / r$ matrices $\tilde{D}(\vec{q})$ of dimension $3 r \times 3 r$ instead of one matrix $\tilde{D}$ of dimension $3 N \times 3 N$ is enormous.

We can now express the displacements in the new basis

$$
\bar{u}_{\alpha}(d, \vec{l} \mid t)=\sqrt{\frac{r}{N}} \sum_{j=1}^{3 r} \sum_{\vec{q}}^{B Z} Q_{j}(\vec{q}) e_{j}(\alpha, d \mid \vec{q}) \mathrm{e}^{-\mathrm{i}\left(\vec{q} \cdot \vec{l}-\omega_{j}(\vec{q}) t\right)} .
$$

The $3 N$ expansion coefficients $Q_{j}(\vec{k})$ are complex numbers with amplitude and phase determined by the initial conditions. They are called the normal coordinates.

We make the transition to quantum mechanics in the new basis by defining the operators

$$
\begin{aligned}
\mathbf{Q}_{j}(\vec{q}) & =\sqrt{\frac{r}{N}} \sum_{\alpha, d}^{3, r} e_{j}^{*}(\alpha, d \mid \vec{q}) \sum_{l} \mathrm{e}^{-\mathrm{i} \vec{q} \cdot \vec{l}} \overline{\mathbf{u}}_{\alpha}(d, \vec{l}) \\
& =\sqrt{\frac{r}{N}} \sum_{\alpha, d}^{3, r} \sqrt{m_{d}} e_{j}^{*}(\alpha, d \mid \vec{q}) \sum_{l} \mathrm{e}^{-\mathrm{i} \vec{q} \cdot \vec{l}} \mathbf{u}_{\alpha}(d, \vec{l}),
\end{aligned}
$$




$$
\begin{aligned}
\mathbf{P}_{j}(\vec{q}) & =\sqrt{\frac{r}{N}} \sum_{\alpha, d}^{3, r} e_{j}^{*}(\alpha, d \mid \vec{q}) \sum_{l} \mathrm{e}^{-\mathrm{i} \vec{q} \cdot \vec{l}} \overrightarrow{\mathbf{p}}_{\alpha}(d, \vec{l}) \\
& =\sqrt{\frac{r}{N}} \sum_{\alpha, d}^{3, r} \frac{e_{j}^{*}(\alpha, d \mid \vec{q})}{\sqrt{m_{d}}} \sum_{l} \mathrm{e}^{-\mathrm{i} \vec{q} \cdot \vec{l}} \mathbf{p}_{\alpha}(d, \vec{l}) .
\end{aligned}
$$

The normal coordinate operators, i.e. the position operators in the basis of the eigenvectors of the dynamical matrices $\tilde{D}(\vec{q})$ are the Fourier transforms of the weighted atomic displacements. The same holds for their conjugated moment operators.

From the normal coordinates we can construct the ladder operators using the expressions (9.37). For the creation operators

$$
\mathbf{a}_{j}^{+}(\vec{q})=\sqrt{\frac{r}{N}} \sum_{\alpha, d, \vec{l}} \mathrm{e}^{\mathrm{i} \vec{q} \cdot \vec{l}} e_{j}(\alpha, d \mid \vec{q}) \cdot\left[\sqrt{\frac{m_{d} \omega_{j}(\vec{q})}{2 \hbar}} \mathbf{u}_{\alpha}(d, \vec{l})-\mathrm{i} \sqrt{\frac{1}{2 \hbar m_{d} \omega_{j}(\vec{q})}} \mathbf{p}_{\alpha}(d, \vec{l})\right]
$$

and for the annihilation operator

$$
\mathbf{a}_{j}(\vec{q})=\sqrt{\frac{r}{N}} \sum_{\alpha, d, \vec{l}} \mathrm{e}^{-\mathrm{i} \vec{q} \cdot \vec{l}} e_{j}^{*}(\alpha, d \mid \vec{q}) \cdot\left[\sqrt{\frac{m_{d} \omega_{j}(\vec{q})}{2 \hbar}} \mathbf{u}_{\alpha}(d, \vec{l})+\mathrm{i} \sqrt{\frac{1}{2 \hbar m_{d} \omega_{j}(\vec{q})}} \mathbf{p}_{\alpha}(d, \vec{l})\right] .
$$

These operators are in a certain manner the Fourier transforms of the local creation and annihilation operators $\mathbf{a}^{+}(d, \vec{l})$ and $\mathbf{a}(d, \vec{l})$.

Inverting these relations we express the position and momentum operators in terms of the creation and annihilation operators.

$$
\begin{aligned}
\mathbf{u}_{\alpha}(\vec{l}, d) & =\sqrt{\frac{r}{N}} \sum_{j, \vec{q}} \sqrt{\frac{\hbar}{2 m_{d} \omega_{j}(\vec{q})}} \cdot\left[e_{j}(\alpha, d \mid \vec{q}) \mathrm{e}^{\mathrm{i} \vec{q} \cdot \vec{l}} \cdot \mathbf{a}_{j}(\vec{q})+e_{j}^{*}(\alpha, d \mid \vec{q}) \mathrm{e}^{-\mathrm{i} \vec{q} \cdot \vec{l}} \cdot \mathbf{a}_{j}^{+}(\vec{q})\right] \\
& =\sqrt{\frac{r}{N}} \sum_{j, \vec{q}} \sqrt{\frac{\hbar}{2 m_{d} \omega_{j}(\vec{q})}} \cdot e_{j}(\alpha, d \mid \vec{q}) \cdot\left(\mathbf{a}_{j}(\vec{q})+\mathbf{a}_{j}^{+}(-\vec{q})\right) \mathrm{e}^{\mathrm{i} \vec{q} \cdot \vec{l}}
\end{aligned}
$$

and

$$
\begin{aligned}
\mathbf{p}_{\alpha}(\vec{l}, d) & =-\mathrm{i} \sqrt{\frac{r}{N}} \sum_{j, \vec{q}} \sqrt{\frac{\hbar \omega_{j}(\vec{q}) m_{d}}{2}} \cdot\left[e_{j}(\alpha, d \mid \vec{q}) \mathrm{e}^{\mathrm{i} \vec{q} \cdot \vec{l}} \cdot \mathbf{a}_{j}(\vec{q})-e_{j}^{*}(\alpha, d \mid \vec{q}) \mathrm{e}^{-\mathrm{i} \vec{q} \cdot \vec{l}} \cdot \mathbf{a}_{j}^{+}(\vec{q})\right] \\
& \left.=-\mathrm{i} \sqrt{\frac{r}{N}} \sum_{j, \vec{k}} \sqrt{\frac{\hbar \omega_{j}(\vec{q}) m_{d}}{2}} \cdot e_{j}(\alpha, d \mid \vec{k}) \cdot\left(\mathbf{a}_{j}(\vec{q})-\mathbf{a}_{j}^{+}(-\vec{q})\right]\right) \mathrm{e}^{\mathrm{i} \vec{q} \cdot \vec{l}} \cdot
\end{aligned}
$$

The second equalities rely on $\vec{e}_{j}(\vec{q})=\vec{e}_{j}(-\vec{q})$. They are only valid for crystals with inversion symmetry.

Replacing the expressions (9.65) in the Hamiltonian (9.13) we obtain in perfect analogy to expression (9.40)

$$
\mathbf{H}=\sum_{\vec{q}} \sum_{j=1}^{3 r} \hbar \omega_{j}(\vec{q})\left(\mathbf{a}_{j}^{+}(\vec{q}) \mathbf{a}_{j}(\vec{q})+\frac{1}{2}\right)
$$


the Hamiltonian is thus diagonalised.

We are left with identifying the eigenfunctions. For a wave vector $\vec{q}$ these eigenfunctions are characterised by the occupation of the various phonon branches. Formally

$$
\left|\psi_{n_{1}(\vec{q}), \ldots, n_{3 r}(\vec{q})}\right\rangle \equiv\left|n_{1}(\vec{q}), \ldots, n_{3 r}(\vec{q})\right\rangle=\prod_{j=1}^{3 r} \frac{\left(\hat{a}_{j}^{+}\right)^{n_{j}(\vec{q})}}{\sqrt{n_{j}(\vec{q}) !}}|0, \ldots, 0\rangle .
$$

The integer

$$
n_{j}(\vec{q})=\left\langle\mathbf{a}_{j}^{+}(\vec{q}) \mathbf{a}_{j}(\vec{q})\right\rangle
$$

indicates for each oscillator characterised by the wave vector $\vec{q}$ and the branch index $j$ the level of excitation. A complete basis is obtained by the direct products of the functions

$$
\left|n_{1}(\vec{q}), \ldots, n_{j}(\vec{q})\right\rangle
$$

taken over all wave vectors $\vec{q}$. The energy of the system in a superposition of stationary states is given as

$$
E=\sum_{\vec{q}}^{B z} \sum_{j=1}^{3 r} \hbar \omega_{j}\left(n_{j}(\vec{q})+\frac{1}{2}\right) .
$$

Language becomes very heavy when being obliged to speak about the occupation level $n_{j}(\vec{q})$ of oscillators. For this reason and in perfect analogy with other field theories the excitations are considered quasi-particles. Each oscillator is a reservoir of these particles called phonons. We will often use the word phonon in a slightly sloppy way, i.e. indiscriminate of whether the system has lattice periodicity or not. Creating a phonon of type $(j, \vec{q})$ corresponds to increasing $n(j, \vec{q})$ by one. Annihilating a phonon of type $(j, \vec{q})$ amounts to decreasing $n(j, \vec{q})$ by one. One should, however, not forget that each excited state has its own personality described by the wave functions of a harmonic oscillator expressed in the normal coordinates $\mathbf{Q}_{j}$. E.g. creating a phonon from the ground state does not at all produce the same changes as creating a phonon from an already excited state. To bring this message home we remind the reader about the form of the wave functions $u_{n}(x)$ of a simple one-dimensional harmonic quantum oscillator. They are written as

$$
u_{n}(x)=\left(\frac{\alpha}{2^{n} n ! \sqrt{\pi}}\right) \mathrm{e}^{-(\alpha x)^{2} / 2} H_{n}(\alpha x)
$$

with $H_{n}(\xi)$ Hermite functions

$$
\begin{aligned}
& H_{0}(\xi)=1, \\
& H_{1}(\xi)=2 \xi, \\
& H_{2}(\xi)=4 \xi^{2}-2, \\
& H_{3}(\xi)=8 \xi^{3}-12 \xi,
\end{aligned}
$$

and

$$
\alpha=\sqrt{\frac{m \omega}{\hbar}} .
$$



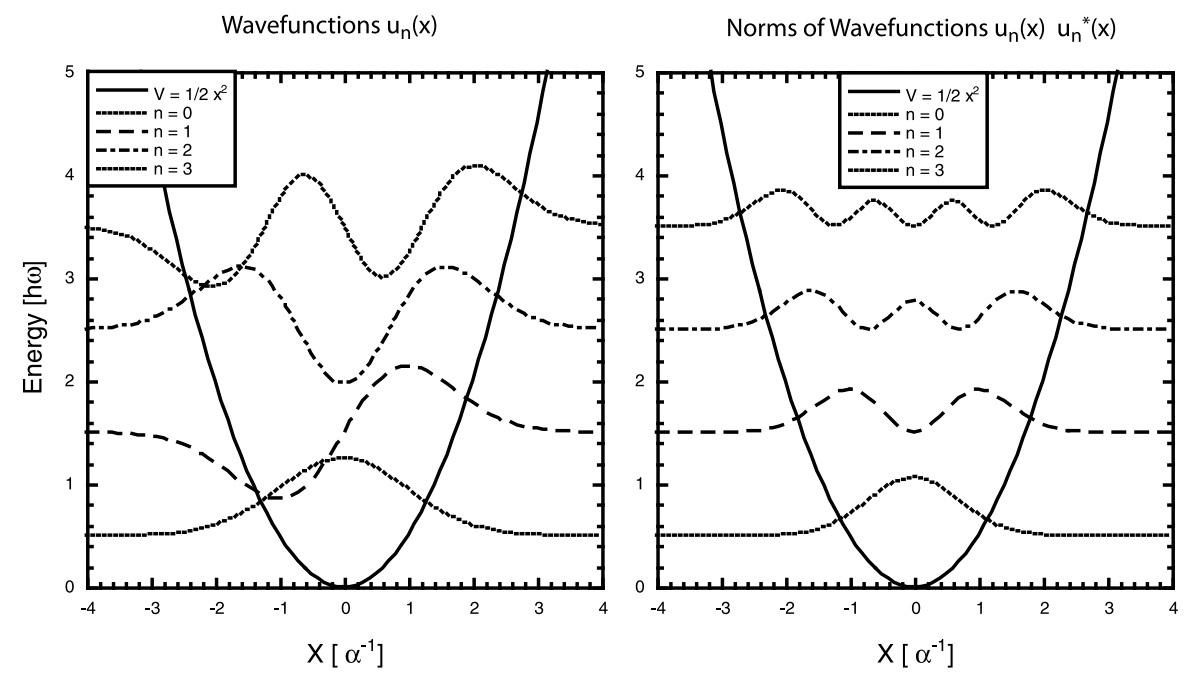

Fig. 46. Harmonic oscillator wave functions as well as their norm. The residence probability moves from the centre to the borders as the excitation level $n$ increases. The narrower the potential the more confined are the wave functions. The intrinsic confinement length can be calculated as $l_{\text {conf }}=2 \sqrt{2 \ln (2)} \alpha^{-1}=2 \sqrt{2 \ln (2)} \sqrt{(\hbar / m \omega)}$.

These functions are depicted in Fig. 46. For phonons the coordinate $x$ has to be replaced by the normal coordinates $Q$.

\subsection{Dynamic structure factor}

We now have all the necessary tools at our disposition to calculate the dynamical structure factor $S(\vec{Q}, \omega)$ for a harmonic system. Our starting point is expression (8.7), which we repeat here for convenience

$$
S(\vec{Q}, \omega)=\sum_{\kappa, \kappa^{\prime}} b_{\kappa} b_{\kappa^{\prime}}^{*} S_{\kappa, \kappa^{\prime}}(\vec{Q}, \omega)
$$

with the partial scattering functions defined as

$$
S_{\kappa, \kappa^{\prime}}(\vec{Q}, \omega)=\frac{1}{2 \pi \hbar} \sum_{j \in\left\{j_{\kappa}\right\}, j^{\prime} \in\left\{j_{\kappa^{\prime}}\right\}} \int_{-\infty}^{\infty} \mathrm{d} t\left\langle\mathrm{e}^{-\mathrm{i} \vec{Q} \cdot \overrightarrow{\mathbf{R}}_{j^{\prime}}(t=0)} \mathrm{e}^{\mathrm{i} \vec{Q} \cdot \overrightarrow{\mathbf{R}}_{j}(t)}\right\rangle \mathrm{e}^{-\mathrm{i} \omega t}
$$

Since the system is harmonic the movements of the ions can be described as vibrations around their equilibrium positions $\vec{R}_{j}^{0}, j=1, \ldots, N$. Attention, here we introduce an important change in notation. From now on $\vec{R}_{j}^{0}$ designates the equilibrium position and is no longer an abbreviation for $\vec{R}_{j}(t=0)$. In general the equilibrium positions do not coincide with the positions of the ions at $t=0$, which means that $\vec{R}_{j}^{0} \neq \vec{R}_{j}(t=0)$. The displacement of the ions are expressed as relative displacements

$$
\overrightarrow{\mathbf{u}}_{j}(t)=\overrightarrow{\mathbf{R}}_{j}(t)-\vec{R}_{j}^{0}, \quad j=1, \ldots, N
$$

Thus

$$
\left\langle\mathrm{e}^{-\mathrm{i} \vec{Q} \cdot \overrightarrow{\mathbf{R}}_{j^{\prime}}(t=0)} \mathrm{e}^{\mathrm{i} \vec{Q} \cdot \overrightarrow{\mathbf{R}}_{j}(t)}\right\rangle=\left\langle\mathrm{e}^{-\mathrm{i} \vec{Q} \cdot\left(\vec{R}_{j^{\prime}}^{0}+\overrightarrow{\mathbf{u}}_{j^{\prime}}(t=0)\right)} \mathrm{e}^{\mathrm{i} \vec{Q} \cdot\left(\vec{R}_{j}^{0}+\overrightarrow{\mathbf{u}}_{j}(t)\right)}\right\rangle .
$$


At this point of the calculation we profit from the fact that the equilibrium positions are simple vectors and, therefore, commute with all the operators. Hence

$$
\left\langle\mathrm{e}^{-\mathrm{i} \vec{Q} \cdot\left(\vec{R}_{j^{\prime}}^{0}+\overrightarrow{\mathbf{u}}_{j^{\prime}}(t=0)\right)} \mathrm{e}^{\mathrm{i} \vec{Q} \cdot\left(\vec{R}_{j}^{0}+\overrightarrow{\mathbf{u}}_{j}(t)\right)}\right\rangle=\mathrm{e}^{-\mathrm{i} \vec{Q} \cdot\left(\vec{R}_{j^{\prime}}^{0}-\vec{R}_{j}^{0}\right)}\left\langle\mathrm{e}^{-\mathrm{i} \vec{Q} \cdot \overrightarrow{\mathbf{u}}_{j^{\prime}}(t=0)} \mathrm{e}^{\mathrm{i} \vec{Q} \cdot \overrightarrow{\mathbf{u}}_{j}(t)}\right\rangle
$$

In order to ease our notation, we want $\overrightarrow{\mathbf{u}}_{j}$ to signify $\overrightarrow{\mathbf{u}}_{j}(t=0)$ as long as time is not explicitly mentioned.

The harmonic approximation is based on the assumption that the displacements are small compared to the interatomic distances. It is, therefore, logical to expand expression (9.77) in terms of the displacements. To perform this expansion, without being obliged to give attention to the time ordering of non-commuting variables, it is desirable to turn the expression into a simple exponential of an expectation value. In a first step we convert the product of exponentials into a single exponential. We achieve this by using a general relation of operator algebra

$$
\exp \mathbf{A} \cdot \exp \mathbf{B}=\exp (\mathbf{A}+\mathbf{B}+\mathbf{C})
$$

with

$$
\mathbf{C}=\frac{1}{2}[\mathbf{A}, \mathbf{B}]+\frac{1}{12}[[\mathbf{A}, \mathbf{B}], \mathbf{B}]+\frac{1}{12}[[\mathbf{B}, \mathbf{A}], \mathbf{A}]+\cdots .
$$

In order to apply this relation to our case we set

$$
\begin{aligned}
& \mathbf{A}:=-\mathrm{i} \vec{Q} \cdot \overrightarrow{\mathbf{u}}_{j^{\prime}}, \\
& \mathbf{B}:=\mathrm{i} \vec{Q} \cdot \overrightarrow{\mathbf{u}}_{j}(t) .
\end{aligned}
$$

In Section 9.4 we have learned that the ionic displacement can be expressed in terms of creation and annihilation operators (see expression (9.44))

$$
\mathbf{u}(\alpha, j)=\sum_{i} \sqrt{\frac{\hbar}{2 m_{j} \omega_{i}}}\left[e_{i}(\alpha, j) \mathbf{a}_{i}+e_{i}^{*}(\alpha, j) \mathbf{a}_{i}^{+}\right]
$$

with $\omega_{i}$ the frequencies and $e_{i}(\alpha, j)(\alpha=x, y, z, j=1, \ldots, N)$ the eigenvectors of the $3 N$ vibrations. All the operators in this expression are formulated in the Schrödinger picture, i.e. they are time independent.

The expectation value

$$
\left\langle\mathrm{e}^{-\mathrm{i} \vec{Q} \cdot\left(\vec{R}_{j^{\prime}}^{0}+\overrightarrow{\mathbf{u}}_{j^{\prime}}(t=0)\right)} \mathrm{e}^{\mathrm{i} \vec{Q} \cdot\left(\vec{R}_{j}^{0}+\overrightarrow{\mathbf{u}}_{j}(t)\right)}\right\rangle
$$

is formulated in terms of the Heisenberg operators $\overrightarrow{\mathbf{u}}_{j}(t)$. In order to evaluate this expression in the basis of the vibrational eigenstates we must express the annihilation and creation operators of the vibrations equally as Heisenberg operators. Formally this is achieved via

$$
\begin{aligned}
& \mathbf{a}_{i}(t)=\exp \left(\mathrm{i} \mathbf{H} \frac{t}{\hbar}\right) \mathbf{a}_{i} \exp \left(-\mathrm{i} \mathbf{H} \frac{t}{\hbar}\right) \\
& \mathbf{a}_{i}^{+}(t)=\exp \left(\mathrm{i} \mathbf{H} \frac{t}{\hbar}\right) \mathbf{a}_{i}^{+} \exp \left(-\mathrm{i} \mathbf{H} \frac{t}{\hbar}\right) .
\end{aligned}
$$

The Heisenberg operators satisfy the relation (see references [10,37])

$$
\frac{\partial}{\partial t} \mathbf{A}(t)=\frac{\mathrm{i}}{\hbar}[\mathbf{H}, \mathbf{A}(t)]
$$


In the case of vibrations the Hamiltonian is written as (expression (9.40))

$$
\mathbf{H}=\sum_{i=1}^{3 N} \hbar \omega_{i}\left(\mathbf{a}_{i}^{+} \mathbf{a}_{i}+\frac{1}{2}\right)
$$

Using the commutation relation

$$
\left[\mathbf{a}_{i}, \mathbf{a}_{i^{\prime}}^{+}\right]=\delta_{i, i^{\prime}}
$$

we find

$$
\begin{gathered}
\frac{\partial}{\partial t} \mathbf{a}_{i}(t)=\frac{\mathrm{i}}{\hbar}\left[\mathbf{H}, \mathbf{a}_{i}(t)\right]=-\mathrm{i} \omega_{i} \cdot \mathbf{a}_{i}(t), \\
\frac{\partial}{\partial t} \mathbf{a}_{i}^{+}(t)=\frac{\mathrm{i}}{\hbar}\left[\mathbf{H}, \mathbf{a}_{i}^{+}(t)\right]=\mathrm{i} \omega_{i} \cdot \mathbf{a}_{i}^{+}(t),
\end{gathered}
$$

and hence

$$
\begin{aligned}
& \mathbf{a}_{i}(t)=\mathbf{a}_{i}(t=0) \exp \left(-\mathrm{i} \omega_{i} t\right)=\mathbf{a}_{i} \cdot \exp \left(-\mathrm{i} \omega_{i} t\right), \\
& \mathbf{a}_{i}(t)^{+}=\mathbf{a}_{i}^{+}(t=0) \exp \left(\mathrm{i} \omega_{i} t\right)=\mathbf{a}_{i}^{+} \cdot \exp \left(\mathrm{i} \omega_{i} t\right) .
\end{aligned}
$$

This leads to

$$
\mathbf{u}_{\alpha}(j ; t)=\sum_{i} \sqrt{\frac{\hbar}{2 m_{j} \omega_{i}}}\left[e_{i}(\alpha, j) \mathbf{a}_{i} \exp \left(-\mathrm{i} \omega_{i} t\right)+e_{i}^{*}(\alpha, j) \mathbf{a}_{i}^{+} \exp \left(\mathrm{i} \omega_{i} t\right)\right] .
$$

The operators $\overrightarrow{\mathbf{u}}(t)$ for any time $t$ remain a linear combination of the creation and annihilation operators. The commutators of these operators at different times are, therefore, simple numbers. Hence the series of commutators in (9.79) is truncated after the first term leaving us with

$$
\exp \mathbf{A} \cdot \exp \mathbf{B}=\exp \left(\mathbf{A}+\mathbf{B}+\frac{1}{2}[\mathbf{A}, \mathbf{B}]\right)=\exp \left(\frac{1}{2}[\mathbf{A}, \mathbf{B}]\right) \exp (\mathbf{A}+\mathbf{B})
$$

Thus we have successfully rewritten the correlation functions of the expression (9.77) in an exponential form. In order to expand this expression, we need to do a little more. It is necessary to turn the expectation value of the exponential function into an exponential function of an expectation value. In other words, the \langle\rangle should be transferred to the exponent. This operation is possible using the Bloch identity.

Let $\mathbf{L}$ be any linear combination of phonon annihilation $\mathbf{a}$ and creation $\mathbf{a}^{+}$operators.

$$
\mathbf{L}=\sum_{j} a_{j} \mathbf{a}_{j}+b_{j} \mathbf{a}_{j}^{+}
$$

The Bloch identity teaches us that

$$
\langle\exp (\mathbf{L})\rangle=\exp \left(\frac{1}{2}\left\langle\mathbf{L}^{2}\right\rangle\right)
$$

The reader anxious to find the prove of this identity can consult the books by Squires [62] or Lovesey [39]. 
As $\overrightarrow{\mathbf{u}}(j ; t)$ is expressed (see (9.92)) as a linear combination of the form (9.94) the Bloch identity is clearly applicable to expression (9.77). We find

$$
\begin{aligned}
\langle\exp \mathbf{A} \cdot \exp \mathbf{B}\rangle & =\left\langle\exp \left(\mathbf{A}+\mathbf{B}+\frac{1}{2}[\mathbf{A}, \mathbf{B}]\right)\right\rangle \\
& =\exp \left(\frac{1}{2}[\mathbf{A}, \mathbf{B}]\right)\langle\exp (\mathbf{A}+\mathbf{B})\rangle \\
& =\exp \left(\frac{1}{2}[\mathbf{A}, \mathbf{B}]\right) \exp \left(\frac{1}{2}\left\langle(\mathbf{A}+\mathbf{B})^{2}\right\rangle\right) \\
& =\exp \left(\frac{1}{2}\left\langle(\mathbf{A}+\mathbf{B})^{2}+[\mathbf{A}, \mathbf{B}]\right\rangle\right) \\
& =\exp \left(\frac{1}{2}\left\langle\mathbf{A}^{2}+\mathbf{B}^{2}+\mathbf{A B}+\mathbf{B A}+\mathbf{A B}-\mathbf{B A}\right\rangle\right) \\
& =\exp \left(\frac{1}{2}\left\langle\mathbf{A}^{2}+\mathbf{B}^{2}+2 \mathbf{A B}\right\rangle\right),
\end{aligned}
$$

or in an explicit manner

$$
\begin{aligned}
& \left\langle\exp \left(-\mathrm{i} \vec{Q} \cdot \overrightarrow{\mathbf{u}}_{j^{\prime}}\right) \exp \left(\mathrm{i} \vec{Q} \cdot \overrightarrow{\mathbf{u}}_{j}(t)\right)\right\rangle \\
& \quad=\exp \left(\frac{1}{2}\left\langle-\left(\vec{Q} \cdot \overrightarrow{\mathbf{u}}_{j^{\prime}}\right)^{2}-\left(\vec{Q} \cdot \overrightarrow{\mathbf{u}}_{j}(t)\right)^{2}+2\left(\vec{Q} \cdot \overrightarrow{\mathbf{u}}_{j^{\prime}}\right)\left(\vec{Q} \cdot \overrightarrow{\mathbf{u}}_{j}(t)\right)\right\rangle\right) \\
& \quad=\exp \left(-\frac{1}{2}\left\langle\left(\vec{Q} \cdot \overrightarrow{\mathbf{u}}_{j^{\prime}}\right)^{2}\right\rangle\right) \exp \left(-\frac{1}{2}\left\langle\left(\vec{Q} \cdot \overrightarrow{\mathbf{u}}_{j}(t)\right)^{2}\right\rangle\right) \exp \left(\left\langle\left(\vec{Q} \cdot \overrightarrow{\mathbf{u}}_{j^{\prime}}\right)\left(\vec{Q} \cdot \overrightarrow{\mathbf{u}}_{j}(t)\right)\right\rangle\right) .
\end{aligned}
$$

We would like to emphasise that this result is only valid in the harmonic approximation due to the fact that we have invoked the Bloch identity. As we have done for the operators $\vec{R}_{j}(t)$ we will denote the displacements $\vec{u}_{j}(t)$ without explicitly indicating their operator character unless we intend to explicitly emphasis this character.

\subsection{Debye-Waller factor}

The quantity

$$
W_{j}(\vec{Q}):=\frac{1}{2}\left\langle\left(\vec{Q} \cdot \vec{u}_{j}\right)^{2}\right\rangle
$$

is proportional to the square of the projection of the average displacement of the ion $j$ along the direction $\vec{Q}$.

We call $W_{j}(\vec{Q})$ the Debye-Waller function of the particle $j$ and

$$
\exp \left(-W_{j}(\vec{Q})\right)=\exp \left(-\frac{1}{2}\left\langle\left(\vec{Q} \cdot \vec{u}_{j}\right)^{2}\right\rangle\right)
$$

its Debye-Waller factor $[15,69]$. We make this explicit distinction between the Debye-Waller function and the Debye-Waller factor in order to facilitate later discussions. 
With this definition the partial scattering functions (9.74) become

$$
\begin{aligned}
S_{\kappa, \kappa^{\prime}}(\vec{Q}, \omega) & =\frac{1}{2 \pi \hbar} \sum_{j \in\left\{j_{\kappa}\right\}, j^{\prime} \in\left\{j_{\kappa^{\prime}}\right\}} \int_{-\infty}^{\infty} \mathrm{d} t\left\langle\mathrm{e}^{-\mathrm{i} \vec{Q} \cdot \vec{R}_{j^{\prime}}(t=0)} \mathrm{e}^{\mathrm{i} \vec{Q} \cdot \vec{R}_{j}(t)}\right\rangle \mathrm{e}^{-\mathrm{i} \omega t} \\
& =\frac{1}{2 \pi \hbar} \sum_{j \in\left\{j_{\kappa}\right\}, j^{\prime} \in\left\{j_{\kappa^{\prime}}\right\}} \int_{-\infty}^{\infty} \mathrm{d} t \mathrm{e}^{-\mathrm{i} \vec{Q} \cdot\left(\vec{R}_{j^{\prime}}^{0}-\vec{R}_{j^{0}}^{0}\right)}\left\langle\mathrm{e}^{-\mathrm{i} \vec{Q} \cdot \vec{u}_{j^{\prime}}(t=0)} \mathrm{e}^{\mathrm{i} \vec{Q} \cdot \vec{u}_{j}(t)}\right\rangle \\
& =\frac{1}{2 \pi \hbar} \sum_{j \in\left\{j_{\kappa}\right\}, j^{\prime} \in\left\{j_{\kappa^{\prime}}\right\}} \int_{-\infty}^{\infty} \mathrm{d} t \mathrm{e}^{-\mathrm{i} \vec{Q} \cdot\left(\vec{R}_{j^{\prime}}^{0}-\vec{R}_{j}^{0}\right)} \mathrm{e}^{-W_{j^{\prime}}(\vec{Q})} \mathrm{e}^{-W_{j}(\vec{Q})} \mathrm{e}^{\left\langle\left(\vec{Q} \cdot \vec{u}_{j^{\prime}}\right)\left(\vec{Q} \cdot \vec{u}_{j}(t)\right)\right\rangle} .
\end{aligned}
$$

Using expressions (9.44) and (9.47) and respecting the commutation relations for ladder operators ${ }^{95}$ the mean square amplitude of particle $j$ in the direction $\alpha$ is calculated as

$$
\begin{aligned}
\overline{u(\alpha, j)^{2}} & =\left\langle\mathbf{u}(\alpha, j) \mathbf{u}(\alpha, j)^{+}\right\rangle \\
& =\sum_{i=1}^{3 N} \frac{\left|e_{i}(\alpha, j)\right|^{2} \hbar}{2 m_{j} \omega_{i}}\left\langle\mathbf{a}_{i} \mathbf{a}_{i}^{+}+\mathbf{a}_{i}^{+} \mathbf{a}_{i}\right\rangle \\
& =\sum_{i=1}^{3 N} \frac{\left|e_{i}(\alpha, j)\right|^{2} \hbar}{2 m_{j} \omega_{i}}\left(1+2\left\langle\mathbf{a}_{i}^{+} \mathbf{a}_{i}\right\rangle\right) \\
& =\sum_{i=1}^{3 N} \frac{\left|e_{i}(\alpha, j)\right|^{2} \hbar}{2 m_{j} \omega_{i}}\left(2 n\left(\omega_{i}\right)+1\right)
\end{aligned}
$$

with

$$
2 n\left(\omega_{i}\right)+1=2 \frac{1}{\exp \left(\hbar \omega_{i} /\left(k_{\mathrm{B}} T\right)\right)-1}+1=\operatorname{coth}\left(\frac{1}{2} \frac{\hbar \omega_{i}}{k_{\mathrm{B}} T}\right) .
$$

We can immediately use this result to determine the Debye-Waller function in terms of the vibrational eigenvectors and eigenfrequencies

$$
2 W_{j}(\vec{Q})=\left\langle\left(\vec{Q} \cdot \vec{u}_{j}\right)^{2}\right\rangle=\sum_{\alpha=x, y, z} \sum_{j=1}^{3 N} \frac{\left|Q_{\alpha} \cdot e_{i}(\alpha, j)\right|^{2} \hbar}{2 m_{j} \omega_{i}} \operatorname{coth}\left(\frac{1}{2} \frac{\hbar \omega}{k_{\mathrm{B}} T}\right) .
$$

\footnotetext{
${ }^{95}$ The commutator of ladder operators is given as
}

$$
\left[\mathbf{a}_{j}, \mathbf{a}_{j^{\prime}}^{+}\right]=\delta_{j, j^{\prime}} .
$$

For the expectation values we retain the general rule that

$$
\left\langle\mathbf{a}_{j} \mathbf{a}_{j^{\prime}}\right\rangle=\left\langle\mathbf{a}_{j}^{+} \mathbf{a}_{j^{\prime}}^{+}\right\rangle=0
$$

for any value of $j$ and $j^{\prime}$ while

$$
\left\langle\mathbf{a}_{j} \mathbf{a}_{j^{\prime}}^{+}\right\rangle=\left\langle\mathbf{a}_{j}^{+} \mathbf{a}_{j^{\prime}}\right\rangle=0
$$

for $j \neq j^{\prime}$. 
It is clear that this average does not depend on time. Thus the expectation value (9.97) simplifies to

$$
\left\langle\mathrm{e}^{-\mathrm{i} \vec{Q} \cdot \vec{u}_{j^{\prime}}} \mathrm{e}^{\mathrm{i} \vec{Q} \cdot \vec{u}_{j}(t)}\right\rangle=\mathrm{e}^{-W_{j^{\prime}}(\vec{Q})} \mathrm{e}^{-W_{j}(\vec{Q})} \mathrm{e}^{\left\langle\left(\vec{Q} \cdot \vec{u}_{j^{\prime}}\right)\left(\vec{Q} \cdot \vec{u}_{j}(t)\right)\right\rangle} .
$$

The Debye-Waller function is in general specific to an atomic site $j$ and depends in addition on the direction of $\vec{Q}$. The symmetry of the site can restrain the form of the Debye-Waller function. In the extreme case it becomes isotropic.

If we group the ions into symmetrically equivalent classes $\{\kappa\}$ then the mean square displacement can be expressed elegantly by introducing the concept of partial densities of states

$$
F_{\kappa}(\omega)=\frac{1}{3 N_{\kappa}} \sum_{j=1}^{3 N} \sum_{n \in\{\kappa\}} \sum_{\alpha=1}^{3}\left|e_{j}(\alpha, n)\right|^{2} \delta\left(\omega-\omega_{j}\right)=\frac{1}{3 N_{\kappa}} \sum_{j=1}^{3 N} \sum_{n \in\{\kappa\}}\left|\vec{e}_{j}(n)\right|^{2} \delta\left(\omega-\omega_{j}\right) .
$$

The sum over the atoms includes the totality of ions within an equivalence class $\{\kappa\}$. Normalisation has been chosen such that

$$
\int_{0}^{\infty} F_{\kappa}(\omega) \mathrm{d} \omega=1
$$

In analogy

$$
g(\omega)=\frac{1}{3 N} \sum_{j=1}^{3 N} \delta\left(\omega-\omega_{j}\right)
$$

is the total density-of-states with

$$
\int_{0}^{\infty} g(\omega) \mathrm{d} \omega=1
$$

Replacing expression (9.105) in expression (9.101) we get

$$
\overline{u(\alpha, j \in\{\kappa\})^{2}}=\frac{\hbar}{2 m_{\kappa}} \int_{0}^{\infty} \frac{F_{\kappa}(\omega)}{\omega}(2 n(\omega)+1) \mathrm{d} \omega .
$$

In particular, for a monatomic cubic system the Debye-Waller function can be expressed as a function of the total density of states

$$
2 W=\overline{u^{2}}=\frac{\hbar Q^{2}}{2 m} \int_{0}^{\infty} \frac{g(\omega)}{\omega}(2 n(\omega)+1) \mathrm{d} \omega .
$$

The physical significance of the Debye-Waller function becomes obvious when we look at the probability $f(\vec{u} \cdot \hat{Q})$ of finding an ion in the direction of $\hat{Q}$ with a displacement lying between $\vec{u} \cdot \hat{Q}$ and $\vec{u} \cdot \hat{Q}+\mathrm{d}(\vec{u} \cdot \hat{Q})$. If the dynamics is harmonic this probability can be calculated from the wave functions of the harmonic oscillator. Bloch [62] showed that the function $f(\vec{u} \cdot \hat{Q})$ is a Gaussian

$$
f(\vec{u} \cdot \hat{Q})=C \exp \left(-\frac{(\vec{u} \cdot \hat{Q})^{2}}{2 \sigma(\hat{Q})^{2}}\right)
$$




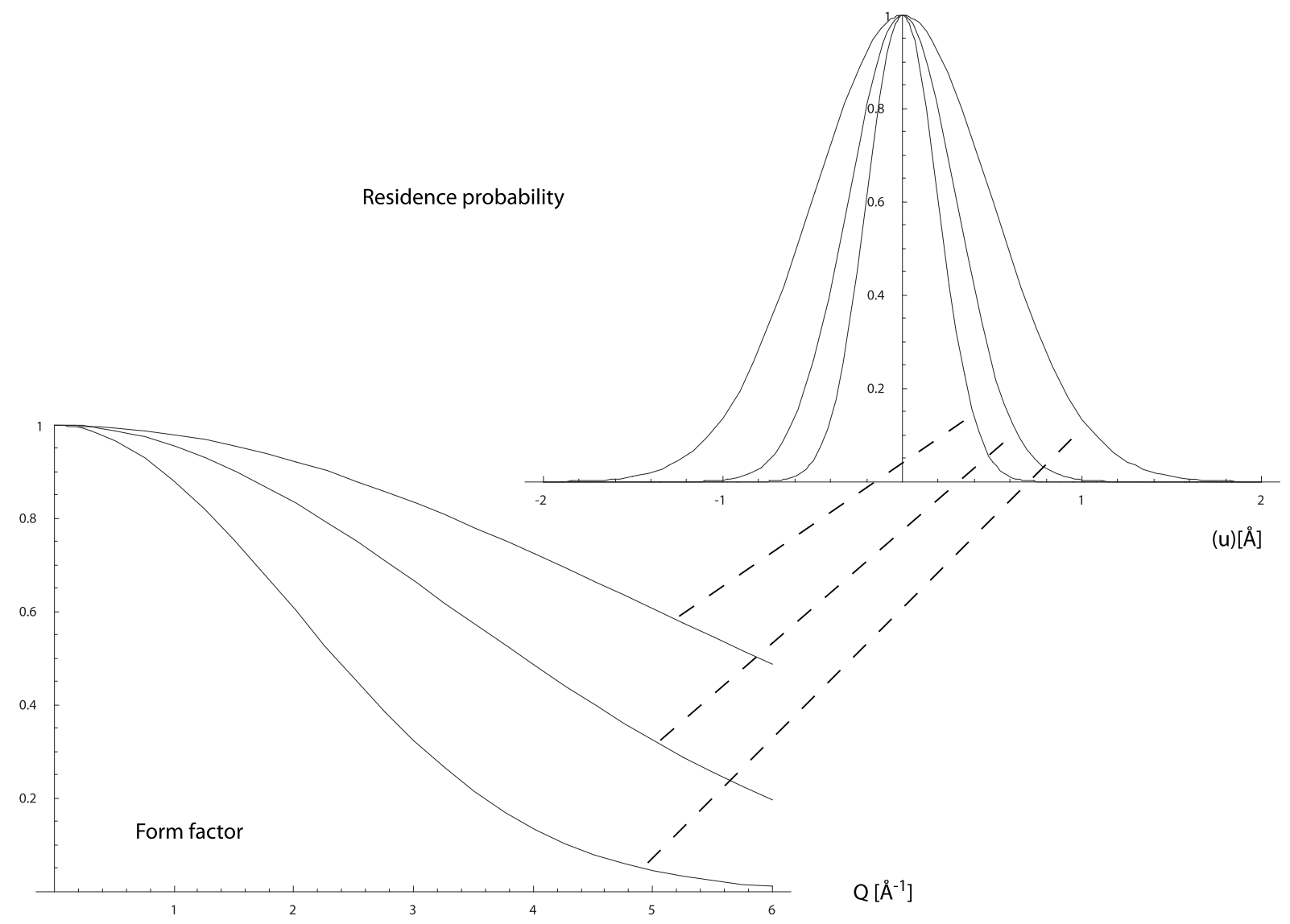

Fig. 47. Above: residence probability along the direction $\vec{Q}$ for different values of $\sigma^{2}=\overline{(\vec{u} \cdot \hat{Q})^{2}}$. Below: Corresponding form factors.

with $\sigma(\hat{Q})^{2}=\overline{(\vec{u} \cdot \hat{Q})^{2}}$ the mean square displacement along the $\hat{Q}$ direction and $C$ a normalisation constant.

The Fourier transform of the function $f(\vec{u} \cdot \hat{Q})$ is found to be

$$
\int_{-\infty}^{\infty} \exp \left(-\frac{(\vec{u} \cdot \hat{Q})^{2}}{2 \sigma^{2}}\right) \exp (-\mathrm{i} \vec{Q} \cdot \vec{u}) \mathrm{d}(\vec{u} \cdot \hat{Q})=\sqrt{2 \pi \sigma^{2}} \exp \left(-\frac{1}{2}(\vec{Q} \cdot \vec{u})^{2}\right)
$$

Hence the expression

$$
\exp \left(-\frac{1}{2}(\vec{Q} \cdot \vec{u})^{2}\right)
$$

corresponds to the form factor of the ionic wave function. If the ion is strongly de-localised, which means that the vibrational amplitudes are large, then the form factor will decrease quickly with $|\vec{Q}|$. If to the contrary the ion is strongly localised then the form factor will tend to a constant (see Fig. 47).

\subsection{Coherent elastic scattering}

The exponential

$$
\exp \left(\left\langle\left(\vec{Q} \cdot \overrightarrow{\mathbf{u}}_{j^{\prime}}\right)\left(\vec{Q} \cdot \overrightarrow{\mathbf{u}}_{j}(t)\right)\right\rangle\right)
$$


can now be expanded as a function of the expectation value ${ }^{96}$

$$
\aleph_{j^{\prime}, j}(\vec{Q}, t):=\left\langle\left(\vec{Q} \cdot \vec{u}_{j^{\prime}}\right)\left(\vec{Q} \cdot \vec{u}_{j}(t)\right)\right\rangle .
$$

The first term is independent of time and thus persist at $t=\infty$. It will give rise to elastic scattering (see Section 8.9). Starting from expression (9.74) for the partial dynamic structure factors and using

$$
\int_{-\infty}^{\infty} \exp (\mathrm{i} \omega t) \mathrm{d} t=2 \pi \hbar \delta(\hbar \omega)
$$

we find

$$
S_{\kappa, \kappa^{\prime}}^{\mathrm{el}}(\vec{Q}, \omega)=\sum_{j \in\left\{j_{\kappa}\right\}, j^{\prime} \in\left\{j_{\kappa^{\prime}}\right\}} \exp \left(-W_{j^{\prime}}(\vec{Q})-W_{j}(\vec{Q})\right) \exp \left(-\mathrm{i} \vec{Q} \cdot\left(\vec{R}_{j^{\prime}}^{0}-\vec{R}_{j}^{0}\right)\right) \delta(\hbar \omega) .
$$

When the diffracting system is a crystal, the equilibrium positions are defined with respect to a primitive cell (see Section 9.5)

$$
\vec{R}_{j}^{0}=\vec{R}_{d}^{0}(\vec{l})=\vec{l}+\vec{d}
$$

For elastic scattering $k_{i}=k_{f}$. The coherent partial differential cross section becomes

$$
\begin{aligned}
& \left(\frac{\mathrm{d}^{2} \sigma}{\mathrm{d} \Omega \mathrm{d} E_{f}}\right)_{\mathrm{coh}}^{\mathrm{el}} \\
& =S_{\mathrm{coh}}(\vec{Q}, \hbar \omega=0) \\
& =\sum_{\kappa, \kappa^{\prime}} \bar{b}_{\kappa} \bar{b}_{\kappa^{\prime}}^{*} S_{\kappa, \kappa^{\prime}}(\vec{Q}, \hbar \omega=0) \\
& =\sum_{\vec{l} \vec{l}^{\prime}} \exp \left(\mathrm{i} \vec{Q} \cdot\left(\vec{l}-\vec{l}^{\prime}\right)\right) \sum_{d, d^{\prime}} \bar{b}_{d} \bar{b}_{d^{\prime}}^{*} \exp \left(-W_{d}(\vec{Q})\right) \exp \left(-W_{d^{\prime}}(\vec{Q})\right) \exp (\overrightarrow{\mathrm{i}} \vec{Q} \cdot \vec{d}) \exp \left(-\mathrm{i} \vec{Q} \cdot \vec{d}^{\prime}\right) \delta(\hbar \omega) \\
& =\frac{N}{r} \sum_{\vec{l}} \exp (\overrightarrow{\mathrm{i}} \vec{Q} \cdot \vec{l}) \sum_{d, d^{\prime}} \bar{b}_{d} \bar{b}_{d^{\prime}}^{*} \exp \left(-W_{d}(\vec{Q})\right) \exp \left(-W_{d^{\prime}}(\vec{Q})\right) \exp (\mathrm{i} \vec{Q} \cdot \vec{d}) \exp \left(-\mathrm{i} \vec{Q} \cdot \vec{d}^{\prime}\right) \delta(\hbar \omega) \\
& =\frac{N}{r} \sum_{\vec{l}} \exp (\mathrm{i} \vec{Q} \cdot \vec{l})\left|\sum_{d} \bar{b}_{d} \exp \left(-W_{d}(\vec{Q})\right) \exp (\mathrm{i} \vec{Q} \cdot \vec{d})\right|^{2} \delta(\hbar \omega),
\end{aligned}
$$

where we have used

$$
\sum_{\vec{l}} \exp \left(\mathrm{i} \vec{Q} \cdot\left(\vec{l}-\vec{l}^{\prime}\right)\right)=\sum_{\vec{l}} \exp (\mathrm{i} \vec{Q} \cdot \vec{l})
$$

exploiting the translation symmetry of the system. ${ }^{97}$ The expression

$$
F(\vec{Q}):=\sum_{d} \bar{b}_{d} \cdot \exp \left(-W_{d}(\vec{Q})\right) \cdot \exp (\mathrm{i} \vec{Q} \cdot \vec{d})
$$

\footnotetext{
${ }^{96}$ This expectation value is of such importance for our discussion that we introduce a specific symbol (the aleph $\aleph$ ) to denote it.

${ }^{97}$ In a crystal all primitive cells are equivalent. The choice of origin should not influence a function that only depends on the distance between the primitive cells.
} 
is called the the (nuclear) form factor of the primitive cell. The analog quantity in the diffraction from a grating is the Fourier transform of the motif multiplied with the Fourier transform of the individual slits (see Section 4.5).

If we introduce an explicit basis of primitive translations

$$
\vec{l}=l_{1} \vec{a}_{1}+l_{2} \vec{a}_{2}+l_{3} \vec{a}_{3}
$$

we can define a basis of the reciprocal lattice via

$$
\begin{aligned}
& \vec{b}_{1}=2 \pi \frac{\vec{a}_{2} \times \vec{a}_{3}}{\vec{a}_{1} \cdot\left(\vec{a}_{2} \times \vec{a}_{3}\right)}, \\
& \vec{b}_{2}=2 \pi \frac{\vec{a}_{3} \times \vec{a}_{1}}{\vec{a}_{1} \cdot\left(\vec{a}_{2} \times \vec{a}_{3}\right)}, \\
& \vec{b}_{3}=2 \pi \frac{\vec{a}_{1} \times \vec{a}_{2}}{\vec{a}_{1} \cdot\left(\vec{a}_{2} \times \vec{a}_{3}\right)} .
\end{aligned}
$$

This reciprocal lattice is composed of Brillouin zones that are centred on the reciprocal lattice vectors

$$
\vec{G}=h \vec{b}_{1}+k \vec{b}_{2}+l \vec{b}_{3}
$$

By definition

$$
\vec{l} \cdot \vec{G}=2 \pi n, \quad n \text { integer. }
$$

Expressing $\vec{Q}$ as a vector in reciprocal space the sum over the lattice vectors $\vec{l}$ can be written in the form

$$
\sum_{\vec{l}} \exp (\mathrm{i} \vec{Q} \cdot \vec{l})=\frac{(2 \pi)^{3}}{V_{\text {unit cell }}} \sum_{\vec{G}} \delta(\vec{Q}-\vec{G}) .
$$

We arrive at the final result for elastic scattering

$$
\left(\frac{\mathrm{d}^{2} \sigma}{\mathrm{d} \Omega \mathrm{d} E_{f}}\right)_{\text {coh }}^{\mathrm{el}}=N_{\text {unit cell }} \frac{(2 \pi)^{3}}{V_{\text {unit cell }}}|F(\vec{Q})|^{2} \sum_{\vec{G}} \delta(\vec{Q}-\vec{G}) \cdot \delta(\hbar \omega) .
$$

$N_{\text {unit cell }}=N / r$ is the number of primitive cells in the crystal.

Hence we obtain Bragg peaks for scattering directions $\vec{Q}$ that correspond to reciprocal lattice vectors $\vec{G}$. The intensity of the peaks is modulated by the form factor $F(\vec{Q})$. The situation is identical to the case of three-dimensional optical gratings that we have discussed in Section 4.6. The result coincides with what we had found for a crystal where the positions of the atoms had been held fixed (see Section 6.5)

$$
\left(\frac{\mathrm{d}^{2} \sigma}{\mathrm{d} \Omega \mathrm{d} E_{f}}\right)_{\text {coh }}^{\mathrm{el}}=\left|\sum_{d} \bar{b}_{d} \mathrm{e}^{\mathrm{i} \vec{Q} \cdot \vec{d}}\right|^{2} \delta(\hbar \omega)
$$

apart from the presence of the Debye-Waller factors. This is not surprising as when the positions are fixed (Dirac $\delta$-functions) the form factors turn into constants. Fixed atoms are nevertheless unphysical. Quantum fluctuations will smear out the wave function even for the lowest temperatures. 


\subsection{Refractive index and coherent reflection}

Before treating the next higher order of perturbation we will make a short but important detour. The expression (9.128) limits coherent elastic scattering in a periodic lattice strictly to Bragg scattering. The situation will not be altered by the higher order terms as these will systematically lead to inelastic contributions. This cannot be the full truth. We know very well from optics that light is not only diffracted but equally refracted. Given that the matter wave equation is in the stationary picture identical to the Helmholtz equation (see Section 3.14) refraction phenomena have to exist equally for particle waves. This is confirmed by experiment.

To capture refraction in a theoretical framework we will go back to the original Schrödinger equation ${ }^{98}$

$$
\left[-\frac{\hbar^{2}}{2 m} \Delta+V(\vec{r})\right] \phi(\vec{r})=E \phi(\vec{r})
$$

and try to solve the equation directly for a periodic suite of Fermi potentials

$$
V(\vec{r})=\frac{2 \pi \hbar^{2}}{m}\left\langle\hat{\rho}_{\mathrm{b}}(\vec{r}, t)\right\rangle=\frac{2 \pi \hbar^{2}}{m}\left\langle\sum_{d} \sum_{\vec{l}} \bar{b}_{d} \cdot \delta\left(\vec{r}-\vec{R}_{d}(\vec{l} ; t)\right)\right\rangle
$$

with $\hat{\rho}_{\mathrm{b}}$ the scattering length density operator [62]. The expectation value is to be taken with respect to the phonon eigenstates. Expressing the $\delta$-function via its Fourier transform (see (8.25))

$$
\delta\left(\vec{r}-\vec{R}_{d}(\vec{l} ; t)\right)=\frac{1}{(2 \pi)^{3}} \int \mathrm{d}^{3} Q \exp \left(\mathrm{i} \vec{Q} \cdot\left(\vec{r}-\vec{R}_{d}(\vec{l} ; t)\right)\right)
$$

we obtain

$$
V(\vec{r})=\frac{\hbar^{2}}{4 m \pi^{2}} \sum_{d} \sum_{\vec{l}} \bar{b}_{d} \int \mathrm{d}^{3} Q\left\langle\exp \left(\mathrm{i} \vec{Q} \cdot\left(\vec{r}-\vec{R}_{d}(\vec{l} ; t)\right)\right\rangle .\right.
$$

Using the displacement operators

$$
\vec{u}_{d}(l ; t)=R_{d}(\vec{l} ; t)-R_{d}^{0}(\vec{l})=R_{d}(\vec{l} ; t)-\vec{l}-\vec{d}
$$

the potential $V(\vec{r})$ becomes

$$
\begin{aligned}
& \frac{\hbar^{2}}{4 m \pi^{2}} \sum_{d} \sum_{\vec{l}} \bar{b}_{d} \int \mathrm{d}^{3} Q\left\langle\exp \left(\mathrm{i} \vec{Q} \cdot\left(\vec{r}-\left(\vec{u}_{d}(\vec{l} ; t)+\vec{R}_{d}^{0}(\vec{l})\right)\right)\right\rangle\right. \\
& \quad=\frac{\hbar^{2}}{4 m \pi^{2}} \sum_{d} \sum_{\vec{l}} \bar{b}_{d} \int \mathrm{d}^{3} Q \cdot \exp (\mathrm{i} \vec{Q} \cdot \vec{r}) \cdot \exp \left(-\mathrm{i} \vec{Q} \cdot \vec{R}_{d}^{0}(\vec{l})\right) \cdot\left\langle\exp \left(-\mathrm{i} \vec{Q} \cdot \vec{u}_{d}(\vec{l} ; t)\right)\right\rangle \\
& \quad=\frac{\hbar^{2}}{4 m \pi^{2}} \sum_{d} \sum_{\vec{l}} \bar{b}_{d} \int \mathrm{d}^{3} Q \cdot \exp (\mathrm{i} \vec{Q} \cdot \vec{r}) \cdot \exp (-\mathrm{i} \vec{Q} \cdot \vec{l}) \cdot \exp (-\mathrm{i} \vec{Q} \cdot \vec{d}) \cdot\left\langle\exp \left(-\mathrm{i} \vec{Q} \cdot \vec{u}_{d}(\vec{l} ; t)\right)\right\rangle \\
& =\frac{\hbar^{2}}{4 m \pi^{2}} \int \mathrm{d}^{3} Q \cdot \exp (\mathrm{i} \vec{Q} \cdot \vec{r}) \cdot \sum_{\vec{l}} \exp (-\mathrm{i} \vec{Q} \cdot \vec{l}) \cdot \sum_{d} \vec{b}_{d} \exp (-\mathrm{i} \vec{Q} \cdot \vec{d}) \cdot\left\langle\exp \left(-\mathrm{i} \vec{Q} \cdot \vec{u}_{d}(\vec{l} ; t)\right)\right\rangle .
\end{aligned}
$$

\footnotetext{
${ }^{98} \mathrm{To}$ avoid confusion with the displacement operators we opt for a change of notation with respect to Section 3.1 and call the wave function $\phi(\vec{r})$.
} 
To evaluate the expectation value we use the Bloch identity. This leads us to

$$
\left\langle\exp \left(-\mathrm{i} \vec{Q} \cdot \vec{u}_{d}(\vec{l} ; t)\right)\right\rangle=\exp \left(-\frac{1}{2} Q^{2} \cdot\left\langle u_{d}(\vec{l} ; t)^{2}\right\rangle\right)=\exp \left(-W_{d}(\vec{Q})\right)
$$

As the expectation value does no longer depend on the lattice vector $\vec{l}$ we may execute the sum over the lattice vector using (9.127)

$$
\sum_{\vec{l}} \exp (-\mathrm{i} \vec{Q} \cdot \vec{l})=\frac{(2 \pi)^{3}}{V_{\text {unit cell }}} \sum_{\vec{G}} \delta(\vec{Q}-\vec{G})
$$

The potential becomes

$$
\begin{aligned}
V(\vec{r}) & =\frac{2 \pi \hbar^{2}}{m V_{\text {unit cell }}} \sum_{\vec{G}} \int \mathrm{d}^{3} Q \exp (\mathrm{i} \vec{Q} \cdot \vec{r}) \cdot \delta(\vec{Q}-\vec{G}) \cdot \sum_{d} \bar{b}_{d} \exp (-\mathrm{i} \vec{Q} \cdot \vec{d}) \cdot \exp \left(-W_{d}(\vec{Q})\right) \\
& =\frac{2 \pi \hbar^{2}}{m V_{\text {unit cell }}} \sum_{\vec{G}} \exp (\mathrm{i} \vec{G} \cdot \vec{r}) \cdot \sum_{d} \bar{b}_{d} \exp (-\mathrm{i} \vec{G} \cdot \vec{d}) \cdot \exp \left(-W_{d}(\vec{G})\right) \\
& =\frac{2 \pi \hbar^{2}}{m} \frac{1}{V_{\text {unit cell }}} \sum_{\vec{G}} \exp (\mathrm{i} \vec{G} \cdot \vec{r}) \cdot F(\vec{G})
\end{aligned}
$$

with the nuclear form factor of the primitive cell $F(\vec{Q})$ defined via (9.120). This is the result that we would have intuitively expected. The potential is periodic with the thermal smearing of the positions described by the DebyeWaller factors. We are now looking for the solutions of the Schrödinger equation for this potential

$$
\Delta \phi(\vec{r})+k_{0}^{2} \phi(\vec{r})=\sum_{\vec{G}} \exp (\mathrm{i} \vec{G} \cdot \vec{r}) \cdot \tilde{F}_{\vec{G}}
$$

with the renormalised form factors

$$
\tilde{F}_{\vec{G}}:=\frac{4 \pi}{V_{\text {unit cell }}} F(\vec{G})
$$

$k_{0}$ is the wave vector of the neutron outside the sample

$$
\frac{\hbar^{2}}{2 m} k_{0}^{2}=E
$$

We know from general symmetry considerations that the one-particle solutions to a periodic potential have the Bloch form, i.e. they can be written as

$$
\phi(\vec{r})=\xi(\vec{r}) \cdot \mathrm{e}^{\mathrm{i} \vec{k} \cdot \vec{r}}
$$

with $\xi(\vec{r})$ possessing the lattice periodicity

$$
\xi(\vec{r})=\xi(\vec{r}+\vec{l}), \quad \forall \vec{l}
$$


A lattice periodic function can be expanded in exponentials of reciprocal lattice vectors

$$
\xi(\vec{r})=\sum_{\vec{G}} a_{\vec{G}} \cdot \exp (-\mathrm{i} \vec{G} \cdot \vec{r})
$$

The wave function, therefore, takes the general form

$$
\phi(\vec{r})=\sum_{\vec{G}} a_{\vec{G}} \cdot \exp (\mathrm{i}(\vec{k}-\vec{G}) \cdot \vec{r}) .
$$

Solving the Schrödinger equation amounts to determining the wave vector $\vec{k}$ inside the sample together with the set of expansion coefficients $a_{\vec{G}}(\vec{k})$ (which are, as explicitly indicated here, a function of $\vec{k}$ ). Substituting (9.145) into (9.130) leads us to the defining equations of $a_{\vec{G}}(\vec{k})$

$$
\begin{aligned}
& \sum_{\vec{G}} a_{\vec{G}}\left[k_{0}^{2}-(\vec{k}-\vec{G})^{2}\right] \cdot \exp (\mathrm{i}(\vec{k}-\vec{G}) \cdot \vec{r}) \\
& =\sum_{\vec{G}^{\prime}} \tilde{F}_{\vec{G}^{\prime}} \exp \left(-\mathrm{i} \vec{G}^{\prime} \cdot \vec{r}\right) \sum_{\vec{G}} a_{\vec{G}} \cdot \exp (\mathrm{i}(\vec{k}-\vec{G}) \cdot \vec{r}) \\
& =\sum_{\vec{G}^{\prime}} \tilde{F}_{\vec{G}^{\prime}} \sum_{\vec{G}} a_{\vec{G}} \cdot \exp \left(\mathrm{i}\left(\vec{k}-\left(\vec{G}-\vec{G}^{\prime}\right)\right) \cdot \vec{r}\right) \\
& =\sum_{\vec{G}} \sum_{\vec{G}^{\prime}} \tilde{F}_{\vec{G}^{\prime}} \cdot a_{\vec{G}+\vec{G}^{\prime}} \cdot \exp (\mathrm{i}(\vec{k}-\vec{G}) \cdot \vec{r}) .
\end{aligned}
$$

Equating terms proportional to

$$
\exp (\mathrm{i}(\vec{k}-\vec{G}) \cdot \vec{r})
$$

yields

$$
a_{\vec{G}}\left[k_{0}^{2}-(\vec{k}-\vec{G})^{2}\right]=\sum_{\vec{G}^{\prime}} \tilde{F}_{\vec{G}^{\prime}} a_{\vec{G}+\vec{G}^{\prime}}
$$

The expansion coefficients $a_{\vec{G}}(\vec{k})$ are, therefore, all coupled. The system of coupled equations is difficult to solve for an arbitrary scattering direction $\vec{k}$. The cases of interest fortunately arise when $\vec{k}$ is close to $\vec{k}_{0}$ or close to a reciprocal lattice vector $\vec{G}$.

Let us consider the first case where $\vec{k} \approx \vec{k}_{0}$. In this case the expression (9.147) reads for $\vec{G}=0$

$$
a_{0}=\frac{1}{\left[k_{0}^{2}-k^{2}\right]} \sum_{\vec{G}^{\prime}} \tilde{F}_{\vec{G}^{\prime}} a_{\vec{G}^{\prime}}
$$

As $k_{0}^{2}-k^{2}$ will be a very small number the coefficient $a_{0}$ will dominate all the others. Neglecting those we get

$$
a_{0}=\frac{1}{\left[k_{0}^{2}-k^{2}\right]} \tilde{F}_{0} a_{0}
$$


This allows defining the refractive index in the usual way as the ratio of the $k$ vectors outside and inside the material

$$
n:=\frac{k}{k_{0}}=\sqrt{1-\frac{\tilde{F}_{0}}{k_{0}^{2}}}
$$

We may exploit the fact that $k$ is very close to $k_{0}$ to approximate the square root expression via $\sqrt{1-x} \approx 1-\frac{1}{2} x$ for small $x$. This leads to

$$
n \approx \frac{\tilde{F}_{0}}{2 k_{0}^{2}}
$$

For $\vec{Q}=0$ the renormalised form factor $\tilde{F}_{0}$ is given as

$$
\tilde{F}_{0}=\frac{4 \pi}{V_{\text {unit cell }}} \sum \bar{b}_{d}=4 \pi \cdot \rho \cdot \bar{b}
$$

Therefore,

$$
n=1-\frac{1}{2 \pi} \rho \lambda^{2} \bar{b}
$$

The scattering length in this expression is averaged over the entire unit cell. The neutron refractive index is, therefore, a concept related to coherent scattering. As the size of the primitive cell does not enter into the final expressions the result does not depend on periodicity and holds thus for arbitrary structures.

The quantity

$$
\rho \bar{b}=\frac{1}{V} \sum_{i \in V} \bar{b}_{i}
$$

is called the scattering length density (SLD) of the material. Values of $b$ are typically in the range of $10^{-15} \mathrm{~m}$ and $N \approx 10^{29}$ per $\mathrm{m}^{3}$. For $\lambda=1 \AA$ we can estimate the order of magnitude of the expression

$$
\lambda^{2} \rho \bar{b} \approx 10^{-6}
$$

The index of refraction of thermal and even cold neutrons is, therefore, extremely close to one in particular if compared to the refractive index of ordinary light. This smallness reflects the weak interaction of neutrons with matter.

The refractive index is responsible for neutron reflection at a surface. The mathematical framework for describing reflection can be transposed one-to-one from light optics. Thus total reflection is observed if the angle subtended by the beam satisfies the condition

$$
\cos \Theta_{c}=n
$$

with $\Theta$ defined in Fig. 48. Total reflection thus occurs if $n<1$. This is the case if

$$
1-\left(\sin \Theta_{c}\right)^{2}=\left(1-\frac{\lambda^{2}}{2 \pi} \rho \cdot \bar{b}\right)^{2} \approx 1-\frac{\lambda^{2}}{\pi} \rho \cdot \bar{b}
$$


Straight rectangular neutron guide

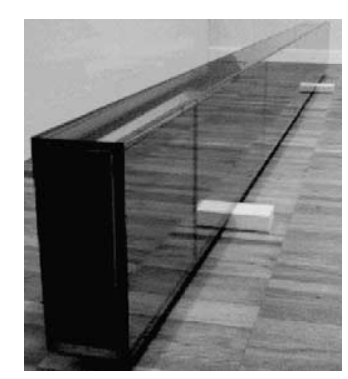

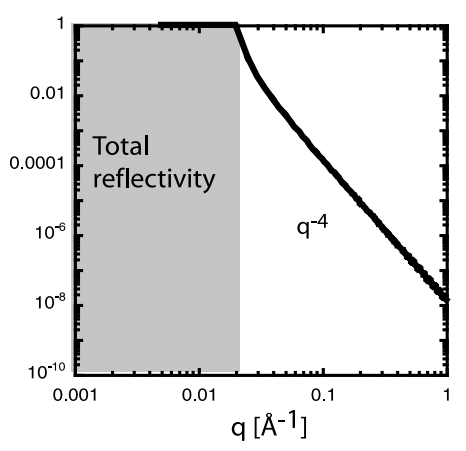

surface normal

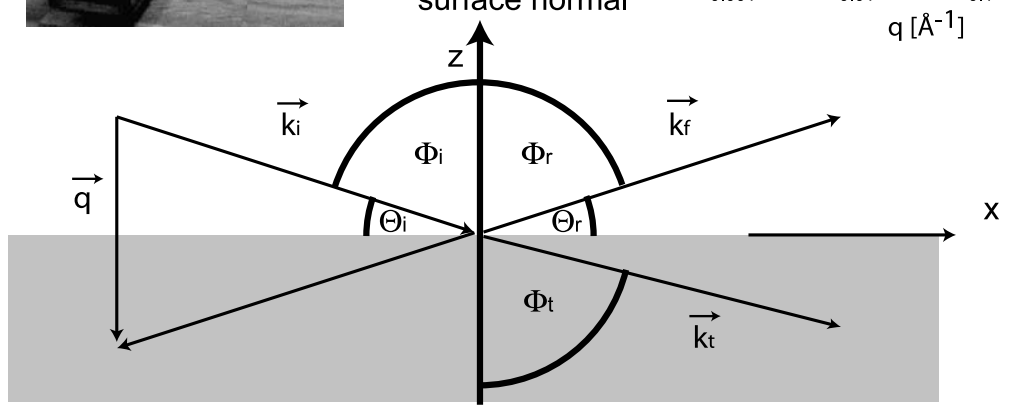

Fig. 48. Neutron reflection. The reflectivity is one below the critical momentum transfer $q_{c}$ and drops off rapidly with larger $q$ (that is, with larger reflection angles). Natural $\mathrm{Ni}$ is the reference coating material for standard neutron guides. Super-mirror guides are guides featuring a multi-layer coating that pushes the critical angle to higher values.

and thus

$$
\sin \Theta_{c}=\sqrt{\frac{\rho \cdot \bar{b}}{\pi}} \lambda .
$$

$\Theta_{c}$ is called the critical angle of the material. Total external reflection $(n<1$ for neutrons in a material that possesses positive scattering length like $\mathrm{Ni}$ ) inside a channel is used to transport neutrons over large distances. Nickel, due to its large coherent scattering cross section and thus rather large critical angle of $\Theta_{c}=0.1^{\circ} / \AA$, is a material of choice for these neutron guide applications. The transport is the more efficient the longer the wave length as due to the increased critical angle at longer wave length larger divergences can be tolerated.

The reflectivity $R$ is given as the ratio of the reflected to the incoming intensity. Its dependence on the refraction angle $\Phi_{i}$ is given by the Fresnel equations

$$
R=\left|\frac{n_{1} \cos \Phi_{i}-n_{2} \cos \Phi_{t}}{n_{1} \cos \Phi_{i}+n_{2} \cos \Phi_{t}}\right|^{2}=\left|\frac{n_{1} \cos \Phi_{i}-n_{2} \sqrt{1-\left(n_{1} / n_{2} \sin \Phi_{i}\right)^{2}}}{n_{1} \cos \Phi_{i}+n_{2} \sqrt{1-\left(n_{1} / n_{2} \sin \Phi_{i}\right)^{2}}}\right|^{2} .
$$

It can be directly derived from the fact that for specular reflection (i) $\Phi_{i}=\Phi_{t}$ and (ii)

$$
\frac{\sin \Phi_{i}}{\sin \Phi_{t}}=\frac{n_{2}}{n_{1}}
$$

This is Snell's law that derives directly from the continuity of the wave function at the interface. Please note that the refraction angles $\Phi$ are defined with respect to the surface normal while the scattering angles $\Theta$ are defined with respect to the surface (see Fig. 48). 
If the medium on the reflection side is vacuum then $n_{1}=1$ and $n_{2}=n$. The Fresnel equations can be expressed conveniently as a function of the length of the scattering vector

$$
q=\frac{4 \pi}{\lambda} \sin \Theta
$$

We obtain

$$
R=\left[\frac{q-\sqrt{q^{2}-q_{c}^{2}}}{q+\sqrt{q^{2}-q_{c}^{2}}}\right]^{2}
$$

with the length of the critical scattering vector given by

$$
q_{c}=\frac{4 \pi}{\lambda} \sin \Theta_{c}
$$

For $q \gg q_{c}$ this expression simplifies to

$$
R=16 \frac{\pi^{2}}{q^{4}}(\rho \cdot \bar{b})^{2} .
$$

This so-called Fresnel decay is a property of a planar surface. We could have obtained this result calculating the scattering amplitude in the Born approximation as the Fourier transform of the scattering length density $\rho$

$$
R \propto \frac{1}{q^{2}}\left|\int_{0}^{-\infty} \mathrm{d} z \rho \mathrm{e}^{\mathrm{i} q z}\right|^{2} \propto \frac{1}{q^{4}},
$$

where we have transformed the original 3-dimensional integration into an integration along the direction normal to the surface. At angles close to the critical angle, i.e. when the scattering becomes important, this result diverges from the actual form given by the Fresnel expression (9.160). This is not surprising as the Born approximation breaks down close to total reflection. A technique known as the distorted-wave Born approximation (DWBA) is required to treat the problem $[11,60]$.

The intensity at small angles is often observed to scale as

$$
I(q) \propto \frac{1}{q^{4}}
$$

This is known as Porod's law. Its origin can be traced back to scattering from surfaces or interfaces in inhomogeneous materials [60]. Porod's law is, therefore, directly related to the Fresnel formula (9.160). For Porod's law to be applicable the objects that create the interfaces have to be large with respect to $q^{-1}$. Porod scattering allows in principle to extract the ratio of surface area $S$ over volume $V$ of these objects.

Let us finish the discussion with a few words concerning transmission into a denser medium. For $q<q_{c}$ the total incident intensity is reflected. There is, therefore, no energy flow across the surface. Despite this fact the neutron wave function inside the medium is not vanishing but of the form

$$
\phi_{t}(\vec{r}) \propto \mathrm{e}^{\mathrm{i}\left(\vec{k}_{t} \cdot \vec{r}-\omega \cdot t\right)}
$$

The wave function inside the medium is thus defined by the wave vector $\vec{k}_{t}$. If we choose the geometry such that the scattering takes place in the $x-z$ plane and if we place the surface normal parallel to $\hat{z}$ then the phase factor of 
the wave function reads

$$
\vec{k}_{t} \cdot \vec{r}=k_{t} \cdot x \cdot \sin \Phi_{t}-k_{t} \cdot z \cdot \cos \Phi_{t}=k_{t} x \sin \Phi_{t}-\mathrm{i} k_{t} z \sqrt{\frac{\sin ^{2} \Phi_{i}}{n^{2}}-1},
$$

where for $\Phi>\Phi_{c}$ we have used Snell's law in the form

$$
\cos \Phi_{t}=\sqrt{1-\frac{\sin ^{2} \Phi_{i}}{n^{2}}}=\mathrm{i} \sqrt{\frac{\sin ^{2} \Phi_{i}}{n^{2}}-1}
$$

The fact that the phase has a finite imaginary component implies that the wave function decays exponentially into the medium. This wave is called evanescent. The penetration depth is given by

$$
l_{\mathrm{p}}=\frac{\lambda_{t}}{2 \pi}\left(\frac{\sin ^{2} \Phi_{i}}{n^{2}}-1\right)^{-1 / 2}
$$

which can be expressed as a function of $q_{c}$

$$
l_{\mathrm{p}}=\frac{2}{\sqrt{q_{c}^{2}-q^{2}}}
$$

In the case of $\Theta<\Theta_{c}$ we speak of grazing incidence scattering. The volume probed is defined by the penetration of the evanescent wave

$$
\phi_{e}(z) \propto \mathrm{e}^{-1 / 2 \sqrt{q_{c}^{2}-q^{2}} \cdot z}, \quad q<q_{c}
$$

On a perfect silicon surface this penetration is e.g. about $100 \AA$ at $q=0$. The penetration depth diverges at $q=q_{c}$.

If the wave vector $\vec{k}$ comes to lie close to a reciprocal lattice vector $\vec{G}$ then the associated expansion coefficient $a_{\vec{G}}$ becomes comparable to $a_{0}$ and we get Bragg scattering. The formalism of this so-called dynamical scattering theory is quite powerful and allows exact predictions of the width and intensity of Bragg scattering that cannot be obtained within the Born approximation. E.g. finite penetration is responsible for the finite wavelength acceptance [12] of Bragg scattering from a perfect crystal $(\Delta d \rightarrow 0)$ even for an ideally collimated beam $(\Delta \Theta \rightarrow 0)$. If we perform a rocking curve about the Bragg angle $\Theta_{\vec{G}}$ then we get total reflection over a plateau. The reflectivity curve is described by the Ewald function ${ }^{99}$ (shown in Fig. 49) [38]

$$
R(y)= \begin{cases}1, & |y| \leqslant 1 \\ 1-\sqrt{\frac{y^{2}-1}{y^{2}},} & |y|>1\end{cases}
$$

with

$$
y=\frac{E-E_{0}}{E_{0}} \frac{\pi V_{\text {unit cell }}}{\lambda^{2}\left|F_{\vec{G}}\right|} .
$$

\footnotetext{
${ }^{99}$ The reflectivity depends on the exact diffraction configuration and in particular on how the crystal is cut with respect to the lattice planes. If the crystal is e.g. cut such that the incident beam is grazing the surface then total surface reflection has to be taken into account, which modifies the Ewald function. For details see [71].
} 


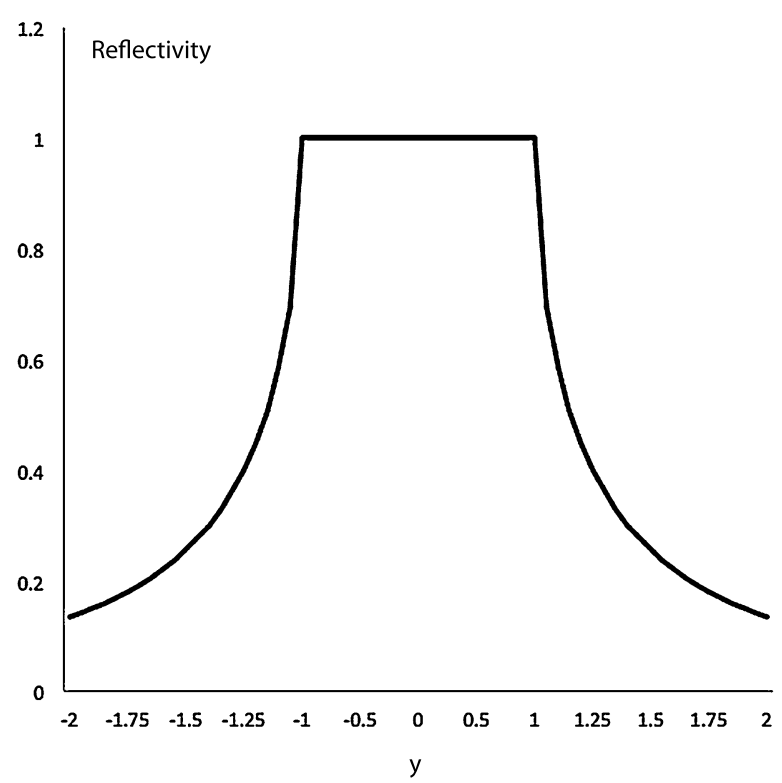

Fig. 49. Ewald function describing the total reflection region around a Bragg peak. The variable $y$ is defined in the text. The width $\Delta y=4 / \sqrt{3}$ of the curve is called the Darwin width.

$E_{0}$ denotes the neutron energy at the centre of the Bragg reflection. The FWHM of the Ewald function is given by

$$
y_{D}=\frac{\Delta E}{E_{0}}=\frac{4}{\sqrt{3}} \frac{\lambda^{2}\left|F_{\vec{G}}\right|}{\pi V_{\text {unit cell }}} .
$$

This width is called the Darwin width. This effect cannot be described by the Bragg equation as the Bragg equation is derived on the assumption of an infinitely large homogeneously illuminated scattering volume. In the case of silicon the Darwin width amounts to a few seconds of arc. We will discuss further effects related to dynamical theory in Section 11. The discussion will always stay qualitative. For an in-depth formal treatment of dynamical scattering theory, which was pioneered by Goldberger and Seitz [30], the reader is referred to the literature.

\subsection{Coherent scattering from vibrations}

After this short detour into the world of dynamical scattering theory we come back to the expansion of the scattering function in terms of phonon displacement operators. The next term in the expansion of

$$
\exp \left(\left\langle\left(\vec{Q} \cdot \vec{u}_{j^{\prime}}\right)\left(\vec{Q} \cdot \vec{u}_{j}(t)\right)\right\rangle\right)
$$

is proportional to

$$
\aleph_{j^{\prime}, j}(\vec{Q}, t)=\left\langle\left(\vec{Q} \cdot \vec{u}_{j^{\prime}}\right)\left(\vec{Q} \cdot \vec{u}_{j}(t)\right)\right\rangle .
$$

It involves the motion of the ions and will, therefore, produce inelastic scattering. We can calculate $\aleph_{j^{\prime}, j}(\vec{Q}, t)$ using expression (9.92) for $\vec{u}_{j}(t)$.

$$
\begin{aligned}
& \left\langle\left(\vec{Q} \cdot \vec{u}_{j^{\prime}}\right)\left(\vec{Q} \cdot \vec{u}_{j}(t)\right)\right\rangle \\
& \quad=\sum_{\alpha, \beta} Q_{\alpha} Q_{\beta}\left\langle\mathbf{u}_{\alpha}\left(j^{\prime} ; t=0\right) \mathbf{u}_{\beta}(j ; t)\right\rangle
\end{aligned}
$$




$$
\begin{aligned}
= & \frac{\hbar}{2} \sum_{\alpha, \beta} \frac{Q_{\alpha} Q_{\beta}}{\sqrt{m_{j} m_{j^{\prime}}}} \sum_{i, i^{\prime}} \sqrt{\frac{1}{\omega_{i} \omega_{i^{\prime}}}} \\
& \times\left\langle\left[e_{i}\left(\alpha, j^{\prime}\right) \mathbf{a}_{i}+e_{i}^{*}\left(\alpha, j^{\prime}\right) \mathbf{a}_{i}^{+}\right]\left[e_{i^{\prime}}(\beta, j) \mathbf{a}_{i^{\prime}} \exp \left(-\mathrm{i} \omega_{i^{\prime}} t\right)+e_{i^{\prime}}^{*}(\beta, j) \mathbf{a}_{i^{\prime}}^{+} \exp \left(\mathrm{i} \omega_{i^{\prime}} t\right)\right]\right\rangle .
\end{aligned}
$$

This expression can be simplified considerably if we exploit the rules for calculating expectation values of products involving creation and annihilation operators

$$
\begin{aligned}
& \left\langle\mathbf{a}_{i} \mathbf{a}_{i^{\prime}}\right\rangle=0, \\
& \left\langle\mathbf{a}_{i}^{+} \mathbf{a}_{i^{\prime}}^{+}\right\rangle=0, \\
& \left\langle\mathbf{a}_{i}^{+} \mathbf{a}_{i^{\prime}}\right\rangle=n\left(\omega_{i}\right) \delta_{i, i^{\prime}}, \\
& \left\langle\mathbf{a}_{i} \mathbf{a}_{i^{\prime}}^{+}\right\rangle=\left(1+n\left(\omega_{i}\right)\right) \delta_{i, i^{\prime}},
\end{aligned}
$$

with $n\left(\omega_{i}\right)$ the Bose-Einstein occupation factor of the oscillator with frequency $\omega_{i}$. Thus

$$
\begin{aligned}
\left\langle\left(\vec{Q} \cdot \vec{u}_{j^{\prime}}\right)\left(\vec{Q} \cdot \vec{u}_{j}(t)\right)\right\rangle & \\
= & \frac{\hbar}{2} \sum_{\alpha, \beta} \frac{Q_{\alpha} Q_{\beta}}{\sqrt{m_{j} m_{j^{\prime}}}} \sum_{i, i^{\prime}} \sqrt{\frac{1}{\omega_{i} \omega_{i^{\prime}}}} \times \delta_{i, i^{\prime}} \\
& \times\left\{\left[e_{i}\left(\alpha, j^{\prime}\right) e_{i^{\prime}}^{*}(\beta, j)\left\langle\mathbf{a}_{i} \mathbf{a}_{i^{\prime}}^{+}\right\rangle \exp \left(\mathrm{i} \omega_{i^{\prime}} t\right)\right]+\left[e_{i}^{*}\left(\alpha, j^{\prime}\right) e_{i^{\prime}}(\beta, j)\left\langle\mathbf{a}_{i}^{+} \mathbf{a}_{i^{\prime}}\right\rangle \exp \left(-\mathrm{i} \omega_{i^{\prime}} t\right)\right]\right\} \\
= & \frac{\hbar}{2} \sum_{\alpha, \beta} \frac{Q_{\alpha} Q_{\beta}}{\sqrt{m_{j} m_{j^{\prime}}}} \sum_{i} \frac{1}{\omega_{i}} \\
& \times\left\{\left[e_{i}\left(\alpha, j^{\prime}\right) e_{i}^{*}(\beta, j)\left(1+n\left(\omega_{i}\right)\right) \exp \left(\mathrm{i} \omega_{i} t\right)\right]+\left[e_{i}^{*}\left(\alpha, j^{\prime}\right) e_{i}(\beta, j) n\left(\omega_{i}\right) \exp \left(-\mathrm{i} \omega_{i} t\right)\right]\right\} \\
= & \frac{\hbar}{2} \sum_{i}\left\{\left[\frac{A_{i}\left(\vec{Q} ; j^{\prime}, j\right)}{\omega_{i}}\left(1+n\left(\omega_{i}\right)\right) \exp \left(\mathrm{i} \omega_{i} t\right)\right]+\left[\frac{A_{i}\left(\vec{Q} ; j, j^{\prime}\right)}{\omega_{i}} n\left(\omega_{i}\right) \exp \left(-\mathrm{i} \omega_{i} t\right)\right]\right\}
\end{aligned}
$$

with

$$
A_{i}\left(\vec{Q} ; j^{\prime}, j\right):=\frac{\left(\vec{Q} \cdot \vec{e}_{i}\left(j^{\prime}\right)\right) \cdot\left(\vec{Q} \cdot \vec{e}_{i}(j)\right)^{*}}{\sqrt{m_{j^{\prime}} m_{j}}}
$$

Inserting this in the expression (9.100) the partial scattering functions are found.

$$
\begin{aligned}
& S_{\kappa, \kappa^{\prime}}^{1-\mathrm{ph}}(\vec{Q}, \omega) \\
& =\frac{1}{2 \pi \hbar} \sum_{j \in\left\{j_{\kappa}\right\}, j^{\prime} \in\left\{j_{\kappa^{\prime}}\right\}} \mathrm{e}^{-\mathrm{i} \vec{Q} \cdot\left(\vec{R}_{j^{\prime}}^{0}-\vec{R}_{j}^{0}\right)} \mathrm{e}^{-W_{j^{\prime}}(\vec{Q})} \mathrm{e}^{-W_{j}(\vec{Q})} \int_{-\infty}^{\infty} \mathrm{d} t\left\langle\left(\vec{Q} \cdot \vec{u}_{j^{\prime}}\right)\left(\vec{Q} \cdot \vec{u}_{j}(t)\right)\right\rangle \\
& =\frac{1}{4 \pi} \sum_{j \in\left\{j_{\kappa}\right\}, j^{\prime} \in\left\{j_{\kappa^{\prime}}\right\}} \mathrm{e}^{-\mathrm{i} \vec{Q} \cdot\left(\vec{R}_{j^{\prime}}^{0}-\vec{R}_{j}^{0}\right)} \mathrm{e}^{-W_{j^{\prime}}(\vec{Q})} \mathrm{e}^{-W_{j}(\vec{Q})} \\
& \quad \times \int_{-\infty}^{\infty} \mathrm{d} t \sum_{i}\left\{\left[\frac{A_{i}\left(\vec{Q} ; j^{\prime}, j\right)}{\omega_{i}}\left(1+n\left(\omega_{i}\right)\right) \exp \left(\mathrm{i} \omega_{i} t\right)\right]+\left[\frac{A_{i}\left(\vec{Q} ; j, j^{\prime}\right)}{\omega_{i}} n\left(\omega_{i}\right) \exp \left(-\mathrm{i} \omega_{i} t\right)\right]\right\}
\end{aligned}
$$




$$
\begin{aligned}
& =\frac{1}{2} \sum_{j \in\left\{j_{\kappa}\right\}, j^{\prime} \in\left\{j_{\kappa^{\prime}}\right\}} \mathrm{e}^{-\mathrm{i} \vec{Q} \cdot\left(\vec{R}_{j^{\prime}}^{0}-\vec{R}_{j^{0}}^{0}\right)} \mathrm{e}^{-W_{j^{\prime}}(\vec{Q})} \mathrm{e}^{-W_{j}(\vec{Q})} \\
& \quad \times \sum_{i}\left\{\left[\frac{A_{i}\left(\vec{Q} ; j^{\prime}, j\right)}{\omega_{i}}\left(1+n\left(\omega_{i}\right)\right) \delta\left(\omega-\omega_{i}\right)\right]+\left[\frac{A_{i}\left(\vec{Q} ; j, j^{\prime}\right)}{\omega_{i}} n\left(\omega_{i}\right) \delta\left(\omega+\omega_{i}\right)\right]\right\},
\end{aligned}
$$

where we have used again

$$
\int_{-\infty}^{\infty} \exp (\mathrm{i} \omega t) \mathrm{d} t=2 \pi \delta(\omega)
$$

With this result we can calculate the coherent scattering function

$$
\begin{aligned}
& S_{\text {coh }}^{1-\mathrm{ph}}(\vec{Q}, \omega) \\
& =\sum_{\kappa, \kappa^{\prime}} \bar{b}_{\kappa} \cdot \bar{b}_{\kappa^{\prime}}^{*} S_{\kappa, \kappa^{\prime}}^{1-\mathrm{ph}}(\vec{Q}, \omega) \\
& =\frac{1}{2} \sum_{\kappa, \kappa^{\prime}} \bar{b}_{\kappa} \cdot \bar{b}_{\kappa^{\prime}}^{*} \sum_{j \in\left\{j_{\kappa}\right\}, j^{\prime} \in\left\{j_{\kappa^{\prime}}\right\}} \mathrm{e}^{-\mathrm{i} \vec{Q} \cdot\left(\vec{R}_{j^{\prime}}^{0}-\vec{R}_{j}^{0}\right)} \mathrm{e}^{-W_{j^{\prime}}(\vec{Q})} \mathrm{e}^{-W_{j}(\vec{Q})} \\
& \quad \times \sum_{i}\left\{\left[\frac{A_{i}\left(\vec{Q} ; j^{\prime}, j\right)}{\omega_{i}}\left(1+n\left(\omega_{i}\right)\right) \delta\left(\omega-\omega_{i}\right)\right]+\left[\frac{A_{i}\left(\vec{Q} ; j, j^{\prime}\right)}{\omega_{i}} n\left(\omega_{i}\right) \delta\left(\omega+\omega_{i}\right)\right]\right\} .
\end{aligned}
$$

An alternative way of writing this expression is

$$
S_{\operatorname{coh}}^{1-p h}(\vec{Q}, \omega)=\frac{1}{2} \sum_{i} \frac{\left|F_{i}(\vec{Q})\right|^{2}}{\omega_{i}}\left\{\left[\left(1+n\left(\omega_{i}\right)\right) \delta\left(\omega-\omega_{i}\right)\right]+\left[n\left(\omega_{i}\right) \delta\left(\omega+\omega_{i}\right)\right]\right\}
$$

with

$$
\begin{aligned}
\left|F_{i}(\vec{Q})\right|^{2} & :=\sum_{\kappa, \kappa^{\prime}} \frac{\bar{b}_{\kappa} \cdot \vec{b}_{\kappa^{\prime}}^{*}}{\sqrt{m_{\kappa^{\prime}} m_{\kappa}}} \sum_{j \in\left\{j_{\kappa}\right\}, j^{\prime} \in\left\{j_{\kappa^{\prime}}\right\}} \mathrm{e}^{-\mathrm{i} \vec{Q} \cdot \vec{R}_{j^{\prime}}^{0}} \mathrm{e}^{\mathrm{i} \vec{Q} \cdot \vec{R}_{j}^{0}} \mathrm{e}^{-W_{j^{\prime}}(\vec{Q})} \mathrm{e}^{-W_{j}(\vec{Q})}\left(\vec{Q} \cdot \vec{e}_{i}\left(j^{\prime}\right)\right) \cdot\left(\vec{Q} \cdot \vec{e}_{i}(j)\right)^{*} \\
& =\left|\sum_{\kappa} \frac{\bar{b}_{\kappa}}{\sqrt{m_{\kappa}}} \sum_{j \in\left\{j_{\kappa}\right\}} \mathrm{e}^{-\mathrm{i} \vec{Q} \cdot \vec{R}_{j}^{0}} e^{-W_{j}(\vec{Q})}\left(\vec{Q} \cdot \vec{e}_{i}(j)\right)\right|^{2}
\end{aligned}
$$

The function $F_{i}(\vec{Q})$ can be considered the form factor of the vibration $\left(\omega_{i}, \vec{e}_{i}\right)$.

The Dirac functions in energy assure that the scattering of neutrons only takes place when the energy transfer is $\pm \hbar \omega$, i.e. when it corresponds to a vibrational frequency. When $\hbar \omega$ is positive ${ }^{100}$ the neutron will loose energy in the scattering process. As a compensation an excitation is created in the sample. The occupation level of a vibration is indicated by the factor $n(\omega)$ (see Fig. 50). Since the creation of an excitation is proportional to $n\left(\omega_{i}\right)+1$ this down-scattering process is possible ${ }^{101}$ even at very low temperatures when the system is in its ground state and

\footnotetext{
${ }^{100}$ Attention: This convention is not always respected in the literature. It is always useful to add 'loss' or 'gain' in energy of the neutron.

${ }^{101}$ Naturally under the condition that the kinematic conditions can be fulfilled (see Section 6.4).
} 


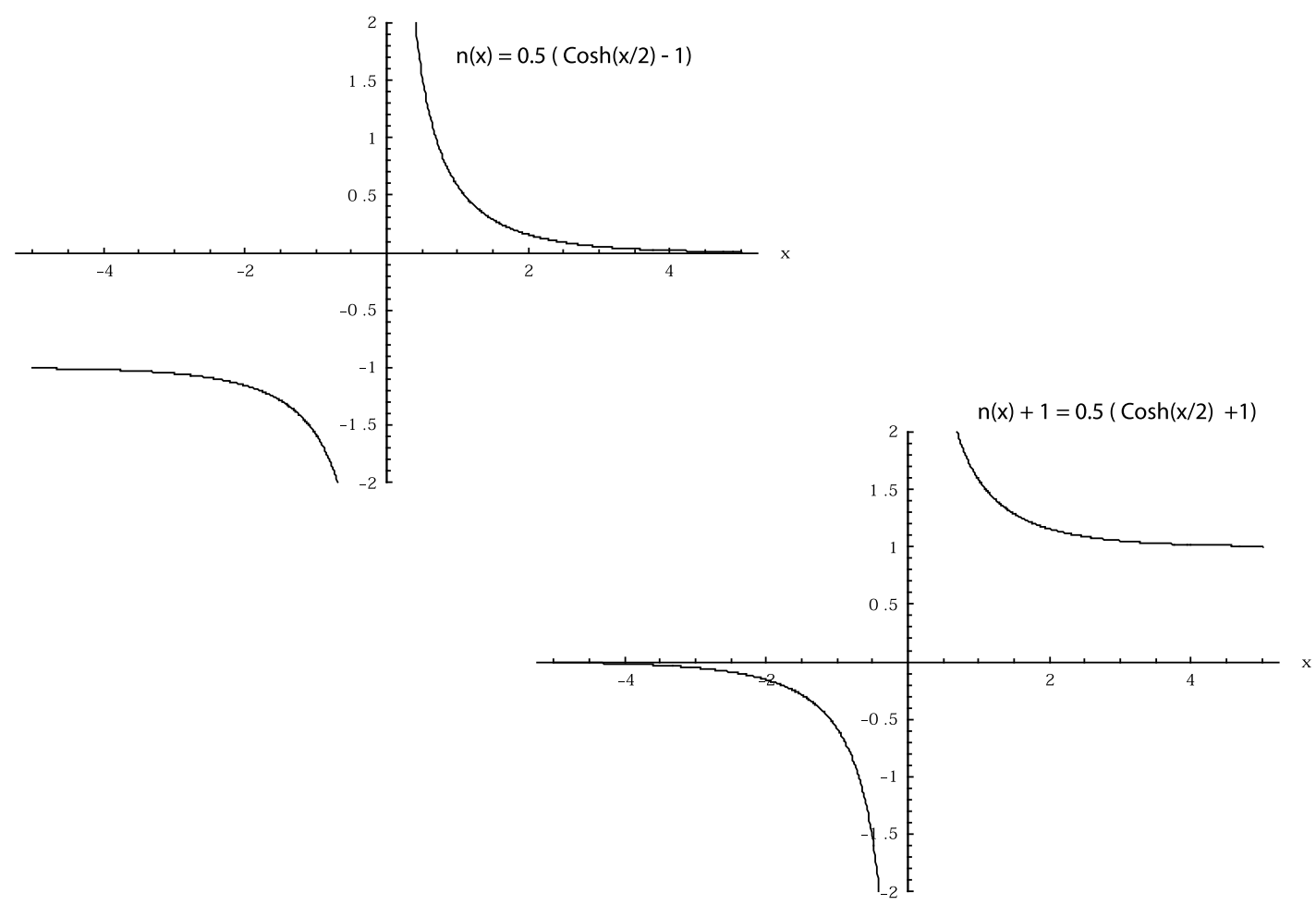

Fig. 50. Occupation factor $n(x)=(1 / 2)(\cosh (x / 2)-1)$ and $n(x)+1=(1 / 2)(\cosh (x / 2)+1)$ with $x=(\hbar \omega) /\left(k_{\mathrm{B}} T\right)$. We note that $n(x)+1=n(-x)$. In our notation $\hbar \omega>0$ correspond to an energy loss for the neutron. The " 1 " in $n(x)+1$ is the mathematical expression for the fact that it is always possible to create an excited state. When $\hbar \omega<0$ the neutron gains energy and the scattering will not be possible when $\hbar \omega \gg k_{\mathrm{B}} T$. But attention, this statement is only true when the system is in its equilibrium state. Hydrogen as well as deuterium are examples of systems well known for taking a long time before equilibrating at low temperatures. The deviation of the balance between signals on the gain and loss side from the value predicted for thermal equilibrium can serve as a measure of the non-equilibrium character of such systems [27].

$n(\omega)$ is close to zero. For $\hbar \omega<0$ the neutron gains energy in the scattering process. This process only happens when the corresponding oscillator is in an excited state.

The scattering intensity depends among other things on the terms

$$
\frac{\left(\vec{Q} \cdot \vec{e}_{i}\left(j^{\prime}\right)\right) \cdot\left(\vec{Q} \cdot \vec{e}_{i}(j)\right)^{*}}{\sqrt{m_{j^{\prime}} m_{j}}}\left(1+n\left(\omega_{i}\right)\right), \quad \omega>0
$$

and

$$
\frac{\left(\vec{Q} \cdot \vec{e}_{i}(j)\right) \cdot\left(\vec{Q} \cdot \vec{e}_{i}\left(j^{\prime}\right)\right)^{*}}{\sqrt{m_{j^{\prime}} m_{j}}} n\left(\omega_{i}\right), \quad \omega<0 .
$$

Comparing these expressions to the expectation value of the displacements $(9.101)$

$$
\left\langle\mathbf{u}(\alpha, j) \mathbf{u}(\alpha, j)^{+}\right\rangle=\sum_{i=1}^{3 N} \frac{\left|e_{i}(\alpha, j)\right|^{2} \hbar}{2 m_{j} \omega_{i}}\left(2 n\left(\omega_{i}\right)+1\right)
$$


we conclude that the scattering intensity of a vibration is strongly linked to the amplitude (projected onto the momentum transfer $\vec{Q}$ ). ${ }^{102}$ In other words, the neutrons measure the fluctuation amplitudes. Since fluctuations include zero point motion inelastic scattering is assured even at very low temperatures.

\subsection{1. $f$ sum rule for vibrations}

In order to give a concrete application for the $f$ sum rule (expression (8.187) in Section 8.12) we will now calculate the first moment of the vibrational scattering function. Since the Hamiltonian for a harmonic system only contains interactions that depend on the position operators the conditions of validity laid out in Section 8.12 are assured.

Starting from (9.185) we find

$$
\begin{aligned}
\int_{-\infty}^{\infty} \mathrm{d}(\hbar \omega)(\hbar \omega) S_{\mathrm{coh}}^{1-\mathrm{ph}}(\vec{Q}, \omega) & =\frac{\hbar^{2}}{2} \sum_{i}\left|F_{i}(\vec{Q})\right|^{2} \int_{-\infty}^{\infty} \mathrm{d} \omega \frac{\omega}{\omega_{i}}\left\{\left[\left(1+n\left(\omega_{i}\right)\right) \delta\left(\omega-\omega_{i}\right)\right]+\left[n\left(\omega_{i}\right) \delta\left(\omega+\omega_{i}\right)\right]\right\} \\
& =\frac{\hbar^{2}}{2} \sum_{i}\left|F_{i}(\vec{Q})\right|^{2}\left\{\left(1+n\left(\omega_{i}\right)-n\left(\omega_{i}\right)\right\}\right. \\
& =\frac{\hbar^{2}}{2} \sum_{i}\left|F_{i}(\vec{Q})\right|^{2}
\end{aligned}
$$

Hence the first moment is equal to the sum of the vibrational form factors squared. This sum can be calculated following (9.186) as

$$
\begin{aligned}
& \sum_{i}\left|F_{i}(\vec{Q})\right|^{2} \\
& \quad=\sum_{\kappa, \kappa^{\prime}} \frac{\bar{b}_{\kappa} \cdot \bar{b}_{\kappa^{\prime}}^{*}}{\sqrt{m_{\kappa^{\prime}} m_{\kappa}}} \sum_{j \in\left\{j_{\kappa}\right\}, j^{\prime} \in\left\{j_{\kappa^{\prime}}\right\}} \mathrm{e}^{-\mathrm{i} \vec{Q} \cdot \vec{R}_{j^{\prime}}^{0}} \mathrm{e}^{\mathrm{i} \vec{Q} \cdot \vec{R}_{j}^{0}} \mathrm{e}^{-W_{j^{\prime}}(\vec{Q})} \mathrm{e}^{-W_{j}(\vec{Q})} \sum_{i}\left(\vec{Q} \cdot \vec{e}_{i}\left(j^{\prime}\right)\right) \cdot\left(\vec{Q} \cdot \vec{e}_{i}(j)\right)^{*}
\end{aligned}
$$

The eigenvectors form a complete basis set, in which all the ionic movements can be expressed. They formally have to satisfy the completeness relation

$$
\sum_{i} \vec{e}_{i}\left(\alpha, j^{\prime}\right) \vec{e}_{i}(\beta, j)^{*}=\delta_{\alpha, \beta} \delta_{j, j^{\prime}}
$$

As a consequence

$$
\begin{aligned}
\sum_{i}\left(\vec{Q} \cdot \vec{e}_{i}\left(j^{\prime}\right)\right) \cdot\left(\vec{Q} \cdot \vec{e}_{i}(j)\right)^{*} & =\sum_{\alpha, \beta} Q_{\alpha} Q_{\beta} \sum_{i} \vec{e}_{i}\left(\alpha, j^{\prime}\right) \vec{e}_{i}(\beta, j)^{*} \\
& =\sum_{\alpha, \beta} Q_{\alpha} Q_{\beta} \delta_{\alpha, \beta} \delta_{j, j^{\prime}} \\
& =Q^{2} \delta_{j, j^{\prime}}
\end{aligned}
$$

\footnotetext{
${ }^{102}$ According to the expression (9.101) the temperature factors are connected to the mean square amplitudes of a vibration $\left(n\left(\omega_{i}\right)+1\right)+n\left(\omega_{i}\right) \propto\left\langle u_{i}^{2}\right\rangle$.
} 
and hence

$$
\begin{aligned}
\int_{-\infty}^{\infty} \mathrm{d}(\hbar \omega)(\hbar \omega) S_{\text {coh }}^{1-\mathrm{ph}}(\vec{Q}, \omega) & =\frac{\hbar^{2}}{2} \sum_{i}\left|F_{i}(\vec{Q})\right|^{2} \\
& =\frac{\hbar^{2} Q^{2}}{2} \sum_{\kappa} N_{\kappa} \frac{\bar{b}_{\kappa} \cdot \bar{b}_{\kappa}^{*}}{m_{\kappa}} \mathrm{e}^{-2 W_{\kappa}(\vec{Q})} \\
& =\sum_{\kappa} N_{\kappa} \bar{b}_{\kappa} \cdot \bar{b}_{\kappa}^{*} \mathrm{e}^{-2 W_{\kappa}(\vec{Q})} E_{\kappa}^{r}(Q)
\end{aligned}
$$

with as before the recoil energy of the ion $\kappa$ defined as

$$
E_{\kappa}^{r}(Q):=\frac{\hbar^{2} Q^{2}}{2 m_{\kappa}}
$$

This expression for the vibrational part of the first moment can be compared with that obtained for the total first moment of the partial scattering function (8.187)

$$
\int_{-\infty}^{\infty} \mathrm{d}(\hbar \omega)(\hbar \omega) S_{\kappa, \kappa^{\prime}}(\vec{Q}, \omega)=\left.\frac{\hbar^{2}}{2} \frac{\partial}{\partial t} K_{\kappa, \kappa^{\prime}}(\vec{Q}, t)\right|_{t=0}=\delta_{\kappa, \kappa^{\prime}} N_{\kappa} E_{\kappa}^{r}(Q)
$$

We note that the difference between the two expressions resides in the presence of the Debye-Waller factor in the vibrational part of the first moment. The Debye-Waller factor introduces a temperature dependence in

$$
\int_{-\infty}^{\infty} \mathrm{d}(\hbar \omega)(\hbar \omega) S_{\operatorname{coh}}^{1-\mathrm{ph}}(\vec{Q}, \omega)
$$

Due to this dependence the vibrational first moment systematically falls short of the total first moment

$$
\int_{-\infty}^{\infty} \mathrm{d}(\hbar \omega)(\hbar \omega) S_{\mathrm{coh}}(\vec{Q}, \omega)
$$

This short-fall can be explained by the neglect of multi-phonon contributions that we will address in Section 10 .

The sum rule teaches us without any further calculation that for any scattering vector $\vec{Q}$ all the atoms contribute to the inelastic signal. The intensity of this contribution depends, apart from the scattering lengths, on the inverse of the mass and on the inverse of the frequencies of the vibrational modes, in which the atom participates. If we had wanted, we could have practically derived the form of the vibrational scattering just from the sum rule.

\subsection{Coherent scattering of a phonon in a crystal}

The expressions obtained up to now are valid for any harmonic system. They may seem complex but they allow the exact calculation of inelastic scattering based only on a knowledge of the vibrational frequencies and eigenvectors. Naturally, the calculation of all frequencies and eigenvectors is a real challenge in an extended amorphous system. Extracting information on the character of the modes without employing calculations, although not completely impossible, remains a rather arbitrary process for complex amorphous systems. The best one can hope for is to identify coarse classes of motion (acoustic, optic, rigid motion of structural units etc.) from the $Q$ dependence of a given band of frequencies [6,7]. 
The situation becomes much more favourable when working with crystals. Due to the translational symmetry the displacement operators are written as (see expression (9.65))

$$
\begin{aligned}
\mathbf{u}_{\alpha}(\vec{l}, d) & =\sqrt{\frac{r}{N}} \sum_{i, \vec{q}} \sqrt{\frac{\hbar}{2 m_{d} \omega_{i}(\vec{q})}}\left[e_{i}(\alpha, d \mid \vec{q}) \mathrm{e}^{\mathrm{i} \vec{q} \cdot \vec{l}} \mathbf{a}_{i}(\vec{q})+e_{i}^{*}(\alpha, d \mid \vec{q}) \mathrm{e}^{-\mathrm{i} \vec{q} \cdot \vec{l}} \mathbf{a}_{i}^{+}(\vec{q})\right] \\
& =\sqrt{\frac{r}{N}} \sum_{i, \vec{q}} \sqrt{\frac{\hbar}{2 m_{\kappa} \omega_{i}(\vec{q})}} e_{i}(\alpha, \kappa \mid \vec{q})\left(\mathbf{a}_{i}(\vec{q})+\mathbf{a}_{i}^{+}(-\vec{q})\right) \mathrm{e}^{\mathrm{i} \vec{q} \cdot \vec{l}}
\end{aligned}
$$

where the second line is subject to the condition $e_{j}(\vec{q})=e_{j}(-\vec{q})$. With this expression for the displacement operator we can calculate

$$
\aleph_{j^{\prime}, j}(\vec{Q}, t)=\aleph_{\vec{l}^{\prime}, \overrightarrow{l,}, d^{\prime}, d}(\vec{Q}, t) .
$$

We obtain

$$
\begin{aligned}
& \left\langle\left(\vec{Q} \cdot \overrightarrow{\mathbf{u}}\left(\vec{l}^{\prime}, d^{\prime}\right)\right)(\vec{Q} \cdot \overrightarrow{\mathbf{u}}(\vec{l}, d ; t))\right\rangle \\
& =\frac{\hbar}{2} \frac{r}{N} \sum_{\alpha, \beta} \frac{Q_{\alpha} Q_{\beta}}{\sqrt{m_{d^{m}} m_{d^{\prime}}}} \sum_{i, i^{\prime}} \sum_{\vec{q}, \vec{q}^{\prime}} \sqrt{\frac{1}{\omega_{i}(\vec{q}) \omega_{i^{\prime}}\left(\vec{q}^{\prime}\right)}} \times \delta_{i, i^{\prime}} \times \delta_{\vec{q}, \vec{q}^{\prime}} \\
& \times\left\{\left[e_{i}\left(\alpha, d^{\prime} \mid \vec{q}\right) e_{i^{\prime}}^{*}\left(\beta, d \mid \vec{q}^{\prime}\right) \exp \left(\mathrm{i}\left(\vec{q} \cdot \vec{l}^{\prime}-\vec{q}^{\prime} \cdot \vec{l}\right)\right) \exp \left(\mathrm{i} \omega_{i^{\prime}} t\right)\right]\left\langle\mathbf{a}_{i}\left(\vec{q}^{\prime}\right) \mathbf{a}_{i^{\prime}}^{+}(\vec{q})\right\rangle\right. \\
& \left.+\left[e_{i}^{*}\left(\alpha, d^{\prime} \mid \vec{q}\right) e_{i^{\prime}}\left(\beta, d \mid \vec{q}^{\prime}\right) \exp \left(\mathrm{i}\left(-\vec{q} \cdot \vec{l}^{\prime}+\vec{q}^{\prime} \cdot \vec{l}\right)\right) \exp \left(-\mathrm{i} \omega_{i^{\prime}} t\right)\right]\left\langle\mathbf{a}_{i}^{+}(\vec{q}) \mathbf{a}_{i^{\prime}}\left(\vec{q}^{\prime}\right)\right\rangle\right\} \\
& =\frac{\hbar}{2} \frac{r}{N} \sum_{\alpha, \beta} \frac{Q_{\alpha} Q_{\beta}}{\sqrt{m_{d^{m} d^{\prime}}}} \sum_{i, \vec{q}} \frac{1}{\omega_{i}(\vec{q})} \\
& \times\left\{\left[e_{i}\left(\alpha, d^{\prime} \mid \vec{q}\right) e_{i}^{*}(\beta, d \mid \vec{q}) \exp \left(\mathrm{i}\left(\vec{q} \cdot\left(\vec{l}^{\prime}-\vec{l}\right)\right)\right)\left(1+n\left(\omega_{i}\right)\right) \exp \left(\mathrm{i} \omega_{i} t\right)\right]\right. \\
& \left.+\left[e_{i}^{*}\left(\alpha, d^{\prime} \mid \vec{q}\right) e_{i}(\beta, d \mid \vec{q}) \exp \left(-\mathrm{i}\left(\vec{q} \cdot\left(\vec{l}^{\prime}-\vec{l}\right)\right)\right) n\left(\omega_{i}\right) \exp \left(-\mathrm{i} \omega_{i} t\right)\right]\right\} \\
& =\frac{\hbar}{2} \frac{r}{N} \sum_{i, \vec{q}}\left\{\left[\frac{A_{i, \vec{q}}\left(\vec{Q} ; d^{\prime}, d\right)}{\omega_{i}(\vec{q})}\left(1+n\left(\omega_{i}\right)\right) \exp \left(\mathrm{i}\left(\vec{q} \cdot\left(\vec{l}^{\prime}-\vec{l}\right)\right)\right) \exp \left(\mathrm{i} \omega_{i} t\right)\right]\right. \\
& \left.+\left[\frac{A_{i, \vec{q}}\left(\vec{Q} ; d, d^{\prime}\right)}{\omega_{i}(\vec{q})} n\left(\omega_{i}\right) \exp \left(-\mathrm{i}\left(\vec{q} \cdot\left(\vec{l}^{\prime}-\vec{l}\right)\right)\right) \exp \left(-\mathrm{i} \omega_{i} t\right)\right]\right\}
\end{aligned}
$$

with

$$
A_{i, \vec{q}}\left(\vec{Q} ; d^{\prime}, d\right):=\frac{\left(\vec{Q} \cdot \vec{e}_{i}\left(d^{\prime} \mid \vec{q}\right)\right)\left(\vec{Q} \cdot \vec{e}_{i}(d \mid \vec{q})^{*}\right)}{\sqrt{m_{d^{m} d^{\prime}}}}
$$

In the case of a crystal we have a maximum of $r$ different atoms in our system, with $r$ being the number of atoms in the primitive cell. Without any supplementary information concerning the potential symmetries of the crystal 
we should consider them all as distinct. The partial dynamical structure factors become

$$
\begin{aligned}
S_{d, d^{\prime}}^{1-\mathrm{ph}}(\vec{Q}, \omega) & \frac{1}{2 \pi \hbar} \sum_{\vec{l} \vec{l}^{\prime}} \mathrm{e}^{-\mathrm{i} \vec{Q} \cdot\left(\overrightarrow{l^{\prime}}+\vec{d}^{\prime}-\vec{l}-\vec{d}\right)} \mathrm{e}^{-W_{d^{\prime}}(\vec{Q})} \mathrm{e}^{-W_{d}(\vec{Q})} \int_{-\infty}^{\infty} \mathrm{d} t\left\langle\left(\vec{Q} \cdot \overrightarrow{\mathbf{u}}\left(\vec{l}^{\prime}, d^{\prime}\right)\right)(\vec{Q} \cdot \overrightarrow{\mathbf{u}}(\vec{l}, d ; t))\right\rangle \\
= & \frac{r}{4 \pi N} \mathrm{e}^{-W_{d^{\prime}}(\vec{Q})} \mathrm{e}^{-W_{d}(\vec{Q})} \mathrm{e}^{-\mathrm{i} \vec{Q} \cdot\left(\vec{d}^{\prime}-\vec{d}\right)} \sum_{\vec{l}, \vec{l}^{\prime}} \mathrm{e}^{-\mathrm{i} \vec{Q} \cdot\left(\overrightarrow{l^{\prime}}-\vec{l}\right)} \\
& \times \sum_{i, \vec{q}}\left\{\left[\frac{A_{i, \vec{q}^{\prime}}\left(\vec{Q} ; d^{\prime}, d\right)}{\omega_{i}(\vec{q})}\left(1+n\left(\omega_{i}(\vec{q})\right)\right) \exp \left(\mathrm{i}\left(\vec{q} \cdot\left(\vec{l}^{\prime}-\vec{l}\right)\right)\right) \int_{-\infty}^{\infty} \mathrm{d} t \exp \left(\mathrm{i} \omega_{i}(\vec{q}) t\right)\right]\right. \\
& \left.+\left[\frac{A_{i, \vec{q}}\left(\vec{Q} ; d, d^{\prime}\right)}{\omega_{i}(\vec{q})} n\left(\omega_{i}(\vec{q})\right) \exp \left(-\mathrm{i}\left(\vec{q} \cdot\left(\overrightarrow{l^{\prime}}-\vec{l}\right)\right)\right) \int_{-\infty}^{\infty} \mathrm{d} t \exp \left(-\mathrm{i} \omega_{i}(\vec{q}) t\right)\right]\right\} \\
= & \frac{(2 \pi)^{3}}{2 V_{\text {unit cell }}} \mathrm{e}^{-W_{d^{\prime}}(\vec{Q})} \mathrm{e}^{-W_{d}(\vec{Q})} \mathrm{e}^{-\mathrm{i} \vec{Q} \cdot\left(\overrightarrow{d^{\prime}}-\vec{d}\right)} \\
& \times \sum_{i, \vec{q}} \sum_{\vec{G}}\left\{\left[\frac{A_{i, \vec{q}}\left(\vec{Q} ; d^{\prime}, d\right)}{\omega_{i}(\vec{q})} \cdot\left(1+n\left(\omega_{i}(\vec{q})\right) \cdot \delta(\vec{q}-\vec{Q}-\vec{G}) \cdot \delta\left(\omega-\omega_{i}(\vec{q})\right)\right]\right.\right. \\
& \left.+\left[\frac{A_{i, \vec{q}}\left(\vec{Q} ; d, d^{\prime}\right)}{\omega_{i}(\vec{q})} \cdot n\left(\omega_{i}(\vec{q})\right) \cdot \delta(\vec{q}+\vec{Q}-\vec{G}) \cdot \delta\left(\omega+\omega_{i}(\vec{q})\right)\right]\right\}
\end{aligned}
$$

where we have used the relations (9.119) and (9.127). With this result we can calculate the coherent scattering function

$$
\begin{aligned}
S_{\text {coh }}^{1-\mathrm{ph}}(\vec{Q}, \omega)= & \sum_{d, d^{\prime}} \bar{b}_{d} \cdot \bar{b}_{d^{\prime}}^{*} S_{d, d^{\prime}}^{1-\mathrm{ph}}(\vec{Q}, \omega) \\
= & \frac{(2 \pi)^{3}}{V_{\text {unit cell }}} \sum_{d, d^{\prime}} \overline{b_{d}} \cdot{\overline{b_{d}^{\prime}}}^{*} \mathrm{e}^{-W_{d}(\vec{Q})} \mathrm{e}^{-W_{d^{\prime}}(\vec{Q})} \mathrm{e}^{\mathrm{i} \vec{Q} \cdot \vec{d}} \mathrm{e}^{-\mathrm{i} \vec{Q} \cdot \overrightarrow{d^{\prime}}} \\
& \times \sum_{i, \vec{q}} \sum_{\vec{G}}\left\{\left[\frac{A_{i, \vec{q}}\left(\vec{Q} ; d^{\prime}, d\right)}{\omega_{i}(\vec{q})} \cdot\left(1+n\left(\omega_{i}(\vec{q})\right)\right) \cdot \delta(\vec{q}-\vec{Q}-\vec{G}) \cdot \delta\left(\omega-\omega_{i}(\vec{q})\right)\right]\right. \\
& \left.+\left[\frac{A_{i, \vec{q}}\left(\vec{Q} ; d, d^{\prime}\right)}{\omega_{i}(\vec{q})} \cdot n\left(\omega_{i}(\vec{q})\right) \cdot \delta(\vec{q}+\vec{Q}-\vec{G}) \cdot \delta\left(\omega+\omega_{i}(\vec{q})\right)\right]\right\} .
\end{aligned}
$$

The intrinsic structure of this expression becomes clearer when written as

$$
\begin{aligned}
S_{\text {coh }}^{1-\mathrm{ph}}(\vec{Q}, \omega)= & \frac{(2 \pi)^{3}}{2 V_{\text {unit cell }}} \sum_{\vec{G}} \sum_{i, \vec{q}} \frac{\mid F_{i}\left(\vec{Q}|\vec{q}|^{2}\right.}{\omega_{i}(\vec{q})} \\
& \times\left[\left(1+n\left(\omega_{i}(\vec{q})\right)\right) \cdot \delta(\vec{q}-(\vec{Q}+\vec{G})) \cdot \delta\left(\omega-\omega_{i}(\vec{q})\right)\right. \\
& \left.+n\left(\omega_{i}(\vec{q})\right) \cdot \delta(\vec{q}+(\vec{Q}+\vec{G})) \cdot \delta\left(\omega+\omega_{i}(\vec{q})\right)\right]
\end{aligned}
$$


with

$$
\begin{aligned}
\left|F_{i}(\vec{Q} \mid \vec{q})\right|^{2} & =\sum_{d, d^{\prime}}^{r} \frac{\bar{b}_{d} \cdot \bar{b}_{d^{\prime}}^{*}}{\sqrt{m_{d^{m}} m_{d^{\prime}}}} \mathrm{e}^{-W_{d}(\vec{Q})} \mathrm{e}^{-W_{d^{\prime}}(\vec{Q})}\left(\vec{Q} \cdot \vec{e}_{i}\left(d^{\prime} \mid \vec{q}\right)\right)\left(\vec{Q} \cdot \vec{e}_{i}(d \mid \vec{q})\right)^{*} \mathrm{e}^{\mathrm{i} \vec{Q} \cdot \vec{d}} \mathrm{e}^{-\mathrm{i} \vec{Q} \cdot \vec{d}^{\prime}} \\
& =\left|\sum_{d=1}^{r} \frac{\overline{b_{d}}}{\sqrt{m_{d}}} \mathrm{e}^{-W_{d}(\vec{Q})}\left(\vec{Q} \cdot \vec{e}_{i}(d \mid \vec{q})\right) \mathrm{e}^{-\mathrm{i} \vec{Q} \cdot \vec{d}}\right|
\end{aligned}
$$

The function $F_{i}(\vec{Q} \mid \vec{q})$ is called the phonon form factor of the branch $i$ and wave vector $\vec{q}$. The expression found for the scattering from a phonon does not explicitly depend on the number of scatterers $N$. This may be surprising at first glance. An increase in sample volume should in the Born approximation lead to a proportional increase in scattering. This increase is nevertheless implicit in expression (9.202), which is proportional to the number of phonons. This number is governed by the density of $\vec{q}$ vectors in the first Brillouin zone. Due to the periodic boundary conditions a larger crystal will have shorter distances between neighbouring $\vec{q}$ vectors. The total number of modes corresponds to $3 N$ where $N$ is the number of atoms in the crystal.

The fundamental laws of physics require the conservation of momentum. The change in momentum of the neutron $\left(\hbar \vec{Q}=\hbar \vec{k}_{i}-\hbar \vec{k}_{f}\right)$ during the scattering process should then be compensated by an opposite change in momentum of the sample. This observation also holds for an amorphous system. The Dirac functions

$$
\begin{aligned}
& \delta(\vec{q}-(\vec{Q}+\vec{G})) \quad \text { and } \\
& \delta(\vec{q}+(\vec{Q}+\vec{G}))
\end{aligned}
$$

that we find in the scattering function (9.202) of the crystal imply that the coherent scattering is also subjected to the conservation of the crystal momentum. In order for the scattering to take place their must exist a reciprocal lattice vector $\vec{G}$ such that ${ }^{103}$

$$
\begin{aligned}
& \vec{Q}=\vec{q}+\vec{G} \text { or } \\
& \vec{Q}=-\vec{q}+\vec{G} .
\end{aligned}
$$

So clearly, during the scattering process the change in momentum of the neutron

$$
\hbar \vec{Q}=\hbar \vec{k}_{i}-\hbar \vec{k}_{f}
$$

should be absorbed (9.206) or provided (9.207) by the phonon

$$
\hbar \vec{q}
$$

up to an reciprocal lattice vector $\hbar \vec{G}$ (see Fig. 51). One talks about the creation or emission of a phonon as well as the annihilation or absorption of a phonon. Neutron scattering is in this way identical to the scattering of Bloch electrons. The conservation of crystal momentum is a constraint imposed on all particle scattering processes in a periodic lattice potential.

To illustrate the expressions for the coherent scattering we are inspired by Squires [62] and recall that a phonon represents a propagating wave. In a reference frame that moves along $\vec{q}$ with the phase velocity of the phonon $\left(\omega_{i}(\vec{q}) /|\vec{q}|\right)$ the motion of the atoms appear frozen. The structure of the crystal appears to be modulated in a sinusoidal manner. It is known (for example in incommensurate systems) that a static modulation introduces satellites

\footnotetext{
${ }^{103}$ Going from $(9.204),(9.205)$ to $(9.206),(9.207)$ we use the fact that the reciprocal lattice is a Bravais lattice where there exists for every vector $\vec{G}$ a vector $-\vec{G}$.
} 

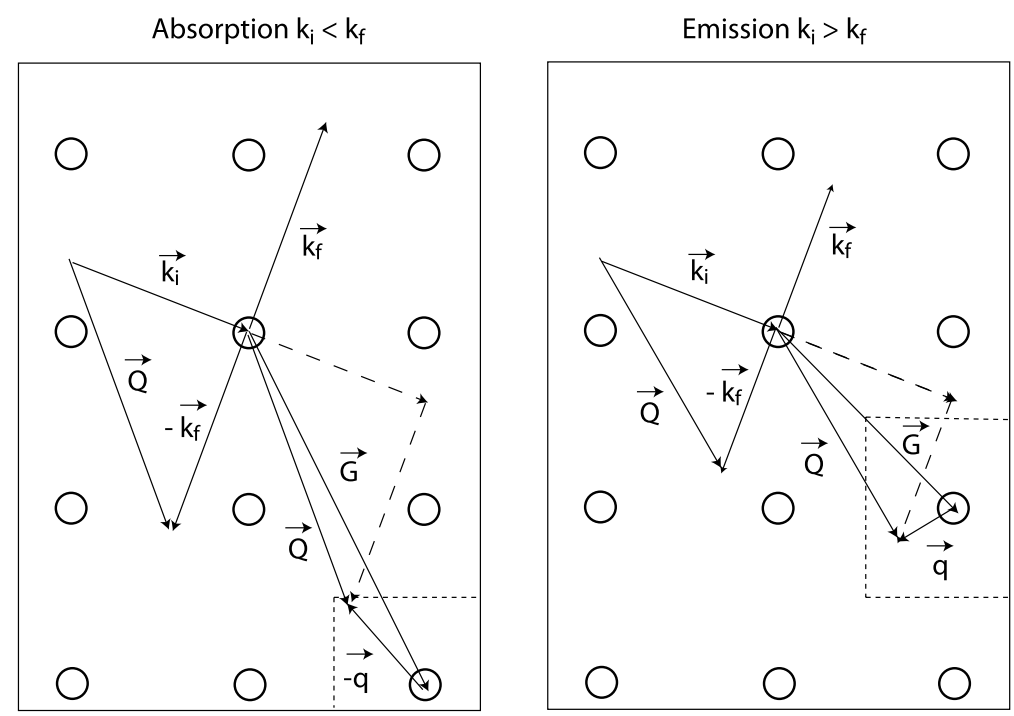

Fig. 51. Schematic representation of the conservation of crystal momentum when absorbing (9.207) or emitting a phonon (9.206).

to the Bragg peaks at positions $\vec{G} \pm \vec{q}$. This brings us to the expressions (9.206) and (9.207). The intensity of the satellites are given by the amplitude squared of the modulation. The transformation from the moving reference system back to the reference system of the laboratory teaches us that the satellites correspond to an inelastic scattering process. The corresponding energy transfers are give by the expressions

$$
\begin{aligned}
& \frac{\hbar}{2 m_{n}}\left(k_{i}^{2}-k_{f}^{2}\right)=\hbar \omega_{i}(\vec{q}), \\
& \frac{\hbar}{2 m_{n}}\left(k_{f}^{2}-k_{i}^{2}\right)=\hbar \omega_{i}(\vec{q}) .
\end{aligned}
$$

The intensity of the scattering is driven by the amplitude of the modulation.

The conservation of crystal momentum has important consequences for the scattering of neutrons. Let us consider an incident beam described by the wave vector $\vec{k}_{i}$ and a direction of detection described by $\hat{k}_{f}$. The inelastic scattering will be zero except for values of $k_{f}$ corresponding to energy transfers defined in the expressions (9.208) and (9.209). Given that the phonon spectrum is discrete for a given $\vec{q}$ the scattering will also be discrete (see Fig. 52). Each inelastic peak corresponds to the emission or the absorption of a phonon, where the frequency is given by the relations (9.206)-(9.209). The only unknown is the branch index. By performing a large number of measurements sweeping a large area of $(\vec{Q}, \omega)$ space it is a priori possible to determine the dispersion relations in quite complex samples.

The relations (9.206)-(9.209) limit the observation of a phonon mode to the accessible kinematic region as defined by the experimental configuration. A good example to illustrate these limitations is the measurement of an acoustic mode in the first Brillouin zone. ${ }^{104}$ The accessible region for a fixed $k_{i}$ is defined by (see Section 6.4 and Fig. 53)

$$
\hbar \omega(Q) \leqslant \frac{\hbar}{2 m}\left(k_{i}^{2}-\left(Q-k_{i}\right)^{2}\right)
$$

\footnotetext{
${ }^{104}$ This is often called Brillouin scattering
} 
$\Gamma$ point at $Q=(6,1,1) * 2 \pi / a$

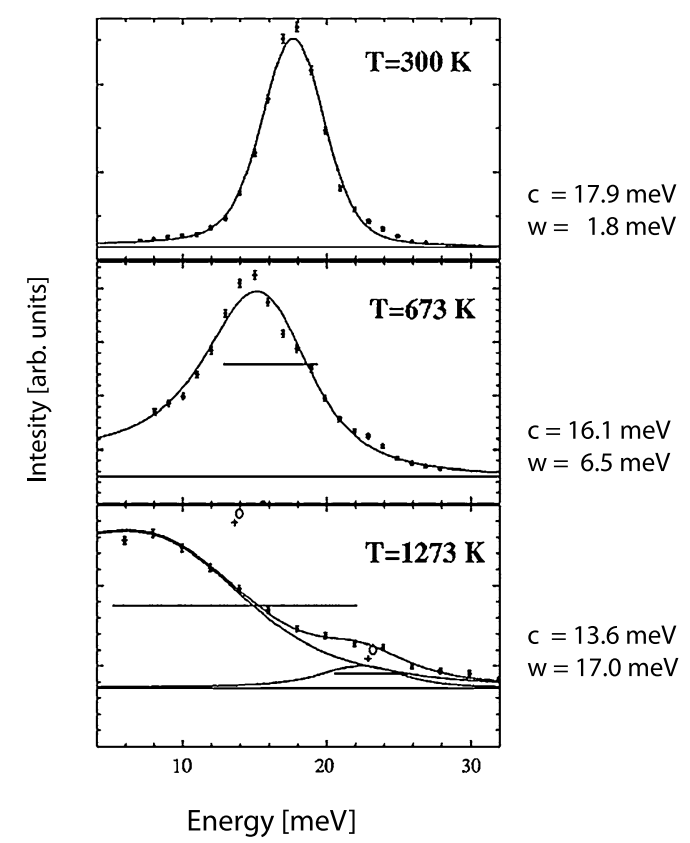

$X$ point at $Q=(7,0,0) * 2 \pi / a$

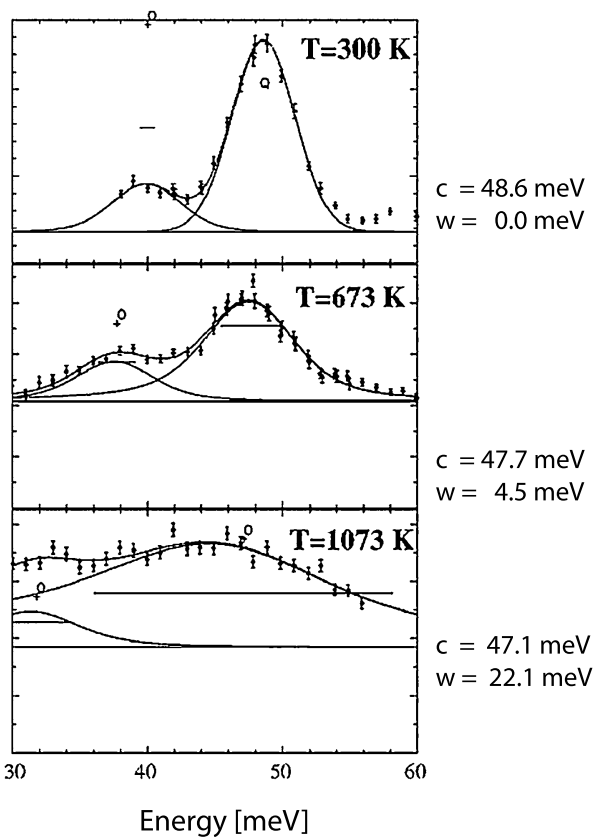

Fig. 52. Example of phonon peaks obtained with a three axis spectrometer in the super-ionic conductor $\mathrm{CaF}_{2}$. The measurements are done in such a way that $\vec{Q}$ stays constant. We are speaking of a constant $Q$ scan. The respective values of $\vec{Q}$ can be found above the figures. The peaks can be described as damped harmonic oscillators (see Section 10.7). The centres and the widths are indicated to the right of the peaks. They are also included graphically as horizontal lines in the figures. At $300 \mathrm{~K}$ the widths are given by the resolution of the instrument. At higher temperatures the peaks soften and spread. It is also noticed that the background level increases significantly with temperature due to multi-phonon scattering (see Section 10.4). A full set of such measurements leads to the dispersion relations shown in Fig. 45 (images courtesy of K. Schmalzl).

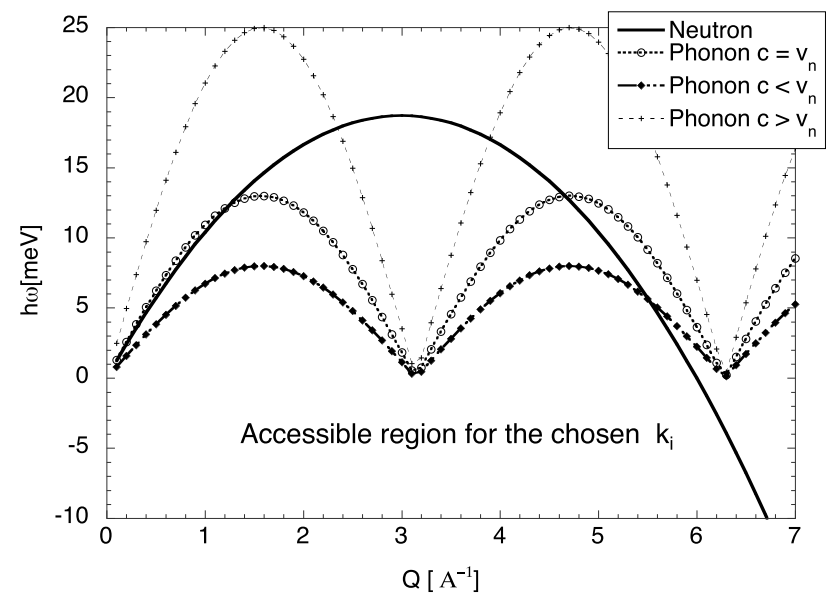

Fig. 53. In order for a phonon to contribute to the cross section the experimental configuration should permit to satisfy both the conservation of energy and the conservation of crystal momentum. In phonon creation mode $\left(k_{i}>k_{f}\right)$ this corresponds to simultaneously respecting (9.208) and (9.206). In the first Brillouin zone this is possible if the speed of sound $c_{\text {sound }}$ is inferior to the speed of the incoming neutron $v_{n}$. The situation shown here corresponds to a fixed $k_{i}$ of $3 \AA^{-1}$ (as in Fig. 31). 
The slope of the envelope of this domaine at $Q=0$ is calculated as

$$
\left(\frac{\mathrm{d}(\hbar \omega(Q))}{\mathrm{d} Q}\right)_{Q=0}=\frac{\hbar}{m} k_{i}=v_{n},
$$

where $v_{n}$ is the velocity of the incident neutron. The slope of the phonon dispersion relation that we would like to measure is given by the speed of sound $c_{\text {sound }}$. If $c_{\text {sound }}<v_{n}$ the mode does not contribute to the scattering by phonon creation, i.e. for $\omega>0$. There are a priori no constraints to the scattering by phonon annihilation. However, the scattering angles become smaller and smaller as $c_{\text {sound }}$ increases. The signal risks to be drowned in the background created by the direct beam. In a crystal this problem can be avoided by working in higher Brillouin zones (see Fig. 53). This possibility does not exist in an amorphous system. The situation is completely different in the case of X-rays where the speed of sound is always smaller than the speed of light.

The relations (9.206)-(9.209) are necessary but not sufficient conditions for phonon scattering. In order for scattering to take place it is also necessary that the form factor $F_{i}(\vec{Q} \mid \vec{q})$ is sufficiently strong for the signal to become observable. A simple example that illustrates this fact are transverse phonons in the first Brillouin zone. Transverse phonons are characterised by the fact that all atomic motions are perpendicular to $\vec{q}$. This translates into the following condition for the eigenvectors

$$
\vec{e}_{i}(d \mid \vec{q}) \cdot \vec{q}=0 \quad \forall d
$$

In the first Brillouin zone $\vec{G}=0$ and hence $\vec{Q}=\vec{q}$.

$$
\left|F_{i}(\vec{Q} \mid \vec{q})\right|^{2}=\left|\sum_{d=1}^{r} \frac{\overline{b_{d}}}{\sqrt{m_{d}}} \mathrm{e}^{-W_{d}(\vec{Q})}\left(\vec{q} \cdot \vec{e}_{i}(d \mid \vec{q})\right) \mathrm{e}^{-\mathrm{i} \vec{Q} \cdot \vec{d}}\right|=0 .
$$

Hence the transverse modes do not contribute to the signal in the first Brillouin zone.

In practice one should be aware of the fact that the character of a mode is in general mixed in complex materials. However, symmetries can introduce systematic extinction for certain directions in the reciprocal space, and not only in the first Brillouin zone. Along these so-called high-symmetry directions the form factor may, therefore, be rigorously zero for some of the phonons. We are not going to pursue the discussion of these selection rules that can be derived using group theory. The interested reader can consult the literature $[19,45,54]$.

The $\left|F_{i}(\vec{Q} \mid \vec{q})\right|^{2}$ factor determines the intensity with which a phonon can be observed for a given $\vec{Q}$. Among other things it is a function of the eigenvectors $\vec{e}_{i}(d \mid \vec{q})(d=1, \ldots, r)$ of the phonon. In general it is very difficult to obtain the $\vec{e}_{i}(d \mid \vec{q})(d=1, \ldots, r)$ from a measurement of $\left|F_{i}(\vec{Q} \mid \vec{q})\right|^{2}$. The determination of $\vec{e}_{i}(d \mid \vec{q})(d=1, \ldots, r)$ is rather the exception than the rule [65]. It is nevertheless possible to obtain interesting information on the character of the phonon mode from even limited measurements of $\left|F_{i}(\vec{Q} \mid \vec{q})\right|^{2}$. A particular promising case are strongly polarised modes. The scattering of neutrons is very sensitive to the projection of the atomic movements onto $\vec{Q}$. Mathematically this fact is expressed by the presence of the scalar products

$$
\left(\vec{Q} \cdot \vec{e}_{i}(d \mid \vec{q})\right)
$$

in the phonon form factor. If we choose our experimental configuration in such a way that either

$$
\vec{Q} \| \vec{q}
$$

or

$$
\vec{Q} \perp \vec{q}
$$




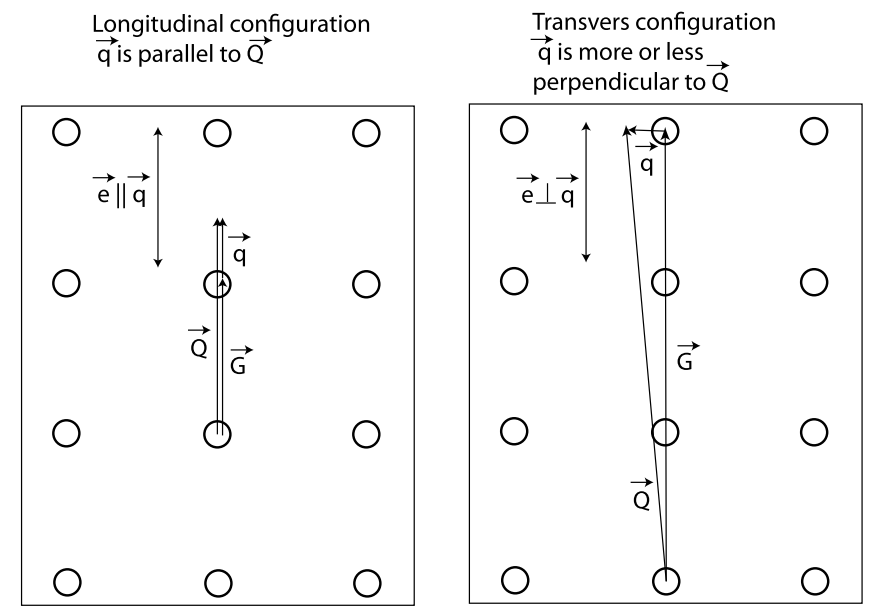

Fig. 54. In the phonon form factor the components of the eigenvector $\vec{e}_{i}(d \mid \vec{q})(d=1, \ldots, r)$ are projected onto $\vec{Q}$. The visibility of a mode depends strongly on this projection. This dependence allows us to specifically select modes via adapted experimental configuration. If $\vec{Q}$ is parallel to $\vec{q}$ the transverse modes, for which $\vec{e}_{i}(d \mid \vec{q})(d=1, \ldots, r)$ are all perpendicular to $\vec{q}$ (and hence in this configuration also perpendicular to $\vec{Q}$ ) will be invisible. In this configuration it is, therefore, assured that only the longitudinal components of the movements are measured. This configuration is shown in the figure to the left. It can be realised in pure form. The transverse configuration (see figure to the right) will for high-symmetry directions generally keep a mixed character as it is not possible to render $\vec{Q}$ and $\vec{q}$ perfectly perpendicular. The longitudinal component diminishes, however, for a given $\vec{q}$ with $\vec{Q}$. Hence it is preferable to work in distant zones of reciprocal space.

then we can separate the longitudinal modes

$$
e_{i}(d \mid \vec{q}) \| \vec{q}
$$

from the transverse modes

$$
e_{i}(d \mid \vec{q}) \perp \vec{q}
$$

(see Fig. 54).

Due to the presence of the factor $Q^{2}$ in $\left|F_{i}(\vec{Q} \mid \vec{q})\right|^{2}$ it is preferable to work in higher Brillouin zones provided that the intensity gain due to $Q^{2}$ is not eaten up by the Debye-Waller factors

$$
\mathrm{e}^{-W_{d}(\vec{Q})}
$$

that depend on the Debye-Waller functions $W_{d}(\vec{Q})$ and, therefore, decrease with $Q$. As we will see in Section 10 the intensity at large $Q$ values is transferred to scattering processes involving several phonons.

\subsection{Incoherent elastic scattering}

We now turn our attention to incoherent scattering. Our starting point is the partial differential cross section (6.21)

$$
\left(\frac{\mathrm{d} \sigma}{\mathrm{d} \Omega \mathrm{d} E_{f}}\right)_{\text {inc }}=\frac{k_{f}}{k_{i}} \frac{1}{2 \pi \hbar} \sum_{\lambda_{i}} p\left(\lambda_{i}\right) \sum_{j}^{N}\left(\overline{b_{\kappa}^{2}}-\left(\bar{b}_{\kappa}\right)^{2}\right) \int_{-\infty}^{\infty} \mathrm{d} t\left\langle\lambda_{i}\left|\mathrm{e}^{-\mathrm{i} \vec{Q} \cdot \vec{R}_{j}^{0}} \mathrm{e}^{\mathrm{i} \vec{Q} \cdot \vec{R}_{j}(t)}\right| \lambda_{i}\right\rangle \mathrm{e}^{-\mathrm{i} \omega t}
$$


that we can express in terms of the incoherent scattering function

$$
S_{\mathrm{inc}}(\vec{Q}, \omega)=\sum_{\kappa}\left(\overline{b_{\kappa}^{2}}-\left(\bar{b}_{\kappa}\right)^{2}\right) S_{\kappa}^{s}(\vec{Q}, \omega)
$$

The partial self scattering functions are defined as

$$
S_{\kappa}^{s}(\vec{Q}, \omega)=\frac{1}{2 \pi \hbar} \sum_{j \in\left\{j_{\kappa}\right\}} \int_{-\infty}^{\infty} \mathrm{d} t\left\langle\mathrm{e}^{-\mathrm{i} \vec{Q} \cdot \vec{R}_{j}(t=0)} \mathrm{e}^{\mathrm{i} \vec{Q} \cdot \vec{R}_{j}(t)}\right\rangle \mathrm{e}^{-\mathrm{i} \omega t}
$$

The expectation value involving a single atom

$$
\left\langle\mathrm{e}^{-\mathrm{i} \vec{Q} \cdot \vec{R}_{j}(t=0)} \mathrm{e}^{\mathrm{i} \vec{Q} \cdot \vec{R}_{j}(t)}\right\rangle
$$

is just a special case of the expectation value involving pairs of atoms

$$
\left\langle\mathrm{e}^{-\mathrm{i} \vec{Q} \cdot \vec{R}_{j^{\prime}}(t=0)} \mathrm{e}^{\mathrm{i} \vec{Q} \cdot \vec{R}_{j}(t)}\right\rangle .
$$

We, therefore, can rely on the formalism developed in the section devoted to coherent response. The Bloch identity teaches us that

$$
\left\langle\mathrm{e}^{-\mathrm{i} \vec{Q} \cdot \overrightarrow{\mathbf{u}}_{j}} \mathrm{e}^{\mathrm{i} \vec{Q} \cdot \overrightarrow{\mathbf{u}}_{j}(t)}\right\rangle=\mathrm{e}^{-2 W_{j}(\vec{Q})} \mathrm{e}^{\left\langle\left(\vec{Q} \cdot \overrightarrow{\mathbf{u}}_{j}\right)\left(\vec{Q} \cdot \overrightarrow{\mathbf{u}}_{j}(t)\right)\right\rangle} .
$$

The first term in the expansion of this expression in terms of

$$
\aleph_{j, j}(\vec{Q}, t)=\left\langle\left(\vec{Q} \cdot \vec{u}_{j}\right)\left(\vec{Q} \cdot \vec{u}_{j}(t)\right)\right\rangle
$$

gives the incoherent elastic scattering

$$
\left(\frac{\mathrm{d}^{2} \sigma}{\mathrm{d} \Omega \mathrm{d} E_{f}}\right)_{\mathrm{inc}}^{\mathrm{el}}=\sum_{\kappa}\left(\overline{b_{\kappa}^{2}}-\left(\overline{b_{\kappa}}\right)^{2}\right) S_{\kappa}(\vec{Q}, \hbar \omega=0)=\sum_{\kappa} \sum_{j \in\left\{j_{\kappa}\right\}}\left(\overline{b_{\kappa}^{2}}-\left(\bar{b}_{\kappa}\right)^{2}\right) \mathrm{e}^{-2 W_{j}(\vec{Q})} \delta(\hbar \omega) .
$$

If for a class of atoms $\{\kappa\}$ all the occupied sites are equivalent then the Debye-Waller factor does only depend on the type $\kappa$ but not on the site $j$. We get

$$
\left(\frac{\mathrm{d}^{2} \sigma}{\mathrm{d} \Omega \mathrm{d} E_{f}}\right)_{\mathrm{inc}}^{\mathrm{el}}=\sum_{\kappa} \sum_{j \in\left\{j_{\kappa}\right\}}\left(\overline{b_{\kappa}^{2}}-\left(\overline{b_{\kappa}}\right)^{2}\right) \mathrm{e}^{-2 W_{\kappa}(\vec{Q})} \delta(\hbar \omega)=\sum_{\kappa} N_{\kappa} \frac{\sigma_{\mathrm{inc}}(\kappa)}{4 \pi} \mathrm{e}^{-2 W_{\kappa}(\vec{Q})} \delta(\hbar \omega),
$$

with $N(\kappa)$ the number of atoms of type $\kappa$. This is equivalent to the expression that we would find in the case of fixed atoms (see Section 6.5). The only difference is again the presence of the $\exp \left(-2 W_{\kappa}(\vec{Q})\right.$ ) factors in $(9.217)$. The $\vec{Q}$ dependence introduced by them reflects the fact that the atoms are distributed about their equilibrium positions due to thermal motion. Thus the incoherent scattering ceases to be isotropic and is directed towards the front. This tendency is the more pronounced the higher the temperature. It is nevertheless still present at very low temperature due to zero point fluctuations. This fact has to be taken into account if an incoherent scatter like vanadium is used for calibration purposes. 


\subsection{Diffuse scattering}

It is important to distinguish between incoherent scattering and diffuse scattering. The term diffuse scattering is employed when confronted with chemical disorder, i.e. with disorder in the occupation of an atomic site $\vec{R}_{j}$ by one or several chemical elements. A typical example are solid solutions. In a solid solution the positions $\vec{R}_{j}$ in the primitive cell of a crystal is occupied in completely random manner by different chemical elements. The difference with respect to incoherent scattering lies in the fact that the physical quantities that we would like to study with neutron scattering rely heavily on the chemical composition of the sample but not on its nuclear composition. Hence the diffuse scattering signal is of direct interest to us. It gives precious information on the chemical composition (type of disorder, distribution and correlations among the chemical elements that occupy a site). Incoherent scattering would allow us to obtain similar information on the distribution of isotopes. Unfortunately, the distribution of isotopes or of nuclear spins is in general of lesser interest. Hence incoherent scattering could be considered as a pure annoyance. In practice this is not true. It allows us to extract information on the time evolution of a single scatterer with much more ease than from coherent scattering. We will demonstrate this in the following section.

How can we include chemical disorder in our formalism? Following the reasoning that conducted us to incoherent scattering we have to take all possible arrangements into account when calculating statistically averaged expectation values

$$
S(\vec{Q}, \omega)=\left\{\sum_{j, j^{\prime}=1}^{N}\left(b_{j} b_{j^{\prime}}^{*}\right) S_{j, j^{\prime}}(\vec{Q}, \omega)\right\}_{\mathrm{av}} .
$$

The sums should be evaluated over the coherence volumes that we have discussed in section (4.9). Unlike incoherent scattering the averaging imposed by chemical disorder not only affects the product of scattering lengths $\left(b_{j} b_{j^{\prime}}^{*}\right)$ but equally the partial scattering functions

$$
S_{j, j^{\prime}}(\vec{Q}, \omega)=\frac{1}{2 \pi \hbar} \sum_{\lambda_{i}} p\left(\lambda_{i}\right) \int_{-\infty}^{\infty} \mathrm{d} t\left\langle\lambda_{i}\left|\mathrm{e}^{-\mathrm{i} \vec{Q} \cdot \vec{R}_{j^{\prime}}^{0}} \mathrm{e}^{\mathrm{i} \mathrm{Q} \cdot \vec{R}_{j}(t)}\right| \lambda_{i}\right\rangle \mathrm{e}^{-\mathrm{i} \omega t}
$$

It is evident that $S_{j, j^{\prime}}(\vec{Q}, \omega)$ will depend in detail on the chemical elements that occupy the sites $j$ and $j^{\prime}$. Apart from the mass the interactions that determine the distance between atoms as well as the excitation spectrum depend on the atom type. Therefore, in order to describe the diffuse scattering we rely on a concrete model of statistical disorder.

The simplest model assumes that structure and chemical occupation are completely uncorrelated. In other words, we can model the structure by a lattice with lattice positions $\vec{R}_{j}$. The disorder is taken into account by introducing occupation factors at each site. Under these conditions the elastic scattering function can be written as

$$
S(\vec{Q}, \omega)=\sum_{j, j^{\prime}=1}^{N} \overline{\left(f_{j} f_{j^{\prime}}\right)} S_{j, j^{\prime}}^{\mathrm{el}}(\vec{Q}, \omega)
$$

with

$$
f_{j}:=b_{j} \mathrm{e}^{-W_{j}(\vec{Q})}
$$

and following (9.116)

$$
S_{j, j^{\prime}}^{\mathrm{el}}(\vec{Q}, \omega)=\sum_{j, j^{\prime}} \exp \left(-\mathrm{i} \vec{Q} \cdot\left(\vec{R}_{j^{\prime}}^{0}-\vec{R}_{j}^{0}\right)\right) \delta(\hbar \omega) .
$$


This expression is formally identical to (6.1). Thus we can immediately give the results for the averages:

$$
\begin{array}{ll}
j \neq j^{\prime}: & \overline{\left(f_{j} f_{j^{\prime}}\right)}={\overline{f_{j}}}^{2}, \\
j=j^{\prime}: & \overline{\left(f_{j} f_{j}\right)}=\overline{f_{j}^{2}},
\end{array}
$$

with

$$
\begin{aligned}
& \overline{f_{j}}=\sum_{\kappa} c_{j}^{\kappa} \mathrm{e}^{-W_{j}^{\kappa}(\vec{Q})} \sum_{i} p_{j}^{i} \cdot b_{j}^{i}=\sum_{\kappa} c_{j}^{\kappa} \overline{b_{j}} \mathrm{e}^{-W_{j}^{\kappa}(\vec{Q})}, \\
& \overline{f_{j}^{2}}=\sum_{\kappa} c_{j}^{\kappa} \mathrm{e}^{-2 W_{j}^{\kappa}(\vec{Q})} \sum_{i} p_{j}^{i} \cdot\left(f_{j}^{i}\right)^{2}=\sum_{\kappa} c_{j}^{\kappa} \mathrm{e}^{-2 W_{j}^{\kappa}(\vec{Q})} \overline{b_{j}^{2}} .
\end{aligned}
$$

As in the Section 6.1 the coefficients $p_{j}^{i}$ give the probability of the scattering length of the element at site $\vec{R}_{j}$ having the value $b_{j}^{i}$. The $c_{j}^{\kappa}$ factors give the fractional occupancy (concentrations) at site $j$ for the atoms of type $\kappa$. It follows that

$$
\begin{aligned}
& \sum_{i} p_{j}^{i}=1, \\
& \sum_{\kappa} c_{j}^{\kappa}=1 .
\end{aligned}
$$

We are now able to write down the expressions for the cross sections.

Let us start with the incoherent cross section. Following (9.217) it is given by

$$
\left(\frac{\mathrm{d}^{2} \sigma}{\mathrm{d} \Omega \mathrm{d} E_{f}}\right)_{\mathrm{inc}}^{\mathrm{el}}=\sum_{\kappa} N_{\kappa} \frac{\sigma_{\mathrm{inc}}(\kappa)}{4 \pi} \mathrm{e}^{-2 W_{\kappa}(\vec{Q})} \delta(\hbar \omega)=N \sum_{\kappa} c_{\kappa} \frac{\sigma_{\mathrm{inc}}(\kappa)}{4 \pi} \mathrm{e}^{-2 W_{\kappa}(\vec{Q})} \delta(\hbar \omega)
$$

where we have assumed that the Debye-Waller factors depend exclusively on the type of atom independent of the site, which is not always the case. To illustrate this result we consider a very simple case. Two types of atoms be distributed in an random fashion on a Bravais lattice. This could e.g. describe a metallic alloy. In this case

$$
\begin{aligned}
\left(\frac{\mathrm{d}^{2} \sigma}{\mathrm{d} \Omega \mathrm{d} E_{f}}\right)_{\mathrm{inc}}^{\mathrm{el}} & =N\left(c_{1} \sigma_{\mathrm{inc}}(1) \mathrm{e}^{-2 W_{1}(\vec{Q})}+c_{2} \sigma_{\mathrm{inc}}(2) \mathrm{e}^{-2 W_{2}(\vec{Q})}\right) \frac{\delta(\hbar \omega)}{4 \pi} \\
& =N\left((1-c) \sigma_{\mathrm{inc}}(1) \mathrm{e}^{-2 W_{1}(\vec{Q})}+c \sigma_{\mathrm{inc}}(2) \mathrm{e}^{-2 W_{2}(\vec{Q})}\right) \frac{\delta(\hbar \omega)}{4 \pi}
\end{aligned}
$$

with $c_{1}$ and $c\left(:=c_{2}\right)$ denoting the fractional concentrations of the two elements. The random occupation of the lattice sites has, therefore, no effect on the incoherent response apart from the fact that the incoherent cross sections $\sigma_{\kappa}$ have to be properly weighted by the concentrations. As a matter of fact, we do not even need the formalism involving the occupation factors $f_{j}$ in order to obtain this result.

Let us now turn our attention towards coherent elastic scattering. From the expressions (9.220) and (9.116) the total scattering function is found to be

$$
\begin{aligned}
\left(\frac{\mathrm{d}^{2} \sigma}{\mathrm{d} \Omega \mathrm{d} E_{f}}\right)_{\text {total }}^{\mathrm{el}} & =S^{\mathrm{el}}(\vec{Q}, \omega) \\
& =\sum_{j, j^{\prime}=1}^{N} \overline{\left(f_{j} f_{j^{\prime}}\right)} S_{j, j^{\prime}}(\vec{Q}, \hbar \omega=0)
\end{aligned}
$$




$$
\begin{aligned}
& =\sum_{j \neq j^{\prime}} \overline{f_{j} f_{j^{\prime}}^{*}} \exp \left(-\mathrm{i} \vec{Q} \cdot\left(\vec{R}_{j^{\prime}}^{0}-\vec{R}_{j}^{0}\right)\right) \delta(\hbar \omega)+\sum_{j} \overline{f_{j}^{2}} \delta(\hbar \omega) \\
& =\sum_{j, j^{\prime}} \overline{f_{j} f_{j^{\prime}}^{*}} \exp \left(-\mathrm{i} \vec{Q} \cdot\left(\vec{R}_{j^{\prime}}^{0}-\vec{R}_{j}^{0}\right)\right) \delta(\hbar \omega)+\sum_{j}\left(\overline{f_{j}^{2}}-\overline{f_{j}} \overline{f_{j}^{*}}\right) \delta(\hbar \omega) .
\end{aligned}
$$

The first term of this expression corresponds to the scattering function of the average structure, i.e. of the structure that we obtain by placing on each site a fictitious element possessing the average scattering length and the average Debye-Waller factor. In the case of a crystal, the Bragg peaks produced by this term persist and do not change position. Only their intensity will be affected by the disorder. The second term closely resembles incoherent scattering. Its $\vec{Q}$-dependence is limited to that of the Debye-Waller factors. If we subtract from this expression the expression (9.229) that was found for the incoherent scattering we obtain what is known as Laue monotonic scattering. From an experimental point of view ${ }^{105}$ diffuse scattering of this type is practically indistinguishable from incoherent scattering. ${ }^{106}$

In order to further illustrate this general discussion we return to our example of two types of elements distributed on a Bravais lattice. The averages of the scattering length are calculated as

$$
\begin{aligned}
& \bar{f}=(1-c) \mathrm{e}^{-W_{1}(\vec{Q})} \overline{b_{1}}+c \mathrm{e}^{-W_{2}(\vec{Q})} \overline{b_{2}}, \\
& \overline{f^{2}}=(1-c) \mathrm{e}^{-2 W_{1}(\vec{Q}) \overline{\left(b_{1}\right)^{2}}}+c \mathrm{e}^{-2 W_{2}(\vec{Q}) \overline{\left(b_{2}\right)^{2}} .}
\end{aligned}
$$

For the total elastic cross section we find

$$
\left(\frac{\mathrm{d}^{2} \sigma}{\mathrm{d} \Omega \mathrm{d} E_{f}}\right)_{\mathrm{coh}}^{\mathrm{el}}=f^{2}\left|\sum_{l} \exp (\mathrm{i} \vec{Q} \cdot \vec{l})\right|^{2} \delta(\hbar \omega)+N\left(\overline{f^{2}}-\bar{f}^{2}\right) \delta(\hbar \omega)
$$

The first part of this expression gives rise to the Bragg peaks. The second part deserves to be looked at more carefully. Using the expressions (9.225) and (9.226) and neglecting for the sake of clarity the Debye-Waller factors we get

$$
\begin{aligned}
\left(\overline{f^{2}}-\bar{f}^{2}\right)= & (1-c) \overline{b_{1}^{2}}+c \overline{b_{j}^{2}}-\left((1-c) \overline{b_{1}}+c \overline{b_{2}}\right)^{2} \\
= & \left\{(1-c)\left(\overline{b_{1}^{2}}-{\overline{b_{1}}}^{2}\right)+c\left(\overline{b_{2}^{2}}-{\overline{b_{2}}}^{2}\right)\right\} \\
& +\left\{(1-c){\overline{b_{1}}}^{2}+c{\overline{b_{2}}}^{2}-(1-c)^{2}{\overline{b_{1}}}^{2}-c^{2}{\overline{b_{2}}}^{2}+\left(c-c^{2}\right) \overline{b_{1} b_{2}}\right\} \\
= & \left\{(1-c)\left(\overline{b_{1}^{2}}-{\overline{b_{1}}}^{2}\right)+c\left(\overline{b_{2}^{2}}-{\overline{b_{2}}}^{2}\right)\right\} \\
& +\left\{\left(c-c^{2}\right){\overline{b_{1}}}^{2}+\left(c-c^{2}\right){\overline{b_{2}}}^{2}+\left(c-c^{2}\right) \overline{b_{1} b_{2}}\right\} \\
= & \left\{(1-c)\left(\overline{b_{1}^{2}}-{\overline{b_{1}}}^{2}\right)+c\left(\overline{b_{2}^{2}}-{\overline{b_{2}}}^{2}\right)\right\}+\left\{c(c-1)\left(\overline{b_{1}}-\overline{b_{2}}\right)^{2}\right\} .
\end{aligned}
$$

The expression

$$
N\left\{(1-c)\left(\overline{b_{1}^{2}}-{\overline{b_{1}}}^{2}\right)+c\left(\overline{\bar{b}_{2}^{2}}-{\overline{b_{2}}}^{2}\right)\right\}
$$

\footnotetext{
${ }^{105}$ If the incoherent scattering is only due to the nuclear spins then polarisation analysis can be used to separate the spin-incoherent from the diffuse scattering.

${ }^{106}$ This statement is true to the point where one often voluntarily confuses the diffuse scattering and the incoherent scattering in multicomponent amorphous systems [4]. The coherent scattering cross section is then defined as the total variance of the distribution of scattering lengths $b$, due to isotope, nuclear spin and chemical disorder. In this context one talks of incoherent scattering even for X-rays, which objectively does not make sense if we use the strict definition that we gave for incoherent scattering in Section 6.1.
} 
is nothing but the incoherent scattering weighted with the particle concentrations. Apart from the neglected DebyeWaller factors it is identical to (9.230). The diffuse scattering is included in the term

$$
N\left\{c(c-1)\left(\overline{b_{1}}-\overline{b_{2}}\right)^{2}\right\} .
$$

Like the incoherent scattering the diffuse scattering is independent of $\vec{Q}$ apart from the dependence coming with the Debye-Waller factors. It is observed whenever there exists a variation of the scattering lengths among the atoms that share a lattice site, and this even if all the atoms involved scatter purely coherently.

In general the simple models employed here do not match reality. Chemical disorder, even in its very dilute form, introduces structural distortions and thus influences the positions of the atoms and creates correlations among neighbours. The Bragg scattering is, therefore, better described by expressions of the form

$$
\left(\frac{\mathrm{d}^{2} \sigma}{\mathrm{d} \Omega \mathrm{d} E_{f}}\right)_{\text {coh }}^{\mathrm{el}}=f^{2}\left|\sum_{l} \exp \left(\mathrm{i} \vec{Q} \cdot\left(\vec{l}+\vec{u}_{\text {static }}(\vec{l})\right)\right)\right|^{2} \delta(\hbar \omega)
$$

where we have introduced the disorder induced distortions in the form of static displacements

$$
\vec{l} \rightarrow \vec{l}+\vec{u}_{\text {static }}(\vec{l})
$$

The choice of the variable $u$ has been done on purpose in order to emphasise the similarity with displacements having their origin in vibrations. From this analogy we can conjecture for weak distortions that disorder can be well represented by effective Debye-Waller factors. For a Gaussian distribution of distortions this static DebyeWaller factor has the same form as the one obtained for vibrations

$$
\exp \left(\frac{1}{2} \overline{\left(\vec{Q} \cdot \vec{u}_{\text {static }}\right)^{2}}\right)=\exp \left(\frac{1}{6} Q^{2} \overline{u_{\text {static }}^{2}}\right)
$$

Between Bragg peaks diffuse scattering is observed as the square of the Fourier transform of the distortions. These distortion fields can nicely be related to sets of frozen phonons ${ }^{107}$ and thus can be described by eigenvectors. This is a subject that we are not going to develop further.

\subsection{Incoherent scattering from one phonon}

After this small detour to diffuse scattering we now return to the discussion of incoherent scattering. The next higher term in the expansion of (9.215) contains the correlation function

$$
\begin{aligned}
\aleph_{j, j}(\vec{Q}, t) & =\left\langle\left(\vec{Q} \cdot \vec{u}_{j}\right)\left(\vec{Q} \cdot \vec{u}_{j}(t)\right)\right\rangle \\
& =\frac{\hbar}{2} \sum_{i}\left\{\left[\frac{A_{i}(\vec{Q} ; j)}{\omega_{i}}\left(1+n\left(\omega_{i}\right)\right) \exp \left(\mathrm{i} \omega_{i} t\right)\right]+\left[\frac{A_{i}(\vec{Q} ; j)}{\omega_{i}} n\left(\omega_{i}\right) \exp \left(-\mathrm{i} \omega_{i} t\right)\right]\right\}
\end{aligned}
$$

with

$$
A_{i}(\vec{Q} ; j):=\frac{\left(\vec{Q} \cdot \vec{e}_{i}(j)\right) \cdot\left(\vec{Q} \cdot \vec{e}_{i}(j)\right)^{*}}{m_{j}}=\frac{\left|\vec{Q} \cdot \vec{e}_{i}(j)\right|^{2}}{m_{j}} .
$$

\footnotetext{
${ }^{107} \mathrm{We}$ imagine that the atomic displacements corresponding to the eigenvector of a particular phonon are suddenly frozen in time.
} 
This expression allows us to calculate the incoherent scattering function for the atom type $\kappa$ as

$$
\begin{aligned}
S_{\kappa}^{1-\mathrm{ph}}(\vec{Q}, \omega) & =\sum_{j \in\left\{j_{\kappa}\right\}} \frac{\mathrm{e}^{-2 W_{j}(\vec{Q})}}{2 \pi \hbar} \int_{-\infty}^{\infty} \mathrm{d} t\left\langle\left(\vec{Q} \cdot \vec{u}_{j}\right)\left(\vec{Q} \cdot \vec{u}_{j}(t)\right)\right\rangle \mathrm{e}^{-\mathrm{i} \omega t} \\
& =\sum_{j \in\left\{j_{\kappa}\right\}} \frac{\mathrm{e}^{-2 W_{j}(\vec{Q})}}{2} \sum_{i} \frac{\left|\vec{Q} \cdot \vec{e}_{i}(j)\right|^{2}}{m_{\kappa} \omega_{i}}\left\{\left[\left(1+n\left(\omega_{i}\right)\right) \delta\left(\omega-\omega_{i}\right)\right]+\left[n\left(\omega_{i}\right) \delta\left(\omega+\omega_{i}\right)\right]\right\},
\end{aligned}
$$

and from there the partial differential cross section

$$
\begin{aligned}
& \left(\frac{\mathrm{d}^{2} \sigma}{\mathrm{d} \Omega \mathrm{d} E_{f}}\right)_{\mathrm{inc}}^{1-\mathrm{ph}} \\
& \quad=\frac{k_{f}}{k_{i}} \sum_{\kappa}\left(\overline{b_{\kappa}^{2}}-\left(\bar{b}_{\kappa}\right)^{2}\right) S_{\kappa}(\vec{Q}, \hbar \omega) \\
& \quad=\frac{k_{f}}{k_{i}} \sum_{\kappa} \frac{\sigma_{\mathrm{inc}}}{8 \pi m_{\kappa}} \sum_{j \in\left\{j_{\kappa}\right\}} \mathrm{e}^{-2 W_{j}(\vec{Q})} \sum_{i} \frac{\left|\vec{Q} \cdot \vec{e}_{i}(j)\right|^{2}}{\omega_{i}}\left\{\left[\left(1+n\left(\omega_{i}\right)\right) \delta\left(\omega-\omega_{i}\right)\right]+\left[n\left(\omega_{i}\right) \delta\left(\omega+\omega_{i}\right)\right]\right\} .
\end{aligned}
$$

We recognise the functional form of the coherent cross section with two Dirac $\delta$-functions.

One of them

$$
\begin{aligned}
\left(\frac{\mathrm{d}^{2} \sigma}{\mathrm{d} \Omega \mathrm{d} E_{f}}\right)_{\text {inc }}^{1-\mathrm{ph}(+)} & =\frac{k_{f}}{k_{i}} \sum_{\kappa}\left(\overline{b_{\kappa}^{2}}-\left(\bar{b}_{\kappa}\right)^{2}\right) S_{\kappa}(\vec{Q}, \hbar \omega) \\
& =\frac{k_{f}}{k_{i}} \sum_{\kappa} \frac{\sigma_{\mathrm{inc}}(\kappa)}{8 \pi m_{\kappa}} \sum_{j \in\left\{j_{\kappa}\right\}} \mathrm{e}^{-2 W_{j}(\vec{Q})} \sum_{i} \frac{\left|\vec{Q} \cdot \vec{e}_{i}(j)\right|^{2}}{\omega_{i}}\left[\left(1+n\left(\omega_{i}\right)\right) \delta\left(\omega-\omega_{i}\right)\right]
\end{aligned}
$$

corresponds to the creation of a vibrational excitation and the other

$$
\begin{aligned}
\left(\frac{\mathrm{d}^{2} \sigma}{\mathrm{d} \Omega \mathrm{d} E_{f}}\right)_{\text {inc }}^{1-\mathrm{ph}(-)} & =\frac{k_{f}}{k_{i}} \sum_{\kappa}\left(\overline{b_{\kappa}^{2}}-\left(\bar{b}_{\kappa}\right)^{2}\right) S_{\kappa}(\vec{Q}, \hbar \omega) \\
& =\frac{k_{f}}{k_{i}} \sum_{\kappa} \frac{\sigma_{\mathrm{inc}}(\kappa)}{8 \pi m_{\kappa}} \sum_{j \in\left\{j_{\kappa}\right\}} \mathrm{e}^{-2 W_{j}(\vec{Q})} \sum_{i} \frac{\left|\vec{Q} \cdot \vec{e}_{i}(j)\right|^{2}}{\omega_{i}}\left[n\left(\omega_{i}\right) \delta\left(\omega+\omega_{i}\right)\right]
\end{aligned}
$$

to its annihilation.

\subsubsection{Relation with the partial density of states in cubic or isotropic systems}

The general discussion of the incoherent one-phonon scattering has to end here. For an arbitrary system, hence a priori anisotropic, the eigenvectors depend explicitly on the directions $\vec{Q}$, onto which they are projected. This is particularly evident in a stratified system like graphite. ${ }^{108}$ A mode that describes a vibration of atoms in the layers has no equivalent vibration perpendicular to the layers, at least not at the same frequency. The situation changes

${ }^{108}$ Graphite is actually a bad example here since it only scatters coherently. 
if the system is cubic and monatomic. For any eigenvector two other modes with the same energy must exist such that the three vectors $\vec{e}_{i_{m}}(j)(m=1,2,3)$ form an orthonormal system. The sum of the projections onto $\vec{Q}$ gives 109

$$
\sum_{i_{m}}\left|\vec{Q} \cdot \vec{e}_{i_{m}}(j)\right|^{2}=\frac{1}{3} Q^{2}\left|\vec{e}_{i}(j)\right|^{2}=\frac{1}{3} Q^{2}
$$

It is tempting to think that when averaging over all $\vec{Q}$ directions, for example when working with powders, all systems boil down to the cubic monatomic case. This is unfortunately not true. Certainly, for a powder as well as for an amorphous system the scattering function has to be independent of the direction of $\vec{Q}$. For a given band of frequencies $] \omega, \omega+\Delta \omega]$ the average over all the projections of the eigenvectors onto $\vec{Q}$ has necessarily the form

$$
\left\{\sum_{i}\left|\vec{Q} \cdot \vec{e}_{i}(j)\right|^{2}\right\}_{\left.\left.\omega_{i} \in\right] \omega, \omega+\Delta \omega\right]}=f(Q, \omega)\left\{\sum_{i}\left|\vec{e}_{i}(j)\right|^{2}\right\}_{\left.\left.\omega_{i} \in\right] \omega, \omega+\Delta \omega\right]}
$$

The problem is that the function $f(Q, \omega)$ is system dependent. In practice it is found that even systems of low dimensionality such as nano-tubes can be interpreted correctly by assuming that $f(Q, \omega)=f(Q)$, which means tat $f(q, \omega)=f(q)$, and thus independent of $\omega$.

Inspired by the monatomic cubic system we are going to use the relation

$$
f(Q, \omega)=\frac{1}{3} Q^{2}
$$

and, therefore,

$$
\left\{\sum_{i}\left|\vec{Q} \cdot \vec{e}_{i}(j)\right|^{2}\right\}_{\left.\left.\omega_{i} \in\right] \omega, \omega+\Delta \omega\right]}=\frac{1}{3} Q^{2}\left\{\sum_{i}\left|\vec{e}_{i}(j)\right|^{2}\right\}_{\left.\left.\omega_{i} \in\right] \omega, \omega+\Delta \omega\right]}
$$

In order to ease the notation and to give a more precise significance to expression (9.246) we introduce the atomspecific density of states

$$
F_{j}(\omega):=\sum_{i}\left|\vec{e}_{i}(j)\right|^{2} \delta\left(\omega-\omega_{i}\right)
$$

It gives a measure of the number of modes that are present in a particular energy band and in which the atom $j$ participates. With the help of these density of states the differential cross section can be written as

$$
\begin{aligned}
\left(\frac{\mathrm{d}^{2} \sigma}{\mathrm{d} \Omega \mathrm{d} E_{f}}\right)_{\mathrm{inc}}^{1-\mathrm{ph}(+)} & =\frac{k_{f}}{k_{i}} \frac{Q^{2}}{3} \sum_{\kappa} \frac{\sigma_{\mathrm{inc}}(\kappa)}{8 \pi m_{\kappa}} \sum_{j \in\left\{j_{\kappa}\right\}} \mathrm{e}^{-2 W_{j}(\vec{Q})} \frac{F_{j}(\omega)}{\omega}(1+n(\omega)), \quad \omega>0, \\
\left(\frac{\mathrm{d}^{2} \sigma}{\mathrm{d} \Omega \mathrm{d} E_{f}}\right)_{\mathrm{inc}}^{1-\mathrm{ph}(-)} & =\frac{k_{f}}{k_{i}} \frac{Q^{2}}{3} \sum_{\kappa} \frac{\sigma_{\mathrm{inc}}(\kappa)}{8 \pi m_{\kappa}} \sum_{j \in\left\{j_{\kappa}\right\}} \mathrm{e}^{-2 W_{j}(\vec{Q})} \frac{F_{j}(-\omega)}{-\omega} n(-\omega) \\
& =\frac{k_{f}}{k_{i}} \frac{Q^{2}}{3} \sum_{\kappa} \frac{\sigma_{\mathrm{inc}}(\kappa)}{8 \pi m_{\kappa}} \sum_{j \in\left\{j_{\kappa}\right\}} \mathrm{e}^{-2 W_{j}(\vec{Q})} \frac{F_{j}(\omega)}{\omega}(n(\omega)+1), \quad \omega<0,
\end{aligned}
$$

${ }^{109}$ This relation is particularly evident if $\vec{e}_{i_{1}}(j)$ is parallel to $\vec{Q}$. In this specific case $\left|\vec{Q} \cdot \vec{e}_{i_{1}}(j)\right|^{2}=Q^{2}$ and $\left|\vec{Q} \cdot \vec{e}_{i_{2}}(j)\right|^{2}=\left|\vec{Q} \cdot \vec{e}_{i_{3}}(j)\right|^{2}=0$. 
where we have used the fact that $n(-x)=n(x)+1$ (see Fig. 50) and defined

$$
F_{j}(-\omega):=F_{j}(\omega), \quad \omega \geqslant 0 .
$$

With this convention we are no longer obliged to make an explicit distinction between the expression for energy gain and energy loss. ${ }^{110}$ Until now the index $\kappa$ referred to a chemical element. This classification was adapted because the coherent and incoherent scattering cross sections depended on the atom type. At this point it becomes useful not to distinguish any longer between different types of atoms but between atoms that are symmetrically inequivalent. The concept of symmetrical equivalence is well defined in crystals. In amorphous system all the atoms of the same chemical element can often be considered to be equivalent. The expressions for the incoherent cross section simplify further when introducing the partial density of states for the symmetrical equivalent atoms distinguished by the index $\kappa$

$$
F_{\kappa}(\omega):=\frac{1}{3 N_{\kappa}} \sum_{j \in\left\{j_{\kappa}\right\}} \sum_{i}\left|\vec{e}_{i}(j)\right|^{2} \delta\left(\omega-\omega_{i}\right),
$$

with $N_{\kappa}$ being the number of atoms of this type. The partial density of states can be evaluated referring to any one single member of the class of equivalent atoms

$$
F_{\kappa}(\omega):=\frac{1}{3} \sum_{i}\left|\vec{e}_{i}\left(j \in\left\{j_{\kappa}\right\}\right)\right|^{2} \delta\left(\omega-\omega_{i}\right)
$$

For the cross section the following expression is found

$$
\left(\frac{\mathrm{d}^{2} \sigma}{\mathrm{d} \Omega \mathrm{d} E_{f}}\right)_{\text {inc }}^{1-\mathrm{ph}}=\frac{k_{f}}{k_{i}} Q^{2} \frac{1}{8 \pi} \sum_{\kappa} N_{\kappa} \frac{\sigma_{\mathrm{inc}}(\kappa)}{m_{\kappa}} \mathrm{e}^{-2 W_{\kappa}(\vec{Q})} F_{\kappa}(\omega) \frac{(1+n(\omega))}{\omega} .
$$

In a crystal the number of inequivalent atoms is at maximum equal to the number of atoms in the primitive cell $r$. Thus the result can be written as

$$
\left(\frac{\mathrm{d}^{2} \sigma}{\mathrm{d} \Omega \mathrm{d} E_{f}}\right)_{\text {inc }}^{1-\mathrm{ph}}=\frac{k_{f}}{k_{i}} Q^{2} \frac{N}{8 \pi r} \sum_{d} \frac{\sigma_{\text {inc }}(d)}{m_{d}} \mathrm{e}^{-2 W_{\kappa}(\vec{Q})} F_{d}(\omega) \frac{(1+n(\omega))}{\omega} .
$$

We would like to note that in order to arrive at this expression we have at no stage of the development used the fact that in a crystal the eigenvectors are classified according to the wave vector $\vec{q}$. The translational symmetry, that is at

$$
\begin{aligned}
& \text { incoherent scattering is given as } \\
& \qquad\left(\frac{\mathrm{d}^{2} \sigma}{\mathrm{d} \Omega \mathrm{d} E_{f}}\right)_{\mathrm{inc}}^{1-\mathrm{ph}(+)}=\frac{k_{f}}{k_{i}} \frac{Q^{2}}{3} \sum_{\kappa} \frac{\sigma_{\text {inc }}(\kappa)}{8 \pi m_{\kappa}} \sum_{j \in\left\{j_{\kappa}\right\}} \mathrm{e}^{-2 W_{j}(\vec{Q})} \frac{F_{j}(\omega)}{\omega}(1+n(\omega)), \quad \omega>0, \\
& \left(\frac{\mathrm{d}^{2} \sigma}{\mathrm{d} \Omega \mathrm{d} E_{f}}\right)_{\text {inc }}^{1-\mathrm{ph}(-)}=\frac{k_{f}}{k_{i}} \frac{Q^{2}}{3} \sum_{\kappa} \frac{\sigma_{\text {inc }}(\kappa)}{8 \pi m_{\kappa}} \sum_{j \in\left\{j_{\kappa}\right\}} \mathrm{e}^{-2 W_{j}(\vec{Q})} \frac{F_{j}(\omega)}{\omega} n(\omega), \quad \omega<0,
\end{aligned}
$$

${ }^{110}$ We would nevertheless like to emphasise that a competing convention exist. The frequency $\omega$ is always assumed to be positive and the

which also can be written as

$$
\left(\frac{\mathrm{d}^{2} \sigma}{\mathrm{d} \Omega \mathrm{d} E_{f}}\right)_{\text {inc }}^{1-\mathrm{ph}( \pm)}=\frac{k_{f}}{k_{i}} \frac{Q^{2}}{3} \sum_{\kappa} \frac{\sigma_{\text {inc }}(\kappa)}{8 \pi m_{\kappa}} \sum_{j \in\left\{j_{\kappa}\right\}} \mathrm{e}^{-2 W_{j}(\vec{Q})} \frac{F_{j}(\omega)}{\omega}\left[\frac{1}{2}\left(\cosh \left(\frac{\hbar \omega}{2 k_{\mathrm{B}} T}\right) \pm 1\right)\right] .
$$


the origin of this classification, has no added value in the case of incoherent scattering besides reducing equivalence classes. This should not surprise us. The vectors $\vec{q}$ define the relative phase of the movement of the atoms. Since there is no interference between the wavelets originating from different atoms in the case of incoherent scattering phase relations do not enter the game. As a consequence, and unlike for coherent scattering incoherent scattering is not subjected to selection rules that invoke the conservation of crystal momentum.

The expression for the incoherent partial differential cross section is even simpler in the case of a Bravais lattice, i.e. when we are dealing with only one atom per primitive cell

$$
\left(\frac{\mathrm{d}^{2} \sigma}{\mathrm{d} \Omega \mathrm{d} E_{f}}\right)_{\text {inc }}^{1-\mathrm{ph}}=\frac{k_{f}}{k_{i}} Q^{2} \frac{N}{8 \pi} \frac{\sigma_{\text {inc }}}{m} \mathrm{e}^{-2 W(\vec{Q})} \frac{g(\omega)}{\omega}(1+n(\omega))
$$

with

$$
\begin{aligned}
g(\omega) & =\frac{1}{3 N} \sum_{j} \sum_{i}\left|\vec{e}_{i}(j)\right|^{2} \delta\left(\omega-\omega_{i}\right) \\
& =\frac{1}{3 N} \sum_{i} \delta\left(\omega-\omega_{i}\right) \sum_{j}\left|\vec{e}_{i}(j)\right|^{2} \\
& =\frac{1}{3 N} \sum_{i} \delta\left(\omega-\omega_{i}\right)
\end{aligned}
$$

the vibrational density of states (see Fig. 55 for a concrete example of an experimental density of states obtained on a monatomic incoherent scatterer). In contrast to the expression (9.202) for coherent one-phonon scattering the expression (9.262) is explicitly proportional to the number of scatterers $N$. The origin of this explicit dependence has its origin in our convention for normalising the density of states $g(\omega)$. Combining (9.262) and (9.263) it becomes immediately obvious that the scattering is proportional to the number of modes, which again is proportional to the number of atoms in the system.

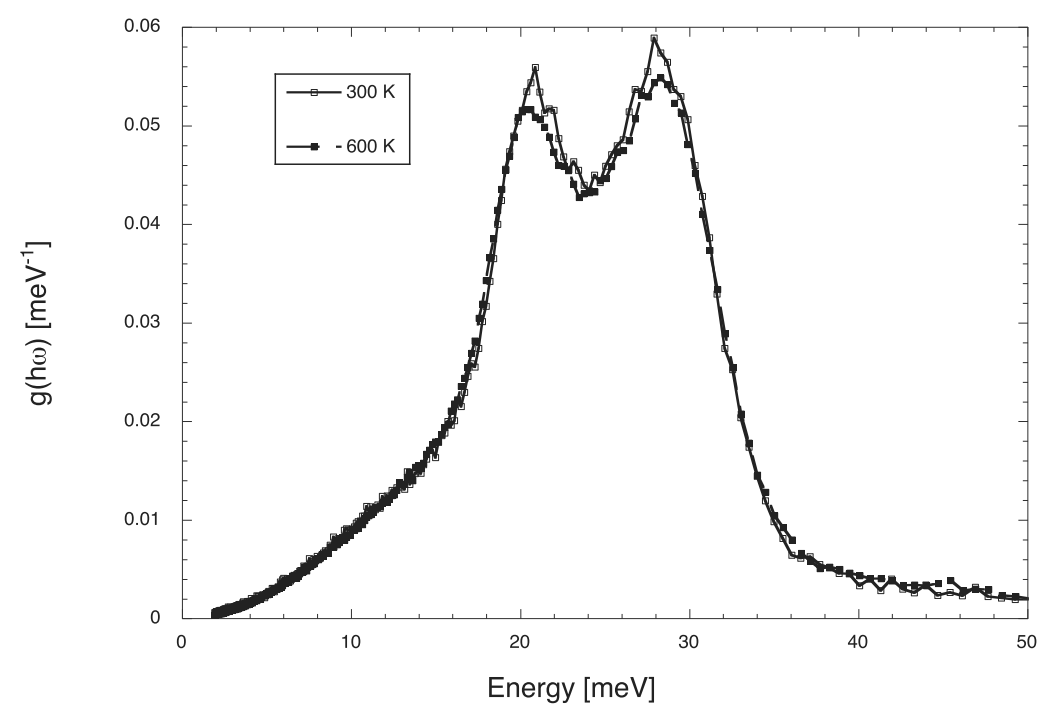

Fig. 55. The density of states of Vanadium at $300 \mathrm{~K}$ and $600 \mathrm{~K}$ obtained from incoherent one-phonon scattering as measured on the instrument IN6 (see Fig. 58) with an incident wave length of $\lambda=4.16 \AA$. The correction of the multi-phonon contribution has been made using the procedure described in Section 10.5 (see Fig. 61). For a harmonic material, such as is Vanadium at these temperatures, the density of states is more or less invariant with $T$. 


\subsubsection{Ratio in intensity between inelastic and elastic scattering}

The expressions that we have derived give the scattering intensity in absolute units. To get a feeling for the size of inelastic signals it is interesting to compare them to the elastic signal as a reference. We will perform this exercise for the simplest possible case, which is a monatomic incoherent scatterer on a cubic lattice like Vanadium. For such a system the results found in the previous section and in particular the expression (9.262) can be rigorously applied. According to (9.217) the elastic scattering is given by

$$
\left(\frac{\mathrm{d} \sigma}{\mathrm{d} \omega}\right)_{\mathrm{inc}}^{\mathrm{el}}=N \sigma_{i} \mathrm{e}^{-2 W(Q)}
$$

If we concentrate on the low-frequency part of the spectrum then we know that the modes have their origin in acoustic vibrations. These are represented by the well known linear dispersion relations

$$
\omega=\vec{q} \cdot c_{j, \hat{q}}^{\text {sound }}
$$

with $c_{j, \hat{k}}^{\text {sound }}$ the speed of sound along the direction $\hat{q}$ for the acoustic branch $j$. The density of states in this region can then be calculated as

$$
g(\omega)=\left(\frac{V}{N}\right)\left(\frac{2 \pi^{2}}{c_{\text {sound }}^{3}}\right) \omega^{2}=\frac{3 \omega^{2}}{\omega_{D}^{3}}
$$

where $c_{\text {sound }}$ represents the velocity of sound averaged over all directions and all branches. $\omega_{D}$ is the Debye frequency of the material as defined via expression (9.266). $V$ is the volume occupied by the $N$ atoms.

In order to apply Eq. (9.262) we need to take into account the thermal occupation factors. For the excitation energies

$$
\hbar \omega \ll k_{\mathrm{B}} T: \quad n(\omega) \rightarrow \hbar \omega / k_{\mathrm{B}} T .
$$

Hence for the inelastic cross section in neutron energy gain we find

$$
\begin{aligned}
\left(\frac{\mathrm{d}^{2} \sigma}{\mathrm{d} E_{f} \mathrm{~d} \Omega}\right)_{\mathrm{inc}} & =\frac{3 N \sigma_{i}}{8 \pi} \frac{k_{f}}{k_{i}} \frac{\hbar^{2} Q^{2}}{2 m} \mathrm{e}^{-2 W(\vec{Q})}\left(\frac{k_{\mathrm{B}} T}{\left(\hbar \omega_{D}\right)^{3}}\right) \\
& =\frac{k_{f}}{k_{i}} 3 N \frac{\sigma_{i}}{8 \pi} \mathrm{e}^{-2 W(\vec{Q})}\left(\frac{E_{r}(Q) \cdot\left(k_{\mathrm{B}} T\right)}{\left(\hbar \omega_{D}\right)^{3}}\right),
\end{aligned}
$$

with the recoil energy of the atom defined in Section 8.10. We will meet $E_{r}(Q)$ again several times in the discussion of multi-phonons. This expression is proportional to the temperature but is no longer dependent on $\omega$. This statement holds for all three dimensional systems, which is to say for all systems whose density of states is proportional to $\omega^{2}$. We see, in particular, that the decrease in the number of phonon states as $\omega$ tends to zero compensates for the divergence of the factor $n(\omega / \omega) .^{111}$

This gives the ratio between the inelastic and elastic signal that we were looking for.

$$
\left(\frac{\mathrm{d} \sigma}{\mathrm{d} \Omega}\right)_{\mathrm{inc}}^{\mathrm{el}} / \int_{0}^{\hbar \omega_{l}}\left(\frac{\mathrm{d}^{2} \sigma}{\mathrm{d}(\hbar \omega) \mathrm{d} \Omega}\right)_{\mathrm{inc}}^{\mathrm{el}} \mathrm{d} \hbar \omega \approx \frac{4 \pi}{3}\left(\frac{\left(\hbar \omega_{D}\right)^{3}}{E_{r}(Q)\left(\hbar \omega_{l}\right)\left(k_{\mathrm{B}} T\right)}\right),
$$

where we have used $k_{i} \approx k_{f}$ for small energy transfers. Thus the ratio is defined by the characteristic energy $\hbar \omega_{D}$ of the vibrational spectrum, the thermal energy $k_{\mathrm{B}} T$ and the integration interval $\hbar \omega_{l}$. These values are all in the

\footnotetext{
${ }^{111} n(\omega) \rightarrow\left(\hbar \omega /\left(k_{\mathrm{B}} T\right)\right)^{-1}$ for $\omega \rightarrow 0-\varepsilon$ (see Fig. 50).
} 
range between zero and $100 \mathrm{meV}$. The Debye energies are often around $40 \mathrm{meV}$, while $k_{\mathrm{B}} T$ at room temperature is around $30 \mathrm{meV}$. Taking $Q=2 \AA^{-1}$ and an atomic mass of $50 \mathrm{amu}$, the recoil energy $\hbar^{2} Q^{2} / 2 m$ is about $0.3 \mathrm{meV}$. This simple estimation gives inelastic intensities (integrated over an energy range from 0 to $10 \mathrm{meV}$ ) that are 100 to 1000 times smaller than what is collected in the elastic channel. This explains why it is so important to keep the background as low as possible for inelastic experiments. Note that the Debye-Waller factor, since it effects the elastic and inelastic intensities in the same way, is not present in this ratio.

\subsubsection{Generalised density of states}

The systems that are of scientific interest are often complex and expression (9.262) is, therefore, not valid for them. This is unfortunate as this expression allows for a simple interpretation of experimental results in terms of a physical quantity of great interest, the density of states. From a purely formal point of view there is no problem in postulating an expression similar to (9.262) for a crystal with several atoms in the primitive cell. Starting from expression (9.260)

$$
\left(\frac{\mathrm{d}^{2} \sigma}{\mathrm{d} \Omega \mathrm{d} E_{f}}\right)_{\text {inc }}^{1-\mathrm{ph}(+)}=\frac{k_{f}}{k_{i}} Q^{2} \frac{N}{8 \pi} \mathrm{e}^{-2 W(\vec{Q})} \frac{G(\omega)}{\omega}(1+n(\omega)),
$$

where we have introduced the generalised density of states

$$
G(\omega):=\sum_{d=1}^{r} \frac{\sigma_{\text {inc }}(d)}{m_{d}} F_{d}(\omega)
$$

and under the condition that the Debye-Waller factors

$$
\mathrm{e}^{-2 W_{d}(\vec{Q})} \approx \mathrm{e}^{-2 W(\vec{Q})}
$$

are more or less independent of $d$. In the generalised density of states $G(\omega)$ the partial density of states $F_{d}(\omega)$ corresponding to different atoms in the primitive cell are weighted with what is called their respective scattering power

$$
\frac{\sigma_{\text {inc }}(d)}{m_{d}}
$$

We have in principle the possibility of isolating the different contributions of the generalised density of states provided that their exists a way of modifying the contrast. The situation is similar to isolating partial correlation functions (see Section 8.4), of which the partial densities of states are the direct manifestation. Contrast variation is possible if a chemical element possesses several isotopes with very different scattering lengths. This is the case for hydrogen and deuterium. Care should, however, be applied in this specific case, which is often exploited in soft matter and biology. The masses of the two isotopes are very different. This difference has a significant influence on the frequencies of those modes, in which either hydrogen or deuterium participate. The most obvious example is water. The spectrum of the librations softens drastically upon the exchange of light water $\mathrm{H}_{2} \mathrm{O}$ by heavy water $\mathrm{D}_{2} \mathrm{O}^{112}$

If we succeed in isolating the partial density of states then we are in a position to determine the vibrational density of states $g(\omega)$. This is from a physics point of view the most interesting quantity since it enters into the thermodynamical calculations. Identifying the partial density of states is unfortunately rarely possible. In most cases we have to satisfy ourselves with the generalised density of states.

\footnotetext{
${ }^{112}$ It should not be forgotten that besides the masses, the scattering characteristics of hydrogen and deuterium are very different (see Section 6.3). Deuterium is a coherent scatterer with a non-negligible incoherent cross section.
} 
In the light of the approximation that we were obliged to introduce in order to reach expression (9.269) the statement that incoherent scattering gives direct access to $g(\omega)$ should be taken with a lot of precaution. It is certainly true that even in the case of polyatomic systems it is always possible to write the scattering function formally as

$$
S(\vec{Q}, \omega)=A(\vec{Q}, \omega) G_{\mathrm{eff}}(\omega)
$$

with, in analogy to expression (9.262),

$$
\left.A(\vec{Q}, \omega):=\frac{\hbar}{4 \pi} \frac{\left(\sigma_{\mathrm{inc}}(j) \mathrm{e}^{-2 W_{j}(Q)}\right)_{\mathrm{av}}}{2 M_{\mathrm{av}}} \frac{Q^{2}}{\omega}[n(\omega)+1)\right]
$$

and

$$
M_{\mathrm{av}}=\frac{1}{N} \sum_{j} m_{j} \quad \text { and } \quad\left(\bar{b}^{2} \mathrm{e}^{-2 W_{j}(Q)}\right)_{\mathrm{av}}=\frac{1}{N} \sum_{j} \sigma_{\text {inc }}(j) \exp \left(-2 W_{j}(Q)\right) .
$$

The effective density of states $G_{\text {eff }}(\omega)$ is well-defined from an mathematical point of view. Unfortunately, it can be very different from the true vibrational density of states $g(\omega)$, and even worse also from the generalised density of states $G \omega)$. In the case where the goal itself is to verify a given dynamical model, the correct procedure is to calculate the generalised density of states $G(\omega)$ from the theory, or even better to proceed directly to a calculation of the scattering function $S(\vec{Q}, \omega)$ and to compare those quantities with the experimental data.

Without a theoretical model one should be aware of the errors introduced by blindly identifying the effective density of states $G_{\text {eff }}(\omega)$ with the true vibrational density of states $g(\omega)$. We find this source of error sufficiently important to illustrate it with a simple but telling example: the dynamics of ice. Ice is a typical molecular crystal and has several excitation bands (see Fig. 56). At low frequencies ( $\hbar \omega<40 \mathrm{meV}$ ) the excitations correspond to

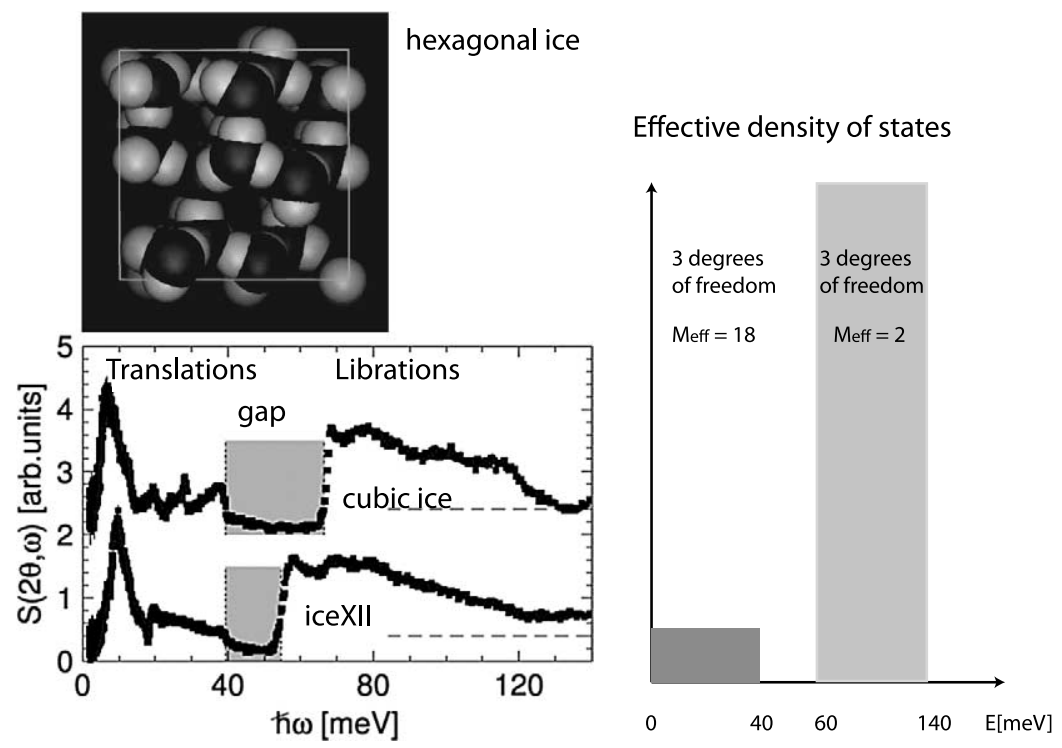

Fig. 56. Inelastic intensity of two $\mathrm{H}_{2} \mathrm{O}$ phases of ice measured at the instrument TOSCA at ISIS [36]. In ice the translational modes are well separated from the librational modes. Due to the fact that for librations the movement is limited to the two hydrogen atoms the inverse effective mass, that enters the intensity calculation is nine times as large as that to be used for the translations. If this fact is not properly taken into account when extracting the vibrational density of states with the help of expression (9.271) the number of modes in the vibrational band is overestimated, in the worst case by a factor of nine. 
the translational centre-of-mass motions of the water molecules. Let us for the moment neglect the fact that the crystalline phases of ice possess more than one molecule in the primitive cell. If the librations that will be discussed below are supposed to be completely decoupled from the translations, then the system can be considered atomic (where the "effective" atom is the molecule $\mathrm{H}_{2} \mathrm{O}$ ) in this low frequency region. The weight of each translational mode in the cross section (9.260) is determined by $2 \sigma_{\text {inc }}(\mathrm{H}) / m\left(\mathrm{H}_{2} \mathrm{O}\right)$. This result is obtained by remembering that the eigenvectors $e_{i}$ are normalised to unity and that the scattering is dominated by the incoherent cross section of hydrogen. The next higher band of excitations contains the librations of the water molecules. Librations are, like translations, external modes, i.e. they do not introduce distortions of the $\mathrm{H}_{2} \mathrm{O}$ molecule. For ideal librations the centre-of-mass is at rest (see the decoupling mentioned above). As the centre-of-mass nearly coincides with the oxygen site we can assume in good approximation that the vibrational modes possess non-zero components only for the hydrogen atoms. The weight of each librational mode in the cross section is determined by $\sigma_{\text {inc }}(\mathrm{H}) / m(\mathrm{H})$. This implies that, even though they will contain the same number of modes, the intensity of a librational band will exceed that of a "hypothetical" translational band covering the same frequency region by a factor 9 . If this fact is ignored and the generalised density of states is blindly interpreted as the true density of states then this mistake will result in an enormous error in this admittedly eminently unfavourable case. This is confirmed by more elaborate calculations using molecular dynamics simulations [13].

\subsection{The incoherent approximation}

As we already mentioned several times the density of states is of great importance for the physical properties of a material. It is in principle accessible through the incoherent scattering signal provided we take the precautions discussed in the previous section. Unfortunately ${ }^{113}$ one is often facing coherent scatterers. If one is dealing with a monatomic crystal then one can always try to measure the entire set of dispersion curves and derive from this information the density of states. This quickly becomes an impractical approach when working with even slightly more complex structures, i.e. with several atoms in the primitive cell. The problem is rooted in the requirement that, in order to determine the density of states, the experiment must capture the modes in their totality. Since coherent scattering produced by phonons is very selective due to the conservation of crystal momentum this requires at minimum the coverage of a symmetrically representative part of the Brillouin zone. Such a survey may still be insufficient as due to the projection of the eigenvectors onto $\vec{Q}$ in the form factor a particular phonon may be invisible despite of the fact that both energy and crystal momentum conservation are fulfilled. To repeat a telling example, the projection onto $\vec{Q}$ is strictly zero for purely transverse modes in the first Brillouin zone. And even if the signal contained contributions from all the modes this will not be a guarantee that we are in a position of calculating the density of states. This will only be the case if we knew the exact weight with which the modes contribute to the signal. This weight depends among other things again on the projection of the eigenvectors onto $\vec{Q}$ and thus calculating this weight implies a prior knowledge of these eigenvectors. We thus need a more efficient method than the extraction of the density-of-states from single crystal data even if that method was very approximative.

Given that an average over all the modes is needed it seems logical to turn towards powder measurements. The possibility of realising such measurements has been discussed quite early (see for example [5]). To better define the problem and to identify the principle sources of error we have to study in a bit more detail the influence of powder averaging on the inelastic cross section. If we limit ourselves to cubic monatomic systems we obtain from (9.202) for the coherent partial differential cross section

$$
\begin{aligned}
\left(\frac{\mathrm{d} \sigma^{2}}{\mathrm{~d}(\hbar \omega) \mathrm{d} \Omega}\right)_{\text {coh }}^{1-\mathrm{ph}}= & \frac{k_{f}}{k_{i}} \frac{\sigma_{\text {coh }}}{4 \pi} \frac{(2 \pi)^{3}}{V_{\text {unit cell }}} \sum_{\vec{G}} \sum_{i, \vec{q}} \mathrm{e}^{-2 W(Q)} \frac{\left|\vec{Q} \cdot \vec{e}_{i}(\vec{q})\right|^{2}}{2 m \omega_{i}(\vec{q})} \\
& \times\left(1+n\left(\omega_{i}(\vec{q})\right)\right) \delta(\vec{q}-(\vec{Q}+\vec{G})) \delta\left(\omega-\omega_{i}(\vec{q})\right),
\end{aligned}
$$

\footnotetext{
${ }^{113}$ Unfortunately, because, as we had discussed in detail, the information content of coherent scattering is always superior to that of incoherent scattering. The problem is related to extracting this information content.
} 
where we only show the part that concerns the emission of a phonon. The part that concerns the absorption can be derived in perfect analogy.

In order to carry out the powder average it is in general preferable to transform the sum over $q$ into an integral ${ }^{114}$

$$
\begin{aligned}
& \left(\frac{\mathrm{d} \sigma^{2}}{\mathrm{~d}(\hbar \omega) \mathrm{d} \Omega}\right)_{\mathrm{coh}}^{1-\mathrm{ph}} \\
& =N \frac{k_{f}}{k_{i}} \frac{\sigma_{\mathrm{coh}}}{8 \pi m} \sum_{i=1}^{3} \int_{\mathrm{BZ}} \mathrm{d}^{3} q \frac{\left.\delta\left(\omega-\omega_{i}(\vec{q})\right)\right)}{\omega_{i}(\vec{q})}\left(1+n\left(\omega_{i}(\vec{q})\right)\right) \\
& \quad \times \sum_{\vec{G}} \mathrm{e}^{-2 W(Q)}\left|\vec{Q} \cdot \vec{e}_{i}(\vec{q})\right|^{2} \delta(\vec{q}-(\vec{Q}+\vec{G})) .
\end{aligned}
$$

For a given energy transfer the vector $\vec{Q}$ is defined in the reference system of the spectrometer by $\vec{k}_{i}$ and $\vec{k}_{f}$. The vectors $\vec{q}$ as well as the eigenvectors $\vec{e}_{i}(\vec{q})$ of the phonons are defined in the reference system of the sample. In a powder this reference changes from one grain to the next. From the perspective of the sample the direction of $\vec{Q}$, therefore fluctuates arbitrarily in all directions. Formally the vector $\vec{Q}$ can be found with equal probability at any point on a sphere with radius $Q$. In order to capture this effect we have to average the cross section over all possible $\vec{Q}$ directions. Let us define

$$
\left(\frac{\mathrm{d} \sigma^{2}}{\mathrm{~d}(\hbar \omega) \mathrm{d} \Omega}\right)_{\mathrm{coh}-|\mathrm{Q}|}^{1-\mathrm{ph}}=\frac{1}{4 \pi Q^{2}} \int_{0}^{\pi} \sin \Theta_{Q} \mathrm{~d} \Theta_{Q} \int_{0}^{2 \pi} \mathrm{d} \Phi_{Q}\left(\frac{\mathrm{d} \sigma^{2}}{\mathrm{~d}(\hbar \omega) \mathrm{d} \Omega}\right)_{\text {coh }}^{1-\mathrm{ph}} .
$$

In this expression the spherical coordinates refer to (as explicitly indicated) $\vec{Q}$ and not $\vec{k}_{f}$ (as in previous sections). Carrying out this average for the expression (9.276) we find

$$
\begin{aligned}
\left(\frac{\mathrm{d} \sigma^{2}}{\mathrm{~d}(\hbar \omega) \mathrm{d} \Omega}\right)_{\mathrm{coh}-|\mathrm{Q}|}^{1-\mathrm{ph}}= & N \frac{k_{f}}{k_{i}} \frac{\sigma_{\mathrm{coh}}}{8 \pi m} \frac{1}{4 \pi Q^{2}} \sum_{i=1}^{3} \int_{\mathrm{BZ}} \mathrm{d}^{3} q \frac{\left.\delta\left(\omega-\omega_{i}(\vec{q})\right)\right)}{\omega_{i}(\vec{q})}\left(1+n\left(\omega_{i}(\vec{q})\right)\right) \\
& \times \sum_{\vec{G}} \mathrm{e}^{-2 W(Q)}\left|\vec{e}_{i}(\vec{q}) \cdot(\vec{q}-\vec{G})\right|^{2} \delta(|\vec{q}-\vec{G}|-Q) \\
= & N \frac{k_{f}}{k_{i}} \frac{\sigma_{\mathrm{coh}}}{8 \pi m} \frac{1}{4 \pi} \sum_{i=1}^{3} \int_{\mathrm{BZ}} \mathrm{d}^{3} q \frac{\left.\delta\left(\omega-\omega_{i}(\vec{q})\right)\right)}{\omega_{i}(\vec{q})}\left(1+n\left(\omega_{i}(\vec{q})\right)\right) \\
& \times \sum_{\vec{G}} \mathrm{e}^{-2 W(Q)}\left|\vec{e}_{i}(\vec{q}) \cdot \hat{k}_{\vec{G}}\right|^{2} \delta\left(\left|\vec{q}_{\vec{G}}\right|-Q\right),
\end{aligned}
$$

where in order to simplify the expression we pose $\vec{q}_{\vec{G}}:=\vec{q}-\vec{G}$. With respect to (9.276) the Dirac functions of the vector $\vec{Q}$ become Dirac functions of the norm $Q$. This makes perfect sense as in a powder we will always find a grain that satisfies the conservation of crystal momentum $\vec{q}_{\vec{G}}=\vec{Q}$ provided that $Q$ equals $\left|\vec{q}_{\vec{G}}\right|$.

$$
\begin{aligned}
& { }^{114} \text { We use the replacement } \\
& \qquad \sum_{\vec{q}} \cdots \rightarrow \frac{N}{V_{\mathrm{Bz}}} \int \mathrm{d}^{3} q \ldots=N \frac{V_{\text {unit cell }}}{(2 \pi)^{3}} \int \mathrm{d}^{3} q \ldots
\end{aligned}
$$


If the scattering had been incoherent then we would have found starting from (9.245)

$$
\begin{aligned}
\left(\frac{\mathrm{d} \sigma^{2}}{\mathrm{~d}(\hbar \omega) \mathrm{d} \Omega}\right)_{\text {inc }-|\mathrm{Q}|}^{1-\mathrm{ph}}= & N \frac{k_{f}}{k_{i}} \frac{\sigma_{\text {inc }}}{8 \pi m} \frac{V_{\text {unit cell }}}{(2 \pi)^{3}} \mathrm{e}^{-2 W(Q)} \\
& \times \sum_{i=1}^{3} \int_{\mathrm{BZ}} \mathrm{d}^{3} q \frac{\left.\delta\left(\omega-\omega_{i}(\vec{q})\right)\right)}{\omega_{i}(\vec{q})}\left(1+n\left(\omega_{i}(\vec{q})\right)\right)\left\{\left|\vec{e}_{i}(\vec{q}) \cdot \vec{Q}\right|^{2}\right\}_{\mathrm{av}} \\
= & N \frac{k_{f}}{k_{i}} \frac{\sigma_{\text {inc }}}{8 \pi m} \frac{1}{4 \pi} \mathrm{e}^{-2 W(Q)} \frac{g(\omega)}{\omega}(1+n(\omega)) .
\end{aligned}
$$

This is a result that we already know (see expression (9.262)). ${ }^{115}$ Compared to the expression (9.279) for incoherent scattering the expression (9.278) for coherent scattering is more selective in terms of the modes that contribute to the integral. In other words, the incoherent scattering is inclusive and the degree of inclusion is solely governed by the incoherent scattering cross sections of the respective atoms. All modes are a priori present in the cross section. The coherent scattering is selective. Even after powder averaging a specific mode will only contribute to the measured cross section if the reciprocal lattice volume covered contains a lattice vector $\vec{G}$ such that $|\vec{q}-\vec{G}|=\vec{Q}$. If $Q$ and $\Delta Q$ are small this volume is small and the number of $\vec{G}$ that are susceptible to meet the condition $|\vec{q}-\vec{G}|=\vec{Q}$ will equally be small (see Fig. 57). In the case of very small $Q$ the volume covered will be contained within the first Brillouin zone and the wave vectors of the modes are submitted to the stringent condition of $|\vec{q}|=Q$. In this scenario the bulk part of the modes will escape observation. The number of reciprocal lattice vectors that enter into the cross section increases significantly with $Q$. Unfortunately very large $Q$ values are not desirable for other reasons. First of all the energy resolution decreases and secondly the multi-phonon contributions become dominant.

The solution consist in using reasonably large $Q$ values and to allow at the same time an important variation $\Delta Q$ in $Q$ in order to assure a better sampling of modes. In practice this variation can be obtained using e.g. a multidetector. For a given energy transfer $\hbar \omega$ the value of $Q$ will vary when going from one detector to another. The experiment then covers an entire shell in reciprocal space that is delimited by the spheres of radius $Q_{\min }(\hbar \omega)$ (interior) and $Q_{\max }(\hbar \omega)$ (exterior). Let us denote by $p(\hbar \omega, Q)$ the probability ${ }^{116}$ that the inelastic scattering proceeds via a channel characterised by the energy transfer $\hbar \omega$ and the norm of the momentum transfer $Q$. Then the coherent cross section integrated over the reciprocal space volume $V$ can be written as

$$
\begin{aligned}
& \left(\frac{\mathrm{d} \sigma^{2}}{\mathrm{~d}(\hbar \omega)}\right)_{\mathrm{coh}-\mathrm{int}}^{1-\mathrm{ph}}=\int_{V}\left(\frac{\mathrm{d} \sigma^{2}}{\mathrm{~d}(\hbar \omega) \mathrm{d} \Omega}\right)_{\mathrm{coh}}^{1-\mathrm{ph}} p_{\hbar \omega}(Q) \mathrm{d}^{3} Q \\
& =4 \pi \int_{Q_{\min }}^{Q_{\max }}\left(\frac{\mathrm{d} \sigma^{2}}{\mathrm{~d}(\hbar \omega) \mathrm{d} \Omega}\right)_{\operatorname{coh}-|\mathrm{Q}|}^{1-\mathrm{ph}} Q^{2} p_{\hbar \omega}(Q) \mathrm{d} Q \\
& =N \frac{k_{f}}{k_{i}} \frac{\sigma_{\mathrm{coh}}}{8 \pi m} \sum_{i=1}^{3} \int_{\mathrm{BZ}} \mathrm{d}^{3} q \frac{\delta\left(\omega-\omega_{i}(\vec{q})\right)}{\omega_{i}(\vec{q})}\left(1+n\left(\omega_{i}(\vec{q})\right)\right) \\
& \times \int_{Q_{\min }}^{Q_{\max }} \sum_{\vec{G}} \mathrm{e}^{-2 W(Q)}\left|\vec{e}_{i}(\vec{q}) \cdot \hat{q}_{\vec{G}}\right|^{2} \delta\left(\left|\vec{q}_{\vec{G}}\right|-Q\right) Q^{2} p_{\hbar \omega}(Q) \mathrm{d} Q
\end{aligned}
$$

$$
\sum_{i=1}^{3} \int_{\mathrm{BZ}} \mathrm{d}^{3} q \ldots=3 V_{\mathrm{Bz}} \int g(\omega) \ldots \mathrm{d} \omega .
$$

${ }^{116}$ This probability depends on the details of the experimental set-up. We will later give the exact expression for this probability in the case of a direct geometry time-of-flight multi-detector spectrometer. 


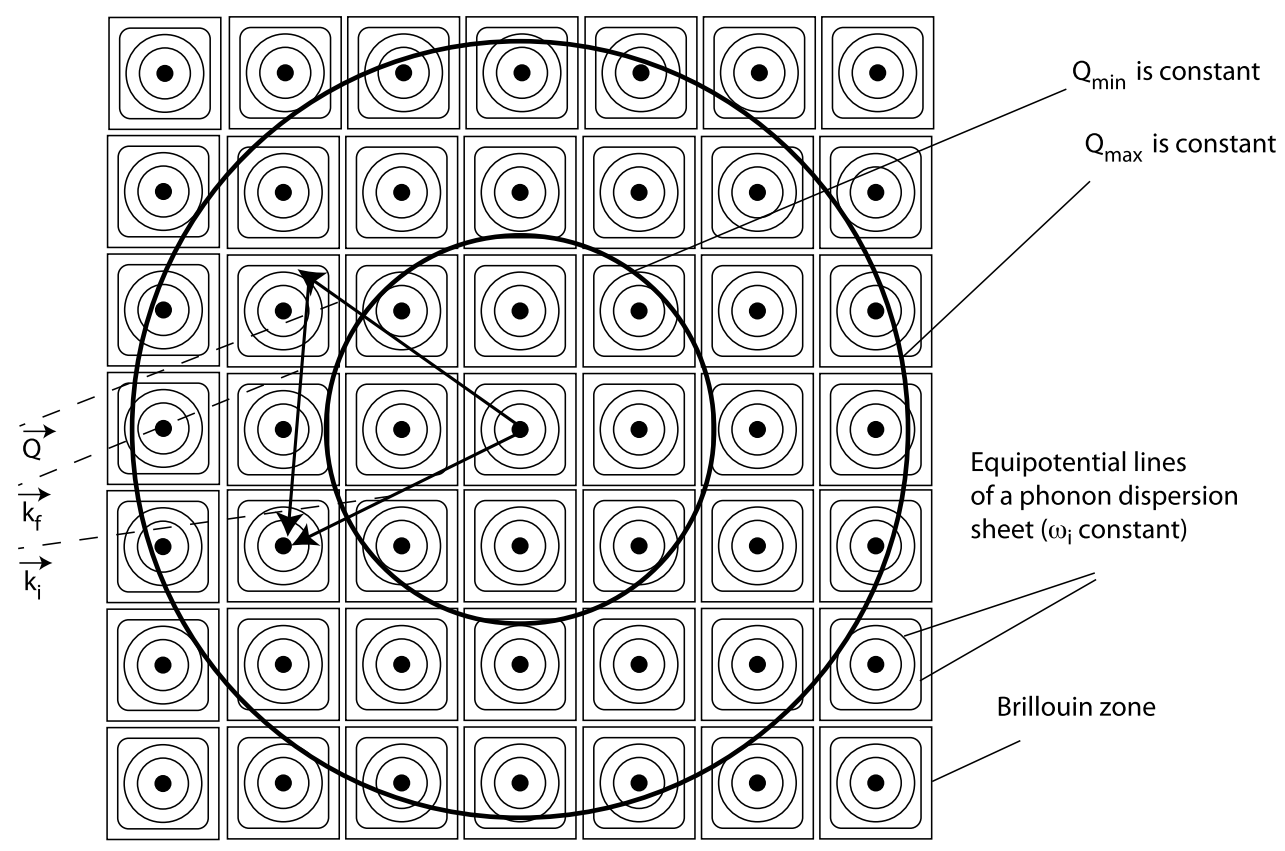

Fig. 57. Part of the reciprocal space for a cubic lattice. The phonon dispersion curves are indicated in a schematic manner by the equipotential lines. If there is only one detector, then $Q$ is fixed by the energy transfer $\hbar \omega$. In a crystal only one point $(\vec{Q}, \hbar \omega)$ is measured. In a powder $\hat{Q}$ scans all possible directions in the reference system of the sample. The measurement is in this way averaged over a sphere with radius $Q$. If the radius of this sphere is small then only a small part of the modes are selected. With a multi-detector instrument (see Fig. 58) the area between the spheres of radius $Q_{\max }$ and $Q_{\min }$ is covered. In the example shown here this area corresponds to about twenty zones in the plane and to about one hundred zones in the three-dimensional volume.

$$
\begin{aligned}
= & N \frac{k_{f}}{k_{i}} \frac{\sigma_{\mathrm{coh}}}{8 \pi m} \sum_{i=1}^{3} \int_{\mathrm{BZ}} \mathrm{d}^{3} q \frac{\delta\left(\omega-\omega_{i}(\vec{q})\right)}{\omega_{i}(\vec{q})}\left(1+n\left(\omega_{i}(\vec{q})\right)\right) \\
& \times \sum_{(\vec{q}-\vec{G}) \in V} \mathrm{e}^{-2 W(|\vec{q}-\vec{G}|)}\left|\vec{e}_{i}(\vec{q}) \cdot \hat{k}_{\vec{G}}\right|^{2}|\vec{q}-\vec{G}|^{2} p_{\hbar \omega}(|\vec{q}-\vec{G}|) .
\end{aligned}
$$

For a given mode $(i, \vec{q})$ the integration of the $\vec{Q}$ dependent terms is then contained in the function

$$
h_{i}(\vec{q}):=\sum_{(\vec{q}-\vec{G}) \in V} \mathrm{e}^{-2 W(|\vec{q}-\vec{G}|)}\left|\vec{e}_{i}(\vec{q}) \cdot \hat{q}_{\vec{G}}\right|^{2}|\vec{q}-\vec{G}|^{2} p_{\hbar \omega}(|\vec{q}-\vec{G}|) .
$$

In order to evaluate this expression further we neglect the $\vec{Q}$ dependence of the Debye-Waller factor. For sufficiently large volumes $V$ we can imagine that the terms appearing in $h_{i}(\vec{q})$ can be replaced by their mean values

$$
h_{i}(\vec{q})=\mathrm{e}^{-2 W(Q)} n \overline{\left|\vec{e}_{i}(\vec{q}) \cdot \hat{k}_{\vec{G}}\right|^{2}|\vec{q}-\vec{G}|^{2} p_{\hbar \omega}(|\vec{q}-\vec{G}|)}
$$

with $n$ the number of terms, which is identical to the number of Brillouin zones contained in the volume $V$

$$
n:=\frac{V}{V_{\mathrm{Bz}}}=V \frac{V_{\mathrm{unit}} \text { cell }}{(2 \pi)^{3}}
$$


In the mean values

$$
\overline{\left|\vec{e}_{i}(\vec{q}) \cdot \hat{q}_{\vec{G}}\right|^{2}|\vec{q}-\vec{G}|^{2} p_{\hbar \omega}(|\vec{q}-\vec{G}|)}
$$

the expressions

$$
\left|\vec{e}_{i}(\vec{q}) \cdot \hat{q}_{\vec{G}}\right|^{2}
$$

only depend on the direction and

$$
|\vec{q}-\vec{G}|^{2} p_{\hbar \omega}(|\vec{q}-\vec{G}|)
$$

only on the norm of $\vec{q}-\vec{G}$. Hence the two parts can be considered as uncorrelated

$$
\overline{\left|\vec{e}_{i}(\vec{q}) \cdot \hat{q}_{\vec{G}}\right|^{2}|\vec{q}-\vec{G}|^{2} p_{\hbar \omega}(|\vec{q}-\vec{G}|)}=\overline{\left|\vec{e}_{i}(\vec{q}) \cdot \hat{q}_{\vec{G}}\right|^{2}} \times \overline{|\vec{q}-\vec{G}|^{2} p_{\hbar \omega}(|\vec{q}-\vec{G}|)} .
$$

For a monatomic cubic system

$$
\overline{\left|\vec{e}_{i}(\vec{q}) \cdot \hat{q}_{\vec{G}}\right|^{2}}=\frac{1}{3}
$$

and in parallel

$$
\overline{|\vec{q}-\vec{G}|^{2} p_{\hbar \omega}(|\vec{q}-\vec{G}|)}=\frac{1}{V} \int_{Q_{\min }(\hbar \omega)}^{Q_{\max }(\hbar \omega)} p_{\hbar \omega}(Q) Q^{2} \mathrm{~d} Q .
$$

This leads to

$$
h_{i}(\vec{q})=\mathrm{e}^{-2 W(Q)} \frac{V_{\text {unit cell }}}{(2 \pi)^{3}} \frac{1}{3} \int_{Q_{\min }(\hbar \omega)}^{Q_{\max }(\hbar \omega)} p_{\hbar \omega}(Q) Q^{2} \mathrm{~d} Q,
$$

and finally to

$$
\begin{aligned}
\left(\frac{\mathrm{d} \sigma^{2}}{\mathrm{~d}(\hbar \omega)}\right)_{\mathrm{coh}-\mathrm{int}}^{1-\mathrm{ph}}= & N \frac{k_{f}}{k_{i}} \frac{\sigma_{\mathrm{coh}}}{8 \pi m} \mathrm{e}^{-2 W(Q)} \frac{1}{3 V_{\mathrm{Bz}}} \sum_{i=1}^{3} \int_{\mathrm{BZ}} \mathrm{d}^{3} q \frac{\delta\left(\omega-\omega_{i}(\vec{q})\right)}{\omega_{i}(\vec{q})}\left(1+n\left(\omega_{i}(\vec{q})\right)\right) \\
& \times \int_{Q_{\min }(\hbar \omega)}^{Q_{\max }(\hbar \omega)} p_{\hbar \omega}(Q) Q^{2} \mathrm{~d} Q \\
= & N \frac{k_{f}}{k_{i}} \frac{\sigma_{\mathrm{coh}}}{8 \pi m} \mathrm{e}^{-2 W(Q)} \frac{g(\omega)}{\omega}(1+n(\omega)) \int_{Q_{\min }(\hbar \omega)}^{Q_{\max }(\hbar \omega)} p_{\hbar \omega}(Q) Q^{2} \mathrm{~d} Q .
\end{aligned}
$$

This result is identical to the expression found when integrating (9.279) for the incoherent scattering. ${ }^{117}$ Hence we can postulate the incoherent approximation as [42]

$$
\left(\frac{\mathrm{d} \sigma^{2}}{\mathrm{~d}(\hbar \omega)}\right)_{\mathrm{coh}-\mathrm{int}}^{1-\mathrm{ph}} \approx\left(\frac{\mathrm{d} \sigma^{2}}{\mathrm{~d}(\hbar \omega)}\right)_{\mathrm{inc}-\mathrm{int}}^{1-\mathrm{ph}} .
$$

${ }^{117}$ For the incoherent scattering the averaging over $Q$ is trivial if we, as done here, neglect the $Q$ dependence of the Debye-Waller factors. In a more realistic calculation, this dependence is taken into account (see Section 10.6). 
The expression to the right refers to a hypothetical incoherent scatterer. Under this approximation the formalism that we have elaborated for incoherent scattering can be employed to obtain a good estimate for the density of states of a coherent scatterer, provided that the region sampled in $\vec{Q}$ is sufficiently large. The validity of this approximation can be tested by comparing values obtained for $g(\omega)$ when sampling over different regions in $\vec{Q}$ space. Another possibility to estimate the errors introduced by the incoherent approximation is computer simulations. Good estimates are already obtained with not very sophisticated dynamical models. In general, as both experiment [55] and simulation [67] show the introduced errors rarely exceed $20 \%$ and this even in the most unfavourable cases.

For time-of-flight spectrometers in direct geometry (see [52] and Fig. 58) with a fixed incident energy and a multi-detector ${ }^{118}$ covering the scattering angles from $\Theta_{\min }$ to $\Theta_{\max }$ the integrated cross section takes the form ${ }^{119}$

$$
\left(\frac{\mathrm{d} \sigma^{2}}{\mathrm{~d}(\hbar \omega)}\right)_{\Theta_{\min }}^{\Theta_{\max }}=\Delta \Phi \int_{\Theta_{\min }}^{\Theta_{\max }} \sin \Theta\left(\frac{\mathrm{d} \sigma^{2}}{\mathrm{~d}(\hbar \omega) \mathrm{d} \Omega}\right) \mathrm{d} \Theta=\Delta \Phi \int_{\Theta_{\min }}^{\Theta_{\max }} \frac{Q}{k_{i} k_{f}}\left(\frac{\mathrm{d} \sigma^{2}}{\mathrm{~d}(\hbar \omega) \mathrm{d} \Omega}\right) \mathrm{d} Q .
$$

The incoherent approximation translates in this case into

$$
\int_{\Theta_{\min }}^{\Theta_{\max }}\left(\frac{\mathrm{d}^{2} \sigma}{\mathrm{d}(\hbar \omega) \mathrm{d} \Omega}\right){ }_{\text {coh }} \sin \Theta \mathrm{d} \Theta \approx \int_{\Theta_{\min }}^{\Theta_{\max }}\left(\frac{\mathrm{d}^{2} \sigma}{\mathrm{d}(\hbar \omega) \mathrm{d} \Omega}\right)_{\text {inc }} \sin \Theta \mathrm{d} \Theta
$$

or in an equivalent manner

$$
\int_{Q_{\min }}^{Q_{\max }}\left(\frac{\mathrm{d}^{2} \sigma}{\mathrm{d}(\hbar \omega) \mathrm{d} \Omega}\right)_{\operatorname{coh}} Q \mathrm{~d} Q \approx \int_{Q_{\min }}^{Q_{\max }}\left(\frac{\mathrm{d}^{2} \sigma}{\mathrm{d}(\hbar \omega) \mathrm{d} \Omega}\right)_{\text {inc }} Q \mathrm{~d} Q
$$

Comparing (9.294) to (9.281) allows us to identify

$$
p_{\hbar \omega}(Q)=\Delta \Phi \frac{Q}{k_{i} k_{f}}
$$

which allows us to calculate

$$
\int_{Q_{\min }(\hbar \omega)}^{Q_{\max }(\hbar \omega)} p_{\hbar \omega}(Q) Q^{2} \mathrm{~d} Q=\frac{\Delta \Phi}{k_{i} k_{f}} \int_{Q_{\min }}^{Q_{\max }} Q^{3} \mathrm{~d} Q=\frac{\Delta \Phi}{4 k_{i} k_{f}}\left(Q_{\max }^{4}-Q_{\min }^{4}\right) .
$$

\footnotetext{
${ }^{118}$ We assume that the data are integrated over the $\Phi$ angle along the Debye-Scherrer cones, that is to say along trajectories with $|Q|=$ constant. If the part of the cone that is covered varies with $\Theta$ this has to be taken into account when normalising the data.

${ }^{119}$ With $\vec{Q}=\vec{k}_{i}-\vec{k}_{f}$ and $\Theta$ the scattering angle, it follows that

$$
Q=\sqrt{k_{i}^{2}+k_{f}^{2}+2 k_{i} k_{f} \cos \Theta},
$$

and hence

$$
\frac{\mathrm{d} Q}{\mathrm{~d} \Theta}=\frac{1}{2 Q} 2 k_{i} k_{f} \sin \Theta,
$$

as well as

$$
Q \mathrm{~d} Q=k_{i} k_{f} \sin \Theta \mathrm{d} \Theta .
$$




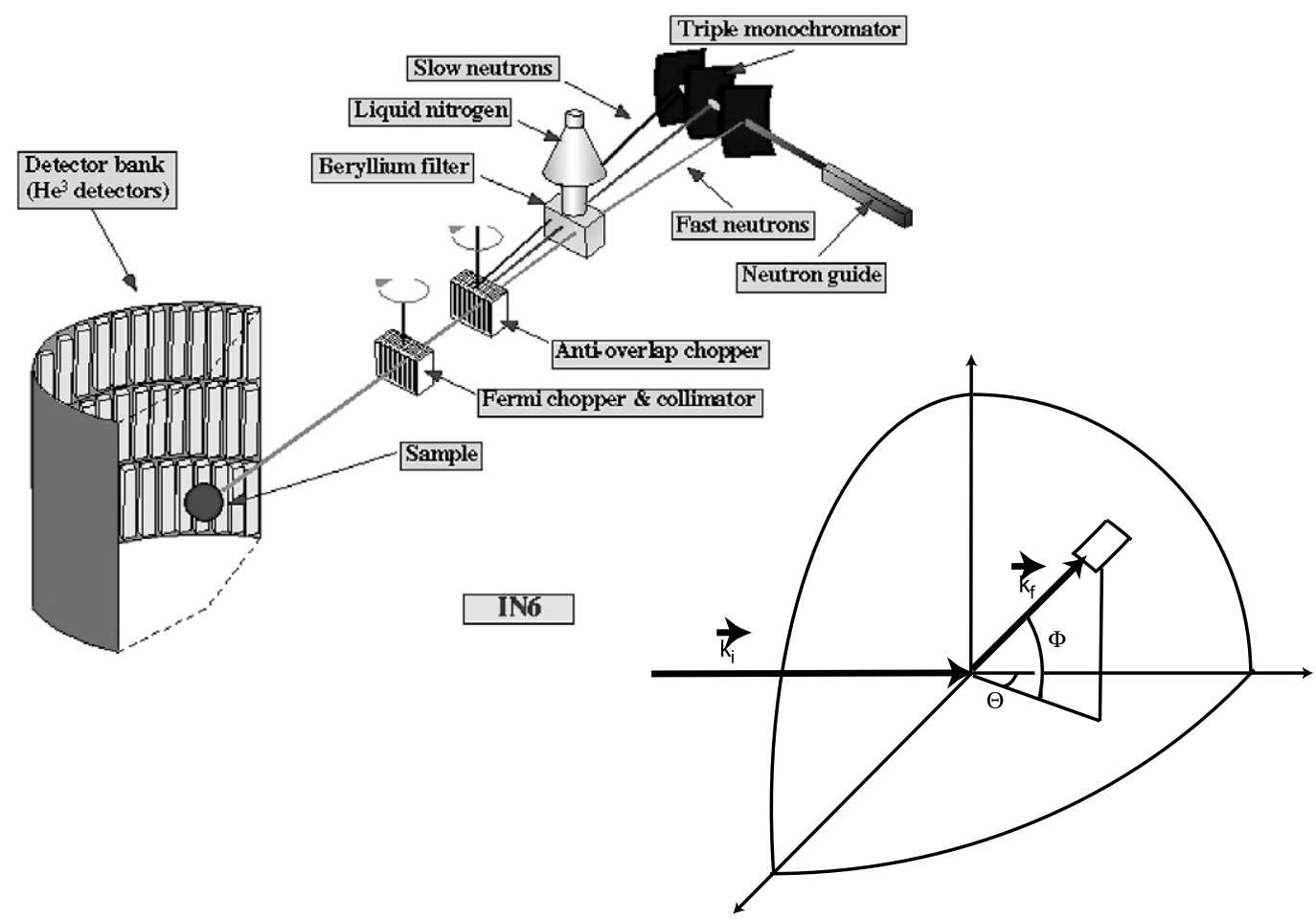

Fig. 58. The IN6 instrument at the ILL is a direct geometry time-of-flight instrument. The incident beam is monochromatic and pulsed, which means that $\vec{k}_{i}$ is constant. $k_{f}$ is determined by the time it takes the neutron to travel from the sample to the detector. It is equipped with 334 detectors arranged in three rows. The detection tubes are arranged in such a way that they maintain the same angle with respect to the scattering along the three rows. The vertical angular opening of the detectors $\Delta \Phi$ is defined by the height. Spectrometers of this type permit to sample reciprocal space for a given energy transfer as indicated in Fig. 57.

This gives, with the help of (9.289), the relation between the integrated cross section and the density of states

$$
\left(\frac{\mathrm{d} \sigma^{2}}{\mathrm{~d}(\hbar \omega)}\right)_{\Theta_{\min }}^{\Theta_{\max }}=\frac{N \Delta \Phi}{k_{i}^{2}} \frac{\sigma_{\mathrm{coh}}}{32 \pi m} \mathrm{e}^{-2 W(Q)}\left(Q_{\max }^{4}-Q_{\min }^{4}\right) \frac{g(\omega)}{\omega}(1+n(\omega)) .
$$

This expression is extremely valuable because it allows to extract the density of states along the trajectories defined by the scattering angles and does not require a preliminary extrapolation to constant $Q$ curves. This is particularly helpful when the data are collected with low incoming energy in up-scattering mode. Using a low incoming energy may be advantageous in terms of resolution and equally because the lower $Q$-values lead to reduced multi-phonon contributions.

\section{Inelastic neutron scattering beyond the one-phonon expressions}

The expressions that we have obtained for the scattering functions contain only one-phonon processes, either in absorption or in emission. Formally this is the consequence of retaining only terms linear in

$$
\aleph_{j^{\prime}, j}(\vec{Q}, t)=\left\langle\left(\vec{Q} \cdot \vec{u}_{j^{\prime}}\right)\left(\vec{Q} \cdot \vec{u}_{j}(t)\right)\right\rangle
$$


when expanding the expression

$$
\exp \left(\aleph_{j^{\prime}, j}(\vec{Q}, t)\right)=\exp \left(\left\langle\left(\vec{Q} \cdot \vec{u}_{j^{\prime}}\right)\left(\vec{Q} \cdot \vec{u}_{j}(t)\right)\right\rangle\right) .
$$

We had motivated this decision with the argument that the ionic displacements are small in the regime of the harmonic approximation. If we had been precise we would have added that small means small with respect to the inverse of the norm of the $\vec{Q}$ vector.

The one-phonon expressions are, therefore, valid provided that (i) the temperature is sufficiently low, (ii) that the system is far from points of instability in the phase diagram and that (iii) the momentum transfer $|\vec{Q}|$ is not too large. When the last condition is violated we should, independently of the validity of the first two conditions, either take higher order contributions into account or, even better, calculate the scattering function directly without phonon expansion. We insist on the fact that this inclusion of higher order effects will be necessary even if the harmonic approximation remains valid. Multi-phonon contributions exist both for harmonic and anharmonic systems.

\subsection{The integral version of the scattering function}

In order to judge the complexity of a calculation without phonon expansion we remind ourselves that in the harmonic approximation

$$
\begin{aligned}
& S_{\kappa, \kappa^{\prime}}(\vec{Q}, \omega) \\
& \quad=\frac{1}{2 \pi N \hbar} \sum_{j \in\left\{j_{\kappa}\right\}, j^{\prime} \in\left\{j_{\kappa^{\prime}}\right\}} \int_{-\infty}^{\infty} \mathrm{d} t \mathrm{e}^{-\mathrm{i} \omega t} \mathrm{e}^{-\mathrm{i} \vec{Q} \cdot\left(\vec{R}_{j^{\prime}}^{0}-\vec{R}_{j}^{0}\right)} \mathrm{e}^{-W_{j^{\prime}}(\vec{Q})} \mathrm{e}^{-W_{j}(\vec{Q})} \mathrm{e}^{\aleph_{j^{\prime}, j}(\vec{Q}, t)}
\end{aligned}
$$

with

$$
2 W_{j}(\vec{Q})=\left\langle\left(\vec{Q} \cdot \vec{u}_{j}\right)^{2}\right\rangle=\sum_{\alpha=x, y, z} \sum_{j=1}^{3 N} \frac{\left|Q_{\alpha} \cdot e_{i}(\alpha, j)\right|^{2} \hbar}{2 m_{j} \omega_{i}} \operatorname{coth}\left(\frac{1}{2} \frac{\hbar \omega}{k_{\mathrm{B}} T}\right),
$$

and

$$
\begin{aligned}
\aleph_{j^{\prime}, j}(\vec{Q}, t)= & \left\langle\left(\vec{Q} \cdot \vec{u}_{j^{\prime}}\right)\left(\vec{Q} \cdot \vec{u}_{j}(t)\right)\right\rangle \\
= & \frac{\hbar}{2} \sum_{\alpha, \beta} \frac{Q_{\alpha} Q_{\beta}}{\sqrt{m_{j} m_{j^{\prime}}}} \sum_{i, i^{\prime}} \sqrt{\frac{1}{\omega_{i} \omega_{i^{\prime}}}} \times \delta_{i, i^{\prime}} \\
& \times\left\{\left[e_{i}\left(\alpha, j^{\prime}\right) e_{i^{\prime}}^{*}(\beta, j)\left\langle\mathbf{a}_{i} \mathbf{a}_{i^{\prime}}^{+}\right\rangle \exp \left(\mathrm{i} \omega_{i^{\prime}} t\right)\right]+\left[e_{i}^{*}\left(\alpha, j^{\prime}\right) e_{i^{\prime}}(\beta, j)\left\langle\mathbf{a}_{i}^{+} \mathbf{a}_{i^{\prime}}\right\rangle \exp \left(-\mathrm{i} \omega_{i^{\prime}} t\right)\right]\right\} \\
= & \frac{\hbar}{2} \sum_{i}\left\{\left[\frac{A_{i}\left(\vec{Q} ; j^{\prime}, j\right)}{\omega_{i}}\left(1+n\left(\omega_{i}\right)\right) \exp \left(\mathrm{i} \omega_{i} t\right)\right]+\left[\frac{A_{i}\left(\vec{Q} ; j, j^{\prime}\right)}{\omega_{i}} n\left(\omega_{i}\right) \exp \left(-\mathrm{i} \omega_{i} t\right)\right]\right\},
\end{aligned}
$$

as well as

$$
A_{i}\left(\vec{Q} ; j^{\prime}, j\right)=\frac{\left(\vec{Q} \cdot \vec{e}_{i}\left(j^{\prime}\right)\right) \cdot\left(\vec{Q} \cdot \vec{e}_{i}(j)\right)^{*}}{\sqrt{m_{j^{\prime}} m_{j}}} .
$$

Hence it is possible to calculate $S_{\kappa, \kappa^{\prime}}(\vec{Q}, \omega)$ numerically provided that the dynamics of the system is known, i.e. provided that it is possible to determine the frequencies and the eigenvectors of all the harmonic modes, for example from ab-initio calculations or from molecular dynamics simulations. 


\subsection{The isotropic harmonic oscillator}

An analytical solution for the scattering function can be found for the simple isotropic harmonic oscillator. ${ }^{120}$ We are going to derive this function motivated by the pedagogical aspect of the exercise. Expression (10.2) is our starting point. When dealing with only one oscillator of frequency $\omega_{0}$, which in addition is assumed to be isotropic, the following expression is found for the Debye-Waller factor

$$
2 W(\vec{Q})=\left\langle(\vec{Q} \cdot \vec{u})^{2}\right\rangle=\frac{Q^{2} \hbar}{2 m \omega_{0}} \operatorname{coth}\left(\frac{1}{2} \frac{\hbar \omega_{0}}{k_{\mathrm{B}} T}\right)
$$

and for the expectation value

$$
\begin{aligned}
\aleph(\vec{Q}, t) & =\langle(\vec{Q} \cdot \vec{u})(\vec{Q} \cdot \vec{u}(t))\rangle \\
& =\frac{Q^{2} \hbar}{2 m \omega_{0}}\left\{\left[\left(1+n\left(\omega_{0}\right)\right) \exp \left(\mathrm{i} \omega_{0} t\right)\right]+\left[n\left(\omega_{0}\right) \exp \left(-\mathrm{i} \omega_{0} t\right)\right]\right\} \\
& =\frac{Q^{2} \hbar}{2 m \omega_{0}} \frac{\cosh \left(\omega_{0}\left(\mathrm{i} t+\hbar /\left(2 k_{\mathrm{B}} T\right)\right)\right)}{\sinh \left(\hbar \omega_{0} /\left(k_{\mathrm{B}} T\right)\right)} .
\end{aligned}
$$

The energy and temperature scales are fixed by $\hbar \omega_{0}$. The amplitude of the Debye-Waller function as well as the expectation value is governed by $Q^{2} \hbar / 2 m \omega_{0}$. For a harmonic oscillator in one dimension the fluctuation in momentum is calculated as

$$
\Delta p=\sqrt{\left\langle n\left|\mathbf{p}^{2}\right| n\right\rangle}=\sqrt{\frac{1}{2} \hbar m \omega_{0}}
$$

for the ground state. Thus,

$$
\frac{Q^{2} \hbar}{2 m \omega_{0}}=\frac{1}{4} \frac{(Q \hbar)^{2}}{(1 / 2) \hbar m \omega_{0}}=\left(\frac{1}{2} \frac{Q \hbar}{\Delta p}\right)^{2} .
$$

Hence the amplitude is governed by the ratio between the (i) the momentum transfer of the neutron and (ii) the momentum fluctuations in the ground state of the harmonic oscillator. This expression could also have been written in terms of energy ratios

$$
\frac{Q^{2} \hbar}{2 m \omega_{0}}=\frac{\hbar^{2} Q^{2} /(2 m)}{\hbar \omega_{0}}=\frac{E_{r}}{\hbar \omega_{0}}
$$

Here

$$
E_{r}(Q)=\frac{\hbar^{2} Q^{2}}{2 m}
$$

is again the well known expression for the recoil energy of a particle with mass $m$.

The ratio $E_{r}(Q) / \hbar \omega_{0}$ can be considered as the principle parameter, that, along with the temperature, governs the dynamic response of a harmonic oscillator.

${ }^{120}$ Analytic solutions for harmonic oscillators are often found in physics. 
If we postulate

$$
\begin{aligned}
& y:=\frac{Q^{2} \hbar}{2 m \omega_{0}} \frac{1}{\sinh \left(\hbar \omega_{0} /\left(k_{\mathrm{B}} T\right)\right)} \\
& z(t):=\omega_{0}\left(\mathrm{i} t+\frac{\hbar}{2 k_{\mathrm{B}} T}\right)
\end{aligned}
$$

then

$$
\langle(\vec{Q} \cdot \vec{u})(\vec{Q} \cdot \vec{u}(t))\rangle=y \cosh (z(t))
$$

and thus

$$
\begin{aligned}
S(\vec{Q}, \omega) & =\frac{1}{2 \pi \hbar} \mathrm{e}^{-2 W(\vec{Q})} \int_{-\infty}^{\infty} \mathrm{d} t \mathrm{e}^{\langle(\vec{Q} \cdot \vec{u})(\vec{Q} \cdot \vec{u}(t))\rangle} \\
& =\frac{1}{2 \pi \hbar} \exp (-2 W(\vec{Q})) \int_{-\infty}^{\infty} \mathrm{d} t \exp (y \cosh (z(t)))
\end{aligned}
$$

At this point the identity

$$
\exp (y \cosh (z(t)))=\sum_{n=-\infty}^{n=\infty} \exp (n z(t)) I_{n}(y)
$$

is used with $I_{n}=I_{-n}$ the Bessel functions of the first kind ${ }^{121}$ (see Fig. 59). Executing the integral with the help of this expression we find

$$
\begin{aligned}
\int_{-\infty}^{\infty} \mathrm{d} t \exp (y \cosh (z(t))) & =\sum_{n=-\infty}^{n=\infty} I_{n}(y) \int_{-\infty}^{\infty} \mathrm{d} t \exp (n z(t)) \\
& =\sum_{n=-\infty}^{n=\infty} I_{n}(y) \exp \left(\frac{\hbar \omega_{0}}{2 k_{\mathrm{B}} T}\right) \int_{-\infty}^{\infty} \mathrm{d} t \exp \left(\mathrm{i} n \omega_{0} t\right) \\
& =\sum_{n=-\infty}^{n=\infty} I_{n}(y) \exp \left(\frac{\hbar \omega}{2 k_{\mathrm{B}} T}\right)(2 \pi \hbar) \delta\left(\hbar \omega-n \hbar \omega_{0}\right) .
\end{aligned}
$$

${ }^{121}$ We have already met the spherical Bessel functions in Section 3.3. Bessel functions are the solutions to the differential equation

$$
x^{2} \frac{\mathrm{d}^{2} y}{\mathrm{~d} x^{2}}+x \frac{\mathrm{d} y}{\mathrm{~d} x}+\left(x^{2}-n^{2}\right) y=0 .
$$

The Bessel functions of the first kind are the solutions defined at $x=0$. They are calculated as follows

$$
I_{n}(x)=\left(\frac{x}{2}\right)^{n} \sum_{p=0}^{\infty} \frac{(-1)^{p}}{2^{2 p} p !(n+p) !} x^{2 p}
$$

The graphical representation of the Bessel functions resembles the sine and cosine functions, the amplitude of which decreases as $1 / \sqrt{x}$. 


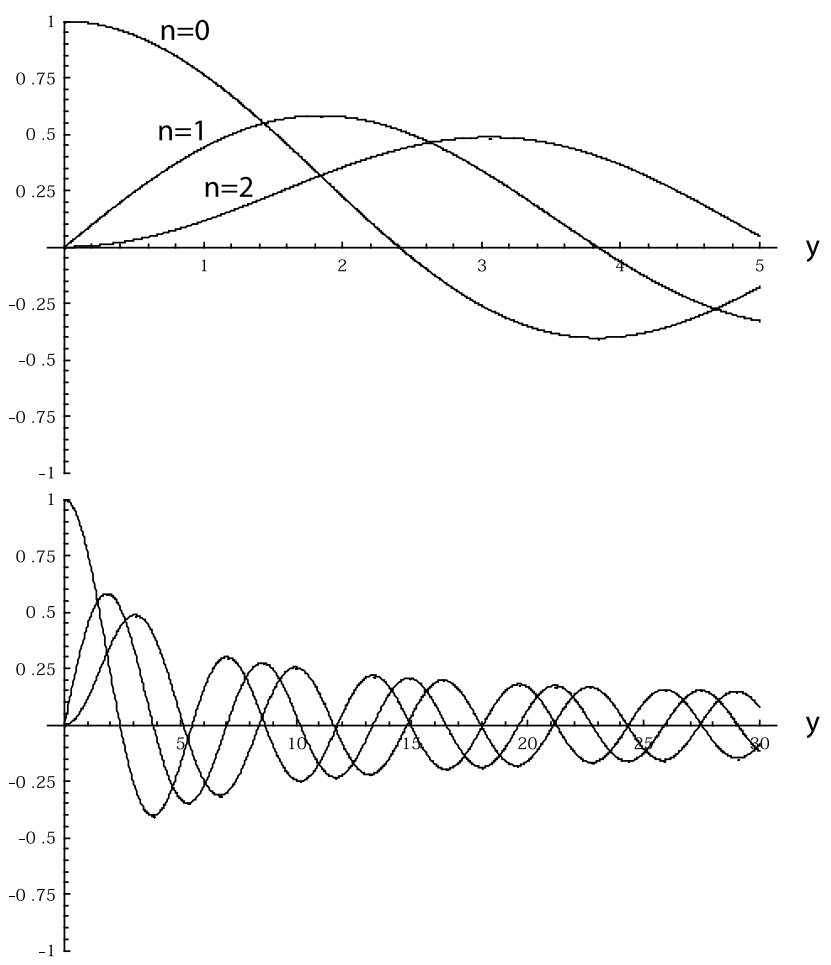

Fig. 59. Bessel functions of the first kind $I_{n}(y)$ for $n=0,1,2$.

This leads us to the scattering function

$$
S(\vec{Q}, \omega)=\exp (-2 W(\vec{Q})) \cdot \exp \left(\frac{\hbar \omega}{2 k_{\mathrm{B}} T}\right) \sum_{n=-\infty}^{n=\infty} I_{n}(y) \delta\left(\hbar \omega-n \hbar \omega_{0}\right) .
$$

Hence the neutron can gain or loose an integer number of energy units $\hbar \omega_{0}$, which is the expected result. If we only keep the $n=0$ term we get the expression for elastic scattering of an isotropic harmonic oscillator. The terms $n \pm 1$ correspond to an increase or a decrease in the level scheme by a single step. The other terms involve scattering processes corresponding to transitions over several levels. We would like to emphasise that we are not dealing with a multiple scattering process in the sense of Section 11.5. The simultaneous creation or annihilation of several vibrations is part of a single scattering process. It does neither depend on the size of the sample nor on the mean free path of the neutrons. It is also not related to anharmonics. With small modifications (see [39]) the result found for the isotropic harmonic oscillator is directly applicable to a system of several Einstein oscillators, i.e. of several decoupled oscillators.

Given that (see Fig. 59)

$$
I_{n}(y) \rightarrow \frac{1}{n !}\left(\frac{y}{2}\right)^{n} \quad \text { for } y \rightarrow 0 \text { and } n>0
$$

the first term in (10.20) dominates for small $Q$ values. The expansion of the scattering function in terms of "phonon" processes is then indicated. 


\subsection{The short time period or impulse approximation}

At the other end of the spectrum, i.e. for $Q \rightarrow \infty$ and thus for $y \rightarrow \infty$, all the $I_{n}(y)$ become comparable (see Fig. 59). The sum in expression (10.20) can therefore no longer be truncated. To identify the mechanism at work at large $Q$ we need another approach, that we will briefly elaborate here.

The scattered waves interfere and their respective phases depend on the relative positions of the atoms, which are a function of time. The time $t_{\text {probe }}$ it typically takes the incident wave front to pass from one atom to another is given by the phase velocity

$$
v_{p}=\frac{\hbar \omega}{\hbar k}=\frac{\hbar k}{2 m}=\frac{1}{2} v_{g}
$$

For example, thermal neutrons $\left(v_{g} \approx 2 \cdot 10^{3} \mathrm{~m} / \mathrm{s}\right)$ cover inter-atomic distances (a few $\AA$ ) in a few tenths of a picosecond. The inter-atomic travel time of the phase front $t_{\text {probe }}$ is then very close to the characteristic phonon time $t_{\mathrm{ph}}$ with frequencies in the $\mathrm{THz}$ range. In other words, the inter-atomic journey of the neutron wave front is well synchronised with the oscillation of the atoms. This is another way of explaining why the observation of phonons is so easy with neutrons. If the incident neutron energy increases $t_{\text {probe }}$ gets shorter and shorter. In order to observe complete oscillation cycles it is necessary to work with a very well-defined frequency of the incident and scattered waves. The observation of interference effects requires a higher and higher relative energy resolution. ${ }^{122}$ In reality it is the opposite trend that the instruments provide. The absolute resolution decreases with the energy of the neutrons.

The fact that the multi-excitation terms are all important for $Q \rightarrow \infty$ tells us that the spectral response is going to shift towards higher energies. The high $Q$ transfers as well as the energy $\hbar \omega$ require the use of high energy neutrons. For a resolution of a few percent we can assume that the observed interferences all come from the short time end of the correlation function $G(\vec{r}, t)$. As a consequence we are going to expand the function

$$
\langle(\vec{Q} \cdot \vec{u})(\vec{Q} \cdot \vec{u}(t))\rangle-\left\langle(\vec{Q} \cdot \vec{u})^{2}\right\rangle=\frac{Q^{2} \hbar}{2 m \omega_{0}}\left\{\frac{\cosh \left(\omega_{0}\left(\mathrm{i} t+\hbar /\left(2 k_{\mathrm{B}} T\right)\right)\right.}{\sinh \left(\hbar \omega_{0} /\left(k_{\mathrm{B}} T\right)\right)}-\operatorname{coth}\left(\frac{1}{2} \frac{\hbar \omega}{k_{\mathrm{B}} T}\right)\right\}
$$

that we had found for the isotropic oscillator in time. If we limit ourselves all together to $G(\vec{r}, t=0)$ we are working in the static approximation. This necessarily gives us a purely elastic response. This response is vanishing in the case of a harmonic oscillator.

Keeping terms up to second order we include both the real and the imaginary part of the function (10.22)

$$
\left\langle(\vec{Q} \cdot \vec{u})(\vec{Q} \cdot \vec{u}(t))-(\vec{Q} \cdot \vec{u})^{2}\right\rangle=\frac{Q^{2} \hbar}{2 m}\left[\mathrm{i} t-\frac{t^{2} \omega_{0}}{2} \operatorname{coth}\left(\frac{1}{2} \frac{\hbar \omega_{0}}{k_{\mathrm{B}} T}\right)\right] .
$$

We are confronted with a Gaussian function shifted along the imaginary axis in the complex plane. The calculation of the correlation function (10.15) involves a Fourier transform of (10.23). With

$$
\int_{-\infty}^{\infty} \mathrm{d} t \exp \left(-a t^{2}+b t\right)=\sqrt{\frac{\pi}{a}} \exp \left(b^{2} / 4 a\right)
$$

\footnotetext{
${ }^{122}$ The increase in resolution is important here. For X-rays the inter-atom trajectory is much shorter $\left(t_{\text {probe }} \approx \AA / c=0.3 \cdot 10^{-18} \mathrm{~s}\right)$. Hence it can be assumed (and this argument was made before third generation synchrotrons came into operation [62]) that X-ray scattering gives only access to instantaneous correlations $(G(\vec{r}, t=0))$. This is obviously no longer true with the introduction of high resolution $\mathrm{X}$-ray spectrometers. The energy of the incident wave is so well defined $\left(E_{i} / \Delta E \approx 10^{7}\right)$, that the interferences can be observed even so the phase shifts are small on the scale of $t_{\text {probe }}$.
} 
the scattering function becomes

$$
S(\vec{Q}, \omega)=\frac{1}{\sqrt{2 \pi \Delta^{2}}} \exp \left(-\frac{\left(\hbar \omega-E_{r}\right)^{2}}{2 \Delta^{2}}\right) .
$$

This is a Gaussian function centred on the recoil energy

$$
E_{r}=\frac{\hbar^{2} Q^{2}}{2 m}
$$

with a width proportional to the Debye-Waller factor

$$
\begin{aligned}
\Delta^{2} & :=\frac{Q^{2} \hbar^{2}}{2 m}\left(\hbar \omega_{0}\right) \operatorname{coth} \frac{\hbar \omega_{0}}{2 k_{\mathrm{B}} T} \\
& =E_{r}\left(\hbar \omega_{0}\right) \operatorname{coth} \frac{\hbar \omega_{0}}{2 k_{\mathrm{B}} T} \\
& =2\left(\hbar \omega_{0}\right)^{2} W(\vec{Q}) .
\end{aligned}
$$

Before interpreting the result (10.23) we will derive it in an alternative way, that is without using the analytical expression (10.22). For very short times the change in position of an atom is given by its instantaneous velocity $\vec{v}_{j}$. We treat this fact in the language of quantum mechanics by writing the Heisenberg operator for the position as

$$
\overrightarrow{\mathbf{R}}_{j}(t)=\overrightarrow{\mathbf{R}}_{j}(t=0)+\frac{\mathbf{P}_{j}(t)}{m_{j}} t .
$$

This approximation is called the impulse approximation. In order to calculate the scattering function (9.74) the correlation function

$$
\begin{aligned}
& \left\langle\exp \left(-\mathrm{i} \vec{Q} \cdot \overrightarrow{\mathbf{R}}_{j^{\prime}}(t=0)\right) \cdot \exp \left(\mathrm{i} \vec{Q} \cdot \overrightarrow{\mathbf{R}}_{j}(t)\right)\right\rangle \\
& \quad=\left\langle\exp \left(-\mathrm{i} \vec{Q} \cdot \overrightarrow{\mathbf{R}}_{j^{\prime}}\right) \cdot \exp \left(\mathrm{i} \vec{Q} \cdot \overrightarrow{\mathbf{R}}_{j}+\mathrm{i} \vec{Q} \cdot \frac{\mathbf{P}_{j}(t)}{m_{j}} t\right)\right\rangle
\end{aligned}
$$

should be evaluated. The character of the operators are emphasised by the writing in bold. Knowing that

$$
\left[\mathbf{R}_{\alpha, j}, \mathbf{P}_{\beta, j^{\prime}}\right]=\mathrm{i} \hbar \delta_{\alpha, \beta} \delta_{j, j^{\prime}}
$$

the identity (9.78) is used to evaluate this expression

$$
\begin{aligned}
& \left\langle\exp \left(-\mathrm{i} \vec{Q} \cdot \overrightarrow{\mathbf{R}}_{j^{\prime}}\right) \cdot \exp \left(\mathrm{i} \vec{Q} \cdot \overrightarrow{\mathbf{R}}_{j}+\mathrm{i} \vec{Q} \cdot \frac{\mathbf{P}_{j}(t)}{m_{j}} t\right)\right\rangle \\
& =\left\langle\exp \left(-\mathrm{i} \vec{Q} \cdot \overrightarrow{\mathbf{R}}_{j^{\prime}}+\mathrm{i} \vec{Q} \cdot \overrightarrow{\mathbf{R}}_{j}+\mathrm{i} \vec{Q} \cdot \frac{\mathbf{P}_{j}(t)}{m_{j}} t+\frac{1}{2}\left[\vec{Q} \cdot \overrightarrow{\mathbf{R}}_{j^{\prime}},\left(\vec{Q} \cdot \overrightarrow{\mathbf{R}}_{j}+\vec{Q} \cdot \frac{\mathbf{P}_{j}(t)}{m_{j}} t\right)\right]\right)\right\rangle \\
& =\exp \left(\mathrm{i} \frac{\hbar Q^{2} t}{2 m_{j}} \delta_{j, j^{\prime}}\right)\left\langle\exp \left(\mathrm{i} \vec{Q} \cdot\left(\overrightarrow{\mathbf{R}}_{j}-\overrightarrow{\mathbf{R}}_{j^{\prime}}\right)+\mathrm{i} \vec{Q} \cdot \frac{\mathbf{P}_{j}(t)}{m_{j}} t\right)\right\rangle .
\end{aligned}
$$

At this point we only keep the terms $j=j^{\prime}$, which means that we consider the response as completely incoherent. In other words, we assume that the resolution of our instrument is in sufficient to observe interference effects at 
these high frequencies having their origin in distinct atoms. The scattering function takes the form

$$
S(\vec{q}, \omega)=\frac{1}{2 \pi \hbar N} \sum_{j} \int_{-\infty}^{\infty} \mathrm{d} t \exp \left(\mathrm{i} t\left(-\omega+\frac{\hbar Q^{2}}{2 m_{j}}\right)\right)\left\langle\exp \left(\mathrm{i} \vec{Q} \cdot \frac{\mathbf{P}_{j}(t)}{m_{j}} t\right)\right\rangle .
$$

For atomes confined in harmonic wells the momentum distribution is given by a Gaussian

$$
\left\langle\exp \left(\mathrm{i} \vec{Q} \cdot \frac{\mathbf{P}_{j}(t)}{m_{j}} t\right)\right\rangle=\exp \left(-t^{2} \frac{\left\langle\left(\vec{Q} \cdot \overrightarrow{\mathbf{P}}_{j}\right)^{2}\right\rangle}{2 m_{j}}\right)
$$

This expression can be verified using the Bloch identity (9.95), which can be applied safely since $\overrightarrow{\mathbf{P}}(\alpha, j)$, in the same way as $\overrightarrow{\mathbf{u}}(\alpha, j)$, is expressed as a linear combination of creation and annihilation operators. We find the same results as before (10.25) but with the width expressed as

$$
\Delta^{2}=\frac{1}{m_{i}}\left\langle\left(\vec{Q} \cdot \overrightarrow{\mathbf{P}}_{j}\right)^{2}\right\rangle
$$

We, therefore, can conclude that the width of the signal is related to the velocity distribution (or momentum distribution) of the particles. Using creation and annihilation operator algebra it is not too difficult to show that (10.34) corresponds indeed to (10.27).

This result can be generalised. If for short times we describe the particles via free particle states with a welldefined momentum $\vec{P}_{j}$, then

$$
\overrightarrow{\mathbf{P}}_{j}\left|\vec{P}_{j}\right\rangle=\vec{P}_{j}\left|\vec{P}_{j}\right\rangle
$$

and, therefore,

$$
\left\langle\exp \left(\mathrm{i} \vec{Q} \cdot \frac{\mathbf{P}_{j}(t)}{m_{j}} t\right)\right\rangle=\sum_{\vec{P}_{j}} n\left(\vec{P}_{j}\right) \exp \left(\mathrm{i} \vec{Q} \cdot \frac{P_{j}}{m_{j}} t\right)
$$

with $n\left(\vec{P}_{j}\right)$ the probability of finding the ion $j$ in the state $\left|\vec{P}_{j}\right\rangle$. The corresponding scattering function (10.33) is calculated as

$$
S(\vec{Q}, \omega)=\sum_{j} \sum_{\vec{p}_{j}} n\left(\vec{p}_{j}\right) \delta\left(\hbar \omega-E_{r}(Q)-\frac{\hbar}{m_{j}}\left(\vec{Q} \cdot \vec{p}_{j}\right)\right) .
$$

Hence scattering experiments at very high energies measure the momentum distribution of the particles.

In Fig. 60 the $S(Q, \omega)$ for a powder of solid deuterium is shown. As we have already mentioned solid deuterium is a quantum solid. The scattering from deuterium is unlike that of hydrogen mainly coherent. The quantum character of this solid implies that there are large fluctuations in the positions despite the low temperature. The mean square displacement $\overline{u^{2}}$ is of the order of $0.25 \AA^{2}$ with the Debye-Waller function being $W(Q)=\frac{1}{6} \overline{u^{2}}$. For $\vec{Q}$ vectors larger than a few $\AA^{-1}$ important multi-phonon contributions should be observed. This is confirmed by the experiment. When $Q$ increases the spectral response shifts towards energies above the one-phonon threshold and its character becomes more and more incoherent. The ratio between the recoil energy and the energy threshold of the phonons (see (8.90)) is approaching one starting from $Q \approx 4 \AA^{-1}$. If we stay with coherent scatterers a more favourable ratio can only be obtained for helium. However even for $\vec{Q}$ values close to $8 \AA^{-1}$ one is still far from the region where the impulse approximation gives good results. In particular, the response does still not 

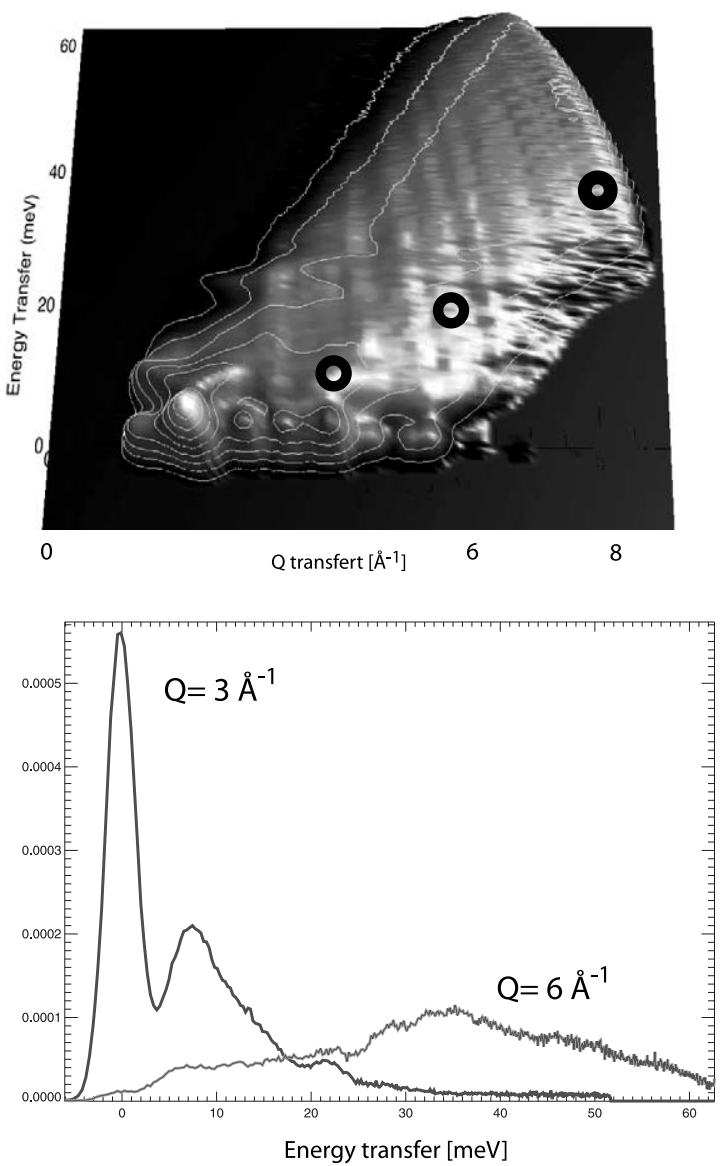

Fig. 60. Illustration of the passage from one phonon scattering to recoil scattering. As an example we use the quantum solid $D_{2}$. For experimental details see [27]. The $\vec{Q}$ and $\omega$ space accessible to the experiment, shown on the top figure, is much larger than the one presented in Fig. 33. This is the advantage of using a much shorter wave length $\left(1.1 \AA^{-1}\right.$ instead of $\left.2.2 \AA^{-1}\right)$. The increase in field of view is coupled to a decrease in resolution. The phonons in solid deuterium have energies that do not exceed $10 \mathrm{meV}$. The intensity observed above this value must then be attributed to (i) transitions between the rotation levels of the molecule $D_{2}$ as well as (ii) multi-phonon scattering. The multi-phonon scattering increases with the momentum transfer $Q$. For very large $Q$ values the inelastic intensity should, according to (10.24), be centred on the recoil energy, which is indicated by the black circles. Due to the contributions from rotational transitions the corresponding parabola is rather a lower limit to the observed scattering. In the lower figure cuts of $S(q, \omega)$ for $Q=3 \AA^{-1}$ and $Q=6 \AA^{-1}$ are shown. We observe that the coherent scattering is shifted from the region of the phonon density of states $(\hbar \omega<10 \mathrm{meV})$ to higher energies, where it step by step looses its internal structure and more and more resembles incoherent scattering. The elastic scattering is completely extinct at large $Q$. The width of the inelastic signal at $Q=6 \AA^{-1}$ is compatible with (10.27) if we use the Debye-Waller function determined experimentally $\left(W(Q) \approx \frac{1}{6} \cdot 0.25 Q[\AA]^{2}\right.$ [27]) and a mean $\hbar \omega$ situated between 5 and $10 \mathrm{meV}$. However the curve at $6 \AA^{-1}$ still does not really resemble a Gaussian. It, in particular, still has structure on an energy scale inferior to the recoil energy.

resemble a Gaussian. In this context it is interesting to have a more detailed look at the approximation (see [31]). The experiments really relevant for this approximation, whose purpose is to measure the momentum distribution, are conducted with very large incident energies at the spallation source ISIS.

\subsection{Multi-phonons in a single crystal}

As soon as the oscillators are coupled and the eigenvectors cease to be trivial the analytic expressions for the simple oscillator are a priori no longer applicable. At very large $\vec{Q}$ values we still may assume that the atoms 
behave as individual particles experiencing the scattering as an impact. We can then hope that the approximation of the scattering function with the recoil function (10.24) continues to give satisfactory results, provided that we proceed via a correct averaging over all the different atom types.

For intermediate $Q$ values we have little choice but to base our treatment on the expansion of the function

$$
\exp \left(\aleph_{j^{\prime}, j}(\vec{Q}, t)\right)=\exp \left(\left\langle\left(\vec{Q} \cdot \vec{u}_{j^{\prime}}\right)\left(\vec{Q} \cdot \vec{u}_{j}(t)\right)\right\rangle\right)
$$

including higher order terms. The second order terms will have the form

$$
\aleph_{j^{\prime}, j}(\vec{Q}, t)^{2}=\left\langle\left(\vec{Q} \cdot \vec{u}_{j^{\prime}}\right)\left(\vec{Q} \cdot \vec{u}_{j}(t)\right)\right\rangle\left\langle\left(\vec{Q} \cdot \vec{u}_{j^{\prime}}\right)\left(\vec{Q} \cdot \vec{u}_{j}(t)\right)\right\rangle .
$$

Expressing the position operators in terms of creation and annihilation operators (9.197) and performing the Fourier transform (9.200) we can show that the scattering function for a harmonic crystal has non-zero contributions only if the conditions

$$
\begin{aligned}
& \hbar \omega=\frac{\hbar^{2}}{2 m}\left(k_{i}^{2}-k_{f}^{2}\right)=\hbar\left( \pm \omega_{i}\left(\vec{q}_{1}\right) \pm \omega_{i^{\prime}}\left(\vec{q}_{2}\right)\right), \\
& \vec{Q}=\vec{k}_{i}-\vec{k}_{f}=\vec{G} \pm \vec{q}_{1} \pm \vec{q}_{2}
\end{aligned}
$$

are met. Hence the scattering involves either (i) the creation of two phonons or (ii) the creation of a phonon combined with the annihilation of a phonon or (iii) the annihilation of two phonons. For each process the energy has to be conserved. The crystal momentum has to be conserved up to a reciprocal lattice vector $\hbar \vec{G}$. Unlike the one-phonon scattering (see (9.208) and (9.209)) the scattering from several phonons is not limited to discrete values of $\hbar \omega_{i}(\vec{q})$. For a given scattering angle and a given energy difference it is in principle always possible to find combinations of $\left(\omega_{i}\left(\vec{q}_{1}\right), \omega_{i^{\prime}}\left(\vec{q}_{2}\right)\right)$ that satisfy $(10.39)$ and $(10.40)$. The number of possible combinations determines to a great deal the intensity of the two-phonon scattering for a given $(\vec{Q}, \omega)$ point. The continuity in the two phonon response complicates considerably its interpretation. For this reason the multi-phonon scattering has little experimental value. Often it is only an undesired background underlying the one-phonon peaks. This noise, which is equally temperature dependent, has to be properly subtracted in order to determine the one-phonon intensity correctly. This is no problem when the instrument resolution is good and the phonon peaks are well separated.

Finding expressions equivalent to (10.40) for the conservation of crystal momentum starting from the $\vec{u}_{j}(t)$ operators becomes very tedious when the number of $\aleph(Q, t)$ factors increases. As we have indicated in Section 9.12 an explicit calculation is also not really necessary. The conservation of momentum is a consequence of the periodicity of the Hamiltonian. It is a fundamental result of quantum mechanics, which stipulates that any symmetry operation $S$ of the system is coupled to a quantum mechanical operator $T_{s}$ that commutes with the Hamiltonian. For a free particle a displacement $\vec{r} \rightarrow \vec{r}+\vec{r}_{0}$ along any direction in space leaves the Hamiltonian invariant. Such a translation changes the phase of the wave function by the amount $\vec{q} \cdot \vec{r}_{0}$. It is, hence, described by the operator

$$
T_{\vec{r}_{0}}=\exp \left(\frac{\mathrm{i}}{\hbar} \vec{p} \cdot \vec{r}_{0}\right)
$$

$T_{\vec{r}_{0}}$ evidently commutes with $H=p^{2} / 2 m$. It, therefore, constitutes a constant of motion. In other words, if the particle starts out in an eigenstate to $T_{\vec{r}_{0}}$ it will stay in that state forever. In the case of several particles only interacting among themselves the translation of the system as a whole will still leave the Hamiltonian invariant. This translation is described by

$$
T_{\vec{r}_{0}}=\exp \left(\frac{\mathrm{i}}{\hbar} \sum_{i} \vec{p}_{i} \cdot \vec{r}_{0}\right) .
$$


The fact that $\left[T_{\vec{r}_{0}}, H\right]=0$ leads to the conservation of the total momentum. It naturally persists in a crystal. It is nevertheless not very useful in the context of scattering. What really interests us is the consequences of the translation by a lattice vector $\vec{R}_{0}$. Unlike arbitrary translations of the system, these particular translations are equivalent to a permutation of particles. They, therefore, create a link both between the displacements

$$
\vec{u}(\vec{R}) \rightarrow \vec{u}\left(\vec{R}-\vec{R}_{0}\right)
$$

and the momenta

$$
\vec{P}(\vec{R}) \rightarrow \vec{P}\left(\vec{R}-\vec{R}_{0}\right)
$$

of the particles. No such relation can be established for arbitrary translations. These relations are valid for all $\vec{R}$ that designate an equilibrium position of an atom. In order to exploit this extra symmetry it is necessary to identify the associated quantum mechanical operator. We are looking for a unitary operator

$$
T\left(\vec{R}_{0}\right)=\exp \left(\mathbf{i K}\left(\vec{R}_{0}\right)\right)
$$

that assures

$$
\begin{aligned}
& \exp \left(\mathrm{iK}\left(\vec{R}_{0}\right)\right) \vec{u}(\vec{R}) \exp \left(-\mathrm{i} \mathbf{K}\left(\vec{R}_{0}\right)\right)=\vec{u}\left(\vec{R}-\vec{R}_{0}\right), \\
& \exp \left(\mathbf{i K}\left(\vec{R}_{0}\right)\right) \vec{P}(\vec{R}) \exp \left(-\mathrm{i} \mathbf{K}\left(\vec{R}_{0}\right)\right)=\vec{P}\left(\vec{R}-\vec{R}_{0}\right) .
\end{aligned}
$$

For the operator $T\left(\vec{R}_{0}\right)$ to fulfil this requirement we choose

$$
\mathbf{K}\left(\vec{R}_{0}\right)\left|\left\{n_{i}(\vec{q})\right\}\right\rangle=\sum_{\vec{q}} \sum_{i} n_{i}(\vec{q})\left(\vec{q} \cdot \vec{R}_{0}\right)\left|\left\{n_{i}(\vec{q})\right\}\right\rangle .
$$

The reader interested in the prove of this assertion can consult [2]. The fact that we write $\mathbf{K}\left(\vec{R}_{0}\right)$ in the basis of phonon eigenstates does not imply that the crystal should be harmonic. $\mathbf{K}\left(\vec{R}_{0}\right)$ is an operator that extracts from a given quantum state that is represented in the basis of phonon eigenvectors the sum of the projections of the wave vector $\vec{q}$ onto $\vec{R}_{0}$. It is called the crystal momentum operator.

The translation operators $T\left(\vec{R}_{0}\right)$ all commute with each other. If the system at a given moment is in an eigenstate of the Hamiltonian, then this state will be an eigenstate of all the individual translation operators $T\left(\vec{R}_{0}\right)$ with the corresponding eigenvalues

$$
\exp \left(\mathrm{i} \sum_{\vec{q}} \sum_{i} n_{i}(\vec{q})\left(\vec{q} \cdot \vec{R}_{0}\right)\right) .
$$

The fact that the $T\left(\vec{R}_{0}\right)$ commute with the Hamiltonian implies that the system has to preserve these eigenvalues. If the occupation of a phonon level changes

$$
n_{i}(\vec{q}) \rightarrow n_{i}^{\prime}(\vec{q})
$$

e.g. due to anharmonic interactions, then

$$
\exp \left(\mathrm{i} \sum_{\vec{q}} \sum_{i} n_{i}(\vec{q})\left(\vec{q} \cdot \vec{R}_{0}\right)\right)=\exp \left(\mathrm{i} \sum_{\vec{q}} \sum_{i} n_{i}^{\prime}(\vec{q})\left(\vec{q} \cdot \vec{R}_{0}\right)\right) \quad \forall \vec{R}_{0} .
$$


In other words

$$
\exp \left(\mathrm{i}\left[\sum_{\vec{q}} \sum_{i} n_{i}(\vec{q}) \vec{q}-n_{i}^{\prime}(\vec{q}) \vec{q}\right] \cdot \vec{R}_{0}\right)=1 \quad \forall \vec{R}_{0}
$$

This is an expression that defines the reciprocal lattice vectors $\vec{G}$ via

$$
\exp \left(\mathrm{i} \vec{G} \cdot \vec{R}_{0}\right)=1 \quad \forall \vec{R}_{0}
$$

We, therefore, conclude

$$
\left[\sum_{\vec{q}} \sum_{i} n_{i}(\vec{q}) \vec{q}-n_{i}^{\prime}(\vec{q}) \vec{q}\right]=\vec{G}
$$

Hence symmetry requires that the crystal momentum is conserved within the system up to a reciprocal lattice vector.

We will now add a neutron (or any other particle that only interacts with the ions, e.g. a Bloch electron) to the system. The presence of the neutron does not perturb the symmetry of the lattice. When translated by $\vec{R}_{0}$ the neutron experiences the same interactions as before just with other ions. The neutron translation operator is given by $(10.41)$

$$
T_{\vec{R}_{0}}^{\mathrm{n}}=\exp \left(\frac{\mathrm{i}}{\hbar} \vec{p} \cdot \vec{R}_{0}\right) .
$$

The symmetry of the lattice implies that the product of $T_{\vec{R}_{0}}^{\mathrm{n}}$ and $T_{\vec{R}_{0}}^{\text {ions }}$ commutes with the Hamiltonian. If the initial state of the crystal is an eigenstate of the harmonic system and that if the neutron is found in the state $\vec{p}=\hbar \vec{k}_{i}$ then the combined system is in an eigenstate of

$$
T_{\vec{R}_{0}}^{\mathrm{n}} T_{\vec{R}_{0}}^{\text {ions }} \quad \forall \vec{R}_{0}
$$

with eigenvalues

$$
\exp \left(\mathrm{i}\left[\vec{k}_{i}+\sum_{\vec{q}} \sum_{i} n_{i}(\vec{q}) \vec{q}\right] \cdot \vec{R}_{0}\right)
$$

Due to the interactions between the neutron and the ions as well as anharmonic forces present in the crystal neither the state of the neutron nor the state of the crystal will be stationary. To the contrary, both of them will evolve in time. Using the same arguments that brought us to (10.49) we derive that between the quantum numbers of the initial and final states the relation following relation holds:

$$
\left[\sum_{\vec{q}} \sum_{i} n_{i}(\vec{q}) \vec{q}-n_{i}^{\prime}(\vec{q}) \vec{q}\right]+\vec{G}=\left(\vec{k}_{f}-\vec{k}_{i}\right) .
$$

The change in momentum of the neutron is thus compensation by a change in the crystal momentum. The change in the crystal momentum corresponds to the changes in the wave vectors $\vec{q}$ of the phonons up to a reciprocal lattice vector $\vec{G}$. This is a very general result. It is valid for harmonic as well as anharmonic crystals and stays valid for an arbitrary number of phonons exchanged. The one-phonon selection rules are just a specific case of the more general expression. 


\subsection{Iterative calculation of multi-phonon contribution for incoherent monatomic systems}

An correction of multi-phonon contributions becomes mandatory when working with powders. In this case the one-phonon response is already continuous as function of energy. It is, therefore, qualitatively indistinguishable from the multi-phonon response. Without a model or lattice dynamical calculation a correction for multi-phonons is difficult. Such a correction is nevertheless essential if we want to extract meaningful physical quantities from the measurements. A rigorous iterative procedure can only de developed for an incoherent monatomic system. ${ }^{123}$ This restriction may seem prohibitive. In practice it is, however, realised that due to the multiple "averaging" of the dispersion relations that is inherent to the calculation of multi-phonon contributions details do not really matter. The method, therefore, gives quite convincing results even for rather complex systems.

For an incoherent monatomic system the expectation value that determines the scattering function (10.2) to first order in the displacements has the form

$$
\begin{aligned}
\aleph_{j, j}(\vec{Q}, t) & =\left\langle\left(\vec{Q} \cdot \vec{u}_{j}\right)\left(\vec{Q} \cdot \vec{u}_{j}(t)\right)\right\rangle \\
& =\frac{\hbar}{2} \frac{Q^{2}}{3 N} \frac{1}{m} \sum_{i}\left\{\left[\frac{1}{\omega_{i}}\left(1+n\left(\omega_{i}\right)\right) \exp \left(\mathrm{i} \omega_{i} t\right)\right]+\left[\frac{1}{\omega_{i}} n\left(\omega_{i}\right) \exp \left(-\mathrm{i} \omega_{i} t\right)\right]\right\},
\end{aligned}
$$

where we have used the expressions (9.241) and (9.250). ${ }^{124}$ The last expression can only be applied to cubic or isotropic systems. The expression (10.52) can be reformulated using the density of states

$$
g(\omega):=\frac{1}{3 N} \sum_{i=1}^{3 N} \delta\left(\omega-\omega_{i}\right)
$$

Which leads to

$$
\begin{aligned}
& \left\langle\left(\vec{Q} \cdot \vec{u}_{j}\right)\left(\vec{Q} \cdot \vec{u}_{j}(t)\right)\right\rangle \\
& \quad=\frac{\hbar}{2} \frac{Q^{2}}{m}\left\{\int_{0}^{\infty} \mathrm{d} \omega \frac{g(\omega)}{\omega}[(1+n(\omega)) \exp (\mathrm{i} \omega t)]+\int_{-\infty}^{0} \mathrm{~d} \omega \frac{g(\omega)}{-\omega}[n(-\omega) \exp (\mathrm{i} \omega t)]\right\} .
\end{aligned}
$$

Using the identity

$$
1+n(\omega)=1+\frac{1}{\exp \left(\hbar \omega /\left(k_{\mathrm{b}} T\right)\right)-1}=\frac{\exp \left(\hbar \omega /\left(k_{\mathrm{b}} T\right)\right)}{\exp \left(\hbar \omega /\left(k_{\mathrm{b}} T\right)\right)-1}=\frac{1}{1-\exp \left(-\hbar \omega /\left(k_{\mathrm{b}} T\right)\right)}=-n(-\omega)
$$

we end up with the relative simple expression

$$
\aleph_{j, j}(\vec{Q}, t)=\left\langle\left(\vec{Q} \cdot \vec{u}_{j}\right)\left(\vec{Q} \cdot \vec{u}_{j}(t)\right)\right\rangle=\frac{\hbar Q^{2}}{2 m} \int_{-\infty}^{\infty} \mathrm{d} \omega \frac{g(\omega)}{\omega} \cdot(n(\omega)+1) \cdot \exp (\mathrm{i} \omega t) .
$$

\footnotetext{
${ }^{123}$ The corresponding method was introduced quite early by A. Sjölander [61].

${ }^{124}$ The factor $(3 N)^{-1}$ comes from the fact that the eigenvectors $e_{i}(\alpha, j)$ have $3 N$ components among which the amplitudes of vibration are distributed.
} 
This expression can alternately be formulated as ${ }^{125}$

$$
\aleph_{j, j}(\vec{Q}, t)=\frac{\hbar Q^{2}}{2 m} \int_{0}^{\infty} \mathrm{d} \omega \frac{g(\omega)}{\omega}\left[\mathrm{i} \sin (\omega t)+\cos (\omega t) \operatorname{coth}\left(\frac{\hbar \omega}{2 k_{\mathrm{b}} T}\right)\right] .
$$

\section{Defining}

$$
f(t):=\frac{2 m}{\hbar Q^{2}} \aleph_{j, j}(\vec{Q}, t)=\int_{-\infty}^{\infty} \mathrm{d} \omega \frac{g(\omega)}{\omega} \cdot(n(\omega)+1) \cdot \exp (\mathrm{i} \omega t)
$$

the scattering function (see (8.7) and (10.2)) can be written as

$$
\begin{aligned}
S(\vec{Q}, \omega) & =\frac{\sigma_{\text {inc }}}{4 \pi} \frac{N}{2 \pi \hbar} \int_{-\infty}^{\infty} \mathrm{d} t \mathrm{e}^{-\mathrm{i} \omega t} \mathrm{e}^{-2 W(\vec{Q})} \mathrm{e}^{\left\langle\left(\vec{Q} \cdot \vec{u}_{j}\right)\left(\vec{Q} \cdot \vec{u}_{j}(t)\right)\right\rangle} \\
& =\frac{\sigma_{\text {inc }}}{4 \pi} \frac{N}{2 \pi \hbar} \int_{-\infty}^{\infty} \mathrm{d} t \mathrm{e}^{-\mathrm{i} \omega t} \exp (-2 W(\vec{Q})) \cdot \exp \left(\frac{\hbar Q^{2}}{2 m} f(t)\right) \\
& =\frac{\sigma_{\text {inc }}}{4 \pi} \frac{N}{2 \pi \hbar} \exp \left(-\frac{\hbar Q^{2}}{2 m} f(0)\right) \sum_{p=0}^{\infty} \frac{1}{p !} \int_{-\infty}^{\infty} \mathrm{d} t \mathrm{e}^{-\mathrm{i} \omega t}\left(\frac{\hbar Q^{2}}{2 m} f(t)\right)^{p}
\end{aligned}
$$

where in the last line we have used the fact that ${ }^{126}$ (see (9.110))

$$
2 W(\vec{Q})=\frac{\hbar Q^{2}}{2 m} \int_{0}^{\infty} \mathrm{d} \omega \frac{g(\omega)}{\omega}(2 n(\omega)+1)=\frac{\hbar Q^{2}}{2 m} f(0)=\aleph_{j, j}(\vec{Q}, t=0) .
$$

125

$$
\begin{aligned}
&(1+n(\omega)) \exp (\mathrm{i} \omega t)+n(\omega) \exp (-\mathrm{i} \omega t) \\
&=(1+n(\omega))(\cos (\omega t)+\mathrm{i} \sin (\omega t))+n(\omega)(\cos (-\omega t)+\mathrm{i} \sin (-\omega t)) \\
&=(1+n(\omega))(\cos (\omega t)+\mathrm{i} \sin (\omega t))+n(\omega)(\cos (\omega t)-\mathrm{i} \sin (\omega t)) \\
&=(1+2 n(\omega)) \cos (\omega t)+\mathrm{i} \sin (\omega t) \\
&= \operatorname{coth}\left(\frac{\hbar \omega}{2 k_{\mathrm{b}} T}\right) \cos (\omega t)+\mathrm{i} \sin (\omega t) .
\end{aligned}
$$

${ }^{126}$ Attention, the density of states are often not defined as a function of $\omega$ but of energy and normalised such that

$$
\int_{0}^{\infty} g(\hbar \omega) \mathrm{d}(\hbar \omega)=1
$$

In this case the expressions should be modified accordingly. For the Debye-Waller function we obtain for example

$$
2 W(\vec{Q})=\frac{\hbar^{2} Q^{2}}{2 m} \int_{0}^{\infty} \mathrm{d}(\hbar \omega) \frac{g(\hbar \omega)}{\hbar \omega}(2 n(\omega)+1)=\frac{\hbar^{2} Q^{2}}{2 m} \int_{0}^{\infty} \mathrm{d}(\hbar \omega) \frac{g(\hbar \omega)}{\hbar \omega} \operatorname{coth}\left(\frac{\hbar \omega}{2 k_{\mathrm{B}} T}\right)
$$


From this we derive the intermediate scattering function

$$
I(\vec{Q}, t)=\frac{\sigma_{\text {inc }}}{4 \pi} N \exp \left(-\frac{\hbar Q^{2}}{2 m} f(0)\right) \sum_{p=0}^{\infty} \frac{1}{p !}\left(\frac{\hbar Q^{2}}{2 m} f(t)\right)^{p} .
$$

If only the first term in the sum is kept, which is equivalent to $I(\vec{Q}, t=0)$, then we end up with the elastic scattering function (to be compared with (9.217))

$$
S_{(p=0)}(\vec{Q}, \omega)=N \frac{\sigma}{4 \pi} \exp (-2 W(\vec{Q})) \delta(\hbar \omega)
$$

The next term gives the one-phonon response (to be compared with (9.262))

$$
\begin{aligned}
S_{(p=1)}(\vec{Q}, \omega) & =\frac{\sigma_{\text {inc }}}{4 \pi} \frac{N}{2 \pi \hbar} \exp (-2 W(\vec{Q})) \int_{-\infty}^{\infty} \mathrm{d} t \mathrm{e}^{-\mathrm{i} \omega t}\left(\frac{\hbar Q^{2}}{2 m} f(t)\right) \\
& =\frac{\sigma_{\text {inc }}}{4 \pi} \frac{N}{2 \pi \hbar} \exp (-2 W(\vec{Q})) \int_{-\infty}^{\infty} \mathrm{d} t \mathrm{e}^{-\mathrm{i} \omega t}\left(\frac{\hbar Q^{2}}{2 m} \int_{-\infty}^{\infty} \mathrm{d} \omega^{\prime} \frac{g\left(\omega^{\prime}\right)}{\omega^{\prime}}\left(n\left(\omega^{\prime}\right)+1\right) \exp \left(\mathrm{i} \omega^{\prime} t\right)\right) \\
& =\frac{\sigma_{\text {inc }}}{4 \pi} N \exp (-2 W(\vec{Q})) \frac{Q^{2}}{2 m} \int_{-\infty}^{\infty} \mathrm{d} \omega^{\prime} \frac{g\left(\omega^{\prime}\right)}{\omega^{\prime}}\left(n\left(\omega^{\prime}\right)+1\right) \delta\left(\omega-\omega^{\prime}\right) \\
& =\frac{N}{8 \pi} Q^{2} \frac{\sigma_{\text {inc }}}{m} \exp (-2 W(\vec{Q})) \frac{g(\omega)}{\omega}(n(\omega)+1) .
\end{aligned}
$$

In order to calculate the contributions from several phonons we exploit the fact that

$$
\int_{-\infty}^{\infty} \mathrm{d} t \mathrm{e}^{-\mathrm{i} \omega t} f(t)^{p}=\int_{-\infty}^{\infty} \mathrm{d} t \mathrm{e}^{-\mathrm{i} \omega t} f(t) \cdot f(t)^{p-1}
$$

is the Fourier transform of a product of functions. Hence this Fourier transform is given by the convolution of the individual Fourier transforms. With

$$
\begin{aligned}
T_{0}(\omega) & :=\frac{1}{2 \pi \hbar} \int_{-\infty}^{\infty} \mathrm{d} t \mathrm{e}^{-\mathrm{i} \omega t}=\frac{1}{\hbar} \delta(\omega)=\delta(\hbar \omega), \\
T_{1}(\omega) & :=\frac{1}{2 \pi \hbar} \int_{-\infty}^{\infty} \mathrm{d} t \mathrm{e}^{-\mathrm{i} \omega t} f(t)=\frac{g(\omega)}{\hbar \omega}(n(\omega)+1) \\
& =\frac{g(\omega)}{2 \hbar \omega}\left(\operatorname{coth}\left(\frac{\hbar \omega}{2 k_{b} T}\right)+1\right),
\end{aligned}
$$

the higher order terms are found in a recursive manner

$$
T_{p}(\omega):=\int_{-\infty}^{\infty} \mathrm{d} t \mathrm{e}^{-\mathrm{i} \omega t} f(t)^{p}=\int_{-\infty}^{\infty} T_{1}\left(\omega-\omega^{\prime}\right) T_{p-1}\left(\omega^{\prime}\right) \mathrm{d} \omega^{\prime} .
$$

This is a very important result. The multi-phonon contributions are found by the repetitive convolution of the density of states (correctly weighted by the occupation of modes) with itself. This constitutes a considerable sim- 
plification with respect to expression (10.59). For the scattering function we find

$$
S(\vec{Q}, \omega)=\frac{\sigma_{\text {inc }}}{4 \pi} N \exp (-2 W(Q)) \sum_{p=0}^{\infty} \frac{1}{p !}\left(\frac{\hbar Q^{2}}{2 m}\right)^{p} T_{p}(\omega) .
$$

As a result it is possible to calculate the total inelastic scattering function up to the highest order of phonons as long as the density of states $g(\omega)$ is known. In practice - and without prior knowledge - the density of states $g(\omega)$ is determined in an iterative manner. First $g(\omega)$ is extracted from the data as if there were no higher order contributions, i.e. by using (10.70). The first estimate of $g(\omega)$ is then inserted into the expressions (10.62) and (10.68). This allows to determine the higher oder contributions to the scattering function via the expressions (10.69) and (10.70), and this up to any desired number of terms $p_{\max }$. The difference between this calculated cross section and the actually measured cross section is used to tune $g(\omega)$. This procedure is continued until convergence is reached thus yielding $g(\omega)$ in a self-consistent way. ${ }^{127}$

\subsubsection{Symmetrised p-phonon terms}

The functions $T_{n}(\omega)$ are not symmetric with respect to $\omega$. Up till now this has not caused problems. It is nevertheless often preferable to work with symmetric functions when doing numerical calculations [50,61]. By defining

$$
T_{p}^{\mathrm{sym}}(\omega):=\mathrm{e}^{-\hbar \omega /\left(2 k_{\mathrm{B}} T\right)} T_{p}(\omega)
$$

we verify that

$$
\begin{aligned}
\int_{-\infty}^{\infty} T_{1}^{\mathrm{sym}}\left(\omega-\omega^{\prime}\right) T_{p-1}^{\mathrm{sym}}\left(\omega^{\prime}\right) \mathrm{d} \omega^{\prime} & =\int_{-\infty}^{\infty} \mathrm{e}^{-\hbar\left(\omega-\omega^{\prime}\right) /\left(2 k_{\mathrm{B}} T\right)} T_{1}\left(\omega-\omega^{\prime}\right) \mathrm{e}^{-\hbar \omega^{\prime} /\left(2 k_{\mathrm{B}} T\right)} T_{p-1}\left(\omega^{\prime}\right) \mathrm{d} \omega^{\prime} \\
& =\mathrm{e}^{-\hbar \omega /\left(2 k_{\mathrm{B}} T\right)} \int_{-\infty}^{\infty} T_{1}\left(\omega-\omega^{\prime}\right) T_{p-1}\left(\omega^{\prime}\right) \mathrm{d} \omega^{\prime} \\
& =\mathrm{e}^{-\hbar \omega /\left(2 k_{\mathrm{B}} T\right)} T_{p}(\omega) \\
& =T_{p}^{\mathrm{sym}}(\omega)
\end{aligned}
$$

Hence the functions $T_{p}^{\mathrm{sym}}(\omega)$ can be determined in the same recursive way as the $T_{p}(\omega)$ starting from

$$
T_{1}^{\mathrm{sym}}(\omega)=\frac{g(\omega)}{2 \hbar \omega \sinh \left(\hbar \omega /\left(2 k_{\mathrm{B}} T\right)\right)} .
$$

$T_{1}^{\mathrm{sym}}(\omega)$ is then symmetric with respect to $\omega$ and all the other $T_{p}^{\mathrm{sym}}(\omega)$, which are obtained by successive convolution, are symmetric as well.

Inserting the functions $T_{p}^{\mathrm{sym}}(\omega)$ into (10.70) the partial differential cross section can be written as

$$
\left(\frac{\mathrm{d}^{2} \sigma_{\text {inc }}}{\mathrm{d}(\hbar \omega) \mathrm{d} \Omega}\right)_{\text {inc }}=\frac{k_{f}}{k_{i}} S(\vec{Q}, \omega)=\frac{N \sigma_{\text {inc }}}{4 \pi} \frac{k_{f}}{k_{i}} \mathrm{e}^{\hbar \omega /\left(2 k_{\mathrm{B}} T\right)} \mathrm{e}^{-2 W(Q)} \sum_{p=1}^{\infty}\left(\frac{\hbar Q^{2}}{2 m}\right)^{p} \frac{T_{p}^{\mathrm{sym}}(\omega)}{p !} .
$$

\subsubsection{Normalised p-phonon terms}

The expressions (10.70) and (10.74) both describe the scattering function as a sequence of terms with $p$-phonons. They have in common that the functions $T_{p}(\omega)$ and $\tilde{T}_{p}(\omega)$ employed for this purpose and which can be considered

${ }^{127}$ This procedure is for example implemented in the software package MUPHOCOR of W. Reichardt [50]. 
as pseudo density of states of $p$ phonons are not normalised. Due to this fact it is not evident to find the physical quantity which controls the impact of each term and thus the convergence of the sequence. We can fix this problem by normalising the pseudo-density of states $T_{p}(\omega)$. From (10.66) we get

$$
\int_{-\infty}^{\infty} \mathrm{d} \omega T_{p}(\omega)=\int_{-\infty}^{\infty} \mathrm{d} \omega \int_{-\infty}^{\infty} \mathrm{d} t \mathrm{e}^{-\mathrm{i} \omega t} f(t)^{p}=\int_{-\infty}^{\infty} \mathrm{d} t f(t)^{p} \delta(t)=f(0)^{p} .
$$

With the identity (10.62) these norms are directly linked to the Debye-Waller functions

$$
f(0)^{p}=\left(2 W(Q) \frac{2 m}{\hbar Q^{2}}\right)^{p} .
$$

By defining

$$
\tilde{T}_{p}(\omega):=\frac{T_{p}(\omega)}{f(0)^{p}}=T_{p}(\omega)\left(\frac{\hbar Q^{2}}{2 m} \frac{1}{2 W(Q)}\right)^{p}
$$

we obtain normalised functions

$$
\int_{-\infty}^{\infty} \mathrm{d} \omega \tilde{T}_{p}(\omega)=1
$$

Inserting (10.77) into (10.70) the scattering function can be expressed as

$$
\begin{aligned}
S(\vec{Q}, \omega) & =\frac{\sigma_{\text {inc }}}{4 \pi} N \exp (-2 W(Q)) \sum_{p=0}^{\infty} \frac{1}{p !}\left(\frac{\hbar Q^{2}}{2 m} f(0)\right)^{p} \tilde{T}_{p}(\omega) \\
& =\frac{\sigma_{\text {inc }}}{4 \pi} N \exp (-2 W(Q)) \sum_{p=0}^{\infty} \frac{(2 W(Q))^{p}}{p !} \tilde{T}_{p}(\omega) .
\end{aligned}
$$

Hence the sequence converges rapidly if the Debye-Waller function for the $Q$ in question is small. This is the case if the mean square displacement $\overline{u^{2}}$ of the atoms is small. When $Q$ or $\overline{u^{2}}$ increase the multi-phonon terms become more and more important. This is the situation that we had already encountered in the discussion of the isotropic harmonic oscillator. Thus the Debye-Waller function is a good indicator for the number of terms that should be taken into account when analysing the data.

\subsection{Integrating the p-phonon terms over angle and $Q$}

The expressions that we have developed so far are valid for a particular $Q$-value. If the incoherent approximation has to be used, or if in order to get decent statistics the data have to be anyway summed over several detector banks, then one wishes to determine $g(\omega)$ from data integrated over $Q$ or scattering angle. The integration of choice is in this context the one that we have presented together with the incoherent approximation (see Eq. (9.295)). If we apply this integration to expression (10.74) using (9.293), we obtain

$$
\begin{aligned}
\left(\frac{\mathrm{d} \sigma}{\mathrm{d}(\hbar \omega)}\right)_{\text {int }} & =\Delta \Phi \int_{\theta_{\min }}^{\theta_{\max }}\left(\frac{\mathrm{d}^{2} \sigma_{\text {inc }}}{\mathrm{d}(\hbar \omega) \mathrm{d} \Omega}\right)_{\mathrm{inc}} \sin \theta \mathrm{d} \theta \\
& =\Delta \Phi \int_{Q_{\min }(\omega)}^{Q_{\max }(\omega)}\left(\frac{\mathrm{d}^{2} \sigma_{\mathrm{inc}}}{\mathrm{d}(\hbar \omega) \mathrm{d} \Omega}\right)_{\mathrm{inc}} \frac{1}{k_{i} k_{f}} Q \mathrm{~d} Q
\end{aligned}
$$




$$
\begin{aligned}
& =\Delta \Phi \frac{N \sigma_{\text {inc }}}{4 \pi} \frac{1}{k_{i}^{2}} \mathrm{e}^{\hbar \omega /\left(2 k_{\mathrm{B}} T\right)} \sum_{p=1}^{\infty}\left(\frac{\hbar}{2 m}\right)^{p} \frac{T_{p}^{\mathrm{sym}}(\omega)}{p !} \int_{Q_{\min }(\omega)}^{Q_{\max }(\omega)} Q^{2 p} \mathrm{e}^{-\gamma Q^{2}} Q \mathrm{~d} Q \\
& =\Delta \Phi \frac{N \sigma_{\text {inc }}}{4 \pi} \frac{1}{k_{i}^{2}} \mathrm{e}^{\hbar \omega /\left(2 k_{\mathrm{B}} T\right)} \sum_{p=1}^{\infty}\left(\frac{\hbar}{2 m}\right)^{p} \frac{T_{p}^{\mathrm{sym}}(\omega)}{p !} \frac{1}{2 \gamma^{p+1}} \int_{Q_{\min }(\omega)}^{Q_{\max }(\omega)}\left(\gamma Q^{2}\right)^{p} \mathrm{e}^{-\gamma Q^{2}} \mathrm{~d}\left(\gamma Q^{2}\right)
\end{aligned}
$$

with

$$
\gamma Q^{2}:=2 W(Q),
$$

where $Q_{\min }$ and $Q_{\max }$ naturally depend on the frequency $\omega$. This expression is rigorously exact for an incoherent scatterer in a monatomic, cubic Bravais lattice.

Integrals of the type

$$
I_{p}(y)=\int_{0}^{y} x^{p} \mathrm{e}^{-x} \mathrm{~d} x,
$$

can be calculated by recursion

$$
I_{p}(y):=\int_{0}^{y} x^{p} \mathrm{e}^{-x} \mathrm{~d} x=-y^{p} \mathrm{e}^{-y}-p \int_{0}^{y} x^{p-1} \mathrm{e}^{-x}=-y^{p} \mathrm{e}^{-y}-p I_{p-1}(y) .
$$

This formula can easily be obtained by partial integration. ${ }^{128}$ The first terms are calculated as

$$
\begin{aligned}
& I_{0}(y)=-\mathrm{e}^{-y}, \\
& I_{1}(y)=-(y+1) \mathrm{e}^{-y}, \\
& I_{2}(y)=-\left(y^{2}+2 y+2\right) \mathrm{e}^{-y}, \\
& I_{3}(y)=-\left(y^{3}+3 y^{2}+6 y+6\right) \mathrm{e}^{-y} .
\end{aligned}
$$

${ }^{128}$ Partial integration is defined on the identity

$$
\int_{a}^{b} u^{\prime}(x) v(x) \mathrm{d} x=[u(x) v(x)]_{a}^{b}-\int_{a}^{b} u(x) v^{\prime}(x) \mathrm{d} x .
$$

Posing

$$
\begin{aligned}
& u(x):=-\mathrm{e}^{-x}, \\
& v(x):=x^{p}
\end{aligned}
$$

it follows that

$$
\int_{a}^{b} x^{p} \mathrm{e}^{-x} \mathrm{~d} x=-\left[x^{p} \mathrm{e}^{-x}\right]_{a}^{b}-n \int_{a}^{b} x^{p-1} \mathrm{e}^{-x}
$$

and hence

$$
I_{p}(y):=\int_{0}^{y} x^{p} \mathrm{e}^{-x} \mathrm{~d} x=-y^{p} \mathrm{e}^{-y}-p \int_{0}^{y} x^{p-1} \mathrm{e}^{-x}=-y^{p} \mathrm{e}^{-y}-p I_{p-1}(y) .
$$


Thus we arrive at the result

$$
\left(\frac{\mathrm{d} \sigma}{\mathrm{d}(\hbar \omega)}\right)_{\text {int }}=\Delta \Phi \frac{N \sigma_{\text {inc }}}{8 \pi} \frac{1}{k_{i}^{2}} \mathrm{e}^{\hbar \omega /\left(2 k_{\mathrm{B}} T\right)} \sum_{p=1}^{\infty}\left(\frac{\hbar}{2 m}\right)^{p} \frac{1}{\gamma^{p+1}}\left(I_{p}\left(\gamma Q_{\max }^{2}\right)-I_{p}\left(\gamma Q_{\min }^{2}\right)\right) \frac{T_{p}^{\mathrm{sym}}(\omega)}{p !} .
$$

If the mean square displacements are small with respect to $Q_{\max }^{-2}$ only the one-phonon term is left

$$
\begin{aligned}
\left(\frac{\mathrm{d} \sigma}{\mathrm{d}(\hbar \omega)}\right)_{\text {int }}^{p=1}= & \Delta \Phi \frac{N \sigma_{\text {inc }}}{8 \pi} \frac{1}{k_{i}^{2}} \mathrm{e}^{-\hbar \omega /\left(2 k_{\mathrm{B}} T\right)}\left(\frac{\hbar}{2 m}\right) \frac{1}{\gamma^{2}}\left(I_{1}\left(\gamma Q_{\max }^{2}\right)-I_{1}\left(\gamma Q_{\min }^{2}\right)\right) T_{1}^{\mathrm{sym}}(\omega) \\
= & \Delta \Phi \frac{N \sigma_{\text {inc }}}{8 \pi} \frac{1}{k_{i}^{2}} \mathrm{e}^{-\hbar \omega /\left(2 k_{\mathrm{B}} T\right)}\left(\frac{\hbar}{2 m}\right) \frac{1}{\gamma^{2}} \\
& \times\left(-\left(1+\gamma Q_{\max }^{2}\right) \mathrm{e}^{-\gamma Q_{\max }^{2}}+\left(1+\gamma Q_{\min }^{2}\right) \mathrm{e}^{\left.-\gamma Q_{\min }^{2}\right) T_{1}^{\mathrm{sym}}(\omega) .}\right.
\end{aligned}
$$

In addition we can calculate the factor

$$
I_{1}\left(\gamma Q^{2}\right)=-\left(1+\gamma Q^{2}\right) \mathrm{e}^{-\gamma Q^{2}} \approx-\left(1+\gamma Q^{2}\right)\left(1-\gamma Q^{2}\right)=-1+\frac{1}{2} \gamma^{2} Q^{4} .
$$

Thus

$$
\begin{aligned}
\left(\frac{\mathrm{d} \sigma}{\mathrm{d}(\hbar \omega)}\right)_{\mathrm{int}}^{p=1} & =\Delta \Phi \frac{N \sigma_{\text {inc }}}{16 \pi} \frac{1}{k_{i}^{2}} \mathrm{e}^{\hbar \omega /\left(2 k_{\mathrm{B}} T\right)}\left(\frac{\hbar}{2 m}\right)\left(Q_{\max }^{4}(\omega)-Q_{\min }^{4}(\omega)\right) T_{1}^{\mathrm{sym}}(\omega) \\
& =\Delta \Phi \frac{N \sigma_{\text {inc }}}{16 \pi} \frac{1}{k_{i}^{2}} \mathrm{e}^{\hbar \omega /\left(2 k_{\mathrm{B}} T\right)}\left(\frac{\hbar}{2 m}\right)\left(Q_{\max }^{4}(\omega)-Q_{\min }^{4}(\omega)\right) \frac{g(\omega)}{2 \hbar \omega \sinh \left(\hbar \omega /\left(2 k_{\mathrm{B}} T\right)\right)} \\
& =\Delta \Phi \frac{N \sigma_{\text {inc }}}{32 \pi m} \frac{1}{k_{i}^{2}}(n(\omega)+1) \frac{g(\omega)}{\omega}\left(Q_{\max }^{4}(\omega)-Q_{\min }^{4}(\omega)\right) .
\end{aligned}
$$

This expression establishes a simple relation between experimental data integrated over $Q \mathrm{~d} Q$ and the density of states $g(\omega)$. It is identical to (9.299) apart from the Debye-Waller factor. It permits to estimate the initial density of states from which the iterative process can be started. A concrete example of how, with the formalism developed here, multi-phonon terms can be calculated in an iterative way starting from the Q integrated data is shown in Fig. 61 for the completely incoherent scatterer vanadium.

The expression (10.96) is also interesting from another point of view. The $Q$ dependence of the Debye-Waller factor was correctly taken into account when deriving this result. In addition, the Debye-Waller factor was kept to the same order $\gamma$ as the multi-phonons. The result is that the Debye-Waller factor disappears completely from the expression for the one-phonon cross section. We draw the following conclusion. If it is not possible to correct for the multi-phonons it is better not to correct for the Debye-Waller factor either since the two partially compensate each other.

We would like to reinforce this point by using the $f$ sum rule. With (see (10.65))

$$
S_{(p=1)}(\vec{Q}, \omega)=\frac{N}{8 \pi} Q^{2} \frac{\sigma_{\text {inc }}}{m} \exp (-2 W(\vec{Q})) \frac{g(\omega)}{\omega}(n(\omega)+1)
$$

the first moment is found to be

$$
\begin{aligned}
\int_{\infty}^{\infty} \mathrm{d}(\hbar \omega)(\hbar \omega) S_{(p=1)}(\vec{Q}, \omega) & =\frac{N}{8 \pi} \hbar^{2} Q^{2} \frac{\sigma_{\text {inc }}}{m} \exp (-2 W(\vec{Q})) \int_{0}^{\infty} \mathrm{d} \omega g(\omega) \\
& =N \overline{b^{2}} \frac{\hbar^{2} Q^{2}}{2 m} \exp (-2 W(\vec{Q}))=N \overline{b^{2}} E_{r}(\vec{Q}) \exp (-2 W(\vec{Q})) .
\end{aligned}
$$




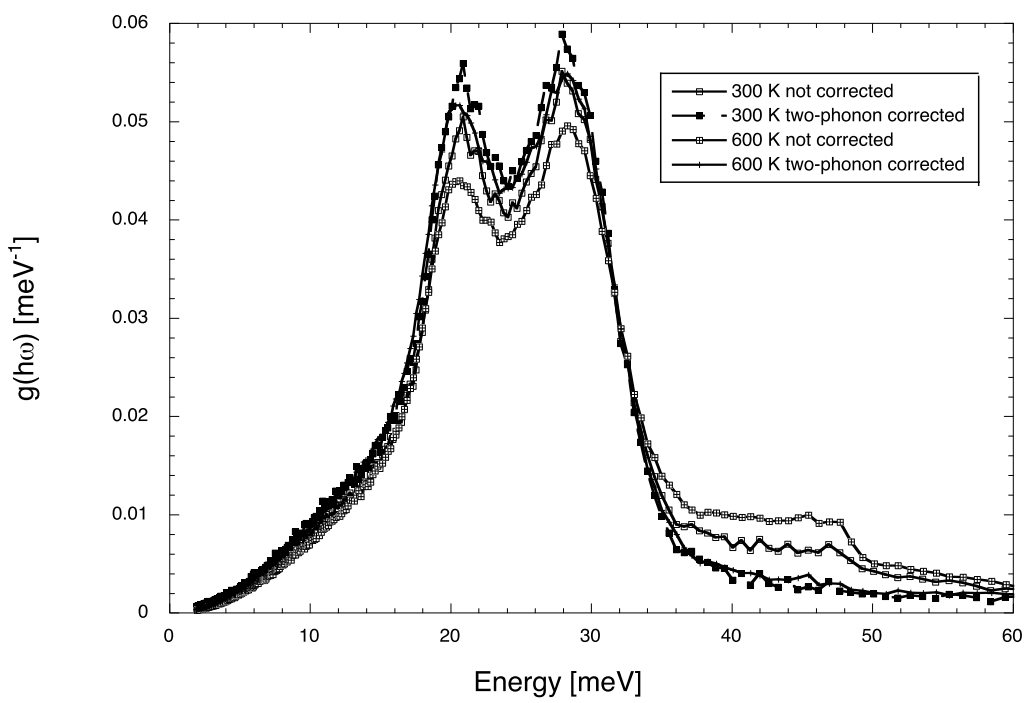

Fig. 61. Density of states for Vanadium at 300 and $600 \mathrm{~K}$ obtained from one-phonon incoherent scattering measured at the instrument IN6. The curves that are corrected for multi-phonons to the second order are compared to the curves where no correction is made. The difference can be attributed to two-phonon processes. The two-phonon contribution increases with temperature. If this correction is not taken into account then one will postulate erroneously the existence of a vibrational band between 35 and $50 \mathrm{meV}$, which in addition would be predicted as highly anharmonic.

The reduction of this moment with respect to the total moment according to (8.187) is due to the Debye-Waller factor. The result is correct if we complete it with the contributions from muliti-phonons. If we decide to ignore the multi-phonon effects it is better to ignore the Debye-Waller factor as well. Then at least the first moment is reproduced correctly.

\subsection{Susceptibility and anharmonic effects}

In Section 8.11 we learned from (8.155) that the scattering function of any system can be written as

$$
S(\vec{Q}, \omega)=\frac{1}{\pi}(1+n(\omega)) \chi_{\vec{Q}}^{\prime \prime}[\omega]
$$

with $\chi_{\vec{Q}}^{\prime \prime}[\omega]$ the generalised susceptibility of a dynamic system. We would now like to apply this relation to vibrations and phonons. If we take the coherent scattering function (9.185)

$$
\begin{aligned}
S_{\mathrm{coh}}^{1-\mathrm{ph}}(\vec{Q}, \omega) & =\frac{1}{2} \sum_{i} \frac{\left|F_{i}(\vec{Q})\right|^{2}}{\omega_{i}}\left\{\left[\left(1+n\left(\omega_{i}\right)\right) \delta\left(\omega-\omega_{i}\right)\right]+\left[n\left(\omega_{i}\right) \delta\left(\omega+\omega_{i}\right)\right]\right\} \\
& =\frac{1}{2}(1+n(\omega)) \sum_{i} \frac{\left|F_{i}(\vec{Q})\right|^{2}}{\omega_{i}}\left\{\left[\delta\left(\omega-\omega_{i}\right)\right]+\left[\delta\left(\omega+\omega_{i}\right)\right]\right\}
\end{aligned}
$$

as a starting point we find

$$
\chi_{\vec{Q}}^{\prime \prime}[\omega]=\frac{\pi}{2} \sum_{i} \frac{\left|F_{i}(\vec{Q})\right|^{2}}{\omega_{i}}\left\{\delta\left(\omega-\omega_{i}\right)+\delta\left(\omega+\omega_{i}\right)\right\} .
$$


The vibrational form factor is given by (9.186)

$$
\left|F_{i}(\vec{Q})\right|^{2}:=\left|\sum_{\kappa} \frac{\overline{b_{\kappa}}}{\sqrt{m_{\kappa}}} \sum_{j \in\left\{j_{\kappa}\right\}} \mathrm{e}^{-\mathrm{i} \vec{Q} \cdot \vec{R}_{j}^{0}} e^{-W_{j}(\vec{Q})}\left(\vec{Q} \cdot \vec{e}_{i}(j)\right)\right|^{2}
$$

Its temperature dependence is contained in the Debye-Waller factor. We should emphasise that the susceptibility defined here refers to fluctuations in the density of the scattering length and not to the atomic density itself.

The expression (10.99) is in perfect analogy with the one derived for the simple harmonic oscillator with frequency $\omega_{0}$ in the limit of vanishing damping (see expression (8.163)). For such an oscillator the susceptibility due to fluctuations of the $x$ position can be calculated as

$$
\chi_{\hat{x}^{+} \hat{x}}^{\prime \prime}[\omega]=\frac{\pi}{2 m \omega_{0}}\left[\left(\delta\left(\omega-\omega_{0}\right)-\delta\left(\omega+\omega_{0}\right)\right]\right.
$$

The expressions easily extend to incoherent scattering. With the incoherent one-phonon scattering function (9.260) given by

$$
S(\vec{Q}, \omega)_{\text {inc }}^{1-\mathrm{ph}(+)}=Q^{2} \frac{1}{8 \pi} \sum_{\kappa} N_{\kappa} \frac{\sigma_{\text {inc }}(\kappa)}{m_{\kappa}} \mathrm{e}^{-2 W_{\kappa}(\vec{Q})} F_{\kappa}(\omega) \frac{(1+n(\omega))}{\omega}
$$

we obtain an expression relating the susceptibility to the partial density of states

$$
\chi_{\vec{Q}}^{\prime \prime}[\omega]=\frac{1}{8} Q^{2} \sum_{\kappa} N_{\kappa} \sigma_{\text {inc }}(\kappa) \frac{\mathrm{e}^{-2 W_{\kappa}(\vec{Q})}}{\omega m_{\kappa}} F_{\kappa}(\omega) .
$$

This expression again depends on temperature only through the Debye-Waller factor. Following the same argument as in the previous section, it is better to neglect this dependence if the multi-phonon contributions are not included. Hence for a harmonic system the susceptibility

$$
\chi_{\vec{Q}}^{\prime \prime}[\omega]=\pi \frac{1}{1+n(\omega)} S_{\mathrm{inc}}(\vec{Q}, \omega)
$$

that is obtained from the measured scattering function should be independent of temperature.

The qualitative affirmation of anharmonicity in the system is often not sufficient for pinning down the origin of the anharmonic effects (for an example see Fig. 62). If we posses a single crystal, which puts us into the position of studying in detail the dispersion curves, we can go one step further. Anharmonic processes have two principle observable consequences. First of all the phonon peaks for a given $\vec{q}$ and a given branch index $i$ obtain a width. Secondly the central frequencies change with temperature.

The susceptibility of a damped mode is given in its most general form [29]

$$
\chi_{(i, \vec{q})}^{\prime \prime}(\omega)=A(i, \vec{q}) \frac{4 \omega_{0}(i, \vec{q}) \Gamma(i, \vec{q} ; \omega)}{\left[\omega^{2}-\omega_{0}(i, \vec{q})^{2}-2 \omega_{0}(i, \vec{q}) \Delta(i, \vec{q} ; \omega)\right]^{2}+4 \omega(i, \vec{q})^{2} \Gamma(i, \vec{q} ; \omega)^{2}} .
$$

Apart from the terms introducing a shift of the centre frequencies this expression resembles strongly the one (see (8.159)) that we had obtained for the simple damped harmonic oscillator in Section 8.11.

The frequency dependence of $\omega_{0}(j, \vec{q})$ and $\Delta(j, \vec{q})$ are a priori unknown which makes this expression impractical for data interpretation. The simplest approximation, that preserves the analytic properties of the scattering function 


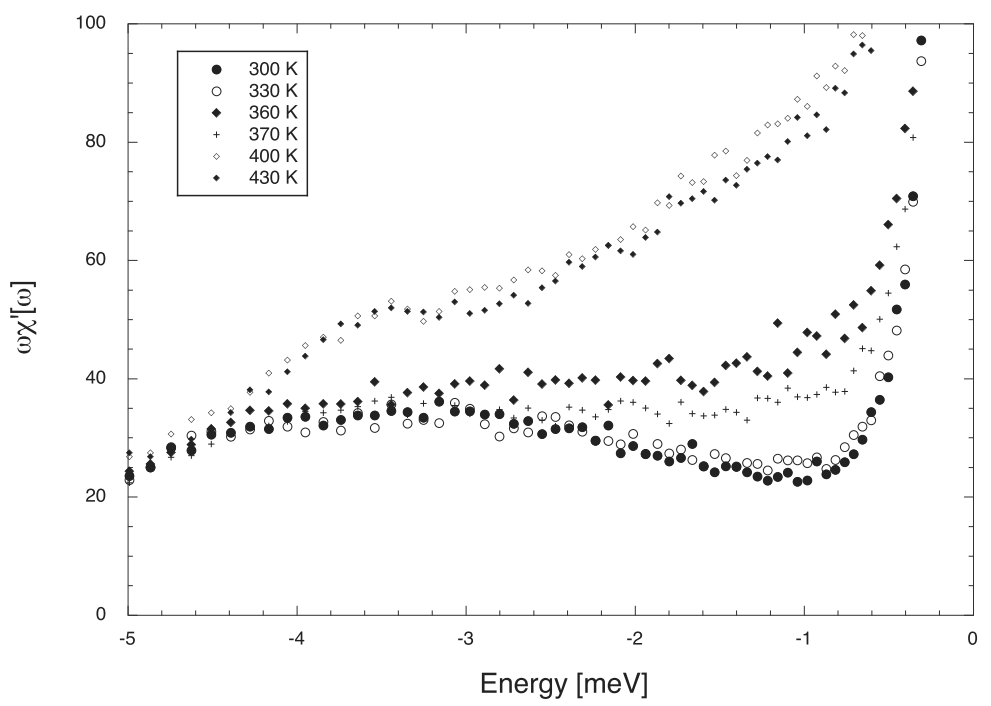

Fig. 62. Susceptibility (in arbitrary units) multiplied by the energy for the polymer $\mathrm{RbC}_{60}$ as a function of temperature upon heating from 300 to $450 \mathrm{~K}$. The measurement was done on the instrument IN6 at the ILL. For details see [55]. For low temperatures the susceptibility is invariant as a function of temperature. When the system starts to de-polymerise, i.e. around $350 \mathrm{~K}$ it becomes strongly anharmonic and the susceptibility evolves. The excess in intensity developing at low energies can be explained with scattering originating from the rotation of the $\mathrm{C}_{60}$ monomers.

like parity is given by [22]

$$
\begin{aligned}
& \Delta(i, \vec{q} ; \omega)=\Delta(i, \vec{q}), \\
& \Gamma(i, \vec{q} ; \omega)=\frac{\omega}{\omega_{0}(j, \vec{q})} \Gamma(i, \vec{q}) .
\end{aligned}
$$

This choice gives us the susceptibility of a damped harmonic oscillator

$$
\chi_{(i, \vec{q})}^{\prime \prime}(\omega)=A(i, \vec{q}) \frac{4 \omega \Gamma(i, \vec{q})}{\left(\omega^{2}-\Omega(i, \vec{q})^{2}\right)^{2}+4 \omega^{2} \Gamma(i, \vec{q})^{2}},
$$

where we have introduced the renormalised frequency $\Omega(i, \vec{q})$ (see Fig. 63).

By introducing the frequencies

$$
\omega_{\text {lor }}(i, \vec{q})^{2}:=\Omega(i, \vec{q})^{2}-\Gamma(i, \vec{q})^{2}
$$

the susceptibility of a damped harmonic oscillator can be written in the alternative form [66]

$$
\chi_{(i, \vec{q})}^{\prime \prime}(\omega)=\frac{A(i, \vec{q})}{\omega_{\text {lor }}(i, \vec{q})}\left[\frac{\Gamma(i, \vec{q})}{\left.\left(\omega-\omega_{\text {lor }}(i, \vec{q})\right)^{2}+\Gamma(i, \vec{q})^{2}\right)}-\frac{\Gamma(i, \vec{q})}{\left.\left(\omega+\omega_{\text {lor }}(i, \vec{q})\right)^{2}+\Gamma(i, \vec{q})^{2}\right)}\right],
$$

that corresponds for $\Gamma(j, \vec{q}) \ll \omega_{\text {lor }}(j, \vec{q})$ to the sum of two Lorentzian functions from the gain and loss part of the spectrum.

This form is interesting since it leads us in the case of $\Gamma(i, \vec{q}) \rightarrow 0$ back to the expression (10.103) for the simple harmonic oscillator, since

$$
\lim _{\varepsilon \rightarrow 0} \frac{1}{\pi} \frac{\varepsilon}{x^{2}+\varepsilon^{2}}=\delta(x) .
$$




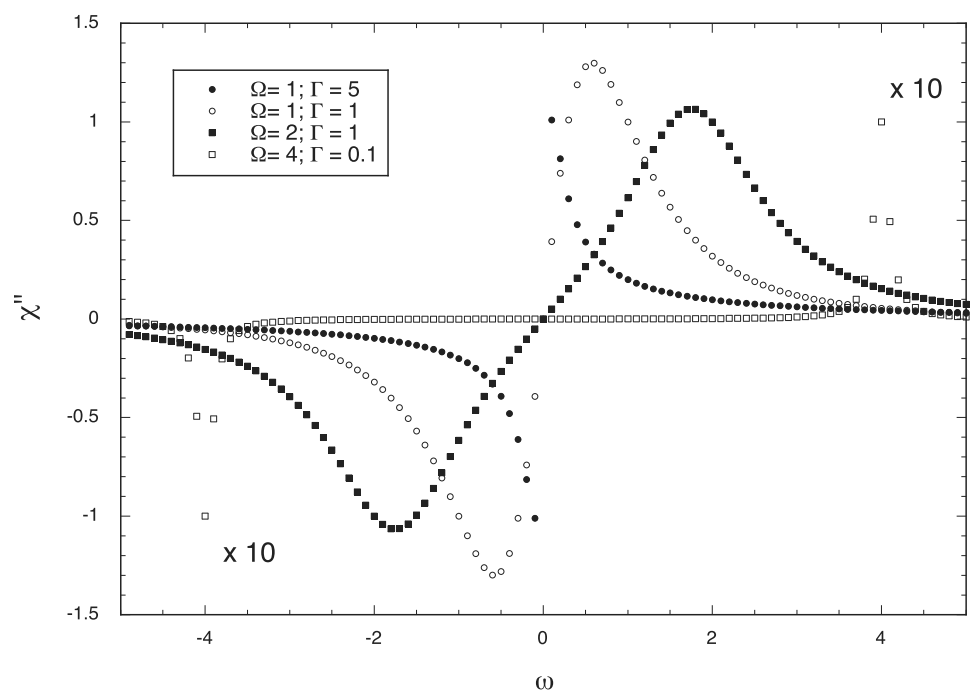

Fig. 63. Susceptibility obtained for a damped harmonic oscillator according to (10.107). For $\Gamma \gg \Omega$ the oscillator is over-damped. For $\Gamma \ll \Omega$ one obtains two Lorentzians.

As a consequence the factor $A(i, \vec{q})$ can be determined by replacing in the expression (10.100) the term (10.101) valid in the limit $\Gamma \rightarrow 0$ - by the expression (10.104). We identify

$$
A(j, \vec{q})=\left|F_{i}(\vec{Q} \mid \vec{q})\right|^{2} .
$$

The phonon form factors $\left|F_{i}(\vec{Q} \mid \vec{q})\right|^{2}$ are defined by (9.203). For the scattering function of an ensemble of damped harmonic oscillators we find

$$
\begin{aligned}
S_{\mathrm{coh}}^{1-\mathrm{ph}}(\vec{Q}, \omega)= & \frac{1}{\pi} \frac{(2 \pi)^{3}}{2 V_{\text {unit cell }}} \sum_{\vec{G}} \sum_{i, \vec{q}} \frac{\left|F_{i}(\vec{Q} \mid \vec{q})\right|^{2}}{\omega_{\text {lor }}(i, \vec{q})}(1+n(\omega)) \\
& \times\left[\delta(\vec{q}-(\vec{Q}+\vec{G})) \frac{\Gamma(i, \vec{q})}{\left.\left(\omega-\omega_{\text {lor }}(i, \vec{q})\right)^{2}+\Gamma(i, \vec{q})^{2}\right)}\right. \\
& \left.-\delta(\vec{q}+(\vec{Q}+\vec{G})) \frac{\Gamma(i, \vec{q})}{\left.\left(\omega+\omega_{\text {lor }}(i, \vec{q})\right)^{2}+\Gamma(i, \vec{q})^{2}\right)}\right] .
\end{aligned}
$$

One should be careful when interpreting the parameters obtained from experimental data by fitting to the expressions (10.107) or (10.111). For small damping the difference between $\Omega$ and $\omega_{\text {lor }}$ is small. When $\Gamma$ increases the frequency $\Omega$ is the more physical parameter. In the case of a soft mode it extrapolates e.g. correctly to zero at the transition temperature [22].

\subsection{Partial summary}

We will again briefly summarise the principle results of the two last sections.

Our starting point were the partial scattering functions defined according to (9.74)

$$
S_{\kappa, \kappa^{\prime}}(\vec{Q}, \omega)=\frac{1}{2 \pi \hbar} \sum_{j \in\left\{j_{\kappa}\right\}, j^{\prime} \in\left\{j_{\kappa^{\prime}}\right\}} \int_{-\infty}^{\infty} \mathrm{d} t\left\langle\mathrm{e}^{-\mathrm{i} \vec{Q} \cdot \vec{R}_{j^{\prime}}(t=0)} \mathrm{e}^{\mathrm{i} \vec{Q} \cdot \vec{R}_{j}(t)}\right\rangle \mathrm{e}^{-\mathrm{i} \omega t}
$$


In a fully relaxed solid the ionic movements are described by the ionic displacements around their equilibrium position

$$
\vec{u}_{j}(t)=\vec{R}_{j}(t)-\vec{R}_{j}^{0}, \quad j=1, \ldots, N .
$$

The expectation value that appears in the expression for the partial scattering functions becomes an expectation value of the displacements (9.77)

$$
\left\langle\mathrm{e}^{-\mathrm{i} \vec{Q} \cdot\left(\vec{R}_{j^{\prime}}^{0}+\vec{u}_{j^{\prime}}(t=0)\right)} \mathrm{e}^{\mathrm{i} \vec{Q} \cdot\left(\vec{R}_{j}^{0}+\vec{u}_{j}(t)\right)}\right\rangle=\mathrm{e}^{-\mathrm{i} \vec{Q} \cdot\left(\vec{R}_{j^{\prime}}^{0}-\vec{R}_{j}^{0}\right)}\left\langle\mathrm{e}^{-\mathrm{i} \vec{Q} \cdot \vec{u}_{j^{\prime}}(t=0)} \mathrm{e}^{\mathrm{i} \vec{Q} \cdot \vec{u}_{j}(t)}\right\rangle .
$$

The algebraic properties of the displacement operators $\overrightarrow{\mathbf{u}}_{j}(t)$ allow us to transform this function in the harmonic approximation into (9.104)

$$
\left\langle\mathrm{e}^{-\mathrm{i} \vec{Q} \cdot \vec{u}_{j^{\prime}}} \mathrm{e}^{\mathrm{i} \vec{Q} \cdot \vec{u}_{j}(t)}\right\rangle=\mathrm{e}^{-W_{j^{\prime}}(\vec{Q})} \mathrm{e}^{-W_{j}(\vec{Q})} \mathrm{e}^{\left\langle\left(\vec{Q} \cdot \vec{u}_{j^{\prime}}\right)\left(\vec{Q} \cdot \vec{u}_{j}(t)\right)\right\rangle}
$$

with $W_{j}(\vec{Q})$ the Debye-Waller functions.

Written in this form it is no longer required to effectuate the statistical average over the exponential functions. The operation \langle\rangle has shifted to the exponent. As a consequence this expression is amenable to an expansion in terms of the expectation value

$$
\aleph_{j^{\prime}, j}(\vec{Q}, t)=\left\langle\left(\vec{Q} \cdot \vec{u}_{j^{\prime}}\right)\left(\vec{Q} \cdot \vec{u}_{j}(t)\right)\right\rangle,
$$

which is bi-linear in $\overrightarrow{\mathbf{u}}_{j}(t)$.

The first term in this expansion describes the elastic scattering. For a crystal the coherent elastic cross section is found as $(9.128)$

$$
\left(\frac{\mathrm{d}^{2} \sigma}{\mathrm{d} \Omega \mathrm{d} E_{f}}\right)_{\text {coh }}^{\text {el }}=N_{\text {unit cell }} \frac{(2 \pi)^{3}}{V_{\text {unit cell }}}|F(\vec{Q})|^{2} \sum_{\vec{G}} \delta(\vec{Q}-\vec{G}) \delta(\hbar \omega),
$$

with the nuclear form factor of the primitive cell

$$
F(\vec{Q}):=\sum_{d} \bar{b}_{d} \exp \left(-W_{d}(\vec{Q})\right) \exp (\overrightarrow{\mathrm{i}} \vec{Q} \cdot \vec{d})
$$

The next term in the expansion provides the contributions from single vibrations (9.185)

$$
S_{\mathrm{coh}}^{1-\mathrm{ph}}(\vec{Q}, \omega)=\frac{1}{2} \sum_{i} \frac{\left|F_{i}(\vec{Q})\right|^{2}}{\omega_{i}}\left\{\left[\left(1+n\left(\omega_{i}\right)\right) \delta\left(\omega-\omega_{i}\right)\right]+\left[n\left(\omega_{i}\right) \delta\left(\omega+\omega_{i}\right)\right]\right\}
$$

with

$$
\left|F_{i}(\vec{Q})\right|^{2}=\left|\sum_{\kappa} \frac{\bar{b}_{\kappa}}{\sqrt{m_{\kappa}}} \sum_{j \in\left\{j_{\kappa}\right\}} \mathrm{e}^{-\mathrm{i} \vec{Q} \cdot \vec{R}_{j}^{0}} \mathrm{e}^{-W_{j}(\vec{Q})}\left(\vec{Q} \cdot \vec{e}_{i}(j)\right)\right|^{2} .
$$

The function $F_{i}(\vec{Q})$ is the vibrational form factor of the mode $i$. It determines the intensity, with which the mode will be observed for a given momentum transfer $\vec{Q}$. For a crystal the translation symmetry adds the conservation 
of crystal momentum (9.202)

$$
\begin{aligned}
S_{\text {coh }}^{1-\mathrm{ph}}(\vec{Q}, \omega)= & \frac{(2 \pi)^{3}}{2 V_{\text {unit cell }}} \sum_{\vec{G}} \sum_{i, \vec{q}} \frac{\left|F_{i}(\vec{Q} \mid \vec{q})\right|^{2}}{\omega_{i}(\vec{q})} \\
& \times\left[\left(1+n\left(\omega_{i}(\vec{q})\right)\right) \delta(\vec{q}-(\vec{Q}+\vec{G})) \delta\left(\omega-\omega_{i}(\vec{q})\right)+n\left(\omega_{i}(\vec{q})\right) \delta(\vec{q}+(\vec{Q}+\vec{G})) \delta\left(\omega+\omega_{i}(\vec{q})\right)\right]
\end{aligned}
$$

with the phonon form factor

$$
\left|F_{i}(\vec{Q} \mid \vec{q})\right|^{2}=\left|\sum_{d=1}^{r} \frac{\overline{b_{d}}}{\sqrt{m_{d}}} \mathrm{e}^{-W_{d}(\vec{Q})}\left(\vec{Q} \cdot \vec{e}_{i}(d \mid \vec{q})\right) \mathrm{e}^{-\mathrm{i} \vec{Q} \cdot \vec{d}}\right|
$$

The discreteness of the coherent one-phonon response permits the determination of the dispersion sheets.

The incoherent scattering for a given $\vec{Q}$ is a continuous function of $\omega$ even for a crystal. For an isotropic system it can be expressed as a function of the partial density of states

$$
\left(\frac{\mathrm{d}^{2} \sigma}{\mathrm{d} \Omega \mathrm{d} E_{f}}\right)_{\text {inc }}^{1-\mathrm{ph}(+)}=\frac{k_{f}}{k_{i}} \frac{Q^{2}}{3} \sum_{\kappa} \frac{\sigma_{\mathrm{inc}}(\kappa)}{8 \pi m_{\kappa}} \sum_{j \in\left\{j_{\kappa}\right\}} \mathrm{e}^{-2 W_{j}(\vec{Q})} \frac{F_{j}(\omega)}{\omega}(1+n(\omega)), \quad \omega>0 .
$$

For a crystal (9.261)

$$
\left(\frac{\mathrm{d}^{2} \sigma}{\mathrm{d} \Omega \mathrm{d} E_{f}}\right)_{\text {inc }}^{1-\mathrm{ph}}=\frac{k_{f}}{k_{i}} Q^{2} \frac{N}{8 \pi r} \sum_{d} \frac{\sigma_{\text {inc }}(d)}{m_{d}} \mathrm{e}^{-2 W_{\kappa}(\vec{Q})} F_{d}(\omega) \frac{(1+n(\omega))}{\omega} .
$$

For a monatomic system this expression can be simplified. The incoherent scattering is directly related to the vibrational density of states $g(\omega)(9.262)$

$$
\left(\frac{\mathrm{d}^{2} \sigma}{\mathrm{d} \Omega \mathrm{d} E_{f}}\right)_{\text {inc }}^{1-\mathrm{ph}}=\frac{k_{f}}{k_{i}} Q^{2} \frac{N}{8 \pi} \frac{\sigma_{\mathrm{inc}}}{m} \mathrm{e}^{-2 W_{d}(\vec{Q})} \frac{g(\omega)}{\omega}(1+n(\omega)) .
$$

The generalisation of this expression to poly-atomic systems is common practice despite the fact that it requires a lot of care.

The one-phonon response is a good approximation for inelastic scattering provided that the displacements of the ions are small with respect to $Q^{-1}$. If this is condition is violated then multi-phonon contributions become important. For monatomic incoherent systems these can be calculated starting from the density of states $g(\omega)$ using (10.70)

$$
S(\vec{Q}, \omega)=\frac{\sigma_{\text {inc }}}{4 \pi} N \exp (-2 W(Q)) \sum_{p=0}^{\infty} \frac{1}{p !}\left(\frac{\hbar Q^{2}}{2 m}\right)^{p} T_{p}(\omega)
$$

with the zero-phonon and one-phonon terms

$$
\begin{aligned}
& T_{0}(\omega):=\frac{1}{2 \pi \hbar} \int_{-\infty}^{\infty} \mathrm{d} t \mathrm{e}^{-\mathrm{i} \omega t}=\frac{1}{\hbar} \delta(\omega)=\delta(\hbar \omega), \\
& T_{1}(\omega):=\frac{1}{2 \pi \hbar} \int_{-\infty}^{\infty} \mathrm{d} t \mathrm{e}^{-\mathrm{i} \omega t} f(t)=\frac{g(\omega)}{\hbar \omega}(n(\omega)+1)=\frac{g(\omega)}{2 \hbar \omega}\left(\operatorname{coth}\left(\frac{\hbar \omega}{2 k_{b} T}\right)+1\right) .
\end{aligned}
$$


This prescription is often extended to poly-atomic and even coherent systems. Caution is again advised.

If the $Q$ transfer is very large then the scattering becomes a collision, under which the atoms recoil. We are in the regime of the impulse approximation (10.25)

$$
S(\vec{Q}, \omega)=\frac{1}{\sqrt{2 \pi \Delta^{2}}} \exp \left(-\frac{\left(\hbar \omega-E_{r}\right)^{2}}{2 \Delta^{2}}\right) .
$$

The dynamic response boils down to a Gaussian centred on the recoil energy

$$
E_{r}=\frac{\hbar^{2} Q^{2}}{2 m}
$$

with a width proportional to the Debye-Waller function

$$
\Delta^{2}=2\left(\hbar \omega_{0}\right)^{2} W(\vec{Q})
$$

It is important to distinguish between multi-phonons and anharmonic effects. Multi-phonons are present when the displacements become large on the length scale of $Q^{-1}$. They add strongly temperature dependent intensity between the one-phonon peaks. Large displacements may also lead the system to explore the anharmonic part of the potential. Anharmonicity of the potential influences all the aspects of the inelastic response. Notably it shifts the frequency of the phonon peaks and gives rise to an intrinsic width of the one-phonon response. These two aspects are captured by the damped harmonic oscillator. Its susceptibility is given by

$$
\chi_{(i, \vec{q})}^{\prime \prime}(\omega)=A(i, \vec{q}) \frac{4 \omega \Gamma(i, \vec{q})}{\left(\omega^{2}-\Omega(i, \vec{q})^{2}\right)^{2}+4 \omega^{2} \Gamma(i, \vec{q})^{2}} .
$$

\section{The particle beam}

Throughout this article we have described the neutron beam by stationary plane waves. This choice was clearly motivated by the necessity of manipulating interference phenomena. The stationary flux is, however, far from the reality of a low-intensity particle beam. Neutrons even for a powerful source arrive individually and at large intervals at the sample. This is in itself not a problem. The wave description remains valid if resolution is properly included in the formalism as we will see in Section 11.6. However, the wave description is unnecessarily cumbersome in situations where interference plays no role. The situation is well known in optics where it is dealt with by switching between light rays and light waves as adequate [32,44]. The theory of light rays is a useful add-on to the theory of light waves. It is applicable when all dimensions of the problem are short in units of the wave length. In the case of particle waves instead of rays we speak of ballistic transport. Like in optics ballistic transport may be used if the wave length is short in relation to the dimensions of the objects that are encountered along the trajectory. For slow neutrons this ratio is close to one when the trajectory leads through matter. ${ }^{129}$ This explains the necessity to work with waves in order to capture the interference effects of scattering. In the following we will see that this simple statement should be further nuanced.

In this section we will first develop the formalism of a particle beam and then integrate this formalism into the description of scattering experiments. We continue to describe the beam in a statistical manner, i.e. in terms of probability distributions. ${ }^{130}$ Statistics is imposed on one hand by quantum mechanics and on the other hand by the inherent uncertainties encountered in neutron production and in neutron transport. The statistical distributions used are also the basis for particle beam simulations [23]. They provide precise definitions of concepts such

\footnotetext{
${ }^{129}$ In condensed matter the dimensions of the objects encountered, which are the atoms, are of the order of a few $\AA$.

${ }^{130}$ For all questions related to statistical notions the reader is referred to text books (e.g. [47]).
} 
as monochromaticity and divergence. We will limit ourselves to the most general description of beam transport without going into any practical detail. Neutron optics is a subject of its own that is not within the scope of this article.

\subsection{Statistical description of the beam}

We have already seen in Section 1.4 that a localised particle should be described by a wave package. This package can be constructed via a superposition of plane waves ${ }^{131}$

$$
\psi(x, t)=\frac{1}{\sqrt{2 \pi}} \int w(k) \mathrm{e}^{\mathrm{i}(k x-\omega(k) t)} \mathrm{d} k .
$$

If the distribution of wave vectors $k$ is a Gaussian we obtain for $t=0$ a spatial width that is inversely proportional to the width in $k$

$$
(\Delta x)_{0}=\frac{1}{2} \frac{1}{(\Delta k)_{0}}
$$

Any corpuscular descriptions of the beam has to take these quantum uncertainties in momentum and position into account. In this spirit the vectors $\vec{r}$ and $\vec{p}(=\hbar \vec{k})$ will in the following be used to denote the average position and momentum of a wave package.

Wave packages are accessible to experimental investigation. Similar to an optical Michelson or Mach-Zender interferometer a neutron interferometer allows superimposing the neutron wave onto itself. This is achieved by division of the amplitude [49]. In the course of a Bragg reflection from a perfect crystal the wave function splits into two parts, one transmitted and the other reflected. By shifting one part in direct or phase space before recombining the parts it is possible to determine the shape of the wave package. Attention, this information is obtained via a series of measurements. Hence, the obtained result is a property of the beam and thus reflects all its imperfections. Figure 64 shows an example.

The neutrons extracted from the moderator can be considered an expanding gas. ${ }^{132}$ Since the neutrons neither interact with each other nor with a thermal bath this gas is not in equilibrium. From a classical point of view the state of the gas is specified by the distribution

$$
p(\vec{k} ;(\vec{r}, t))
$$

which gives the probability of detecting a particle with a propagation vector $\hat{k}=\vec{k} /|\vec{k}|$ and energy $E=\hbar^{2} k^{2} / 2 m$ at a moment $t$ in time at the location $\vec{r}$ in space. This distribution is also called the phase space density of the neutrons. In stationary mode of operation the phase space density does not explicitly depend on time and we can ignore the variable $t$. This is naturally no longer the case when we shape the beam in time.

The probability distribution should be normalised such that the integral

$$
N(t)=\int_{K} \int_{R} p(\vec{k} ;(\vec{r}, t)) \mathrm{d}^{3} k \mathrm{~d}^{3} r
$$

gives the number of neutrons that can be detected at a given moment in the real space element

$$
R=\Delta r_{x} \Delta r_{y} \Delta r_{z}
$$

\footnotetext{
${ }^{131}$ In an attempt to simplify things we limit ourselves again to one dimension.

${ }^{132}$ This description is based on the incoherent nature of the neutron beam starting with its production (attention the word incoherent here does not have the same significance as when used in the scattering context). It is, therefore, not adapted to the treatment of interference phenomena that can be observed by superimposing coherent neutron beams, e.g. produced with the help of beam splitting in a perfect crystal [49].
} 

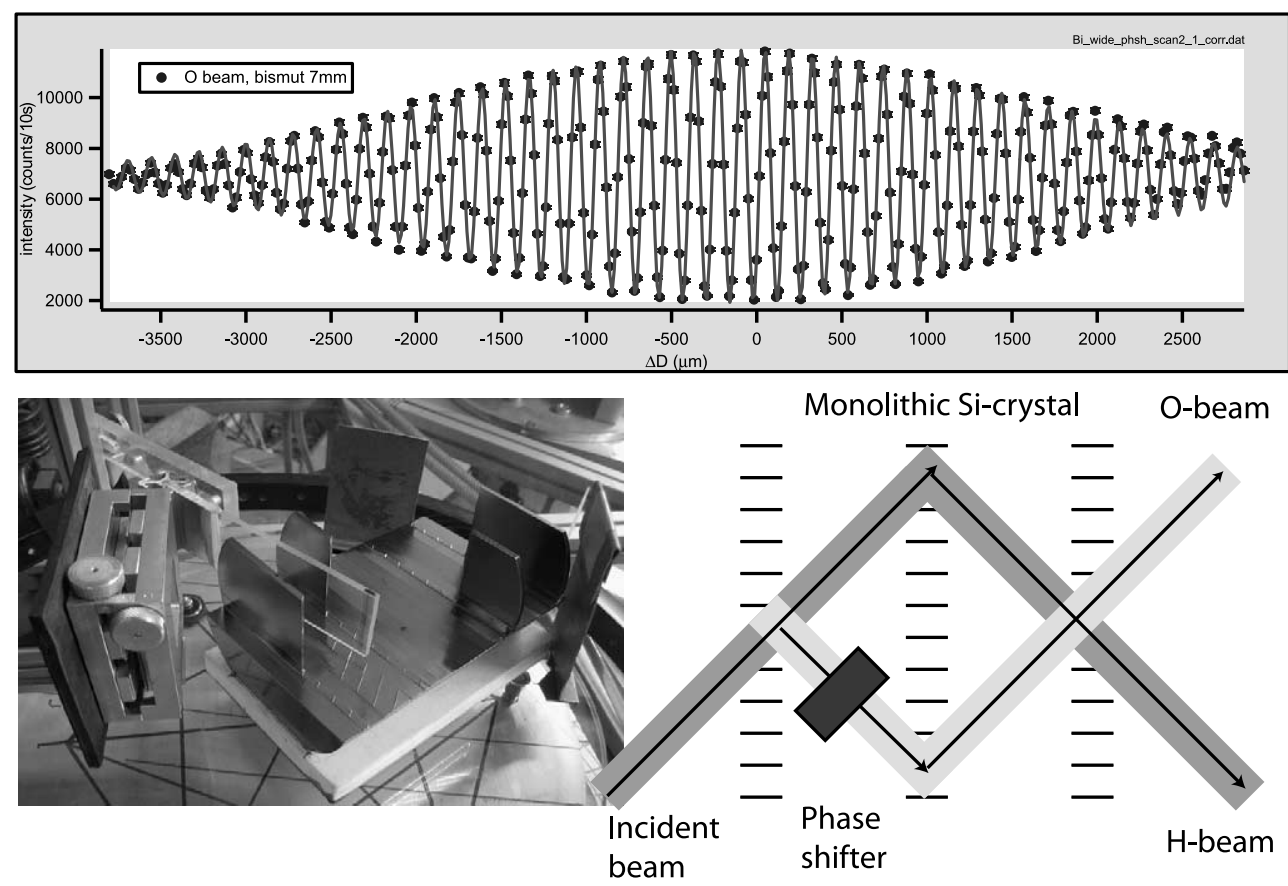

Fig. 64. Interference pattern for neutrons diffracted by a pure silicon crystal. In order to obtain perfectly aligned crystal planes over macroscopic distances the interferometer is cut from one single block of perfect silicon. By introducing material into one of the branches of the interferometer we can change the "optical path" $\Delta D$. This gives rise to the interference pattern shown. In complete analogy with the optical case (see Section 4) one oscillation is equivalent to a change in the optical path length of a wave length, which here is close to $2 \AA$. The wave packages passing through the interferometer possess an extension of about $100 \AA$. This defines the lateral coherence length of the beam. (Figures courtesy of H. Rauch.)

around $\vec{r}$, and that possess wave vectors within the element

$$
K=\Delta k_{x} \Delta k_{y} \Delta k_{z}
$$

around $\vec{k}$. The wave vector space is also called $K$ space. The Heisenberg uncertainty principle imposes that

$$
\Delta r_{\alpha} \cdot \Delta k_{\alpha}>1
$$

for $\alpha=x, y, z$. The $R$ and $K$ volumes thus cannot be arbitrarily small since then $p(\vec{k} ;(\vec{r}, t))$ would no longer be well-defined with respect to their uncertainties. A rigorous mathematical treatment would proceed via a coarse graining of phase space. First configuration space is rendered discrete by decomposing it into cubes of volume $L^{3}$ centred on points $\vec{r}$. The states of the particles found inside the cube are described by cell functions that correspond to plane waves inside the cube and vanish outside. Imposing periodic boundary conditions on the cell functions renders the corresponding wave vectors $\vec{k}$ discrete. Therefore, the decomposition of configuration space into finite volumes transforms the continuous momentum space to a lattice of discrete points. The phase space volume, i.e. the primitive cell of the phase space lattice containing one single state is given as $h^{3}$. As the product of position and momentum are not defined to better accuracy than given by the phase space cell it is assured that the Heisenberg uncertainty principle holds. Details of the coarse graining process can be found in reference [43]. In practice it is rare that the wave vector is better defined than $10^{-4} \AA .{ }^{133}$ Thus we do not take any risks with this definition as

\footnotetext{
${ }^{133}$ This is the value obtained for a backscattering instrument (see [52]) which is optimised for producing a highly monochromatic beam.
} 
long as we limit ourselves to respecting the above $\mu \mathrm{m}$ scale. Below this value $p(\vec{k} ;(\vec{r}, t))$ is considered not to vary significantly.

The real space density of neutrons is found by integrating the phase space density over the wave vector

$$
\rho_{n}(\vec{r}, t)=\int \mathrm{d}^{3} k p(\vec{k} ;(\vec{r}, t))
$$

The particle current that traverses a surface $S$ is determined by the surface integral

$$
I(t)=\int\left[\int_{S} p(\vec{k} ;(\vec{r}, t))\left(\vec{v}_{k} \cdot \mathrm{d} \vec{a}\right)\right] \mathrm{d}^{3} k=\frac{\hbar}{m} \int\left[\int_{S} p(\vec{k} ;(\vec{r}, t))(\vec{k} \cdot \mathrm{d} \vec{a})\right] \mathrm{d}^{3} k,
$$

with $\mathrm{d} \vec{a}$ the infinitesimal surface normal.

In the case of a rectangular surface $A$ perpendicular to $\hat{z}$ expression (11.5) reduces to

$$
I(t)=\frac{\hbar}{m} \int_{A} \mathrm{~d} x \mathrm{~d} y \int p(\vec{k} ;(x, y, t)) k_{z} \mathrm{~d}^{3} k .
$$

If the wave vectors, i.e. the velocities, are distributed homogeneously over the section $A$ we get a further simplification

$$
I(t)=A \frac{\hbar}{m} \int p(\vec{k} ; t) k_{z} \mathrm{~d}^{3} k=A \cdot \rho_{n} \cdot \bar{v}_{z} .
$$

In this final expression we have introduced the average velocity $\bar{v}_{z}$ of the beam along the direction $\hat{z}$. Dividing this expression by the surface area $A$ we get the current density. In an isotropic system, e.g. inside the moderator, the current density integrates to zero. There are as many particles traversing the surface in one direction as in the other. In this case it is usefull to introduce the notion of $f(u x \Phi(t)$

$$
\Phi(t)=\frac{\hbar}{m} \int p(\vec{k} ; t)\left|k_{z}\right| \mathrm{d}^{3} k=\rho_{n} \cdot \overline{\left|v_{z}\right|},
$$

$\Phi(t)$ is the number of particles traversing a unit surface in the direction $\hat{z}$ per second.

A well-defined beam is characterised by narrow probability distributions. E.g. an ideally monochromatic beam only contains neutrons with a single energy. Such a beam has an intensity close to zero. Hence it is preferable that the monochromaticity is not perfect and that the energies of the neutrons are distributed around the nominal value. $^{134}$

$$
E_{0}(\vec{r}, t)=\frac{1}{N} \int_{K} \int_{R} p(\vec{k} ;(\vec{r}, t)) \frac{\hbar^{2} k^{2}}{2 m} \mathrm{~d}^{3} k \mathrm{~d}^{3} r,
$$

with the standard deviation

$$
\Delta E(\vec{r}, t)=\sqrt{\frac{1}{N} \int_{K} \int_{R} p(\vec{k} ;(\vec{r}, t))\left(\frac{\hbar^{2} k^{2}}{2 m}-E_{0}\right)^{2} \mathrm{~d}^{3} k \mathrm{~d}^{3} r .}
$$

$\Delta E$ is the absolute energy width of the beam. The relative width is given by

$$
\frac{\Delta E}{E_{0}}=2 \frac{\Delta \lambda}{\lambda}
$$

\footnotetext{
${ }^{134}$ We will indicate the nominal values by the index zero and the variations (Full Width at Half Maximum) (FWHM) by the prefix $\Delta$.
} 
In general the widths evolves as a function of space and time.

In a similar way it is possible to introduce the direction of the nominal wave vector $\hat{k}_{0}(\vec{r}, t)$. The angular derivations with respect to this direction

$$
\Delta \hat{k}_{x}(\vec{r}, t), \Delta \hat{k}_{y}(\vec{r}, t), \Delta \hat{k}_{z}(\vec{r}, t)
$$

are called the divergences of the beam.

If we want to monitor the beam along its trajectory then we have to formally determine the distribution $p(\vec{k} ;(\vec{r}, t))$ along that trajectory. This is done by counting the number of neutrons in $R(\vec{r})$ that possess wave vectors inside $K(\vec{k})$. The counts will fluctuate about the mean value, which constitutes the ultimate goal of our measurement. Repeating the measurements allows us to reduce the uncertainties. Since the detection probability per unity of time is constant for non-interacting particles the statistical distribution corresponding to the detection is a Poisson distribution [47]. The error on the measurement will then be given by the square root of the number of detected events. Doubling the measurement time will lower the error by a factor of $\sqrt{2}$.

\subsection{Liouville theorem}

As we have already mentioned the neutron beam is not coherent due to the thermal fluctuations in the source. It, therefore, has to be described quantum mechanically by a mixed state (see the detailed discussion in Section 5.6). A mixed state is characterised by the fact that the expectation values of observables $A$ cannot be determined in the form

$$
\langle\hat{A}\rangle=\langle\psi|\hat{A}| \psi\rangle
$$

Let us assume that the neutron beam in its most general form has been prepared such that it can be described at a given moment in time, which we denote by $t_{0}$, by a density operator $\hat{\rho}\left(t_{0}\right)$. Let us further assume that for $t>t_{0}$ the beam evolves as a closed quantum system and that its interaction with the environment is governed by a Hamiltonian $\hat{H}(t)$. In that case quantum mechanics tells us that the time evolution of the density operator $\hat{\rho}(t)$ is given by the quantum Liouville equation

$$
\frac{\partial \hat{\rho}(t)}{\partial t}=-\frac{\mathrm{i}}{\hbar}[\hat{H}(t), \hat{\rho}(t)]
$$

To correctly apply the theorems that follow from this equation it is important to remember its condition of validity. A quantum system can be considered closed if it is not coupled to a larger quantum system. As we have seen in Section 5.6 such a coupling leads to effects of decoherence. ${ }^{135}$ It does not have to be isolated but is allowed to interact with classical systems. E.g. a beam of neutrons travelling down a guide and being reflected from the static potential of the guide coating can be considered a closed quantum system. It remains a closed quantum system when Bragg scattered by a crystal or when following adiabatically a static magnetic field. It ceases to be a closed quantum system if it interacts with microwave radiation or phonons triggering quantum mechanical transitions in the corresponding quantum fields.

We would like to stress that the above equation has been derived for the density operator in the Schrödinger picture. In this context we may recall the equation that governs the evolution of a Heisenberg operator $\hat{A}(t)$

$$
\frac{\partial \hat{A}(t)}{\partial t}=\frac{\mathrm{i}}{\hbar}[\hat{H}(t), \hat{A}(t)] .
$$

\footnotetext{
${ }^{135}$ The importance of the concept of decoherence has been recognised by awarding the 2012 Nobel Prize in physics jointly to Serge Haroche and David J. Wineland "for ground-breaking experimental methods that enable measuring and manipulation of individual quantum systems". These experiments are directly related to the subject of decoherence.
} 
The reader may wish to note the sign change with respect to the quantum Liouville equation. Knowing that the total time derivative of the density operator is calculated as

$$
\frac{\mathrm{d} \hat{\rho}(t)}{\mathrm{d} t}=\frac{\partial \hat{\rho}(t)}{\partial t}+\frac{\mathrm{i}}{\hbar}[\hat{H}(t), \hat{\rho}(t)]
$$

we conclude that

$$
\frac{\mathrm{d} \hat{\rho}(t)}{\mathrm{d} t}=0
$$

This conservation property is known as Liouville's theorem. It implies that phase space density is conserved. The classical version of Liouville's theorem is obtained by replacing the commutator by Poisson brackets

$$
\frac{\partial \rho(t)}{\partial t}=-\{\rho(t), H\}
$$

where $\rho(q, p ; t)$ is the classical distribution function in phase space that determines the probability $\rho(p, q ; t) d^{n} q d^{n} p$ that the system will be found at time $t$ in the infinitesimal phase space volume $d^{n} q d^{n} p$ with $q$ the canonical coordinates and $p$ the conjugated momenta of a Hamiltonian dynamical system. If we use the language of classical mechanics then we may state that particles that start together within a given phase space volume will remain forever united in that volume as they propagate along their respective phase space trajectories. The phase space volume may change in shape but it cannot be compressed. Liouville's theorem has important consequences for designing instruments. It e.g. implies that mirrors can focus a beam in space only at the expense of accepting higher beam divergence, i.e. a compression in configuration space always entails an expansion in particle momenta. To compress the phase space volume it is necessary to couple the beam to external quantum systems like e.g. a phonon bath. This fact is exploited in the case of thermal neutron moderation or in the case of the super thermal production of neutrons presented in Section 6.6.

\subsection{Optical elements and the transfer function}

To simplify the description of neutron transport we decompose the trajectory into optical elements. This approach is applicable provided the passage of the neutron from one element to the next is unidirectional. A typical example is a neutron guide. Neutrons enter the guide upstream and exit the guide downstream after a certain number of reflections. All exits are considered to be definitive. The description in terms of an optical element is even indispensable for all elements of an instrumental setup that require the use of wave theory in order to describe their functionality. This is e.g. the case for a crystal monochromator.

All optical elements in the neutron path change the distribution $p(\vec{k} ; \vec{r}, t)$. The interfaces between optical elements are denoted $S_{i}$ and $S_{f}$, respectively. The distributions before and after an optical element are given by

$$
p\left(\vec{k} ; \vec{r}_{i}, t_{i}\right), \quad \vec{r} \in S_{i}
$$

and

$$
p\left(\vec{k} ; \vec{r}_{f}, t_{f}\right), \quad \vec{r} \in S_{f}
$$

An optical element is fully characterised if we know its transfer function

$$
T\left(\vec{k}_{f}, \vec{k}_{i} ; \vec{r}_{f}, t_{f}, \vec{r}_{i}, t_{i}\right)
$$


that indicates the probability of a given incident current producing a neutron $\vec{k}_{f}$ at the exit. Mathematically the transfer function is defined by the relation

$$
p\left(k_{f} ; \vec{r}_{f}, t_{f}\right) \mathrm{d}^{3} k_{f}=\int T\left(\vec{k}_{f}, \vec{k}_{i} ; \vec{r}_{f}, t_{f}, \vec{r}_{i}, t_{i}\right)\left[\frac{\hbar}{m} \int_{S_{i}}\left(\vec{k}_{i} \cdot \mathrm{d} \vec{a}\right) p\left(\vec{k}_{i} ; \vec{r}_{i}, t_{i}\right)\right] \mathrm{d}^{3} k_{i}
$$

In stationary mode of operation we can neglect the $t$ dependence. For a homogeneous, monochromatic incident beam with zero divergence the current is given according to (11.7) as

$$
\frac{\hbar}{m} \int_{S_{i}}\left(\vec{k}_{i} \cdot \mathrm{d} \vec{a}\right) p\left(\vec{k}_{i} ; \vec{r}_{i}, t_{i}\right)=A \frac{\hbar}{m} k_{z} \rho_{n} \delta\left(\vec{k}_{i}-\vec{k}_{0}\right) .
$$

This leads to

$$
\begin{aligned}
p\left(k_{f} ; \vec{r}_{f}, t_{f}\right) \mathrm{d}^{3} k_{f} & =\rho_{n} A_{i} \frac{\hbar}{m} \int \mathrm{d}^{3} k_{i} T\left(\vec{k}_{f}, \vec{k}_{i} ; \vec{r}_{f}, t_{f}, \vec{r}_{i}, t_{i}\right) k_{z} \delta\left(\vec{k}_{i}-\vec{k}_{0}\right) \\
& =\rho_{n} A \frac{\hbar}{m} k_{z}^{0} T\left(\vec{k}_{f}, \vec{k}_{0}\right) \\
& =\rho_{n}\left(\vec{k}_{0}\right) A v_{z} T\left(\vec{k}_{f}, \vec{k}_{0}\right) \\
& =I_{0} T\left(\vec{k}_{f}, \vec{k}_{0}\right)
\end{aligned}
$$

This confirms that the transfer function acts as an operator that for every optical element associates to an incoming neutron $\vec{k}_{i}$ a probability of ending up as an outgoing neutron $\vec{k}_{f}$. This bears strong similarity with the scattering cross section. This is actually not surprising given that the sample may itself just be considered one of the optical elements of a beam line and that the transfer functions of other optical elements like monochromators have to be calculated using the concepts developed for scattering. This will be the subject of the next section. In order to describe the passage of the beam along a beam line composed of a series of optical elements we have to inject in sequential manner the output distribution of the $n$th element into the $n+1$ st element as input. This procedure allows to develop powerful simulation tools for spectrometer design [23].

\subsection{Small samples}

The sample is a priori an optical element among others. ${ }^{136}$ Its particularity in terms of neutron transport reside in the fact that

- the sample is in general sufficiently small in order for the incident flux to be considered homogeneous over the entire sample area;

- the detectors are placed sufficiently far from the sample so that the direction of the final wave vector can be considered coinciding with the direction of the detector position vector $\left(\hat{k}_{f}=\hat{r}_{f}\right)$.

This allows us to eliminate the variables $\vec{r}_{i}$ and $\vec{r}_{f}$. The influence of the sample on the beam can then de described by the simplified function

$$
f\left(\vec{k}_{f}, \vec{k}_{i}\right) \mathrm{d}^{3} k_{f}
$$

that indicates the probability with which an incident neutron $\vec{k}_{i}$ present in the sample is scattered per unity of time into the element $\mathrm{d}^{3} k_{f}$ about $\vec{k}_{f}$ in $K$ space.

\footnotetext{
${ }^{136}$ The Bragg scattering is used both for rendering the beam monochromatic and as a tool for determining crystal structures.
} 
If we are in the regime of the Born approximation the incident neutron flux is not significantly attenuated by the scattering. In practice this implies that our sample is sufficiently small. The density of neutrons $\rho_{n}$ is in this case homogeneous over the whole sample volume. The cross section is the probability per unity of time and per unity of incident flux of scattering a neutron $\vec{k}_{i}$ into $\mathrm{d}^{3} k_{f}$ around $\vec{k}_{f}$. We find formally

$$
\begin{aligned}
\mathrm{d}^{2} \sigma & =\frac{\rho_{n} V}{\Phi_{i}} f\left(\vec{k}_{f}, \vec{k}_{i}\right) \mathrm{d}^{3} k_{f} \\
& =\frac{\rho_{n} V}{\rho_{n} v_{i}} f\left(\vec{k}_{f}, \vec{k}_{i}\right) \frac{m}{\hbar^{2}} k_{f} \mathrm{~d} \Omega \mathrm{d} E_{f} \\
& =V \frac{m^{2}}{\hbar^{3}} \frac{k_{f}}{k_{i}} f\left(\vec{k}_{f}, \vec{k}_{i}\right) \mathrm{d} \Omega \mathrm{d} E_{f},
\end{aligned}
$$

where we have used

$$
\mathrm{d}^{3} k_{f}=k_{f}^{2} \mathrm{~d} k_{f} \mathrm{~d} \Omega=\frac{m k_{f}}{\hbar^{2}} \mathrm{~d} \Omega \mathrm{d} E_{f}
$$

and

$$
\Phi_{i}=\rho_{n} \frac{\hbar}{m}\left|\vec{k}_{i}\right|=\rho_{n} \frac{\hbar}{m} k_{i}
$$

$\rho_{n}$ is the instantaneous density of neutrons and $V$ is the sample volume. With ${ }^{137}$

$$
\frac{\mathrm{d}^{2} \sigma}{\mathrm{d} \Omega \mathrm{d} E_{f}}=N \frac{\sigma}{4 \pi} \frac{k_{f}}{k_{i}} S(\vec{Q}, \omega)
$$

we get the identity

$$
f\left(\vec{k}_{f}, \vec{k}_{i}\right)=\frac{N}{V} \frac{\hbar^{3}}{m^{2}} \frac{\sigma}{4 \pi} S(\vec{Q}, \omega) .
$$

Hence we find, as expected, the scattering function $S(\vec{Q}, \omega)$. We see in particular that $f\left(\vec{k}_{f}, \vec{k}_{i}\right)$ in the Born approximation only depends on $\vec{Q}$ and $\omega$.

\subsection{Finite size samples and multiple scattering}

The goal of a neutron scattering experiment is to determine the scattering function $S(\vec{Q}, \omega)$. It is the scattering function that gives us, as we have learned in Section 8.2, information about the existence of correlations in the sample. Determining $S(\vec{Q}, \omega)$ is in practice equivalent to the measuring the distributions of neutrons $p(\vec{k} ; \vec{r}, t)$ after the scattering. The precision of the measurement will depend on (i) the preparation of the incident beam and its flux as well as (ii) the detected intensity and the precision of the detection process. The discussion of scattering has up to now been based on the Born approximation. This approach is justified if the scattered wave is a small perturbation of the incident beam and as a consequence does not have to be considered itself a source for secondary scattering

\footnotetext{
${ }^{137}$ Even though it breaks with the general philosophy of this article we will here limit ourselves to monatomic systems. Working with monatomic systems allows us to extract the scattering lengths from the scattering function in the form of the pre factor $\sigma / 4 \pi$. This again makes it possible to normalise the scattering function to the number of scatterers $N$ (for the definitions and the discussion of normalisation of the scattering function see Section 5.9). The generalisation to poly-atomic systems is not difficult to make.
} 
processes. The Born approximation, therefore, imposes that we should work with weakly scattering samples. This constraint translates into a real dilemma for the experimentalist. The neutron flux is fixed, i.e. it is determined by the source as well as the efficiency of the extraction, the transport and the shaping of the neutron beam. This flux is often weak even at the most powerful neutron sources. ${ }^{138}$ In addition, the signal looked for is often not found in the predominant scattering channel. The only variable of the experimental setup that allows the researcher to improve the data statistics without deteriorating the data quality, e.g. in terms of resolution, is often the sample size. Unfortunately, as we will see now, by increasing the sample volume and thereby leaving the range of validity of the Born approximation undesirable second order effects will be produced. These effects have to be put into the balance with the expected gain in statistics.

It is essential to understand these second order effects if we want to eliminate the risk of misinterpreting the experimental data. Working with higher orders of the Born series is in principle possible. However, in practice it turns out a forbiddingly heavy process. Intuitively it should be possible to describe the scattering process as a random walk with the particle propagating freely between successive collisions. For this it is sufficient to mentally break up the sample into regions sufficiently small so that the Born approximation holds. The Born scattering produced by such a region is giving the characteristics of a "collision" in the sense of a transfer function.

Even if this approach is much simpler than working with higher order terms of the Born series it is not without pitfalls. At first glance it may even seem paradoxical. We will dwell for a moment on this statement.

All along our discussion we have underlined that scattering is an interference process. But interference is just the type of phenomena that cannot be described within a corpuscular approach. We recall: The neutron will as a matter wave generate spherical wavelets along its path. These wavelets are characterised by a well-defined phase relation among them. The superposition of these wavelets gives rise to the interference effects. This is the Huygens-Fresnel principle applied to matter waves. From these interferences we derive the correlations existing between scatterers in space and time. The typical example is the Bragg scattering from a single crystal that allows us to determine the structure of the sample. The atomic planes scatter the neutrons of a given wave length along specific directions. If the crystal is very pure these directions are very well defined. Experimentally we can, e.g. observe that Bragg scattering depends on the macroscopic dimensions of a pure crystal such as silicon. The interference effects hence extend over several millimetres (see Figs 66 and 67). In this case we are very far from the validity of the Born approximation. The neutron in this case has to be defined by the superposition of the incident and scattered waves, which due to the periodicity of the lattice take the form of Bloch waves. ${ }^{139}$ This is the foundation of dynamic scattering theory that, as already mentioned in Section 9.9, will not be discussed in this article. However, we would like to emphasise that these interference phenomena over very long distances are observable even for incident wave packets that have a much shorter spatial extension. Hence it is necessary to resist the temptation to consider the size of the incident wave package an upper bound to the size of the interaction zone. It is the capacity of the perfect crystal to select under the given experimental conditions a narrow band of wave vectors from the incident wave package that allows for the observation of interference effects over macroscopic distances.

We can summarise this short discussion as follows: when scattered the neutron interacts with the sample in its totality. In other words, scattering is not a local phenomena. How can we retain under this condition the Born approximation, which we had identified as the basis for the interpretation of neutron scattering, when we are for experimental reasons obliged to work with macroscopic samples? And how can we talk of a mean free path and collision if the scattering is not a local event?

The solution comes, luckily for the experimentalist, from the fact that the vast majority of samples are not perfect. The extreme case is the ideal gas where there are no correlations between the positions of the different atoms. As we have seen in Section 8.10 the scattering can, in this case, be described as the ensemble of collisions with the individual atoms. This characteristic does not make the ideal gas very attractive for neutron scattering experiments. In liquids the correlation length is limited to a few neighbouring atomic layers. The interferences effects due to the

\footnotetext{
${ }^{138}$ It is often said that neutron scattering is a flux limited experimental technique. This is less true for the last generation of spectrometers at powerful sources.

${ }^{139}$ This is the wave function imposed by the symmetry for a particle in a periodic potential.
} 

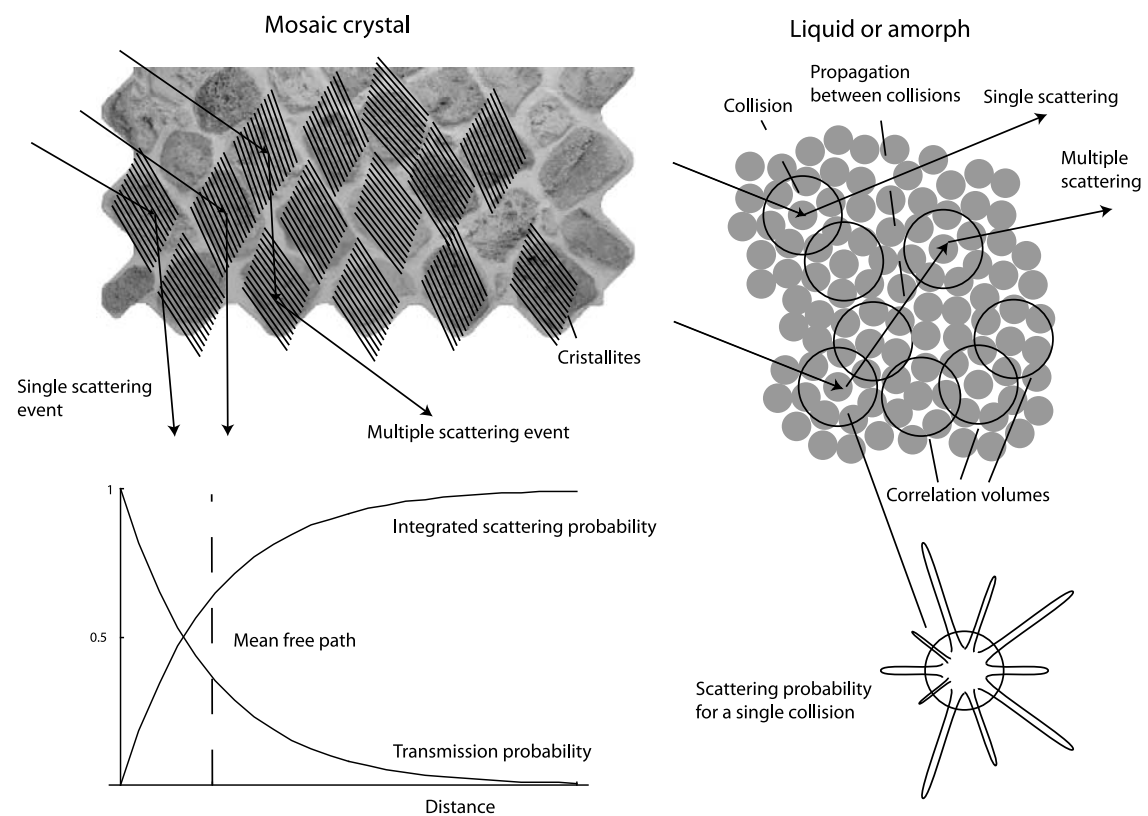

Fig. 65. Schematic representation of neutron scattering from a finite size sample. During the passage the neutron will experience a number of collisions. Each one of them will change the wave vector of the neutron (either the direction, or the energy or both at the same time). The probability of these events is calculated in the Born approximation over the correlation volume that surrounds the location of the collision. After a collision the neutron travels unencumbered a distance $d$. This distance is related to the integrated scattering probability. If the scattering probability is high the collisions are frequent and hence the mean free path is short. In the case of a mosaic crystal the scattering is highly directional. The Bragg lines are found by treating the crystallites in the Born approximation, given that the linear size of the crystallites is smaller than the primary extinction length. In the case of a liquid or an amorphous system the correlation volumes are smaller. Opposite to crystals all points are equivalent given that the liquid is isotropic and homogeneous. In the two cases the mean free path should be much longer that the dimensions of the crystallites or the correlation volumes in order to apply the concept of scattering by collision.

superposition of the scattered wavelets fade out quite quickly with the distance between the scatterers. This allows us to talk about a correlation volume (see Fig. 65). The correlation volume is a property of the sample. It is not to be confounded with the coherence volume that we have discussed in Section 4.9 and which is a property of the neutron beams. In the ideal case the coherence volume is made to match the correlation volume.

The correlation volume of a perfect crystal is macroscopic. In the Born approximation each plane that is added refines the scattering pattern by sharpening the Bragg peaks. We have explicitly demonstrated the validity of this statement for optical lattices (see Section 4.4). Saturation takes place when all of the initial intensity has passed into the scattered wave. At this point the roles are swapped and the scattered wave becomes the incident wave. By enlarging the crystal thickness further the neutron current will again be redirected, this time in the direction of the original incident wave. This "beating" phenomena, that produces the interference fringes already mentioned above is known as the Pendellösung [49]. Its experimental realisation is shown in Figs 66 and 67. The penetration depth at which the incident wave fades is called the primary extinction length. The Born approximation is logically no longer valid for crystals larger than the primary extinction length.

The bulk of the crystals that are found on neutron diffractometers are far from being ideal. ${ }^{140}$ In particular, they have dislocations (see Fig. 65), i.e. the atomic planes are perfectly aligned only within the crystallites. We are speaking of mosaic crystals. The interference effects are per definition very pronounced within a crystallite. The ensemble of crystallites will on the other hand broaden the Bragg peaks by adding reflections at slightly different

\footnotetext{
${ }^{140}$ Crystalline matter is normally not present in single crystalline form. A notable exemption are the huge single crystals of silicon that are grown for the electronic industry. A poly-crystal is composed of several single crystals, that we call crystallites.
} 


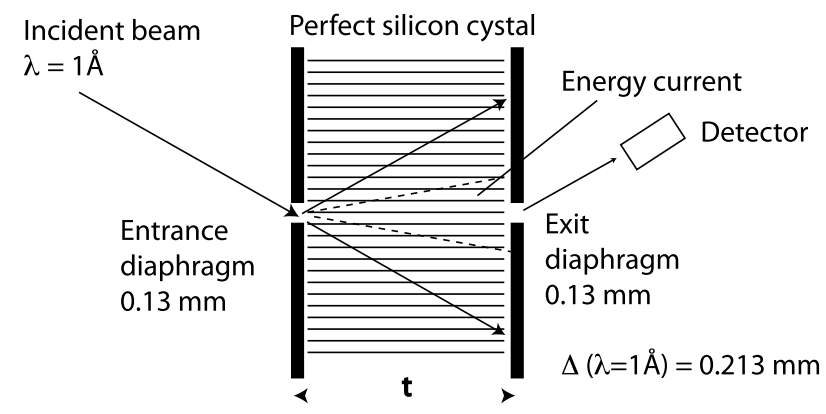

Interference fringes as a function of the position of the exit diaphragm

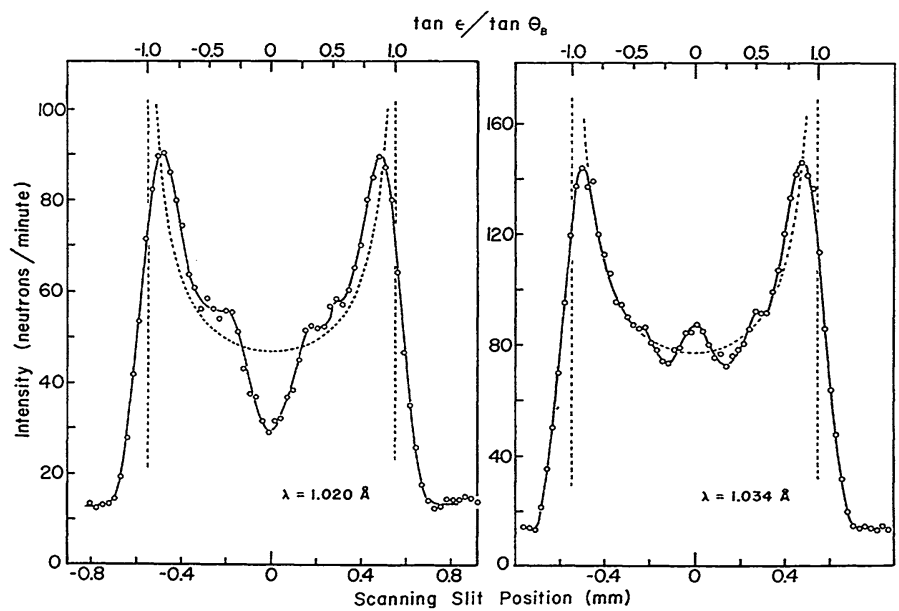

Fig. 66. Schematic illustration of the Shull experiment, which shows the existence of fringes in the Bragg scattering of neutrons (Pendellösung) [59]. A monochromatic neutron beam is submitted to a Bragg reflection by a perfect silicon crystal. For perfect incidence the energy current is directed parallel to the crystalline planes. For a divergent beam the transmission cone can expand until it reaches $\pm \Theta_{\mathrm{Bragg}}$. In order to avoid that this expansion obscures the interferences a good spatial resolution is obtained with slits.

scattering angles and wave lengths. We can thus identify the correlation volume in a mosaic crystal to a good approximation with the size of the volume of the crystallites. If this size is inferior to the extinction length then the Born approximation is applicable at the interior of a crystallite, this facilitates the formalism considerably. At this point we would like to emphasise a fundamental difference between mosaic crystals and liquids or amorphous systems. The liquid is homogeneous. Hence around any point $\vec{r}$ we can draw a sphere that contains always the same correlation volume. For a mosaic crystal the surroundings are completely different depending on whether the point chosen is at the centre of the crystallite or if it is close to one of the boundaries. This inhomogeneity generates particular scattering effects and should be taken into account when analysing the data.

From an practical point of view we can thus only distinguish with difficulty between (i) a scattering process localised within in the correlation volumes and free propagation of the scattered particle from one volume to the next and (ii) a scattering process over the whole sample volume treated with higher order terms in the Born series (note the similarities between Figs 18 and 65). It is, therefore, the final size of the correlation volumes that allows us to imagine the scattering as a process of multiple collisions (see Fig. 65).

The scattering rates and the propagation should be calculated in the Born approximation for the correlation volumes (always under the condition that the linear dimensions of these volumes are smaller than the extinction length). In order to describe the propagation we will first of all introduce the notion of the mean free path, which 


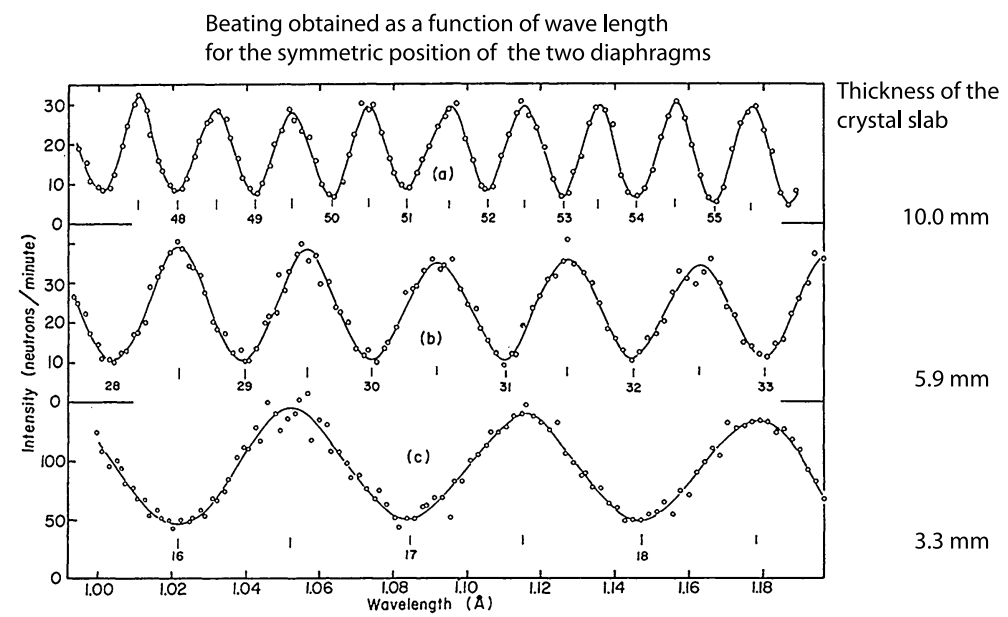

Fig. 67. Same as Fig. 66. The detected reflected flux varies periodically with the thickness of the crystal for a given wave length or in perfect analogy with the wave length for a given thickness of the slab. In silicon for the (111) reflection the Pendellösung period is $\Delta=0.213 \mathrm{~mm}$. The period $\Delta$ can be identified with the primary extinction length. Each fringe corresponds to an extra shift in wave length between the coherent wave packages that propagate in the crystal [59].

is the mean distance that the neutron travels between two collisions. According to (11.24) the probability per unity of time that a neutron with $\vec{k}_{i}$ is scattered is equal to

$$
f\left(\vec{k}_{i}\right)=\int f\left(\vec{k}_{f}, \vec{k}_{i}\right) \mathrm{d}^{3} k_{f}
$$

Thus $f\left(\vec{k}_{i}\right)^{-1}$ is the average time that a neutron spends in the sample without being scattered. During this time the neutron will be displaced by

$$
l\left(\vec{k}_{i}\right)=\frac{v\left(\vec{k}_{i}\right)}{f\left(\vec{k}_{i}\right)} .
$$

$l\left(\vec{k}_{i}\right)$ is the mean free path that we were looking for. Following (11.31) and using expression (11.28)

$$
\begin{aligned}
l\left(\vec{k}_{i}\right)^{-1} & =\frac{1}{v\left(\vec{k}_{i}\right)} \int f\left(\vec{k}_{f}, \vec{k}_{i}\right) \mathrm{d}^{3} k_{f} \\
& =\frac{m}{\hbar k_{i}}\left[\frac{N}{V} \frac{\hbar^{3}}{m^{2}} \frac{\sigma}{4 \pi} \int S(\vec{Q}, \omega) \mathrm{d}^{3} k_{f}\right] \\
& =\frac{N}{V} \frac{k_{f}}{k_{i}} \frac{\sigma}{4 \pi} \int S(\vec{Q}, \omega) \mathrm{d} \Omega \mathrm{d} E_{f} \\
& =\frac{N}{V} \sigma_{\text {tot. }} .
\end{aligned}
$$

The mean free path can, therefore, be calculated from the total cross section. 
The number of neutrons ${ }^{141} n_{s}(z)$ scattered at a point $z$ along the trajectory is proportional to the scattering rate (11.34) as well as to the number of neutrons $n_{t}(z)$ that have been transmitted up to $z$

$$
\mathrm{d} n_{s}(z)=n_{t}(z) \cdot \frac{1}{l} \cdot \mathrm{d} z=n_{t}(z) \cdot \frac{N \sigma_{\text {tot }}}{V} \cdot \mathrm{d} z
$$

with

$$
\mathrm{d} n_{t}(z)=-\mathrm{d} n_{s}(z)
$$

Equation (11.35) has a simple solution (see Fig. 65)

$$
n_{t}(z)=n_{t}(0) \exp \left(-\frac{z}{l}\right)
$$

for the number of neutrons transmitted up to $z$. Thus the number of scattered neutrons up to $z$ is given by

$$
n_{d}(z)=n_{i}(0)\left(1-\exp \left(-\frac{z}{l}\right)\right) .
$$

In the case of very low scattering

$$
z \ll l=\frac{N V}{\sigma_{\text {tot }}}: \quad \frac{n_{d}(z)}{n_{i}(0)}=\frac{\sigma_{\text {tot }}}{N V} z .
$$

Once the neutron is propagated up to a point $\vec{r}$ it will, with a certain probability, undergo a collision. Following this collision the neutron will change its direction and energy. The resulting distribution of wave vectors can be calculated via $f\left(\vec{k}_{f}, \vec{k}_{i}\right)(11.31)$. After the collision the neutron can exit the sample or be scattered a second time and so forth. This is called multiple scattering. Multiple scattering is always an annoyance. It destroys the connection that exists between the measured signal and two-point correlation functions. Multiple scattering could in principle open a way to three point (or even higher) correlation functions. However this has so far not been demonstrated to work in practice (see Section 8.5).

\subsection{Resolution}

The scattering functions define the transfer function of the sample according to (11.31). The intensity registered at the detector per energy interval can thus be obtained via (see also [18])

$$
I\left(E_{f}\right) \mathrm{d} E_{f} \propto \iint p\left(\vec{k}_{i}\right) \cdot S(\vec{Q}, \hbar \omega) \cdot p\left(\vec{k}_{f}\right) \mathrm{d}^{3} k_{i} \mathrm{~d}^{3} k_{f}
$$

The distribution $p\left(\vec{k}_{i}\right)$ has already been introduced. It gives the probability that a neutron with wave vector $\vec{k}_{i}$ is first produced at the source and then finds its way through the optical elements to the sample. The distribution $p\left(\vec{k}_{f}\right)$ describes in perfect analogy the probability of a neutron leaving the sample and finding its way to the detector. It

\footnotetext{
${ }^{141}$ To make sure to distinguish between the number of neutrons $n$ and the number of scatterers $N$ we use small and capital letters, respectively.
} 
can be obtained by creating a virtual population of neutrons at the location of the detector. This population should reflect the detector characteristics concerning resolution in time and space and efficiency of detection. The detector thus plays the role of an inverse source. These neutrons are then propagated back to the sample. They retrace exactly the path that the scattered neutron have taken. ${ }^{142}$

In order to facilitate the discussion the distributions $p\left(\vec{k}_{i}\right)$ and $p\left(\vec{k}_{f}\right)$ are assumed to be homogeneous over the whole sample volume and time independent.

The expression (11.40) can then be written as a convolution [17]

$$
I\left(E_{f}\right) \mathrm{d} E_{f} \propto \iint R\left(\vec{Q}-\vec{Q}_{0}, \hbar \omega-\hbar \omega_{0}\right) \cdot S(\vec{Q}, \hbar \omega) \mathrm{d}^{3} Q \mathrm{~d} \hbar \omega,
$$

with the resolution function defined as

$$
R(\vec{Q}, \hbar \omega)=\iint p\left(\vec{k}_{i}\right) \cdot p\left(\vec{k}_{f}\right) \cdot \delta\left(\vec{Q}-\left(\vec{k}_{i}-\vec{k}_{f}\right)\right) \cdot \delta\left(\hbar \omega-\frac{\hbar^{2}}{2 m}\left(k_{i}^{2}-k_{f}^{2}\right)\right) \mathrm{d}^{3} k_{i} \mathrm{~d}^{3} k_{f} .
$$

$R(\vec{Q}, \hbar \omega)$ measures the number of combinations of $\vec{k}_{i}$ and $\vec{k}_{f}$ that are compatible with the conditions $\vec{k}_{i}-\vec{k}_{f}=\vec{Q}$ and $E_{i}-E_{f}=\hbar \omega$. The variables $\vec{Q}_{0}$ and $\hbar \omega_{0}$ denote the central values of $\vec{Q}$ and $\hbar \omega$. They constitute the centre of gravity of the resolution function, around which the measurement is performed. In order to extract the quantity $S\left(\vec{Q}_{0}, \hbar \omega_{0}\right)$, which finally is what interests us, the measurement should be de-convoluted with the resolution function. In particular the variation in the total number of neutrons in the primary and secondary beam has to be corrected for. These quantities are formally described by the phase space volumes

$$
V_{i}=\int p\left(\vec{k}_{i}\right) \mathrm{d}^{3} k_{i} \quad \text { and } \quad V_{f}=\int p\left(\vec{k}_{f}\right) \mathrm{d}^{3} k_{f}
$$

The de-convolution is a delicate mathematical procedure. In the case where the aim of the measurement consists in verifying a model it is preferable to calculate first the scattering function from the model and then to convolute the result with the resolution function. Ideally we would want to work with as good a resolution as possible, i.e. with resolution functions that sharply peak at $\left(\vec{Q}_{0}, \hbar \omega_{0}\right)$. This implies that the distributions $p\left(\vec{k}_{i}\right)$ and $p\left(\vec{k}_{f}\right)$ are narrow, which leads to a small intensity (see (11.41)). Resolution thus has often to be traded in for flux. A welldesigned spectrometer will give the experimentalist the possibility of adapting the resolution to the requirements of the measurements while sacrificing as little flux as possible.

To give a concrete idea of phase space volumes and resolution we will take a closer look at the practical aspects of Bragg scattering. Bragg scattering was introduced in Sections 4 and 9.8. It is at the basis of crystal monochromators. In reciprocal space the condition for Bragg scattering can be formulated as

$$
\begin{aligned}
& \vec{\tau}=\vec{k}_{f}-\vec{k}_{i}, \quad\left|\vec{k}_{i}\right|=\left|\vec{k}_{f}\right| . \\
& \vec{\tau}_{h k l}=h \vec{a}_{1}^{*}+k \vec{a}_{2}^{*}+l \vec{a}_{3}^{*}
\end{aligned}
$$

denotes a reciprocal lattice vector perpendicular to the lattice planes. Its norm is directly related to the distance $d_{h k l}$ between the planes

$$
\left|\vec{\tau}_{h k l}\right|=2 \pi / d_{h k l} .
$$

Bragg scattering takes places when the vectors $\vec{k}_{i}, \vec{k}_{f}$ and $\vec{\tau}$ form an equilateral triangle with $\tau$ as the basis. As seen in Fig. 68 there is a strong correlation between the length of the $\vec{k}$ vector and the angle it subtends with $\vec{\tau}$. In

\footnotetext{
${ }^{142}$ This is possible since the equations that governs the neutron propagation are symmetric under time reversal in the absence if dissipation.
} 

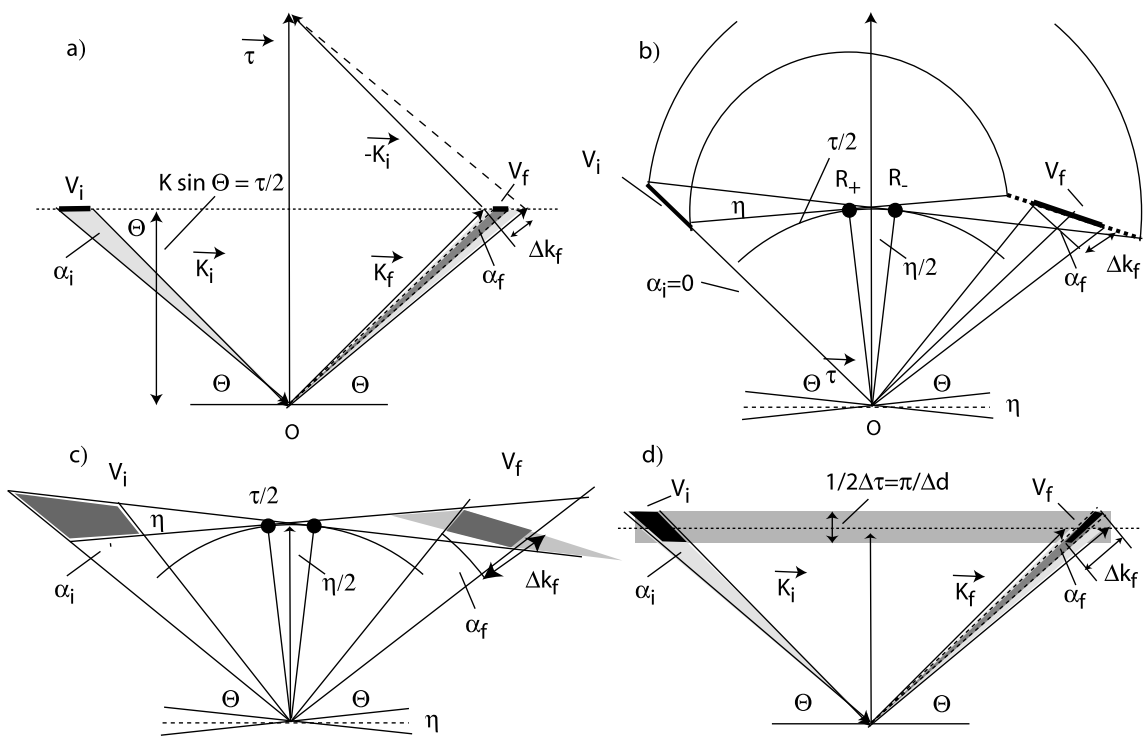

Fig. 68. Description of Bragg scattering in reciprocal space. The conditions for Bragg scattering are fulfilled if the projection of the incident and diffracted wave vectors onto the reciprocal lattice vector $\vec{\tau}$ equal $k \sin \Theta=\tau / 2$. This constraint establishes an exact one-to-one correspondence between $\vec{k}_{i}$ and $\vec{k}_{f}$. The incident beam has necessarily a finite divergence $\alpha_{i}$. In the case of a polychromatic beam this divergence results in a divergent scattered beam. This is indicated by the light grey regions in figure (a). The divergence of the incident beam is translated into a spectral distribution $\Delta k_{f}$ of the diffracted beam. The spectral width can be controlled by reducing the divergence of the diffracted beam with the help of a collimator (the dark grey regions in figure (a)). Thus the phase space volume $V_{i}$ translates into a phase space volume $V_{f}$ defined by the intersection of this region with the line $\tau / 2$. It is immediately obvious that for a given divergence the spectral resolution (monochromaticity) increases with the Bragg angle $\Theta$ and, therefore, depends crucially on the lattice spacing $d$. For a mosaic crystal the orientation of the crystallites varies statistically. The corresponding distribution can be describe by the parameter $\eta$. In our scheme mosaicity corresponds to turning the diagram in figure (a) by $\pm \eta / 2$ around the origin $O$. In figure (b) we show how the element $V_{f}$ can be obtained from $V_{i}$ for an incident beam without divergence. One constructs the images of the shortest and longest $\vec{k}_{i}$ vectors with respect to the lines $O-R_{-}$and $O-R_{+}$, respectively. Note that $V_{f}$ will be inclined with respect to $\vec{k}_{f}$. In figure (c) we show the general situation of an incident beam with divergence $\alpha_{i}$ being diffracted by a mosaic crystal into a final beam of controlled divergence $\alpha_{f}$. The volume $V_{f}$ reflects the final intensity that can be obtained provided that the volume $V_{i}$ is fully filled. An experimental configuration is optimal if $V_{f}$ is maximal for the required $p\left(\vec{k}_{f}\right)$, i.e. for the required $\vec{k}_{f}$ and $\Delta k_{f}$. Figure (d) shows the situation of a crystal with a distribution in lattice spacing $d$. A variation in $d$ can be obtained via a gradient in the chemical composition or via thermal or mechanical treatment introducing distortion.

the case of an ideal crystal only the vectors $\vec{k}_{i}$ and $\vec{k}_{f}$ of a fine slice of phase space can participate in the Bragg scattering. This leads to a tiny diffracted intensity. Only by introducing a distribution of crystallites (mosaic) or of lattice spacing (distortions, displacements) we obtain non-zero phase space volumes and hence real diffracted intensities. ${ }^{143}$

In order to better understand how a distribution of neutrons influences the measurement we will look at the resolution at a slightly different angle. As we have seen the scattering of a neutron can be described in the Born approximation by a superposition of spherical waves

$$
\psi_{\text {out }}(\vec{r}) \propto \sum_{l} b_{l}\left[\frac{\mathrm{e}^{\mathrm{i} k_{f} \cdot\left|\vec{r}-\vec{r}_{l}\right|}}{\left|\vec{r}-\vec{r}_{l}\right|} \cdot \psi_{\text {in }}\left(\vec{r}_{l}\right)\right] \propto \sum_{l} b_{l}\left[\int p\left(\vec{k}_{i}\right) \cdot \frac{\mathrm{e}^{\mathrm{i} k_{f} \cdot\left|\vec{r}-\vec{r}_{l}\right|}}{\left|\vec{r}-\vec{r}_{l}\right|} \cdot \mathrm{e}^{\mathrm{i} \vec{k}_{i} \vec{r}_{l}} \mathrm{~d}^{3} k_{i}\right]
$$

with $\vec{r}_{l}$ being the position of the $l$ th scatterer and $b_{l}$ its scattering length. This is the Huygens-Fresnel principle applied to neutron scattering (see Section 4). The source of the spherical waves is the incident wave function $\psi_{\text {in }}$,

\footnotetext{
${ }^{143}$ In reality Bragg scattering is never ideal due to the extinction effects we already have mentioned.
} 
which in order to take the beam characteristics into account has to be expressed as a weighted superposition of plane waves. As in the case of optical diffraction the scatterers that are found along the wave front, i.e. have positions, for which $\vec{k}_{i} \cdot \vec{r}_{l}$ is constant, will scatter in phase. ${ }^{144}$ Due to the distribution in direction and wave length of $\vec{k}_{i}$ (and $\vec{k}_{f}$ ) the phase coherence is perturbed and the interference patterns are blurred when the distance between the scatterers increases. The resolution function can thus be considered the image of the coherence volume (see Section 4) in reciprocal space [24]. The coherence volume is in a certain way the field of view of the instrument in real space.

The resolutions $\Delta Q_{i}, i=x, y, z$ and $\hbar \Delta \omega$ as well as the coherence volumes increase considerably with the wave length. This is not really surprising due to the fact that the definition of a beam is normally done with respect to its nominal values. As a consequence it is the relative resolutions $\Delta Q / Q$ and $\Delta \omega / \omega$ that are similar over a wide range of instruments. Longer wave length, however, limit the range of accessible $(\vec{Q}, \hbar \omega)$ points. For example, in the case of elastic scattering, where $\left|\vec{k}_{i}\right|=\left|\vec{k}_{f}\right|$, the maximal $Q$ is $2 \vec{k}_{i}$ for $\vec{k}_{f}=-\vec{k}_{i}$. By reducing $\left|\vec{k}_{i}\right|$ we limit the dynamic range of the experimental setup, this means we reduce the field of view in reciprocal space. Large fields of view in real space and in reciprocal space are thus conflicting requirements.

\section{Acknowledgements}

I would like to thank Ursula Bengaard Hansen for having provided a translation into English of the original French article [53] that is at the basis of this work. This facilitated my task enormously. Stéphane Rols, Françoise Leclercq-Hugeux, Andrea Orecchini, Mohamed Zbiri and Alexander Schober for proof reading of the French original. Colin Carlile deserves particular thanks for the extensive proof reading of this article.

The way the theory of non-relativistic scattering is presented here has been hugely inspired by my own teachers in quantum mechanics and solid state physics. Writing this article I relied heavily on the lecture notes of courses on theoretical physics given by D. Strauch, U. Schröder and U. Rössler at the University of Regensburg.

Of equal importance were the monographs on quantum physics by C. Cohen-Tannoudji, B. Diu and F. Laloë, and by O. Hittmair. Concerning the theory of neutron scattering itself I have learned it, as the reader will have certainly realised, from the excellent books of Lovesey and Squires.

\section{References}

[1] P. Ageron, Nuclear Instr. Meth. A 284 (1989), 197.

[2] N.W. Ashcroft and N.D. Mermin, Physique des Solides, EDP Science, 2002 (Solid State Physics, Saunders College, 1975).

[3] E. Balcar and S.W. Lovesey, J. Phys. C: Solid State Phys. 19 (1986), 4605.

[4] A.C. Barnes, H.E. Fischer and P.S. Salmon, J. Phys. IV France 111 (2003), 59.

[5] M.M. Bredov, B.A. Kotov, N.M. Okuneva, V.S. Oskotskii and A.L. Shak-Budagov, Sov. Phys. Solid State 9 (1967), 214.

[6] J.M. Carpenter and C.A. Pelizzari, Phys. Rev. B 12 (1975), 2391.

[7] J.M. Carpenter and C.A. Pelizzari, Phys. Rev. B 12 (1975), 2397.

[8] J. Chadwick, Nature 129 (1932), 312.

[9] J. Chadwick, Proc. Roy. Soc. A 136 (1932), 692.

[10] C. Cohen-Tannoudji, B. Diu and F. Laloë, Quantum Mechanics, Vols 1, 2, Hermann, 2007. (Quantum Mechanics, Vols 1, 2, Wiley VCH, 1977.)

[11] J. Daillant and A. Gibaud, X-ray and Neutron Reflectivity: Principles and Applications, Springer-Verlag, Berlin, 1999.

[12] C.G. Darwin, Phil. Mag. 27 (1914), 315, 675.

[13] J. Dawidowski, F.J. Bermejo and J.R. Granada, Phys. Rev. B 58 (1998), 706-715.

[14] P.G. de Gennes, Physica 25 (1959), 825.

\footnotetext{
${ }^{144}$ As the scattered wave function $\psi_{\text {out }}$ is observed in the detector we must in a rigorous formalism include in the expression (11.45) the shaping of the scattered beam, i.e. the probability that a neutron with wave vector $\vec{k}_{f}$ finds its way into the detector. This would bring us to an expression like (11.41). In the case of optical diffraction we are confronted with a similar problem. The diffracted beam has to be projected onto the observation plane. The lenses that are used fort this purpose will introduce distortions in the image (see Fig. 19).
} 
[15] P. Debye, Interferenz von Röntgenstrahlen und Wärmebewegung, Ann. d. Phys. 348 (1913), 49 (in German).

[16] A.-J. Dianoux and G. Lander (eds), Neutron Data Booklet, Institut Laue-Langevin, Grenoble, France, 2002.

[17] B. Dorner, Acta Cryst. A 28 (1972), 319.

[18] B. Dorner, Journal of Neutron Research 13 (2005), 267.

[19] G. Eckold, Symmetry aspects of excitations, phonons, in: International Tables of Crystallography, Vol. D, Chapter 2.1.

[20] P.A. Egelstaff, Adv. Phys. 11 (1962), 203.

[21] S.A. Egorov and J.L. Skinner, Chem. Phys. Lett. 293 (1998), 469

[22] B. Fåk and B. Dorner, Physica B 234-236 (1997), 1107.

[23] E. Farhi, V. Hugouvieux, M.R. Johnson and W. Kob, J. Comp. Physics 228 (2008), 5251.

[24] J. Felber, R. Gähler, R. Golub and K. Prechtl, Physica B 252 (1998), 34.

[25] E. Fermi, Sul moto dei neutroni nelle sostanze idrogenate (in Italian), Ricerca Scientifica 7 (1936), 13. (English transl.: E. Fermi, Collected Papers, Vol. I, Italy, 1921-1938, Univ. of Chicago Press, Chicago/London, 1962, pp. 980-1016.)

[26] J.H. Ferzinger and A. Leonard, Phys. Rev. 128 (1962), 2188.

[27] A. Frei, E. Gutsmiedl, C. Morkel, A.R. Müller, S. Paul, M. Urban, H. Schober, S. Rols, T. Unruh and M. Hölzel, Phys. Rev. B 80 (2009), 064301.

[28] H.R. Glyde, Excitations in Liquid and Solid Helium, Clarendon Press, Oxford, 1994.

[29] H.R. Glyde and E.C. Svensson, in: Methods of Experimental Physics, Part B, Vol. 23, D.L. Price and K. Sköld, eds, Academic Press, New York, 1987.

[30] M.L. Goldberger and F. Seitz, Phys. Rev. 71 (1947), 294.

[31] J.M.F. Gunn and M. Warner, Z Physik B: Condensed Matter 56 (1984), 13.

[32] E. Hecht, Optics, Addison Wesley, 2001.

[33] O. Hittmair, Lehrbuch der Quantentheorie, Thiemig-Verlag, München, 1972.

[34] J. Jensen and A.R. Mackintosh, Rare Earth Magnetism: Structures and Excitations, Clarendon Press, Oxford, 1991.

[35] Ch. Kittel, Physique de l'état solide, Dunod, 1998. (Solid State Physics, 8th edn, John Wiley and Sons, 2005.)

[36] M.M. Koza, H. Schober, S.F. Parker and J. Peters, Phys. Rev. B 77 (2008), 104306.

[37] M. Le Bellac, Physique Quantique, 2nd edn, EDP Science, 2007.

[38] K.-D. Liss, A. Magerl and W. Gläser, Nuclear Inst. Meth. A 335 (1993), 523.

[39] S.W. Lovesey, Theory of Neutron Scattering from Condensed Matter, Nuclear Scattering, Vol. 1, Polarization Effects and Magnetic Scattering, Vol. 2, Oxford Univ. Press, Oxford, 1984.

[40] A.A. Maradudin, E.W. Montroll, G.H. Weiss and I.P. Ipatova, Theory of Lattice Dynamics in the Harmonic Approximation, Academic Press, New York, 1971.

[41] A. Messiah, Méchanique Quantique, Dunod, Paris, 1959. (Quantum Mechanics, Dover Publications Inc., 2014.)

[42] V.S. Oskotskii, Sov. Phys. Solid State 9 (1967), 420.

[43] R.K. Osborn and S. Yip, Foundations of Neutron Transport Theory, Gordon Breach, New York, 1967.

[44] J.P. Pérez, Optique, Dunod, Paris, 2004.

[45] J.M. Perez-Mato, M. Aroyo, J. Hlinka, M. Quilichini and R. Currat, Phys. Rev. Lett. 81 (1998), 2462.

[46] G. Placzek, Phys. Rev. 86 (1952), 377.

[47] K. Protassov, Analyse statistique des données expérimentales, Collection Grenoble Science, Grenoble, 2002.

[48] A. Rahman, K.S. Singwi and A. Sjölander, Phys. Rev. 126 (1962), 986.

[49] H. Rauch and S. Werner, Neutron Interferometry, Oxford Univ. Press, Oxford, 2000.

[50] W. Reichardt, Bericht des Forschungszentrums Karlsruhe, 1984.

[51] K. Schmalzl, D. Strauch and H. Schober, Phys. Rev. B 68 (2003), 144301.

[52] H. Schober, Neutron instrumentation in neutron applications in Earth, in: Energy and Environmental Sciences, L. Liang, R. Rinaldi and H. Schober, eds, Springer, New York, 2008, pp. 37-104.

[53] H. Schober, Diffusion des neutrons par la matière cristalline ou amorphe non-magnetique, EDP Sciences, Collection SFN 10 (2010), 159-336.

[54] H. Schober, Europ. Phys. J. Web of Conferences 22 (2012), 00012.

[55] H. Schober, A. Tölle, B. Renker, R. Heid and F. Gompf, Phys. Rev. B 56 (1997), 5937-5950.

[56] P. Schofield, Phys. Rev. Lett 4 (1960), 239.

[57] V.F. Sears, Adv. Phys. 24 (1975), 1.

[58] V.F. Sears, Neutron Optics, Oxford Univ. Press, New York, 1989.

[59] C.G. Shull, Phys. Rev. Lett. 21 (1968), 1585.

[60] S.K. Sinha, E.B. Sirota, S. Garoff and H.B. Stanley, Phys. Rev. B 38 (1988), 2297.

[61] A. Sjölander, Arkiv för Fysik 14 (1958), 315.

[62] G.L. Squires, Introduction to the Theory of Thermal Neutron Scattering, Cambridge Univ. Press, Cambridge, 1978.

[63] M. Steiner, J. Low Temp. Phys. 135 (2004), 545.

[64] D. Strauch, Classical Mechanics, Springer, Berlin/Heidelberg, 2009.

[65] D. Strauch and B. Dorner, J. Phys. C: Solid State Phys. 19 (1986), 2853. 
[66] E.F. Talbot, H.R. Glyde, W.G. Stirling and E.C. Svensson, Phys. Rev. B 38 (1988), 11229.

[67] S.N. Taraskin and S.R. Elliott, Phys. Rev. B 55 (1997), 117-123.

[68] L. Van Hove, Phys. Rev. 95 (1954), 249.

[69] I. Waller, Zur Frage der Einwirkung der Wärmebewegung auf die Interferenz von Röntgenstrahlen, Z. Physik A: Hadrons and Nuclei 17 (1923), 398 (in German).

[70] M.J. Yeager, Neutron diffraction analysis of the structure of retinal photoreceptor membranes and rhodopsin, in: Neutron Scattering for the Analysis of Biological Structures, B.P. Schoenborn, ed., Brookhaven National Laboratory, Upton, New York, 1976.

[71] A. Zeilinger and T.J. Beatty, Phys. Rev. B 27 (1993), 7239. 